\title{
Copyright
}

by

Timothy Michael Weinzirl

2013 
The Dissertation Committee for Timothy Michael Weinzirl certifies that this is the approved version of the following dissertation:

\section{Probing Galaxy Evolution By Unveiling the Structure of Massive Galaxies Across Cosmic Time and in Diverse Environments}

Committee:

Shardha Jogee, Supervisor

Volker Bromm

John Kormendy

Milos Milosavljevic

Reynier Peletier

Bev Wills 
Probing Galaxy Evolution By Unveiling the Structure of Massive Galaxies Across Cosmic Time and in Diverse Environments

\author{
by
}

Timothy Michael Weinzirl, B.S., M.A.

\author{
DISSERTATION \\ Presented to the Faculty of the Graduate School of \\ The University of Texas at Austin \\ in Partial Fulfillment \\ of the Requirements \\ for the Degree of \\ DOCTOR OF PHILOSOPHY
}

THE UNIVERSITY OF TEXAS AT AUSTIN

August 2013 
In memory of Grandpa \& Grandma 


\section{Acknowledgments}

There are several people who need to be acknowledged for directly or indirectly contributing to this thesis. First and foremost, I thank my adviser Shardha Jogee for providing access to the Hubble Space Telescope data used in Chapters 3 and 4, for providing introductions to observational and theorist collaborators, and for providing critical commentary and suggestions on every chapter in this thesis. This work would not have been possible without the contributions from my theorist collaborators Sadegh Khochfar, Eyal Neistein, Andreas Burkert, Thorsten Naab, and Ludwig Oser. My Ph.D. Committee members John Kormendy, Bev Wills, Milos Milosavljevic, Reynier Peletier, and Volker Bromm provided additional valuable commentary and suggestions. Graduate coordinator Rachel Walker kindly overviewed the administrative details required to prepare and defend this thesis.

I acknowledge the support and encouragement from my parents, and extended family, especially my late maternal grandparents, regarding my decision to pursue a $\mathrm{Ph} . \mathrm{D}$.

I am grateful that during this thesis I developed not just as a scientist but also as an athlete. My transformation from a skinny-fat 143-pound "bro" into a 204-pound lifter induced a confidence and outlook that made the completion of this thesis much "lighter" than it otherwise would have been. To this end, I further thank the friendly farmers at the Austin Sustainable Food Center Farmers' Market for offering their outstanding products and first-class service every week, rain or shine. The staff at Gregory Gym on the UT campus should be acknowledged for (mostly) staying out of my hair despite my deliberate 
breakage of the rules via the heinous behaviors of deadlifting in stocking feet and chalking my hands where chalk is prohibited.

Finally, I thank my good friend Tom Montemayor for kindly informing me in July 2011 that all graduate students who give a talk at the McDonald Observatory Board of Visitors Meeting pass their Ph.D. exam. 


\section{Probing Galaxy Evolution By Unveiling the Structure of Massive Galaxies Across Cosmic Time and in Diverse Environments}

Publication No.

Timothy Michael Weinzirl, Ph.D.

The University of Texas at Austin, 2013

Supervisor: Shardha Jogee

How galaxies form and evolve is one of the primary outstanding problems in extragalactic astronomy. I conduct a quantitative census of the relative importance of the major structural components (flattened and dynamically cold disk-dominated components versus puffy and dynamically hot spheroidal or triaxial bulges/ellipticals) in massive galaxies over cosmic time and across different environments in order to explore how galaxies evolve under the action of the various assembly mechanisms (major mergers, minor mergers, gas accretion, and internal secular processes) in these different regimes. I perform three inter-related analyses focusing on massive galaxies from $z \sim 0-3$ in both field and rich cluster environments. Important strengths of this thesis include the use of high-resolution, panchromatic imaging from some of the largest and deepest galaxy surveys with the Hubble Space Telescope (HST), Spitzer, and Chandra space telescopes, and also the inclusion of detailed comparisons between the empirical data and hierarchical $\Lambda$ CDM-based models of galaxy evolution. The main results are summarized below.

1) In Chapter 2 (Weinzirl et al. 2009, ApJ, 696, 411), I consider the assembly history 
of high-mass $\left(M_{\star} \geq 10^{10} M_{\odot}\right)$ spirals in the field at $z \sim 0$. Sophisticated 2D structural decompositions, which take into account bulges, disks, and bars, reveal that only a minority (less than 26\%) of massive field spirals host spheroidal or triaxial bulges. Instead, most (74\%) massive field spirals host flattened disky/pseudo bulges with low Sérsic index $n \lesssim 2$ indicative of small-scale inner disks. Furthermore, in $66 \%$ of these spirals, the bulges have a low bulge-to-total stellar mass ratio $(B / T \leq 0.2)$. Massive field spirals overall contain $89 \%$ of their stellar mass in disk-dominated components (large-scale disks, bars, and inner disky/pseudo bulges) with $n \lesssim 2$, while $11 \%$ is in the form of non-disk $n \gtrsim 2$ components, which are likely built by the redistribution of stars during major mergers and possibly to some extent in minor mergers.

Based on detailed comparisons of the $B / T$ ratios predicted by two different theoretical hierarchical semi-analytic models (Khochfar \& Silk 2006; Hopkins et al. 2009b), it seems clear that the abundance of field spirals of low $B / T$ at $z \sim 0$ requires the vast majority of these spirals have not had a major merger since $z \sim 2$. This is consistent with the models, which show that most $(\sim 84-87 \%)$ of massive field spirals have had no major merger over the last 10 Gyr since $z=2$. This implies major mergers are less important than minor mergers and/or secular processes in assembling present-day massive spiral galaxies in the field.

2) In Chapter 3 (Weinzirl et al. 2013), I explore the structure of intermediate and high-mass $\left(M_{\star} \geq 10^{9} M_{\odot}\right)$ galaxies in the central $0.6 \mathrm{Mpc}$ of the Coma cluster at $z=0.02$, the richest nearby galaxy cluster, to constrain the assembly history of galaxies in this rich environment. This work incorporates high-resolution $(0$ '” $1,50 \mathrm{pc}), \mathrm{F} 814 \mathrm{~W}$ ( $I$ band) images from the HST ACS Treasury Survey of the Coma cluster (Carter et al. 2008). 
Structural decompositions are used to assess the frequency of disk-dominated structures, built by dissipative processes, versus spheroidal or triaxial bulge/elliptical components, built by violent stellar processes associated with mergers. After excluding the $2 \mathrm{cDS}$, I find that in the central 0.6 Mpc of the Coma cluster galaxies with stellar mass $M_{\star} \geq 10^{9} M_{\odot}$ have $56 \%$ of the cumulative stellar mass locked up in the high- $n(n \gtrsim 1.7)$ spheroidal or triaxial bulges/ellipticals of E, S0, spiral galaxies while the remaining $44 \%$ is in the form of low$n(n \lesssim 1.7)$ disk-dominated structures (outer disks, inner disks and disky/pseudo bulges, bars). Thus, most of the stellar mass in Coma galaxies appears to have been assembled and shaped through the redistribution of stars during violent major mergers, and possibly to a lesser degree during minor mergers. The significant fraction of stellar mass in diskdominated components underscores the important role by gas-rich dissipative processes that build outer and inner disks even in the rich environment of Coma.

I compare the observed properties of Coma cluster galaxies with predictions from a model of galaxy evolution that combines dark matter halo merger trees from the the Millennium Simulation and semi-analytic models. Among model clusters with total mass $5 \times 10^{14}-10^{16} M_{\odot}$, I find that there is no single cluster that can simultaneously match all the global properties (number density, mass function, halo properties) of the Coma cluster. The predicted model merger rates depend sensitively on whether baryonic or stellar mass is used in defining mergers. I find that the models strongly overpredict the ratio of cold gas mass to stellar mass (by a factor of over 25), and under-predict the mean fraction $f_{\star \text {,hot }}$ of stellar mass locked in hot components over a wide range of galaxy stellar masses. I speculate that the deficiency of hot stellar components in the model is due to ignoring classical bulge production via the merging of star-forming clumps. Adding this process would increase 
$f_{\star, \text { hot }}$ at all stellar masses. At the same time, the model may be overproducing $f_{\star, \text { cold }}$, the fraction of dynamically cold stellar mass in disk-dominated structures. The uncertainty here underscores the need to improve theoretical models by allowing for bulge assembly through modes other than galaxy mergers as well environmental processes, such as ram-pressure stripping and tidal stripping, which impact the cold gas content of galaxies.

3) In Chapter 4 (Weinzirl et al. 2011, ApJ, 743, 87), I study the rest-frame optical structure, star formation rates (SFR), and embedded active galactic nuclei (AGN) of one of the largest and most diverse samples of massive $\left(M_{\star} \geq 5 \times 10^{10} M_{\odot}\right)$ galaxies at $z=2-3$ (10-11 Gyr ago) from the GOODS-NICMOS Survey (Conselice et al. 2011). While most massive galaxies at $z \sim 0$ are morphologically E/S0 galaxies having extended (half-light radius $r_{\mathrm{e}} \sim 4-12 \mathrm{kpc}$ ) sizes and global Sérsic indexes $n \gtrsim 2$, the rest-frame optical structures of the massive galaxies at $z=2-3$ are strikingly offset toward smaller half-light radius $\left(r_{\mathrm{e}} \leq 10 \mathrm{kpc}\right)$ and more disk-dominated global morphologies. Specifically, $\sim 40 \%$ of massive galaxies at $z=2-3$ are "ultra-compact" with $r_{\mathrm{e}} \leq 2 \mathrm{kpc}$; additionally, $\sim 65 \%$ have Sérsic index $n \lesssim 2$ and likely host prominent outer disks. These massive galaxies have SFR that span a wide range (e.g., from $53-1466 M_{\odot} \mathrm{yr}^{-1}$ at $z=2-3$ ) and show a large degree of scatter in the $M_{\star}$-SFR relation of Daddi et al. (2007).

There are interesting correlations between galaxy structure and AGN activity in that most $(\sim 80 \%)$ AGN host galaxies are moderately extended $\left(r_{\mathrm{e}} \sim 2-10 \mathrm{kpc}\right)$, and $\sim 65 \%$ have globally disk-dominated structures (Sérsic index $n \lesssim 2$ ). There is a similar strong link between galaxy structure and inferred star formation rate: among the massive galaxies at $z=2-3$ without AGN and having $\mathrm{SFR} \geq 47 M_{\odot} \mathrm{yr}^{-1}$ (corresponding to the $5 \sigma 24 \mu \mathrm{m}$ flux limit of $30 \mu \mathrm{Jy})$, the majority $(\sim 85 \%)$ have disk-dominated morphologies. 
Moreover, the high SFR tail is populated primarily by systems with disky, $n \lesssim 2$ structures. In contrast, the massive ultra-compact objects at $z=2-3$ are less likely by a factor of $\sim 2$ to have significant $\mathrm{SFR} \geq 47 M_{\odot} \mathrm{yr}^{-1}$.

The rest-frame optical surface brightness and associated stellar mass densities $(\sim$ $10^{10} M_{\odot} \mathrm{pc}^{-2}$ ) in these massive galaxies at $z=2-3$ imply that during their formation phase at $z>2$, star formation proceeded at a pace that could only be fueled by rapid and highly dissipative gas-rich events, such as gas-rich mergers or cold mode accretion. Simulations (e.g., Hopkins et al. 2009a) suggest it is challenging even for gas-rich $\left(f_{\text {gas }}>40 \%\right)$ mergers to produce such a large population of remnants with low Sérsic index $(n \lesssim 2)$. Therefore, cold mode accretion at $z>2$ likely played an important role in forming these massive galaxies.

In order for massive galaxies at $z=2-3$ to evolve into present-day massive $\mathrm{E} / \mathrm{S} 0$ galaxies, they need to significantly increase $\left(n, r_{\mathrm{e}}\right)$. Sérsic index needs to grow to $n \gtrsim 2$ and $r_{\mathrm{e}}$ must increase on average by a factor of $3-4$. Dry major mergers, and especially, dry minor mergers must play an important role in this process. 


\section{Table of Contents}

Acknowledgments $\quad$ v

$\begin{array}{lll}\text { Abstract } & \text { vii }\end{array}$

List of Tables $\quad$ xvii

List of Figures $\quad$ xix

Chapter 1. Introduction 1

1.1 How Do Galaxies Form and Evolve? . . . . . . . . . . . . . . . . . . . . 1

1.2 Quantitative Analysis of Galaxy Structure . . . . . . . . . . . . . . . . . 4

1.3 Relating Galaxy Structure to Physical Processes . . . . . . . . . . . . . . 6

1.4 Thesis Goals and Overview . . . . . . . . . . . . . . . . 16

1.4.1 Probing the Structure and Assembly of Nearby Field Spirals [Chapter 2] 16

1.4.2 Exploring the Structure and Assembly of Galaxies At the Heart of the Coma Cluster $[$ Chapter 3$] \ldots \ldots \ldots$

1.4.3 Structure and Assembly of the Most Massive Galaxies Present a Few Gyr $(z \sim 2-3)$ After the Big Bang $[$ Chapter 4$] \ldots \ldots$

Chapter 2. Probing the Structure and Assembly of Nearby Field Spirals 22

2.1 Introduction . . . . . . . . . . . . . . . . . . 22

2.2 Sample Properties . . . . . . . . . . . . . . . . . . . . . . . . . . . . . . . . . . . . . . 28

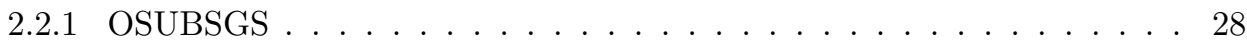

2.2 .2 Stellar Masses . . . . . . . . . . . . . . . . . . . . . . . . 30

2.3 Method and Analysis $\ldots \ldots \ldots \ldots \ldots$

2.3 .1 Image Preparation . . . . . . . . . . . . . . . . . . 33

2.3 .2 Decomposition Steps $\ldots \ldots \ldots 33$

2.3.3 Choosing the Best Fit Between Stage 2 and Stage $3 \ldots \ldots$. . . . . . 37

2.4 Extra Tests to Verify Correctness of Fits . . . . . . . . . . . . . . . 41

2.4.1 Varying $b / a$ as a Function of Radius . . . . . . . . . . . . . 41 
2.4 .2 Fitting Artificially Simulated Images _ . . . . . . . . . . . . . 42

2.4.3 Using $1 D$ Decomposition To Generate Guesses for Bulge Parameters 43

2.4.4 Parameter Coupling . . . . . . . . . . . . . . . . . . . . . 44

2.5 Results and Discussion . . . . . . . . . . . . . . . . . . 47

2.5.1 Impact of Bars in $2 D$ Decomposition $\ldots \ldots \ldots \ldots . \ldots 47$

2.5.2 Mass in Bulges, Disks, and Bars . . . . . . . . . . . . . . . . . . 49

2.5.3 Distribution of Bulge Index and $B / T \ldots \ldots \ldots \ldots \ldots$

2.5.4 Comparison With Other Work . . . . . . . . . . . . . . . . 53

2.5 .5 Bar Strength . . . . . . . . . . . . . . . . . . . . . 54

2.5.6 Bar Fraction as a Function of $B / T$ and Bulge Index . . . . . . . . 57

2.5 .7 Formation of Bulges . . . . . . . . . . . . . . . 58

2.5.8 Comparison of $B / T$ With Hierarchical Models of Galaxy Evolution . . 62

2.6 Summary . . . . . . . . . . . . . . . . . . . . . . . . 71

\section{Chapter 3. Exploring the Structure and Assembly of Galaxies At the Heart} of the Coma Cluster $\quad 115$

3.1 Introduction . . . . . . . . . . . . . . . . . . . . . . . . 115

3.1.1 Roadmap for the Reader . . . . . . . . . . . . . . . . . . . . . . . . 121

3.2 Data and Sample Selection . . . . . . . . . . . . . . . . . . . 122

3.2.1 Selection of Bright Cluster Members . . . . . . . . . . . . . . . . . 123

3.2 .2 Calculation of Stellar Masses . . . . . . . . . . . . . . . . . . 124

3.2.3 Selection of Final Sample of Massive Galaxies . . . . . . . . . . . . 127

3.3 Method and Analysis . . . . . . . . . . . . . . . . . . . . 127

3.3.1 Overview of Structural Decomposition Philosophy . . . . . . . . . . . 127

3.3.1.1 Uncertainties Due to Dwarf Galaxies . . . . . . . . . . . . . 132

3.3.2 Special Steps for Structural Decomposition in Cluster Environments . 133

3.3.3 Structural Decomposition Scheme and Galaxy Classification System 136

3.3.4 Details of Structural Decomposition _ . . . . . . . . . . . . . . 139

3.3.4.1 Single Sérsic Fits . . . . . . . . . . . . . . . . . 139

3.3.4.2 Multi-Component Fits ． . . . . . . . . . . . . . . 140

3.3.4.3 Nuclear Point Sources . . . . . . . . . . . . . . . . . . . . . . . 144

3.3.4.4 cD Galaxies . . . . . . . . . . . . . . . . . . 145

3.4 Empirical Results on Galaxy Structure . . . . . . . . . . . . . . . . 147 
3.4.1 Galaxy Types and Morphology-Density Relation in the Center of Coma147

3.4.2 Census of Low- $n$ Disk-Dominated Structures Versus High- $n$ Spheroidal or Triaxial Bulges/Ellipticals . . . . . . . . . . . . . . . . . 149

3.4.3 Stellar Mass Distributions Within S0, E, and Spiral Galaxy Structures 152

3.4.4 Scaling Relations for Outer Disks and Bulges . . . . . . . . . . . 155

3.4.5 Environmental Processes in Coma . . . . . . . . . . . . . . . . 158

3.5 Comparison of Empirical Results With Theoretical Predictions . . . . . . . . 164

3.5.1 Overview of the Models . . . . . . . . . . . . . . . . . . . 164

3.5.2 The Mass Function and Cumulative Number Density in Coma . . . . . 168

3.5.3 Global Properties of Model Clusters Versus Coma . . . . . . . . . . . . 170

3.5.4 Strong Dependence of Results on Mass Ratio Used to Define Mergers . 174

3.5.5 Cold Gas Mass in Coma Galaxies Versus Model Galaxies . . . . . . . . 175

3.5.6 Data Versus Model Predictions for Stellar Mass in Dynamically Hot and ColdComponents . . . . . . . . . . . . . 176

3.6 Summary \& Conclusions . . . . . . . . . . . . . . . . . . . . . . . . . 181

\section{Chapter 4. Structure and Assembly of the Most Massive Galaxies Present} a Few Gyr $(z \sim 2-3)$ After the Big Bang 217

4.1 Introduction . . . . . . . . . . . . . . . . . . . . . . 217

4.2 Data and Sample . . . . . . . . . . . . . . . . . . . . . . 221

4.2.1 Observations and Pointing Selections for GNS . . . . . . . . . . 221

4.2.2 Our Sample of Massive Galaxies at $\mathrm{z}=1-3 \ldots \ldots$. . . . . . . . . 222

4.2.3 Properties and Selection Biases in the Sample . . . . . . . . . . 226

4.3 Structural Properties of Massive Galaxies . . . . . . . . . . . . . . . . . 229

4.3 .1 Structural Decomposition . . . . . . . . . . . . . . . . . . . . 229

4.3.2 Derived Structural Properties at $z=2-3 \ldots \ldots$. . . . . . 232

4.3.3 Impact of Systematic Effects on Structural Properties . . . . . . . . 235

4.3.3.1 Artificial Redshifting . . . . . . . . . . . . . . . 237

4.4 Star Formation Activity . . . . . . . . . . . . . . . . . . . . . . 244

4.4.1 Matching GNS Galaxies to MIPS $24 \mu \mathrm{m}$ Counterparts . . . . . . . . . 244

4.4 .2 Star Formation Rates . . . . . . . . . . . . . . . . . . . . . . . 246

4.4.3 Relation Between Star Formation and Structure . . . . . . . . . . . 248

4.5 Constraints On Cold Gas Content . . . . . . . . . . . . . . . . . . . 250 
4.6 AGN in massive galaxies at $z=1-3 \ldots \ldots \ldots \ldots \ldots$

4.6 .1 Frequency of AGN . . . . . . . . . . . . . . . . . 253

4.6.2 Relation Between AGN Activity and Structure . . . . . . . . . . . . . 254

4.7 Discussion . . . . . . . . . . . . . . . . . . . . . . 255

4.7.1 Do Massive Galaxies With $n \leq 2$ at $z=2-3$ Host Disks? . . . . . . . 255

4.7.2 Formation of Massive Galaxies By $z=2-3 \ldots 260$

4.7.3 Transformation of Massive Galaxies at $z=2-3$ Into Present-Day E

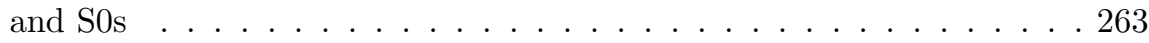

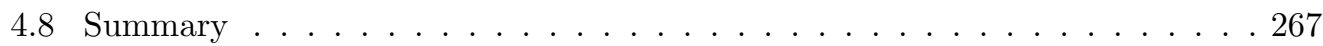

Chapter 5. Summary, Implications, and Future Work 295

5.1 Key Results . . . . . . . . . . . . . . . . . . . . 296

5.1.1 Probing the Structure and Assembly of Nearby Field Spirals . . . . . . 296

5.1.2 Exploring the Structure and Assembly of Galaxies At the Heart of the Coma Cluster . . . . . . . . . . . . . . . . . 298

5.1.3 Structure and Assembly of the Most Massive Galaxies Present a Few Gyr $(z \sim 2-3)$ After the Big Bang . . . . . . . . . . . . . . 301

5.2 What Has Been Learned About Galaxy Formation/Evolution? . . . . . . . . 303

5.3 Outlook and Future Work . . . . . . . . . . . . . . . . . . . . . 310

5.3.1 Dynamics and Gas Content of Massive Galaxies at $z=2-3 \ldots 310$

5.3.2 Chemical Evolution and Stellar Populations of Nearby Galaxies . . . . 311

5.3.3 Future Progress for Hierarchical Models . . . . . . . . . . . . . . . . . 312

$\begin{array}{ll}\text { Appendices } & 318\end{array}$

$\begin{array}{lll}\text { Appendix A. } & \text { Appendix to Chapter } 3 & 319\end{array}$

A.1 Using GALFIT . . . . . . . . . . . . . . . . . . . . . . . . . . . . . 319

A.2 Identifying Core Ellipticals . . . . . . . . . . . . . . . . . . . 321

A.3 Systematic Effects on $n_{\text {disk_max }} \ldots \ldots$. . . . . . . . . . . . . . 323

$\begin{array}{lll}\text { Appendix B. Appendix to Chapter } 4 & 326\end{array}$

B.1 PSF Modeling . . . . . . . . . . . . . . . . . . . . . 326

B.2 Tests on Robustness of Fits and Parameter Coupling . . . . . . . . . . . . . 327

B.3 Recovery of Parameters From Simulated Images _ . . . . . . . . . . . 330

B.4 Tests on MGC Fits . . . . . . . . . . . . . . . . . . . . 331 
Vita

366

xvi 


\section{List of Tables}

2.1 Sample $\mathrm{S} 1$ of Bright $\left(M_{B} \leq-19.3\right)$ Low-to-Moderately Inclined $\left(i \leq 70^{\circ}\right)$

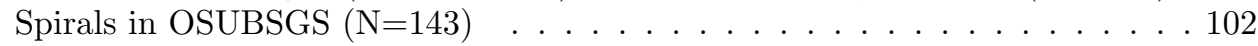

2.1 Sample $\mathrm{S} 1$ of Bright $\left(M_{B} \leq-19.3\right)$ Low-to-Moderately Inclined $\left(i \leq 70^{\circ}\right)$ Spirals in OSUBSGS $(\mathrm{N}=143) \quad \ldots \ldots \ldots$. . . . . . . . . . . . . . . . .

2.1 Sample S1 of Bright $\left(M_{B} \leq-19.3\right)$ Low-to-Moderately Inclined $\left(i \leq 70^{\circ}\right)$ Spirals in OSUBSGS $(\mathrm{N}=143) \quad \ldots \ldots \ldots \ldots$. . . . . . . . . 104

2.2 Parameters From 2D Luminosity Decomposition for Sample S1 (N=143) . . 105

2.2 Parameters From 2D Luminosity Decomposition for Sample S1 (N=143) . . 106

2.2 Parameters From 2D Luminosity Decomposition for Sample S1 (N=143) . . 107

2.2 Parameters From 2D Luminosity Decomposition for Sample S1 (N=143) . . 108

2.3 Decomposition For NGC 4643 . . . . . . . . . . . . . . . . . . . . . 109

2.4 Decomposition For NGC 4548 . . . . . . . . . . . . . . . . . . 109

2.5 Decomposition For NGC 4902 . . . . . . . . . . . . . . . . . 110

2.6 Checking GALFIT robustness with different input guesses for Stage 3 . . . 110

2.7 Parameter Coupling . . . . . . . . . . . . . . . . . . . . . . . . . . . 111

2.8 Mass Breakdown of Galactic Structures . . . . . . . . . . . . . . . . . . 112

$2.9 B / T$ and bulge $n$ in bright/high mass spirals . . . . . . . . . . . . . 112

2.10 Bar Fraction as a Function of $B / T$ and Bulge Index . . . . . . . . . . . 112

$2.11 B / T$ : Data versus Hierarchical Models of Galaxy Evolution . . . . . . . . . . 113

2.12 Comparison of model merger fraction since $z \leq 4$ and bulge Sérsic index . . . 114

3.1 Properties of Cored Ellipticals . . . . . . . . . . . . . . . . . . . 188

3.2 Single Sérsic Profile Structural Parameters ～. . . . . . . . . . . . . . . . 189

3.2 Single Sérsic Profile Structural Parameters ～. . . . . . . . . . . . . . . . 190

3.3 Distribution of Best-Fit Structural Decompositions for Stellar Mass $M_{\star} \geq 10^{9}$ $M_{\odot} \ldots \ldots \ldots \ldots \ldots 1$

3.4 Structural Parameters For the Best Model _ . . . . . . . . . . . . . . . 192

3.4 Structural Parameters For the Best Model . . . . . . . . . . . . . . . . . . . 193

3.5 Morphology-Density Relation . . . . . . . . . . . . . . . . . . . . . . . . . 194 
3.6 Mass Breakdown Among Galaxies With $M_{\star} \geq 10^{9} M_{\odot}$ in the Central 0.6 Mpc of Coma, Excluding the Two cDs . . . . . . . . . . . . . . . . . . . . . . 194

3.7 Mass Breakdown Among Galaxy Components Separated by Sérsic Index . . . 195

3.8 Distribution of Bulge Sérsic Index in S0s . . . . . . . . . . . . . . . . . . . 195

4.1 Rest-Frame Optical Sérsic Index $n$ and $r_{e}$ in Massive $\left(M_{\star} \geq 5 \times 10^{10} M_{\odot}\right)$ Galaxies . . . . . . . . . . . . . . . . . . . . . . . . 290

4.2 Fit of Massive Galaxies to $r_{e} / r_{e, z \sim 0}=\alpha(1+z)^{\beta}$ Over $z=0-3 \ldots 291$

4.3 Fraction of Massive $\left(M_{\star} \geq 5 \times 10^{10} M_{\odot}\right)$ Galaxies With $24 \mu \mathrm{m}$ Detections . . 292

4.4 Summary of AGN Detection and Properties . . . . . . . . . . . . . . . 293

4.5 Summary of Kolmogorov-Smirnov Test on Ellipticity . . . . . . . . . . . . . . 294 


\section{List of Figures}

1.1 This visual representation of the Hubble Sequence (Hubble 1926) highlights the difference between elliptical and disk galaxies. Barred and unbarred galaxies constitute parallel branches in the sequence. [Credit: NASA, STScI,

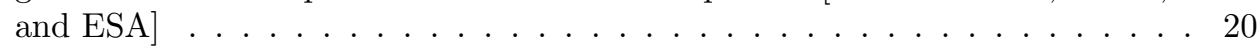

1.2 This figure shows the shape of the Sérsic profile. The Sérsic index $n$ controls the shape of the profile. At larger Sérsic index $n$, the profile center becomes steeper, and the outer wing becomes flatter. . . . . . . . . . . .

2.1 Our final sample S1 consists of 143 bright $\left(M_{B} \leq-19.3\right)$ low-to-moderately inclined $\left(i \leq 70^{\circ}\right)$ spirals in the OSUBSGS survey. The distribution of absolute $B$-band magnitude for the sample of bright spirals in the OSUBSGS survey is shown in the top panel before (solid line) and after (shaded greyscale) the cut to remove highly inclined $\left(i>70^{\circ}\right)$ spirals. The distribution of Hubble types for the sample is shown in the bottom panel before (solid line) and after (shaded greyscale) the cut to remove highly inclined $\left(i>70^{\circ}\right)$ spirals.

2.2 The luminosity function of the full OSUBSGS sample is compared with the $B$-band Schechter luminosity function (SLF). The former is calculated as described in $\S 2.2 .1$ using equation (2.1). The parameters for the SLF are $\Phi^{*}=5.488 \times 10^{-3} \mathrm{Mpc}^{-3}, \alpha=-1.07$, and $M_{B}^{*}=-20.5$ (Efstathiou, Ellis \& Peterson 1988), corresponding to $H_{0}=70 \mathrm{~km} / \mathrm{s} \mathrm{Mpc}^{-1}$. . . . . . . . . . .

2.3 Out of our final sample S1 of 143 bright $\left(M_{B} \leq-19.3\right)$ low-to-moderately inclined $\left(i \leq 70^{\circ}\right)$ OSUBSGS spirals, stellar masses could be estimated for 126 galaxies. Their stellar mass distribution is shown, as determined in $\S 2.2 .2$. Most have stellar masses $M_{\star} \geq 1.0 \times 10^{10} M_{\odot}$. This sample of 126 galaxies is referenced henceforth as the sample $\mathrm{S} 2 . \ldots \ldots \ldots$. . . . . . . .

2.4 An overview of the method of decomposition. All images are subjected to Stages 1, 2, and 3. Either the best fit of Stage 2 or Stage 3 is chosen as the best model. . . . . . . . . . . . . . . . . . .

2.5 Complete $2 D$ decomposition for NGC 4643. Note the prominent bar residuals in the residual for the Stage 1 and Stage 2 bulge-disk decomposition. This is a case where the prominent bar causes the Stage 2 bulge-disk fit to artificially extend the bulge and inflate the $B / T$. The disk fitted in Stage 2 has a low surface brightness and is very extended, well beyond the real disk: the $b / a$ and $P A$ of the fitted disk is shown as contours. Stage 3 bulge-disk-bar decomposition provides the best model. The $\chi^{2}$ for the Stage 1, Stage 2, and Stage 3 residual images are 7360.7, 7284.8, and 2111.59, respectively. See Table 2.3 for the fit parameters. . . . . . . . . . . . . . . 
2.6 The complete $2 D$ decomposition for NGC 4548 . This is an extreme example where the prominent bar results in an extended bulge and inflated $B / T$ in the Stage 2 bulge-disk fit. Like NGC 4643 in Figure 2.5, the disk fitted in Stage 2 has a low surface brightness and is very extended: its $b / a$ and $P A$ are shown as contours. Stage 3 bulge-disk-bar decomposition provides the best model. The $\chi_{\nu}^{2}$ for the Stage 1, Stage 2, and Stage 3 residual images are 7076.1, 6301.3, and 3260.4, respectively. See Table 2.4 for the fit parameters.

2.7 This plot shows the data image, Stage 2 model, and Stage 3 model for NGC 4902. The Stage 2 bulge is too bright and is extended along the major axis of the bar $(B / T=31.2 \%$ and $b / a=0.45)$. In Stage 3 , the bulge and bar are fit with distinct components $(B / T=5.59 \%$, bulge $b / a=0.68$, Bar $/ T=9.97 \%$, bar $b / a=0.22)$. All other fit parameters appear in Table 2.5. . . . . . . . . 81

2.8 The data images and Stage 3 bulge-disk-bar decomposition models of NGC 5427 and NGC 7412 are shown. The Stage 3 models each distinctly show a false bar component, which is not present in the data images. The false components can be inspired by prominent spiral arms, such as those present in these galaxies. Such cases are flagged during the visual inspection of fits and the Stage 3 bulge-disk-bar decomposition is discarded in favor of the Stage 2 bulge-disk decomposition. . . . . . . . . . . . . . . . 8

2.9 This plot compares the $B / T$ obtained by fitting the bulge of NGC 4548 with a Sérsic model of constant $b / a$ as opposed to a Sérsic model varying $b / a$. To mimic a Sérsic model with varying $b / a$ in GALFIT, the bulge was fitted with ten concentric Sérsic profiles with fixed $r_{\mathrm{e}}$, each separated by 0.75 ". The top two panels show the run of $b / a$ and $P A$ of the ten concentric Sérsic profiles. The bottom panel shows the cumulative $B / T$ calculated by summing all models with $r \leq r_{\mathrm{e}}$. The bulge $b / a(0.88), P A(-66.5)$, and $B / T(13 \%)$ from the original Sérsic fit of constant $b / a$ (Table 2.4) are indicated with horizontal lines on the 3 panels. . . . . . . . . . . . . . . 83

2.10 An elementary test is to determine if GALFIT can recover the known parameters of artificial noisy images. Noisy images were simulated by taking parametric model images (left panels) produced by GALFIT, and adding noise and sky background (right panels). The noisy images were then fitted to see if the original known parameters can be recovered. See $\S 2.4 .2$ for

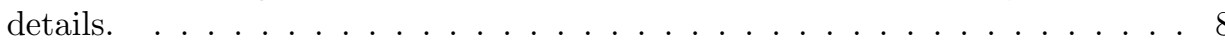

2.11 The plots compare recovered versus original model parameters for the simulated images discussd in $\S 2.4 .2$. The vertical axis limits demonstrate the range explored for each parameter. The dotted line shows $y=x$ for comparison. Except for some extreme cases where the images were highly distorted by noise, all parameters were recovered to within a few percent. . . . . . . .

2.12 The ratio of model-to-recovered parameter is plotted against mean surface brightness inside the disk scalelength, $\mu=\operatorname{mag}+2.5 \times \log _{10}(2 \times \pi \times b / a \times$ $h^{2}$ ), for the simulated images discussed in $\S 2.4$.2. Surface brightness is not photometrically calibrated and is shown for a zeropoint of $0 . \ldots$. . . . 86 
2.13 The top, middle, and bottom panels show stellar mass for bulges, disks, and bars, respectively, along the Hubble sequence. Values are shown for sample $\mathrm{S} 2$ of 126 galaxies in Fig. 2.3. The legend in each panel indicates the type of decomposition used for each data point. . . . . . . . . . . . .

2.14 The individual and mean $B / T$ (left panels) and bulge Sérsic index (right panels) are plotted as a function of Hubble type for the sample S1 of bright galaxies, and as a function of galaxy stellar mass for sample S2. The error bars indicate the standard deviation of the population around the mean in each bin. The legend in each panel indicates the type of decomposition used for each data point. The mean $B / T$ and bulge index in barred galaxies differ systematically from unbarred galaxies, but there is a large overlap in the individual values. As many as $\sim 69 \%$ of bright spiral galaxies have $B / T \leq 0.2$; these bulges are pervasive and exist across the Hubble sequence. Furthermore, as many as $\sim 76 \%$ of bright spirals have low $n \leq 2$ bulges. Such bulges exist in barred and unbarred galaxies across a wide range of Hubble types. . . . . . . . . . . . . . . . . 88

2.15 The relation between $B / T$ and bulge index is shown. In the top panel, galaxies are coded according to bar class. The legend indicates the type of decomposition used for each data point. In the lower panel, galaxies are coded according to Hubble type. A striking $\sim 76 \%$ of bright spirals have low $n \leq 2$ bulges. Such bulges exist in barred and unbarred galaxies across a wide range of Hubble types, and their $B / T$ range from 0.01 to 0.4 , with most having $B / T \leq 0.2$. A moderate fraction $(\sim 22 \%)$ have intermediate $2<n<4$ bulges. These exist in barred and unbarred $\mathrm{S} 0 /$ a to Sd galaxies, and their $B / T$ spans a wide range $(0.05$ to 0.5$)$. Only $(\sim 1 \%)$ have $n \geq 4$. . . . . .

$2.16 \mathrm{~B} / T$ is plotted against $\mathrm{Bar} / T$ and sorted by bulge Sérsic index. There are six galaxies with $\mathrm{Bar} / T \geq 0.3 . \ldots \ldots \ldots \ldots$

2.17 The top two rows show $H$-band images of barred galaxies, which have early RC3 Hubble types, but yet have $B / T \leq 0.2$. The bottom row shows $H$ band images of unbarred galaxies, which have late RC3 Hubble types, but yet have $B / T \sim 0.4$. The Hubble types assigned to these objects more reflect disk smoothness and spiral arm topology than $B / T$. All images are from OSUBSGS with characteristics as described in $\S 2.2 . \quad \ldots \ldots \ldots$. . . . . . 91

$2.18 B / D$ is plotted against Hubble type. The legend indicates the type of decomposition used for each data point. The mean values for barred and unbarred together in each bin are shown. . . . . . . . . . . . . . . . .

2.19 The properties of bars are shown. The error bars indicate the standard deviation of the population around the mean in each bin. The legend in each panel indicates the type of decomposition used for each data point. Upper left: Mean and individual Bar/T plotted against Hubble type. Upper right: Mean and individual bar Sérsic indices plotted against Hubble type. Lower left: Bar $/ T$ plotted against total galaxy stellar mass. The mean Bar/ $T$ in bins of stellar mass is indicated. Lower right: Bar Sérsic index plotted against total galaxy stellar mass. . . . . . . . . . . . . . . . . . 
2.20 Bar strength is plotted against Hubble type and the properties of bars are compared with bulges. The legend in each panel indicates the type of decomposition used for each data point. Upper left: Bar strength, the product of $\mathrm{Bar} / T$ and peak bar ellipticity $e_{b a r}$ from MJ07 is plotted against Hubble type. Upper right: Bar/T is plotted against bar Sérsic index. Lower left: Bar $/ T$ is plotted against bulge Sérsic index. Lower right: $\mathrm{Bar} / T$ is plotted against $B / T$. In the first plot, mean bar strong is calculated for each Hubble type. In the latter three plots, mean Bar/ $T$ is calculated for bins along the ordinate axis. The error bars indicate the standard deviation of the population around the mean in each bin. . . . . . . . . . . . . . . . . . .

2.21 $\mathrm{H}$-band OSUBSGS images of spirals with prominent bars of Bar/T $(>0.3)$ are shown. An interesting example is the oval or lens galaxy NGC 1317: the bar has a low ellipticity, but its $B / T$ is large because it is extended and massive. Such bars/lenses may exert significant gravitational torques although they are not very elongated. . . . . . . . . . . . . . .

2.22 Shown is the distribution of Sérsic indices $n$ for remnants of 1:1 gas-rich major mergers in the simulations of Hopkins et al. 2009a: they lie in the range of $2<n<6$. Specifically, $\sim 22 \%$ of the remnants have classical $n \geq 4$, as much as $20 \%$ have low $n \leq 2.5$, while $50 \%$ have $n \leq 3$. Almost none have $n \leq 2$. [Figure: courtesy of Phil Hopkins] . . . . . . . . . . . . . . . 96

2.23 For those high mass $\left(M_{\star} \geq 1.0 \times 10^{10} M_{\odot}\right)$ galaxies in the theoretical models that experienced a major merger (see $\S 2.5 .8$ ), the $B / T$ of the remnant at $z \sim 0$ is plotted against the redshift $z_{\text {last }}$ of the last major merger. Systems where the last major merger occurred at earlier times have had more time to grow a disk and thus have a lower $B / T$ at $z \sim 0$. The dispersion in the present-day $B / T$ at a given $z_{\text {last }}$ is due to the different times spent by a galaxy in terms of being a satellite versus a central galaxy in a DM halo, since the cooling of gas and the growth of a disk is stopped when a galaxy becomes a satellite. In the model, a high mass galaxy that has undergone a major merger at $z \leq 2$ has a present-day $B / T>0.2$. In effect, a high mass spiral can have a present-day $B / T \leq 0.2$ only if its last major merger occurred at $z>2$ (lookback times $>10 \mathrm{Gyr}$ ). 
2.24 The cumulative fraction $F$ of high mass $\left(M_{\star} \geq 1.0 \times 10^{10} M_{\odot}\right)$ spirals with present-day $B / T \leq$ the $\mathrm{x}$-axis value is shown for the data (colored lines/ points) and for the theoretical model (black lines/points) described in $\S 2.5 .8$. Model and data spirals are defined as systems with $B / T \leq 0.75$. The magenta line shows $F$ from the data, while the other two colored lines break this $F$ in terms of bar class (top panel) or bulge $n$ (lower panel). The black dashed line shows $F$ from all model galaxies, while the black dotted line and black dots show the contribution of model galaxies that experienced, respectively, no major merger and one or more major mergers since $z \leq 4$. Major mergers are defined here as those with $M_{1} / M_{2} \geq 1 / 4$. In the model, the fraction $(\sim 1.6 \%$; see Table 2.11$)$ of high mass spirals, which have undergone a major merger since $z \leq 4$ and host a bulge with a present-day $B / T \leq 0.2$ is a factor of over 30 smaller than the observed fraction $(\sim 66 \%)$ of high mass spirals with present-day $B / T \leq 0.2$. Thus, bulges built via major mergers since $z \leq 4$ seriously fail to account for most of the low $B / T \leq 0.2$ bulges present in two-thirds of high-mass spirals. . . . . . . . . . . . . .

2.25 This figure is similar to Fig. 2.24, except that the model now defines major mergers as those with mass ratio $M_{1} / M_{2} \geq 1 / 6 \ldots \ldots \ldots$. . . . . . . . .

2.26 This figure is similar to Fig. 2.24, except that here spirals are considered to be systems with a $B / T \leq 0.55$ rather than 0.75 in the models, and a corresponding cut is applied to the data points. . . . . . . . . . 100

2.27 This figure is similar to Fig. 2.24, except that $B / T$ of all the observed galaxies has been multiplied by a factor of two, in order to test what would happen in the case where the $M / L$ ratio of the bulge in $H$-band is twice as high as that of the disk and bar. This could happen in an extreme example where the dominant bulge stellar population was much older (e.g. $12 \mathrm{Gyr}$ ) than the age of the dominant disk stellar population (e.g., 3 Gyr). In such a case, the fraction of high mass spirals with $B / T \leq 0.2$ would change from $\sim 66 \%$ in Fig. 2.24 to $\sim 50 \%$. However, the main conclusion that bulges built by major mergers since $z \leq 4$ cannot account for most of the low $B / T \leq 0.2$ bulges, present in a large percentage $(\sim 55 \%)$ of spirals still holds. $\ldots \ldots \ldots 1$

3.1 This figure shows in panels (a) to (d) the luminosity, stellar mass, and $g-r$ color, respectively, for the 447 galaxies in sample S1 having F814W $\leq 24$, locations within the central $0.6 \mathrm{Mpc}$ of the Coma cluster, and cluster membership rating 0-3. See Section 3.2.1 for details. In panel (b), the two most massive sources are $\mathrm{cD}$ galaxies, and the arrows indicate their adopted stellar masses are lower limits (Section 3.2.2). The solid line in panel (d) is the color-luminosity break between the red and blue sequence of galaxies from Blanton et al. (2005), which we convert from luminosity to stellar mass using the relations of Bell et al. (2003). . . . . . . . . . . . . . . . . 196 
3.2 Visually determined Hubble types from the catalog of N. Trentham et al. (in preparation), with updates from Marinova et al. (2012), are plotted against stellar mass. As discussed in Section 3.2.3, we apply a mass cut (dashed line) to focus on galaxies with stellar mass $M_{\star} \geq 10^{9} M_{\odot}$. This effectively removes from the working sample most dwarf/irregular and very low surface brightness (VLSB) galaxies. . . . . . . . . . . . . . . . . . . 197

3.3 Overview of the method of decomposition. All galaxies are subjected to Stage 1 , and most are further processed in Stage 2. A galaxy best fit with a single Sérsic profile plus point source (if needed) is interpreted as a photometric elliptical or photometric disk. A galaxy with extra coherent structure that cannot be described with a single Sérsic profile is subjected to a multiplecomponent fit in Stage 2 and, if needed, Stage 3. Figure 3.4 describes Stage 2 and Stage 3 in more detail. . . . . . . . . . . . . . . . . . . . . 198

3.4 Multi-component decompositions are broken into two phases, Stage 2 and Stage 3. A galaxy without an extended outer disk is interpreted as a photometric E, while a galaxy with such a disk is labeled either an S0 or spiral. When evidence for a large scale bar is found in a galaxy with an outer disk, Stage 3 is used to model the bar component. . . . . . . . . . . . . . . . . 199

3.5 Overview of galaxy classification system. Galaxies are deemed to be best represented by a either a single or multi-component Sérsic profile (plus point source, if needed). Galaxies fitted with a single Sérsic profile are further interpreted as a pure disk (if Sérsic index $n \leq n_{\text {disk_max }}$ ) or photometric elliptical (if Sérsic index $n>n_{\text {disk_max }}$ ). When a multi-component Sérsic profile is required, the galaxy is either an unbarred/barred S0 or spiral, or a photometric $\mathrm{E}$ with inner and outer components. S0s and spirals must have an outer component $\mathrm{C} 2$ with Sérsic index $n \leq n_{\text {disk_max }}$. The inner component $\mathrm{C} 1$ can have any $n$. If the outer component $\mathrm{C} 2$ has Sérsic index $n>n_{\text {disk_max }}$, the galaxy is a photometric elliptical with inner component $\mathrm{C} 1$ of any $n$. The value of $n_{\text {disk_max }}$ is set to 1.66 based on several considerations. We determine $n_{\text {disk_max }}$ to be the maximum Sérsic index of the outer disk in spiral and S0 galaxies showing clear signs of an outer disk, such as bars, spiral arms, rings, or high inclination. . . . . . . . . . . . . . . . 200

3.6 This figure compares our results for the single Sérsic fits (with no points source) with those obtained by Hoyos et al. (2011) using GALFIT on the same data. The sources in our sample requiring one Sérsic component are labeled separately from sources requiring two or three Sérsic profiles. Our derived magnitudes, $r_{\mathrm{e}}$, and $n$ for the sources requiring one Sérsic profile agree well with the parameters from Hoyos et al. (2011), with the one exception being cD galaxy NGC 4874 (COMAi125935.698p275733.36) with $n \sim 3$. Note that cD galaxy NGC 4889 requires only one Sérsic profile but it is not included here as it is not in the Hoyos et al. (2011) sample. See Section 3.3.4.1 for additional details. 
3.7 This figure shows examples $\mathrm{C} 1$ to $\mathrm{C} 6$ where a single Sérsic model (+ point source if needed) does not provide a good fit to coherent galaxy structure that is best modeled with one or more additional Sérsic profiles. Such residual structure includes central compact structures (C2, C3, C4, C5, C6), rings (C3, C4), annuli and extended components (C1, C4), and bars/ovals (C5, C6). These systems are better fitted by models with multiple Sérsic components (see Figures 3.8 and 3.9). Columns 1 and 3 show the input $I$-band images. Columns 2 and 4 show the residuals after subtracting the

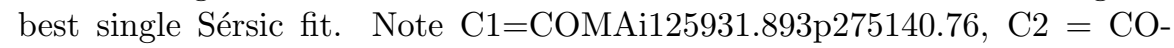
MAi125935.286p275149.13, C3 = COMAi13021.673p275354.81, C4 = COMAi13014.746p28228.69, C5 = COMAi125833.134p272151.73, and C6 = COMAi125930.824p275303.05. . . . . . . . . . . . . . . 202

3.8 This figure shows how some of the galaxies (C2=COMAi125935.286p275149.13 and C4=COMAi13014.746p28228.69) poorly fitted by a single Sérsic model (plus point source if needed) in Stage 1 can be better fitted by two Sérsic models (plus point source if needed) in Stage 2. Each row shows the data, residual after Stage 1, and the residual after Stage 2. Galaxy C2 is best-fit as having an inner disk $(n=0.31)$ and an outer elliptical structure $(n=2.08)$. Galaxy C4 is best fit with an inner bulge $n=3.68$ and an outer disk $(n=0.47) . \quad$. . 203

3.9 This figure shows two examples of barred galaxies (C5=COMAi125833.134p272151.73

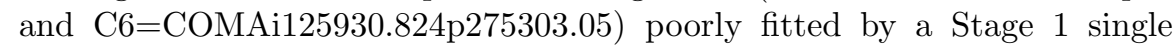
Sérsic model that are better fitted by a Stage 3 triple Sérsic (plus point source if needed) model. Column 1 shows the data images while columns 2 and 3 show the residuals after the Stage 1 and Stage 3 model, respectively. . 204

3.10 This figure shows the decomposition of moderately inclined, barred galaxy COMAi125950.105p275529.44, in which we measure the highest outer disk Sérsic index $n=1.66$. Thus, this galaxy sets the empirically determined upper limit on disk Sérsic index, $n_{\text {disk_max }}=1.66$. Column 1 shows the data images while columns 2 and 3 show the residuals for the Stage 2 and Stage 3 model, respectively. The bar signature is clearly present in the residuals. . . 205

3.11 The distribution of Sérsic index for outer disks in S0/spiral galaxies. The vertical line represents the empirically determined upper limit, $n_{\text {disk_max }}$, in the Sérsic index of outer disks. The dash-dotted and dashed lines show the distributions for barred and unbarred outer disks, respectively. See Section 3.3.4.2 for details. . . . . . . . . . . . . . . . . . . 206

3.12 This figure compares residuals after fitting a single Sérsic model (top row) versus the best fit double Sérsic + nuclear point source model (bottom row) for an elliptical (COMAi13030.954p28630.22), S0 (COMAi13021.673p275354.81), and spiral (COMAi13041.193p28242.34). The nuclear point source is visible in the residuals in the top row. . . . . . . . . . . . . . . . . 207

3.13 This panel shows the relation between total galaxy luminosity and point source luminosity for objects having a nuclear point source in the final, best structural decomposition. . . . . . . . . . . . . . . 208 
3.14 Panels (a) and (b) compare the properties of large-scale disks ( $r_{\mathrm{e}}$, luminosity) with galaxy $M_{\star}$. Massive $5 \times 10^{9} \leq M_{\star} \leq 6.2 \times 10^{10} M_{\odot} \mathrm{S} 0 /$ spiral galaxies from the central 0.6 Mpc of Coma as well as low-density environments (LDEs) are considered. The LDE sample is from Gadotti (2009) and includes galaxies in SDSS Data Release 2 that are face-on $(b / a \geq 0.9)$ and have redshift $0.02 \leq$ $z \leq 0.07$. In panel (b), the $i$-band luminosity represents the ACS F $814 \mathrm{~W}$ photometry for Coma galaxies and the SDSS $i$-band photometry for the LDE galaxies. Panel (c) compares $i$-band mass-to-light ratio $(M / L)_{i}$ with galaxy $M_{\star}$. For Coma, the galaxy wide mass-to-light ratio is plotted, while for LDEs the outer disk $(M / L)_{i}$ is shown. In all panels, the mean values in galaxy $M_{\star}$ for Coma and LDEs are slightly offset for readability. The error bars on the mean values represent the $1 \sigma$ standard deviation. This figure demonstrates that at a given galaxy stellar mass, the average disk half-light radius $r_{\mathrm{e}}$ in the $i$-band is smaller in the central 0.6 Mpc of Coma compared with LDEs. . 209

3.15 This figure is similar to Figure 3.14, except that it emphasizes S0/spiral galaxy bulges. See Section 3.4.4 and Figure 3.14 for extra details on the sample from Gadotti (2009). Panels (a), (b), (c), and (d) show bulge size $\left(r_{\mathrm{e}}\right)$, bulge $i$-band luminosity, bulge Sérsic index, and bulge-to-disk light ratio $(B / D)$, respectively, versus galaxy $M_{\star}$. In all panels, the error bars on the mean values represent the $1 \sigma$ standard deviation, and in all panels the mean values in galaxy $M_{\star}$ for Coma and LDEs are slightly offset for readability. This figure demonstrates that at a given galaxy stellar mass, there is no systematic offset between bulges in Coma and LDEs. . . . . . . . . . . . . 210

3.16 This figure shows a comparison of bulges in massive $\left(5 \times 10^{9} \leq M_{\star} \leq\right.$ $\left.6.2 \times 10^{10} M_{\odot}\right) \mathrm{S} 0 /$ spiral galaxies in low-density environments (LDEs) from Gadotti (2009) versus galaxies in the central 0.6 Mpc of Coma. See Section 3.4.4 and Figure 3.14 for extra details on the sample from Gadotti (2009). Bulges in Coma are divided into groups of low Sérsic index $\left(n \leq n_{\text {disk_max }}\right)$ and high Sérsic index $\left(n>n_{\text {disk_max }}\right)$. Panel (a) shows bulge-to-disk light ratio $(B / D)$ versus bulge Sérsic index. Panels (b) and (c) show bulge and disk $i$-band luminosity, respectively, versus bulge Sérsic index. In each panel, the solid line represents the fit to Coma bulges of all Sérsic $n$, and the dashed line is the corresponding fit to bulges of all Sérsic $n$ from LDEs. The offset in $B / D$ in panel (a) appears to be driven by the offset in disk luminosity in panel $(\mathrm{c}) . \ldots \ldots \ldots 211$ 
3.17 This figure shows how the global parameters of the Coma cluster compare with all 160 clusters in the Millennium simulation having a halo mass in the range $5 \times 10^{14}-10^{16} M_{\odot}$. The solid lines and black data points represent the simulated clusters. In panel (a), the virial mass and virial radius adopted for Coma are $2.8 h_{73}^{-1} \mathrm{Mpc}$ and $1.3 \times 10^{15} h_{73}^{-1} M_{\odot}$ (Section 3.5.2). The open circle is the model cluster having the best match to the projected galaxy number density of Coma. In panels (b) and (c), the cumulative projected galaxy number density and the galaxy mass stellar function of Coma at projected radius $R_{\mathrm{p}} \leq R_{\mathrm{vir}}$ are based on data from the NYU Value-Added Galaxy Catalog (NYU-VAGC, Blanton et al. 2005). In panel (b), the dotted line represents the cumulative galaxy number density of the model cluster best matching the Coma halo parameters. In panel (c), for the Coma galaxy stellar mass function, we measure a slope $\alpha=-1.16$ and characteristic mass $M^{*}=1.17 \times 10^{11} M_{\odot}$. The last mass bin in the global mass function for Coma contains the two $\mathrm{cD}$ galaxies, and the arrow on this bin indicates the adopted stellar masses for the cDs are lower limits. The simulations are based on a model that produces a reasonable match to the galaxy stellar mass function of Li \& White (2009) averaged over all environments at $0.001<z<0.5$ (dashed line). However, they cannot produce a model cluster that simultaneously matches multiple global properties (halo properties, galaxy number density, and galaxy stellar mass function), of Coma, our local benchmark for one of the richest nearby galaxy clusters. . . . . . . . . . . . . . . . . . 212

3.18 This figure shows the three sets of simulated model clusters (30 model clusters in total) chosen to best match, separately, the cumulative galaxy number density (Column 1), the galaxy stellar mass function (Column 2), and halo parameters (halo mass and virial radius, Column 3). The solid lines and solid circles in each panel represent the different simulated clusters. Rows 1, 2, and 3 show how the different simulated clusters compare with the global properties (halo mass and radius, cumulative galaxy number density, and galaxy stellar mass function) determined with data from the NYU Value-Added Galaxy Catalog (NYU-VAGC, Blanton et al. 2005) for Coma in Section 3.5.2. No model cluster simultaneously matches all three global properties. . . . . . . . 213

3.19 This figure highlights the effect that the definition of the merger mass ratio $M_{1} / M_{2}$ has on certain galaxy properties (merger history and $B / T$ ), for a representative model cluster (see Section 3.5.4). Note we require a major merger to have $M_{1} / M_{2} \geq 1 / 4$. The left column is the manifestation of the model cluster when $M_{1} / M_{2}$ refers to the stellar mass ratio, and in the right column the merger mass ratio represents cold gas plus stars. The two mass ratio definitions lead to vastly different merger histories and significantly affect the resulting distribution of $B / T$. . . . . . . . . . . . . . . . . . 214 
3.20 The top panel shows the ratio of cold gas to stellar mass $\left(M_{\text {cold_gas }} / M_{\star}\right)$ for the best cluster model matched by cumulative galaxy number density (see Figure 3.18, column 1). The error bars represent the $1 \sigma$ standard deviation around the mean value. The bottom panel shows the ratio of observed cold gas $\left(H I+H_{2}\right)$ to stellar mass $\left(M_{H I+H_{2}} / M_{\star}\right)$ for galaxies studied by Boselli et al. (1997) that are part of or near the Coma cluster. The dashed line is the median ratio $(0.04)$ for Coma cluster galaxies, and the solid line is the median ratio $(0.09)$ for the non-cluster galaxies. At $10^{10} \lesssim M_{\star} \lesssim 10^{11} M_{\odot}$, the model predicts a cold gas to stellar mass ratio that is a factor $\sim 25-87$ times higher than the median value observed in Coma cluster galaxies. . . . 215

3.21 Top: The mean ratio of stellar mass fraction in dynamically hot components $\left(f_{\star, h o t}=M_{\star, h o t} / M_{\star}\right)$ is plotted as a function of total galaxy $M_{\star}$. In the data, $M_{\star, h o t}$ is taken as the stellar mass of any high $n>n_{\text {disk_max }}$ classical bulge/elliptical component in the galaxy, excluding the $\mathrm{cD}$ galaxies (see Section 3.2.2). The model shown here is the best cluster model matched by cumulative galaxy number density (see Figure 3.18, column 1). For this model, the solid line takes $M_{\star, h o t}$ as the stellar mass built during major mergers in Equation 3.7, namely (MAJOR_ACC+MAJORSF), while the dashed line considers $M_{\star, h o t}$ as (MAJOR_ACC+MAJORSF+MINOR_ACC), where the last term represents the stellar mass accreted during minor mergers. Bottom: The mean stellar mass fraction in dynamically cold flattened components $\left(f_{\star, \text { cold }}=M_{\star, \text { cold }} / M_{\star}\right)$ is plotted as a function of total galaxy stellar mass $M_{\star}$. In the data, $M_{\star, \text { cold }}$ is taken as the stellar mass of any low $n \leq n_{\text {disk_max }}$ disk-dominated component in the galaxy. The model is represented by the solid and dashed lines. With the solid line we take $M_{\star, \text { cold }}$ to be the mass of the outer disk $M_{\star, \text { outer_disk }}$, which is given by $\left(M_{\star}-M_{\star, \text { Bulge,model }}\right)$. For the dotted line, we consider $M_{\star, \text { cold }}$ to be the mass $M_{\star, \text { all_disk }}$ of inner and outer disks. We compute the latter mass as the sum $\left(M_{\star, \text { outer_disk }}+M I N O R S F\right)$, where MINORSF was defined in Equation 3.7 as the mass of stars formed via induced SF during minor mergers. In both panels, only the central 0.6 Mpc of the clusters is considered. The error bars represent the $1 \sigma$ standard deviation on the mean. The mean values for Coma are slightly offset in $M_{\star}$ for readability. The main conclusion is that the best-matching simulated clusters are underpredicting the mean fraction of $f_{\star, \text { hot }}$ and overpredicting $f_{\star, \text { cold }}$ over a wide range in galaxy stellar mass. . . . . . . . . . . . . . . . 216

4.1 The distribution of apparent $H$ (F160W), $V$ apparent magnitude, stellar mass, and redshift for the final, complete sample of 166 galaxies with $M_{\star} \geq$

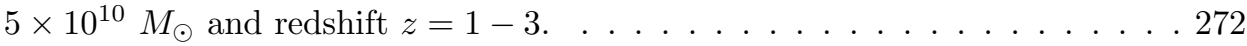


4.2 We compare the galaxy stellar mass functions for GNS over $z=1-3$ with those from other studies that are based on $K$ or IRAC-selected samples (Kajisawa et al. 2009; Marchesini et al. 2009; Pérez-González et al. 2008; Fontana et al. 2006). The vertical line in each plot marks the mass cut $\left(M_{\star} \geq 5 \times 10^{10} M_{\odot}\right)$ for the GNS-based sample used in this paper. We include the data points with error bars from the other studies, where available, along with each Schechter function fit. Some studies (Kajisawa et al. 2009; Marchesini et al. 2009) present results for multiple sets of SED-modeling assumptions, and in these cases we show the results for the assumptions that most closely match those used for GNS by Conselice et al. (2011). For Kajisawa et al. (2009), we show the mass function calculated with Bruzual \& Charlot (2003) stellar templates. For Marchesini et al. (2009), we show the stellar mass functions calculated with Bruzual \& Charlot 2003 templates, metallicities of $0.2,1$, and $2.5 Z_{\odot}$, a Kroupa IMF, and a Calzetti extinction law, but in the above plot, we scale their mass functions by +0.2 dex along the $x$-axis to convert their Kroupa IMF to a Salpeter IMF. For the GNS mass functions, in comparison, the best metallicity is determined on a galaxy-bygalaxy basis from a set of discrete values spanning 0.005 to $2.5 Z_{\odot}$. The error bars for Marchesini et al. (2009) take into account the uncertainties due to cosmic variance, Poisson error, photometric redshifts, and stellar SED templates. The error bars from Pérez-González et al. (2008) account for Poisson error and uncertainty in photometric redshifts. In comparison, the error bars on the GNS mass functions show only Poisson error. . . . . . . . . . . . 273

4.3 For all galaxies detected in the GOODS-NICMOS Survey (GNS) over $z=$ $1-3$, the rest-frame $U-V$ color is plotted against $M_{\star}$ for different redshift bins. Blue systems are preferentially at low masses, while the most massive $\left(M_{\star} \geq 1 \times 10^{11} M_{\odot}\right)$ galaxies are preferentially red. The vertical line denotes $M_{\star}=5 \times 10^{10} M_{\odot}$, the mass cut we adopt for our final sample of 166 galaxies. 274

4.4 NICMOS F160W images for representative GNS galaxies with $M_{\star} \geq 5 \times 10^{10}$ $M_{\odot}$ at $z=2-3$. The Sérsic index $n$ and effective radius $r_{e}$ referenced here are based on fitting single Sérsic components to the NICMOS images, as described in $§ 4.3$. The top panel shows example systems with Sérsic index $n>2$ and half-light radii $r_{e} \leq 4 \mathrm{kpc}$. The middle panel shows examples with $n \leq 2$ and $r_{e} \leq 8 \mathrm{kpc}$. The majority ( $\sim 82 \%$; Table 4.1$)$ of the massive GNS galaxies have $r_{e} \leq 4 \mathrm{kpc}$. In such systems, structural features are generally hard to discern due to resolution effects, so that systems appear fairly featureless (top 4 rows). In the small fraction of massive galaxies at $z=2-3$ with large $r_{e}>4 \mathrm{kpc}$, one can discern some structural features such as an elongated bar-like feature or a combination of a central condensation surrounded by a more extended lower surface brightness component, reminiscent of a bulge and disk (row 5). The bottom panel (row 6) contains systems that appear morphologically disturbed. . . . . . . . . . . . . . . . . 275 
4.5 The $B$-band Sérsic index $n$ and effective radius $r_{e}$ derived from single Sérsic profile fits to massive $\left(M_{\star} \geq 5 \times 10^{10} M_{\odot}\right)$ galaxies are plotted for the three redshift bins listed in Table 4.1. In the top row, the black points represent fits to $z \sim 0$ galaxies by Allen et al. (2006) on $B$-band images of galaxies from the Millennium Galaxy Catalog (Liske et al. 2003). The lower two rows are based on our fits to the NIC3 F160W images of massive GNS galaxies at $z=1-2$ and $z=2-3$. Note that the massive galaxies at $z=2-3$ are strikingly offset toward lower $\left(n, r_{e}\right)$ compared with the massive $z \sim 0$ galaxies, and have five times more low $n \leq 2$ disky systems (see also Figure 4.6). The black dashed line represents the typical half-width half maximum of the NICMOS3 PSF at $z=1-3$ of $\sim 1.2 \mathrm{kpc} \ldots \ldots \ldots \ldots \ldots \ldots \ldots$

4.6 Left column: The distributions of rest-frame optical Sérsic index and effective radius $r_{e}$ based on single Sérsic profile fits to massive $\left(M_{\star} \geq 5 \times 10^{10} M_{\odot}\right)$ galaxies are plotted for the three redshift bins listed in Table 4.1: at $z \sim 0$ (solid line), based on the fits of Allen et al. (2006) on $B$-band images of galaxies from the Millennium Galaxy Catalog (Liske et al. 2003), and at $z=1-2$ (dash-dotted line) and $z=2-3$ (dashed line), based on our fits to the NIC3 F160W images of massive GNS galaxies. Note that a significant fraction $(39.0 \pm 5.56 \%)$ of massive $\left(M_{\star} \geq 5 \times 10^{10} M_{\odot}\right)$ galaxies at $z=2-3$ have $r_{e} \leq 2 \mathrm{kpc}$, compared with only $0.52 \pm 0.37 \%$ at $z \sim 0$. Note also that most $(64.9 \pm 5.4 \%)$ of massive galaxies at $z=2-3$ have low $n \leq 2$ (disky) structures compared with only $13.0 \pm 1.7 \%$ at $z \sim 0$. Right column: Same as left column but for the mass range $M_{\star} \geq 1 \times 10^{11} M_{\odot} . \ldots \ldots 277$

4.7 Left column: The top panel shows mean extinction-corrected rest-frame $B$ band surface brightness within the effective radius $\left.\left(<\mu_{\mathrm{e}}\right\rangle\right)$ for massive $\left(M_{\star} \geq 5 \times 10^{10} M_{\odot}\right)$ galaxies for the three redshift bins listed in Table 4.1. The solid line is for $z \sim 0$ MGC galaxies. The dash-dotted line $(z=1-2)$ and the dashed line $(z=2-3)$ are based on our fits to the NIC3 F160W images of massive GNS galaxies. The GNS galaxies at $z=2-3$ have a mean surface brightness of $16.8 \mathrm{mag} / \operatorname{arcsec}^{2}$ and are systematically brighter than the $z \sim 0$ MGC galaxies, which have a mean surface brightness of 21.3 $\mathrm{mag} / \operatorname{arcsec}^{2}$. In the bottom panel, surface brightness within the effective radius is plotted against effective radius $r_{e}$ for the same redshift bins. Right column: The same plots are repeated for galaxies with $M_{\star} \geq 1 \times 10^{11} M_{\odot}$. Surface brightness is calculated with the extinction-corrected rest-frame $B$ band light and is defined as $\left\langle\mu_{\mathrm{e}}\right\rangle=B_{\text {corr }}+2.5 \log _{10}\left(2 \pi \mathrm{r}_{\mathrm{e}}^{2}\right)-10 \log _{10}(1+\mathrm{z})$, where $B_{\text {corr }}$ is the extinction-corrected, rest-frame $B$ apparent magnitude. $\quad .278$ 
4.8 Top row: The black points show the massive $\left(M_{\star} \geq 5 \times 10^{10} M_{\odot}\right) z \sim 0$ galaxies from MGC described earlier in Figure 4.5. The magenta points denote the SDSS-based sample S1 of 255 representative massive $\left(M_{\star} \geq 5 \times\right.$ $\left.10^{10} M_{\odot}\right) z \sim 0$ galaxies used in the redshifting experiment. Note the $(n$, $r_{e}$ ) distribution of S1 covers the same parameter space as that of the MGC sample. This is also shown quantitatively in Figure 4.9. Row 2: We show as blue squares the $\left(n, r_{e}\right)$ distribution obtained after redshifting $\mathrm{S} 1$ to $z=2.5$ and 're-observing' it with NIC3/F160W as in the GNS survey. We assume a surface brightness evolution of 2.5 magnitudes and brighten each redshifted galaxy by this amount. The actual observed $\left(n, r_{e}\right)$ distributions of the massive galaxies at $z=2-3$ in the GNS survey are significantly offset toward lower values compared with the redshifted galaxies. The black dashed line represents the typical half-width half max of the NICMOS3 PSF at $z=1-3$ of $\sim 1.2 \mathrm{kpc} . \ldots \ldots \ldots \ldots \ldots$

4.9 This figure illustrates the same information as in Figure 4.8 but in more quantitative terms. It shows the $n$ and $r_{e}$ distributions for the full MGC sample of massive $z \sim 0$ galaxies (black line) and the representative sample $\mathrm{S} 1$ of 255 galaxies used in the redshifting experiment (magenta line). Sample S1 does a good job of matching the full MGC sample and is typically within $\pm 10 \%$ for a given bin. We also contrast the $\left(n, r_{e}\right)$ values after redshfiting S1 to $z=2.5$ (blue line) with the actual distribution observed in the massive the GNS galaxies at $z=2-3$ (red line). While $64.9 \pm 5.4 \%$ and $39.0 \pm 5.6 \%$ of the massive $z=2-3$ galaxies have $n \leq 2$ and $r_{e} \leq 2 \mathrm{kpc}$, respectively, the corresponding fractions for the redshifted sample are $10.6 \pm 1.9 \%$ and $1.2 \pm 0.7 \%$. The results shown here are for galaxies with $M_{\star} \geq 5 \times 10^{10} M_{\odot}$, but a similar result is obtained for $M_{\star} \geq 1 \times 10^{11} M_{\odot}$. . . . . . . . . 280 
4.10 Left column: The panels compare the rest-frame optical structural parameters (Sérsic index $n$ and effective radius $\left.r_{e}\right)$ of massive $\left(M_{\star} \geq 5 \times 10^{10} M_{\odot}\right)$ elliptical and S0 galaxies at $z \sim 0$ with the structural parameters recovered after these galaxies were artificially redshifted to $z=2.5$, brightened by 2.5 magnitudes in surface brightness, and re-observed with NIC3/F160W. At $z \sim 0$, the structural parameters were measured from $g$-band images, while at $z=2.5$ they are measured from the artificially redshifted images in the NIC3/F160W band, so that all parameters are measured in the rest-frame blue optical light. The black lines represent equality, while the shaded area represents the regime of $n \leq 2$ and $r_{e} \leq 2 \mathrm{kpc}$, where $64.9 \pm 5.4 \%$ and $39.0 \pm 5.6 \%$, respectively, of massive GNS galaxies at $z=2-3$ lie (Table 4.1 and Figure 4.8). The plots show that the Sérsic index $n$ and effective radius $r_{e}$ of the massive $z \sim 0 E$ and SOs may be lower or higher after redshifting out to $z=2.5$, but they do not, in general, drop to values as low as $n \leq 2$ and $r_{e} \leq 2 \mathrm{kpc}$, and avoid the shaded area. Right column: Same as left column, but this time for massive $\left(M_{\star} \geq 5 \times 10^{10} M_{\odot}\right) z \sim 0$ spiral galaxies. The galaxies are coded by bulge-to-total light ratio $(B / T) . B / T$ was measured with bulge-disk and bulge-disk-bar decomposition of the $z \sim 0 \mathrm{~g}$-band images. The top plot shows that it is mainly massive $z \sim 0$ late-type spirals of low $B / T$ that yield Sérsic index $n$ as low as $n \leq 2$ after redshifting, and populate the shaded area where $64.9 \pm 5.4 \%$ of massive GNS galaxies at $z=2-3$ lie. However, as shown by this lower plot, the local massive spirals have much larger $r_{e}\left(r_{e} \gg 2 \mathrm{kpc}\right)$ and after artificial redshifting avoid the shaded area where $39.0 \pm 5.6 \%$ of the massive GNS galaxies at $z=2-3$ lie. . . . . . 281

4.11 Top left: The $f_{24 \mu m}$ distribution for the massive $\left(M_{\star} \geq 5 \times 10^{10} M_{\odot}\right)$ GNS galaxies with reliable MIPS $24 \mu \mathrm{m}$ counterpart. Upper right: The inferred $L_{I R}$ distribution over 8-1000 $\mu \mathrm{m}$. Lower left: The inferred $\mathrm{SFR}_{\mathrm{IR}}$ distribution based on $L_{I R}$, which is estimated using the Chary \& Elbaz (2001) templates, with a correction at $\mathrm{L}_{I R}>6 \times 10^{11} L_{\odot}$. Lower right: $\mathrm{SFR}_{\mathrm{IR}}$ versus $M_{\star}$. For sources containing an AGN, the measured $L_{I R}$ and $\mathrm{SFR}_{\mathrm{IR}}$ are upper limits. The upper right and bottom panels use different coding for sources identified in $\S 4.6$ as hosting an AGN. . . . . . . . . . . . . . . . . . . . . . 282

4.12 The left-hand panel shows $\mathrm{SFR}_{\mathrm{IR}}$ versus $M_{\star}$ at $z=2-3$. The AGN candidates are coded as triangle symbols, and their $\mathrm{SFR}_{\mathrm{IR}}$ likely overestimate their true SFR. The completeness limit in $\mathrm{SFR}_{\mathrm{IR}}$ (corresponding to the limiting $24 \mu \mathrm{m}$ flux of $\sim 30 \mu \mathrm{Jy}$ ) is shown as a black solid line. The black dots represent SFR from UV measurements by Daddi et al. (2007) for $z \sim 2$; the diagonal green line is their corresponding SFR-mass correlation at $z \sim 2$. The right-hand panel shows mean $\mathrm{SFR}_{\mathrm{IR}}$ in the different redshift bins for sources with $\mathrm{SFR}_{\mathrm{IR}}$ above the detection limit. The error bars are the $1 \sigma$ standard deviation around the mean. The black line shows average UV-based SFR versus redshift for a galaxy with $5 \times 10^{10} M_{\odot}$, as calculated by Drory \& Alvarez (2008). . . . . . . . . . . . . . . . . . . . . 283 
4.13 Left column: $\mathrm{SFR}_{\mathrm{IR}}$ is plotted versus $M_{\star}$, for all galaxies with $M_{\star} \geq 5 \times 10^{10}$ $M_{\odot}$, in different redshift bins spanning 1-1.4 Gyr in cosmic time. Data are sorted according to the Sérsic index $n$ calculated in $\S 4.3 .1$. AGN candidates (see §4.6) are labeled with red x's. Galaxies with $\mathrm{SFR}_{\mathrm{IR}}$ below the detection limit (shown as a horizontal line) are shown with downward pointing arrows because they are upper limits. At $z=2-3$ the majority $(84.6 \pm 10.0 \%)$ of massive non-AGN galaxies with $S F R_{\mathrm{IR}}$ above the detection limit have $n \leq 2$ (disky) structures. Right column: For non-AGN sources, histograms show the fraction of massive galaxies in each redshift bin with a given $\mathrm{SFR}_{\mathrm{IR}}$ for separate ranges of $n$. The vertical black lines mark the $\mathrm{SFR}_{\mathrm{IR}}$ detection limit. For sources to the left of the line, we plot upper limits for $\mathrm{SFR}_{\mathrm{IR}}$. The high SFR tail in each redshift bin is populated primarily by systems with low $n \leq 2$ (disky) structures. . . . . . . . . . . . . . . . . . . . . . . . . 284

4.14 Same as Figure 4.13, but now the data are sorted by half-light radius $r_{e}$. Note that only a small fraction of the ultra-compact $\left(r_{e} \leq 2 \mathrm{kpc}\right)$ galaxies have $\mathrm{SFR}_{\mathrm{IR}}$ above the $5 \sigma$ detection limit. Some ultra-compact galaxies have high $\mathrm{SFR}_{\mathrm{IR}}$, but, on average, their mean $\mathrm{SFR}_{\mathrm{IR}}$ are lower than in more extended systems. . . . . . . . . . . . . . . . . . . . . 285

4.15 Left column: For galaxies with $\mathrm{SFR}_{\mathrm{IR}}$ above the $5 \sigma$ detection limit, the distributions of cold gas surface density $\left(\Sigma_{\text {gas }}\right)$, cold gas mass $M_{\text {gas }}$, and cold gas fraction $\left(f_{\text {gas }}\left(r_{c}\right)\right)$ within the circularized optical half-light radius $r_{c}$ are shown for different redshift ranges. $\Sigma_{\text {gas }}$ is calculated using a SchmidtKennicutt law (Kennicutt 1998) with power-law index 1.4 a normalization factor of $2.5 \times 10^{-4}$. The cold gas fraction $\left(f_{\text {gas }}\left(r_{c}\right) \equiv M_{\text {gas }} /\left(M_{\text {gas }}+M_{\star}\right)\right)$ is calculated relative to the total baryonic mass within $r_{c}$. Right column: Same as left column except that only non-AGN sources are shown. . . . . . . . . . 286

4.16 Top: For galaxies with $\mathrm{SFR}_{\mathrm{IR}}$ above the $5 \sigma$ detection limit, the mean cold gas fraction $\left(f_{\text {gas }}\left(r_{c}\right) \equiv M_{\text {gas }} /\left(M_{\text {gas }}+M_{\star}\right)\right)$ within the circularized optical halflight radius $r_{c}$ is shown in three redshift bins for all galaxies with $5 \times 10^{10}$ $M_{\odot} \leq M_{\star}<10^{11} M_{\odot}$ and $M_{\star} \geq 10^{11} M_{\odot}$. The error bars indicate the $1 \sigma$ scatter in gas fraction and redshift. Bottom: Same as the top except that only non-AGN sources are shown. . . . . . . . . . . . . . . . . 28

4.17 The upper and lower-left panels show single Sérsic index $n$ versus effective radius $r_{e}$ for the $49 \mathrm{AGN}$ candidates selected either based on X-ray properties, mid-IR power-law, or IR-to-optical excess. The lower-right panel shows the median Sérsic index and $r_{e}$ in each redshift bin. . . . . . . . . . . . 288

4.18 In the top panels, the deconvolved ellipticity $(1-b / a)$ measured by GALFIT is shown for massive $\left(M_{\star} \geq 5 \times 10^{10} M_{\odot}\right)$ GNS galaxies at $z=2-3$ with $n \leq 2$ and $n>2$. The bottom panels show the deconvolved ellipticity for similarly massive E/S0 and Spiral galaxies as measured with GIM2D by Allen et al. (2006). . . . . . . . . . . . . . . . . . . . . . . 289 
5.1 This figure shows the morphology breakdown of massive $\left(M_{\star} \geq 5 \times 10^{10} M_{\odot}\right)$ galaxies at $z \sim 0$ from the Millennium Galaxy Catalog (Liske et al. 2003; Driver et al. 2005). . . . . . . . . . . . . . . . . . . . . . . . . 314

5.2 This figure shows how the surface mass densities within the central $3 \mathrm{kpc}$ of massive galaxies at $z=1-3$ (Chapter 4) compare with similarly massive galaxies at $z \sim 0$. The surface mass densities at $z=1-3$ are calculated assuming a conservative mass-to-light ratio $(M / L)$ of 1 . For the $z \sim 0$ galaxies, the $B$-band $M / L$ ratio from Driver et al. (2006) is applied. The median $B$-band $M / L$ ratio is $\sim 5$. Surface densities are determined from PSF-deconvolved model images of the galaxies. . . . . . . . . . . . . . . . 315

5.3 This figure, adapted from Figure 1 of Oser et al. (2012), shows the projected stellar half-mass radius versus stellar mass for galaxies at $z=1-4$ from the simulations of Oser et al. (2012). The galaxies at $z=0$ have stellar mass $M_{\star}>6.3 \times 10^{10} M_{\odot}$ (denoted by the vertical dashed line) and are taken from Nipoti et al. (2009); for these galaxies, the diagonal lines indicates the observed size-mass relation and the associated $1 \sigma$ scatter. Quiescent galaxies have solid symbols, other galaxies have open symbols. The elliptical annulus highlights the parameter space in size/stellar mass that this simulation is

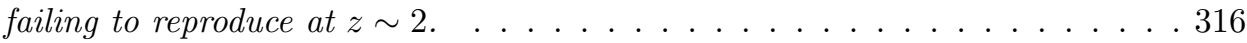

5.4 This figure demonstrates that nearly all the massive $z \sim 2$ galaxies made by Ceverino \& Dekel (in prep.), who use the model of Ceverino, Dekel, \& Bournaud (2010), have global non-disk structures with Sérsic index $n \gtrsim 2$.

B.1 For four representative galaxies with $n \sim 1-4$, the first and second columns show the difference $\chi_{\min }^{2}-\chi_{\min , 0}^{2}$ versus $n$ and $r_{e}$, respectively. $\chi_{\min , 0}^{2}$ is the minimum $\chi^{2}$ obtained when all parameters are freely fit, and $\chi_{\min }^{2}$ is the minimum $\chi^{2}$ when $n$ is held at discrete values $(0.5-10)$. The $r_{e}$ in the second column are the best-fit results for a given $n$ and $\chi_{\min }^{2}$. The red stars mark the best-fit $n_{\min , 0}$ and $r_{e, \min , 0}$ corresponding to $\chi_{\min , 0}^{2}$. The insets in rows 3 and 4 of column 1 show a magnified view around the minimum in $\chi_{\min }^{2}-\chi_{\min , 0}^{2}$. Note that for galaxies with $n_{\min , 0}<2$ (rows 1 and 2 ), $\chi_{\min }^{2}-\chi_{\min , 0}^{2}$ rises sharply at higher $n>n_{\min , 0}$, thereby making it unlikely that a higher $n>2$ would provide a similarly good fit. . . . . . . . . . . . . . 334

B.2 The quantity $\chi_{\min }^{2}-\chi_{\min , 0}^{2}$ from Figure B.1 is shown for all massive GNS galaxies well fitted with a single Sérsic profile. The top panel evaluates $\chi_{\min }^{2}-$ $\chi_{\min , 0}^{2}$ at $n_{\min , 0}-1$, and the bottom panel evaluates $\chi_{\min }^{2}-\chi_{\min , 0}^{2}$ at $n_{\min , 0}+1$, where $n_{\min , 0}$ is the best-fit Sérsic index corresponding to $\chi_{\min , 0}^{2} \ldots \ldots 335$

B.3 For the simulations described in $\S$ B.3, the difference between input and output Sérsic index $n$ and effective radius $r_{e}$ are plotted against effective surface brightness $\mu_{e}$, the surface brightness at $r_{e}$. The vertical lines correspond to the range in $\mu_{e}$ in the NIC3/F160W band for the massive galaxies at $z=1-3$

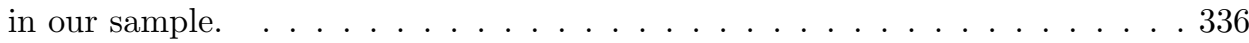


B.4 Top row: We demonstrate for a subset of $z \sim 0$ galaxies in the MGC catalog that the GIM2D-based $\left(n, r_{e}\right)$ values from Allen et al. (2006) are not biased to higher values compared with our GALFIT-based fits for the same galaxies. All fits are performed on the $B$-band images from MGC. Bottom row: We show the effects of adding a point source in the GALFIT models fitted to the $z \sim 0$ MGC galaxies. The values obtained using a model made of a Sérsic component plus a point source are plotted along the y-axis, while the $\mathrm{x}$-axis shows the values obtained with a single Sérsic component. The values of $r_{e}$ are not changed systematically. The Sérsic index is lowered by the addition of the point source, but only $20 \%$ of sources with $n>2$ in the single Serrsic fit have $n \leq 2$ after including the point source. . . . . . . . . . . 337 


\section{Chapter 1}

\section{Introduction}

\subsection{How Do Galaxies Form and Evolve?}

The formation of galaxies has been a classic problem in astrophysics ever since it was demonstrated in the 1920's (e.g., Hubble 1926) that "spiral nebulae" are actually external "island universes", and that the Milky Way is just one of many such galaxies. Contemporary galaxy formation models combine the well-established $\Lambda$ Cold Dark Matter $(\Lambda \mathrm{CDM})$ cosmology (Blumenthal et al. 1984), which describes behavior of dark matter on very large scales, with baryonic physics to model galaxy formation. The initial conditions in the early universe were such that pockets of dark matter decoupled from the Hubble flow before collapsing into virialized halos. Meanwhile, gas cooled and aggregated in the interiors of the dark matter halos to form rotating disks, which are the building blocks of galaxies (Steinmetz \& Navarro 2002; Cole et al. 2000).

Galaxies grow and evolve through a variety of processes. Hierarchical mass assembly occurs via stars/gas delivered from the combination of mergers with other galaxies and also

gas accretion. At the same time, internal secular processes (Kormendy 1993a; Kormendy \& Kennicutt 2004; Jogee, Scoville, \& Kenney 2005) redistribute material, particularly gas, within a galaxy. This occurs when a stellar bar or globally oval structure in a non-interacting galaxy drives the gas inflow into the inner kpc via shocks and gravitational torques.

The precise role of a merger depends on both the mass ratio of the two progenitor 
galaxies and the fraction of gas. A merger is considered "major" when the two progenitors have a high merger mass ratio (e.g., $M_{1} / M_{2} \geq 1 / 4$ ). The merger mass ratio $M_{1} / M_{2}$ can refer to the ratio of stellar mass, the ratio of cold gas plus stellar mass, or to the ratio of dark matter halo masses. The common practice in observational studies to date has been to use a stellar mass ratio as this is most readily measured for a large number of galaxies. Theoretical studies tend to favor the use of halo mass or baryonic mass baryonic mass (cold gas plus stars). There is a need for a consistent definition between observational and theoretical work because the predictions of models are sensitive to the adopted merger ratio definition. We discuss this issue more in Chapter 3. Also note the advent of ALMA and future large-area spectroscopic studies like HETDEX will enable observational studies to get a better handle on baryonic and halo masses.

Observational measures of major merger rates have high uncertainties, but in general it seems clear that major mergers were more frequent at earlier epochs (Conselice et al. 2003; Conselice 2009; Bluck et al. 2012). Minor mergers with lower mass ratios (e.g., $\left.1 / 10 \leq M_{1} / M_{2}<1 / 4\right)$ are intrinsically less destructive, more frequent (Jogee et al. 2009; Lotz et al. 2011), and have longer merger timescales.

In the traditional view of gas accretion, all the smooth gas accreted by a dark matter halo is shock heated to the virial temperature $T_{v i r}\left(\sim 10^{6}-10^{7} \mathrm{~K}\right)$ of the halo as it enters the virial radius (Rees \& Ostriker 1977; White \& Rees 1978). New theoretical work (e.g., Birnboim \& Dekel 2003; Dekel \& Birnboim 2006; Ocvirk et al. 2008 Dekel et al. 2009a, b; Kereš et al. 2005, 2009; Brooks et al. 2009) show that a significant fraction of baryonic matter accreted by dark matter halos remains unshocked and at temperatures $\left(\sim 10^{4}-10^{5} \mathrm{~K}\right)$ much less than $T_{v i r}$. In particular, there exists cold-mode accretion, 
whereby dense cosmological filaments penetrate the virial radius and deliver cold unshocked gas to the central galaxy. For high-mass $\left(M_{\text {halo }}>10^{12} M_{\odot}\right)$ galaxies, halo gas accreting quasi-spherically is shock heated to form a hot halo, and therefore cold-mode accretion is particularly important as a source of cold unshocked gas to help fuel star formation. In highmass galaxies capable of supporting a virial shock, cold-mode accretion is only available for a limited time $(z \gtrsim 2)$. This is because, as cosmic time passes, the cross section of the cold stream relative to the halo rises. When the density of the cold stream is comparable to the density of the halo (at around $z \sim 2$ ), the cold filaments cannot enter the halo without being shocked (e.g., Dekel \& Birnboim 2006). For lower mass galaxies that are incapable of supporting a virial shock, gas accretion from both the halo and dense filaments is dominated by cold unshocked gas at all epochs. While cold-mode accretion arises naturally in hydrodynamical simulations, there is only limited observational evidence that this process is actually occurs (e.g., Rauch et al. 2011; Conselice et al. 2013).

Galaxy environment (e.g., halo mass, local galaxy number density) also plays an important role in galaxy evolution. There is naturally a continuum in galaxy environment, but the two extremes are the low-density field, where galaxy number density is $0.1-1$ galaxies $\mathrm{Mpc}^{-3}$, and the cores of rich clusters where the number density is 1,000-10,000 galaxies $\mathrm{Mpc}^{-3}$. The morphology-density relation (Dressler 1980) is the well-known dependence of galaxy morphology on environment. In clusters, the frequency of elliptical and S0 galaxies exceeds that of spiral galaxies, (e.g., 65\%:35\% in Virgo, McDonald et al. 2009). The reverse is true in the field where the ratio is more like 20\%:80\% (Dressler 1980). The dependence of galaxy morphology on environment is due to a combination of initial conditions (e.g., accelerated formation timescale and different merger rates) in the cluster and cluster-specific 
processes, such as ram-pressure stripping (Gunn \& Gott 1972; Fujita \& Nagashima 1999), galaxy harassment (Barnes \& Hernquist 1991; Moore et al. 1996, 1998, 1999; Hashimoto et al. 1998; Gnedin 2003), and strangulation (Larson et al. 1980), that can transform one galaxy morphology into another by altering star formation and galaxy structure.

The relative importance of galaxy assembly mechanisms (major mergers, minor mergers, gas accretion, and secular processes) plays a profound role on the observable properties of a galaxy, such as structure, star formation history, and the presence or absence of active galactic nuclei (AGN). Although we do not have a firm grasp on the relative importance of these assembly processes, we can indirectly learn about them by studying the observable properties of galaxies. Galaxy structure, in particular, encodes important information about the assembly history of galaxies. By looking back in time at different epochs, it is possible to learn when the main baryonic components of galaxies (bulges, disks, and bars) form and also by which assembly mechanisms. This thesis focuses on the quantitative analysis of galaxy structure in different epochs and different environments.

\subsection{Quantitative Analysis of Galaxy Structure}

It has become clear in the last two decades that the stars in galaxies are arranged in two very distinct kinds of structures assembled via very different formation pathways, namely

disks (flat and rotation-dominated components) and bulges/ellipticals (spheroidal/triaxial dispersion-dominated systems). Disks are notably more complicated because they can feature embedded spiral arms, bars, ovals/lenses, and rings. These two fundamental components underlie the backbone of the Hubble Sequence (Hubble 1926, Figure 1.1), which describes the morphologies of present-day galaxies. 
The structural properties of galaxy components, such as bulges, disks, and bars can be derived through the decomposition of the $1 D$ or $2 D$ light distribution while taking into account the point spread function (PSF). Structural decomposition in $1 D$ has been in use for decades (e.g., Kormendy 1977) and has since been a standard tool in the study of galaxy structure (e.g., Burstein 1979a, b; Boroson 1981; Kent 1985; Baggett et al. 1998). 2D fitting techniques work directly on the original $2 D$ image while taking into account the point spread function (PSF). The residuals in the $2 D$ approach assist in quantifying non-axisymmetric structures like bars and spiral arms, as well as asymmetries like shells, arcs, and clumps. Some authors conclude that the $2 D$ techniques are more accurate at quantifying galaxy structure (e.g., Byun \& Freeman 1995).

Early studies of galaxy structure analyzed S0 and spiral galaxies in terms of a bulge and disk component (i.e., bulge-disk decomposition, Burstein 1979a, b; Boroson 1981; Kent 1985; Baggett et al. 1998; Allen et al. 2006; Byun \& Freeman 1995; de Jong 1996; Simard 1998; Wadadekar et al. 1999). More recent structural analyses, however, have been including the bar in $1 D$ (Jogee et al. 2004; Marinova \& Jogee 2007; Kormendy \& Bender 2012) and $2 D$ techniques (Laurikainen et al. 2005, 2007; Reese et al. 2007; Gadotti 2009; Weinzirl et al. 2009, 2013). Accounting for bars in $2 D$ decomposition is critical for obtaining a reliable characterization of the bulge (e.g., Laurikainen et al. 2005, 2007; Weinzirl et al. 2009).

In structural decomposition, the galaxy light profile is with fitted with analytical profiles for the different structural components (e.g., bulge and disk). A generalized profile commonly used is the Sérsic (1968) profile

$$
I(r)=I_{e} \exp \left[-\kappa\left(\left(\frac{r}{r_{\mathrm{e}}}\right)^{1 / n}-1\right)\right]
$$


where $I_{e}$ is the pixel surface brightness at effective radius $r_{\mathrm{e}}, n$ is the Sérsic index, and $\kappa$ is a non-free parameter coupled to $n$. Figure 1.2 shows how the Sérsic profile looks for different values of $n$.

Luminous elliptical galaxies are well know to have high Sérsic index $n=4$ (de Vaucouleurs 1948, 1953). The large-scale disks of S0s/spirals, in comparison, are commonly fitted with a version of the Sérsic profile (called an exponential profile) with $n$ fixed to 1 (Freeman 1970). Bulges in S0s/spirals, which are defined photometrically as the excess light above the inner extrapolation of the large-scale disk, were originally assumed to also have $n=4$ (Simien \& de Vaucouleurs 1986). Work in the 1990's and early 2000's (e.g., Andredakis \& Sanders 1994; Andredakis et al. 1995; de Jong 1996; Balcells et al. 2003), however, showed that bulges in late-type spirals commonly were better represented by Sérsic index $n=1$ than by $n=4$. Now, it is understood that bulges collectively show a wide range in Sérsic index, from $<1$ to $\sim 4$, and also it is now standard to fit bulges with a generalized Sérsic profile.

\subsection{Relating Galaxy Structure to Physical Processes}

In this section, I describe the distinct formation mechanisms of the two basic building blocks of galaxies: disk-dominated structures and spheroidal or triaxial bulges/ellipticals. I further associate these structures with different ranges of Sérsic index.

\section{A1. Disk-dominated components:}

Disk-dominatd structures include both large-scale and small-scale inner disks (also called disky/pseudo bulges), and they can contain embedded non-axisymmetric structures (spiral arms, bars, ovals/lenses, and rings). Large-scale disks form as gas with 
angular momentum dissipates energy and settles into a thin disk to conserve angular momentum. The source of this gas can be halo gas accretion, cold-mode accretion, or residual gas that is not consumed by star formation in a major or minor merger. Within a gas disk, gravitational instabilities and gas collisions will give rise to star formation. As noted in Section 1.2, large-scale disks are associated with Sérsic index $n \sim 1$.

Inner small-scale disks arise due to gas inflows into the inner kiloparsec triggered by internal secular processes such as bar-driven gas inflows (Kormendy 1993a; Kormendy \& Kennicutt 2004; Jogee, Scoville, \& Kenney 2005) or by external processes such as tidal interactions and minor mergers (Hernquist \& Mihos 1995). Small-scale disks are likewise in general associated with low Sérsic $n \lesssim 2$ (Kormendy \& Fisher 2005; Fisher \& Drory 2008). In the context of elliptical galaxies forming in mergers with dissipation, the inner disks may be called "extra-light" components (Hopkins et al. 2009a; Kormendy et al. 2009).

\section{A2. Spheroidal or triaxial bulges/ellipticals:}

Spheroidal or triaxial bulges/ellipticals are formed by the redistribution of stars during galaxy collisions. During major mergers, stars experience the extreme process known as violent relaxation (Binney \& Merrifield 1987) in which a rapidly varying gravitational potential causes the stars to lose memory of any initial preferred angular momentum vector. This process is less important in the larger galaxy during a minor merger, but it still affects the mass in the smaller progenitor.

In early simulations focusing on gas-poor mergers, the major merger of two spiral galaxies with stellar mass ratio $M_{1} / M_{2} \geq 1 / 4$ would inevitably destroy pre-existing 
stellar disks by violent relaxation, producing a remnant spheroidal or triaxial bulge or elliptical having a puffed-up distribution of stars with a high ratio of ordered-torandom motion $(V / \sigma)$ and a steep de Vaucouleurs $r^{1 / 4}$ (i.e., Sérsic $n=4$ ) surface brightness profile (Toomre 1977; Barnes 1988). Thus, in the traditional view of major mergers, the entirety of the remnant is a violently relaxed, dispersion-dominated "classical" bulge/elliptical with Sérsic index $n \sim 4$. In the next point (A3) I consider how this view has been revised with the latest hydrodynamical simulations.

A3. Impact of major mergers on galaxy structure:

The latest generation of hydrodynamical models of galaxy formation (e.g., Robertson et al. 2006; Naab et al. 2006; Governato et al. 2007; Hopkins et al. 2009a, b) have shown that major mergers are not $100 \%$ efficient at violently relaxing stars. Firstly, while it is reasonably accurate to assume that all pre-existing stars in a 1:1 merger are violently relaxed, this is strictly not true for mergers of lower mass ratio. The fraction of stars that is violently relaxed in the major merger actually depends on the merger mass ratio $M_{1} / M_{2}$ (Hopkins et al. 2009b). Secondly, when the baryonic gas fraction $\left(f_{\text {gas }}\right)$ in the progenitors is non-zero, gas will be funneled to the center of the remnant where it creates after a starburst an inner, small-scale disk (Hopkins et al. 2009a, b) or "extra-light" component. In the case of a moderate-to-high gas fraction $\left(f_{\text {gas }} \sim 20-80 \%\right)$, the merger remnant features a significant newly-built extended stellar disk, which forms from the residual gas in the late merger phases, that is added to any surviving remnant of the initial stellar disk. Thus, depending on the merger mass ratio and available gas supply, a major merger remnant can have both a violently relaxed spheroidal or triaxial bulge and a large-scale/small-scale disk 
(Naab et al. 2006; Robertson et al. 2006; Hopkins et al. 2009a, b).

Structural analysis of major merger remnants in hydrodynamical simulations (e.g., Hopkins et al. 2009a) find that the Sérsic index of the remnants is typically high, in the range of $2<n<6$, for moderate-to-high gas fractions ( $f_{\text {gas }} \sim 20-80 \%$ ).

\section{A4. Impact of minor mergers on galaxy structure:}

While less destructive than major mergers, minor mergers (mass ratio $1 / 10 \leq M_{1} / M_{2}<$ 1/4) are more frequent (Jogee et al. 2009; Lotz et al. 2011) and can have a significant net effect on galaxy structure (Bournaud, Jog, \& Combes 2007; Naab et al. 2009). After the satellite galaxy (mass $M_{2}$ ) enters the dark matter halo of the primary galaxy (mass $M_{1}$ ), dynamical friction pulls the satellite down into the disk of the primary galaxy on a timescale that depends on $\mathrm{M}, V_{c}, \mathrm{R}$ and is a few Gyr for typical values (Jogee 2006). The satellite loses angular momentum, which goes into dynamically heating the larger galaxy. Once in the disk, the satellite induces warps and drives large amplitude non-axisymmetries (Quinn et al. 1993; Hernquist \& Mihos 1995; Mihos et al. 1995; review by Jogee 2006). The satellite is tidally stripped of material while moving through the disk, and if it survives long enough it will eventually fall into the center of the disk, where the remainder of mass $M_{2}$ violently relaxes and then forms/joins the bulge (Hopkins et al. 2009b). If the primary galaxy does not have a prominent bulge, the satellite can violently relax a mass belonging to the primary galaxy as large as the satellite's remaining mass. In the case of a bulge-dominated primary galaxy, the bulge stars are merely heated by the satellite (Hopkins et al. 2009b).

Minor mergers affect the structure of the primary galaxy in two important ways. First, the large amplitude non-axisymmetry (e.g., a stellar bar) induced in the pri- 
mary galaxy drives cold gas from the outer disk into the central few hundred parsecs via a combination of gravitational torques and shocks. This redistributed gas forms a small-scale disk in the center of the galaxy. Secondly, provided the satellite reaches the center of the primary, some stellar mass (particularly that belonging to the satellite) is violently relaxed (Hopkins et al. 2009b). The effect of minor mergers on galaxy structure, and especially the impact on bulge Sérsic index, depends on the relative importance between the gas dissipation and violent relaxation aspects of minor mergers. This is currently a key unknown that is receiving much attention from the theoretical community.

Some initial work (Aguerri et al. 2001; Eliche-Moral et al. 2006; Naab \& Trujillo 2006) has explored with collisionless simulations (i.e., without gas dissipation) the effect of a single minor merger on the structure of a disk-dominated galaxy. Aquerri et al. (2001) studies bulge growth due to satellite accretion on a galaxy with $B / T=0.33$ and and exponential $(n=1)$ bulge profile. They show that a single minor merger event with mass ratio 1:6 to 1:18 can produce a remnant galaxy with a more massive bulge having Sérsic index $n \sim 1.5-2.5$. Eliche-Moral et al. (2006) conduct similar experiments while initially setting the bulge Sérsic index $n$ to 0.9 , showing that the bulge mass and bulge Sérsic index of the primary galaxy increases to $n \sim 1.0-1.9$ Naab \& Trujillo (2006) find that 1:6 mergers of disk galaxies produce remnant bulges with Sérsic index $n \sim 1$. It seems plausible that if gas dissipation were included, the bulge final Sérsic index from these simulations could be even lower due to the presence of an inner, small-scale disk.

The affect of multiple successive minor mergers is likewise being explored as well. 
Minor mergers have a dynamical heating effect, and successive minor mergers are potentially destructive to large-scale disks. Bournaud, Jog, \& Combes (2007) study the effects of repeated minor mergers on galaxy structure. They demonstrate that after successive minor mergers a disk galaxy will eventually transform into a dispersion dominated elliptical galaxy with a Sérsic index $n \sim 4$. Naab et al. (2009) additionally show that compact spheroid galaxies at high-redshift $(z=3)$ can grow significantly in size and Sérsic index by $z \sim 0$ due to successive minor mergers.

A5. Relationship between Sérsic index $n$, galaxy structure, and physical processes:

Structural decomposition yields a Sérsic index $n$ for each component of a galaxy. These components must then be associated with or/and interpreted as flattened diskdominated components (defined in point A1), spheroidal or triaxial bulges/ellipticals (defined in point A2), or other intermediate structures. In this work I separate structures within massive $\left(M_{\star} \geq 10^{9} M_{\odot}\right)$ galaxies into two main groups: flattened diskdominated components $(n \lesssim 2)$ and puffy non-disk components $(n \gtrsim 2)$.

(a) Disk-dominated components with $n \lesssim 2$ :

There is substantial observational evidence that associates disk-dominated components with Sérsic indexes $n \lesssim 2$. The large-scale disks of spiral galaxies in the field are well known to be characterized by an exponential light profile profile (Sérsic index $n \sim 1$ ) over 4-6 disk scalelengths (Freeman 1970, de Jong 1996). Furthermore, careful 2D decompositions of barred S0 galaxies in the Coma cluster (Weinzirl et al. 2013, Chapter 3), in which the Sérsic index of the outer disk is a free parameter, show that the Sérsic index of the outer disk is $n \lesssim 1.7$. 
On smaller scales, flattened, rotationally supported inner disks with high $V / \sigma$ (i.e., disky/pseudo bulges) have been associated with low Sérsic index $n \lesssim 2$ (Kormendy 1993a; Kormendy \& Kennicutt 2004; Jogee, Scoville, \& Kenney 2005; Athanassoula 2005; Kormendy \& Fisher 2005; Kormendy \& Fisher 2005; Fisher \& Drory 2008; Fabricius et al. 2012).

Fisher \& Drory (2008) study all spiral galaxies in the Northern hemisphere that have imaging with $H S T$ and distance $<40$ Mpc. Disky pseudobulges are identified visually based on the presence of disk signatures such as nuclear spirals, nuclear rings, nuclear bars, and nuclear patchiness. Figure 9 of Fisher \& Drory (2008) shows $\sim 90 \%$ of disky pseudobulges have bulge Sérsic index $n \lesssim 2$, while $\sim 90 \%$ of classical bulges have $n \gtrsim 2$.

Fabricius et al. (2012) explores the major-axis kinematics of 45 S0-Scd galaxies with high-resolution spectroscopy. They demonstrate, for the first time, a systematic agreement between the shape of the velocity dispersion profile and the bulge type as indicated by the Sérsic index. Low Sérsic index bulges have both increased rotational support (higher $\left\langle V^{2}>/<\sigma^{2}>\right.$ values) and on average lower central velocity dispersions. Classical bulges have centrally peaked velocity dispersion profiles while disky pseudobulges in general have flat dispersion profiles and even at times show drops in the central velocity dispersion. This correlation holds if visual morphology is used for the bulge classification instead of the Sérsic index.

At high $(z \sim 2)$ redshift, where it is not yet possible to fully resolve galaxy substructures, it has become conventional to use the global Sérsic index $n \lesssim 2$ 
in massive galaxies to separate disk-dominated versus bulge-dominated galaxies (e.g., Ravindranath et al. 2004; van der Wel et al. 2011; Weinzirl et al. 2011). Weinzirl et al. (2011) further explore the distributions of ellipticities $(1-b / a)$ for the massive $z \sim 2$ galaxies with low $(n \leq 2)$ and high $(n>2)$ global Sérsic index. They find that galaxies with low global Sérsic index $n \leq 2$ have a long tail of high projected ellipticities and their distribution of projected ellipticities is much more similar to massive $z \sim 0$ spirals than to massive $z \sim 0$ ellipticals. One question that cannot be fully answered from Weinzirl et al. (2011) is whether the flattening is due to anisotropic velocity dispersions or rotation. However, the above suggests the anisotropy in elliptical galaxies cannot account for the degree of flattening in massive $z \sim 2$ galaxies with low Sérsic index.

In the rest of this work, I denote structures with $n \lesssim 2$ as disk-dominated components, and I further assume that they form via gas-rich dissipative processes.

(b) Non-disk components with $n \gtrsim 2$ :

Galaxy components with $n \gtrsim 2$ include spheroidal or triaxial bulges/ellipticals. Traditionally, in the "classical" case, spheroidal or triaxial bulges/ellipticals were considered to have a Sérsic index $n \sim 4$ and were associated with complete violent relaxation in equal mass (mass ratio 1:1), dry major mergers. However, more generally, the latest hydrodynamical simulations (e.g., Robertson et al. 2006; Naab et al. 2006; Governato et al. 2007; Hopkins et al. 2009a, b) show that in major mergers with gas fractions in the range $f_{\text {gas }} \sim 20-80 \%$, the stellar remnant has Sérsic $2<n<6$ (see A3 for details).

As discussed in A4, the impact of minor mergers on Sérsic $n$ is poorly studied. 
Our limited understanding from simulations suggests that typically single minor mergers produce stellar remnants with $n<2$ (e.g., Eliche-Moral et al. 2006; Naab \& Trujillo et al. 2006; point A4), but repeated minor mergers may in some cases lead to $n \gtrsim 2$. (Bournaud, Jog, \& Combes 2007; Naab et al. 2009).

Therefore, in the rest of this work, I denote components with Sérsic index $n \gtrsim 2$ as non-disk components and assume that such structures are built by the redistribution of stars during major mergers, and possibly, to a lesser degree, during minor mergers.

(c) Caveat regarding dwarf elliptical galaxies:

The term "dwarf elliptical" (dE) is often used to describe elliptical galaxies fainter than $M_{B}=-18$ (Sandage \& Binggeli 1984). Local Group dwarf galaxies are not particularly flattened with $b / a \sim 0.7$, yet they have Sérsic $n \sim 0.5-1$ (e.g., Caldwell 1999; Jerjen et al. 2000). There has been much debate over the true nature of such galaxies. Early studies (Wirth \& Gallagher 1984; Sandage et al. 1985; Binggeli et al. 1988) suggested elliptical and dE galaxies may follow separate luminosity functions, and therefore represent different families of galaxies, that happen to overlap for $-15 \gtrsim M_{B} \gtrsim-18$. A central question is what implications do such objects have on our interpretation of galaxy structure based on Sérsic index? Here, we discuss the different interpretations of $\mathrm{dE}$ galaxies and quantify many such systems are in sample S2.

Several papers (e.g., Kormendy 1985; Kormendy et al. 2009; Kormendy \& Bender 2012) demonstrate that elliptical and dE galaxies separate cleanly in global galaxy parameter correlations for $r_{\mathrm{e}}$, surface brightness $\mu_{\mathrm{e}}$ at $r_{\mathrm{e}}$, and luminosity. The 
differences in structural parameter correlations between elliptical and dE galaxies are interpreted to mean separate formation mechanisms and that elliptical and $\mathrm{dE}$ galaxies are fundamentally different classes of objects. Elliptical galaxies form in major galaxy mergers while $\mathrm{dE}$ galaxies are late-type galaxies stripped of gas by non-merger processes. Kormendy et al. (2009) and Kormendy \& Bender (2012) advocate that such defunct late types should be called spheroidals (Sph) rather than dwarf ellipticals to avoid association with elliptical and "true" dwarf elliptical galaxies (i.e., galaxies that are actually miniature versions of ellipticals, like M32).

An alternate perspective is that dEs and ellipticals represent a continuous population (e.g., Sandage \& Binggeli 1984; Graham \& Guzmán 2003). The reasoning is that $\mathrm{dE}$ galaxies form a linear sequence with giant ellipticals for parameters including concentration (Sérsic index), central surface brightness $\left(\mu_{0}\right)$, luminosity, color, metallicity, age, and dynamics (Graham \& Guzmán 2003; Graham 2013 and references therein).

Graham \& Guzmán (2003) argue that the curved or dichotomous appearance of global parameter correlations involving $r_{\mathrm{e}}$, surface surface brightness $\mu_{\mathrm{e}}$ at $r_{\mathrm{e}}$, or average surface brightness $\left\langle\mu_{\mathrm{e}}\right\rangle$ within $r_{\mathrm{e}}$ is a consequence of the wellestablished linear correlations between luminosity-Sérsic-index and luminosity- $\mu_{0}$ correlations that are continous for $\mathrm{dE}$ and ellipticals.

It is beyond the scope of this thesis to comment further on the interpretation of $\mathrm{dE} /$ spheroidal galaxies. Furthermore, this issue does not significantly affect this thesis as I am concerned primarily with massive $\left(M_{\star} \geq 10^{9} M_{\odot}\right)$ non-dwarf 
galaxies.

\subsection{Thesis Goals and Overview}

This thesis constitutes a quantitative census of the relative importance of the major structural components (flattened, and dynamically cold components versus puffy and dynamically hot spheroidal or triaxial bulges/ellipticals) in massive galaxies over cosmic time, from the present day to 2 billion years after the Big Bang and across different galaxy environments, namely the field and a rich cluster. The purpose is to explore how galaxies evolve under the action of the various assembly mechanisms (major mergers, minor mergers, gas accretion, and internal secular processes) in these different regimes.

This thesis has two key strengths that should be highlighted. First, parts of this thesis are based on panchromatic (X-ray, optical, and infrared) data from some of the largest and deepest surveys by Hubble Space Telescope (HST), Spitzer, and Chandra. Secondly, this thesis makes extensive and meaningful comparisons with hierarchical $\Lambda$ CDMbased models to assess the level of agreement/disagreement and to provide constraints and direction for future theoretical work. My analysis is described in Chapters 2-4, and a brief primer on each chapter is provided below. Chapter 5 provides a summary of the main results and discusses both the implications and the future work that will build upon this thesis.

\subsubsection{Probing the Structure and Assembly of Nearby Field Spirals [Chapter 2]}

This work, which originally appeared as Weinzirl et al. (2009), is included here as Chapter 2. This work was necessary to address two important problems. Around year 20062007, there were anecdotal concerns that the seemingly high fraction of quasi-bulgeless and bulgeless galaxies was in conflict with a hierarchical $\Lambda C D M$ universe. The crux of the issue 
was that if most $\mathrm{S} 0 /$ spiral galaxies had experienced a relatively recent major merger, then their morphology should feature a prominent spheroidal or triaxial bulge with moderateto-high Sérsic $n$ and with a significant bulge-to-total light ratio $B / T>0.2$. In reality, the $B / T$ ratios of present-day S0s/spirals depend on the merger histories of hierarchical models, which vary as a function of environment and redshift (e.g., Cole et al. 2000; Khochfar \& Burkert 2001).

Thus, this problem required not only an observational assessment of the abundance of low $B / T$ and bulgeless galaxies, which was partly in place (Böker et al. 2002; Kautsch et al. 2006; Barazza, Jogee \& Marinova 2007,2008; Kormendy \& Fisher 2008), but also a rigorous comparison with the predictions of hierarchical models, which had not yet been attempted by anyone. The purpose of the model comparison would be to assess the level of agreement/disagreement between observations and theory in addition to understanding what aspect of the hierarchical models was driving that agreement/disagreement.

The unique contributions of this work are two-fold. First, it establishes quantitatively the distribution of $B / T$ in a statistically significant sample of spiral galaxies. Secondly, the structural properties of large-scale disks and bulges are applied to constraining the assembly histories of their massive host spirals. Specifically, I make detailed comparisons with the $B / T$ ratios predicted by two different theoretical semi-analytic models (Khochfar \& Silk 2006b; Hopkins et al. 2009b). Note, however, that the semi-analytic models predict only $B / T$ and detailed tests of the distribution of bulge structure (i.e., Sérsic index $n$ ) were not possible as of 2009 . 


\subsubsection{Exploring the Structure and Assembly of Galaxies At the Heart of the Coma Cluster [Chapter 3]}

It is necessary to extend studies of galaxy assembly to rich cluster environments because hierarchical models of galaxy formation predict differences in the galaxy merger history as a function of galaxy mass and environment (e.g., Cole et al. 2000; Khochfar \& Burkert 2001). Also, the relative importance of physical processes other than mergers (e.g. ram pressure stripping, galaxy harassment, strangulation), which impact disk growth in galaxies, are a strong function of environment.

In Chapter 3, I accomplish this task using high-resolution (50 pc) data from the HST ACS Treasury Survey of the Coma cluster (Carter et al. 2008), focusing on the structure of the most massive $\left(M_{\star} \geq 10^{9} M_{\odot}\right)$ galaxies in the central $0.6 \mathrm{Mpc}$ of the cluster. Coma is a special environment because it is the richest nearby galaxy cluster, with central galaxy number density reaching values as high as 10,000 galaxies $\mathrm{Mpc}^{-3}$, over an order of magnitude higher than in the Virgo cluster (Binggeli et al. 1987). I make a detailed structural analysis of these galaxies to measure the fraction of stellar mass in low- $n \lesssim 1.7$ flattened disk-dominated components (large-scale disks, disky/pseudo bulges, bars, and inner disks) versus high- $n \gtrsim 1.7$ non-disk components that include spheroidal or triaxial bulges/ellipticals and other merger remnants. As in Chapter 2, I make a detailed comparison with hierarchical models, in this case using simulated clusters that are based on dark matter halo merger histories from the Millennium Simulation (Springel et al. 2005) and baryonic physics from semi-analytic models (Neistein \& Weinmann 2010). 


\subsubsection{Structure and Assembly of the Most Massive Galaxies Present a Few Gyr $(z \sim 2-3)$ After the Big Bang [Chapter 4]}

In Chapter 4, which was published as Weinzirl et al. (2011), I investigate the most

massive $\left(M_{\star} \geq 5 \times 10^{10} M_{\odot}\right)$ galaxies just 2-6 Gyr $(z=1-3)$ after the Big Bang using restframe optical imaging from the GOODS-NICMOS survey (Conselice et al. 2011) combined with ancillary data from Chandra and Spitzer. Studies of such high-redshift galaxies are important because it is has been suggested from previous work based on smaller sample sizes that galaxies at early epochs are different both in size and structure from present-day galaxies of similar stellar mass. This work is a significant contribution because it is based on one of the largest and most diverse samples of massive galaxies having space-based restframe optical imaging. In addition to characterizing the rest-frame optical structure of these galaxies, I also investigate how star-formation rate and black hole activity correlate with galaxy structure. 


\section{Edwin Hubble's Classification Scheme}

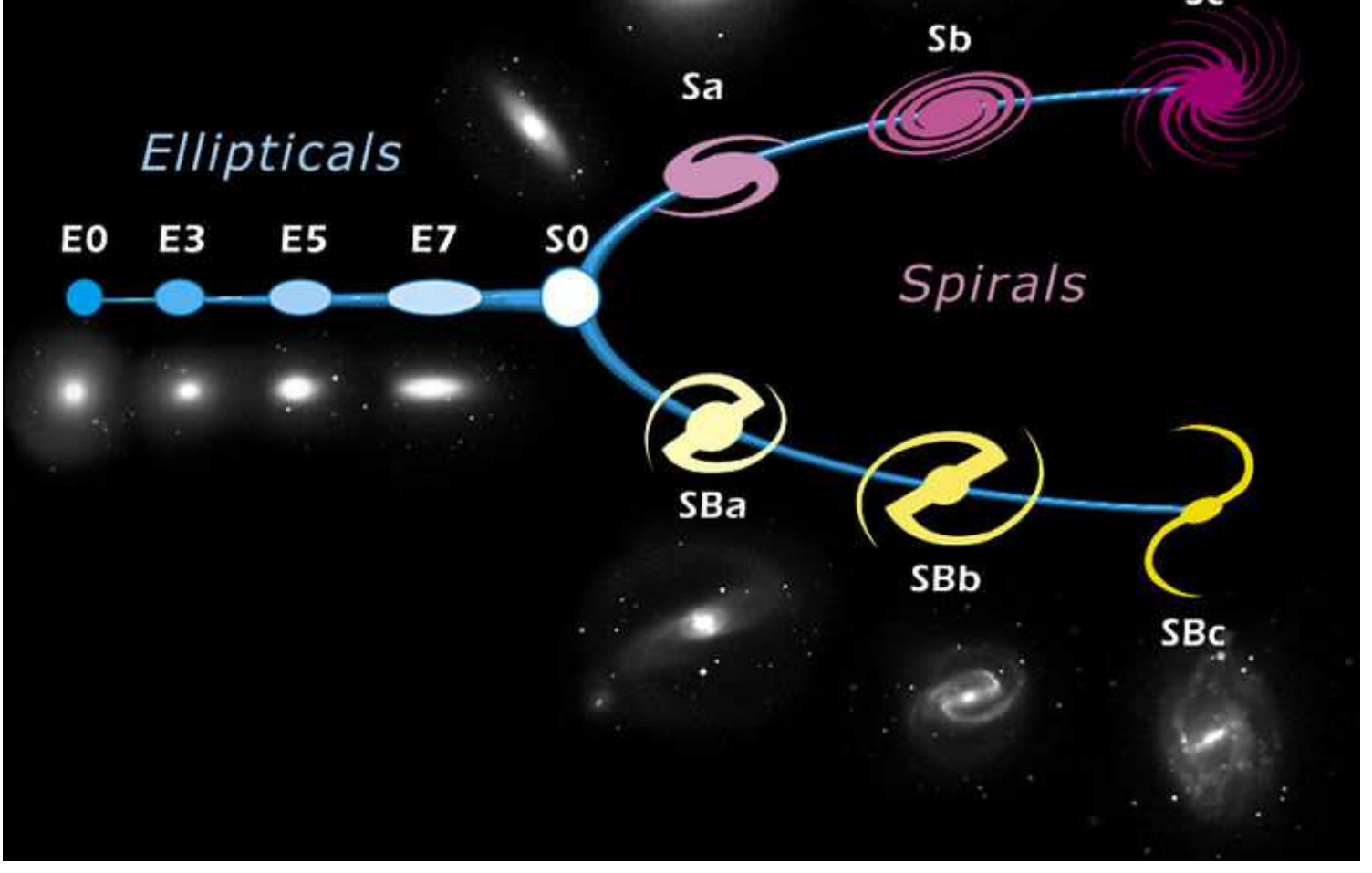

Figure 1.1 This visual representation of the Hubble Sequence (Hubble 1926) highlights the difference between elliptical and disk galaxies. Barred and unbarred galaxies constitute parallel branches in the sequence. [Credit: NASA, STScI, and ESA] 


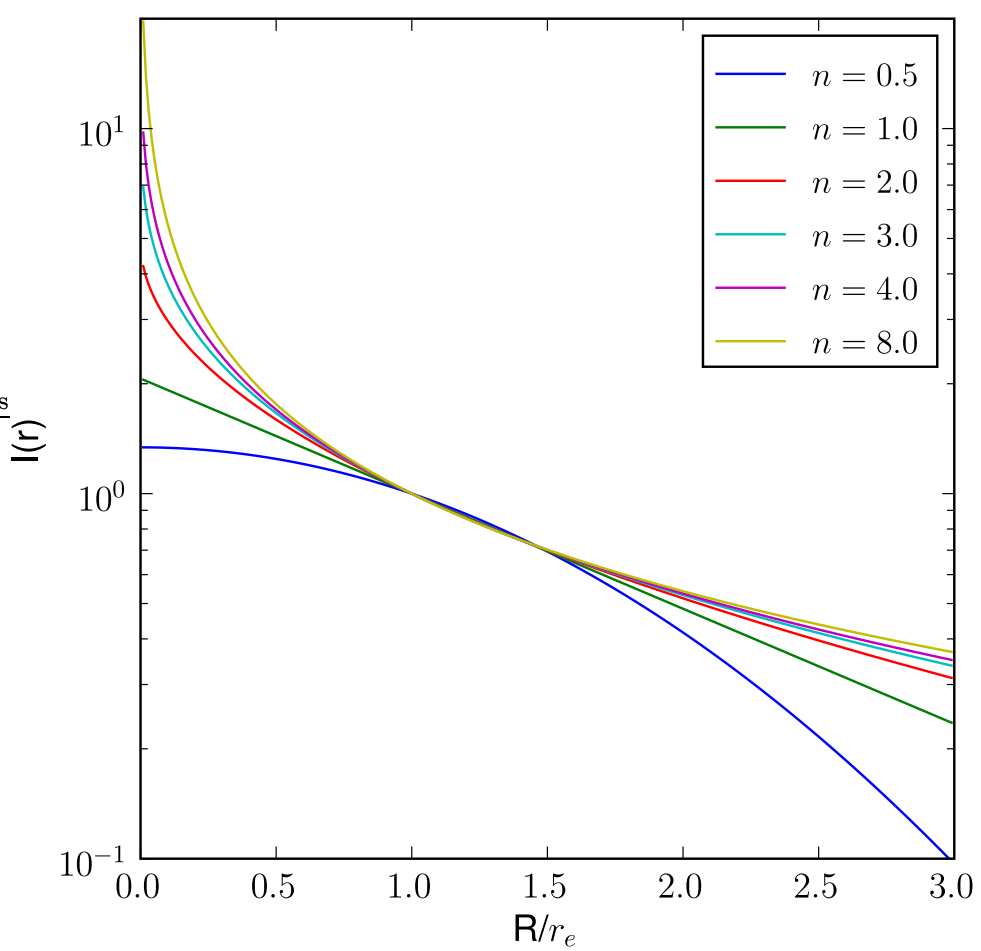

Figure 1.2 This figure shows the shape of the Sérsic profile. The Sérsic index $n$ controls the shape of the profile. At larger Sérsic index $n$, the profile center becomes steeper, and the outer wing becomes flatter. 


\section{Chapter 2}

\section{Probing the Structure and Assembly of Nearby Field Spirals}

1

\subsection{Introduction}

The formation of galaxies is a classic problem in astrophysics. Contemporary galaxy formation models combine the well-established $\Lambda$ Cold Dark Matter $(\Lambda \mathrm{CDM})$ cosmology, which describes behavior of dark matter on very large scales, with baryonic physics to model galaxy formation. In the early Universe, pockets of dark matter decoupled from the Hubble flow, collapsed into virialized halos, and then clustered hierarchically into larger structures. Meanwhile, gas aggregated in the interiors of the halos to form rotating disks, which are the building blocks of galaxies (Steinmetz \& Navarro 2002; Cole et al. 2000). Such disks are typically destroyed during major mergers of galaxies with mass ratio $M_{1} / M_{2}>1 / 4$ (e.g. Steinmetz \& Navarro 2002; Naab \& Burkert 2003; Burkert \& Naab 2004; Mihos \& Hernquist 1996). When the mass ratio is close to unity, the remnant is a spheroid with properties close to that of a classical bulge, namely a steep de Vaucouleurs $r^{1 / 4}$ surface brightness profile

\footnotetext{
${ }^{1}$ This chapter has been previously published as Weinzirl, T., Jogee, S., Khochfar, S., Burkert, A., \& Kormendy, J. 2009, ApJ, 696, 411.

Co-author contributions: Jogee, S. supervised the research. Burkert A. and Khochfar, S. provided predictions from hierarchical models of galaxy formation. Burkert, A., Jogee, S., Khochar, S., and Kormendy, J. provided editorial comments.
} 
and a high ratio of ordered-to-random motion $(v / \sigma)$. We shall return to this point in $\S 2.5$. Within this hierarchical framework, the disk of spiral galaxies forms when gas of higher specific angular momentum subsequently accretes around the bulge (Steinmetz \& Navarro 2002; Burkert \& Naab 2004).

$\Lambda$ CDM-based simulations of galaxy formation face several challenges. One issue is the angular momentum problem; simulated galaxy disks have smaller scalelengths and, therefore, less specific angular momentum than their counterparts in nature (Navarro \& Steinmetz 2000; Burkert \& D'Onghia 2004; D'Onghia et al. 2006). A second issue is the problem of bulgeless or low bulge-to-total mass ratio $(B / T)$ spirals. Within the $\Lambda \mathrm{CDM}$ paradigm, galaxies that had a past major merger at a time when its mass was a fairly large fraction of its present-day mass are expected to have a significant bulge with large $B / T$ and high Sérsic index. Depending on the merger history and hence the fraction of spiral galaxies that fulfill this criterion (see $\S 2.5 .8$ ) we can end up with a small or large fraction of present-day galaxies with low $B / T$.

There is rising evidence that low $B / T$ and bulgeless galaxies are quite common in the local Universe, especially in low mass or late-type galaxies. Late-type Sd galaxies often harbor no bulge (Böker et al. 2002). Kautsch et al. (2006) and Barazza, Jogee \& Marinova (2007, 2008) also find from the analysis of several thousand late-type SDSS galaxies that 15-20\% of such disk galaxies out to $z \sim 0.03$ appear bulgeless. Of the 19 local galaxies $(D<8 \mathrm{Mpc})$ with circular velocity $V_{c}>150 \mathrm{~km} \mathrm{~s}^{-1}, 11(58 \%)$ have pseudobulges instead of merger-built classical bulges (Kormendy \& Fisher 2008).

Theoretical work by Koda et al. (2007) conclude the survival of disk-dominated systems in a $\Lambda$ CDM universe is compatible with observational constraints provided classical 
bulges form only in mergers where $M_{1} / M_{2}>0.3$ and the primary halo has virial velocity $V_{v i r}>55 \mathrm{~km} \mathrm{~s}^{-1}$.

Evidence also suggests that bulges with low $B / T$ and low Sérsic index $n$ may be common even in high mass and/or early-type spirals. Balcells et al. (2003) report that early-type disk galaxies tend to have $n<3$ and often from 1 to 2 . Laurikainen et al. (2007) find barred and unbarred early-type disk galaxies to have mean $B / T$ between 0.25 and 0.35 , while later Hubble types have $B / T<0.2$; they also find mean bulge Sérsic indices to be $\sim 2.5$ or less across the Hubble sequence. Graham \& Worley (2008) report low $B / D$ ratios across the Hubble sequence based on bulge-disk decomposition of $K$-band images of local spiral galaxies. They suggest that these low values are problematic for $\Lambda \mathrm{CDM}$ simulations, but no quantitative assessment of the extent of the problems is presented.

These emerging statistics on the fraction of bulgeless $(B / T \sim 0)$ galaxies, and galaxies with low $B / T$ and low $n$ bulges provide important first constraints. More work is needed to fully explore the the distribution of bulge properties in both high and low mass galaxies. In particular, we need to explore how the observed distributions of bulge $B / T$ and $n$ compare with the predictions from $\Lambda \mathrm{CDM}$-based simulations of galaxy evolution. To the best of our knowledge, few such quantitative comparisons have been attempted, so that it remains unclear how serious the problem of low $B / T$ galaxies is. This study is an attempt to derive robust observational constraints on bulge properties in high mass spirals and to attempt such a comparison with models.

Completely resolving the issue of low $B / T$ systems will require understanding the different types of bulges and their formation pathways. Bulges are commonly divided in several groups: classical bulges, boxy/peanut bulges, and 'pseudobulges' or disky bulges. 
Classical bulges are believed to be built by major mergers $\left(M_{1} / M_{2} \geq 1 / 4\right)$ and the associated violent relaxation of stars. They are associated with modest-to-high bulge Sérsic indices, in the range $2<n<6$ (Hopkins et al. 2009a; Springel et al. 2005; Robertson et al. 2006; $\S 2.5 .8)$. Boxy/peanut bulges are believed to be the result of vertical resonances and buckling instabilities in bars, which are viewed at high inclination (Combes \& Sanders 1981; Combes et al. 1990; Pfenniger \& Norman 1990; Bureau \& Athanassoula 2005; Athanassoula 2005; Martinez-Valpuesta et al. 2006). Pseudobulges or disky bulges are believed to form as a result of gas inflow into the central kiloparsec and subsequent star formation building a compact disky, high $v / \sigma$ stellar component (Kormendy 1993a; Jogee 1999; Kormendy \& Kennicutt 2004, hereafter KK04; Jogee, Scoville, \& Kenney 2005; Athanassoula 2005; Kormendy \& Fisher 2005). Pseudobulges tend to have a bulge $n<2.5$ (Kormendy \& Fisher 2005; Fisher \& Drory 2008).

One possibility for the formation of disky bulges or pseudobulges is the idea of secular evolution (Kormendy 1993a; KK04; Jogee, Scoville, \& Kenney 2005), where a stellar bar or globally oval structure in a non-interacting galaxy drives the gas inflow into the inner kpc via shocks and gravitational torque. Another idea for building disky bulges is that the gas inflow into the inner kiloparsec is driven by external non-secular processes, such as tidal interaction and minor mergers. The gas inflow in such cases can be caused by a tidally induced non-axisymmetric feature, such as a bar (e.g., Quinn et al. 1993; Hernquist \& Mihos 1995), and by tidal torques from the companion. The subsequent central star formation can still form a compact high $v / \sigma$ stellar component, aka a pseudobulge.

Throughout this paper, we avoid making any a priori assumptions about the origin of different types of bulges by simply referring to them according to their bulge Sérsic index 
$n$ or bulge-to-total mass ratio $(B / T)$. We consider bulges of high $(n \geq 4)$, intermediate $(2<n<4)$ and low $(n \leq 2)$ index, as well as those of low or high $B / T$.

The structural properties of galaxy components, such as bulges, disks, and bars can be derived through the decomposition of the $2 D$ light distribution, taking into account the PSF. Many early studies have performed only two component $2 D$ bulge-disk decomposition (e.g., Allen et al. 2006; Byun \& Freeman 1995; de Jong 1996; Simard 1998; Wadadekar et al. 1999), ignoring the contribution of the bar, even in strongly barred galaxies. However, recent work has shown that it is important to include the bar in $2 D$ decomposition of barred galaxies, else the $B / T$ ratio can be artificially inflated, and bulge properties skewed (e.g., Laurikainen et al. 2005, 2007). Furthermore, since most $(\geq 60 \%)$ bright spiral galaxies are barred in the NIR (Eskridge et al. 2000; Laurikainen et al. 2004; Marinova \& Jogee 2007, hereafter MJ07; Menendez-Delmestre et al. 2007), the inclusion of the bar is quite important. This has led to several recent studies, where $2 D$ bulge-disk-bar decomposition are being performed (e.g. Laurikainen et al. 2007; Reese et al. 2007; Gadotti \& Kauffmann 2007).

Another advantage of bulge-disk-bar decomposition over bulge-disk decomposition is that the former allows us to constrain the properties of the bar itself. Bars provide the most important internal mechanism for redistributing angular momentum in baryonic and dark matter components (e.g. Weinberg 1985; Debattista \& Sellwood 1998, 2000; Athanassoula 2002; Berentzen, Shlosman, \& Jogee 2006). They efficiently drive gas inflows into the central kpc, feed central starbursts (Elmegreen 1994; Knapen et al. 1995; Hunt \& Malakan 1999; Jogee et al. 1999; Jogee, Scoville, \& Kenney 2005; Jogee 2006) and lead to the formation of disky or pseudobulges (see above). Furthermore, the prominence of strong bars out to 
$z \sim 1$ over the last 8 Gyr (Jogee et al. 2004; Sheth et al. 2008) suggest that bars have been present over cosmological times and can shape the dynamical and secular evolution of disks. Thus, quantifying bar properties, such as the fractional light and mass ratio (Bar/T), can yield insight into these processes.

In this paper, we constrain the properties of bulges and bars along the Hubble sequence, and compare our results with $\Lambda$ CDM-based simulations of galaxy evolution. In $\S 2.2$, we define our complete sample of 143 bright $\left(M_{B} \leq-19.3\right)$ low-to-moderately inclined $\left(i \leq 70^{\circ}\right)$ spirals from the Ohio State University Bright Spiral Galaxy Survey (OSUBSGS; Eskridge et al. 2002), which is widely used as the local reference sample for bright spirals by numerous studies (e.g., Eskridge et al. 2000; Block et al. 2002; Buta et al. 2005; MJ07 ; Laurikainen et al. 2004, 2007). In $\S 2.3$, we perform $2 D$ bulge-disk and bulge-disk-bar decompositions of $H$-band images using GALFIT (Peng et al. 2002), and derive fractional light ratios $(B / T, \operatorname{Bar} / T, \operatorname{Disk} / T)$, as well as Sérsic indices and half light radii or scale lengths. Tests to verify the robustness of our decompositions are presented in $\S 2.4$. In $\S 2.5$, we present our results. Specifically, the total stellar mass present in bulges, disks, and bars is calculated ( $\S 2.5 .2)$. In $\S 2.5 .3$, the distribution of bulge Sérsic index $n$ and $B / T$ as a function of galaxy Hubble type and stellar mass is presented, and the surprising prevalence of bulges with low Sérsic index $n$ and low $B / T$ is established. A comparison with other works is presented in $\S$ 2.5.4. We examine how Bar/T and bar fraction (the fraction barred disks) change as a function of host galaxy properties in $\S 2.5 .5$. In $\S 2.5 .8$, we compare our observed distribution of bulge $B / T$ and $n$ in high mass $\left(M_{\star} \geq 1.0 \times 10^{10} M_{\odot}\right)$ spirals with predictions from $\Lambda \mathrm{CDM}$ cosmological semi-analytical models. $\S 2.6$ summarizes our results. 


\subsection{Sample Properties}

\subsubsection{OSUBSGS}

Our dataset is derived from the $182 \mathrm{H}$-band images from the public data release of the Ohio State University Bright Spiral Galaxy Survey (OSUBSGS; Eskridge et al. 2002). These galaxies are a subset of the RC3 catalog that have $m_{B} \leq 12$, Hubble types $0 \leq T \leq 9$ (S0/a to $\mathrm{Sm}$ ), $D_{25} \leq 6^{\prime} .5$, and $-80^{\circ}<\delta<+50^{\circ}$. Imaging of OSUBSGS galaxies spans optical and near infrared (NIR) wavelengths with $B V R J H K$ images available for most galaxies. OSUBSGS images were acquired on a wide range of telescopes with apertures ranging from $0.9-2.4 \mathrm{~m}$. The $J H K$ data were acquired with a variety of telescopes and detectors, but mainly with the $1.8 \mathrm{~m}$ Perkins reflector at Lowell Observatory and the CTIO $1.5 \mathrm{~m}$ telescope with the OSIRIS detector, having 18.5 micron pixels (Depoy et al. 1993). Pixel scale is dependent on the telescope and for these observations ranged between 1 $1.50^{\prime \prime} /$ pix. Exposure times were heterogeneous, but the total observing time per object was typically between 10-15 minutes in $H$. The resulting limiting $H$-band surface brightness is $\sim 20 \mathrm{mag} \operatorname{arcsec}^{-2}$. The typical limiting surface brightnesses of the images $\sim 26$ mag $\operatorname{arcsec}^{-2}$ in $B$-band and $\sim 20 \mathrm{mag} \operatorname{arcsec}^{-2}$ in $H$-band (Eskridge et al. 2002). The seeing depends on observing time and location. We find the $H$-band images have seeing of $\sim 3^{\prime \prime}$.

We choose to use the NIR images rather than optical ones for several reasons. Firstly, NIR images are better tracers of the stellar mass than optical images, and the massto-light ratio is less affected by age or dust gradients. Secondly, obscuration by dust and SF are minimized in the NIR, compared with the optical. As the $K$-band images are of poor quality, we settle on using the $H$-band images.

The OSUBSGS is widely used as the local reference sample for bright spirals by 
numerous studies (e.g. Eskridge et al. 2000; Block et al. 2002; Buta et al. 2005; MJ07; Laurikainen et al. 2004, 2007). Thus, there are numerous complementary results that we can use or compare with. In particular, MJ07 have identified bars in this sample using quantitative criteria based on ellipse fitting, and characterized their sizes, position angles, and ellipticities.

OSUBSGS is a magnitude-limited survey with objects whose distances range up to $\sim 60$ Mpc. Faint galaxies are inevitably missed at larger distances, resulting in the absolute magnitude distribution in Figure 2.1. We compare the $B$-band LF of this sample with a Schechter (Schechter 1976) LF (SLF) with $\Phi^{*}=5.488 \times 10^{-3} \mathrm{Mpc}^{-3}, \alpha=-1.07$, and $M_{B}^{*}=-20.5$ (Efstathiou, Ellis \& Peterson 1988) in Figure 2.2. The volume used to determine the number density in each magnitude bin is

$$
V_{\max }=\frac{4 \pi}{3} d_{\max }^{3}(M)
$$

where

$$
d_{\max }(M)=10^{1+0.2\left(m_{c}-M\right)}
$$

is the maximum distance out to which a galaxy of absolute magnitude $M$ can be observed given the cutoff magnitude $m_{c}$. If the SLF is representative of the true LF, then Figure 2.2 suggests that the OSUBSGS sample is seriously incomplete at $M_{B}>-19.3$, while at the brighter end (-19.3 to -23) the shape of its LF matches fairly well the SLF. We thus conclude that the sample is reasonably complete for bright $\left(M_{B} \leq-19.3\right.$ or $\left.L_{B}>0.33 L^{*}\right)$ galaxies.

We exclude highly inclined $\left(i>70^{\circ}\right)$ galaxies for which structural decomposition does not yield accurate results. Thus, our final sample S1 consists of 143 bright $\left(M_{B} \leq\right.$ -19.3) low-to-moderately inclined $\left(i \leq 70^{\circ}\right)$ spirals with Hubble types mainly in the range 
S0/a to Sc (Figure 2.1). Of the 126 for which we could derive stellar masses (see $\S 2.2 .2$, most have stellar masses $M_{\star} \geq 1.0 \times 10^{10} M_{\odot}$ (Figure 2.3). Table 2.1 summarizes the morphologies, luminosities, and stellar masses of the sample. Note that there are few galaxies of late Hubble types (Scd or later) and we do not draw any conclusions on such systems from our study. In a future paper, we will tackle galaxies of lower mass and later Hubble types.

\subsubsection{Stellar Masses}

We derive global stellar masses for most of the OSUBSGS sample galaxies using the relation between stellar mass and rest-frame $B-V$ color from Bell et al. (2003). Using population synthesis models, the latter study calculates stellar $M / L$ ratio as a function of color using functions of the form $\log _{10}(M / L)=a_{\lambda}+b_{\lambda} \times$ Color $+C$, where $a_{\lambda}$ and $b_{\lambda}$ are bandpass dependent constants and $\mathrm{C}$ is a constant that depends on the stellar initial mass function (IMF). For the $V$ band Bell et al. (2003) find $a_{\lambda}=-0.628$ and $b_{\lambda}=1.305$; assuming a Kroupa (1993) IMF, they find $C=-0.10$. This yields an expression for the stellar mass in $M_{\odot}$ for a given $B-V$ color:

$$
M_{\star}=v_{\text {lum }} 10^{-0.628+1.305(B-V)-0.10},
$$

where

$$
v_{\text {lum }}=10^{-0.4(V-4.82)} .
$$

Here, $v_{l u m}$ is the luminosity parametrized in terms of absolute $V$ magnitude.

How reliable are stellar masses determined from this procedure? Clearly, the above relationship between $M_{\star}$ and $B-V$ cannot apply to all galaxies, and must depend on the assumed stellar IMF, and range of ages, dust, and metallicity. However, it is encouraging to 
note that several studies (Bell et al. 2003; Drory et al. 2004; Salucci, Yegorova, \& Drory 2008)

find generally good agreement between masses based on broad-band colors and those from spectroscopic (e.g. Kauffmann et al. 2003) and dynamical (Drory et al. 2004) techniques. Typical errors are within a factor of two to three. Salucci et al. (2008) derive disk masses with both photometric and kinematic methods and find the two methods are equivalent on average. For a sample of 18, they find an rms scatter of 0.23 dex, while on an individual basis the deviation can be as high as 0.5 dex.

We used this relation to compute stellar masses for 126 of $143(88 \%)$ objects. The remainder did not have $B-V$ colors available in the Hyperleda database or RC3. The mass distribution is summarized in Figure 2.3. Individual masses are listed in Table 2.1. This sample of 126 galaxies is referenced henceforth as sample S2.

\subsection{Method and Analysis}

The structural properties of galaxy components, such as bulges, disks, and bars can be derived through the decomposition of the 2D light distribution, taking into account the PSF. There are several algorithms for $2 D$ luminosity decomposition, including GIM2D (Simard et al. 2002), GALFIT (Peng et al. 2002), and BUDDA (de Souza et al. 2004). The latter two allow bulge-disk-bar decomposition, while the former only allows bulge-disk decomposition.

Most previous work has addressed $2 D$ bulge-disk decomposition only. Allen et al. (2006), for example, performed bulge-disk decomposition of $B$-band images with GIM2D on 10,095 galaxies from the Millennium Galaxy Catalog (Liske et al. 2003; Driver et al. 2005). However, recent work (e.g., Laurikainen et al. 2005) has shown that the $B / T$ ratio can be 
artificially inflated in a barred galaxy unless the bar component is included in the $2 D$ decomposition. The fact that most $(\geq 60 \%)$ bright spiral galaxies are barred in the NIR (Eskridge et al. 2000; Laurikainen et al. 2004; MJ07; Menendez-Delmestre et al. 2007), further warrants the inclusion of the bar. Another advantage of bulge-disk-bar decomposition is that it allows us to constrain the properties of the bar itself, and to constrain scenarios of bar-driven evolution (see $\S 2.1$ ).

Motivated by these considerations, several studies have tackled the problem of $2 D$ bulge-disk-bar decomposition. Laurikainen et al. $(2005,2007)$ have developed a $2 D$ multicomponent decomposition code designed to model bulges, disks, primary and secondary bars, and lenses; they apply Sérsic functions to bulges and use either Sérsic or Ferrers functions to describe bars and lenses. Reese et al. (2007) have written a non-parametric algorithm to model bars in $\sim 70 I$-band images. Gadotti \& Kauffmann (2007) are performing $2 D$ bulge-disk-bar and bulge-disk decomposition of 1000 barred and unbarred galaxies from SDSS with the BUDDA software.

In this study, we perform $2 D$ two-component bulge-disk decomposition and threecomponent bulge-disk-bar decomposition of the OSUBSGS sample with GALFIT. We note that Laurikainen et al. (2007) have also performed bulge-disk-bar decomposition on the OSUBSGS sample. However, there are also important complementary differences between our study and theirs. The decomposition algorithm and tests on the robustness performed in our study are different (see $\S 2.3$ and $\S 2.4$ ). Furthermore, unlike Laurikainen et al. (2007), we also compare the bulge-to-total ratio $(B / T)$ with predictions from hierarchical models of galaxy evolution (§ 2.5), and also present the distribution of bar-to-total ratio (Bar/T). 


\subsubsection{Image Preparation}

Running GALFIT on an image requires initial preparation. The desired fitting region and sky background must be known, and the PSF image, bad pixel mask (if needed), and pixel noise map must be generated. We addressed these issues as follows: (1) The GALFIT fitting region must be large enough to include the outer galaxy disk, as well as some sky region. Since cutting out empty regions of sky can drastically reduce GALFIT run-time, a balance was sought between including the entire galaxy and some decent sky region, while excluding large extraneous blank sky areas. (2) It is possible for GALFIT to fit the sky background, but this is not recommended. When the sky is a free parameter, the wings of the bulge Sérsic profile can become inappropriately extended, resulting in a Sérsic index that is too high. Sky backgrounds were measured separately and designated as fixed parameters; (3) GALFIT requires a PSF image to correct for seeing effects. Statistics of many stars in each frame can be used to determine an average PSF. However, many of our images contain merely a few stars. Instead, a high $\mathrm{S} / \mathrm{N}$ star from each frame was used as a PSF. (4) We used ordered lists of pixel coordinates to make bad pixel masks, which are useful for blocking out bright stars and other image artifacts. (5) We had GALFIT internally calculate pixel noise maps for an image from the noise associated with each pixel. Noise values are determined from image header information concerning gain, read noise, exposure time, and the number of combined exposures.

\subsubsection{Decomposition Steps}

Figure 2.4 summarizes our method of decomposition, which we now detail. GALFIT requires initial guesses for each component it fits. It uses a Levenberg-Marquardt downhill- 
gradient algorithm to determine the minimum $\chi^{2}$ based on the input guesses. GALFIT continues iterating until the $\chi^{2}$ changes by less than 5e-04 for five iterations (Peng et al. 2002). We recognize that a drawback to any least-squares method is that a local minimum, rather than a global minimum, in $\chi^{2}$ space may be converged upon. We explore this possibility with multiple tests described in $\S 2.4$. We adopted an iterative process, involving three separate invocations of GALFIT, to perform 1-component, 2-component, and 3-component decomposition:

1. Stage 1 (single Sérsic): In Stage 1, a single Sérsic ${ }^{2}$ component is fitted to the galaxy. This serves the purpose of measuring the total luminosity, which is conserved in later Stages, and the centroid of the galaxy, which is invariant in later fits.

2. Stage 2 (exponential plus Sérsic): In Stage 2, the image is fit with the sum of an exponential disk and a Sérsic component. During the Stage 2 fit, the disk $b / a$ and $P A$ are held constant at values, which we take from the published ellipse fits of MJ07, as well as ellipse fits of our own. This procedure reduces the number of free parameters in the fit by fixing the disk $b / a$ and $P A$, which are easily measurable parameters. It also prevents GALFIT from confusing the disk and bar, and artificially stretching the disk along the bar PA in an attempt to mimic the bar. As initial guesses for the Sérsic component in Stage 2, the output of Stage 1 is used. The Sérsic component in Stage 2 usually represents the bulge, in which case Stage 2 corresponds to a standard bulge-disk decomposition.

\footnotetext{
${ }^{2}$ The functional form for the Sérsic profile is $\Sigma(r)=\Sigma_{e} \exp \left[-\kappa\left(\left(\frac{r}{r_{\mathrm{e}}}\right)^{1 / n}-1\right)\right] . \Sigma_{e}$ is the pixel surface brightness at effective radius $r_{\mathrm{e}}$, and $n$ is the Sérsic index. $\kappa$ is a coupled to $n$ and is not a free parameter.
} 
However, in a few rare cases, where the galaxy has just a bar and a disk, the Sérsic component in Stage 2 represents a bar. The latter is recognizable by a low Sérsic index and large half-light radius.

3. Stage 3 (exponential plus two Sérsic components): In Stage 3, a three-component model consisting of an exponential disk, a Sérsic bulge, and a Sérsic bar is fit. As suggested by Peng et al. (2002), the bar can be well described by an elongated, lowindex Sérsic $(n<1)$ profile. As in Stage 2, the disk $b / a$ and $P A$ are held constant at values predetermined from ellipse fits. We provide initial guesses for the bar $b / a$ and $P A$, based on ellipse fits of the images from MJ07 or analysis of the images in DS9. We provide GALFIT with input guesses for the bulge parameters, based on the output from Stage 2. In principle, it is also possible to generate reasonable guess parameters for the bulge and disk from a bulge-disk decomposition on a $1 D$ profile taken along a select PA. As described in $\S 2.4 .3$, we also experiment with initial guesses derived in this way, and find that the final convergence solution is the same. We also note that GALFIT fixes the bulge $b / a$ and does not allow it to vary with radius, while real bulges may have a varying $b / a$. We tested the impact of fixed and varying bulge $b / a$ on the derived $B / T(\S 2.4 .1)$ and find that there is no significant change in $B / T$.

For objects with central point sources, the bulge Sérsic index in the Stage 2 and Stage 3 models can be inadvertently overestimated unless an extra nuclear component is added to the model. Balcells et al. (2003) note that for galaxies imaged both from the ground and HST, the combination of unresolved central sources and seeing effects mimic high-index bulge Sérsic profiles in the ground images. Depending on sample and resolution, the frequency of central sources can range from 50\% to $90 \%$ (Ravindranath et al. 2001; 
Böker et al. 2002; Balcells et al. 2003). Ravindranath et al. (2001) find a frequency of 50\% in early type (E, S0, S0/a) galaxies, while Böker et al. (2002) measure a frequency of $75 \%$ for spirals with Hubble types Scd to Sm. Balcells et al. (2003) determine a frequency of $84 \%$ for S0-Sbc galaxies imaged with HST. Our dataset most closely resembles the latter sample, so we might expect that, as an upper limit, a similar fraction of our galaxies will need to be corrected with an extra compact component.

We added point sources as third or fourth components to the initial models. For those cases where the model successfully converged with the extra component, the images were visually inspected to verify the presence of a central bright source. Sometimes, the model converged to significantly different and unreasonable parameters for all components. Other times, the model would converge to a very dim point source without changing any of the other model parameters. Where new model parameters were not unreasonable and not identical to the case without the point source, the new model was adopted. This was the case for 111 of $143(78 \%)$ of our sample. The point sources contribute less than $1 \%, 3 \%$, and $5 \%$ of the total luminosity $55 \%, 86 \%$, and $95 \%$ of the time, respectively. As the point sources take up such a small fraction of the light distribution, their contribution is folded back into $B / T$ in all cases where a point source was modeled. Inclusion of the point source reduced bulge index by $\sim 0.8$ for both barred and unbarred galaxies. Such a change is expected based on the above discussion. The decline in bulge index caused a minor decrease in $B / T$; on the mean, this change was $1.04 \%$ for barred galaxies and $0.32 \%$ for unbarred galaxies. For barred galaxies, this light most primarily added to $D / T$ rather than Bar $/ T$.

It is important to recognize the physical significance of the added nuclear components. We began by determining which objects show evidence for AGN activity. The sample 
was checked against the catalog of Ho et al. (1997), the Véron Catalog of Quasars \& AGN, 12th Edition (Véron-Cetty \& Véron 2006), and NED. Of the 111 objects fit with point sources, $43(39 \%)$ contain AGN. An additional 20 (18\%) possess HII nuclei according to Ho et al. (1997) and visibly show bright compact nuclei. The remaining 48 (43\%) probably contain neither AGN nor HII nuclei, but could house nuclear star clusters. For these objects, we visually inspected the images to ensure there was a bright compact source at the center. We are confident that the fitted point source components have physical counterparts in the data images.

GALFIT also allows a diskiness/boxiness parameter to be added to any Sérsic or exponential profile. We did not use this parameter for any bulge or disk profiles. Bars in general have boxy isophotes, and we could have included the diskiness/boxiness parameter in the bar profiles. However, it was found that adding boxiness to the bar did not change any model parameters, including fractional luminosities $B / T, D / T$, and Bar $/ T$, by more than a small percentage, even though the appearance of the residual images improved in some cases due to the change in bar shape. Consequently, we chose to neglect bar boxiness altogether.

\subsubsection{Choosing the Best Fit Between Stage 2 and Stage 3}

All objects in our sample were subjected to Stages 1, 2, and 3. Depending on whether a galaxy with a bulge is unbarred or barred, its best fit should be taken from the Stage 2 bulge-disk decomposition or the Stage 3 bulge-disk-bar decomposition, respectively. For objects with prominent bars, it is obvious that the Stage 3 model provides the best fit. However, it is more difficult to decide between Stage 2 versus Stage 3 fits in galaxies which 
host weak bars with no strong visual signature. In practice, we therefore applied the set of criteria below to each galaxy in order to select between the Stage 2 bulge-disk decomposition and Stage 3 bulge-disk-bar decomposition. Table 2.1 lists the model chosen for each galaxy. Table 2.2 summarizes the model parameters from the best fits.

For completeness, we note that for the few rare galaxies (see $\S 2.3 .2$ ) that have just a bar and a disk, the choice of a final solution is between the Stage 2 bar-disk decomposition and Stage 3 bulge-disk-bar decomposition. The same guidelines below can be used to identify the best model.

1. GALFIT calculates a $\chi^{2}$ and $\chi_{\nu}^{2}$ for each model. It was found that $\chi^{2}$ almost universally declines between the Stage 2 and Stage 3 fits for a given object. This is because in the Stage 3 fit, five extra free parameters (bar luminosity, $r_{\mathrm{e}}$, Sérsic index, $b / a$, and $P A)$ are added with the Sérsic bar component, allowing GALFIT to almost always make a lower $\chi^{2}$ model during Stage 3 . However, this does not necessarily mean that the solution in Stage 3 is more correct physically. Thus, an increasing $\chi^{2}$ was interpreted as a sign that the Stage 3 fit should not be adopted, but a decreasing $\chi^{2}$ was not considered as a sufficient condition to adopt Stage 3 .

2. In cases with prominent bars, a symmetric light distribution due to unsubtracted bar light was often found in the Stage 1 and Stage 2 bulge-disk residuals. This was strong evidence that the Stage 3 bulge-disk-bar fit be selected. NGC 4643 is shown in Figure 2.5 because it has a particularly striking bar residual; the corresponding fit parameters appear in Table 2.3. Note that in all figures and tables, we adopt the convention that PA values are positive/negative if they are measured from North 
counterclockwise/clockwise.

3. The Stage 2 and Stage 3 models were selected only so long as the model parameters were all well behaved. In unbarred galaxies, the Stage 3 model parameters might be unphysically large or small, in which case the Stage 2 fit was favored. Conversely, in galaxies with prominent bars, the bulge component of the Stage 2 bulge-disk fit tends to grow too extended in size. Addition of a bar in the Stage 3 bulge-disk-bar fit removes this artifact, giving a more physical solution. An extreme example of this situation is the barred galaxy NGC 4548, which has a prominent bar and a faint disk. The Stage 2 fit, based on a Sérsic bulge and exponential disk, is highly inadequate to describe the bulge, disk, and the bar because it leads to an extremely extended bulge. The Stage 3 bulge-disk-bar fit, however, yields a believable fit with a prominent bar. The results of Stage 1, Stage 2, and Stage 3 are displayed in Figure 2.6 and Table 2.4.

4. Not all barred galaxies had unphysical Stage 2 models. Instead, the bulge could be stretched along the $P A$ of the bar, giving the bulge a lower Sérsic index and larger effective radius. A Stage 3 model that returned the bulge to a size and shape more representative of the input image was favored over the Stage 2 fit. Figure 2.7 and Table 2.5 demonstrate this behavior in NGC 4902. We distinguish this effect from cases like NGC 4548 (Figure 2.6 and Table 2.4) where the Stage 2 fit is completely wrong.

5. In cases where there was no bar, GALFIT can sometimes be enticed into fitting a bar to any existing spiral arms, rings, or the clumpy disks of late-type spirals. Stage 3 fits in these cases could be discarded by noting the resulting discrepancies in appearance 
between the galaxy images and the Stage 3 model images. Examples of false bars are shown in Figure 2.8 .

After fitting the whole sample and picking the best fit from either the Stage 2 bulge-disk decomposition or the Stage 3 bulge-disk-bar decomposition, we also performed the following extra tests. For our sample S1 of 143 bright $\left(M_{B} \leq-19.3\right)$ low-to-moderately inclined $\left(i \leq 70^{\circ}\right)$ spirals in the OSUBSGS survey, we determine the fraction (75 of 143 or $\sim 52 \%$ ) of spiral galaxies where a bulge-disk-bar decomposition was picked as the best fit for the $H$-band image. There are also eight galaxies with pure bar-disk fits. The $H$-band bar fraction, which is defined as the fraction of disk galaxies that are barred, is therefore $58.0 \pm 4.13 \%$ (83 of 143$)$. We then compared our results $(58.0 \% \pm 4.13 \%)$ with the $H$-band bar fraction (60\%) determined from ellipse fits of the OSUBSGS sample by MJ07, with a slightly more conservative inclination cut $\left(i \leq 60^{\circ}\right)$. The two numbers are in excellent agreement. As a further check to our fits, we compare the bar and unbarred classification for individual galaxies from our fits with those from MJ07, which were based on ellipse fits. Of the 73 galaxies that we classify as barred, and that are mutually fitted by MJ07, 54 (74\%) are also classified as barred by MJ07. The remaining 19 (26\%) galaxies are mainly weakly barred (with Bar/T below 0.08). Their RC3 optical types are weakly barred AB (10), barred B (7), and unbarred A (2).

In most previous bulge-disk and bulge-disk-bar $2 D$ decomposition, the issue of parameter coupling and the systematic exploration of local versus global minima in $\chi^{2}$ have been ignored. Quantifying how the parameters are coupled is important in measuring error bars for the model parameters. With $2 D$ models containing several free parameters, this 
is not an easy task. Although we also do not address this problem in rigorous detail, we describe in $\S 2.4 .4$ simple test that explores parameter coupling in our models.

\subsection{Extra Tests to Verify Correctness of Fits}

\subsubsection{Varying $b / a$ as a Function of Radius}

Models generated with GALFIT do not allow the $b / a$ of the bulge, disk, or bar to vary with radius. Since real bulges may have a varying $b / a$, it is legitimate to investigate the impact of fixing the bulge $b / a$ on the estimated $B / T$. We therefore performed the following test on NGC 4548. To mimic a model bulge of varying $b / a$, we fitted the bulge light of NGC 4548 with ten concentric Sérsic profiles of increasing $r_{\mathrm{e}}$ and varying $b / a$. The $r_{\mathrm{e}}$ of the outermost profile comes from the original bulge model (see Table 2.4) where $b / a$ was kept constant with radius. The separation in $r_{\mathrm{e}}$ between adjacent profiles is 0.5 pixels $(0.75 ")$. The luminosity, Sérsic index, $b / a$, and $P A$ of each profile were free parameters. The disk and bar components were fixed to the values in Table 2.4, as the emphasis was on the change in the bulge.

Figure 2.9 compares the $B / T$ obtained by fitting the bulge of NGC 4548 with a Sérsic model of constant $b / a$ as opposed to a Sérsic model varying $b / a$. The bulge $b / a$ (0.88), $P A(-66.5)$, and $B / T$ (13\%) from the original Sérsic fit of constant $b / a$ (Table 2.4) are indicated with horizontal lines on the 3 panels. The top two panels show the run of $b / a$ (0.85 to 1.0$)$ and $P A\left(-90^{\circ}\right.$ to $\left.+90^{\circ}\right)$ of the ten concentric Sérsic profiles. It can be seen that the Sérsic indices of the ten bulge models were generally higher toward the center and declined at larger $r_{\mathrm{e}}$, indicating that the 'fitted bulge is more concentrated at the center. The bottom panel shows the cumulative $B / T$ calculated by summing all models with $r \leq r_{\mathrm{e}}$. 
The last point representing the summed $B / T$ from all ten components is $14.5 \%$, in good agreement with the $13.0 \%$ value from the Sérsic fit of constant $b / a$. Thus, using a Sérsic model of constant $b / a$, does not have any significant adverse impact on our derived $B / T$ in NGC 4548.

\subsubsection{Fitting Artificially Simulated Images}

An elementary test is to determine if GALFIT can recover the known parameters of artificially simulated noisy images. The images were simulated by taking parametric model images produced by GALFIT, and adding noise to the images with the PyFITS module for Python (Barrett \& Bridgman 1999). Noise in each pixel was calculated by adding in

quadrature the noise due to the source, sky, and read noise. The standard deviation of pixel noise in electrons was computed as

$$
\sigma=\sqrt{T_{\text {source }}+T_{\text {sky }}+T_{\text {read }}^{2}},
$$

where $T_{\text {source }}$ is the number of electrons due to the source, $T_{s k y}$ is the number of electrons due to the sky, and $T_{\text {read }}$ is the detector read noise. The contribution due to detector dark current was very small and therefore neglected. The offset added to each pixel was drawn from a normal distribution centered at zero with standard deviation $\sigma$.

Our test sample consisted of 40 models (20 bulge-disk and 20 bulge-disk-bar) with noise added as described. Thirty of the images included point sources as extra components. The range explored for each parameter in the model images is a fair representation of the parameter space covered by our full sample (e.g., $B / T$ ranges from $0.02-0.70$, the bulge index ranges from $\sim 0-5$, and the full range of possible bulge and bar PA was also tested $\left(-90^{\circ}\right.$ to $\left.\left.+90^{\circ}\right)\right)$. In terms of surface brightness, the models span five magnitudes in mean 
surface brightness inside the disk scalelength. Examples of the noise-added models are shown in Figure 2.10.

The noisy images were subjected to the $2 D$ decomposition procedure outlined in Figure 2.4. GALFIT reproduced the model (bulge, disk, bar, and point source) parameters quite closely for the majority of the test cases. Figure 2.11 compares the recovered versus original model parameters. Except for some extreme cases where the images were highly distorted by noise, all parameters were recovered to within a few percent. Figure 2.12 plots the ratio of model-to-recovered parameter against mean surface brightness inside the disk scalelength; there is no strong trend in error with dimming surface brightness. This suggests our decompositions are effective across the parameter space spanned by our sample. The overall success of this test is evidence that GALFIT is able to converge to the absolute minimum in $\chi^{2}$ space for our bulge-disk and bulge-disk-bar decompositions when the input is the sum of parametric functions.

\subsubsection{Using $1 D$ Decomposition To Generate Guesses for Bulge Parameters}

It is important to verify that GALFIT converges to the same solution even if the initial guesses for the bulge parameters in Stage 2 and 3 are different. Bulge-disk decomposition from $1 D$ profiles provides an alternative means of generating initial guesses. While $1 D$ bulge-disk decompositions of radial profiles along the bar major axis can be influenced by the bar, decomposition of cuts along the bar minor axis will not be influenced as heavily. The resulting bulge and disk parameters should be adequate guesses for Stage 3 of our $2 D$ decomposition method.

We tested the robustness of our Stage 3 fits by extracting initial guesses for the bulge 
and disk using $1 D$ decomposition along the bar minor axis. The nonlinear least-squares algorithm designed to perform the $1 D$ decomposition simultaneously fits the sky-subtracted profiles with the sum of a Sérsic bulge and an exponential disk, while ignoring the PSF. The results from the $1 D$ decomposition include a bulge magnitude, $r_{\mathrm{e}}$, Sérsic index, disk magnitude, and disk scalelength.

The robustness of several bulge-disk-bar fits were tested by using the results of the $1 D$ decomposition as input to Stage 3 . The $1 D$ decompositions do not provide information about the axis ratio $(b / a)$ or $P A$, so these parameters for the bulge were estimated by eye; for the disk, the $b / a$ and $P A$ were fixed to the values determined by ellipse fitting, as described in $\S 2.3 .2$. The initial bar parameters were unchanged from the earlier Stage 3 fits. In all cases, the new models were identical to the Stage 3 models. As an example, Table 2.6 compares Stage 3 input derived from 1D decomposition and GALFIT for NGC 4548 and NGC 4643. In each case, both sets of input reproduced the same results.

\subsubsection{Parameter Coupling}

Assessing the coupling between model parameters is complicated when models have a large number of free parameters. A standard approach is to calculate confidence regions using multi-dimensional ellipsoids for a given $\Delta \chi^{2}$ contour. As the errors in the GALFIT models are not normally distributed, but are instead dominated by the systematics of galaxy structure, this approach does not yield meaningful results because of the ambiguity in assessing which $\Delta \chi^{2}$ contour levels are statistically significant.

We carry out a simple test for representative galaxies to determine not only the effects of parameter coupling, but also the the effect parameter coupling has on model 
parameter errors, paying particularly close attention to $B / T, D / T$, and $B a r / T$, as they are of primary interest. We perform this test on four representative galaxies (NGC 3885, NGC 4151, NGC 4643, and NGC 7213). Two are barred (NGC 4151 and NGC 4643). Two have high $(n>2)$ bulge indices (NGC 4643 and NGC 7213), and the other two have low $(n<2)$ bulge indices.

We fit two and three-component bulge-disk and bulge-disk-bar models using fixed bulge indices of $n=1$ and $n=4$. The initial inputs to these fits were the same as those used to generate the model in which bulge $n$ is a free parameter. We then had GALFIT re-fit the models with bulge index as a free parameter using these output model parameters as input initial guesses. We compare the output of these two fits with the the best fit as selected in $\S 2.3 .3$, in which bulge $n$ is a free parameter. Ideally, the re-fits should converge to the same final parameters as the selected best fit.

Table 2.7 displays the outcome of this test for the four representative systems. For barred galaxies NGC 4643 and NGC 4151, the $n=1$ and $n=4$ re-fits converged to the same $\chi^{2}$ as when bulge $n$ is initially left a free parameter. $B / T, D / T$, and $B a r / T$ are precisely equal for NGC 4643, while for NGC 4151 there is a small dispersion of $0.1-0.2 \%$. For unbarred galaxies NGC 3885 and NGC 7213, the $n=1$ re-fits again converged to the same $\chi^{2}$ and model parameters as when bulge $n$ is initially left a free parameter. This is not true for the $n=4$ re-fits. When the $n=4$ condition is enforced, the bulges in these cases become too extended and luminous while the $\chi^{2}$ drop below those when bulge $n$ is initially left a free parameter. During the $n=4$ re-fits, $B / T$ increases further at the expense of $D / T$ and the $\chi^{2}$ remain unchanged or decrease further. The $B / D$ ratio from the $n=4$ re-fit for NGC 3885 is 1.3 , roughly 3.5 times higher than when $n$ is free. Given that NGC 3885 is an 
S0/a galaxy with a bulge embedded in a smooth extended disk, the latter $B / D$ is arguably too large. For NGC 7213 , the $n=4$ re-fit yields starkly unphysical values. The $B / D$ ratio is 11.3 , and $r_{\mathrm{e}} / h$, the ratio between bulge effective radius and disk scalelength is 9.0. The lower $\chi^{2}$ in these cases cannot be taken seriously as the bulges are too luminous and the resulting $B / D$ ratios do not match the data images.

As illustrated by the above discussion and Table 2.7, this test shows that in some cases (e.g., NGC 4643 and NGC 4151) GALFIT converges to the similar model parameters and $B / T, D / T$, and Bar $/ T$ while starting from highly different initial input guesses (e.g. bulge $n=1$, bulge $n=4$, and bulge $n$ based on the Stage 2 fits) in different regions of parameter space. For NGC 3885 and NGC 7213, however, during the $n=4$ re-fits GALFIT converged to models that were unphysical and different compared with the reasonable models generated with input guesses corresponding to the bulge fixed at $n=1$ or the bulge $n$ based on output from Stage 1. In effect, when the initial input guesses were very different from the data images, the resulting models were found, in spite of the lower $\chi^{2}$, to be unphysical through comparison with the input data images. We emphasize that for all sources analyzed in the paper, the data, converged model output, and residuals are always inspected before adopting the best final fit (see $\S 2.3 .3$ ).

Table 2.7 also provides hints as to how the model parameters are coupled to bulge index. As suggested in the above discussion, fixing the bulge index to $n=4$ leads to a more extended and luminous bulge, causing bulge $r_{\mathrm{e}}$ and $B / T$ to rise without fail for increasing bulge index. The disk is coupled with the bulge such that increasing bulge index, bulge $r_{\mathrm{e}}$, and $B / T$ yields a reduction in $D / T$. At the same time, disk scalelength either increases (NGC 3885 and NGC 4643) or decreases (NGC 3885 and NGC 4151); in the latter two 
cases, the disk becomes very compact and the bulge quite extended. The behavior of the bar is coupled with both the bulge and disk. In the case of NGC 4643, as bulge index is raised to $n=4$, bar $r_{\mathrm{e}}$ becomes slightly larger, but Bar $/ T$ falls by a factor of 2.6 as light is redistributed from the bar and disk to the bulge. Bar index also declines as the bar assumes a flatter profile. For NGC 4151, bar $r_{\mathrm{e}}$ again increases slightly, but this time Bar/T rises by a factor of 1.7 as light is transferred from the disk to the bulge and bar.

Based on the above test, we stress that GALFIT was able to overcome this parameter coupling in the cases where the input guess parameters well-reflected the data images.

\subsection{Results and Discussion}

\subsubsection{Impact of Bars in $2 D$ Decomposition}

From the Stage 2 bulge-disk decomposition and Stage 3 bulge-disk-bar decompositions, which we performed on all objects $(\S 2.3 .2)$ we saw firsthand the effects of adding a bar to the fit of a barred galaxy. We summarize below some of these effects in order to underscore the importance of including a bar component in the $2 D$ luminosity decomposition of barred galaxies

1. During the Stage 2 bulge-disk decomposition of a barred galaxy, the luminosity which comes from the galaxy's disk, bulge, and bar gets distributed only between two model components: the model bulge and disk. Since the disk $b / a$ and $P A$ are measured independently and held constant during the fits, the Stage 2 model tends to distort the bulge in order to fit the bar. Thus, the bulge in the Stage 2 bulge-disk decomposition of a barred galaxy can be artificially long or too bright and extended. When a model bar component is added in the Stage 3 bulge-disk-bar decomposition of a barred galaxy, 
it forces a reshuffling of the luminosity between the three components. Generally, the bulge declines in luminosity, whereas light can be either taken from, or added back, to the disk.

2. We find that the inclusion of a bar component in the Stage 3 bulge-disk-bar decomposition of a barred galaxy reduces the bulge fractional luminosity $B / T$, compared with the Stage 2 bulge-disk decomposition. For our 75 barred galaxies, the reductions correspond to factors of less than two, 2 to 4 , and above 4 , in $36 \%, 25 \%$, and $39 \%$ of barred galaxies, respectively. The larger changes in $B / T$ occur in very strongly barred galaxies, where a prominent bar cause the Stage 2 bulge-disk decomposition to overestimate the bulge. For instance, $B / T$ declines in both of NGC 4643 (Figure 2.5 and Table 2.3) and NGC 4548 (Figure 2.6 and Table 2.4). In the latter case, $B / T$ is reduced by a factor of $\sim 5$ between Stage 2 and Stage 3 . These examples underscore the importance of including bars in $2 D$ luminosity decomposition of very strongly barred galaxies.

3. The scalelength of the disk is generally unchanged by including the bar. NGC 4548 (Figure 2.6 and Table 2.4) is a good example. Sometimes, however, the disk from the Stage 2 bulge-disk decomposition of a barred galaxy is erroneous due to a poor fit. The disk parameters from the Stage 3 bulge-disk-bar decomposition are quite different in such cases. NGC 4643 (Figure 2.5 and Table 2.3) illustrates this behavior.

We find that for our sample S1 of 143 bright $\left(M_{B} \leq-19.3\right)$ low-to-moderately inclined $\left(i \leq 70^{\circ}\right)$ spirals (Figure 2.1) in the OSUBSGS survey, 75 of 143 or $\sim 52 \%$ are better fit with a Stage 3 bulge-disk-bar decomposition than a Stage 2 bulge-disk decomposition. 
There are also eight galaxies with pure bar-disk fits. As stated in $\S 2.3 .3$, the resulting $H$ band bar fraction $(58.0 \% \pm 4.13 \%$ or 83 of 143$)$ is in excellent agreement with the $H$-band bar fraction of $60 \%$ reported by MJ07 based on ellipse fits of the OSUBSGS sample, with a slightly more conservative inclination cut $\left(i \leq 60^{\circ}\right)$.

\subsubsection{Mass in Bulges, Disks, and Bars}

The fractional $H$-band luminosities in the bulge, disk, and bar $(B / T, D / T$, Bar $/ T)$ of each galaxy can be considered as a fractional mass if we assume that the same mass-tolight $(M / L)$ ratio can be used to convert the $H$-band luminosities of both the numerator $(B$, $D$, or Bar) and the denominator $(T)$ terms into a stellar mass. This is not an unreasonable assumption as the $H$-band $M / L$ ratio is not very sensitive to differences in dust or age that might exist between the bulge, disk, and bar. The uncertainties in $M / L$ can be estimated by looking at population synthesis models. Charlot, Worthey, \& Bressan (1996) find that for idealized galaxies with a single generation of stars, the uncertainties in $M / L$ ratio due to different input stellar models and spectra are roughly $\pm 35 \%$ for a fixed metallicity and IMF. Furthermore, as the age of a stellar population varies from $\sim 0.5$ Gyr to 10 Gyr, the $K$-band $M / L$ ratio rises by a factor of $\sim 2$ to 3 (Charlot 1996). Asymptotic giant branch (AGB) stars dominate the NIR light for ages between 0.1 and 1 Gyr, while red giant branch (RGB) and super-giant branch (SGB) stars dominate between 1 Gyr and 10 Gyr.

In this paper, we convert the $B / T$ light ratio determined from $H$-band images to a $B / T$ mass ratio by assuming a constant mass-to-light $(M / L)$ in the $H$-band for both the bulge and the rest of the galaxy. However, Moorthy \& Holtzman (2006) present line strengths of bulges and inner disks for 38 spirals with Hubble types S0 to Sc. They show $76 \%$ 
of spirals have negative metallicity gradients. $B-K$ color gradients are shown to largely match metallicity gradients and are likewise negative outward, indicating bulge $M / L$ is higher than in the disk and bar. If bulges are much older than the disks, then our prescription would underestimate the true $B / T$ mass ratio. If we assume an extreme case where bulges are $\sim 12$ Gyr and the disk light is dominated by a young 3 Gyr population, our assumption of a constant $H$-band $M / L$ ratio would underestimate the true $B / T$ by a factor of $\leq 2$ (Charlot 1996). In several sections of the paper (e.g., $\S 2.5 .3, \S 2.5 .8$ ), we illustrate how our main results would change if the true $B / T$ was higher by up to a factor of two. On the other hand, central regions of galaxies may harbor intense episodes of star formation. If the bulge is younger than the disk and happens to have star formation and a significant young population of massive stars, then our prescription could overestimate the true $B / T$ mass ratio. This would make our current results on the high fraction of low $B / T$ bulges even stronger.

Using the total galaxy stellar mass from $\S 2.2 .2$, the fractional masses can be converted into absolute masses. (We do not convert the $H$-band luminosity directly into a mass as the $H$-band images do not have photometric calibration). The results are shown in Table 3.6. For our sample S1 of 143 bright $\left(M_{B} \leq-19.3\right)$ low-to-moderately inclined $\left(i \leq 70^{\circ}\right)$ spirals with a mass-weighted mean Hubble type of Sab-Sb, we find that $\sim 70 \%$ of the stellar mass is in disks, $\sim 10 \%$ is in stellar bars and $\sim 20 \%$ is in bulges (with $\sim 11 \%$ in $n>2$ bulges and $\sim 9 \%$ in $n \leq 2$ bulges). Thus while bulges with $n \leq 2$ are highly ubiquitous (see next section), they only account for a small fraction of the total stellar mass budget.

Figure 2.13 shows the stellar mass for bulges, disks, and bars along the Hubble 
sequence.

It is useful to compare our results with those of Driver et al. (2006), who performed bulge-disk decomposition of $B$-band images with GIM2D on 10,095 galaxies from the Millennium Galaxy Catalog (Liske et al. 2003; Driver et al. 2005). They found $68.6 \%$ of the stellar mass to be in disks, and $32.6 \%$ in bulges (with $30.8 \%$ in high $n$ bulges, and $1.8 \%$ in low $n \leq 2$ bulges). Their study thus finds a higher stellar mass fraction in all bulges (32.6\% vs our $18.9 \%$ ), and in high $n$ bulges ( $30.8 \%$ vs our $10.4 \%)$, and a lower fraction in low $n \leq 2$ bulges ( $1.8 \%$ vs our $8.4 \%)$, and disks+bars $(68.6 \%$ vs our $71.6 \%+9.6 \%)$. This difference can be attributed to the fact that the Driver et al. (2006) study did not perform bulge-disk-bar fits and thus, their $B / T$ ratios may be skewed to higher values.

\subsubsection{Distribution of Bulge Index and $B / T$}

Figure 2.14 shows the individual and mean $B / T$ and bulge Sérsic index, plotted, as a function of Hubble type and galaxy stellar mass. Barred and unbarred galaxies are shown separately. Figure 2.15 shows the relationship between bulge index and $B / T$.

We first consider the $B / T$ values in Figure 2.14. The mean $B / T$ in barred galaxies is lower than in unbarred galaxies, but there is a large overlap in the individual values. The offset in the mean $B / T$ of barred and unbarred galaxies reported here, agrees with the result of Laurikainen et al. (2007; see $\S 2.5 .4)$ on the same sample. We also note that $B / T$ does not correlate with Bar/T (Fig. 2.16): aside from the six galaxies with large Bar/T (>0.3), most galaxies have low-to-moderate Bar/T and a wide range of $B / T$ is seen at each Bar/T. This is reassuring and suggests that the bar fit is not arbitrarily biasing the $B / T$ values. The distribution of Bar/T is further discussed in $\S 2.5 .5$. 
How does the $B / T$ vary as a function of Hubble type and galaxy stellar mass? Bulges with very high $B / T(>0.4)$ exist primarily in galaxies with high mass $\left(M_{\star}>6 \times 10^{10} M_{\odot}\right)$ and early types (S0/a to Sab). Bulges with very low $B / T(<0.1)$ lie primarily in lower mass galaxies with later morphologies (Sb to $\mathrm{Sc})$. It is striking that $\sim 69 \%$ of bright $\left(M_{B} \leq-19.3\right)$ low-to-moderately inclined $\left(i \leq 70^{\circ}\right)$ spirals have $B / T \leq 0.2$ : these bulges are pervasive and exist across the whole spectrum of S0/a to Sd. The results are summarized in Table 2.9. We shall return to this point in $\S 2.5 .8$. We note again that these $B / T$ mass ratios were calculated assuming a constant $M / L$ ratio in the $H$-band for the bulge and disk components. As noted in $\S 2.5 .2$, if the bulge in these high mass spirals is much younger (older) than the disk and bar, then the $B / T$ can be overestimated (underestimated) by up to a factor of two, and the limiting value of 0.2 for the $B / T$ cited in the above fraction, would have to be modified in the extreme case to 0.1 and 0.4 , respectively.

Some of the low $B / T \leq 0.2$ values for six barred S0/a and Sa galaxies in Figure 2.14 may at first look suspicious. Balcells et al. (2007a) report the mean $B / T$ for S0 galaxies to be 0.25 , so much smaller $B / T$ are potentially worrisome. OSUBSGS $H$-band images of these objects in Figure 2.17 show a smooth extended disk around the bulge. It should be noted that Hubble types were originally assigned on a combination of criteria including disk smoothness and spiral arm topology in addition to the prominence of the bulge. It is likely that these galaxies were assigned early Hubble types due to their smooth extended disks, in spite of their low bulge-to-disk ratio.

Similarly, some of the high $B / T \sim 0.4$ bulges in three of the Sc galaxies may at first seem odd. However, again, visual inspection of their image (Figure 2.17) reveals prominent spiral arms and clumpiness, which may explain why they were assigned late Hubble types. 
How does the bulge Sérsic index $n$ vary as a function of Hubble type, and galaxy stellar mass (Figure 2.14), as well as $B / T$ (Figure 2.15)? The results are summarized in Table 2.9. Only a small fraction $(\sim 1 \%)$ of bright spirals have high $n \geq 4$ bulges; such bulges lie primarily in S0/a to Sab, and have a large $B / T>0.2$. A moderate fraction $(\sim 22 \%)$ have intermediate $2<n<4$ bulges; these exist in barred and unbarred S0/a to Sd, and their $B / T$ spans a wide range (0.03 to 0.5$)$ with a mean of 0.29 . A strikingly large fraction

( 76\%) of bright spirals have low $n \leq 2$ bulges; such bulges exist in barred and unbarred galaxies across a wide range of Hubble types, and their B/T varies from 0.01 to 0.4 , with most having $B / T \leq 0.2$.

\subsubsection{Comparison With Other Work}

As an independent check of our decomposition method, we compare our results with independently published decompositions.

We find our mean $H$-band $B / D$ (Figure 2.18) ratios are comparable to the $K$ band $B / D$ derived with the $1 D$ bulge-disk decompositions of Graham (2001) and Trujillo et al. (2002). Like Graham (2001) and Trujillo et al. (2002), we find $B / D$ is widely variable with Hubble type and that mean $B / D$ steadily declines from Sa through Scd galaxies. Graham (2001) and Trujillo et al. (2002) find bulge indices are widely variable within a Hubble type, but they are in general $>1$ for early types and $<1$ for late types. We likewise find wide scatter in bulge index with $n<1$ bulges existing in both early and late types. Figure 2.18 is also in good agreement with the more recent results of Graham \& Worley (2008), who have calculated, as a function of morphology, inclination and dust-corrected $B / D$ and bulge Sérsic indices. They find $B / D$ values are typically $<1 / 3$. 
Another meaningful comparison can be made with Laurikainen et al. (2007) who, using their own $2 D$ decomposition code, fit a hybrid sample containing some OSUBSGS galaxies combined with additional S0 galaxies. One difference between their work and ours is that they typically model bars with a Ferrers function, but may sometimes use a Sérsic profile, while we use only the latter. Also, they include additional components to model secondary bars or inner disks. They report a distinct offset in the mean $B / T$ between barred and unbarred galaxies, which we confirm in Figure 2.14. Their mean $B / T$ are similar to ours, and they conclude that pseudobulges exist throughout the Hubble sequence. The Sérsic indices derived by Laurikainen et al. (2007) are likewise similar, on the mean, to ours for both barred and unbarred systems. They likewise find $n \leq 2$ bulges across early and late Hubble type galaxies.

Balcells et al. (2003) emphasized that bulges typically have indices $\sim 3$ or lower. Our results in Figure 2.14 are consistent. We find a low frequency $(\sim 1 \%)$ of high $n \geq 4$ bulges, with most bulges having $n \leq 3$.

\subsubsection{Bar Strength}

Stellar bars exert gravitational torques on the gaseous component and are particularly efficient in driving gas from the outer disk into the inner kiloparsec (see $\S 2.1$ ). Thus, it would be natural to have a measure of bar strength that is sensitive to the strength of the gravitational torque and hence measures both the shape and mass of the bar.

Many measures of bar strength have been formulated. The $Q_{b}$ method of (Block et al. 2002; Buta et al. 2003; Buta et al. 2005) measures directly the gravitational torque at a single point along the bar. This method requires a value of scaleheight for the disk 
and a model of the potential to be made from the image. In the bar/inter-bar contrast method of Elmegreen \& Elmegreen (1985) and Elmegreen et al. (1996), bar strength is parameterized as the ratio between peak surface brightness in the bar region and the minimum surface brightness in the inter-bar region. Elmegreen \& Elmegreen (1985) and Elmegreen et al. (1996) also characterize bar strength with the maximum amplitude of the $m=2$ mode from Fourier decomposition. When ellipse fitting is applied, the maximum ellipticity of the bar, $e_{b a r}$, can be used to characterize bar strength (e.g. MJ07). This constitutes a partial measure of bar strength only, however, as it offers no information about mass of the bar.

Bulge-disk-bar decomposition in the $H$-band provides another partial measure of bar strength through the $H$-band Bar/T light ratio, which is a measure of the Bar/T mass ratio under the assumption that the $H$-band $M / L$ ratio is the same for the bar and the rest of the galaxy, as discussed in $\S 2.5 .2$. Figures 2.19 and 2.20 explore the derived bar properties.

The upper left panel of Figure 2.19 plots the individual and mean Bar/T against Hubble type. There is a wide range $(\sim 0.03$ to $\sim 0.47)$ in the individual $\mathrm{Bar} / T$ at a given Hubble type. The mean Bar/T remains fairly constant with Hubble type from Sa to Sb, but shows a possible weak decline by about 0.1 from $\mathrm{Sb}$ to Sc. Their number statistics are too small to make any robust statement for later Hubble types. We also note that six systems have high Bar/T above 0.3; these are displayed in Figure 2.21.

Bar Sérsic indices are mostly below unity. Neither the individual, nor the mean bar Sérsic index, show any trend with Hubble type or with stellar mass, for Sa to Sc galaxies (Fig. 2.19). Thus, the steepness of the bar profile does not seem to depend on the Hubble type. Is the bar mass ratio and its mass profile related? There is a wide range in the 
individual Bar/T at a given bar Sérsic index (Fig. 2.20). The mean Bar $/ T$ rises with bar index out to a bar index of $\sim 0.6$, and then flattens out. This suggests that on the mean, bars of lower Bar/T have flatter profiles.

Is there a relation between the bar strength and the bulge present in a galaxy? There is a wide range in the individual Bar/ $T$ at a given $B / T$, and at a given bulge Sérsic index (Fig. 2.20). The mean Bar/T shows a weak decline for bulge Sérsic indices above two. Similarly the mean Bar/T shows a weak rise from 0.1 to 0.25 as $B / T$ rises out to 0.15 , after which the trend flattens or reverses.

Both Bar/T and maximum bar ellipticity $e_{b a r}$ are partial measures of bar strength. Figure 2.19 shows mean Bar/T may scale weakly with Hubble type. The bars with highest $e_{b a r}$ (i.e, thin bars) are often termed strong bars, and $e_{b a r}$ has been shown to correlate with $Q_{b}$. Total bar strength should scale with both bar mass and bar ellipticity. Does bar strength have a dependence on Hubble type? The upper left panel of Figure 2.20 plots the product of Bar $/ T$ and $e_{b a r}$, as determined by MJ07 for galaxies mutually classified as barred, against Hubble type. There is a wide range in Bar $/ T \times e_{b a r}$ in each bin, and mean bar strength shows no definite trend with Hubble type. We note that bars with high Bar/T and high $e_{b a r}$ should exert the largest gravitational torque and be most effective at driving gas inflows. A nice example is the oval or lens galaxy NGC 1317 (Figure 2.21); the bar has a low ellipticity, but its $B / T$ is large as it is extended and massive. Such bars or lenses may exert significant gravitational torques although they are not very elongated. 


\subsubsection{Bar Fraction as a Function of $B / T$ and Bulge Index}

As outlined in $\S 2.5 .3$, we found that as many as $\sim 76 \%$ of bright spirals have bulges with $n \leq 2$; such bulges exist in barred and unbarred galaxies across a wide range of Hubble types, and their spread in $B / T$ is from 0.01 to 0.4 , with most having $B / T \leq 0.2$. The variation of the bar fraction as a function of $B / T$ and bulge $n$ can provide important constraints on bulge formation scenarios $(\S 2.5 .8)$. Table 2.10 shows our results. The bar fraction declines with bulge index; $\sim 65 \%$ of the spirals with low $n \leq 2$ bulges host bars while intermediate $2<n<4$ bulges have a lower bar fraction $(\sim 38 \%)$. The high $n \geq 4$ bulges in the sample are unbarred, so the bar fraction is $0 \%$. Systems with low $B / T$ are more likely to be barred. For $B / T \leq 0.2$, the bar fraction is high $(\sim 68 \%)$. Systems with $0.2<B / T<0.4$ and $B / T \geq 0.4$ have lower bar fraction $(\sim 42 \%$ and $\sim 17 \%)$.

Overall, Table 2.10 shows bulges with low $n \leq 2$ and low $B / T \leq 0.2$ preferentially exist in barred galaxies. This is consistent with earlier work (Odewahn 1996; Barazza et al. 2008; Marinova et al. 2009; Aguerri et al. 2009) where an enhanced optical bar fraction is seen is galaxies with late Hubble types or low $B / D$. It may be tempting to infer this result to mean the formation pathway of two-thirds of low- $B / T$ bulges is related to bars in that spontaneous or/and tidally induced bars play a role in bulge formation (with the remaining one-third of such bulges may have been formed either by mechanisms like retrograde minor mergers or short-lived bars). We caution that this type of cause-effect relationship is not the only scenario consistent with this result. It may also be possible that bar instabilities are favored in galaxies with low $B / T$ and no inner Lindblad resonances (ILR). Under these conditions, the swing amplifier model with a feedback loop (Julian \& Toomre 1966; Toomre 1981; Binney \& Tremaine 1987) may be responsible for bar formation and partly account 
for the high bar fraction in galaxies of low $B / T$.

\subsubsection{Formation of Bulges}

Our observational results provide some interesting challenges for models of galaxy evolution that try to address the origin of present-day bulges. Any successful model must be able to account for the observed distribution of bulge $B / T$ and $n$ in high mass $\left(M_{\star} \geq\right.$ $\left.1.0 \times 10^{10} M_{\odot}\right)$ spirals, as shown in Table 2.9 and Table 2.10. In particular, the following results must be reproduced:

1. In terms of the overall distribution of bulge $n$, as much as $(\sim 74 \%)$ of high mass spirals have bulge $n \leq 2$ : such bulges exist in barred and unbarred galaxies and their $B / T$ ranges from 0.01 to 0.4 , with most having $B / T \leq 0.2$ (Table 2.9). A moderate fraction ( $\sim 24 \%)$ of high mass spirals have $2<n<4$, and just $(\sim 2 \%)$ have $n \geq 4$.

2. Theoretical models often make more robust predictions on the bulge-to-total mass ratio $B / T$ than on the bulge index $n$, so we consider the empirical $B / T$ distribution in detail. We note that as much as $\sim 66 \%$ of high mass spirals have bulges with $B / T \leq 0.2$ (Table 2.9). In terms of bar fraction, $\sim 68 \%$ are barred (Table 2.10 ).

3. The fraction of bars rises among spirals with low bulge index $n$. About $63 \%$ of spirals with low $n \leq 2$ bulges host bars, while the bar fraction in spirals with $2<n<4$ bulges (44\%) is two-thirds as large (Table 2.10).

In a hierarchical Universe, there are several physical processes that contribute to the assembly of bulges: major mergers, minor mergers, and secular evolution. We briefly describe these, expanding on our introduction in $\S 2.1$. 
Major mergers, defined as those with mass ratio $M_{1} / M_{2} \geq 1 / 4$, typically destroy the extended outer stellar disks during violent relaxation, leaving behind a classical bulge. Such bulges are associated with modest-to-high bulge Sérsic indices, in the range $2<n<6$ (Hopkins et al. 2009a; Springel et al. 2005; Robertson et al. 2006; § 2.5.8) in simulations. This trend is also consistent with the fact that among ellipticals, high luminosity ones tend to have a Sérsic index $n>4$, while low luminosity ones tend to have $2 \leq n \leq 3.5$ (Caon et al. 1993; Kormendy et al. 2008). The final Sérsic index depends on the amount of residual gas the settles into a somewhat disky component. Simulations by Hopkins et al. (2009a) find that the Sérsic indices of remnants from 1:1 gas-rich major mergers lie in the range of $2<n<4$, with most above 2.5 (see Fig. 2.22). This body of evidence strongly suggests that many bulges with $n>2$ might have a major merger origin.

Minor mergers, typically defined as those with mass ratio $1 / 10<M_{1} / M_{2}<1 / 4$, do not destroy the stellar disk of the primary system, but can contribute to building bulges via three pathways. Firstly, a fraction $F_{\text {sat }}$ of the satellite's stellar mass can end up in the central region of the primary galaxy. The value of $F_{\text {sat }}$ depends on how centrally concentrated the in-falling satellite is. Typically, the more diffuse outer stellar body is tidally stripped, while the central core sinks by dynamical friction to the central region (e.g., Quinn et al. 1993; Walker et al. 1996). Secondly, a non-axisymmetric feature (e.g., a stellar bar or bar-like feature) can be induced in the main disk, and gravitational torques exerted by the feature can drive gas into the inner kpc (e.g., Hernquist \& Mihos 1995; Jogee 2006 and references therein), where subsequent SF forms a compact high $v / \sigma$ stellar component, or disky pseudobulge. Most of the gas inflow happens during the merger phase and large gas inflow rates (e.g., $\gg 1 M_{\odot}$ per year) may be generated. Thirdly, gas inflow 
can also be caused by direct tidal torques from the companion (e.g., Hernquist \& Mihos 1995; Eliche-Moral et al. 2006). It is to be noted that in the simulations by Hernquist \& Mihos (1995), the gas inflow generated by non-axisymmetric features (e.g., bar-like features) in the inner part of the disk is much larger than that caused by direct tidal torques from the satellite. In the recent work of Eliche-Moral et al. (2006), N-body simulations of minor mergers followed by fits of 1D Sérsic+exponential models to the remnants, suggest that the bulge Sérsic index and $B / D$ ratio can grow as a result of the central re-concentration of stellar disk material in the primary system by tidal forces. Minor mergers are frequent under $\Lambda \mathrm{CDM}$, and the likelihood of multiple successive minor mergers occurring during the formation of a galaxy is high. Bournaud, Jog, \& Combes (2007) study the effects of repeated minor mergers on galaxy structure. They show that a disk galaxy undergoing successive minor mergers will eventually transform into an elliptical galaxy with an $r^{1 / 4}$-law profile and high $V / \sigma$. However, galaxy growth is not completely merger-driven and the efficiency of minor mergers at creating ellipticals must be regulated by other mechanisms (e.g., cold gas accretion).

In addition, the process of secular evolution can build a disky bulge (pseudobulge) between merger events. Here a stellar bar or globally oval structure in a non-interacting galaxy drives gas inflow into the inner kpc, where subsequent star formation forms a compact high $v / \sigma$ stellar component (e.g., Kormendy 1993a; Jogee 1999; KK04; Jogee, Scoville, \& Kenney 2005; Athanassoula 2005; Kormendy \& Fisher 2005; Kormendy 2008). This process is different from that of minor mergers in the sense that it happens in the quiescent phase of the galaxy, between minor or major merger events. The prevalence of pseudobulges in galaxies of different Hubble types is discussed in KK04, and select examples of S0 galaxies 
with pseudobulges are also shown in Kormendy \& Cornell (2004) and KK04.

The present-day bulge mass can be written as the sum of mass contributed from each process:

$$
M_{\text {bulge }}=M_{\text {bulge }} \times\left(f_{\mathrm{maj}}+f_{\min 1}+f_{\min 2}+f_{\min 3}+f_{\mathrm{sec}}\right),
$$

where

- $f_{\text {maj }}$ is the percentage of the bulge stellar mass, which is built by major mergers,

- $f_{\min 1}$ is the percentage of the bulge stellar mass, which is built during minor mergers from stars of the satellite. This depends on the fraction $F_{\text {sat }}$ of the satellite's stellar mass, which ends up in the central region of the primary galaxy during each minor merger.

- $f_{\min 2}$ is the percentage of the bulge stellar mass, which is built during minor mergers from gas inflow caused by a tidally induced bar.

- $f_{\min 3}$ is the percentage of the stellar mass, which is built during minor mergers from gas inflow caused by tidal torques from the companion.

- $f_{\text {sec }}$ is the percentage of the stellar mass, which is built secularly between merger events from gas inflow caused by bars or ovals

In $\S 2.5 .8$, we compare our derived distribution of bulge $n$ and $B / T$ with hierarchical models that model major and minor mergers, but not secular evolution. The main goal of the model is to see whether bulges built via major mergers can account for the large fraction of high mass spirals with bulges of low $B / T$ or/and low $n$. A secondary goal is to see if a first 
order simplified prescription for minor mergers can broadly account for the observations. We stress here that bulge-building during minor mergers is modeled in a very simple way: all the stars in the satellite are assumed to contribute to the bulge of the larger galaxy (i.e., $F_{\text {sat }}=100 \%$ ), and bulge-building via gas inflow driven through tidal torques and via gravitational torques from induced bars are ignored (i.e., $f_{\min 2}=0$, and $f_{\min 3}=0$ ). Furthermore, the models entirely ignore secular evolution between mergers. In a future paper, these extra terms will be addressed and a comprehensive picture built of the relative importance of minor mergers and secular processes in making present-day bulges.

\subsubsection{Comparison of $B / T$ With Hierarchical Models of Galaxy Evolution}

We compare our data with the predictions from cosmological semi-analytical models based on Khochfar \& Burkert (2005) and Khochfar \& Silk (2006b). We briefly describe the models first. The merger trees of dark matter (DM) halos are derived by using the extended Press-Schechter formalism (Press \& Schechter 1974), as in Somerville \& Kolatt (1999). When two DM halos merge, the merger time scale of the galaxies is calculated by considering the timescale it would take the satellite galaxies to reach the central galaxy at the center of the halo via dynamical friction (e.g., Kauffmann et al. 1999; Springel et al. 2001). The baryonic physics, which includes radiative cooling, star formation, and feedback from supernovae, is treated via semi-analytic prescriptions (see Khochfar \& Silk 2006b and references therein). Baryonic mass inside the dark matter halos is divided between hot gas, cold gas, and stars. The hot gas is initially shock-heated to the halo virial temperature. As the gas radiatively cools, it settles down into a rotationally supported disk at the halo center. Cold disk gas is allowed to fragment and subsequently form stars according to the Schmidt-Kennicutt law (Kennicutt 1998). Star formation is regulated by feedback from 
supernovae using the prescription in Kauffmann et al. (1999).

Major mergers are typically considered as those with stellar mass ratio $M_{1} / M_{2} \geq$ 1/4. In the simulations, one assumes that during a major merger any existing stellar disk is destroyed, gas is converted to stars with some star formation efficiency (SFE), and all stars present undergo violent relaxation to form a bulge. Therefore, the bulge-to-total stellar mass ratio $(B / T)$ of a bulge immediately after a major merger is always one. Note that the SFE during a major merger is not assumed to be $100 \%$ as there is mounting evidence from SPH simulations (Springel \& Hernquist 2005; Cox et al. 2008) that not all cold gas is converted to stars. Instead, the burst efficiency defined by Cox et al. (2008) is applied to control the fraction of stars formed due to the interaction. This efficiency is dependent on the relative masses of merging galaxies and is expressed as

$$
e=e_{1: 1}\left(\frac{M_{\text {Satellite }}}{M_{\text {Primary }}}\right)^{\gamma}
$$

where $e_{1: 1}$ is the burst efficiency for a 1:1 merger and $\gamma$ fixes the dependence on mass ratio; Cox et al. (2008) find $e_{1: 1}=0.55$ and $\gamma=0.69$. The remaining fraction (1-e) of gas is added to the gaseous disk and can start making stars.

As stated above, immediately after a major merger, the remnant is a bulge with a $B / T$ equal to one. As time proceeds, $B / T$ falls because a stellar disk grows around the bulge as hot gas in the halo cools, settles into a disk, and forms stars. The formation of stars by any residual cold gas left at the end of the major merger also helps to grow the disk. Thus $B / T$ falls until the next major merger happens, at which point $B / T$ is reset to one in the models.

The bulge may also grow in stellar mass due to minor mergers. Minor mergers are 
defined as mergers with mass ratio $1 / 10<M_{1} / M_{2}<1 / 4$, and the stellar disk of the large companion is not destroyed during such mergers. The models assume that during minor mergers, all the stars in the satellite are added to the bulge of the host, while the gas settles in the disk. When DM halos grow by accretion or minor mergers, the hot gas that comes in with a satellite is immediately stripped and added to the hot gas component of the host. The cold gas in the disk of the satellite is only added to the cold gas of the host if they merge. Until they merge the satellite is using up its own cold gas to make stars.

Fig. 2.23 shows the relationship between the present-day $B / T$ of a a high mass $\left(M_{\star} \geq 1.0 \times 10^{10} M_{\odot}\right)$ spiral and the redshift $z_{\text {last }}$ of its last major merger. As expected, systems where the last major merger occurred at earlier times, have had more time to grow a disk and have a lower $B / T$. The dispersion in the present-day $B / T$ at a given $z_{\text {last }}$ is due to the different times spent by a galaxy in terms of being a satellite versus a central galaxy in a DM halo, since the cooling of gas and the growth of a disk is stopped when a galaxy becomes a satellite. Thus, galaxies that became a satellite galaxy shortly after their last major merger stayed at high $B / T$. Conversely, galaxies that continued to be a central galaxy for a long time after their last major merger will have low $B / T$.

The present-day $B / T$ of a high mass $\left(M_{\star} \geq 1.0 \times 10^{10} M_{\odot}\right)$ spiral depends on its major merger history. In particular, we note from Fig. 2.23 that a high mass $\left(M_{\star} \geq\right.$ $\left.1.0 \times 10^{10} M_{\odot}\right)$ galaxy, which has undergone a past major merger since $z \leq 2$ will end up hosting a present-day $B / T>0.2$. In effect, a high mass spiral can have a present-day $B / T \leq 0.2$ only if its last major merger occurred at $z>2$ (lookback times $>10$ Gyr).

The predicted distribution of present-day $B / T$ depends on the galaxy merger history in the models and it is relevant to ask how well the latter is constrained observationally. 
Over the redshift range $z \sim 0.24$ to 0.80 (lookback times of 3 to 7 Gyr), recent studies by Jogee et al. $(2008,2009)$ find that among high mass $\left(M_{\star} \geq 2.5 \times 10^{10} M_{\odot}\right)$ galaxies, $\sim 10 \%$ of galaxies are undergoing mergers of mass ratio $>1 / 10$, and $\sim 3 \%$ are undergoing major mergers of mass ratio $>1 / 4$. These findings agree within a factor of less than $\sim 2$ with the merger rates from the models of Khochfar \& Burkert (2001) over $z \sim 0.24$ to 0.80. At higher redshifts, the empirical merger rate/fraction is uncertain due to relatively modest volumes and bandpass shifting effects, but there is a general trend towards higher merger fractions at higher redshifts (e.g., Conselice et al. 2003). The models used here (Khochfar \& Burkert 2001) agree with this trend and predict that $\sim 13.5 \%$ and $\sim 20 \%$ of high mass $\left(M_{\star} \geq 1.0 \times 10^{10} M_{\odot}\right)$ spirals have undergone major mergers since $z \leq 2$ and $z \leq 4$, respectively (see Table 2.11 ).

The contribution of galaxies with different merger histories to the present-day $B / T$ distribution are shown in Table 2.11. The top and middle parts of the table describe systems with and without major mergers since $z \leq 2$ and $z \leq 4$, respectively. In the model, $\sim 13.5 \%$ of present-day high mass $\left(M_{\star} \geq 1.0 \times 10^{10} M_{\odot}\right)$ spirals, experienced a major merger since $z \leq 2$, causing most of them $(\sim 11.2 \%)$ to have a present-day high $B / T>0.4$ and a negligible fraction $(\sim 0.1 \%)$ to have a low present-day $B / T \leq 0.2$. In contrast, the remaining $\sim 86.5 \%$ spirals experienced no major merger at $z \leq 2$, and most (67.2\%) of them have a present-day low $B / T \leq 0.2$. If the comparisons are extended to systems without a major merger since $z \leq 4$, the numbers are very similar (see middle part of Table 2.11).

Table 2.11 shows that there is good agreement between the model and data for the fraction of high mass spirals with present-day low $B / T \leq 0.2(\sim 67 \%$ in the model versus $\sim 66 \%$ in the data). The model contribution to low $B / T \leq 0.2$ comes almost entirely from 
galaxies, which have not had a major merger since $z \leq 2$ (see column 4 in Table 2.11). In fact, most of these galaxies have not even had a major merger since $z \leq 4$, as illustrated by the bottom part of Table 2.11. In the model, the fraction ( 1.6\%; column 3 of Table 2.11) of high mass spirals, which have undergone a major merger since $z \leq 4$ and host a bulge with a present-day $B / T \leq 0.2$, is a factor of over thirty smaller than the observed fraction ( $\sim 66 \%)$ of high mass spirals with $B / T \leq 0.2$. Thus, bulges built via major mergers since $z \leq 4$ seriously fail to account for the bulges present in $\sim 66 \%$ of high mass spirals. These results are also illustrated in Fig. 2.24, which shows the comparison between data and models for the cumulative fraction of high mass spirals as a function of present-day $B / T$.

It is also interesting to note from Table 2.11 that although the models reproduce well the frequency of bulges with present-day low $B / T \leq 0.2$, they tend to over-produce the frequency of present-day high $B / T>0.4$ systems by nearly a factor of two $(\sim 14 \%$ in the model versus $\sim 8 \%$ in the data; see columns 6 and 2 in middle part of Table 11). Most of this overproduction stems from major mergers at $z \leq 4(\sim 13 \%$; column 4 in middle part of Table 2.11). This suggests that major mergers, as currently modeled here, are building bulges too efficiently.

One possible solution to this problem might relate to the suggestion by Hopkins et al. (2009c) that the efficiency of bulge-building during the major merger of two spirals depends not only on the mass ratio $M_{1} / M_{2}$ of the merger, but also depends on the cold gas mass fraction $f_{\text {gas }}$ in the disk. In their semi-analytic models, the entire stellar mass of the satellite violently relaxes, but the fraction of stellar mass in the primary disk that violently relaxes and adds to the bulge is $M_{1} / M_{2}$. This differs with our models where the entire primary stellar disk is always destroyed in a major merger. Furthermore, the fraction $F$ of 
the total gas mass, which loses angular momentum, falls to the nucleus, and is transformed into stellar mass in a nuclear starburst, is $\sim\left(1-f_{\text {gas }}\right) \times\left(M_{1} / M_{2}\right)$. In particular, $F$ is lower for more gas-rich disks, causing a suppression of the burst efficiency in gas-rich systems, and a reduction in the stellar mass that ends up in the bulge built during the major merger. The predictions for the distribution of $B / T$ from the Hopkins et al. (2009c) models are shown in the bottom of Table 2.11, with major mergers considered as those with baryonic galaxygalaxy mass ratio $>/ 1 / 3$. Due to the reduced stellar mass that ends up in the bulge after a major merger, the Hopkins et al. (2009c) models tend to yield lower $B / T$ after such a merger. Thus, the models produce overall fewer high $B / T>0.4$ systems, and more intermediate $0.2<B / T<0.4$ and low $B / T \leq 0.2$ systems (see column 6 in Table 2.11). Nonetheless, the predictions are not very different from the models by Khochfar \& Burkert (2005) and Khochfar \& Silk (2006b), which we use in this paper. In particular, the conclusion that the large frequency of high mass $\left(M_{\star} \geq 1.0 \times 10^{10} M_{\odot}\right)$ spirals with low $B / T \leq 0.2$ bulges can only be accounted for by spirals without a major merger since $z \leq 2$ also holds with the Hopkins et al. (2009c) models.

Thus, we conclude that the observed large frequency $(\sim 66 \%)$ of high mass $\left(M_{\star} \geq\right.$ $\left.1.0 \times 10^{10} M_{\odot}\right)$ spirals with low present-day $B / T \leq 0.2$ can be accounted for in our and other hierarchical models by high mass spirals, which have not undergone a major merger since $z \leq 2$, and most of which have not even experienced a major merger over the last 12 Gyr since $z \leq 4$. In the models, most of these present-day low $B / T \leq 0.2$ bulges are built by minor mergers since $z \leq 4$. As noted earlier, our models explore bulge-building via minor and major mergers, but do not explicitly incorporate secular processes (see $§ 2.5 .7$ ). In practice, secular processes may contribute to the building of present-day low $B / T \leq 0.2$ 
bulges, and are particularly relevant at $z \leq 2$, where major mergers cannot build such bulges.

For completeness, we further explore how sensitive are our results to other assumptions made in the data and models of Khochfar \& Burkert (2005) and Khochfar \& Silk (2006b):

- How sensitive are the results to the mass ratio used to separate major and minor mergers? Fig. 2.25 is similar to Fig. 2.24 except that the model now defines major mergers as those with mass ratio $M_{1} / M_{2} \geq 1 / 6$. In this case, about $30 \%$ of the model spirals undergo major mergers since $z \leq 4$ rather than $\sim 20 \%$. The overall model $F$ (black dashed line) now under-predicts the data $F$ by about $10 \%$ for $B / T>0.2$. However, the main conclusion that bulges built by major mergers since $z \leq 4$ cannot account for most of the low $B / T \leq 0.2$ bulges, present in a large percentage $(\sim 66 \%)$ of spirals still holds.

- How sensitive are the results to the $B / T$ cut used to define spirals? Fig. 2.26 is similar to Fig. 2.24 except that here spirals are considered to be systems with a $B / T \leq 0.55$ rather than 0.75 in the models, and a corresponding cut is applied to the data points. The results are similar to Fig. 2.24

- How sensitive are the results to our assumed constant $H$-band mass-to-light $(M / L)$ for the bulge, disk, and bar? Fig. 2.27 is similar to Fig. 2.24 except that the $B / T$ of all the observed galaxies has been multiplied by a factor of two, in order to test what would happen in the case where the $M / L$ ratio of the bulge in $H$-band is twice as high as that of the disk and bar. This could happen in an extreme example where the dominant bulge stellar population was much older (e.g. 12 Gyr) than the age of 
the dominant disk stellar population (e.g., 3 Gyr). In such a case, the fraction of high mass spirals with $B / T \leq 0.2$ would change from $\sim 66 \%$ in Fig. 2.24 to $\sim 55 \%$, and deviate from the model overall model $F$ (black dashed line) by $\sim 20 \%$. However, the main conclusion that bulges built by major mergers since $z \leq 4$ cannot account for most of the low $B / T \leq 0.2$ bulges, present in a large percentage $(\sim 55 \%)$ of spirals still holds.

Finally, it is important to note that so far we have compared the data and model only in terms of bulge $B / T$, but not in terms of bulge index $n$ or in terms of bar fraction. In effect, we have only shown that the models reproduce a subset of the results outlined in points (1)-(3) of $\S 2.5 .7$. Since the semi-analytic models do not produce a distribution of bulge index $n$, we resort to presenting only an indirect comparison in Table 2.12. We assume that bulges, which form in major mergers have a bulge $n>2.5$. This assumption is based the evidence presented in $\S 2.5 .7$. Thus, in Test 1 of Table 2.12, we compare the fraction $(\sim 66 \%)$ of galaxies in the semi-analytic models having $B / T \leq 0.2$ and no major merger since $z \leq 4$, with the observed fraction $(\sim 65 \%)$ of galaxies with $B / T \leq 0.2$ and bulge $n \leq 2.5$. There is close agreement between the two values. In Test 2 of Table 2.12, the fraction $(\sim 12.7 \%)$ of model galaxies with $B / T>0.4$ and no major merger since $z \leq 4$ is a factor of $\sim 3$ higher than the fraction $(\sim 3.5 \%)$ of high mass spirals with $B / T>0.4$ and bulge $n>2.5$. Similarly, the fraction $(\sim 1.6 \%)$ of model galaxies with $B / T \leq 0.2$ and a past major merger since $z \leq 4$ is also a factor of $\sim 2$ higher than the observed fraction $(\sim 0.9 \%)$ of high mass spirals with $B / T \leq 0.2$ and bulge $n>2.5$ (Test 3 , Table 2.12). Thus, in terms of bulge $B / T$ and $n$, there is good agreement between data and model for Test 1 (involving model galaxies with no major mergers since $z \leq 4$ ). This suggests that the vast majority of 
bulges with $B / T \leq 0.2$ and $n \leq 2.5$ likely formed in galaxies having had no major merger since $z \leq 4$. However, the agreement is less good for Tests 2 and 3 (involving model galaxies with major mergers since $z \leq 4)$ and this suggests that the models may be building bulges a little bit too efficiently during a major merger, in agreement with the conclusion reached earlier.

What about the role of bars in the formation of these bulges of low $B / T$ and low $n$ ? A detailed direct comparison with the semi-analytic models is not possible as the role of bars is not yet modeled, but related comparisons are possible. First, it is important to note that bar-driven gas inflow into the inner $\mathrm{kpc}$ and the subsequent building of disky stellar components or 'pseudobulges' (see § 2.1) can happen in both isolated galaxies and in minor mergers $(\S 2.5 .7)$, since bars can be spontaneously induced in an isolated disk or tidally induced during an interaction or minor merger. The triggering of a bar is favored in a prograde interaction or minor merger. Thus, bulge-building via induced bars is more likely to happen in prograde rather than retrograde minor mergers. Statistically about half of minor mergers might be prograde or prograde-like, and half retrograde. Thus, one would expect bars to be induced in only half of the minor mergers. If this assumption is correct and if most of the mass in bulges with present-day $B / T \leq 0.2$ is formed in minor mergers, then one would expect only about half of these bulges to host bars. This is close to what is observed, as shown by Table 2.10 . We see that $\sim 63 \%$ of high mass spirals with low $n \leq 2$ bulges and $\sim 68 \%$ of spirals with low $B / T \leq 0.2$ bulges host bars. This suggests that in high mass spirals, spontaneous and/or tidally induced bars may play a part in forming up to two-thirds of $B / T \leq 0.2$ or $n \leq 2$ bulges. The remaining one-third of such bulges may have been formed either by mechanisms that do not involve bars (e.g., retrograde minor 
mergers) or by bars that are not long-lived.

\subsection{Summary}

The properties of galaxy components (bulges, disks, and bars) in the local Universe provide key constraints for models of galaxy evolution. Most previous $2 D$ decompositions have focused on two-component bulge-disk decomposition, and ignored the contribution of the bar even in strongly barred galaxies. However, as shown by this work and other recent studies (e.g., Laurikainen et al. 2005; Laurikainen et al. 2007; Reese et al. 2007), it is important to include the bar component in the $2 D$ decomposition, in order to correctly estimate the bulge-to-total ratio $(B / T)$ and disk properties. In this paper we have developed an iterative $2 D$, bulge-disk-bar decomposition technique using GALFIT and applied it to $H$-band images to a complete sample (S1) of 143 bright $\left(M_{B} \leq-19.3\right)$ low-to-moderately inclined $\left(i \leq 70^{\circ}\right)$ spirals from the OSU Bright Spiral Galaxy Survey (OSUBSGS). The sample has primarily spirals with Hubble type S0/a to Sc and stellar mass $M_{\star} \geq 1.0 \times 10^{10} M_{\odot}$. We performed two-component bulge-disk decomposition, as well as three-component bulge-diskbar decomposition on the $2 D$ light distribution of all galaxies, taking into account the PSF. We use an exponential profile for the disk, and Sérsic profiles for the bulge and bar. A number of quantitative indicators, including bar classification from ellipse fits, are used to pick either the bulge-disk-bar decomposition or bulge-disk decomposition, as the best final fit for a galaxy. Our main results are the following.

1. We find that it is necessary to include the bar component in $2 D$ decomposition of barred galaxies, otherwise, the bulge-to-total ratio $(B / T)$ will be overestimated and the disk properties may be skewed. Examples of the effect of including the bar are 
shown for the prominently barred galaxies NGC 4643 (Figure 2.5, Table 2.3) and NGC 4548 (Figure 2.6, Table 2.4).

2. We find that out of the 143 low-to-moderately inclined $\left(i \leq 70^{\circ}\right)$ spirals in our sample, 75 of 143 or $\sim 52 \%$ are better fit with a Stage 3 bulge-disk-bar decomposition than a Stage 2 bulge-disk decomposition. There are also eight galaxies with pure bar-disk fits. The resulting $H$-band bar fraction, defined as the fraction of disk galaxies that are barred, is $58.0 \pm 4.13 \%$ ( 84 of 143 ). This fraction is in excellent agreement with the $H$-band bar fraction of $60 \%$ reported by MJ07, based on ellipse fits of the same OSUBSGS sample, with a more conservative inclination cut $\left(i \leq 60^{\circ}\right)$.

3. $H$-band images tend to trace the overall mass fairly well and are not overly impacted by extinction and age gradients. We therefore assume a constant mass-to-light $(M / L)$ in the $H$-band for the bulge, disk, and bar, and assume their $H$-band light fraction is a measure of their mass fraction. For our sample S1 of 143 bright $\left(M_{B} \leq-19.3\right)$ low-to-moderately inclined $\left(i \leq 70^{\circ}\right)$ spirals with a mass-weighted mean Hubble type of Sab-Sb, we find that $71.6 \%$ of the stellar mass is in disks, $9.6 \%$ is in stellar bars and $18.9 \%$ is in bulges (with $10.4 \%$ in $n>2$ bulges and $8.4 \%$ in $n \leq 2$ bulges).

If disks and bars are much younger (e.g., $\sim 3$ Gyr old ) than bulges (e.g., $\sim 12$ Gyr old), then our prescription would underestimate the true $B / T$ by a factor of $\leq 2$. On the other hand, if the bulge is younger than the disk and happens to harbor a significant young population of massive stars, then our prescription will overestimate the true $B / T$ mass ratio, and make our current results on the high fraction of low $B / T$ bulges (see point 4 below) even stronger. 
4. We explore the relationship between $B / T$, bulge Sérsic index, and Hubble types (Fig. 2.14 \& Fig. 2.15). Only a small fraction $(\sim 1 \%)$ of bright spirals have high $n \geq 4$ bulges; such bulges lie primarily in S0/a to Sab, and have a large $B / T>0.2$. A moderate fraction $(\sim 22 \%)$ have intermediate $2<n<4$ bulges; these exist in barred and unbarred S0/a to Sd, and their $B / T$ spans a wide range (0.03 to 0.5$)$ with a mean of 0.29. Finally, a strikingly large fraction ( $\sim 76 \%$ ) of bright spirals have low $n \leq 2$ bulges; such bulges exist in barred and unbarred galaxies across a wide range of Hubble types, and their $B / T$ varies from 0.01 to 0.4 , with most having $B / T \leq 0.2$.

5. Bulges with very high $B / T(>0.4)$ exist primarily in galaxies with high mass $\left(M_{\star}>\right.$ $\left.6 \times 10^{10} M_{\odot}\right)$ and early types $(\mathrm{S} 0 /$ a to Sab). Bulges with very low $B / T(<0.1)$ lie primarily in lower mass galaxies with later morphologies ( $\mathrm{Sb}$ to $\mathrm{Sc}$ ). As many as $\sim 69 \%$ of bright spirals have bulges with $B / T \leq 0.2$ : these bulges are pervasive and exist across the whole spectrum of S0/a to Sd (Figure 2.14).

6. Modeling bars with $2 D$ decomposition allows us to measure bar properties and the bar-to-total ratio $(\mathrm{Bar} / T)$, which is a measure of bar strength. There is a wide range $(\sim 0.03$ to $\sim 0.47)$ in the individual Bar/T at a given Hubble type. The mean Bar/T remains fairly constant with Hubble type from $\mathrm{Sa}$ to $\mathrm{Sb}$, but shows a possible weak decline by about 0.1 from Sb to Sc (See Figure 2.19 and Figure 2.20). The bar fraction (Table 2.10 ) declines with $B / T$; it is highest $(\sim 68 \%$ ) for bright spirals with $B / T \leq 0.2$, and lower $(\sim 36 \%)$ by nearly a factor of two in spirals with $B / T>0.2$.

It may be tempting to infer this to mean the formation of two-thirds of low- $B / T$ bulges is related to spontaneous or/and tidally induced bars. Such a cause-effect relationship is not the only scenario consistent with this result. It may also be possible 
that bar instabilities are favored in galaxies with low $B / T$ and no inner Lindblad resonances. Under these conditions, the swing amplifier model with a feedback loop may be responsible for bar formation and partly account for the high bar fraction in galaxies of low $B / T$.

7. We compare the observed distribution of bulge $B / T$ and $n$ in high mass $\left(M_{\star} \geq 1.0 \times\right.$ $\left.10^{10} M_{\odot}\right)$ spirals with predictions from a set of $\Lambda \mathrm{CDM}$ cosmological semi-analytical models (Table 2.11, Table 2.12, and Figs. 2.24 to 2.27). Major mergers are considered as those with stellar mass ratio $M_{1} / M_{2} \geq 1 / 4$. In the models, a high mass spiral can have a bulge with a present-day low $B / T \leq 0.2$ only if it did not undergo a major merger since $z \leq 2$ (Fig. 2.23). The model merger history shows that only $\sim 13.5 \%$ and $\sim 20 \%$ of the high mass spirals experience major mergers since $z \leq 2$ and $z \leq 4$, respectively. The fraction $(\sim 1.6 \%)$ of high mass spirals which have undergone a major merger since $z \leq 4$ and host a bulge with a present-day low $B / T \leq 0.2$ is $a$ factor of over thirty smaller than the observed fraction $(\sim 66 \%)$ of high mass spirals with $B / T \leq 0.2$ (Table 2.11 ). Thus, bulges built via major mergers since $z \leq 4$, over the last 12 Gyr, seriously fail to account for most of the low $B / T \leq 0.2$ bulges present in two-thirds of high-mass spirals. The overall picture that emerges is that the observed large frequency $(\sim 66 \%)$ of high mass $\left(M_{\star} \geq 1.0 \times 10^{10} M_{\odot}\right)$ spirals with low present-day $B / T \leq 0.2$ can be accounted for in our hierarchical models by high mass spirals, which have not undergone a major merger since $z \leq 2$, and most of which have not even experienced a major merger since $z \leq 4$. Most of these present-day low $B / T \leq 0.2$ bulges are likely to have been built by a combination of minor mergers and/or secular processes since $z \leq 4$. 

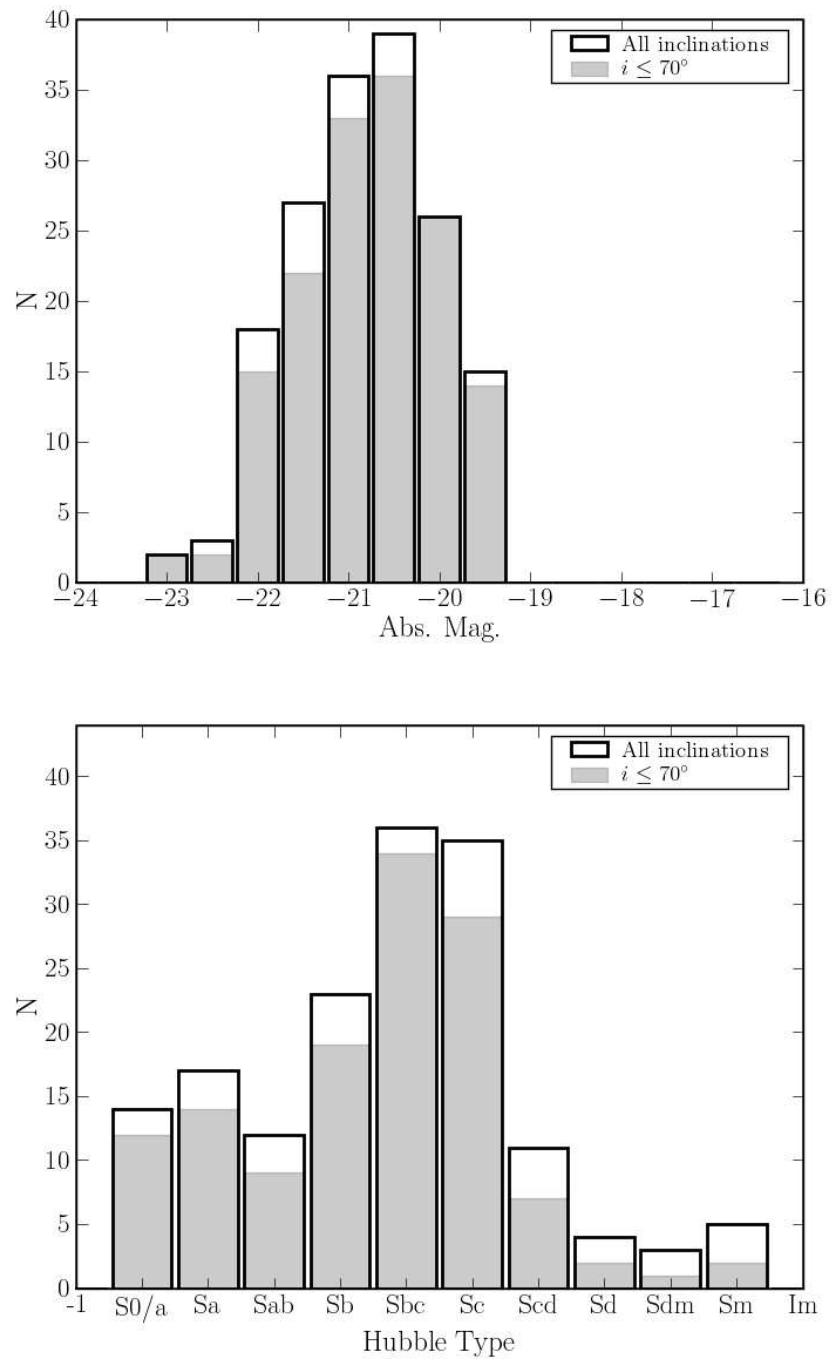

Figure 2.1 Our final sample S1 consists of 143 bright $\left(M_{B} \leq-19.3\right)$ low-to-moderately inclined $\left(i \leq 70^{\circ}\right)$ spirals in the OSUBSGS survey. The distribution of absolute $B$-band magnitude for the sample of bright spirals in the OSUBSGS survey is shown in the top panel before (solid line) and after (shaded greyscale) the cut to remove highly inclined $\left(i>70^{\circ}\right)$ spirals. The distribution of Hubble types for the sample is shown in the bottom panel before (solid line) and after (shaded greyscale) the cut to remove highly inclined $\left(i>70^{\circ}\right)$ spirals. 


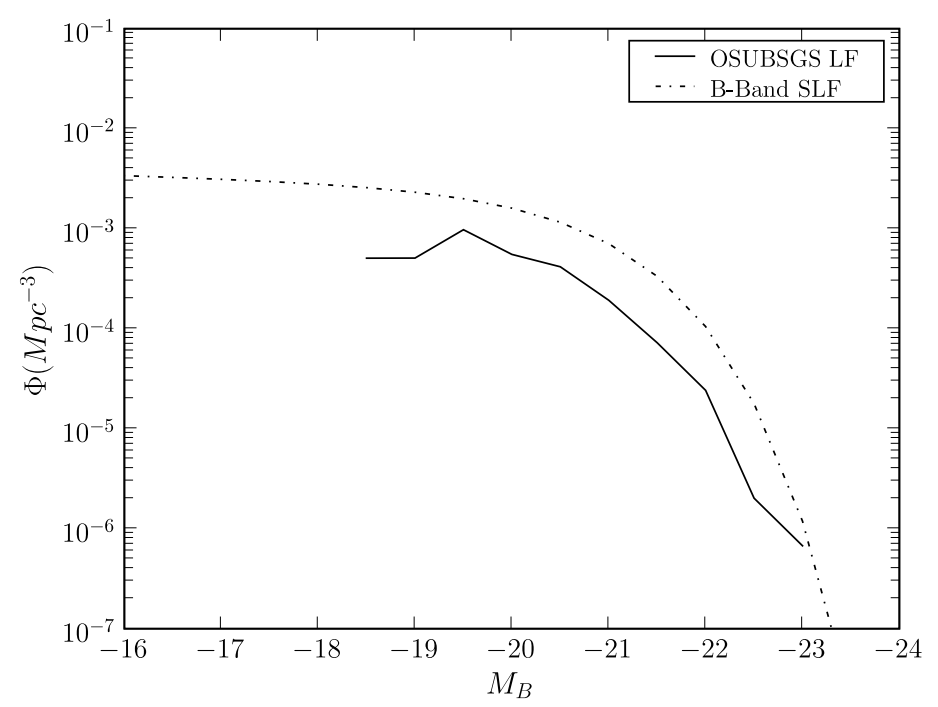

Figure 2.2 The luminosity function of the full OSUBSGS sample is compared with the $B$ band Schechter luminosity function (SLF). The former is calculated as described in $\S 2.2 .1$ using equation (2.1). The parameters for the SLF are $\Phi^{*}=5.488 \times 10^{-3} \mathrm{Mpc}^{-3}, \alpha=-1.07$, and $M_{B}^{*}=-20.5$ (Efstathiou, Ellis \& Peterson 1988), corresponding to $H_{0}=70 \mathrm{~km} / \mathrm{s} \mathrm{Mpc}^{-1}$. 


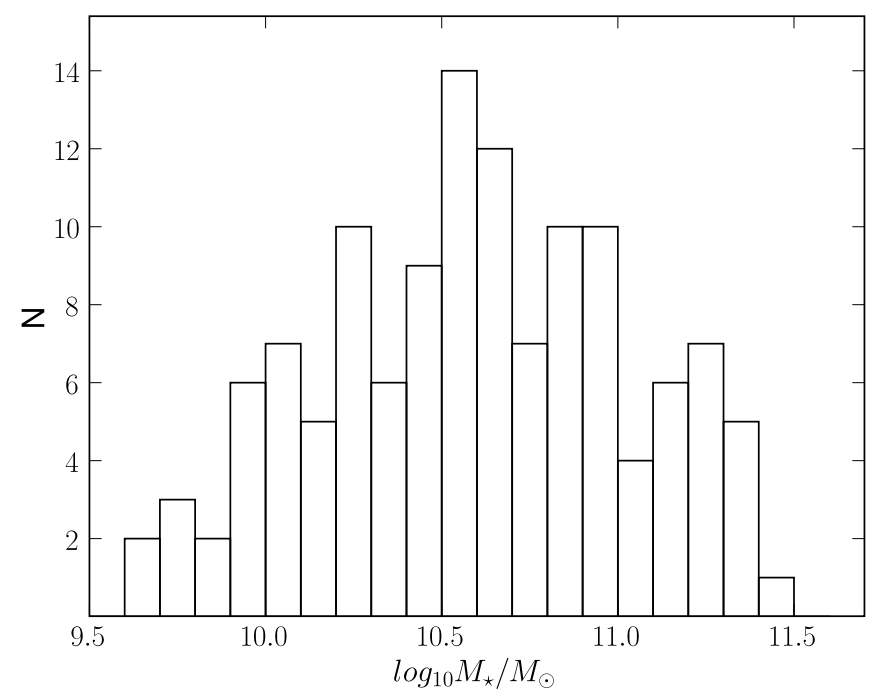

Figure 2.3 Out of our final sample S1 of 143 bright $\left(M_{B} \leq-19.3\right)$ low-to-moderately inclined $\left(i \leq 70^{\circ}\right)$ OSUBSGS spirals, stellar masses could be estimated for 126 galaxies. Their stellar mass distribution is shown, as determined in $\S 2.2 .2$. Most have stellar masses $M_{\star} \geq 1.0 \times 10^{10} M_{\odot}$. This sample of 126 galaxies is referenced henceforth as the sample S2. 


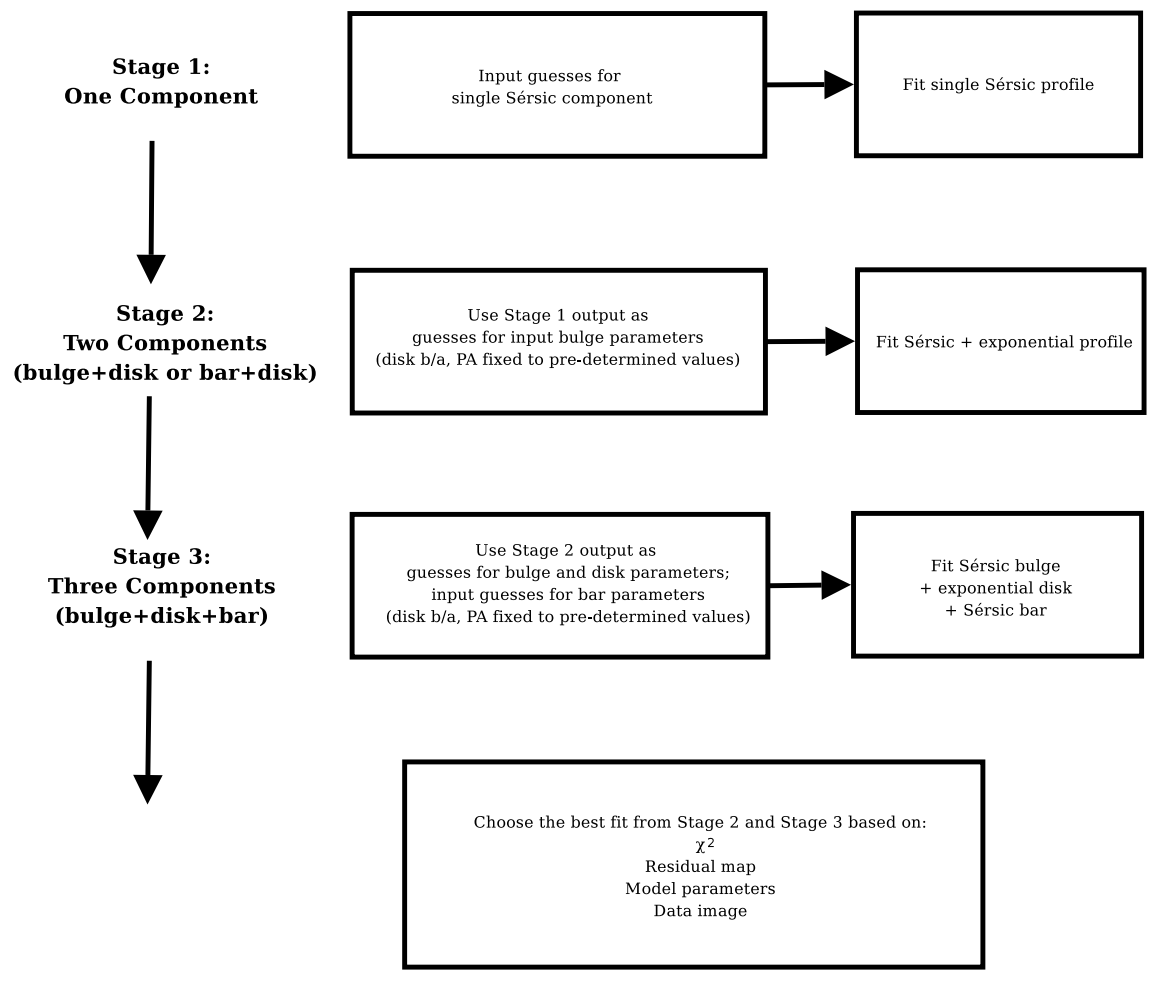

Figure 2.4 An overview of the method of decomposition. All images are subjected to Stages 1,2 , and 3. Either the best fit of Stage 2 or Stage 3 is chosen as the best model. 


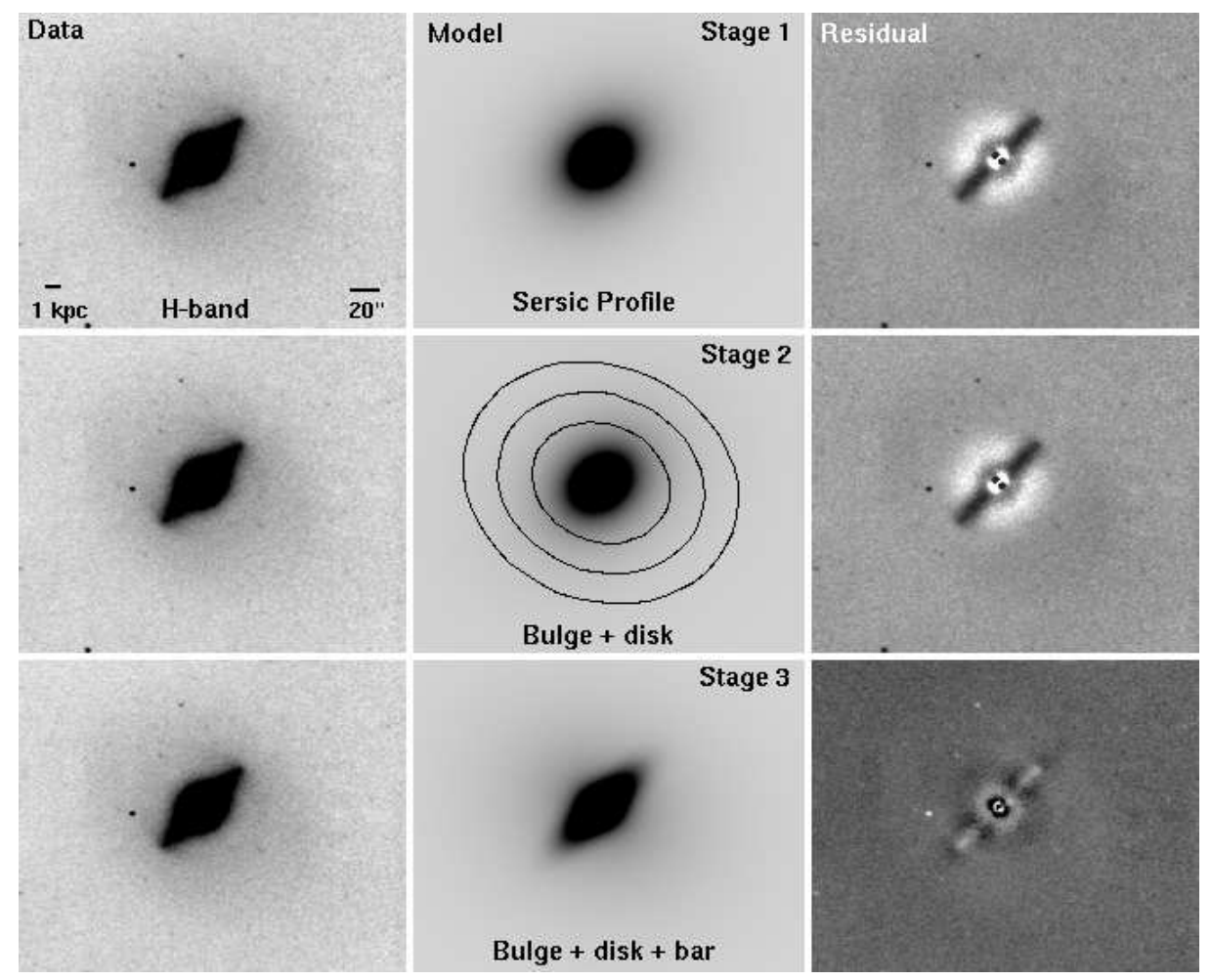

Figure 2.5 Complete $2 D$ decomposition for NGC 4643. Note the prominent bar residuals in the residual for the Stage 1 and Stage 2 bulge-disk decomposition. This is a case where the prominent bar causes the Stage 2 bulge-disk fit to artificially extend the bulge and inflate the $B / T$. The disk fitted in Stage 2 has a low surface brightness and is very extended, well beyond the real disk: the $b / a$ and $P A$ of the fitted disk is shown as contours. Stage 3 bulge-disk-bar decomposition provides the best model. The $\chi^{2}$ for the Stage 1, Stage 2, and Stage 3 residual images are 7360.7, 7284.8, and 2111.59, respectively. See Table 2.3 for the fit parameters. 


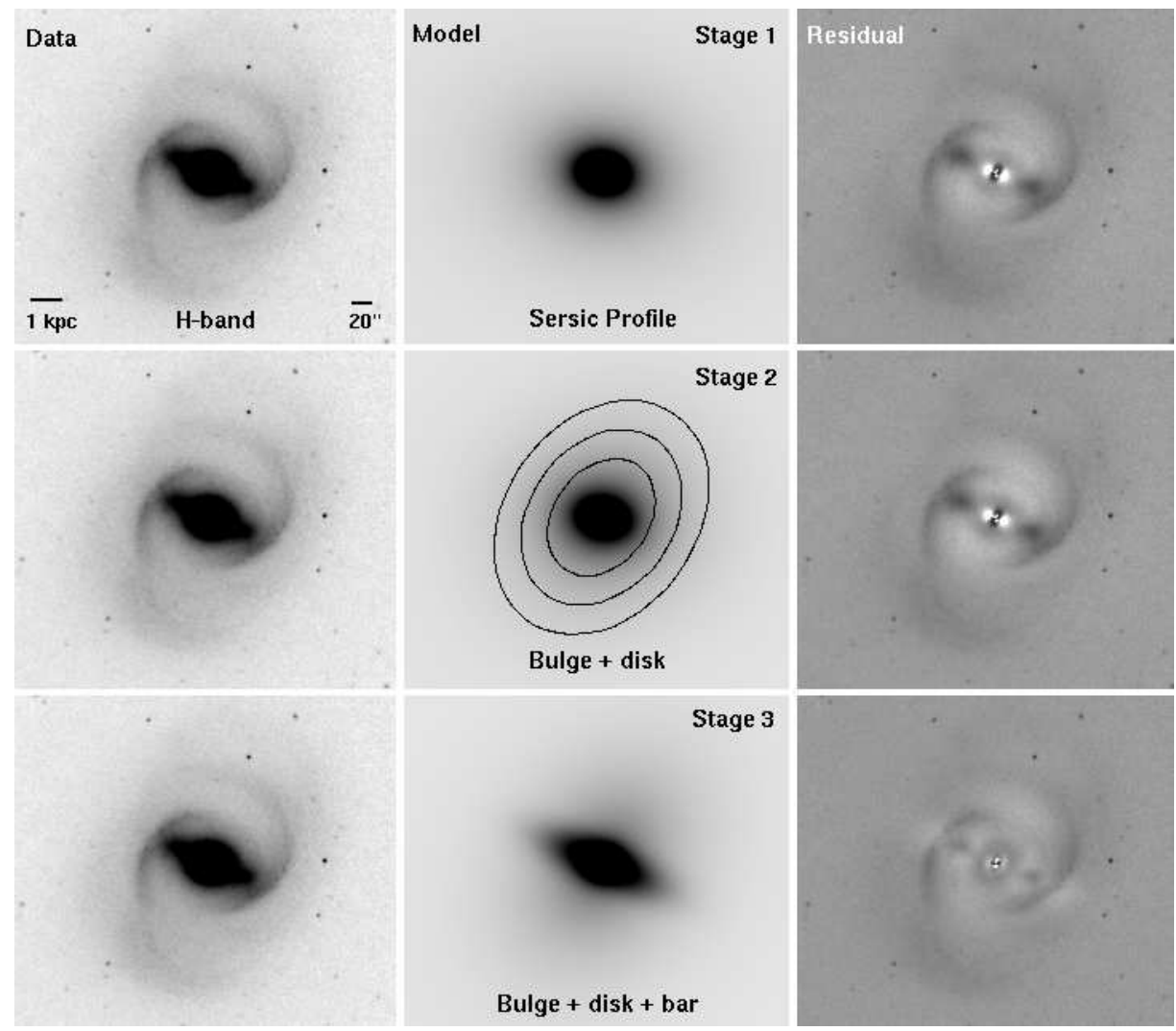

Figure 2.6 The complete $2 D$ decomposition for NGC 4548. This is an extreme example where the prominent bar results in an extended bulge and inflated $B / T$ in the Stage 2 bulge-disk fit. Like NGC 4643 in Figure 2.5, the disk fitted in Stage 2 has a low surface brightness and is very extended: its $b / a$ and $P A$ are shown as contours. Stage 3 bulge-diskbar decomposition provides the best model. The $\chi_{\nu}^{2}$ for the Stage 1, Stage 2, and Stage 3 residual images are 7076.1, 6301.3, and 3260.4, respectively. See Table 2.4 for the fit parameters. 


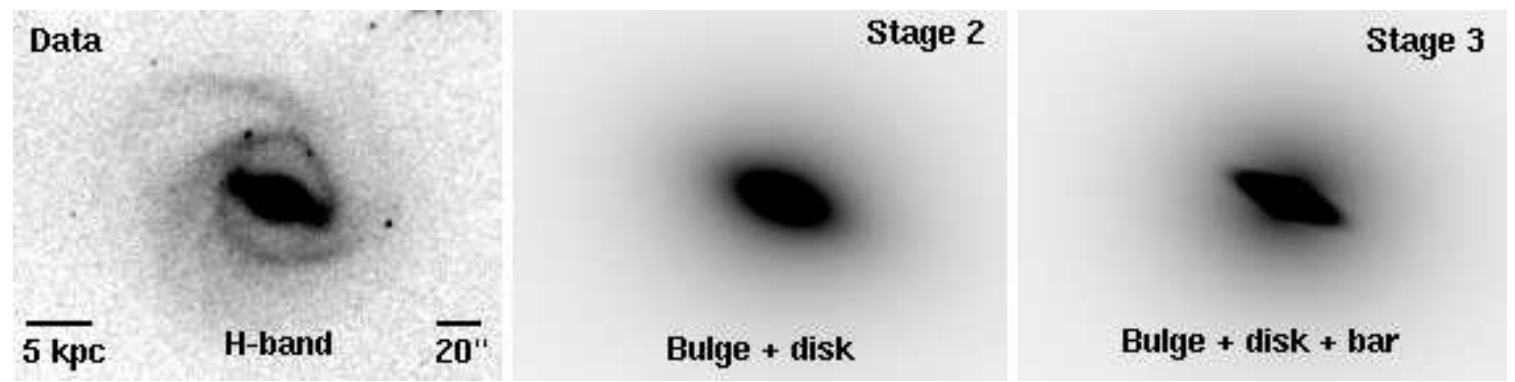

Figure 2.7 This plot shows the data image, Stage 2 model, and Stage 3 model for NGC 4902. The Stage 2 bulge is too bright and is extended along the major axis of the bar $(B / T=31.2 \%$ and $b / a=0.45)$. In Stage 3, the bulge and bar are fit with distinct components $(B / T=5.59 \%$, bulge $b / a=0.68$, Bar $/ T=9.97 \%$, bar $b / a=0.22$ ). All other fit parameters appear in Table 2.5. 

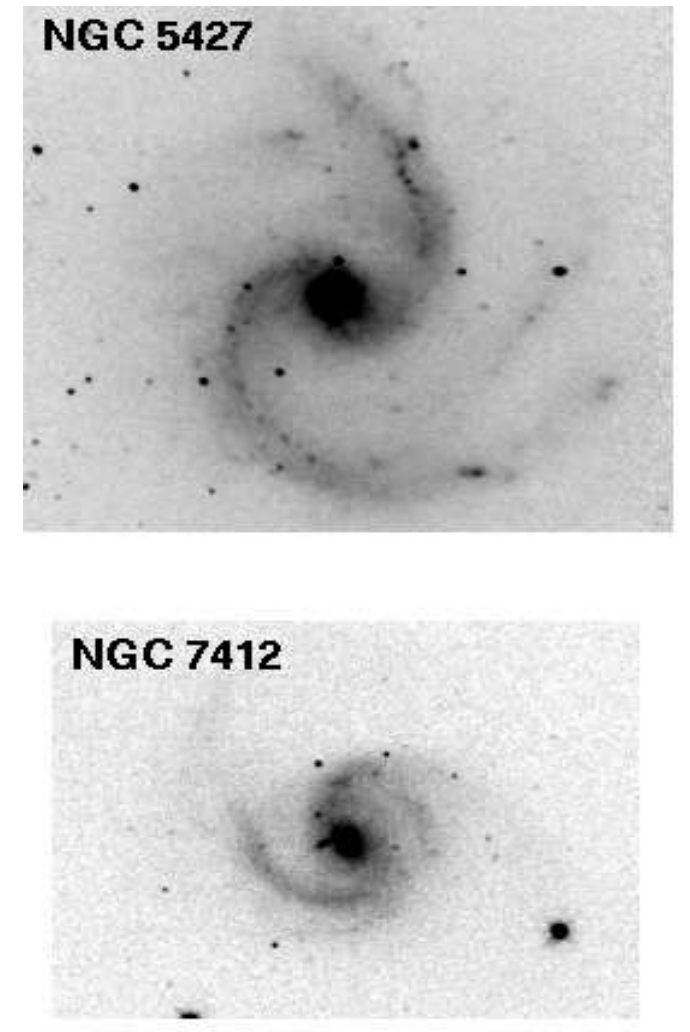

Figure 2.8 The data images and Stage 3 bulge-disk-bar decomposition models of NGC 5427 and NGC 7412 are shown. The Stage 3 models each distinctly show a false bar component, which is not present in the data images. The false components can be inspired by prominent spiral arms, such as those present in these galaxies. Such cases are flagged during the visual inspection of fits and the Stage 3 bulge-disk-bar decomposition is discarded in favor of the Stage 2 bulge-disk decomposition. 

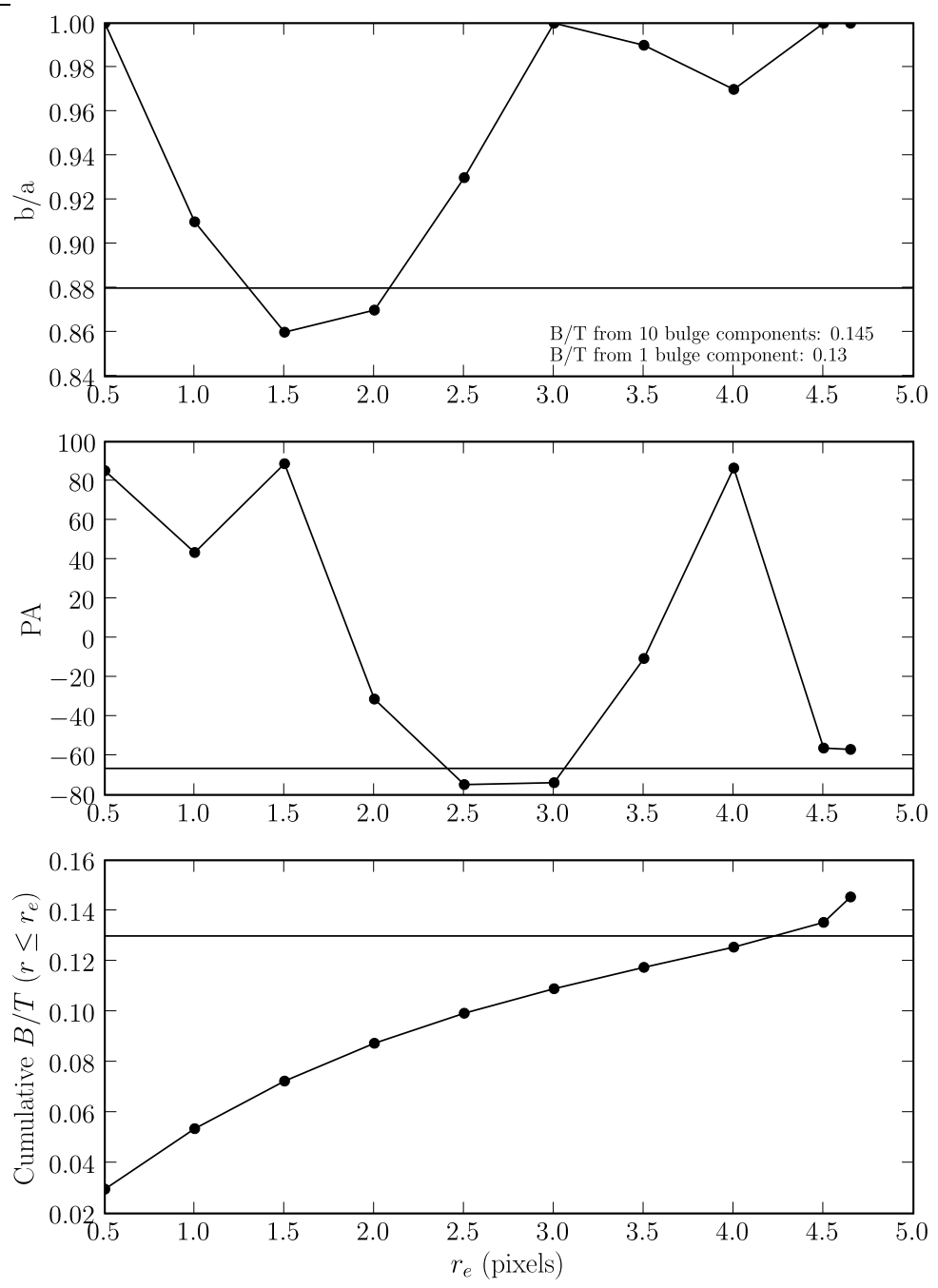

Figure 2.9 This plot compares the $B / T$ obtained by fitting the bulge of NGC 4548 with a Sérsic model of constant $b / a$ as opposed to a Sérsic model varying $b / a$. To mimic a Sérsic model with varying $b / a$ in GALFIT, the bulge was fitted with ten concentric Sérsic profiles with fixed $r_{\mathrm{e}}$, each separated by 0.75 ". The top two panels show the run of $b / a$ and $P A$ of the ten concentric Sérsic profiles. The bottom panel shows the cumulative $B / T$ calculated by summing all models with $r \leq r_{\mathrm{e}}$. The bulge $b / a(0.88), P A(-66.5)$, and $B / T(13 \%)$ from the original Sérsic fit of constant $b / a$ (Table 2.4) are indicated with horizontal lines on the 3 panels. 

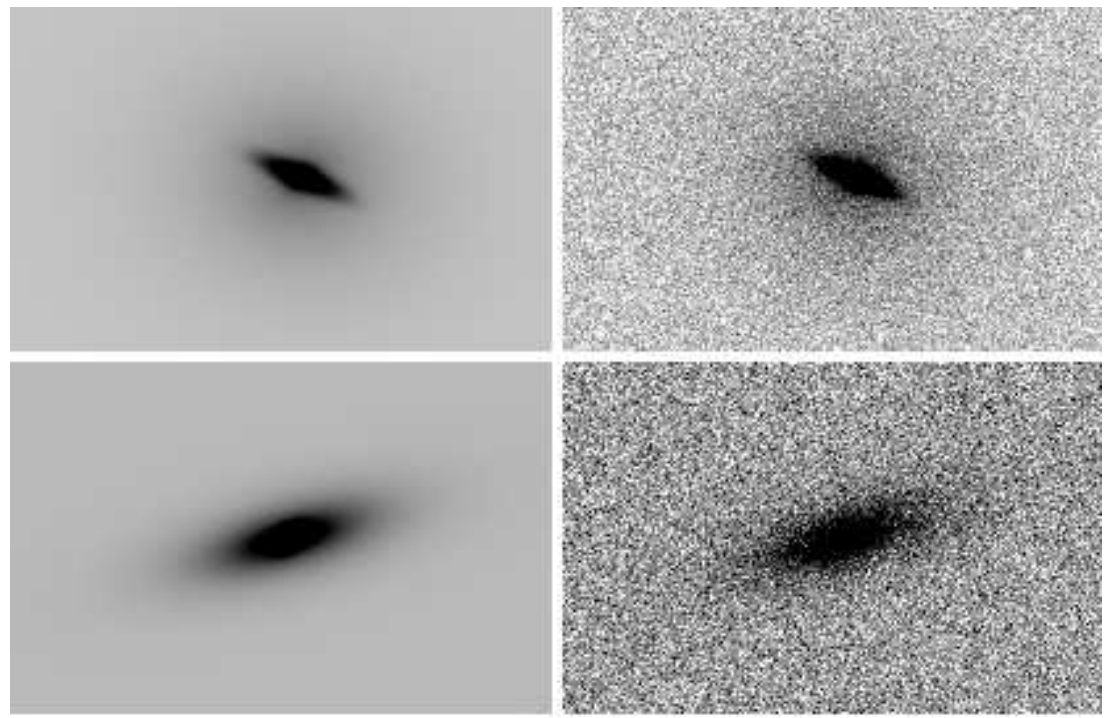

Figure 2.10 An elementary test is to determine if GALFIT can recover the known parameters of artificial noisy images. Noisy images were simulated by taking parametric model images (left panels) produced by GALFIT, and adding noise and sky background (right panels). The noisy images were then fitted to see if the original known parameters can be recovered. See $\S 2.4 .2$ for details. 

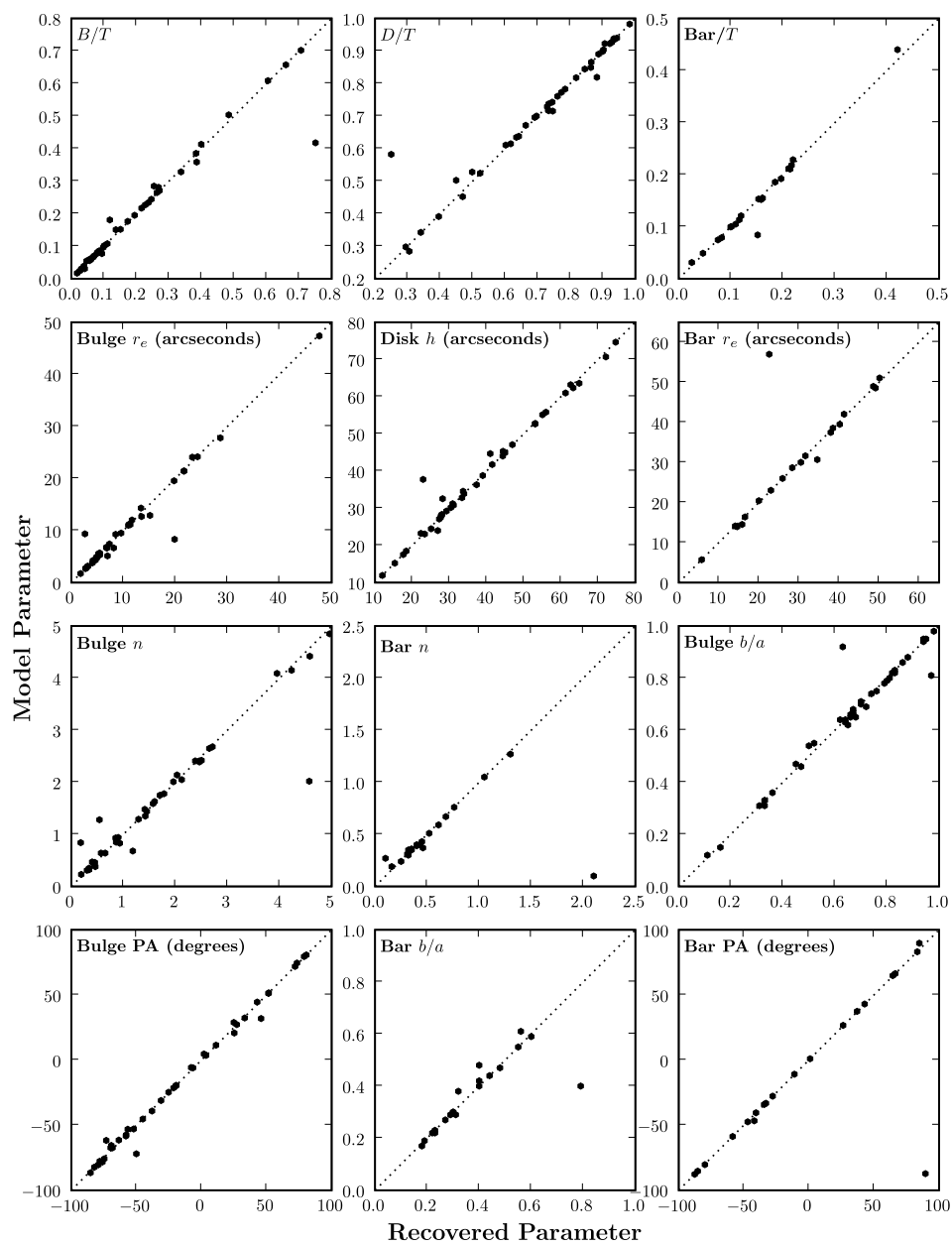

Figure 2.11 The plots compare recovered versus original model parameters for the simulated images discussd in $\S 2.4 .2$. The vertical axis limits demonstrate the range explored for each parameter. The dotted line shows $y=x$ for comparison. Except for some extreme cases where the images were highly distorted by noise, all parameters were recovered to within a few percent. 

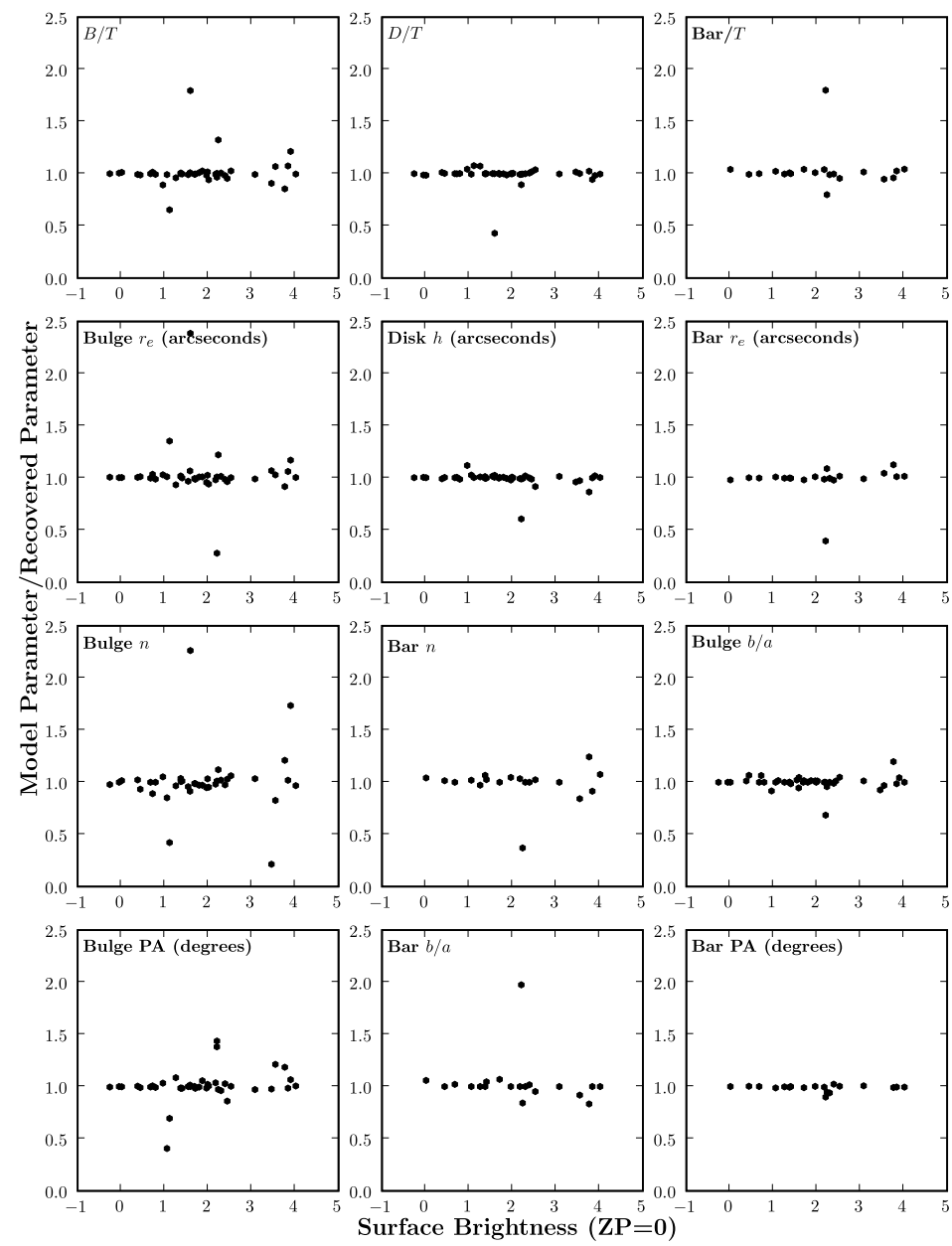

Figure 2.12 The ratio of model-to-recovered parameter is plotted against mean surface brightness inside the disk scalelength, $\mu=m a g+2.5 \times \log _{10}\left(2 \times \pi \times b / a \times h^{2}\right)$, for the simulated images discussed in $\S 2.4 .2$. Surface brightness is not photometrically calibrated and is shown for a zeropoint of 0 . 

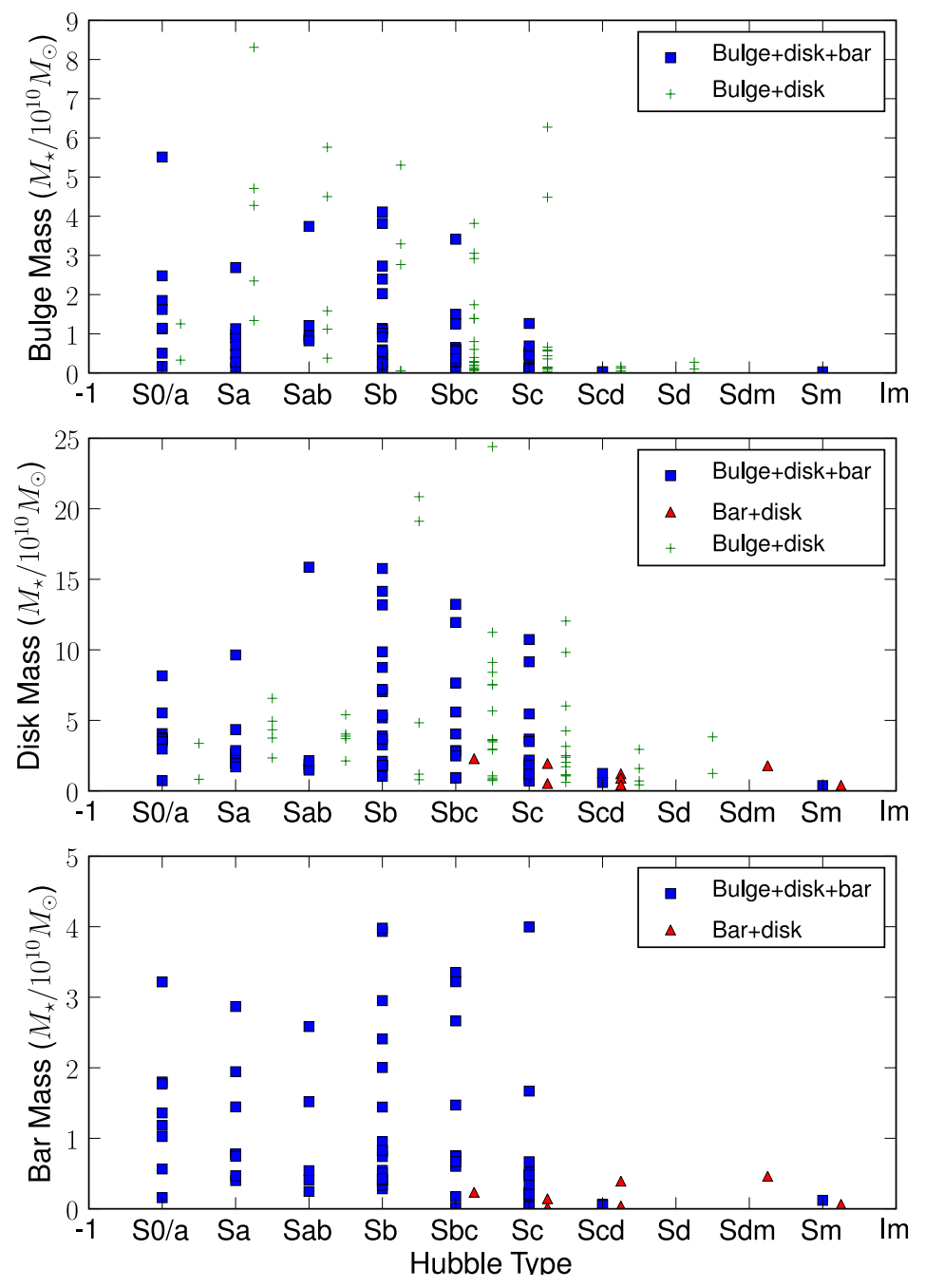

Figure 2.13 The top, middle, and bottom panels show stellar mass for bulges, disks, and bars, respectively, along the Hubble sequence. Values are shown for sample S2 of 126 galaxies in Fig. 2.3. The legend in each panel indicates the type of decomposition used for each data point. 

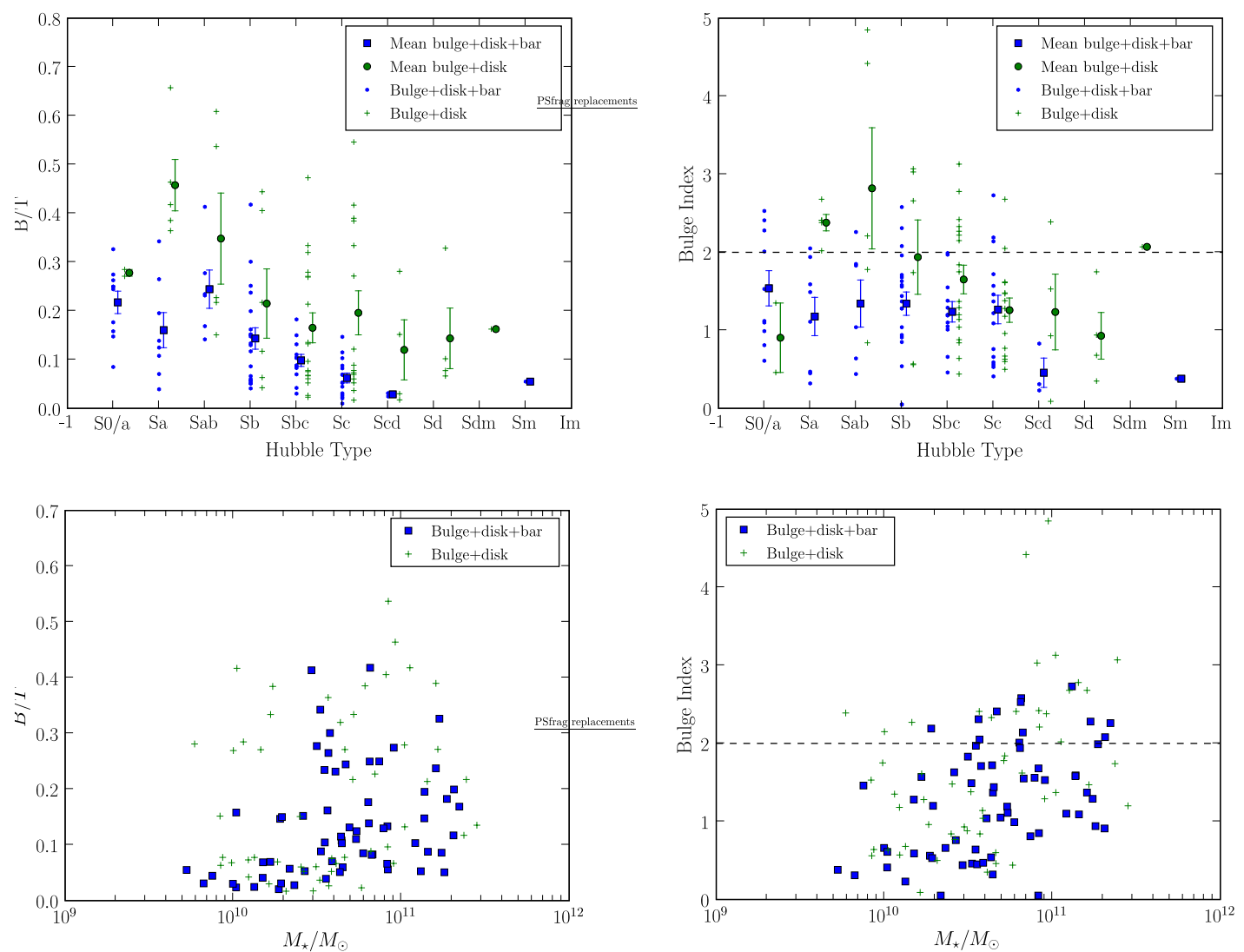

Figure 2.14 The individual and mean $B / T$ (left panels) and bulge Sérsic index (right panels) are plotted as a function of Hubble type for the sample S1 of bright galaxies, and as a function of galaxy stellar mass for sample S2. The error bars indicate the standard deviation of the population around the mean in each bin. The legend in each panel indicates the type of decomposition used for each data point. The mean $B / T$ and bulge index in barred galaxies differ systematically from unbarred galaxies, but there is a large overlap in the individual values. As many as $\sim 69 \%$ of bright spiral galaxies have $B / T \leq 0.2$; these bulges are pervasive and exist across the Hubble sequence. Furthermore, as many as $\sim 76 \%$ of bright spirals have low $n \leq 2$ bulges. Such bulges exist in barred and unbarred galaxies across a wide range of Hubble types. 

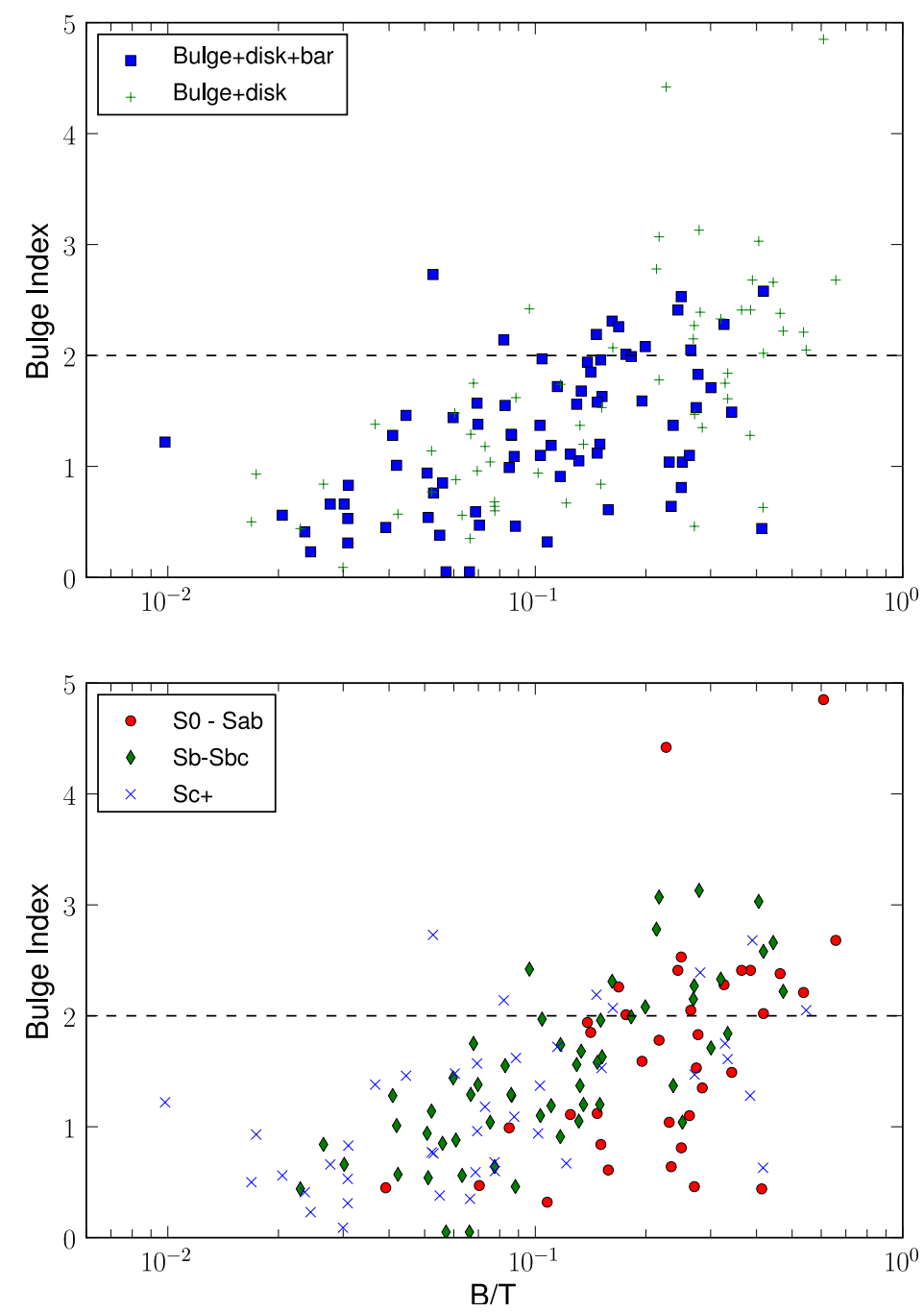

Figure 2.15 The relation between $B / T$ and bulge index is shown. In the top panel, galaxies are coded according to bar class. The legend indicates the type of decomposition used for each data point. In the lower panel, galaxies are coded according to Hubble type. A striking $\sim 76 \%$ of bright spirals have low $n \leq 2$ bulges. Such bulges exist in barred and unbarred galaxies across a wide range of Hubble types, and their $B / T$ range from 0.01 to 0.4 , with most having $B / T \leq 0.2$. A moderate fraction $(\sim 22 \%)$ have intermediate $2<n<4$ bulges. These exist in barred and unbarred $\mathrm{S} 0 / \mathrm{a}$ to $\mathrm{Sd}$ galaxies, and their $B / T$ spans a wide range (0.05 to 0.5$)$. Only $(\sim 1 \%)$ have $n \geq 4$. 


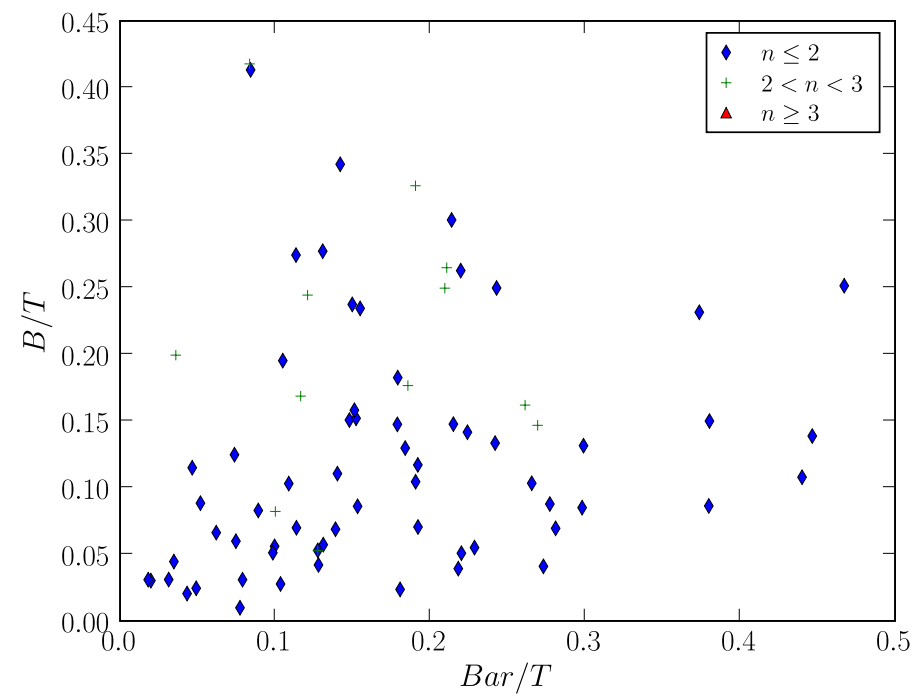

Figure $2.16 B / T$ is plotted against Bar $/ T$ and sorted by bulge Sérsic index. There are six galaxies with $\mathrm{Bar} / T \geq 0.3$. 


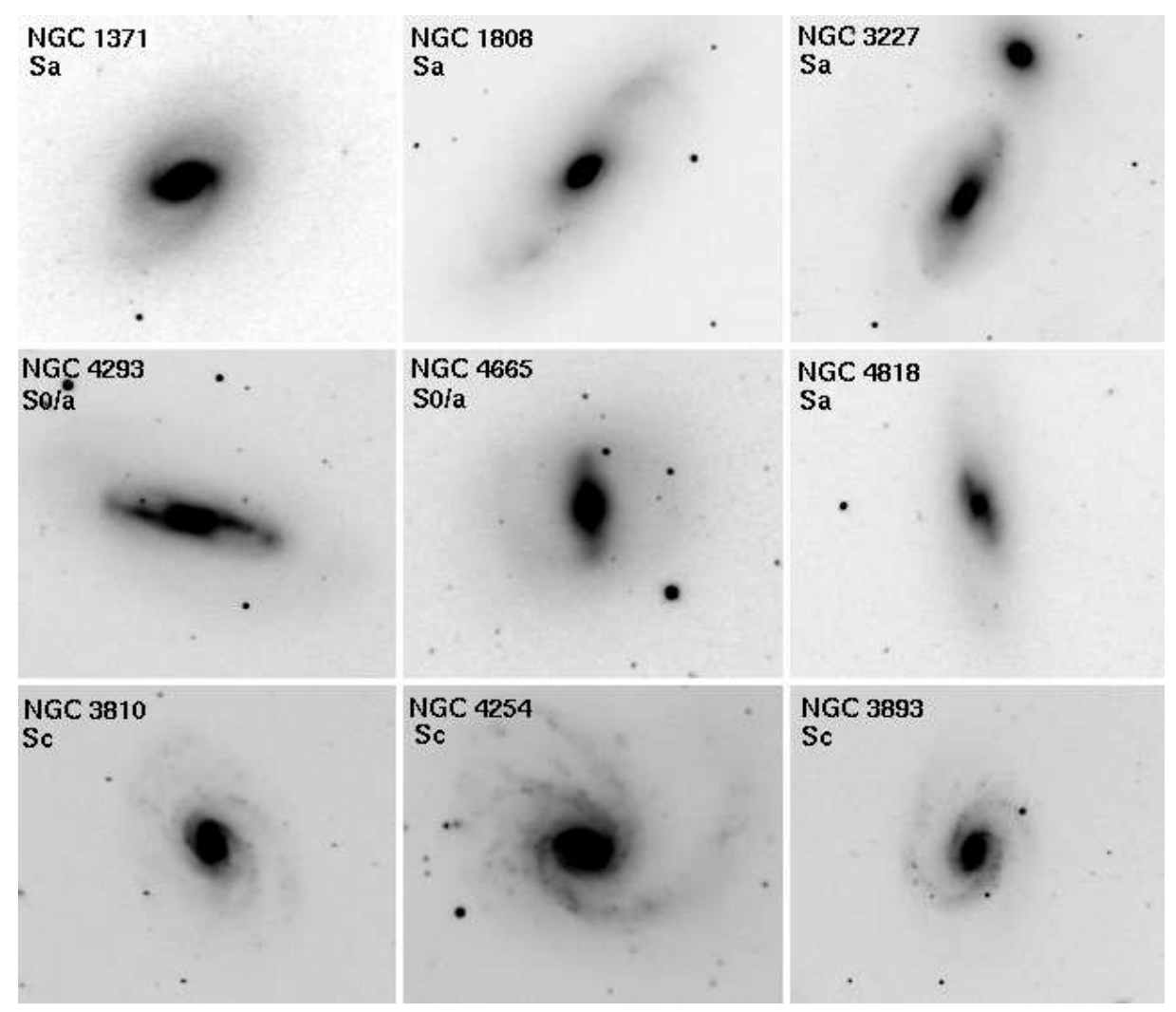

Figure 2.17 The top two rows show $H$-band images of barred galaxies, which have early RC3 Hubble types, but yet have $B / T \leq 0.2$. The bottom row shows $H$-band images of unbarred galaxies, which have late RC3 Hubble types, but yet have $B / T \sim 0.4$. The Hubble types assigned to these objects more reflect disk smoothness and spiral arm topology than $B / T$. All images are from OSUBSGS with characteristics as described in $\S 2.2$. 


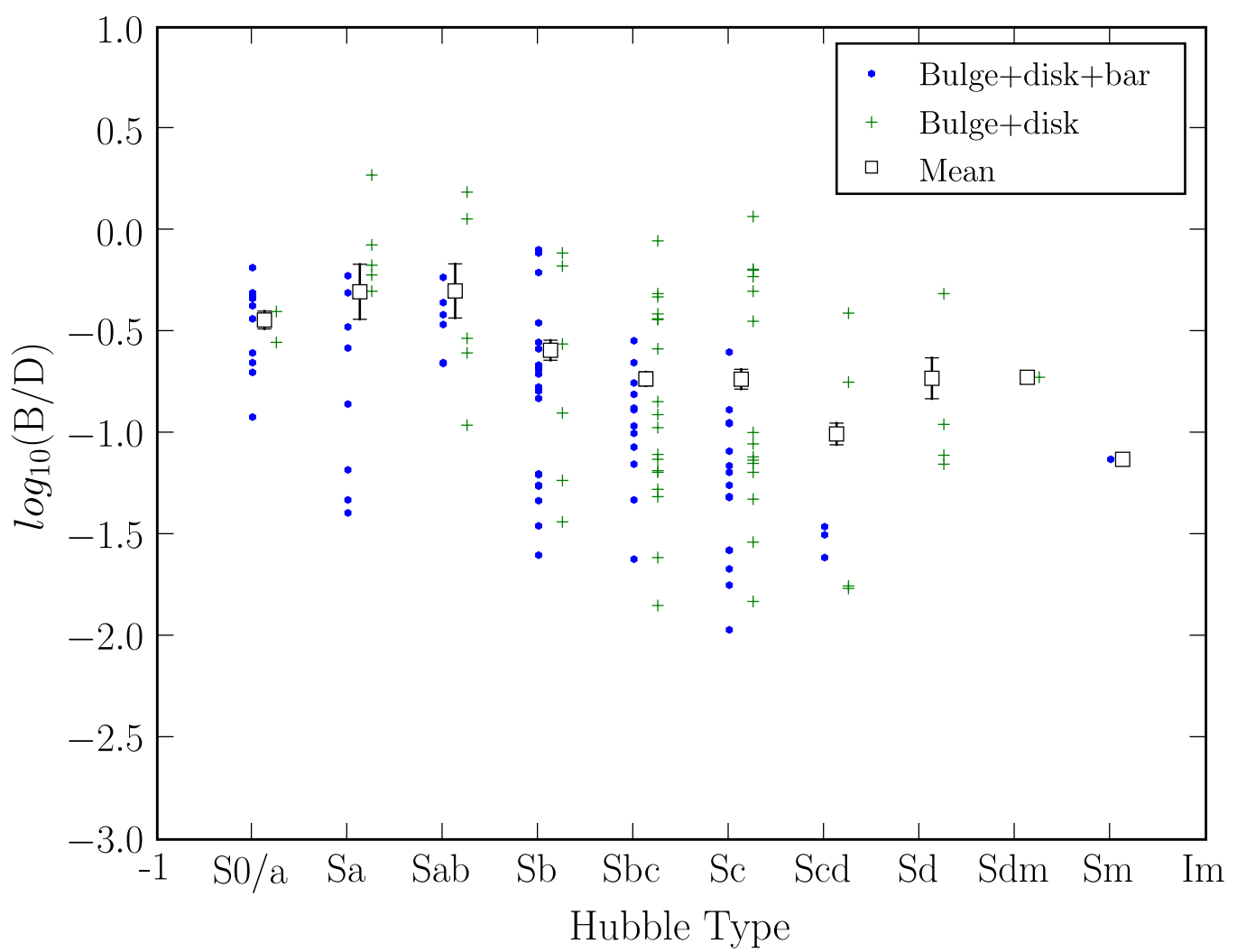

Figure $2.18 \mathrm{~B} / \mathrm{D}$ is plotted against Hubble type. The legend indicates the type of decomposition used for each data point. The mean values for barred and unbarred together in each bin are shown. 

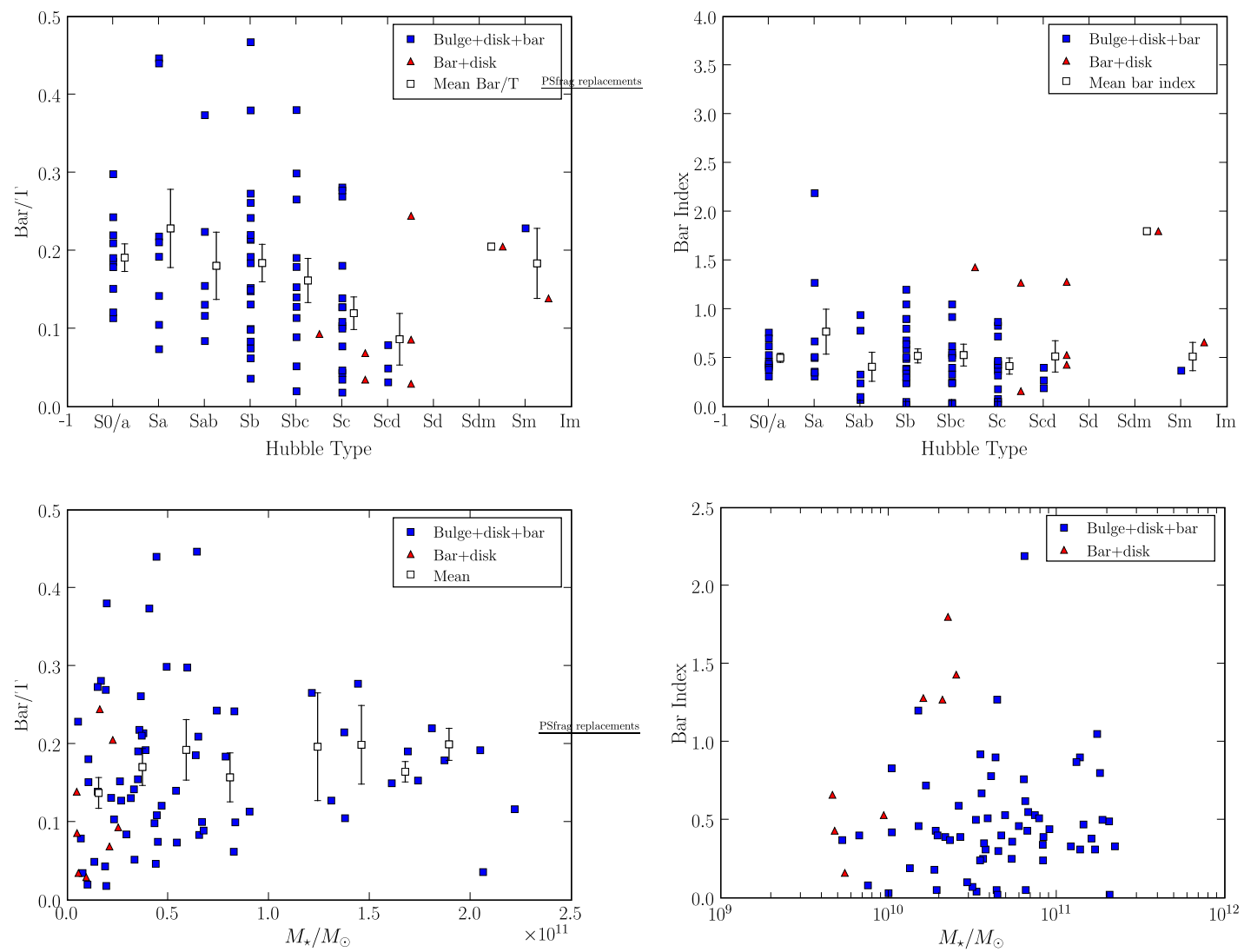

Figure 2.19 The properties of bars are shown. The error bars indicate the standard deviation of the population around the mean in each bin. The legend in each panel indicates the type of decomposition used for each data point. Upper left: Mean and individual Bar/T plotted against Hubble type. Upper right: Mean and individual bar Sérsic indices plotted against Hubble type. Lower left: Bar/T plotted against total galaxy stellar mass. The mean Bar $/ T$ in bins of stellar mass is indicated. Lower right: Bar Sérsic index plotted against total galaxy stellar mass. 

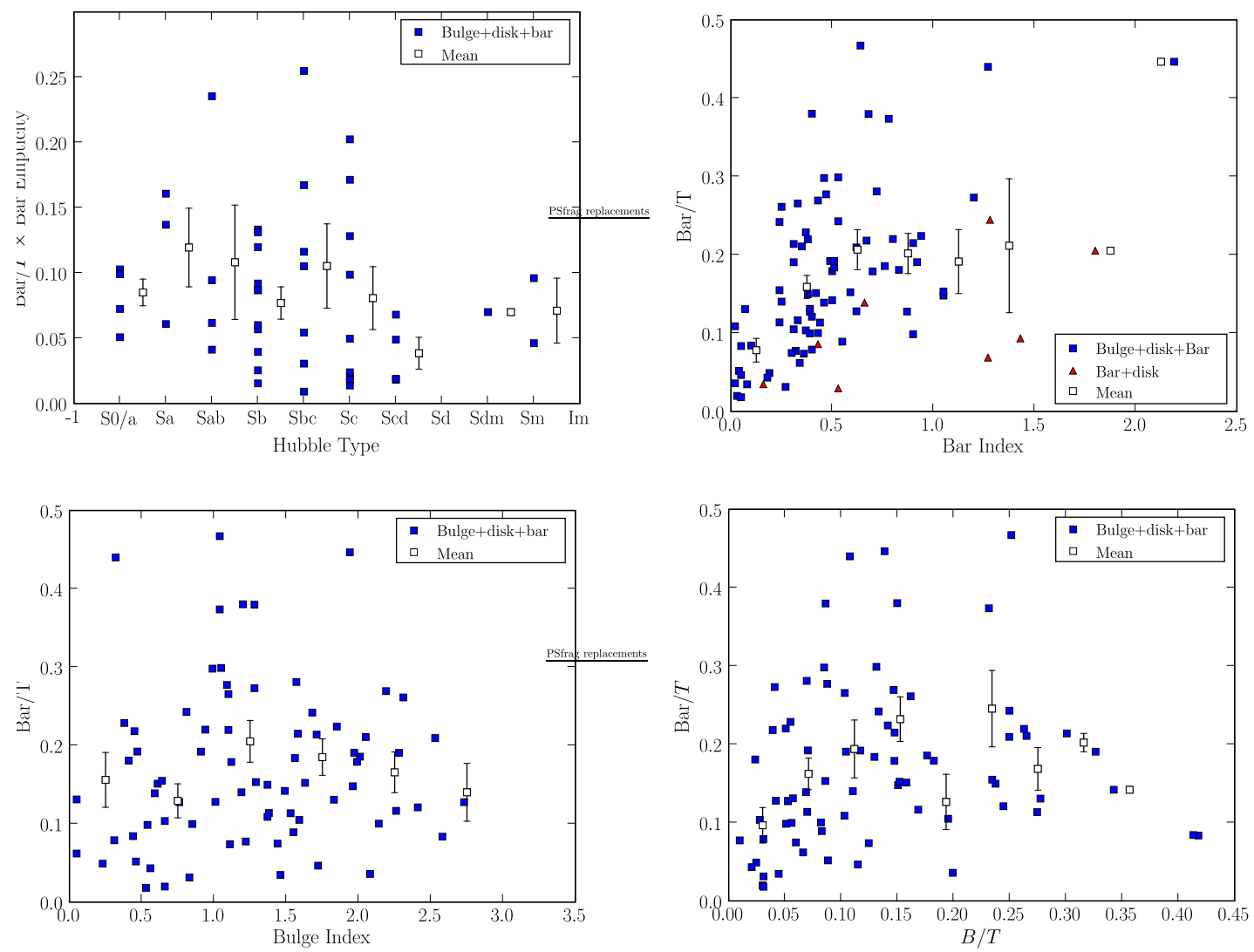

Figure 2.20 Bar strength is plotted against Hubble type and the properties of bars are compared with bulges. The legend in each panel indicates the type of decomposition used for each data point. Upper left: Bar strength, the product of Bar/T and peak bar ellipticity $e_{b a r}$ from MJ07 is plotted against Hubble type. Upper right: Bar/T is plotted against bar Sérsic index. Lower left: Bar $/ T$ is plotted against bulge Sérsic index. Lower right: Bar $/ T$ is plotted against $B / T$. In the first plot, mean bar strong is calculated for each Hubble type. In the latter three plots, mean Bar $/ T$ is calculated for bins along the ordinate axis. The error bars indicate the standard deviation of the population around the mean in each bin. 

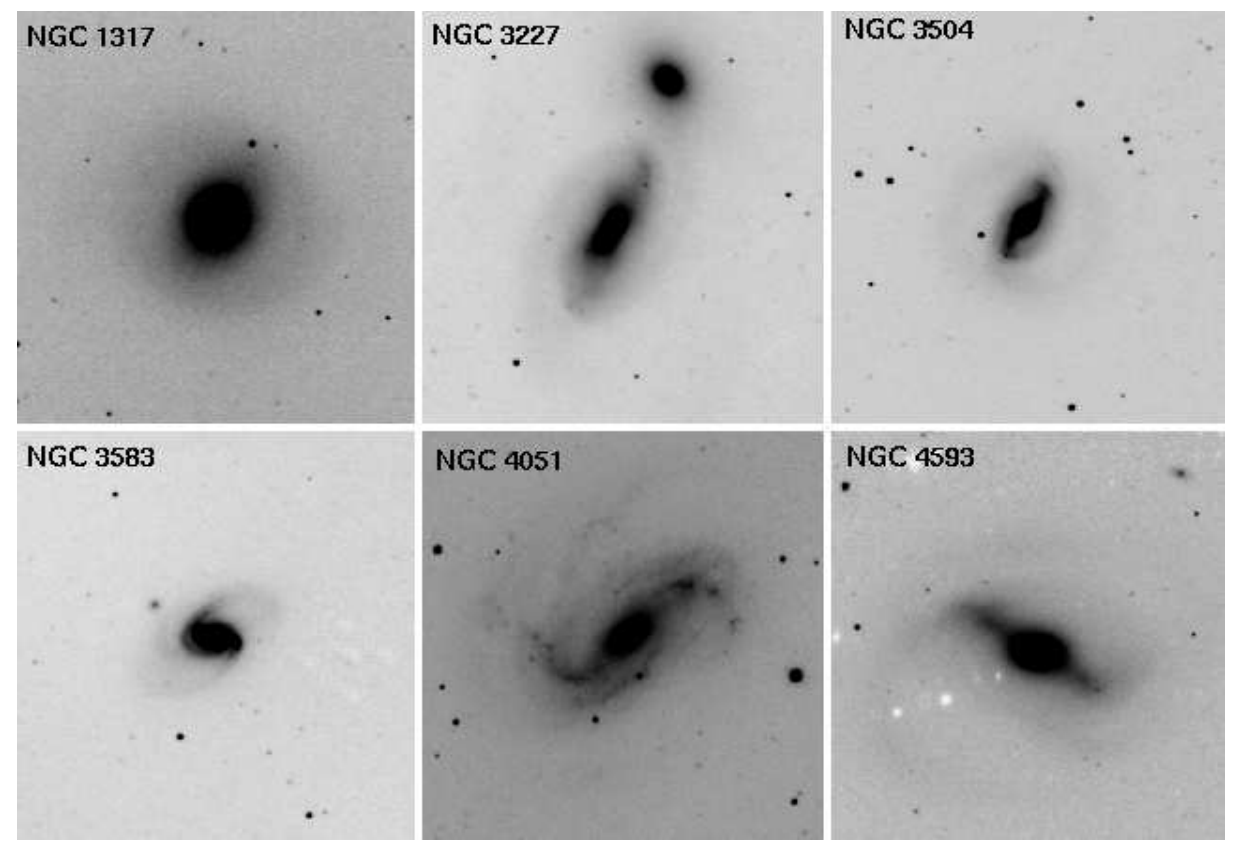

Figure $2.21 \mathrm{H}$-band OSUBSGS images of spirals with prominent bars of Bar/T $(>0.3)$ are shown. An interesting example is the oval or lens galaxy NGC 1317: the bar has a low ellipticity, but its $B / T$ is large because it is extended and massive. Such bars/lenses may exert significant gravitational torques although they are not very elongated. 

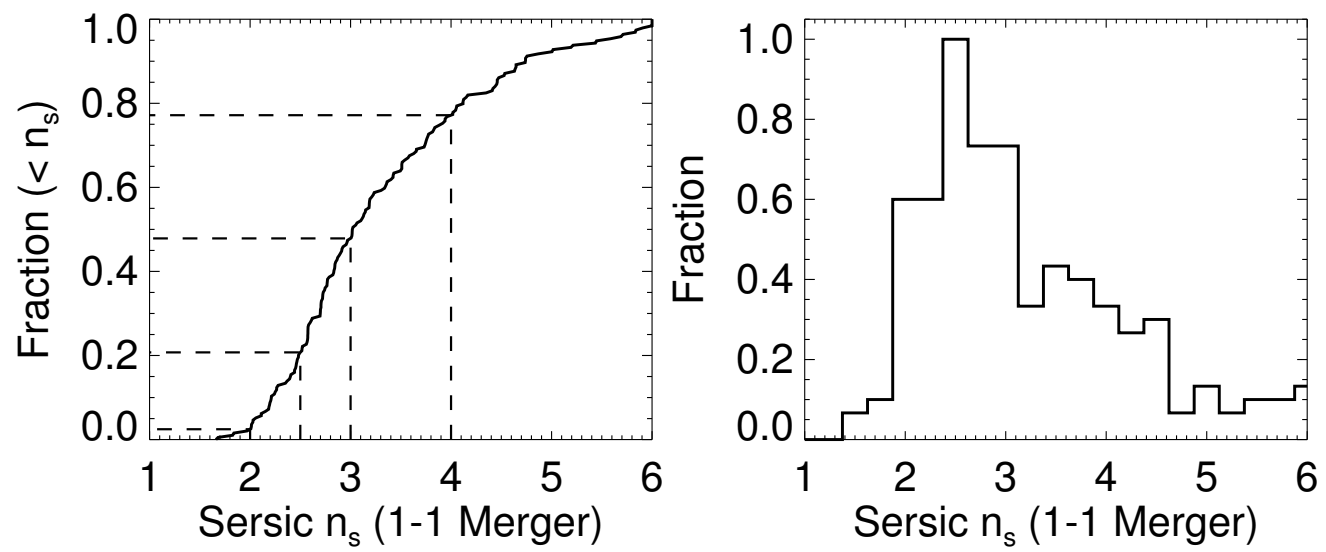

Figure 2.22 Shown is the distribution of Sérsic indices $n$ for remnants of 1:1 gas-rich major mergers in the simulations of Hopkins et al. 2009a: they lie in the range of $2<n<6$. Specifically, $\sim 22 \%$ of the remnants have classical $n \geq 4$, as much as $20 \%$ have low $n \leq 2.5$, while $50 \%$ have $n \leq 3$. Almost none have $n \leq 2$. [Figure: courtesy of Phil Hopkins] 


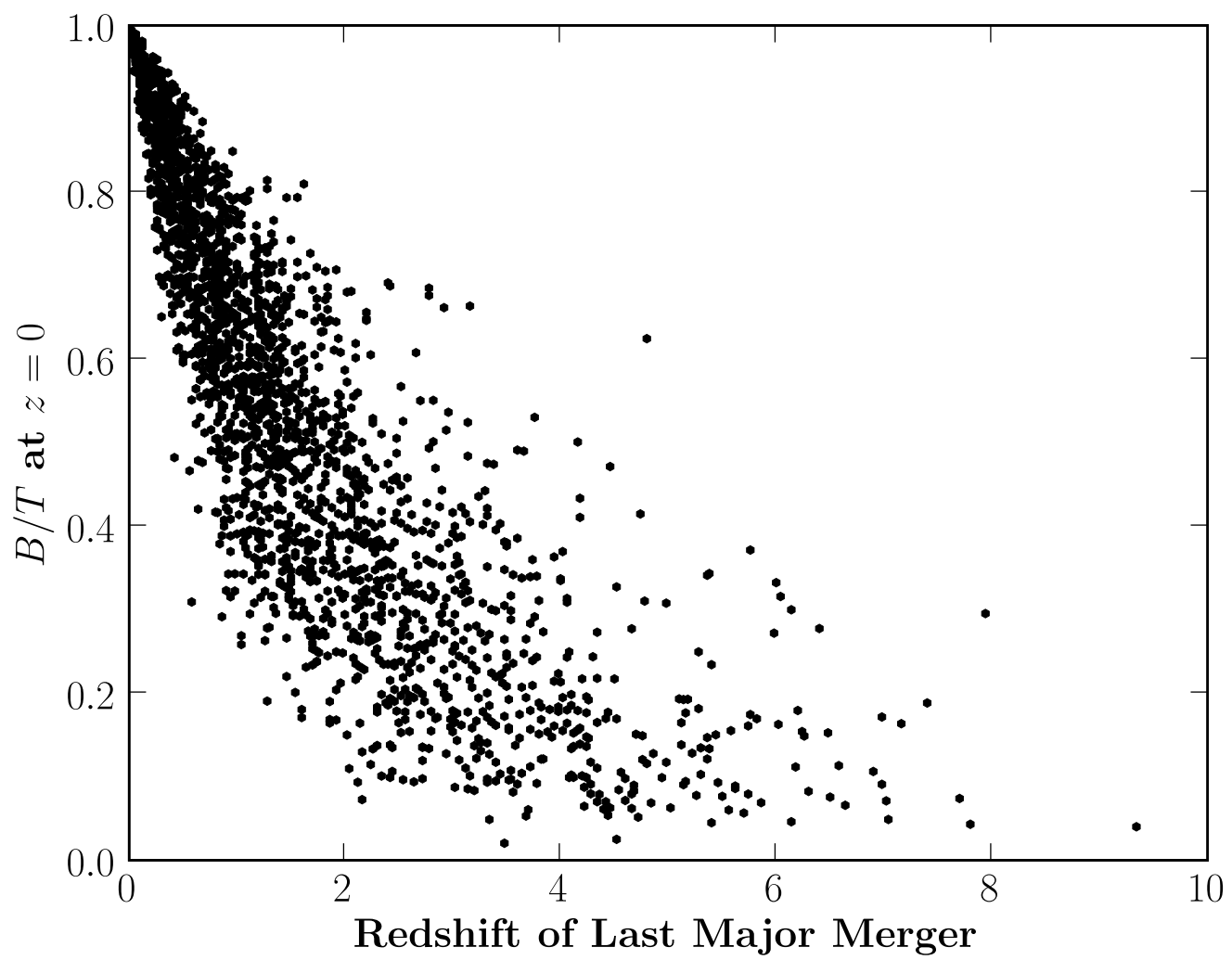

Figure 2.23 For those high mass $\left(M_{\star} \geq 1.0 \times 10^{10} M_{\odot}\right)$ galaxies in the theoretical models that experienced a major merger (see $\S 2.5 .8$ ), the $B / T$ of the remnant at $z \sim 0$ is plotted against the redshift $z_{\text {last }}$ of the last major merger. Systems where the last major merger occurred at earlier times have had more time to grow a disk and thus have a lower $B / T$ at $z \sim 0$. The dispersion in the present-day $B / T$ at a given $z_{\text {last }}$ is due to the different times spent by a galaxy in terms of being a satellite versus a central galaxy in a DM halo, since the cooling of gas and the growth of a disk is stopped when a galaxy becomes a satellite. In the model, a high mass galaxy that has undergone a major merger at $z \leq 2$ has a present-day $B / T>0.2$. In effect, a high mass spiral can have a present-day $B / T \leq 0.2$ only if its last major merger occurred at $z>2$ (lookback times $>10 \mathrm{Gyr}$ ). 

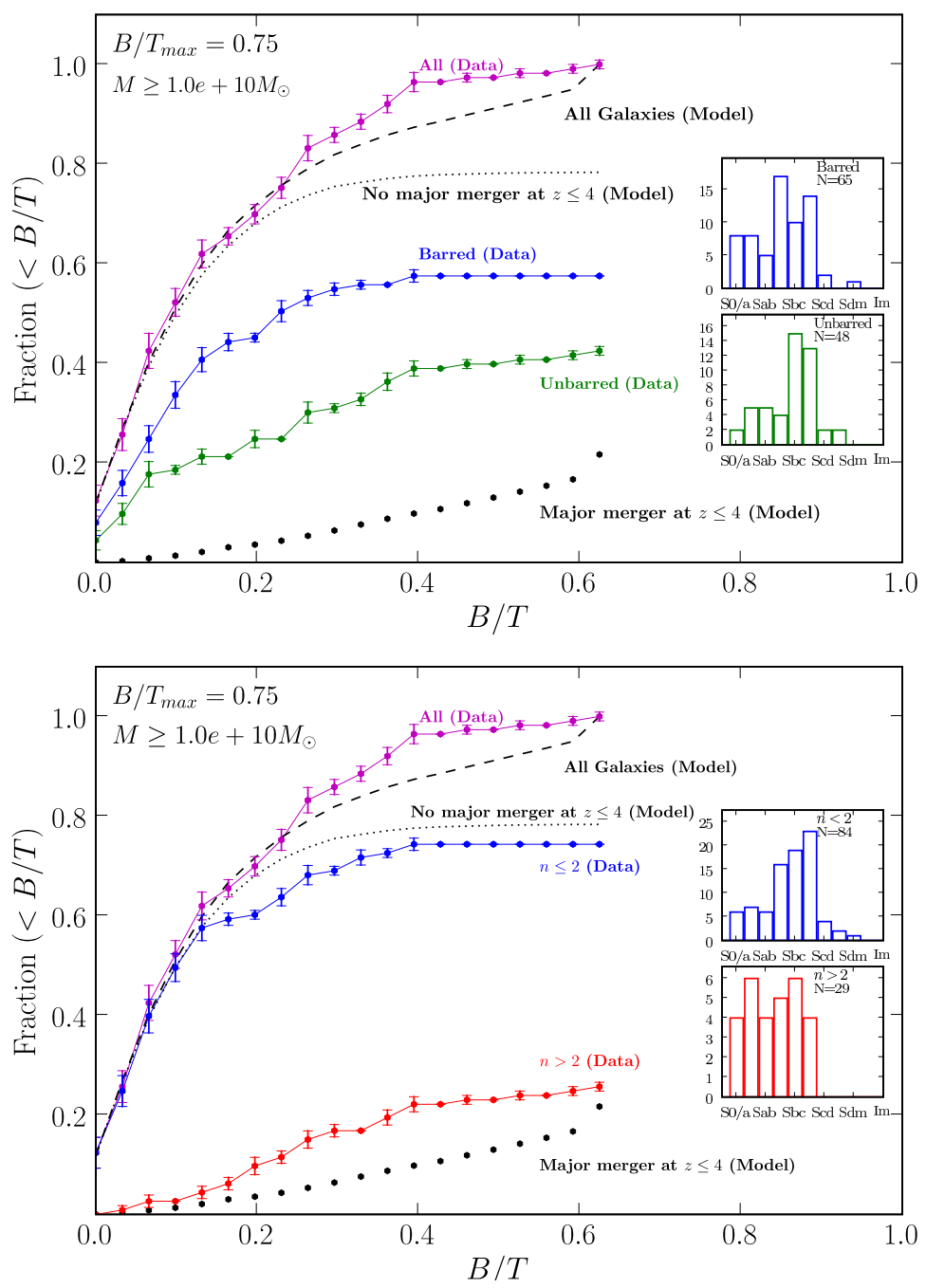

Figure 2.24 The cumulative fraction $F$ of high mass $\left(M_{\star} \geq 1.0 \times 10^{10} M_{\odot}\right)$ spirals with present-day $B / T \leq$ the $\mathrm{x}$-axis value is shown for the data (colored lines/ points) and for the theoretical model (black lines/points) described in $\S 2.5 .8$. Model and data spirals are defined as systems with $B / T \leq 0.75$. The magenta line shows $F$ from the data, while the other two colored lines break this $F$ in terms of bar class (top panel) or bulge $n$ (lower panel). The black dashed line shows $F$ from all model galaxies, while the black dotted line and black dots show the contribution of model galaxies that experienced, respectively, no major merger and one or more major mergers since $z \leq 4$. Major mergers are defined here as those with $M_{1} / M_{2} \geq 1 / 4$. In the model, the fraction $(\sim 1.6 \%$; see Table 2.11$)$ of high mass spirals, which have undergone a major merger since $z \leq 4$ and host a bulge with a present-day $B / T \leq 0.2$ is a factor of over 30 smaller than the observed fraction $(\sim 66 \%)$ of high mass spirals with present-day $B / T \leq 0.2$. Thus, bulges built via major mergers since $z \leq 4$ seriously fail to account for most of the low $B / T \leq 0.2$ bulges present in two-thirds of high-mass spirals. 

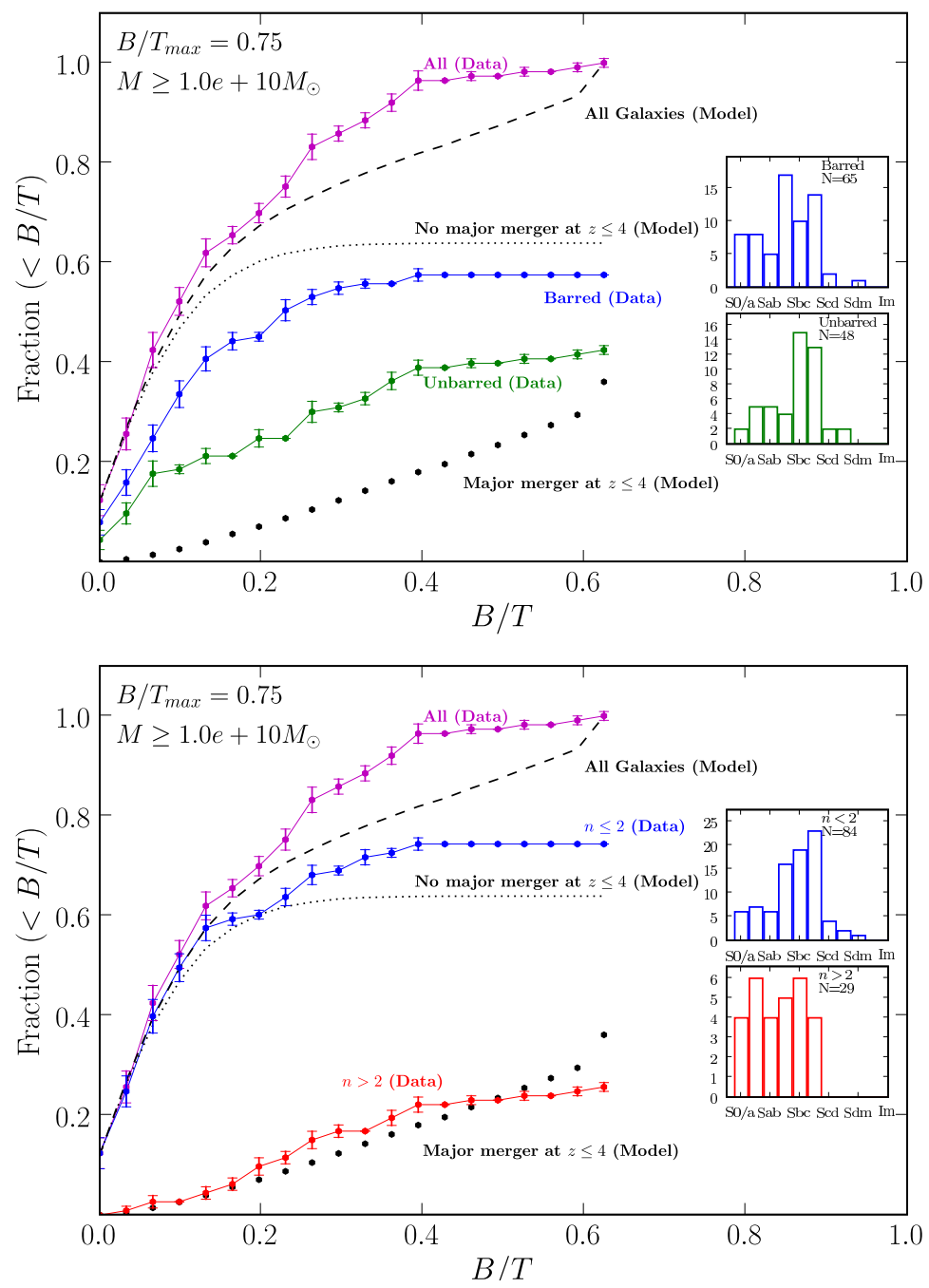

Figure 2.25 This figure is similar to Fig. 2.24, except that the model now defines major mergers as those with mass ratio $M_{1} / M_{2} \geq 1 / 6$. 

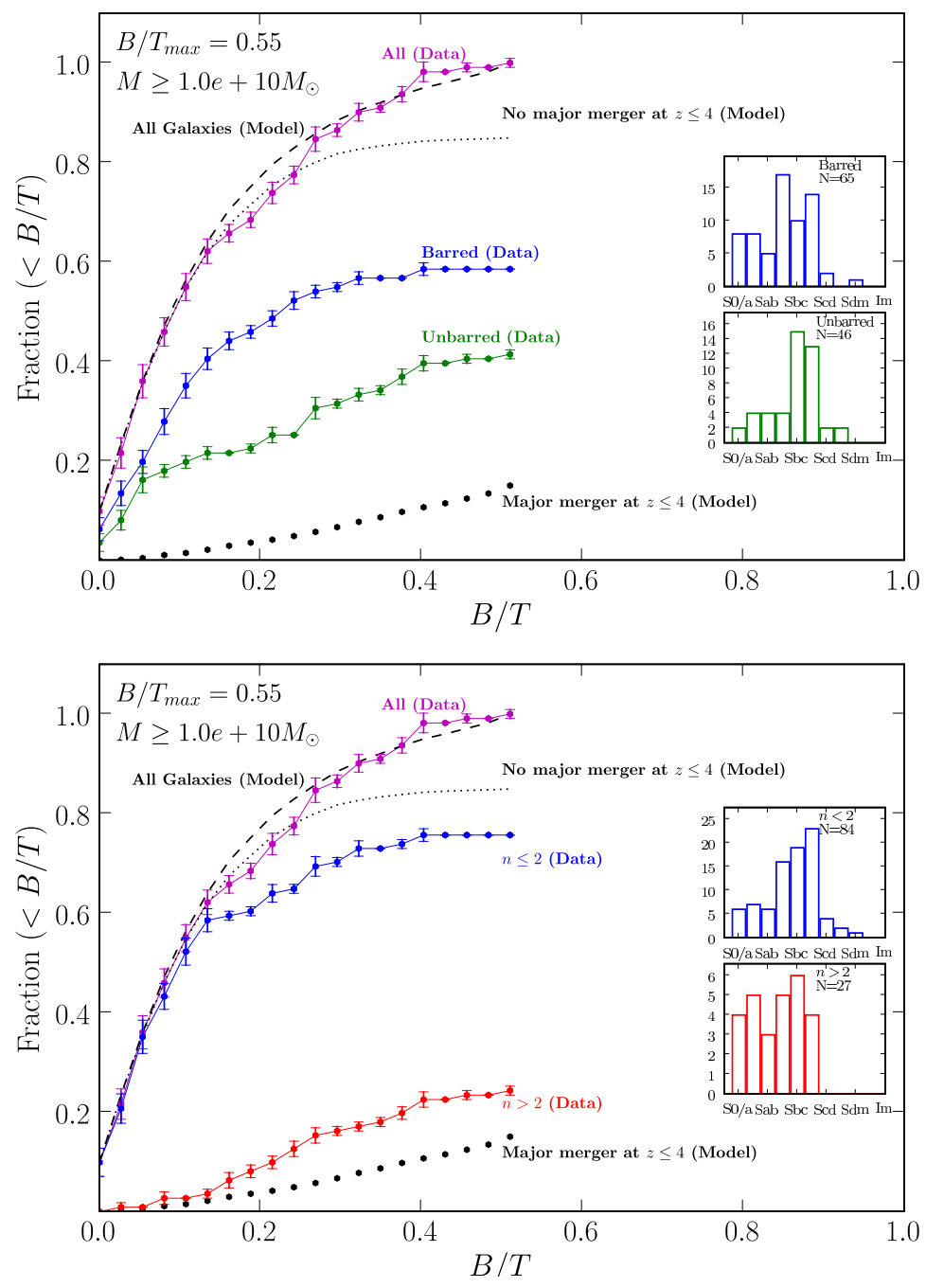

Figure 2.26 This figure is similar to Fig. 2.24, except that here spirals are considered to be systems with a $B / T \leq 0.55$ rather than 0.75 in the models, and a corresponding cut is applied to the data points. 

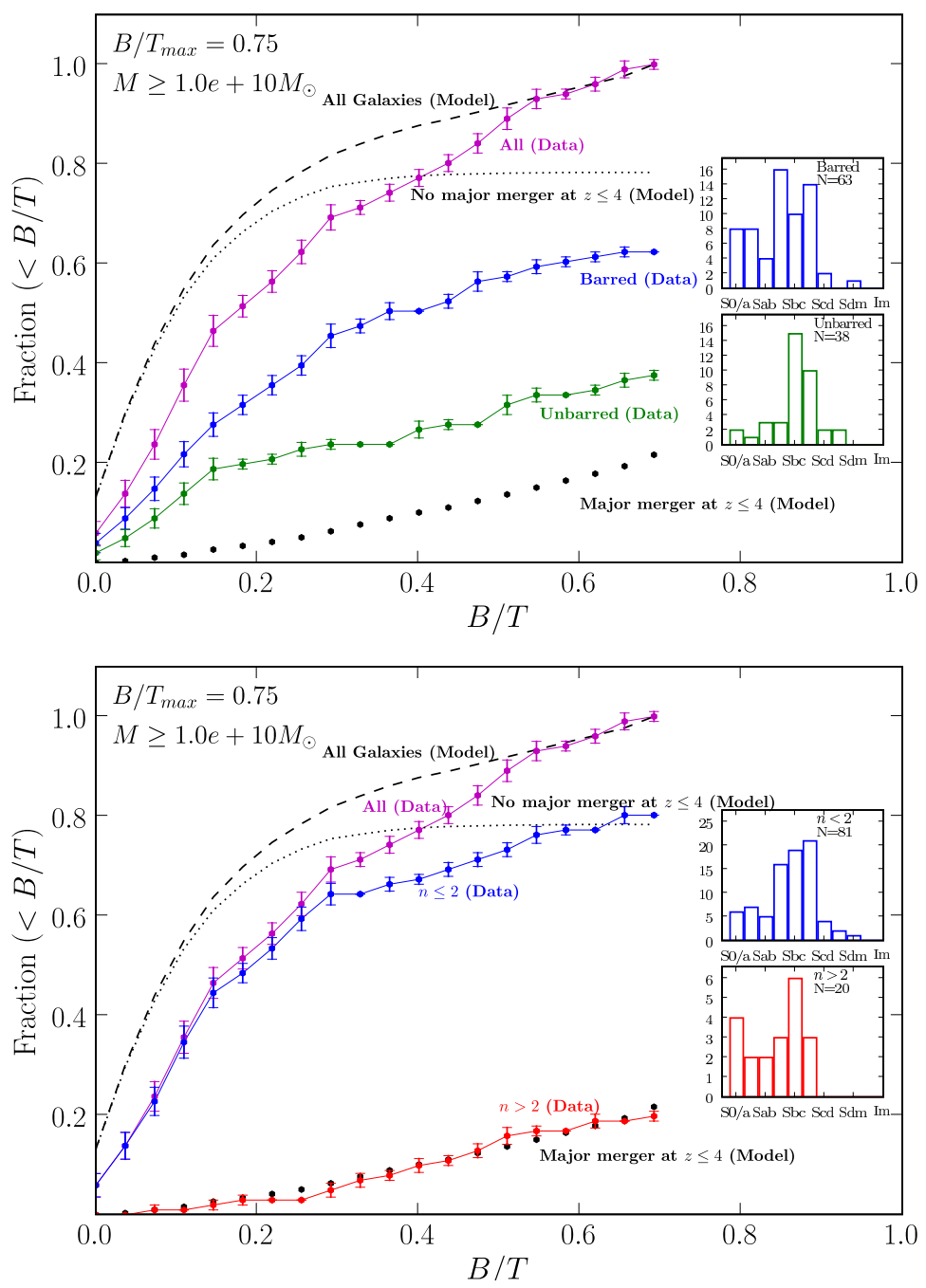

Figure 2.27 This figure is similar to Fig. 2.24, except that $B / T$ of all the observed galaxies has been multiplied by a factor of two, in order to test what would happen in the case where the $M / L$ ratio of the bulge in $H$-band is twice as high as that of the disk and bar. This could happen in an extreme example where the dominant bulge stellar population was much older (e.g. 12 Gyr) than the age of the dominant disk stellar population (e.g., $3 \mathrm{Gyr}$ ). In such a case, the fraction of high mass spirals with $B / T \leq 0.2$ would change from $\sim 66 \%$ in Fig. 2.24 to $\sim 50 \%$. However, the main conclusion that bulges built by major mergers since $z \leq 4$ cannot account for most of the low $B / T \leq 0.2$ bulges, present in a large percentage $(\sim 55 \%)$ of spirals still holds. 
Table 2.1. Sample S1 of Bright $\left(M_{B} \leq-19.3\right)$ Low-to-Moderately Inclined $\left(i \leq 70^{\circ}\right)$ Spirals in OSUBSGS $(\mathrm{N}=143)$

\begin{tabular}{|c|c|c|c|c|c|c|c|}
\hline $\begin{array}{c}\text { Galaxy Name } \\
\text { (1) }\end{array}$ & $\begin{array}{c}\text { Best Fit } \\
(2)\end{array}$ & $\begin{array}{l}\text { Hubble Type } \\
\text { (RC3) } \\
\text { (3) }\end{array}$ & $\begin{array}{c}\text { Bar Type } \\
\text { (RC3) } \\
(4)\end{array}$ & $\begin{array}{c}D \\
(\mathrm{Mpc}) \\
(5)\end{array}$ & $\begin{array}{c}M_{B} \\
(\mathrm{mag}) \\
(6)\end{array}$ & $\begin{array}{c}B-V \\
(\operatorname{mag}) \\
(7)\end{array}$ & $\begin{array}{c}M_{\star} \\
\left(M_{\odot}\right) \\
(8)\end{array}$ \\
\hline IC0239 & $\mathrm{PSF}+\mathrm{Bulge}+\mathrm{Disk}+\mathrm{Bar}$ & $\mathrm{SAB}(\mathrm{rs}) \mathrm{cd}$ & $\mathrm{AB}$ & 15.21 & -19.3 & 0.70 & $1.34 \mathrm{e}+10$ \\
\hline IC 4444 & Bulge + Disk & $\mathrm{SAB}(\mathrm{rs}) \mathrm{bc}$ & $\mathrm{AB}$ & 28.82 & -20.9 & 0.64 & $4.35 \mathrm{e}+10$ \\
\hline IC5325 & PSF+Bulge+Disk & $\mathrm{SAB}(\mathrm{rs}) \mathrm{bc}$ & $\mathrm{AB}$ & 19.39 & -19.6 & 0.56 & $9.78 \mathrm{e}+09$ \\
\hline NGC0210 & Bulge+Disk+Bar & $\mathrm{SAB}(\mathrm{s}) \mathrm{b}$ & $\mathrm{AB}$ & 21.75 & -20.4 & 0.71 & $3.77 \mathrm{e}+10$ \\
\hline NGC0278 & PSF+Bulge+Disk & $\mathrm{SAB}(\mathrm{rs}) \mathrm{b}$ & $\mathrm{AB}$ & 12.64 & -19.5 & 0.64 & $1.24 \mathrm{e}+10$ \\
\hline NGC0289 & $\mathrm{PSF}+\mathrm{Bulge}+\mathrm{Disk}+\mathrm{Bar}$ & $\mathrm{SAB}(\mathrm{rs}) \mathrm{bc}$ & $\mathrm{AB}$ & 20.79 & -20.2 & 0.73 & $3.33 \mathrm{e}+10$ \\
\hline NGC0428 & $\mathrm{PSF}+\mathrm{Bulge}+\mathrm{Disk}+\mathrm{Bar}$ & $\mathrm{SAB}(\mathrm{s}) \mathrm{m}$ & $\mathrm{AB}$ & 15.96 & -19.4 & 0.44 & $5.28 \mathrm{e}+09$ \\
\hline NGC0488 & PSF+Bulge+Disk & $\mathrm{SA}(\mathrm{r}) \mathrm{b}$ & $\mathrm{A}$ & 31.39 & -21.8 & 0.87 & $2.44 \mathrm{e}+11$ \\
\hline NGC0578 & $\mathrm{PSF}+\mathrm{Bulge}+\mathrm{Disk}+\mathrm{Bar}$ & $\mathrm{SAB}(\mathrm{rs}) \mathrm{c}$ & $\mathrm{AB}$ & 20.89 & -20.5 & 0.51 & $1.87 \mathrm{e}+10$ \\
\hline NGC0613 & $\mathrm{PSF}+\mathrm{Bulge}+\mathrm{Disk}+\mathrm{Bar}$ & $\mathrm{SB}(\mathrm{rs}) \mathrm{bc}$ & $\mathrm{B}$ & 18.75 & -20.8 & 0.68 & $4.92 \mathrm{e}+10$ \\
\hline NGC1042 & PSF+Bulge+Disk & $\mathrm{SAB}(\mathrm{rs}) \mathrm{cd}$ & $\mathrm{AB}$ & 17.89 & -20.2 & 0.54 & $1.63 \mathrm{e}+10$ \\
\hline NGC1073 & $\mathrm{PSF}+$ Bulge+Disk + Bar & $\mathrm{SB}(\mathrm{rs}) \mathrm{c}$ & B & 16.29 & -19.9 & 0.50 & $1.04 \mathrm{e}+10$ \\
\hline NGC1084 & PSF+Bulge+Disk & $\mathrm{SA}(\mathrm{s}) \mathrm{c}$ & A & 18.32 & -20.6 & 0.58 & $2.64 \mathrm{e}+10$ \\
\hline NGC1087 & Bar + Disk & $\mathrm{SAB}(\mathrm{rs}) \mathrm{c}$ & $\mathrm{AB}$ & 20.36 & -20.6 & 0.52 & $2.09 \mathrm{e}+10$ \\
\hline NGC1187 & $\mathrm{PSF}+\mathrm{Bulge}+\mathrm{Disk}+\mathrm{Bar}$ & $\mathrm{SB}(\mathrm{r}) \mathrm{c}$ & $\mathrm{B}$ & 17.46 & -20.2 & 0.56 & $1.66 \mathrm{e}+10$ \\
\hline NGC1241 & $\mathrm{PSF}+\mathrm{Bulge}+\mathrm{Disk}+\mathrm{Bar}$ & $\mathrm{SB}(\mathrm{rs}) \mathrm{b}$ & $\mathrm{B}$ & 28.50 & -21.7 & 0.85 & $2.05 \mathrm{e}+11$ \\
\hline NGC1300 & $\mathrm{PSF}+$ Bulge+Disk+Bar & $\mathrm{SB}(\mathrm{rs}) \mathrm{bc}$ & $\mathrm{B}$ & 20.14 & -20.9 & 0.68 & $5.39 \mathrm{e}+10$ \\
\hline NGC1302 & $\mathrm{PSF}+\mathrm{Bulge}+\mathrm{Disk}+\mathrm{Bar}$ & $(\mathrm{R}) \mathrm{SB}(\mathrm{r}) 0$ & $\mathrm{~B}$ & 21.43 & -20.2 & 0.89 & $6.37 \mathrm{e}+10$ \\
\hline NGC1309 & $\mathrm{PSF}+\mathrm{Bulge}+\mathrm{Disk}$ & $\mathrm{SA}(\mathrm{s}) \mathrm{bc}$ & $\mathrm{A}$ & 27.86 & -20.5 & 0.44 & $1.46 \mathrm{e}+10$ \\
\hline NGC1317 & Bulge+Disk+Bar & $\mathrm{SAB}(\mathrm{r}) \mathrm{a}$ & $\mathrm{AB}$ & 18.11 & -20.2 & 0.89 & $6.42 \mathrm{e}+10$ \\
\hline NGC1350 & $\mathrm{PSF}+\mathrm{Bulge}+\mathrm{Disk}+\mathrm{Bar}$ & $\left(\mathrm{R}^{\prime}\right) \mathrm{SB}(\mathrm{r}) \mathrm{ab}$ & $\mathrm{B}$ & 18.11 & -21.1 & 0.87 & $1.38 \mathrm{e}+11$ \\
\hline NGC1371 & $\mathrm{PSF}+\mathrm{Bulge}+\mathrm{Disk}+\mathrm{Bar}$ & $\mathrm{SAB}(\mathrm{rs}) \mathrm{a}$ & $\mathrm{AB}$ & 18.32 & -20.0 & 0.90 & $5.43 \mathrm{e}+10$ \\
\hline NGC2196 & PSF+Bulge+Disk & $\left(\mathrm{R}^{\prime}\right) \mathrm{SA}(\mathrm{s}) \mathrm{a}$ & A & 30.86 & -21.0 & 0.81 & $9.22 \mathrm{e}+10$ \\
\hline NGC2442 & $\mathrm{PSF}+$ Bulge+Disk+Bar & $\mathrm{SAB}(\mathrm{s}) \mathrm{bc} ; \mathrm{pec}$ & $\mathrm{AB}$ & 18.32 & -20.8 & 0.82 & $8.28 \mathrm{e}+10$ \\
\hline NGC2559 & Bulge+Disk+Bar & $\mathrm{SB}(\mathrm{s}) \mathrm{bc} ; \mathrm{pec}$ & $\mathrm{B}$ & 21.43 & -21.0 & - & - \\
\hline NGC2566 & Bulge+Disk & $\left(\mathrm{R}^{\prime}\right) \mathrm{SB}(\mathrm{rs}) \mathrm{ab} ; \mathrm{pec}$ & $\mathrm{B}$ & 22.61 & -20.7 & 0.81 & $6.98 \mathrm{e}+10$ \\
\hline NGC2775 & $\mathrm{PSF}+\mathrm{Bulge}+\mathrm{Disk}$ & $\mathrm{SA}(\mathrm{r}) \mathrm{ab}$ & $\mathrm{A}$ & 18.21 & -20.6 & 0.90 & $9.46 \mathrm{e}+10$ \\
\hline NGC3059 & Bar + Disk & $\mathrm{SB}(\mathrm{rs}) \mathrm{c}$ & $\mathrm{B}$ & 15.86 & -20.1 & 0.68 & $2.52 \mathrm{e}+10$ \\
\hline NGC3166 & Bulge+Disk+Bar & $\mathrm{SAB}(\mathrm{rs}) 0$ & $\mathrm{AB}$ & 23.57 & -20.2 & 0.93 & $7.41 \mathrm{e}+10$ \\
\hline NGC3169 & $\mathrm{PSF}+\mathrm{Bulge}+\mathrm{Disk}$ & $\mathrm{SA}(\mathrm{s}) \mathrm{a} ; \mathrm{pec}$ & $\mathrm{A}$ & 21.11 & -20.3 & 0.85 & $6.09 \mathrm{e}+10$ \\
\hline NGC3223 & PSF+Bulge+Disk & $\mathrm{SA}(\mathrm{s}) \mathrm{b}$ & $\mathrm{A}$ & 40.82 & -21.9 & 0.82 & $2.36 \mathrm{e}+11$ \\
\hline NGC3227 & $\mathrm{PSF}+$ Bulge+Disk+Bar & $\mathrm{SAB}(\mathrm{s}) \mathrm{a} ; \mathrm{pec}$ & $\mathrm{AB}$ & 22.07 & -20.1 & 0.82 & $4.42 \mathrm{e}+10$ \\
\hline NGC3261 & $\mathrm{PSF}+\mathrm{Bulge}+\mathrm{Disk}+\mathrm{Bar}$ & $\mathrm{SB}(\mathrm{rs}) \mathrm{b}$ & $\mathrm{B}$ & 35.79 & -21.3 & - & - \\
\hline NGC3275 & $\mathrm{PSF}+\mathrm{Bulge}+\mathrm{Disk}+\mathrm{Bar}$ & $\mathrm{SB}(\mathrm{r}) \mathrm{ab}$ & B & 45.43 & -21.2 & - & - \\
\hline NGC3319 & $\mathrm{PSF}+\mathrm{Bar}+\mathrm{Disk}$ & $\mathrm{SB}(\mathrm{rs}) \mathrm{cd}$ & $\mathrm{B}$ & 12.32 & -19.4 & 0.41 & $4.76 \mathrm{e}+09$ \\
\hline NGC3338 & $\mathrm{PSF}+\mathrm{Bulge}+\mathrm{Disk}+\mathrm{Bar}$ & $\mathrm{SA}(\mathrm{s}) \mathrm{c}$ & A & 24.43 & -20.6 & 0.59 & $2.67 \mathrm{e}+10$ \\
\hline NGC3423 & Bulge + Disk & $\mathrm{SA}(\mathrm{s}) \mathrm{cd}$ & $\mathrm{A}$ & 11.68 & -19.5 & 0.45 & $5.89 \mathrm{e}+09$ \\
\hline NGC3504 & Bulge+Disk+Bar & (R)SAB(s)ab & $\mathrm{AB}$ & 21.43 & -20.5 & 0.72 & $4.06 \mathrm{e}+10$ \\
\hline NGC3513 & Bulge+Disk+Bar & $\mathrm{SB}(\mathrm{rs}) \mathrm{c}$ & $\mathrm{B}$ & 18.21 & -19.9 & 0.43 & $7.52 \mathrm{e}+09$ \\
\hline
\end{tabular}


Table 2.1 (cont'd)

\begin{tabular}{|c|c|c|c|c|c|c|c|}
\hline $\begin{array}{c}\text { Galaxy Name } \\
\text { (1) }\end{array}$ & $\begin{array}{c}\text { Best Fit } \\
(2)\end{array}$ & $\begin{array}{c}\text { Hubble Type } \\
\text { (RC3) } \\
\text { (3) }\end{array}$ & $\begin{array}{c}\text { Bar Type } \\
\text { (RC3) } \\
(4)\end{array}$ & $\begin{array}{c}D \\
(\mathrm{Mpc}) \\
(5)\end{array}$ & $\begin{array}{c}M_{B} \\
(\mathrm{mag}) \\
(6)\end{array}$ & $\begin{array}{c}B-V \\
(\mathrm{mag}) \\
(7)\end{array}$ & $\begin{array}{c}M_{\star} \\
\left(M_{\odot}\right) \\
(8)\end{array}$ \\
\hline NGC3583 & Bulge+Disk+Bar & $\mathrm{SB}(\mathrm{s}) \mathrm{b}$ & $\mathrm{B}$ & 36.43 & -20.8 & - & - \\
\hline NGC3596 & $\mathrm{PSF}+\mathrm{Bulge}+$ Disk & $\mathrm{SAB}(\mathrm{rs}) \mathrm{c}$ & $\mathrm{AB}$ & 24.64 & -19.6 & - & - \\
\hline NGC3646 & $\mathrm{PSF}+\mathrm{Bulge}+\mathrm{Disk}$ & Ring & - & 59.79 & -22.9 & 0.65 & $2.82 \mathrm{e}+11$ \\
\hline NGC3675 & $\mathrm{PSF}+\mathrm{Bulge}+$ Disk & $\mathrm{SA}(\mathrm{s}) \mathrm{b}$ & $\mathrm{A}$ & 13.71 & -20.2 & - & - \\
\hline NGC3684 & PSF+Bulge+Disk & $\mathrm{SA}(\mathrm{rs}) \mathrm{bc}$ & $\mathrm{A}$ & 25.07 & -19.4 & 0.62 & $1.00 \mathrm{e}+10$ \\
\hline NGC3686 & $\mathrm{PSF}+\mathrm{Bulge}+\mathrm{Disk}+\mathrm{Bar}$ & $\mathrm{SB}(\mathrm{s}) \mathrm{bc}$ & $\mathrm{B}$ & 25.18 & -19.6 & 0.57 & $9.97 \mathrm{e}+09$ \\
\hline NGC3705 & $\mathrm{PSF}+\mathrm{Bulge}+\mathrm{Disk}+\mathrm{Bar}$ & $\mathrm{SAB}(\mathrm{r}) \mathrm{ab}$ & $\mathrm{AB}$ & 18.21 & -19.9 & 0.79 & $3.15 \mathrm{e}+10$ \\
\hline NGC3726 & $\mathrm{PSF}+\mathrm{Bulge}+\mathrm{Disk}+\mathrm{Bar}$ & $\mathrm{SAB}(\mathrm{r}) \mathrm{c}$ & $\mathrm{AB}$ & 18.21 & -20.6 & 0.49 & $1.93 \mathrm{e}+10$ \\
\hline NGC3810 & PSF+Bulge+Disk & $\mathrm{SA}(\mathrm{rs}) \mathrm{c}$ & $\mathrm{A}$ & 18.11 & -20.1 & 0.58 & $1.72 \mathrm{e}+10$ \\
\hline NGC3885 & PSF+Bulge+Disk & $\mathrm{SA}(\mathrm{s}) 0$ & $\mathrm{~A}$ & 29.79 & -19.6 & 0.95 & $4.62 \mathrm{e}+10$ \\
\hline NGC3887 & $\mathrm{PSF}+\mathrm{Bulge}+\mathrm{Disk}+\mathrm{Bar}$ & $\mathrm{SB}(\mathrm{r}) \mathrm{bc}$ & $\mathrm{B}$ & 20.68 & -20.4 & - & - \\
\hline NGC3893 & $\mathrm{PSF}+\mathrm{Bulge}+\mathrm{Disk}$ & $\mathrm{SAB}(\mathrm{rs}) \mathrm{c}$ & $\mathrm{AB}$ & 18.21 & -19.9 & - & - \\
\hline NGC3938 & $\mathrm{PSF}+\mathrm{Bulge}+\mathrm{Disk}$ & $\mathrm{SA}(\mathrm{s}) \mathrm{c}$ & $\mathrm{A}$ & 18.21 & -20.0 & 0.52 & $1.23 \mathrm{e}+10$ \\
\hline NGC3949 & $\mathrm{PSF}+\mathrm{Bulge}+$ Disk & $\mathrm{SA}(\mathrm{s}) \mathrm{bc}$ & $\mathrm{AB}$ & 18.21 & -19.9 & 0.45 & $8.66 \mathrm{e}+09$ \\
\hline NGC4027 & Bar+Disk & $\mathrm{SB}(\mathrm{s}) \mathrm{dm}$ & $\mathrm{B}$ & 27.43 & -20.6 & 0.54 & $2.25 \mathrm{e}+10$ \\
\hline NGC4030 & PSF+Bulge+Disk & $\mathrm{SA}(\mathrm{s}) \mathrm{bc}$ & $\mathrm{A}$ & 27.75 & -20.8 & - & - \\
\hline NGC4051 & $\mathrm{PSF}+\mathrm{Bulge}+\mathrm{Disk}+\mathrm{Bar}$ & $\mathrm{SAB}(\mathrm{rs}) \mathrm{bc}$ & $\mathrm{AB}$ & 18.21 & -20.0 & 0.65 & $1.95 \mathrm{e}+10$ \\
\hline NGC4062 & $\mathrm{PSF}+\mathrm{Bulge}+\mathrm{Disk}$ & $\mathrm{SA}(\mathrm{s}) \mathrm{c}$ & $\mathrm{A}$ & 10.39 & -19.5 & 0.76 & $2.07 \mathrm{e}+10$ \\
\hline NGC4123 & $\mathrm{PSF}+\mathrm{Bulge}+\mathrm{Disk}+\mathrm{Bar}$ & $\mathrm{SB}(\mathrm{r}) \mathrm{c}$ & $\mathrm{B}$ & 17.68 & -19.8 & 0.61 & $1.50 \mathrm{e}+10$ \\
\hline NGC4145 & $\mathrm{PSF}+\mathrm{Bulge}+\mathrm{Disk}$ & $\mathrm{SAB}(\mathrm{rs}) \mathrm{d}$ & $\mathrm{AB}$ & 22.18 & -20.1 & 0.51 & $1.34 \mathrm{e}+10$ \\
\hline NGC4151 & $\mathrm{PSF}+\mathrm{Bulge}+\mathrm{Disk}+\mathrm{Bar}$ & $\left(\mathrm{R}^{\prime}\right) \mathrm{SAB}(\mathrm{rs}) \mathrm{ab}$ & $\mathrm{AB}$ & 21.75 & -20.1 & 0.73 & $2.93 \mathrm{e}+10$ \\
\hline NGC4212 & PSF+Bulge+Disk & $\mathrm{SAc}$ & $\mathrm{A}$ & 18.00 & -20.4 & 0.67 & $3.28 \mathrm{e}+10$ \\
\hline NGC4254 & $\mathrm{PSF}+\mathrm{Bulge}+\mathrm{Disk}$ & $\mathrm{SA}(\mathrm{s}) \mathrm{c}$ & $\mathrm{A}$ & 18.00 & -22.6 & 0.57 & $1.61 \mathrm{e}+11$ \\
\hline NGC4293 & $\mathrm{PSF}+\mathrm{Bulge}+\mathrm{Disk}+\mathrm{Bar}$ & $(\mathrm{R}) \mathrm{SB}(\mathrm{s}) 0$ & $\mathrm{~B}$ & 18.21 & -20.1 & 0.90 & $5.94 \mathrm{e}+10$ \\
\hline NGC4303 & Bulge + Disk + Bar & $\mathrm{SAB}(\mathrm{rs}) \mathrm{bc}$ & $\mathrm{AB}$ & 16.29 & -21.8 & 0.53 & $6.76 \mathrm{e}+10$ \\
\hline NGC4314 & $\mathrm{PSF}+\mathrm{Bulge}+\mathrm{Disk}+\mathrm{Bar}$ & $\mathrm{SB}(\mathrm{rs}) \mathrm{a}$ & $\mathrm{B}$ & 10.39 & -19.8 & 0.85 & $3.69 \mathrm{e}+10$ \\
\hline NGC4394 & $\mathrm{PSF}+\mathrm{Bulge}+\mathrm{Disk}+\mathrm{Bar}$ & $(\mathrm{R}) \mathrm{SB}(\mathrm{r}) \mathrm{b}$ & $\mathrm{B}$ & 18.00 & -19.4 & 0.85 & $2.61 \mathrm{e}+10$ \\
\hline NGC4414 & $\mathrm{PSF}+\mathrm{Bulge}+\mathrm{Disk}+\mathrm{Bar}$ & $\mathrm{SA}(\mathrm{rs}) \mathrm{c}$ & $\mathrm{A}$ & 10.39 & -20.0 & 0.84 & $4.38 \mathrm{e}+10$ \\
\hline NGC4450 & $\mathrm{PSF}+\mathrm{Bulge}+\mathrm{Disk}+\mathrm{Bar}$ & $\mathrm{SA}(\mathrm{s}) \mathrm{ab}$ & $\mathrm{A}$ & 18.00 & -21.9 & 0.82 & $2.22 \mathrm{e}+11$ \\
\hline NGC4487 & $\mathrm{PSF}+\mathrm{Bulge}+\mathrm{Disk}+\mathrm{Bar}$ & $\mathrm{SAB}(\mathrm{rs}) \mathrm{cd}$ & $\mathrm{AB}$ & 21.32 & -19.6 & - & - \\
\hline NGC4490 & PSF+Bulge+Disk & $\mathrm{SB}(\mathrm{s}) \mathrm{d} ; \mathrm{pec}$ & $\mathrm{B}$ & 8.36 & -21.7 & 0.43 & $4.10 \mathrm{e}+10$ \\
\hline NGC4527 & Bulge + Disk + Bar & $\mathrm{SAB}(\mathrm{s}) \mathrm{bc}$ & $\mathrm{AB}$ & 14.46 & -21.5 & 0.86 & $1.87 \mathrm{e}+11$ \\
\hline NGC4548 & $\mathrm{PSF}+\mathrm{Bulge}+\mathrm{Disk}+\mathrm{Bar}$ & $\mathrm{SB}(\mathrm{rs}) \mathrm{b}$ & $\mathrm{B}$ & 18.00 & -20.8 & 0.81 & $7.85 \mathrm{e}+10$ \\
\hline NGC4593 & $\mathrm{PSF}+\mathrm{Bulge}+\mathrm{Disk}+\mathrm{Bar}$ & (R)SB(rs)b & $\mathrm{B}$ & 42.32 & -20.8 & - & - \\
\hline NGC4618 & $\mathrm{PSF}+\mathrm{Bar}+\mathrm{Disk}$ & $\mathrm{SB}(\mathrm{rs}) \mathrm{m}$ & $\mathrm{B}$ & 7.82 & -19.3 & 0.44 & $4.62 \mathrm{e}+09$ \\
\hline NGC4643 & Bulge+Disk + Bar & $\mathrm{SB}(\mathrm{rs}) 0$ & B & 27.54 & -19.9 & 0.96 & $6.49 \mathrm{e}+10$ \\
\hline NGC4647 & Bulge+Disk & $\mathrm{SAB}(\mathrm{rs}) \mathrm{c}$ & $\mathrm{AB}$ & 18.00 & -19.8 & 0.65 & $1.67 \mathrm{e}+10$ \\
\hline NGC4651 & $\mathrm{PSF}+\mathrm{Bulge}+$ Disk & $\mathrm{SA}(\mathrm{rs}) \mathrm{c}$ & $\mathrm{A}$ & 18.00 & -19.6 & 0.57 & $1.05 \mathrm{e}+10$ \\
\hline NGC4654 & Bulge + Disk & $\mathrm{SAB}(\mathrm{rs}) \mathrm{cd}$ & $\mathrm{AB}$ & 18.00 & -20.6 & 0.60 & $2.99 \mathrm{e}+10$ \\
\hline NGC4665 & $\mathrm{PSF}+\mathrm{Bulge}+\mathrm{Disk}+\mathrm{Bar}$ & $\mathrm{SB}(\mathrm{s}) 0$ & $\mathrm{~B}$ & 19.18 & -19.3 & - & - \\
\hline NGC4689 & PSF + Bulge+Disk & $\mathrm{SA}(\mathrm{rs}) \mathrm{bc}$ & $\mathrm{A}$ & 18.00 & -20.7 & 0.65 & $3.84 \mathrm{e}+10$ \\
\hline NGC4691 & $\mathrm{PSF}+\mathrm{Bulge}+\mathrm{Disk}+\mathrm{Bar}$ & $(\mathrm{R}) \mathrm{SB}(\mathrm{s}) 0 ; \mathrm{pec}$ & $\mathrm{B}$ & 24.11 & -19.6 & 0.58 & $1.04 \mathrm{e}+10$ \\
\hline NGC4698 & PSF+Bulge+Disk & $\mathrm{SA}(\mathrm{s}) \mathrm{ab}$ & $\mathrm{A}$ & 18.00 & -19.9 & 0.91 & $5.15 \mathrm{e}+10$ \\
\hline NGC4699 & Bulge+Disk+Bar & $\mathrm{SAB}(\mathrm{rs}) \mathrm{b}$ & $\mathrm{AB}$ & 27.54 & -21.5 & 0.89 & $2.06 \mathrm{e}+11$ \\
\hline NGC4772 & $\mathrm{PSF}+\mathrm{Bulge}+\mathrm{Disk}+\mathrm{Bar}$ & $\mathrm{SA}(\mathrm{s}) \mathrm{a}$ & $\mathrm{A}$ & 17.46 & -19.4 & 0.92 & $3.30 \mathrm{e}+10$ \\
\hline NGC4775 & PSF+Bulge+Disk & $\mathrm{SA}(\mathrm{s}) \mathrm{d}$ & $\mathrm{A}$ & 28.50 & -20.4 & - & - \\
\hline NGC4781 & PSF+Bulge+Disk & $\mathrm{SB}(\mathrm{rs}) \mathrm{d}$ & $\mathrm{B}$ & 24.11 & -20.8 & - & - \\
\hline NGC4818 & $\mathrm{PSF}+\mathrm{Bulge}+\mathrm{Disk}+\mathrm{Bar}$ & $\mathrm{SAB}(\mathrm{rs}) \mathrm{ab} ; \mathrm{pec}$ & $\mathrm{AB}$ & 23.04 & -19.7 & 0.89 & $3.88 \mathrm{e}+10$ \\
\hline NGC4856 & $\mathrm{PSF}+\mathrm{Bulge}+\mathrm{Disk}+\mathrm{Bar}$ & $\mathrm{SB}(\mathrm{s}) 0$ & B & 22.61 & -20.2 & 0.99 & $9.04 \mathrm{e}+10$ \\
\hline NGC4902 & $\mathrm{PSF}+\mathrm{Bulge}+\mathrm{Disk}+\mathrm{Bar}$ & $\mathrm{SB}(\mathrm{r}) \mathrm{b}$ & $\mathrm{B}$ & 42.00 & -21.4 & 0.69 & $8.33 e+10$ \\
\hline NGC4930 & $\mathrm{PSF}+\mathrm{Bulge}+\mathrm{Disk}+\mathrm{Bar}$ & $\mathrm{SB}(\mathrm{rs}) \mathrm{b}$ & $\mathrm{B}$ & 37.50 & -21.2 & 0.90 & $1.61 \mathrm{e}+11$ \\
\hline NGC4939 & PSF+Bulge+Disk & $\mathrm{SA}(\mathrm{s}) \mathrm{bc}$ & $\mathrm{A}$ & 47.46 & -22.2 & 0.64 & $1.43 \mathrm{e}+11$ \\
\hline NGC4941 & PSF+Bulge+Disk & $(\mathrm{R}) \mathrm{SAB}(\mathrm{r}) \mathrm{ab}$ & $\mathrm{AB}$ & 6.86 & -19.4 & 0.84 & $2.50 \mathrm{e}+10$ \\
\hline NGC4995 & $\mathrm{PSF}+$ Bulge+Disk + Bar & $\mathrm{SAB}(\mathrm{rs}) \mathrm{b}$ & $\mathrm{AB}$ & 30.00 & -20.6 & 0.87 & $8.25 \mathrm{e}+10$ \\
\hline NGC5054 & Bulge + Disk & $\mathrm{SA}(\mathrm{s}) \mathrm{bc}$ & $\mathrm{A}$ & 29.25 & -21.1 & 0.76 & $8.31 \mathrm{e}+10$ \\
\hline
\end{tabular}


Table 2.1 (cont'd)

\begin{tabular}{|c|c|c|c|c|c|c|c|}
\hline $\begin{array}{c}\text { Galaxy Name } \\
\text { (1) }\end{array}$ & $\begin{array}{c}\text { Best Fit } \\
(2)\end{array}$ & $\begin{array}{c}\text { Hubble Type } \\
\text { (RC3) } \\
\text { (3) }\end{array}$ & $\begin{array}{c}\text { Bar Type } \\
\text { (RC3) } \\
(4)\end{array}$ & $\begin{array}{c}D \\
(\mathrm{Mpc}) \\
(5)\end{array}$ & $\begin{array}{c}M_{B} \\
(\mathrm{mag}) \\
(6)\end{array}$ & $\begin{array}{c}B-V \\
(\mathrm{mag}) \\
(7)\end{array}$ & $\begin{array}{c}M_{\star} \\
\left(M_{\odot}\right) \\
(8)\end{array}$ \\
\hline NGC5085 & Bulge + Disk & $\mathrm{SA}(\mathrm{s}) \mathrm{c}$ & $\mathrm{A}$ & 30.96 & -19.3 & 0.87 & $2.54 \mathrm{e}+10$ \\
\hline NGC5101 & $\mathrm{PSF}+\mathrm{Bulge}+\mathrm{Disk}+\mathrm{Bar}$ & $(\mathrm{R}) \mathrm{SB}(\mathrm{rs}) 0$ & $\mathrm{~B}$ & 29.36 & -20.8 & 1.00 & $1.69 \mathrm{e}+11$ \\
\hline NGC5121 & PSF+Bulge+Disk & $\left(\mathrm{R}^{\prime}\right) \mathrm{SA}(\mathrm{s}) \mathrm{a}$ & $\mathrm{A}$ & 23.68 & -19.4 & 0.95 & $3.68 \mathrm{e}+10$ \\
\hline NGC5161 & $\mathrm{PSF}+\mathrm{Bulge}+\mathrm{Disk}$ & $\mathrm{SA}(\mathrm{s}) \mathrm{c}$ & $\mathrm{A}$ & 35.89 & -21.7 & 0.79 & $1.65 \mathrm{e}+11$ \\
\hline NGC5247 & PSF + Bulge+Disk & $\mathrm{SA}(\mathrm{s}) \mathrm{bc}$ & $\mathrm{A}$ & 23.79 & -21.2 & 0.54 & $3.86 \mathrm{e}+10$ \\
\hline NGC5371 & $\mathrm{PSF}+\mathrm{Bulge}+\mathrm{Disk}+\mathrm{Bar}$ & $\mathrm{SAB}(\mathrm{rs}) \mathrm{bc}$ & $\mathrm{AB}$ & 40.50 & -22.1 & 0.70 & $1.74 \mathrm{e}+11$ \\
\hline NGC5427 & $\mathrm{PSF}+$ Bulge+Disk & $\mathrm{SA}(\mathrm{s}) \mathrm{c} ;$ pec & $\mathrm{A}$ & 40.82 & -21.2 & 0.57 & $4.61 \mathrm{e}+10$ \\
\hline NGC5483 & Bulge+Disk+Bar & $\mathrm{SA}(\mathrm{s}) \mathrm{c}$ & $\mathrm{A}$ & 26.46 & -20.3 & - & - \\
\hline NGC5643 & $\mathrm{PSF}+\mathrm{Bulge}+\mathrm{Disk}+\mathrm{Bar}$ & $\mathrm{SAB}(\mathrm{rs}) \mathrm{c}$ & $\mathrm{AB}$ & 18.11 & -20.9 & 0.74 & $6.68 \mathrm{e}+10$ \\
\hline NGC5676 & $\mathrm{PSF}+\mathrm{Bulge}+\mathrm{Disk}$ & $\mathrm{SA}(\mathrm{rs}) \mathrm{bc}$ & $\mathrm{A}$ & 36.96 & -21.5 & 0.68 & $9.01 \mathrm{e}+10$ \\
\hline NGC5701 & $\mathrm{PSF}+\mathrm{Bulge}+\mathrm{Disk}+\mathrm{Bar}$ & (R)SB(rs)0 & $\mathrm{B}$ & 27.96 & -19.9 & 0.88 & $4.67 \mathrm{e}+10$ \\
\hline NGC5713 & PSF + Bulge + Disk & $\mathrm{SAB}(\mathrm{rs}) \mathrm{bc} ; \mathrm{pec}$ & $\mathrm{AB}$ & 32.57 & -21.1 & 0.64 & $5.21 \mathrm{e}+10$ \\
\hline NGC5850 & $\mathrm{PSF}+\mathrm{Bulge}+\mathrm{Disk}+\mathrm{Bar}$ & $\mathrm{SB}(\mathrm{r}) \mathrm{b}$ & $\mathrm{B}$ & 30.54 & -21.5 & 0.79 & $1.37 \mathrm{e}+11$ \\
\hline NGC5921 & $\mathrm{PSF}+\mathrm{Bulge}+\mathrm{Disk}+\mathrm{Bar}$ & $\mathrm{SB}(\mathrm{r}) \mathrm{bc}$ & $\mathrm{B}$ & 27.00 & -20.6 & 0.66 & $3.51 \mathrm{e}+10$ \\
\hline NGC5962 & Bulge + Disk + Bar & $\mathrm{SA}(\mathrm{r}) \mathrm{c}$ & $\mathrm{A}$ & 34.07 & -20.9 & 0.64 & $4.43 e+10$ \\
\hline NGC6215 & $\mathrm{PSF}+$ Bulge+Disk & $\mathrm{SA}(\mathrm{s}) \mathrm{c}$ & $\mathrm{A}$ & 21.96 & -20.4 & 0.54 & $1.84 \mathrm{e}+10$ \\
\hline NGC6221 & Bulge+Disk+Bar & $\mathrm{SB}(\mathrm{s}) \mathrm{bc} ; \mathrm{pec}$ & $\mathrm{B}$ & 20.79 & -21.6 & 0.74 & $1.31 \mathrm{e}+11$ \\
\hline NGC6300 & $\mathrm{PSF}+\mathrm{Bulge}+\mathrm{Disk}+\mathrm{Bar}$ & $\mathrm{SB}(\mathrm{rs}) \mathrm{b}$ & $\mathrm{B}$ & 15.32 & -20.3 & 0.78 & $4.49 \mathrm{e}+10$ \\
\hline NGC6384 & Bulge + Disk & $\mathrm{SAB}(\mathrm{r}) \mathrm{bc}$ & $\mathrm{AB}$ & 28.50 & -21.5 & 0.72 & $1.05 \mathrm{e}+11$ \\
\hline NGC6753 & $\mathrm{PSF}+\mathrm{Bulge}+\mathrm{Disk}+\mathrm{Bar}$ & $(\mathrm{R}) \mathrm{SA}(\mathrm{r}) \mathrm{b}$ & $\mathrm{A}$ & 43.82 & -21.6 & 0.83 & $1.81 \mathrm{e}+11$ \\
\hline NGC6782 & $\mathrm{PSF}+\mathrm{Bulge}+\mathrm{Disk}+\mathrm{Bar}$ & $(\mathrm{R}) \mathrm{SAB}(\mathrm{r}) \mathrm{a}$ & $\mathrm{AB}$ & 54.43 & -21.4 & - & - \\
\hline NGC6902 & $\mathrm{PSF}+\mathrm{Bulge}+\mathrm{Disk}$ & $\mathrm{SA}(\mathrm{r}) \mathrm{b}$ & $\mathrm{A}$ & 38.25 & -21.2 & 0.71 & $8.12 \mathrm{e}+10$ \\
\hline NGC6907 & $\mathrm{PSF}+\mathrm{Bulge}+\mathrm{Disk}+\mathrm{Bar}$ & $\mathrm{SB}(\mathrm{s}) \mathrm{bc}$ & $\mathrm{B}$ & 46.07 & -21.8 & 0.69 & $1.21 \mathrm{e}+11$ \\
\hline NGC7083 & $\mathrm{PSF}+$ Bulge+Disk & $\mathrm{SA}(\mathrm{s}) \mathrm{bc}$ & $\mathrm{A}$ & 41.46 & -21.8 & 0.65 & $1.05 \mathrm{e}+11$ \\
\hline NGC7205 & $\mathrm{PSF}+\mathrm{Bulge}+\mathrm{Disk}$ & $\mathrm{SA}(\mathrm{s}) \mathrm{bc}$ & $\mathrm{A}$ & 21.96 & -20.7 & 0.60 & $3.11 \mathrm{e}+10$ \\
\hline NGC7213 & $\mathrm{PSF}+$ Bulge+Disk & $\mathrm{SA}(\mathrm{s}) \mathrm{a}$ & $\mathrm{A}$ & 23.57 & -21.0 & 0.89 & $1.26 \mathrm{e}+11$ \\
\hline NGC7217 & PSF+Bulge+Disk & $(\mathrm{R}) \mathrm{SA}(\mathrm{r}) \mathrm{ab}$ & A & 17.14 & -20.5 & 0.90 & $8.38 \mathrm{e}+10$ \\
\hline NGC7412 & $\mathrm{PSF}+\mathrm{Bulge}+\mathrm{Disk}+\mathrm{Bar}$ & $\mathrm{SB}(\mathrm{s}) \mathrm{b}$ & $\mathrm{B}$ & 22.61 & -20.2 & 0.53 & $1.50 \mathrm{e}+10$ \\
\hline NGC7479 & $\mathrm{PSF}+\mathrm{Bulge}+\mathrm{Disk}+\mathrm{Bar}$ & $\mathrm{SB}(\mathrm{s}) \mathrm{c}$ & $\mathrm{B}$ & 34.71 & -21.7 & 0.75 & $1.44 \mathrm{e}+11$ \\
\hline NGC7552 & Bulge + Disk + Bar & $\left(\mathrm{R}^{\prime}\right) \mathrm{SB}(\mathrm{s}) \mathrm{ab}$ & $\mathrm{B}$ & 20.89 & -20.5 & 0.68 & $3.49 \mathrm{e}+10$ \\
\hline NGC7723 & $\mathrm{PSF}+\mathrm{Bulge}+\mathrm{Disk}+\mathrm{Bar}$ & $\mathrm{SB}(\mathrm{r}) \mathrm{b}$ & B & 25.39 & -20.5 & 0.73 & $4.32 \mathrm{e}+10$ \\
\hline NGC7727 & PSF+Bulge+Disk & $\mathrm{SAB}(\mathrm{s}) \mathrm{a} ; \mathrm{pec}$ & $\mathrm{AB}$ & 24.96 & -20.8 & 0.91 & $1.13 \mathrm{e}+11$ \\
\hline NGC7741 & Bulge+Disk+Bar & $\mathrm{SB}(\mathrm{s}) \mathrm{cd}$ & B & 13.18 & -19.3 & 0.53 & $6.67 \mathrm{e}+09$ \\
\hline
\end{tabular}

Note. - Columns are: (1) Galaxy name. (2) The best fit chosen based on the criteria outlined in § 2.3.3. (3) Hubble type from RC3 (de Vaucouleurs et al. 1991). (4) RC3 bar type, which is based on visual inspection of optical images and runs as 'B'='strongly barred', 'AB'='weakly barred', and 'A'='unbarred'. (5) The distance in Mpc from the Nearby Galaxies Catalog (Tully 1988) scaled to a Hubble constant of $70 \mathrm{~km} \mathrm{~s}^{-1} \mathrm{Mpc}^{-1}$. Distances are adjusted assuming the Galaxy is retarded by $300 \mathrm{~km} \mathrm{~s} s^{-1}$ from the universal expansion by the mass of the Virgo Cluster. Exceptions are NGC 6753, NGC 6782, and NGC 6907 where distances are from RC3, NGC 3504 where the distance is from Kenney et al. (1993), and NGC 4314 where the distance is from Benedict et al. (1996). (6) Absolute $B$-band magnitude from Hyperleda. Corrections have been applied for galactic and internal extinction. Galactic absorption is computed based on Schlegel et al. (1998). Internal extinction is corrected for following Bottinelli et al. (1995). Kcorrection co-efficients are drawn from RC2 (de Vaucouleurs et al. 1976). (7) $B-V$ color from Hyperleda. Corrections for extinction and k-correction have been applied as in column 6. (8) Stellar mass, calculated as outlined in $\S 2.2 .2$. 
Table 2.2. Parameters From 2D Luminosity Decomposition for Sample S1 (N=143)

\begin{tabular}{|c|c|c|c|c|c|c|c|c|c|c|c|c|c|}
\hline $\begin{array}{c}\text { Galaxy Name } \\
\text { (1) }\end{array}$ & $\begin{array}{c}\text { Best Fit } \\
(2)\end{array}$ & $\begin{array}{c}B / T \\
\% \\
(3)\end{array}$ & $\begin{array}{l}D / T \\
\% \\
(4)\end{array}$ & $\begin{array}{c}\mathrm{Bar} / T \\
\% \\
(5)\end{array}$ & $\begin{array}{c}B / D \\
(6)\end{array}$ & $\begin{array}{c}\underset{/ 1}{\text { Bulge }} r_{\mathrm{e}} \\
(7)\end{array}$ & $\begin{array}{c}\text { Bulge } r_{\mathrm{e}} \\
\text { kpc } \\
(8)\end{array}$ & $\begin{array}{c}\text { Bulge } n \\
(9)\end{array}$ & $\begin{array}{l}\underset{\prime \prime}{\text { Disk h }} \\
(10)\end{array}$ & $\begin{array}{l}\text { Disk h } \\
\text { kpc } \\
(11)\end{array}$ & $\begin{array}{c}\underset{\prime \prime}{\operatorname{Bar}} r_{\mathrm{e}} \\
(12)\end{array}$ & $\begin{array}{c}\text { Bar } r_{\mathrm{e}} \\
\mathrm{kpc} \\
(13)\end{array}$ & $\begin{array}{l}\text { Bar } n \\
(14)\end{array}$ \\
\hline ESO138-10 & PSF+Bulge+Disk & 16.25 & 83.75 & - & 0.19 & 10.94 & 0.83 & 2.07 & 27.10 & 2.06 & - & - & - \\
\hline IC0239 & $\mathrm{PSF}+$ Bulge+Disk + Bar & 2.45 & 92.65 & 4.91 & 0.03 & 6.63 & 0.49 & 0.23 & 34.56 & 2.54 & 13.95 & 1.02 & 0.19 \\
\hline IC4444 & Bulge+Disk & 31.97 & 68.03 & - & 0.47 & 7.94 & 1.10 & 2.33 & 17.67 & 2.45 & - & - & - \\
\hline IC5325 & PSF+Bulge+Disk & 6.79 & 93.21 & - & 0.07 & 15.17 & 1.42 & 1.75 & 23.18 & 2.17 & - & - & - \\
\hline NGC0150 & $\mathrm{PSF}+\mathrm{Bulge}+\mathrm{Disk}+\mathrm{Bar}$ & 5.72 & 81.18 & 13.11 & 0.07 & 4.22 & 0.42 & 0.05 & 33.57 & 3.33 & 17.82 & 1.77 & 0.39 \\
\hline NGC0157 & PSF+Bulge+Disk & 2.29 & 97.71 & - & 0.02 & 3.44 & 0.37 & 0.44 & 31.82 & 3.43 & - & - & - \\
\hline NGC0210 & Bulge+Disk+Bar & 30.07 & 48.54 & 21.39 & 0.62 & 4.81 & 0.50 & 1.71 & 83.21 & 8.71 & 29.41 & 3.08 & 0.31 \\
\hline NGC0278 & PSF+Bulge+Disk & 4.23 & 95.77 & - & 0.04 & 3.46 & 0.21 & 0.57 & 13.48 & 0.82 & & & \\
\hline NGC0289 & $\mathrm{PSF}+$ Bulge+Disk + Bar & 8.83 & 85.99 & 5.18 & 0.10 & 4.91 & 0.49 & 0.46 & 20.28 & 2.03 & 18.90 & 1.89 & 0.04 \\
\hline NGC0428 & $\mathrm{PSF}+$ Bulge+Disk+Bar & 5.50 & 71.64 & 22.86 & 0.08 & 9.27 & 0.71 & 0.38 & 32.55 & 2.51 & 30.70 & 2.36 & 0.37 \\
\hline NGC0488 & PSF+Bulge+Disk & 21.72 & 78.28 & - & 0.28 & 9.91 & 1.49 & 3.07 & 38.94 & 5.87 & - & - & - \\
\hline NGC0578 & $\mathrm{PSF}+\mathrm{Bulge}+$ Disk $+\mathrm{Bar}$ & 2.05 & 93.63 & 4.32 & 0.02 & 4.19 & 0.42 & 0.56 & 40.73 & 4.10 & 14.95 & 1.50 & 0.18 \\
\hline NGC0613 & $\mathrm{PSF}+$ Bulge + Disk + Bar & 13.14 & 56.97 & 29.90 & 0.23 & 6.12 & 0.55 & 1.05 & 44.69 & 4.04 & 62.49 & 5.65 & 0.53 \\
\hline NGC0685 & Bar+Disk & & 96.53 & 3.47 & - & - & - & - & 41.24 & 3.24 & 20.68 & 1.62 & 0.16 \\
\hline NGC0779 & Bulge+Disk+Bar & 16.18 & 57.69 & 26.13 & 0.28 & 6.04 & 0.54 & 2.31 & 38.74 & 3.46 & 29.00 & 2.59 & 0.25 \\
\hline NGC0864 & $\mathrm{PSF}+$ Bulge+Disk $+\mathrm{Bar}$ & 2.77 & 86.88 & 10.35 & 0.03 & 3.52 & 0.36 & 0.66 & 39.54 & 4.08 & 21.07 & 2.17 & 0.37 \\
\hline NGC0908 & PSF+Bulge+Disk & 8.86 & 91.14 & - & 0.10 & 7.18 & 0.66 & 1.62 & 48.96 & 4.50 & - & - & - \\
\hline NGC1042 & PSF+Bulge+Disk & 3.00 & 97.00 & - & 0.03 & 5.91 & 0.51 & 0.09 & 39.83 & 3.43 & - & - & - \\
\hline NGC1073 & $\mathrm{PSF}+$ Bulge+Disk+Bar & 2.36 & 79.58 & 18.06 & 0.03 & 5.47 & 0.43 & 0.41 & 50.02 & 3.93 & 34.24 & 2.69 & 0.83 \\
\hline NGC1084 & PSF+Bulge+Disk & 5.23 & 94.77 & - & 0.06 & 3.24 & 0.29 & 0.77 & 18.68 & 1.65 & - & - & - \\
\hline NGC1087 & Bar+Disk & - & 93.13 & 6.87 & - & - & - & - & 28.55 & 2.80 & 7.79 & 0.76 & 1.27 \\
\hline NGC1187 & $\mathrm{PSF}+$ Bulge + Disk + Bar & 6.94 & 64.96 & 28.10 & 0.11 & 4.81 & 0.41 & 1.57 & 38.20 & 3.22 & 51.85 & 4.37 & 0.72 \\
\hline NGC1241 & $\mathrm{PSF}+$ Bulge+Disk+Bar & 11.70 & 69.10 & 19.21 & 0.17 & 2.61 & 0.36 & 0.91 & 27.69 & 3.79 & 17.89 & 2.45 & 0.49 \\
\hline NGC1300 & $\mathrm{PSF}+$ Bulge+Disk+Bar & 11.04 & 74.94 & 14.02 & 0.15 & 4.63 & 0.45 & 1.19 & 63.53 & 6.16 & 65.89 & 6.39 & 0.25 \\
\hline NGC1302 & $\mathrm{PSF}+$ Bulge+Disk +Bar & 17.65 & 63.79 & 18.57 & 0.28 & 4.96 & 0.51 & 2.01 & 44.92 & 4.63 & 20.44 & 2.11 & 0.76 \\
\hline NGC1309 & PSF+Bulge+Disk & 27.07 & 72.93 & - & 0.37 & 10.86 & 1.45 & 2.27 & 16.67 & 2.23 & - & - & - \\
\hline NGC1317 & Bulge+Disk+Bar & 13.86 & 41.48 & 44.65 & 0.33 & 3.99 & 0.35 & 1.94 & 47.37 & 4.13 & 20.22 & 1.76 & 2.19 \\
\hline NGC1350 & $\mathrm{PSF}+$ Bulge+Disk $+\mathrm{Bar}$ & 19.52 & 69.99 & 10.50 & 0.28 & 7.41 & 0.65 & 1.59 & 52.77 & 4.61 & 51.07 & 4.46 & 0.31 \\
\hline NGC1371 & $\mathrm{PSF}+$ Bulge+Disk+Bar & 12.46 & 80.16 & 7.38 & 0.16 & 5.17 & 0.46 & 1.11 & 29.34 & 2.59 & 18.92 & 1.67 & 0.36 \\
\hline NGC1385 & PSF + Bar+Disk & - & 75.54 & 24.46 & - & - & - & - & 28.69 & 2.59 & 15.63 & 1.41 & 1.28 \\
\hline NGC1511 & Bulge+Disk & 28.47 & 71.53 & - & 0.40 & 27.78 & 2.17 & 1.35 & 23.99 & 1.87 & - & - & - \\
\hline NGC1559 & PSF+Bar+Disk & - & 97.06 & 2.94 & - & - & - & - & 31.16 & 2.30 & 9.84 & 0.73 & 0.53 \\
\hline NGC1703 & PSF+Bulge+Disk & 6.32 & 93.68 & - & 0.07 & 2.83 & 0.25 & 0.56 & 18.24 & 1.64 & - & - & - \\
\hline NGC1792 & PSF+Bulge+Disk & 2.65 & 97.35 & - & 0.03 & 3.72 & 0.26 & 0.84 & 38.50 & 2.71 & - & - & - \\
\hline NGC1808 & $\mathrm{PSF}+\mathrm{Bulge}+\mathrm{Disk}+\mathrm{Bar}$ & 3.92 & 74.27 & 21.82 & 0.05 & 2.78 & 0.16 & 0.45 & 38.78 & 2.17 & 5.71 & 0.32 & 0.67 \\
\hline NGC1964 & $\mathrm{PSF}+$ Bulge + Disk + Bar & 41.78 & 49.87 & 8.35 & 0.84 & 8.31 & 0.86 & 2.58 & 18.59 & 1.92 & 8.05 & 0.83 & 0.05 \\
\hline NGC2090 & Bulge+Disk+Bar & 14.67 & 58.39 & 26.94 & 0.25 & 17.68 & 0.93 & 2.19 & 160.02 & 8.45 & 33.74 & 1.78 & 0.43 \\
\hline NGC2139 & Bulge+Disk & 15.16 & 84.84 & - & 0.18 & 8.27 & 0.95 & 1.53 & 18.12 & 2.09 & - & - & - \\
\hline NGC2196 & $\mathrm{PSF}+\mathrm{Bulge}+\mathrm{Disk}$ & 46.38 & 53.62 & - & 0.86 & 13.42 & 1.99 & 2.38 & 28.38 & 4.21 & - & - & - \\
\hline
\end{tabular}


Table 2.2 (cont'd)

\begin{tabular}{|c|c|c|c|c|c|c|c|c|c|c|c|c|c|}
\hline $\begin{array}{c}\text { Galaxy Name } \\
\text { (1) }\end{array}$ & $\begin{array}{c}\text { Best Fit } \\
(2)\end{array}$ & $\begin{array}{l}B / T \\
\% \\
(3)\end{array}$ & $\begin{array}{l}D / T \\
\% \\
(4)\end{array}$ & $\begin{array}{c}\mathrm{Bar} / T \\
\% \\
(5)\end{array}$ & $\begin{array}{c}B / D \\
(6)\end{array}$ & $\begin{array}{c}\underset{\prime \prime}{\text { Bulge }} r_{\mathrm{e}} \\
(7)\end{array}$ & $\begin{array}{c}\text { Bulge } r_{\mathrm{e}} \\
\text { kpc } \\
(8)\end{array}$ & $\begin{array}{c}\text { Bulge } n \\
(9)\end{array}$ & $\begin{array}{c}\underset{\prime \prime}{\text { Disk h }} \\
(10)\end{array}$ & $\begin{array}{l}\text { Disk h } \\
\text { kpc } \\
(11)\end{array}$ & $\begin{array}{c}\text { Bar } r_{\mathrm{e}} \\
(12)\end{array}$ & $\begin{array}{c}\text { Bar } r_{\mathrm{e}} \\
\mathrm{kpc} \\
(13)\end{array}$ & $\begin{array}{c}\text { Bar } n \\
(14)\end{array}$ \\
\hline NGC2442 & $\mathrm{PSF}+\mathrm{Bulge}+$ Disk $+\mathrm{Bar}$ & 13.33 & 62.47 & 24.19 & 0.21 & 4.78 & 0.42 & 1.68 & 78.46 & 6.93 & 60.15 & 5.31 & 0.24 \\
\hline NGC2559 & Bulge+Disk+Bar & 6.98 & 81.64 & 11.37 & 0.09 & 4.01 & 0.41 & 1.38 & 34.65 & 3.58 & 21.40 & 2.21 & 0.24 \\
\hline NGC2566 & Bulge+Disk & 22.71 & 77.29 & - & 0.29 & 1.74 & 0.19 & 4.42 & 23.05 & 2.51 & - & - & - \\
\hline $\mathrm{NGC} 2775$ & $\mathrm{PSF}+\mathrm{Bulge}+$ Disk & 60.87 & 39.13 & - & 1.56 & 47.35 & 4.16 & 4.85 & 28.09 & 2.47 & - & - & - \\
\hline NGC3059 & Bar+Disk & & 90.68 & 9.32 & - & & & - & 64.22 & 4.91 & 23.94 & 1.83 & 1.43 \\
\hline NGC3166 & Bulge+Disk+Bar & 24.97 & 50.74 & 24.29 & 0.49 & 3.25 & 0.37 & 0.81 & 20.14 & 2.29 & 13.85 & 1.57 & 0.53 \\
\hline NGC3169 & PSF+Bulge+Disk & 38.53 & 61.47 & - & 0.63 & 11.01 & 1.12 & 2.41 & 55.12 & 5.60 & - & - & - \\
\hline NGC3223 & PSF+Bulge+Disk & 11.71 & 88.29 & - & 0.13 & 5.39 & 1.05 & 1.74 & 29.05 & 5.68 & - & - & - \\
\hline NGC3227 & $\mathrm{PSF}+$ Bulge+Disk + Bar & 10.78 & 45.23 & 44.00 & 0.24 & 4.84 & 0.51 & 0.32 & 44.67 & 4.75 & 39.51 & 4.20 & 1.27 \\
\hline NGC3261 & $\mathrm{PSF}+$ Bulge+Disk+Bar & 15.06 & 70.15 & 14.79 & 0.21 & 2.95 & 0.51 & 1.96 & 28.89 & 4.96 & 14.19 & 2.43 & 1.05 \\
\hline NGC3275 & $\mathrm{PSF}+$ Bulge+Disk+Bar & 14.15 & 63.44 & 22.41 & 0.22 & 2.21 & 0.48 & 1.85 & 28.25 & 6.14 & 20.75 & 4.51 & 0.94 \\
\hline NGC3319 & PSF+Bar+Disk & & 91.41 & 8.59 & & - & - & - & 66.08 & 3.93 & 14.60 & 0.87 & 0.43 \\
\hline NGC3338 & $\mathrm{PSF}+$ Bulge+Disk+Bar & 5.29 & 81.96 & 12.75 & 0.06 & 4.39 & 0.52 & 0.76 & 36.42 & 4.28 & 20.62 & 2.42 & 0.39 \\
\hline NGC3423 & Bulge + Disk & 28.10 & 71.90 & - & 0.39 & 24.09 & 1.36 & 2.39 & 31.21 & 1.76 & - & - & - \\
\hline NGC3504 & Bulge+Disk+Bar & 23.15 & 39.49 & 37.37 & 0.59 & 2.37 & 0.24 & 1.04 & 29.55 & 3.05 & 26.02 & 2.69 & 0.78 \\
\hline NGC3513 & Bulge+Disk+Bar & 4.45 & 92.08 & 3.47 & 0.05 & 10.73 & 0.94 & 1.46 & 33.18 & 2.91 & 18.32 & 1.61 & 0.08 \\
\hline NGC3583 & Bulge+Disk+Bar & 8.62 & 53.40 & 37.98 & 0.16 & 1.29 & 0.23 & 1.28 & 21.78 & 3.80 & 14.89 & 2.60 & 0.68 \\
\hline NGC3596 & PSF+Bulge+Disk & 12.15 & 87.85 & - & 0.14 & 5.34 & 0.63 & 0.67 & 18.54 & 2.20 & - & - & - \\
\hline NGC3646 & PSF+Bulge+Disk & 13.53 & 86.47 & - & 0.16 & 3.60 & 1.03 & 1.20 & 26.01 & 7.41 & - & - & - \\
\hline NGC3675 & PSF+Bulge+Disk & 44.42 & 55.58 & - & 0.80 & 28.41 & 1.88 & 2.66 & 47.12 & 3.12 & - & - & - \\
\hline NGC3684 & PSF+Bulge+Disk & 26.92 & 73.08 & - & 0.37 & 11.43 & 1.38 & 2.15 & 15.96 & 1.92 & - & - & - \\
\hline NGC3686 & $\mathrm{PSF}+\mathrm{Bulge}+$ Disk $+\mathrm{Bar}$ & 3.02 & 95.00 & 1.98 & 0.03 & 4.39 & 0.53 & 0.66 & 27.57 & 3.34 & 21.32 & 2.58 & 0.03 \\
\hline NGC3705 & $\mathrm{PSF}+\mathrm{Bulge}+\mathrm{Disk}+\mathrm{Bar}$ & 27.73 & 59.20 & 13.07 & 0.47 & 8.62 & 0.76 & 1.83 & 37.77 & 3.32 & 30.00 & 2.63 & 0.07 \\
\hline NGC3726 & $\mathrm{PSF}+$ Bulge+Disk+Bar & 3.09 & 95.10 & 1.81 & 0.03 & 5.64 & 0.50 & 0.53 & 49.58 & 4.35 & 41.09 & 3.61 & 0.05 \\
\hline NGC3810 & PSF+Bulge+Disk & 38.43 & 61.57 & - & 0.62 & 12.35 & 1.08 & 1.28 & 23.78 & 2.08 & - & - & - \\
\hline NGC3885 & PSF+Bulge+Disk & 27.10 & 72.90 & - & 0.37 & 3.13 & 0.45 & 0.46 & 11.94 & 1.71 & - & - & - \\
\hline NGC3887 & $\mathrm{PSF}+$ Bulge+Disk + Bar & 4.19 & 83.01 & 12.80 & 0.05 & 5.27 & 0.53 & 1.01 & 42.69 & 4.25 & 37.62 & 3.75 & 0.62 \\
\hline NGC3893 & PSF+Bulge+Disk & 54.61 & 45.39 & - & 1.20 & 20.05 & 1.76 & 2.05 & 23.40 & 2.05 & - & - & - \\
\hline NGC3938 & PSF+Bulge+Disk & 7.30 & 92.70 & - & 0.08 & 6.43 & 0.56 & 1.18 & 32.84 & 2.88 & - & - & - \\
\hline NGC3949 & PSF+Bulge+Disk & 7.75 & 92.25 & - & 0.08 & 4.67 & 0.41 & 0.64 & 15.22 & 1.34 & - & - & - \\
\hline NGC4027 & Bar+Disk & & 79.48 & 20.52 & - & - & - & - & 43.11 & 5.68 & 26.50 & 3.49 & 1.80 \\
\hline NGC4030 & PSF+Bulge+Disk & 47.29 & 52.71 & - & 0.90 & 17.18 & 2.29 & 2.22 & 20.67 & 2.76 & - & - & - \\
\hline NGC4051 & $\mathrm{PSF}+$ Bulge+Disk + Bar & 14.99 & 46.99 & 38.02 & 0.32 & 11.29 & 0.99 & 1.20 & 65.81 & 5.78 & 69.69 & 6.12 & 0.40 \\
\hline NGC4062 & PSF+Bulge+Disk & 1.69 & 98.31 & - & 0.02 & 3.62 & 0.18 & 0.50 & 34.98 & 1.76 & - & - & - \\
\hline NGC4123 & $\mathrm{PSF}+$ Bulge+Disk $+\mathrm{Bar}$ & 6.87 & 79.23 & 13.90 & 0.09 & 6.55 & 0.56 & 0.59 & 56.76 & 4.84 & 42.98 & 3.66 & 0.46 \\
\hline NGC4145 & PSF+Bulge+Disk & 7.75 & 92.25 & - & 0.08 & 12.90 & 1.38 & 0.68 & 63.59 & 6.79 & - & - & - \\
\hline NGC4151 & $\mathrm{PSF}+\mathrm{Bulge}+\mathrm{Disk}+\mathrm{Bar}$ & 41.32 & 50.26 & 8.42 & 0.82 & 9.36 & 0.98 & 0.44 & 37.74 & 3.95 & 57.00 & 5.97 & 0.10 \\
\hline NGC4212 & PSF+Bulge+Disk & 3.67 & 96.33 & - & 0.04 & 1.44 & 0.12 & 1.38 & 22.56 & 1.96 & - & - & - \\
\hline NGC4254 & PSF+Bulge+Disk & 38.98 & 61.02 & - & 0.64 & 30.46 & 2.64 & 2.68 & 34.19 & 2.97 & - & - & - \\
\hline
\end{tabular}


Table 2.2 (cont'd)

\begin{tabular}{|c|c|c|c|c|c|c|c|c|c|c|c|c|c|}
\hline $\begin{array}{c}\text { Galaxy Name } \\
\text { (1) }\end{array}$ & $\begin{array}{c}\text { Best Fit } \\
\text { (2) }\end{array}$ & $\begin{array}{c}B / T \\
\% \\
(3)\end{array}$ & $\begin{array}{l}D / T \\
\% \\
(4)\end{array}$ & $\begin{array}{c}\mathrm{Bar} / T \\
\% \\
(5)\end{array}$ & $\begin{array}{c}B / D \\
(6)\end{array}$ & $\begin{array}{c}\underset{/ 1}{\text { Bulge }} r_{\mathrm{e}} \\
(7)\end{array}$ & $\begin{array}{c}\text { Bulge } r_{\mathrm{e}} \\
\text { kpc } \\
(8)\end{array}$ & $\begin{array}{c}\text { Bulge } n \\
(9)\end{array}$ & $\begin{array}{l}\text { Disk h } \\
\quad 1 \\
(10)\end{array}$ & $\begin{array}{l}\text { Disk h } \\
\text { kpc } \\
(11)\end{array}$ & $\begin{array}{c}\underset{\prime \prime}{\operatorname{Bar}} r_{\mathrm{e}} \\
(12)\end{array}$ & $\begin{array}{l}\text { Bar } r_{\mathrm{e}} \\
\mathrm{kpc} \\
(13)\end{array}$ & $\begin{array}{c}\text { Bar } n \\
(14)\end{array}$ \\
\hline NGC4293 & $\mathrm{PSF}+$ Bulge+Disk+Bar & 8.49 & 61.70 & 29.81 & 0.14 & 8.00 & 0.70 & 0.99 & 58.26 & 5.11 & 55.17 & 4.84 & 0.46 \\
\hline NGC4303 & Bulge+Disk+Bar & 8.28 & 82.81 & 8.91 & 0.10 & 2.67 & 0.21 & 1.55 & 44.58 & 3.50 & 32.35 & 2.54 & 0.55 \\
\hline NGC4314 & $\mathrm{PSF}+$ Bulge+Disk+Bar & 26.49 & 52.44 & 21.07 & 0.51 & 11.22 & 0.56 & 2.05 & 62.35 & 3.13 & 48.98 & 2.46 & 0.35 \\
\hline NGC4394 & $\mathrm{PSF}+$ Bulge+Disk+Bar & 15.20 & 69.58 & 15.22 & 0.22 & 4.20 & 0.36 & 1.63 & 41.74 & 3.62 & 28.70 & 2.49 & 0.59 \\
\hline NGC4414 & $\mathrm{PSF}+$ Bulge+Disk+Bar & 11.48 & 83.86 & 4.65 & 0.14 & 3.50 & 0.18 & 1.72 & 21.27 & 1.07 & 17.66 & 0.89 & 0.05 \\
\hline NGC4450 & $\mathrm{PSF}+$ Bulge+Disk+Bar & 16.86 & 71.49 & 11.65 & 0.24 & 8.19 & 0.71 & 2.26 & 47.16 & 4.09 & 36.13 & 3.14 & 0.33 \\
\hline NGC4487 & $\mathrm{PSF}+$ Bulge+Disk+Bar & 3.10 & 93.77 & 3.13 & 0.03 & 6.66 & 0.68 & 0.83 & 27.92 & 2.87 & 14.52 & 1.49 & 0.27 \\
\hline NGC4490 & PSF+Bulge+Disk & 6.64 & 93.36 & & 0.07 & 21.41 & 0.86 & 0.35 & 44.02 & 1.78 & 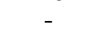 & & \\
\hline NGC4527 & Bulge+Disk+Bar & 18.24 & 63.84 & 17.91 & 0.29 & 5.17 & 0.36 & 1.99 & 55.52 & 3.87 & 41.19 & 2.87 & 0.50 \\
\hline NGC4548 & $\mathrm{PSF}+$ Bulge+Disk + Bar & 12.96 & 68.65 & 18.39 & 0.19 & 6.97 & 0.61 & 1.56 & 58.22 & 5.05 & 44.91 & 3.90 & 0.51 \\
\hline NGC4593 & $\mathrm{PSF}+$ Bulge + Disk + Bar & 25.13 & 28.15 & 46.72 & 0.89 & 5.27 & 1.07 & 1.04 & 33.07 & 6.70 & 41.94 & 8.50 & 0.64 \\
\hline NGC4618 & PSF+Bar+Disk & - & 86.11 & 13.89 & - & - & - & - & 46.22 & 1.75 & 17.05 & 0.64 & 0.66 \\
\hline NGC4643 & Bulge+Disk+Bar & 24.96 & 54.10 & 20.95 & 0.46 & 5.43 & 0.72 & 2.53 & 48.22 & 6.38 & 21.30 & 2.82 & 0.62 \\
\hline NGC4647 & Bulge+Disk & 33.39 & 66.61 & - & 0.50 & 12.90 & 1.12 & 1.61 & 42.63 & 3.70 & - & - & - \\
\hline NGC4651 & PSF+Bulge+Disk & 41.67 & 58.33 & - & 0.71 & 18.52 & 1.61 & 0.63 & 30.46 & 2.64 & - & - & - \\
\hline NGC4654 & Bulge+Disk & 1.74 & 98.26 & - & 0.02 & 3.78 & 0.33 & 0.93 & 30.16 & 2.62 & - & - & - \\
\hline NGC4665 & $\mathrm{PSF}+$ Bulge + Disk + Bar & 14.74 & 67.38 & 17.89 & 0.22 & 6.92 & 0.64 & 1.12 & 50.48 & 4.66 & 30.68 & 2.83 & 0.70 \\
\hline NGC4689 & PSF+Bulge+Disk & 5.22 & 94.78 & - & 0.06 & 6.97 & 0.61 & 1.14 & 41.85 & 3.63 & - & - & - \\
\hline NGC4691 & PSF + Bulge+Disk +Bar & 15.80 & 69.09 & 15.11 & 0.23 & 11.62 & 1.35 & 0.61 & 33.90 & 3.93 & 24.99 & 2.90 & 0.42 \\
\hline NGC4698 & PSF+Bulge+Disk & 21.72 & 78.28 & - & 0.28 & 6.78 & 0.59 & 1.78 & 28.34 & 2.46 & - & - & - \\
\hline NGC4699 & Bulge+Disk+Bar & 19.93 & 76.48 & 3.59 & 0.26 & 2.62 & 0.35 & 2.08 & 15.93 & 2.11 & 13.49 & 1.79 & 0.02 \\
\hline NGC4772 & $\mathrm{PSF}+$ Bulge + Disk + Bar & 34.25 & 51.55 & 14.20 & 0.66 & 9.29 & 0.78 & 1.49 & 58.16 & 4.90 & 52.58 & 4.43 & 0.50 \\
\hline NGC 4775 & PSF+Bulge+Disk & 32.85 & 67.15 & - & 0.49 & 21.43 & 2.94 & 1.75 & 18.48 & 2.53 & - & - & - \\
\hline NGC4781 & PSF+Bulge+Disk & 10.18 & 89.82 & - & 0.11 & 12.02 & 1.39 & 0.94 & 32.82 & 3.81 & - & - & - \\
\hline NGC4818 & PSF + Bulge+Disk +Bar & 7.04 & 73.74 & 19.22 & 0.10 & 2.82 & 0.31 & 0.47 & 36.31 & 4.03 & 16.38 & 1.82 & 0.51 \\
\hline NGC4856 & $\mathrm{PSF}+\mathrm{Bulge}+$ Disk+Bar & 27.44 & 61.22 & 11.35 & 0.45 & 5.95 & 0.65 & 1.53 & 30.61 & 3.33 & 17.07 & 1.86 & 0.44 \\
\hline NGC4902 & $\mathrm{PSF}+$ Bulge+Disk+Bar & 5.59 & 84.44 & 9.97 & 0.07 & 4.31 & 0.87 & 0.85 & 29.24 & 5.88 & 14.13 & 2.84 & 0.39 \\
\hline NGC4930 & PSF+Bulge+Disk+Bar & 23.74 & 61.29 & 14.98 & 0.39 & 6.46 & 1.16 & 1.37 & 45.02 & 8.09 & 32.96 & 5.92 & 0.38 \\
\hline NGC4939 & PSF+Bulge+Disk & 21.37 & 78.63 & - & 0.27 & 9.52 & 2.16 & 2.78 & 32.14 & 7.29 & - & - & - \\
\hline NGC4941 & PSF+Bulge+Disk & 15.08 & 84.92 & - & 0.18 & 4.30 & 0.14 & 0.84 & 23.23 & 0.77 & - & - & - \\
\hline NGC4995 & $\mathrm{PSF}+$ Bulge+Disk+Bar & 6.62 & 87.18 & 6.20 & 0.08 & 4.14 & 0.60 & 0.05 & 19.56 & 2.82 & 18.03 & 2.60 & 0.34 \\
\hline NGC5054 & Bulge + Disk & 9.64 & 90.36 & - & 0.11 & 5.28 & 0.74 & 2.42 & 52.65 & 7.40 & - & - & - \\
\hline NGC5085 & Bulge+Disk & 6.04 & 93.96 & - & 0.06 & 5.53 & 0.82 & 1.48 & 33.88 & 5.04 & - & - & - \\
\hline NGC5101 & $\mathrm{PSF}+$ Bulge+Disk + Bar & 32.63 & 48.31 & 19.06 & 0.68 & 7.61 & 1.07 & 2.28 & 28.39 & 4.00 & 37.85 & 5.34 & 0.31 \\
\hline NGC5121 & PSF+Bulge+Disk & 36.43 & 63.57 & - & 0.57 & 4.68 & 0.53 & 2.41 & 15.62 & 1.78 & - & - & - \\
\hline NGC5161 & PSF+Bulge+Disk & 27.14 & 72.86 & - & 0.37 & 20.54 & 3.54 & 1.47 & 43.98 & 7.57 & - & - & - \\
\hline NGC5247 & PSF+Bulge+Disk & 7.54 & 92.46 & - & 0.08 & 8.46 & 0.97 & 1.04 & 50.85 & 5.82 & - & - & - \\
\hline NGC5371 & $\mathrm{PSF}+$ Bulge+Disk+Bar & 8.60 & 76.08 & 15.32 & 0.11 & 3.13 & 0.61 & 1.29 & 55.81 & 10.83 & 23.03 & 4.47 & 1.05 \\
\hline NGC5427 & PSF+Bulge+Disk & 7.76 & 92.24 & - & 0.08 & 4.74 & 0.93 & 0.60 & 24.59 & 4.81 & - & - & - \\
\hline
\end{tabular}


Table 2.2 (cont'd)

\begin{tabular}{|c|c|c|c|c|c|c|c|c|c|c|c|c|c|}
\hline $\begin{array}{c}\text { Galaxy Name } \\
\text { (1) }\end{array}$ & $\begin{array}{c}\text { Best Fit } \\
\text { (2) }\end{array}$ & $\begin{array}{l}B / T \\
\% \\
(3)\end{array}$ & $\begin{array}{l}D / T \\
\% \\
(4)\end{array}$ & $\begin{array}{c}\mathrm{Bar} / T \\
\% \\
(5)\end{array}$ & $\begin{array}{c}B / D \\
(6)\end{array}$ & $\begin{array}{c}\underset{\prime \prime}{\text { Bulge }} r_{\mathrm{e}} \\
(7)\end{array}$ & $\begin{array}{c}\text { Bulge } r_{\mathrm{e}} \\
\text { kpc } \\
(8)\end{array}$ & $\begin{array}{c}\text { Bulge } n \\
\text { (9) }\end{array}$ & $\begin{array}{c}\underset{\prime \prime}{\text { Disk h }} \\
(10)\end{array}$ & $\begin{array}{l}\text { Disk h } \\
\text { kpc } \\
(11)\end{array}$ & $\begin{array}{c}\text { Bar } r_{\mathrm{e}} \\
(12)\end{array}$ & $\begin{array}{l}\operatorname{Bar} r_{\mathrm{e}} \\
\operatorname{kpc} \\
(13)\end{array}$ & $\begin{array}{c}\text { Bar } n \\
(14)\end{array}$ \\
\hline NGC5483 & Bulge+Disk+Bar & 0.98 & 91.28 & 7.73 & 0.01 & 3.23 & 0.41 & 1.22 & 23.25 & 2.96 & 8.74 & 1.11 & 0.32 \\
\hline NGC5643 & $\mathrm{PSF}+$ Bulge+Disk+Bar & 8.21 & 81.78 & 10.01 & 0.10 & 5.68 & 0.50 & 2.14 & 45.30 & 3.95 & 48.58 & 4.24 & 0.43 \\
\hline NGC5676 & PSF+Bulge+Disk & 6.67 & 93.33 & - & 0.07 & 3.71 & 0.66 & 1.29 & 23.10 & 4.09 & - & - & - \\
\hline NGC5701 & $\mathrm{PSF}+\mathrm{Bulge}+$ Disk$+\mathrm{Bar}$ & 24.43 & 63.47 & 12.09 & 0.38 & 11.13 & 1.50 & 2.41 & 70.67 & 9.50 & 26.01 & 3.50 & 0.40 \\
\hline NGC5713 & PSF+Bulge+Disk & 33.40 & 66.60 & & 0.50 & 15.59 & 2.44 & 1.84 & 18.52 & 2.90 & - & . & \\
\hline NGC5850 & $\mathrm{PSF}+\mathrm{Bulge}+$ Disk+Bar & 14.76 & 63.74 & 21.50 & 0.23 & 6.22 & 0.91 & 1.58 & 72.24 & 10.59 & 46.05 & 6.75 & 0.90 \\
\hline NGC5921 & $\mathrm{PSF}+$ Bulge+Disk+Bar & 10.43 & 70.50 & 19.06 & 0.15 & 2.55 & 0.33 & 1.97 & 40.88 & 5.31 & 35.05 & 4.55 & 0.92 \\
\hline NGC5962 & Bulge+Disk+Bar & 10.30 & 78.82 & 10.88 & 0.13 & 2.33 & 0.38 & 1.37 & 14.56 & 2.38 & 13.85 & 2.26 & 0.02 \\
\hline NGC6215 & $\mathrm{PSF}+\mathrm{Bulge}+\mathrm{Disk}$ & 6.95 & 93.05 & - & 0.07 & 2.55 & 0.27 & 0.96 & 14.02 & 1.48 & - & - & - \\
\hline NGC6221 & Bulge+Disk+Bar & 5.27 & 81.98 & 12.76 & 0.06 & 3.16 & 0.32 & 2.73 & 51.47 & 5.15 & 19.33 & 1.93 & 0.87 \\
\hline NGC6300 & $\mathrm{PSF}+$ Bulge+Disk+Bar & 5.98 & 86.55 & 7.47 & 0.07 & 5.43 & 0.40 & 1.44 & 47.08 & 3.48 & 31.64 & 2.34 & 0.30 \\
\hline NGC6384 & Bulge + Disk & 27.92 & 72.08 & - & 0.39 & 15.66 & 2.14 & 3.13 & 40.05 & 5.49 & - & - & \\
\hline NGC6753 & $\mathrm{PSF}+$ Bulge+Disk+Bar & 5.08 & 72.91 & 22.02 & 0.07 & 1.50 & 0.31 & 0.94 & 19.17 & 4.02 & 7.88 & 1.65 & 0.80 \\
\hline NGC6782 & $\mathrm{PSF}+$ Bulge+Disk+Bar & 26.27 & 51.75 & 21.98 & 0.51 & 3.90 & 1.01 & 1.10 & 27.14 & 7.05 & 19.27 & 5.00 & 0.38 \\
\hline NGC6902 & PSF+Bulge+Disk & 40.55 & 59.45 & - & 0.68 & 12.68 & 2.32 & 3.03 & 30.13 & 5.52 & - & - & - \\
\hline NGC6907 & $\mathrm{PSF}+\mathrm{Bulge}+$ Disk $+\mathrm{Bar}$ & 10.32 & 63.12 & 26.56 & 0.16 & 3.49 & 0.77 & 1.10 & 28.99 & 6.39 & 23.37 & 5.15 & 0.33 \\
\hline NGC7083 & PSF+Bulge+Disk & 13.23 & 86.77 & - & 0.15 & 5.87 & 1.17 & 1.37 & 23.26 & 4.62 & - & - & - \\
\hline NGC7205 & PSF+Bulge+Disk & 6.08 & 93.92 & - & 0.06 & 4.70 & 0.50 & 0.88 & 31.03 & 3.28 & - & - & - \\
\hline NGC7213 & PSF+Bulge+Disk & 65.74 & 34.26 & - & 1.92 & 19.55 & 2.22 & 2.68 & 63.19 & 7.17 & - & - & - \\
\hline NGC7217 & PSF+Bulge+Disk & 53.71 & 46.29 & - & 1.16 & 21.07 & 1.74 & 2.21 & 26.84 & 2.22 & - & - & - \\
\hline NGC7412 & $\mathrm{PSF}+$ Bulge+Disk + Bar & 4.09 & 68.60 & 27.31 & 0.06 & 2.84 & 0.31 & 1.28 & 23.58 & 2.57 & 35.06 & 3.81 & 1.20 \\
\hline NGC7479 & $\mathrm{PSF}+$ Bulge+Disk+Bar & 8.76 & 63.51 & 27.72 & 0.14 & 6.00 & 1.00 & 1.09 & 37.59 & 6.26 & 40.63 & 6.77 & 0.47 \\
\hline NGC7552 & Bulge+Disk+Bar & 23.44 & 61.08 & 15.48 & 0.38 & 2.70 & 0.27 & 0.64 & 17.54 & 1.76 & 42.05 & 4.23 & 0.24 \\
\hline NGC7723 & $\mathrm{PSF}+\mathrm{Bulge}+$ Disk $+\mathrm{Bar}$ & 5.11 & 85.03 & 9.85 & 0.06 & 2.61 & 0.32 & 0.54 & 21.70 & 2.65 & 21.81 & 2.66 & 0.90 \\
\hline NGC7727 & PSF+Bulge+Disk & 41.77 & 58.23 & - & 0.72 & 8.31 & 1.00 & 2.02 & 24.46 & 2.94 & - & - & - \\
\hline NGC7741 & Bulge+Disk+Bar & 3.09 & 89.02 & 7.90 & 0.03 & 9.51 & 0.60 & 0.31 & 60.98 & 3.88 & 30.02 & 1.91 & 0.40 \\
\hline
\end{tabular}

Note. - Columns are: (1) Galaxy name. (2) The best fit chosen based on the criteria outlined in § 2.3.3. (3) Bulge-to-total light ratio. If a PSF is included in the model, the contribution from the PSF is also added in. (4) Disk-to-total light ratio. (5) Bar-to-total light ratio. (6) Bulge-to-disk ratio (7) Bulge effective radius in arcseconds. (8) Bulge effective radius in kpc. (9) Bulge Sérsic index. (10) Disk scalelength in arcseconds. (11) Disk scalelength in kpc. (12) Bar effective 
Table 2.3. Decomposition For NGC 4643

\begin{tabular}{|c|c|c|c|c|c|c|c|}
\hline $\begin{array}{l}\text { Fit } \\
\text { (1) }\end{array}$ & (2) & $\begin{array}{c}r_{\mathrm{e}} \text { or } \mathrm{h} \\
\left(\begin{array}{c}\prime \prime \\
)\end{array}\right) \\
(3)\end{array}$ & $\begin{array}{c}r_{\mathrm{e}} \text { or } \mathrm{h} \\
(\mathrm{kpc}) \\
(4)\end{array}$ & $\begin{array}{l}n \\
(5)\end{array}$ & $\begin{array}{l}b / a \\
(6)\end{array}$ & $\begin{array}{c}\text { Position Angle } \\
(7)\end{array}$ & $\begin{array}{c}\text { Fractional light } \\
\text { (8) }\end{array}$ \\
\hline Stage 1 & Sérsic & 27.90 & 2.66 & 4.44 & 0.80 & -51.03 & $100 \%$ \\
\hline Stage 2 & $\begin{array}{c}\text { Bulge } \\
\text { Disk }\end{array}$ & $\begin{array}{c}23.86 \\
335.88\end{array}$ & $\begin{array}{c}2.30 \\
32.33\end{array}$ & $\begin{array}{l}4.16 \\
1.00\end{array}$ & $\begin{array}{l}0.80 \\
0.84\end{array}$ & $\begin{array}{c}-51.08 \\
66.94\end{array}$ & $\begin{array}{l}34.6 \% \\
65.4 \%\end{array}$ \\
\hline Stage 3 & $\begin{array}{c}\text { Bulge } \\
\text { Disk } \\
\text { Bar }\end{array}$ & $\begin{array}{c}5.43 \\
48.22 \\
21.30\end{array}$ & $\begin{array}{l}0.52 \\
4.64 \\
2.05\end{array}$ & $\begin{array}{l}2.53 \\
1.00 \\
0.62\end{array}$ & $\begin{array}{l}0.90 \\
0.84 \\
0.37\end{array}$ & $\begin{array}{c}60.52 \\
66.94 \\
-45.84\end{array}$ & $\begin{array}{l}25.0 \% \\
54.1 \% \\
20.9 \%\end{array}$ \\
\hline
\end{tabular}

Note. - Columns are: (1) Indicates whether the model is for Stage 1 (pure Sérsic profile), Stage 2 (bulge+disk or bar+disk), or Stage 3 (bulge+disk+bar). (2) One of pure Sérsic profile, Sérsic bulge, exponential disk, or Sérsic bar. (3) Effective radius of the bulge/bar or disk scalelength in arcseconds. (4) Effective radius of the bulge/bar or disk scalelength in kpc. (5) The Sérsic index of the profile. (6) Axis ratio of the component. (7) Position angle in degrees of the component. Positive angles are East of North. (8) The percentage of total galaxy light contributed by the component.

Table 2.4. Decomposition For NGC 4548

\begin{tabular}{|c|c|c|c|c|c|c|c|}
\hline $\begin{array}{l}\text { Fit } \\
(1)\end{array}$ & $(2)$ & $\begin{array}{c}r_{\mathrm{e}} \text { or } \mathrm{h} \\
\left(\begin{array}{c}\prime \prime \\
)\end{array}\right) \\
(3)\end{array}$ & $\begin{array}{c}r_{\mathrm{e}} \text { or } \mathrm{h} \\
(\mathrm{kpc}) \\
(4)\end{array}$ & $\begin{array}{l}n \\
(5)\end{array}$ & $\begin{array}{r}b / a \\
(6)\end{array}$ & $\begin{array}{c}\text { Position Angle } \\
(7)\end{array}$ & $\begin{array}{l}\text { Fractional light } \\
\text { (8) }\end{array}$ \\
\hline Stage 1 & Sérsic & 154.59 & 5.19 & 5.19 & 0.80 & 78.31 & $100 \%$ \\
\hline \multirow[t]{2}{*}{ Stage 2} & Bulge & 57.86 & 1.94 & 4.32 & 0.76 & 75.77 & $61.5 \%$ \\
\hline & Disk & 60.39 & 2.03 & 1.00 & 0.75 & -32.54 & $38.5 \%$ \\
\hline \multirow[t]{3}{*}{ Stage 3} & Bulge & 6.98 & 0.23 & 1.56 & 0.88 & -66.50 & $13.0 \%$ \\
\hline & Disk & 58.22 & 1.96 & 1.00 & 0.75 & -32.54 & $68.6 \%$ \\
\hline & Bar & 44.91 & 1.51 & 0.51 & 0.35 & 66.65 & $18.4 \%$ \\
\hline
\end{tabular}

Note. - Columns are: (1) Indicates whether the model is for Stage 1 (pure Sérsic profile), Stage 2 (bulge+disk or bar+disk), or Stage 3 (bulge+disk+bar). (2) One of pure Sérsic profile, Sérsic bulge, exponential disk, or Sérsic bar. (3) Effective radius of the bulge/bar or disk scalelength in arcseconds. (4) Effective radius of the bulge/bar or disk scalelength in kpc. (5) The Sérsic index of the profile. (6) Axis ratio of the component. (7) Position angle in degrees of the component. Positive angles are East of North. (8) The percentage of total galaxy light contributed by the component. 
Table 2.5. Decomposition For NGC 4902

\begin{tabular}{cccccccc}
\hline \hline Fit & & $\begin{array}{c}r_{\mathrm{e}} \text { or h } \\
\left({ }^{\prime \prime}\right)\end{array}$ & $\begin{array}{c}r_{\mathrm{e}} \text { or h } \\
(\mathrm{kpc})\end{array}$ & $n$ & $b / a$ & Position Angle & Fractional light \\
$(1)$ & $(2)$ & $(3)$ & $(4)$ & $(5)$ & $(6)$ & $(7)$ & $(8)$ \\
\hline Stage 1 & Sérsic & 154.4 & 28.8 & 5.11 & 0.54 & 69.0 & $100 \%$ \\
\hline Stage 2 & Bulge & 11.5 & 2.15 & 1.02 & 0.38 & 68.5 & $17.4 \%$ \\
& Disk & 32.8 & 6.12 & 1.00 & 0.84 & 81.1 & $82.6 \%$ \\
\hline Stage 3 & Bulge & 4.31 & 0.80 & 0.85 & 0.68 & -52.96 & $5.59 \%$ \\
& Disk & 29.2 & 5.45 & 1.00 & 0.84 & 81.1 & $9.9 \%$ \\
& Bar & 14.1 & 2.64 & 0.39 & 0.22 & 66.37 & $9.97 \%$ \\
\hline
\end{tabular}

Note. - Columns are: (1) Indicates whether the model is for Stage 1 (pure Sérsic profile), Stage 2 (bulge+disk or bar+disk), or Stage 3 (bulge+disk+bar). (2) One of pure Sérsic profile, Sérsic bulge, exponential disk, or Sérsic bar. (3) Effective radius of the bulge/bar or disk scalelength in arcseconds. (4) Effective radius of the bulge/bar or disk scalelength in kpc. (5) The Sérsic index of the profile. (6) Axis ratio of the component. (7) Position angle in degrees of the component. Positive angles are East of North. (8) The percentage of total galaxy light contributed by the component.

Table 2.6. Checking GALFIT robustness with different input guesses for Stage 3

\begin{tabular}{|c|c|c|c|c|c|c|c|c|}
\hline$(1)$ & $\begin{array}{c}B / T \\
(2)\end{array}$ & $\begin{array}{c}\text { Bulge } r_{\mathrm{e}} \\
\text { (") } \\
(3)\end{array}$ & $\begin{array}{c}\text { Bulge } n \\
(4)\end{array}$ & $\begin{array}{c}D / T \\
(5)\end{array}$ & $\begin{array}{c}\text { Disk } h \\
(") \\
(6)\end{array}$ & $\begin{array}{c}\operatorname{Bar} / T \\
(7)\end{array}$ & $\begin{array}{c}\text { Bar } r_{\mathrm{e}} \\
(") \\
(8)\end{array}$ & $\begin{array}{c}\text { Bar } n \\
(9)\end{array}$ \\
\hline \multicolumn{9}{|c|}{ NGC 4548} \\
\hline Initial guesses from 1D decomposition & $17.5 \%$ & 7.39 & 1.17 & $63.5 \%$ & 28.4 & $19.0 \%$ & 37.5 & 0.54 \\
\hline Stage 3 Output & $13.0 \%$ & 6.98 & 1.56 & $68.6 \%$ & 58.2 & $18.4 \%$ & 44.9 & 0.51 \\
\hline Initial guesses from from Stage 2 & $11.1 \%$ & 7.50 & 1.70 & $69.9 \%$ & 64.5 & $19.1 \%$ & 37.5 & 0.54 \\
\hline Stage 3 Output & $13.0 \%$ & 6.98 & 1.56 & $68.6 \%$ & 58.2 & $18.4 \%$ & 44.9 & 0.51 \\
\hline \multicolumn{9}{|c|}{ NGC 4643} \\
\hline Initial guesses from 1D decomposition & $33.6 \%$ & 7.18 & 0.86 & $40.4 \%$ & 37.5 & $26.0 \%$ & 22.0 & 0.60 \\
\hline Stage 3 Output & $25.0 \%$ & 5.43 & 2.53 & $54.1 \%$ & 48.2 & $20.9 \%$ & 21.3 & 0.62 \\
\hline Initial guesses from Stage 2 & $24.1 \%$ & 5.30 & 2.5 & $51.8 \%$ & 46.4 & $24.1 \%$ & 22.0 & 0.60 \\
\hline Stage 3 Output & $25.0 \%$ & 5.43 & 2.53 & $54.1 \%$ & 48.2 & $20.9 \%$ & 21.3 & 0.62 \\
\hline
\end{tabular}

Note. - For each galaxy, the rows are: (1) The initial guesses to Stage 3 provided by 1D decomposition. (2) The corresponding output for the input of row (1). (3) The initial guesses to Stage 3 provided by Stage 2. (4) The corresponding output for the input of row (3).

The columns are: (2) Bulge-to-total luminosity fraction. (3) Effective radius of the bulge in arcseconds. (4) The Sérsic index of the profile. (5) Disk-to-total luminosity fraction. (6) Disk scalelength in arcseconds. (7) Bar-to-total luminosity fraction. (8) Bar effective radius in arcseconds. (9) Bar Sérsic index. 
Table 2.7. Parameter Coupling

\begin{tabular}{|c|c|c|c|c|c|c|c|c|c|}
\hline $\begin{array}{c}\text { Type of Fit } \\
\text { (1) }\end{array}$ & $\begin{array}{l}\chi^{2} \\
(2)\end{array}$ & $\begin{array}{c}\text { Bulge } r_{\mathrm{e}} \\
(") \\
(3)\end{array}$ & $\begin{array}{c}\text { Bulge } n \\
\text { (4) }\end{array}$ & $\begin{array}{l}B / T \\
(5)\end{array}$ & $\begin{array}{c}\text { Disk } h \\
(") \\
(6)\end{array}$ & $\begin{array}{c}D / T \\
(7)\end{array}$ & $\begin{array}{c}\text { Bar } r_{\mathrm{e}} \\
(") \\
(8)\end{array}$ & $\begin{array}{c}\text { Bar } n \\
(9)\end{array}$ & $\begin{array}{c}\operatorname{Bar} / T \\
(10)\end{array}$ \\
\hline \multicolumn{10}{|c|}{ NGC 3885} \\
\hline initial input & 11241.33 & 2.32 & 2.00 & $27.7 \%$ & 10.44 & $72.3 \%$ & - & - & - \\
\hline $\mathrm{n}=$ free & 8210.12 & 3.13 & 0.46 & $27.1 \%$ & 11.94 & $72.9 \%$ & - & - & - \\
\hline$n=1$ fixed & [8307.82] & {$[2.96]$} & [1.00] & {$[29.1 \%]$} & {$[12.90]$} & {$[70.9 \%]$} & - & - & - \\
\hline$n=4$ fixed & {$[7373.55]$} & {$[6.28]$} & {$[4.00]$} & {$[53.7 \%]$} & {$[20.45]$} & {$[46.3 \%]$} & - & - & - \\
\hline $\mathrm{n}=1 \mathrm{re}-\mathrm{fit}$ & 8210.12 & 3.13 & 0.46 & $27.1 \%$ & 11.94 & $72.9 \%$ & - & - & - \\
\hline $\mathrm{n}=4 \mathrm{re}-\mathrm{fit}$ & 7258.46 & 7.11 & 4.49 & $57.3 \%$ & 19.87 & $42.7 \%$ & - & - & - \\
\hline \multicolumn{10}{|c|}{ NGC 7213} \\
\hline initial input & 605757.39 & 5.80 & 4.00 & $56.4 \%$ & 9.25 & $43.6 \%$ & - & - & - \\
\hline $\mathrm{n}=$ free & 71170.10 & 19.55 & 2.68 & $65.7 \%$ & 63.19 & $34.3 \%$ & - & - & - \\
\hline$n=1$ fixed & [96312.29] & {$[7.81]$} & {$[1.00]$} & {$[31.4 \%]$} & {$[27.79]$} & {$[68.6 \%]$} & - & - & - \\
\hline$n=4$ fixed & [70998.69] & [41.11] & {$[4.00]$} & {$[95.4 \%]$} & {$[6.75]$} & {$[4.6 \%]$} & - & - & - \\
\hline$n=1$ re-fit & 71170.10 & 19.53 & 2.68 & $65.7 \%$ & 63.16 & $34.3 \%$ & - & - & - \\
\hline $\mathrm{n}=4$ re-fit & 69970.51 & 72.04 & 5.60 & $91.9 \%$ & 7.98 & $8.1 \%$ & - & - & - \\
\hline \multicolumn{10}{|c|}{ NGC 4643} \\
\hline initial input & 7841.46 & 5.34 & 2.50 & $24.1 \%$ & 46.40 & $51.8 \%$ & 22.04 & 0.60 & $24.1 \%$ \\
\hline $\mathrm{n}=$ free & 2111.59 & 5.43 & 2.53 & $25.0 \%$ & 48.22 & $54.1 \%$ & 21.30 & 0.62 & $20.9 \%$ \\
\hline$n=1$ fixed & [3001.98] & {$[2.31]$} & {$[1.00]$} & {$[9.6 \%]$} & {$[53.37]$} & {$[57.0 \%]$} & {$[15.05]$} & {$[0.88]$} & [33.4\%] \\
\hline$n=4$ fixed & [2491.06] & [12.08] & {$[4.00]$} & {$[35.4 \%]$} & {$[87.22]$} & {$[51.7 \%]$} & {$[23.37]$} & {$[0.61]$} & {$[12.9 \%]$} \\
\hline$n=1$ re-fit & 2111.59 & 5.43 & 2.53 & $25.0 \%$ & 48.21 & $54.1 \%$ & 21.30 & 0.62 & $20.9 \%$ \\
\hline $\mathrm{n}=4 \mathrm{re}-\mathrm{fit}$ & 2111.59 & 5.43 & 2.53 & $25.0 \%$ & 48.22 & $54.1 \%$ & 21.30 & 0.62 & $20.9 \%$ \\
\hline \multicolumn{10}{|c|}{ NGC 4151} \\
\hline initial input & 1388891.11 & 7.50 & 0.50 & $24.6 \%$ & 33.00 & $65.0 \%$ & 55.50 & 0.10 & $10.3 \%$ \\
\hline $\mathrm{n}=$ free & 16391.66 & 9.36 & 0.44 & $41.3 \%$ & 37.74 & $50.3 \%$ & 57.00 & 0.10 & $8.4 \%$ \\
\hline $\mathrm{n}=1$ fixed & [18100.67] & [11.58] & {$[1.00]$} & {$[42.2 \%]$} & {$[86.28]$} & {$[41.1 \%]$} & [56.70] & {$[0.17]$} & [16.7\%] \\
\hline $\mathrm{n}=4$ fixed & {$[20051.12]$} & [108.39] & {$[4.00]$} & [51.4\%] & {$[8.21]$} & {$[20.4 \%]$} & [68.23] & {$[0.28]$} & {$[28.2 \%]$} \\
\hline$n=1$ re-fit & 16391.58 & 9.38 & 0.44 & $41.2 \%$ & 38.31 & $49.7 \%$ & 55.57 & 0.12 & $9.0 \%$ \\
\hline $\mathrm{n}=4$ re-fit & 16390.93 & 9.36 & 0.44 & $41.3 \%$ & 38.20 & $49.8 \%$ & 55.88 & 0.11 & $8.8 \%$ \\
\hline
\end{tabular}

Note. - For each galaxy, the rows are: (1) The model outputs (columns 2 to 10) from GALFIT based on the Stage 1 or Stage 2 fits. These outputs are used as initial guesses in the fits for rows 2 and 3. (2) The model outputs (columns 2 to 10) from GALFIT when the bulge $n$ is allowed to vary freely, and the input guesses are based on the parameters in columns 2 to 10 of row 1. (3) The model outputs (columns 2 to 10) from GALFIT when the bulge Sérsic index is held fixed at $n=1$, and the input guesses are based on the parameters in columns 2 to 10 of row 1. The model is not always physically meaningful because the bulge index is fixed. (4) As in row 3 , except that the bulge Sérsic index is now held fixed at $n=4$. The model is not always physically meaningful because the bulge index is fixed. (5) The model outputs (columns 2 to 10) from GALFIT, when the bulge $n$ is allowed to vary freely, but the input guesses are now based on the parameters in columns 2 to 10 of row 3. (6) The model outputs (columns 2 to 10) from GALFIT, when the bulge $n$ is allowed to vary freely, but the input guesses are now based on the parameters in columns 2 to 10 of row 4 . The columns are: (1) The type of fit whose model outputs are shown in columns 2 to 10. (2) The $\chi^{2}$ of the fit. (3) Effective radius of the bulge in arcseconds. (4) The Sérsic index of the bulge (in the case of Stage 2 bulge-disk fits in row 1 and in the case of all fits in rows 2 to 6 ), or the Sérsic index of a single component (in the case of Stage 1 fits in row 1). (5) Bulge-to-total luminosity fraction. (6) Disk scalelength in arcseconds. (7) Disk-to-total luminosity fraction. (8) Bar effective radius in arcseconds. (9) Bar Sérsic index. (10) Bar-to-total luminosity fraction. 
Table 2.8. Mass Breakdown of Galactic Structures

\begin{tabular}{cc}
\hline \hline Structure & Mass \\
\hline Bulges & $18.9 \% \pm 3.49 \%$ \\
Disks & $71.6 \% \pm 4.02 \%$ \\
Bars & $9.58 \% \pm 2.62 \%$ \\
\hline Bulges with $n>2$ & $10.4 \% \pm 2.72 \%$ \\
Bulges with $n \leq 2$ & $8.42 \% \pm 2.47 \%$ \\
\hline
\end{tabular}

Table 2.9. $B / T$ and bulge $n$ in bright/high mass spirals

\begin{tabular}{lc}
\hline \multicolumn{2}{c}{ Sample $\left(M_{B} \leq-19.3\right)$} \\
\hline Fraction of spirals with bulge $n \geq 4$ & $1.40 \% \pm 0.98 \%$ \\
Fraction of spirals with bulge $2<n<4$ & $22.4 \% \pm 3.49 \%$ \\
Fraction of spirals with bulge $n \leq 2$ & $76.2 \% \pm 3.56 \%$ \\
Fraction of spirals with bulge $n>2$ & $23.8 \% \pm 3.56 \%$ \\
Fraction of spirals with $B / T \leq 0.2$ & $68.5 \% \pm 3.88 \%$ \\
Fraction of spirals with $B / T>0.2$ & $31.1 \% \pm 3.88 \%$ \\
Fraction of spirals with $0.2<B / T<0.4$ & $23.1 \% \pm 3.52 \%$ \\
Fraction of spirals with $B / T \geq 0.4$ & $8.39 \% \pm 2.32 \%$ \\
& \\
\hline & Sample $M_{\star} \geq 1 \times 10^{10} M_{\odot}$ \\
\hline & \\
\hline Fraction of spirals with bulge $n \geq 4$ & $1.77 \% \pm 1.24 \%$ \\
Fraction of spirals with bulge $2<n<4$ & $23.9 \% \pm 4.01 \%$ \\
Fraction of spirals with bulge $n \leq 2$ & $74.3 \% \pm 4.11 \%$ \\
Fraction of spirals with bulge $n>2$ & $25.7 \% \pm 4.11 \%$ \\
Fraction of spirals with $B / T \leq 0.2$ & $66.4 \% \pm 4.44 \%$ \\
Fraction of spirals with $B / T>0.2$ & $33.6 \% \pm 4.44 \%$ \\
Fraction of spirals with $0.2<B / T<0.4$ & $25.7 \% \pm 4.11 \%$ \\
Fraction of spirals with $B / T \geq 0.4$ & $7.96 \% \pm 2.55 \%$ \\
\hline
\end{tabular}

Table 2.10. Bar Fraction as a Function of $B / T$ and Bulge Index

\begin{tabular}{lc}
\hline \hline \multicolumn{3}{c}{ Sample $\left(M_{B} \leq-19.3\right)$} \\
\hline Bar fraction in spirals with bulge $n \geq 4$ & $0.00 \% \pm 0.00 \%$ \\
Bar fraction in spirals with bulge $2<n<4$ & $37.5 \% \pm 8.56 \%$ \\
Bar fraction in spirals with bulge $n \leq 2$ & $65.1 \% \pm 4.56 \%$ \\
Bar fraction of spirals with bulge $n>2$ & $35.3 \% \pm 8.20 \%$ \\
Bar fraction of spirals with $B / T \leq 0.2$ & $68.4 \% \pm 4.70 \%$ \\
Bar fraction of spirals with $B / T>0.2$ & $35.6 \% \pm 7.14 \%$ \\
Bar fraction of spirals with $0.2<B / T<0.4$ & $42.4 \% \pm 8.60 \%$ \\
Bar fraction of spirals with $B / T \geq 0.4$ & $16.7 \% \pm 10.8 \%$ \\
\hline & \\
\hline & Sample $M_{\star} \geq 1 \times 10^{10} M_{\odot}$ \\
\hline Bar fraction of spirals with bulge $n \geq 4$ & $0.00 \% \pm 0.00 \%$ \\
Bar fraction of spirals with bulge $2<n<4$ & $44.4 \% \pm 9.56 \%$ \\
Bar fraction of spirals with bulge $n \leq 2$ & $63.1 \% \pm 5.27 \%$ \\
Bar fraction of spirals with bulge $n>2$ & $41.4 \% \pm 9.15 \%$ \\
Bar fraction of spirals with $B / T \leq 0.2$ & $68.0 \% \pm 5.39 \%$ \\
Bar fraction of spirals with $B / T>0.2$ & $36.8 \% \pm 7.83 \%$ \\
Bar fraction of spirals with $0.2<B / T<0.4$ & $41.4 \% \pm 9.15 \%$ \\
Bar fraction of spirals with $B / T \geq 0.4$ & $22.2 \% \pm 13.9 \%$ \\
\hline
\end{tabular}


Table 2.11. B/T: Data versus Hierarchical Models of Galaxy Evolution

\begin{tabular}{|c|c|c|c|c|c|}
\hline (1) & $\begin{array}{l}\text { Data } \\
(2)\end{array}$ & Model & $\begin{array}{c}\text { Model spirals with } \\
\text { major mergers } \\
\text { since redshift } z_{1} \\
(4)\end{array}$ & $\begin{array}{c}\text { Model spirals with } \\
\text { no major merger } \\
\text { since redshfit } z_{1} \\
(5)\end{array}$ & $\begin{array}{l}\text { All model } \\
\text { spirals } \\
(6)\end{array}$ \\
\hline & & & For $z_{1} \leq 2$ & For $z_{1} \leq 2$ & \\
\hline Fraction of spirals with $B / T \leq 0.2$ & $66.4 \% \pm 4.44 \%$ & SK & $0.1 \%$ & $67.2 \%$ & $67.3 \%$ \\
\hline Fraction of spirals with $0.2<B / T \leq 0.4$ & $25.7 \% \pm 4.11 \%$ & SK & $2.2 \%$ & $16.6 \%$ & $18.8 \%$ \\
\hline Fraction of spirals with $0.4<B / T \leq 0.75$ & $8.0 \% \pm 2.5 \%$ & SK & $11.2 \%$ & $2.6 \%$ & $13.8 \%$ \\
\hline Fraction of spirals with $B / T \leq 0.75$ & $100 \%$ & SK & $13.5 \%$ & $86.5 \%$ & $100.0 \%$ \\
\hline Fraction of spirals with $B / T<0.2$ & $66.4 \%+4.44 \%$ & $\mathrm{SK}$ & For $z_{1} \leq 4$ & For $z_{1} \leq 4$ & $673 \%$ \\
\hline Fraction of spirals with $0.2<B / T \leq 0.4$ & $25.7 \% \pm 4.11 \%$ & SK & $\begin{array}{l}1.0 \% \\
5.5 \%\end{array}$ & $\begin{array}{l}05.7 \% \\
13.3 \%\end{array}$ & $\begin{array}{l}07.3 \% \\
18.8 \%\end{array}$ \\
\hline Fraction of spirals with $0.4<B / T \leq 0.75$ & $8.0 \% \pm 2.5 \%$ & SK & $12.6 \%$ & $1.3 \%$ & $13.9 \%$ \\
\hline Fraction of spirals with $B / T \leq 0.75$ & $100 \%$ & SK & $19.8 \%$ & $80.2 \%$ & $100.0 \%$ \\
\hline & & & For $z_{1} \leq 2$ & For $z_{1} \leq 2$ & \\
\hline $\begin{array}{l}\text { Fraction of spirals with } B / T \leq 0.2 \\
\text { Fraction of spirals with } 0.2<B / T \leq 0.4\end{array}$ & $\begin{array}{l}66.4 \% \pm 4.44 \% \\
25.7 \% \pm 4.11 \%\end{array}$ & $\begin{array}{l}\mathrm{PH} \\
\mathrm{PH}\end{array}$ & $\begin{array}{l}3.0 \% \\
7.1 \%\end{array}$ & $\begin{array}{l}67.7 \% \\
13.1 \%\end{array}$ & $\begin{array}{l}70.7 \% \\
20.1 \%\end{array}$ \\
\hline Fraction of spirals with $0.4<B / T \leq 0.75$ & $8.0 \% \pm 2.5 \%$ & $\mathrm{PH}$ & $5.9 \%$ & $3.1 \%$ & $9.0 \%$ \\
\hline Fraction of spirals with $B / T \leq 0.75$ & $100 \%$ & $\mathrm{PH}$ & $15.7 \%$ & $84.0 \%$ & $99.8 \%$ \\
\hline
\end{tabular}

Note. - Column 2 shows the empirical fraction of high mass spirals with a given $B / T$. Columns 3 to 6 show the fraction of high mass spirals with a given $B / T$ in the models, which define spirals as systems with $B / T \leq 0.75$. Column 3 indicates the model used: SK refers to the models by Khochfar \& Burkert (2005) and Khochfar \& Silk (2006b), while PH refers to those by Hopkins et al. (2009c). The models are described in $\$ 2.5 .8$ and have different prescriptions for the amount of mass that gets converted into bulge stars. Column 4 shows the fraction of spirals, which have a given $B / T$ and have had one or more major mergers since redshift $z_{1}$. Column 5 shows the fraction of spirals, which have a given $B / T$ and have had no major merger since redshift $z_{1}$. Column 6 is the sum of column 4 and 5 , and shows the fraction of spirals with a given $B / T$ in the models. It can be directly compared with the data in column 2 . 
Table 2.12. Comparison of model merger fraction since $z \leq 4$ and bulge Sérsic index

\begin{tabular}{|c|c|}
\hline \multicolumn{2}{|l|}{$\begin{array}{l}\text { Stellar mass limit: } 1.0 \times 10^{10} M_{\odot} \\
\text { Model galaxies: } 7674 \\
\text { Observed galaxies: } 113\end{array}$} \\
\hline \multicolumn{2}{|l|}{ Test 1} \\
\hline $\begin{array}{l}\% \text { of model galaxies } \mathrm{w} / B / T \leq 0.2 \text { with no major merger since } z \leq 4 \text { : } \\
\% \text { of observed galaxies } \mathrm{w} / B / T \leq 0.2 \text { and bulge } n \leq 3: \\
\% \text { of observed galaxies } \mathrm{w} / B / T \leq 0.2 \text { and bulge } n \leq 2.5 \text { : } \\
\% \text { of observed galaxies } \mathrm{w} / B / T \leq 0.2 \text { and bulge } n \leq 2 \text { : }\end{array}$ & $\begin{array}{l}5040 / 7674=65.7 \% \pm 0.54 \% \\
75 / 113=66.4 \% \pm 4.44 \% \\
74 / 113=65.5 \% \pm 4.47 \% \\
67 / 113=59.3 \% \pm 4.62 \%\end{array}$ \\
\hline \multicolumn{2}{|l|}{ Test 2} \\
\hline $\begin{array}{l}\% \text { of model galaxies w } / B / T \geq 0.4 \text { and a major merger since } z \leq 4 \text { : } \\
\% \text { of observed spirals } \mathrm{w} / B / T \geq 0.4 \text { and bulge } n>3: \\
\% \text { of observed spirals } \mathrm{w} / B / T \geq 0.4 \text { and bulge } n>2.5 \text { : } \\
\% \text { of observed spirals } \mathrm{w} / B / T \geq 0.4 \text { and bulge } n>2 \text { : }\end{array}$ & $\begin{array}{l}971 / 7674=12.7 \% \pm 0.38 \% \\
2 / 113=1.77 \% \pm 1.24 \% \\
4 / 113=3.54 \% \pm 1.74 \% \\
7 / 113=6.19 \% \pm 2.27 \%\end{array}$ \\
\hline \multicolumn{2}{|l|}{ Test 3} \\
\hline $\begin{array}{l}\% \text { of model galaxies } \mathrm{w} / B / T \leq 0.2 \text { and a major merger since } z \leq 4 \text { : } \\
\% \text { of observed galaxies } \mathrm{w} / B / T \leq 0.2 \text { and bulge } n>3 \text { : } \\
\% \text { of observed galaxies } \mathrm{w} / B / T \leq 0.2 \text { and bulge } n>2.5 \\
\% \text { of observed galaxies } \mathrm{w} / B / T \leq 0.2 \text { and bulge } n>2\end{array}$ & $\begin{array}{l}126 / 7674=1.64 \% \pm 0.15 \% \\
0 / 113=0.00 \% \pm 0.00 \% \\
1 / 113=0.88 \% \pm 0.88 \% \\
8 / 113=7.08 \% \pm 2.41 \%\end{array}$ \\
\hline
\end{tabular}




\section{Chapter 3}

\section{Exploring the Structure and Assembly of Galaxies At the Heart of the Coma Cluster}

1

\subsection{Introduction}

How galaxies form and evolve is one of the primary outstanding problems in extragalactic astronomy. $\Lambda$ CDM-based models of galaxy formation describe well the behavior of dark matter on large scales, and state-of-the art simulations attempt to model galaxy formation by combining a $\Lambda \mathrm{CDM}$ cosmology with baryonic physics. The initial conditions were such that pockets of dark matter decoupled from the Hubble flow in the early Universe before collapsing into virialized halos. Halos clustered hierarchically into progressively larger structures. Meanwhile, in the halo interiors, gas formed rotating disks which undergo star formation (SF) to produce stellar disks (Cole 2000; Steinmetz \& Navarro 2002). The subsequent growth of galaxies is thought to proceed through a combination of major mergers, (e.g. Toomre 1977; Barnes 1988; Khochfar \& Silk 2006b, 2009a), minor mergers (e.g., Oser

\footnotetext{
${ }^{1}$ This chapter has been submitted for publication to Monthly Notices of the Royal Astronomical Society. Co-author contributions: Jogee, S. supervised the research. Khochfar, S. and Neistein, E. provided predictions from hierarchical models of galaxy formation. Marinova, I. provided an updated catalog of galaxy Hubble types. Hoyos, C. provided model point spread functions. Balcells, M., den Brok, M., Goudfrooij, P., Hammer, D., Hoyos, C., Mobasher, B., Peletier, R. F., Trentham, N., and Verdoes Kleijn, G. contributed to the Coma Treasury Survey data products. Balcells, M., den Brok, M., Carter, D., Goudfrooij, P., Hammer, D., Hoyos, C., Jogee, S., Khochfar, S., Kormendy, J., Mobasher, B. Neistein, E., Peletier, R. F., Trentham, N., and Verdoes Kleijn, G. contributed editorial comments.
} 
et al. 2012, Hilz et al. 2013), cold-mode gas accretion (Birnboim \& Dekel 2003; Kereš et al. 2005; Dekel \& Birnboim 2006; Dekel et al. 2009a, b; Kereš et al. 2009; Brooks et al. 2009; Ceverino et al. 2010), and other secular processes (Kormendy \& Kennicutt 2004).

Current galaxy formation models face several challenges. The relative importance of galaxy assembly mechanisms (major mergers, minor mergers, and cold mode accretion) for the SF activity and structure of galaxies is not well known and is actively debated. In terms of SF activity, recent observational (e.g., Bell et al. 2005; Wolf et al. 2005; Jogee et al. 2009; Robaina et al. 2009; López-Sanjuan et al. 2010) and theoretical (e.g. Hopkins et al. 2006b, 2010c) work suggests that major mergers have not contributed significantly to the cosmic star formation rate (SFR) density over the last 8 Gyr $(z<1$; but see Eliche-Moral et al. 2010).

As far as the structure of galaxies is concerned, we are still actively studying and debating the epoch and formation pathway for the main stellar components of galaxies, namely flattened, dynamically cold disk-dominated components (ranging from outer disks to circumnuclear disks) versus puffy, dynamically hot spheroidal or triaxial bulges/ellipticals.

We adopt the widely used definition of a bulge as the excess light above an outer disk in an S0 or spiral galaxy. There are different kinds of bulges: classical bulges, disky pseudobulges, and boxy pseudobulges. Classical bulges are puffy and dynamically hot with spheroidal or triaxial shapes; they live in the elliptical-bulge fundamental plane (Kormendy et al. 2009; Fisher \& Drory 2008, 2010; Kormendy \& Bender 2012). Disky pseudobulges are flattened inner disks with relatively higher ratios of $V / \sigma$. Boxy pseudobulges are end-on or edge (face)-on bars found only in highly inclined edge-on galaxies. For remainder of the paper we refer to classical bulges simply as "bulges" when the context is unambiguous. 
Elliptical galaxies are like classical bulges with no outer disk. They come in two varieties as discussed in Kormendy et al. (2009). These types correspond to the slow and rapid rotators as defined, for example, by the SAURON and ATLAS ${ }^{3 D}$ teams (de Zeeuw et al. 2002; Emsellem et al. 2004, 2007, 2011; Capellari et al. 2007, 2011a, b; Krajnović et al. 2008, 2011; McDermid et al. 2006). We will qualify ellipticals as rotating/disky and nonrotating/boxy ellipticals when we need to refer to this distinction.

On the theoretical front, simulations have changed the traditional view whereby the major merger of two spiral galaxies produces only a bulge or elliptical remnant. In early simulations focusing on gas-poor mergers, the major merger of two spiral galaxies with mass ratio $M_{1} / M_{2} \geq 1 / 4$ would inevitably destroy the pre-existing stellar disks by violent relaxation, producing a remnant bulge or elliptical having a puffed-up distribution of stars with a low ratio of ordered-to-random motion $(V / \sigma)$ and a steep de Vaucouleurs $r^{1 / 4}$ (i.e., Sérsic index $n=4$ ) surface brightness profile (Toomre 1977).

Improved simulations (Robertson et al. 2006; Naab et al. 2006; Governato et al. 2007; Hopkins et al. 2009a, b) significantly revised this picture. In unequal-mass major mergers, violent relaxation of stellar disks is not complete. Rather, the fraction of violently relaxed stars in the major merger scales with merger mass ratio $M_{1} / M_{2}$ (Hopkins et al. 2009b). For major mergers where the progenitors have moderate-to-high gas fractions, some fraction of the gas is driven by gravitational torques to the center of the galaxy where it undergoes star formation to create "extra-light" components (Hopkins et al. 2009a; Kormendy et al. 2009) that are effectively small-scale disks. The gas that remains at large radii augments any pre-existing large scale disk that has not been violently relaxed in the merger. The overall single Sérsic index $n$ of such remnants are typically $2 \lesssim n \lesssim 4$ 
(Hopkins et al. 2009a; Naab et al. 2006; Naab \& Trujillo 2006). The merger remnants made in hydrodynamic simulations with gas fractions roughly consistent with those in the local Universe produce ellipticals that look remarkably like the rotating/disky ellipticals and classical bulges in the nearby Universe. The subsequent accretion of gas from the halo, cold streams, and minor mergers can further build large-scale stellar disks, whose size depends on the specific angular momentum of the accreted gas (Steinmetz \& Navarro 200).

Recent simulations (Bournaud, Elmegreen, \& Elmegreen 2007; Elmegreen et al. 2009) have suggested an alternate bulge formation mode that is a gentler variant of the merger picture. Classical bulges with high Sérsic index $n \simeq 2-4$ result from violent disk instabilities in high-redshift galaxies when a few massive star-forming clumps migrate to the center of the disk and merge. We do not further distinguish between the classical bulges built by major mergers versus violent disk instabilities.

Getting a census of dynamically hot bulges/ellipticals and dynamically cold, flattened disk-dominated components in galaxies provides a powerful way of evaluating the importance of violent stellar processes, such as violent relaxation, which build bulges/ellipticals during major mergers, and possibly minor mergers, versus disks built by dissipative settling of gas accreted through cold streams, halo accretion, and gas-rich mergers. Ideally, such a census would identify stellar disks on different scales, ranging from outer disks of galaxies to disk-dominated central components (often called disky pseudobulges (e.g., Kormendy \& Kennicutt 2004) to probe processes involving gas of different angular momentum.

Numerous observational efforts have been undertaken to derive such a census among galaxies in the field environment. Photometric studies (e.g., Kormendy 1993a; Graham 2001; Balcells et al. 2003, 2007b; Laurikainen et al. 2007; Graham \& Worley 2008; Fisher \& Drory 
2008; Weinzirl et al. 2009; Gadotti 2009; Kormendy et al. 2010) have dissected field galaxies into outer stellar disks and different types of central bulges (classical, disky/boxy pseudobulges see Section 3.3.1 for details) associated with different Sérsic index, and compiled the stellar bulge-to-total ratio $(B / T)^{2}$ of spirals and S0s. It is found that low- $B / T$ and bulgeless galaxies are common in the field at low redshifts, both among low-mass or late-type galaxies (Böker et al. 2002; Kautsch et al. 2006; Barazza et al. 2007, 2008) and among high-mass spirals or early-type spirals (Kormendy 1993a; Balcells et al. 2003, 2007b; Laurikainen et al. 2007; Graham \& Worley 2008; Weinzirl et al. 2009; Gadotti 2009; Kormendy et al. 2010). Balcells et al (2003) highlighted the paucity of $r^{1 / 4}$ profiles in the bulges of early-type disk galaxies. Working on a bigger sample, Weinzirl et al. (2009) report that the majority $(66.4 \pm 4.4 \%)$ of massive $\left(M_{\star} \geq 10^{10} M_{\odot}\right)$ field spirals have bulges with low $B / T$ $(B / T \leq 0.2)$ and low Sérsic index $(n \leq 2)$.

These empirical results can be used to test models of the assembly history of field galaxies. For instance, Weinzirl et al. (2009) find that the results reported above are consistent with hierarchical models of Khochfar \& Silk (2006b) and Hopkins et al. (2009a), which predict that most $(\sim 85 \%)$ massive field spirals have had no major merger since $z=2$. In these models, the stellar $B / T$ is $\sim 1$ at the epoch $z \sim 2$ of the last major merger, but falls to below 0.2 by $z \sim 0$ because the outer disk of these field galaxies grows faster than the bulge, through gas accretion from the halo, cold streams, and minor mergers. However, no theoretical model yet published can make completely bulgeless galaxies with $B / T$ exactly zero. The pure-disk-galaxy problem still remains the biggest unsolved problem in galaxy formation (Kormendy et al. 2010; Peebles \& Nusser 2010; Fisher \& Drory 2011).

\footnotetext{
${ }^{2}$ In this paper, we implicitly refer to $B / T$ as a stellar mass ratio unless otherwise indicated.
} 
It is important to extend such studies from the field environment to rich clusters where the assembly history of galaxies is expected to be quite different. Hierarchical models predict differences in galaxy merger history as a function of galaxy mass, environment, and redshift (Cole et al. 2000; Khochfar \& Burkert 2001). Furthermore, cluster-specific physical processes, such as ram-pressure stripping (Gunn \& Gott 1972; Fujita \& Nagashima 1999), galaxy harassment (Barnes \& Hernquist 1991; Moore et al. 1996, 1998, 1999; Hashimoto et al. 1998; Gnedin 2003), and strangulation (Larson et al. 1980), can alter SF history, and galaxy stellar structure (disks, bulges, bars).

Efforts to establish accurate demographics of galaxy components in clusters are ongoing. In the nearby Virgo cluster, Kormendy et al. (2009) find more than $2 / 3$ of the stellar mass is in classical bulges/ellipticals. Furthermore, there is clear evidence for ongoing environmental effects in Virgo. Some key results are summarized here, but see Kormendy \& Bender (2012) for a more comprehensive review. Spiral galaxies near the center of Virgo show $\mathrm{HI}$ tails interpreted as being stripped by the pervasive hot X-ray-emitting gas (Kenney et al. 2004, 2008; Chung et al. 2007, 2009), which makes sense in light of spirals near the center of Virgo being more HI depeleted than galaxies at the outskirts of the cluster (Cayette et al. 1990, 1994; Chung et al. 2009). Apart from ram-pressure stripping, galaxy harassment appears to have caused dynamical heating and tidal stripping. Kormendy \& Bender (2012) find that many unbarred galaxies have Gaussian $(n \sim 0.5)$ profiles, suggestive of dynamical heating, and they further find transition objects between S0s and spheroidals that look just like the expected end products of dynamical heating. Finally, deep imaging and by Mihos et al. $(2005,2009)$ reveal intracluster light from stars that were freed by gravitational stripping. 
Yet Virgo, with a central projected number density of $360 \mathrm{Mpc}^{-3}$, (Binggeli et al. 1987), is not very rich compared with more typical clusters such as Abell 901/902, where the central number is $1000-1600 \mathrm{Mpc}^{-3}$ (e.g., Heiderman et al. 2009). The Coma cluster at $z=0.02(D=100 \mathrm{Mpc})$ has a central number density $10,000 \mathrm{Mpc}^{-3}$ (The \& White 1986) and is the densest cluster in the local universe. However, ground-based data do not provide high enough resolution $(0,5=250 \mathrm{pc})$ for accurate structural decomposition, constituting an obstacle to earlier work.

In this paper we make use of data from the Hubble Space Telescope (HST) Treasury Survey (Carter et al. 2008) of Coma which provides high-resolution (50 pc) imaging from the Advanced Camera for Surveys (ACS). Our goal is to derive the demographics of galaxy components, in particular classical bulges/ellipticals and flattened disk-dominated components (including both large-scale disks and disky pseudobulges) in the Coma cluster, and to compare the results with the field environment and to theoretical models, in order to constrain the assembly history of galaxies.

All calculations assume a flat $\Lambda \mathrm{CDM}$ cosmology with $\Omega_{\Lambda}=0.7$ and $H_{0}=73 \mathrm{~km} \mathrm{~s}^{-1} \mathrm{Mpc}^{-1}$. We use AB magnitudes throughout the paper, except where indicated otherwise.

\subsubsection{Roadmap for the Reader}

In Section 3.2 we present our mass-complete sample of cluster galaxies with stellar mass $M_{\star} \geq 10^{9} M_{\odot}$. In Section 3.3 we describe our structural decomposition strategy, which is carefully developed to overcome the potential fallacies with structural decomposition in a cluster environment. Sections 3.3.1-3.3.3 pertain to our philosophy and technique of using Sérsic index to separate low- $n$ flattened disk-dominated structures from high- $n$ dynamically 
hotter classical bulges/ellipticals. Section 3.3.4 presents our results for the single and multicomponent structural decompositions.

Readers less interested in the details of the structural analysis may skip to Section 3.4.1 where we quantitatively assign morphologies based on the structural decompositions. We also make a census (Section 3.4.2) of structures built by dissipation versus violent stellar processes typically associated with mergers, explore how stellar mass is distributed in different galaxy components (Section 3.4.3), and consider galaxy scaling relations (Section 3.4.4). In Section 3.4.5, we evaluate and discuss the effect of cluster environmental processes.

In Section 3.5 we compare our empirical results with theoretical predictions based on the Millennium cosmological simulations (Springel et al. 2005) in conjunction with baryonic physics from a semi-analytical model (SAM; Neistein \& Weinmann 2010), after first identifying Coma-like environments in the simulations. Readers not interested in the complete details about the theoretical model can skip to Sections 3.5.3 and 3.5.6.

A discussion and summary of our results appears in Section 3.6.

\subsection{Data and Sample Selection}

This study is based on the data products from the HST-ACS Coma Cluster Treasury Survey (Carter et al. 2008), which provides ACS Wide Field Camera images for 25 pointings spanning $274 \operatorname{arcmin}^{2}$ in the F475W and F814W filters. The total ACS exposure time per pointing is typically 2677 seconds in F475W and 1400 seconds in F814W. Most (19/25) pointings are located within $0.6 \mathrm{Mpc}$ of the central cD galaxy NGC 4874, and the other 6/25 pointings are between 0.9 and $1.75 \mathrm{Mpc}$ southwest of the cluster center. The FWHM 
of the ACS point-spread function (PSF) is $\sim 0^{\prime \prime} 1$ (Hoyos et al. 2011), corresponding to $\sim 50 \mathrm{pc}$ at the $100 \mathrm{Mpc}$ distance to the Coma cluster (Carter et al. 2008).

Hammer et al. (2010) discuss the images and SExtractor source catalogs for Data Release 2.1 (DR2.1). The F814W $5 \sigma$ limit for point sources is 26.8 mag (Hammer et al. 2010), and we estimate the $5 \sigma \mathrm{F} 814 \mathrm{~W}$ surface brightness limit for extended sources within a 0.7 diameter aperture to be $25.6 \mathrm{mag} / \operatorname{arcsec}^{2}$. Several of the ACS images in DR2.1 suffer from bias offsets on the inter-chip and/or inter-quadrant scale that cause difficulty in removing the sky background. We use the updated ACS images reprocessed to reduce the impact of this issue. The DR2.1 images are used when this is issue is not present.

\subsubsection{Selection of Bright Cluster Members}

We select our sample based on the eyeball catalog of N. Trentham et al. (in preparation), with updates from Marinova et al. (2012). This catalog provides visually determined morphologies and cluster membership status for 490 galaxies with an apparent magnitude F814W $\leq 24$ mag. Morphology classifications for the brightest galaxies come from RC3 (de Vaucouleurs et al. 1991) while the faintest galaxies are identified as either dwarf irregular or dwarf elliptical. For intermediate galaxies $(\mathrm{F} 814 \mathrm{~W} \sim 19)$, the classifications are estimates based on optical morphology.

Cluster membership is ranked from 0 to 4 following the method of Trentham \& Tully (2002). Membership class 0 means the galaxy is a spectroscopically confirmed cluster member. The subset of spectroscopically confirmed cluster members was identified based on published redshifts (Colless \& Dunn 1996; Adelman-McCarthy et al. 2008; Mobasher et al. 2001; Chiboucas et al. 2010) and is approximately complete in surface brightness 
at the global half-light radius $\left(\mu_{\mathrm{e}, \mathrm{F} 814 \mathrm{~W}}\right)$ to $\sim 22.5 \mathrm{mag} / \operatorname{arcsec}^{2}$ (den Brok et al. 2011). The remaining galaxies without spectroscopic confirmation are assigned a rating of 1 (very probable cluster member), 2 (likely cluster member), 3 (plausible cluster member), or 4 (likely background object) based on a visual estimation that also considers surface brightness and morphology.

From this catalog, we define a sample S1 of 447 cluster members having F814W $\leq 24$ mag and membership rating 0-3 located within the central 0.6 Mpc of Coma, which is the projected radius probed by the central ACS pointings. To S1 we add the second central cD galaxy NGC 4889, which is not observed by the ACS data. The majority (180) of S1 galaxies have member class 0 , and 30,131, and 106 have member class 1, 2, and 3, respectively.

\subsubsection{Calculation of Stellar Masses}

Here, we calculate stellar masses based on available photometry from ground-based surveys. Stellar masses are a thorny issue due to uncertainties in mass-to-light ratios $(M / L)$ that are driven by the fact that the initial mass function (IMF) in star formation is poorly known as well as degeneracies between age and metallicity. Nonetheless, we use $g r$ photometry from SDSS DR6 (Adelman-McCarthy et al. 2008) or $B V$ photometry from the Canada-France-Hawaii Telescope (CFHT) survey of Adami et al. (2006). For all the galaxies in S1, we adopt the approach below for computing the stellar masses.

We do not use the ACS magnitudes to determine stellar mass. The ACS bands correspond to the $B$ and $I$ bands, and we are not aware of any mass-to-light ratio calibrations for $B-I$ color. We must therefore make use of alternate photometry. Hammer et al. 
(2010) recommend adopting the SDSS photometry for bright $(\mathrm{F} 814 \mathrm{~W} \leq 17)$ sources and the photometry from Adami et al. (2006) for fainter sources. While the ACS and SDSS photometry agree well at bright $(\mathrm{F} 814 \mathrm{~W} \leq 17)$ magnitudes, at fainter magnitudes there is a dispersion indicating that the SDSS flux may be underestimated. At F $814 \mathrm{~W}=18$, the offset is $\leq 0.5 \mathrm{mag}$, but at $\mathrm{F} 814 \mathrm{~W}=20$ it can be as high as 1 mag. Moreover, half (202) of the galaxies in S1 do not have SDSS photometry from DR6, and most of these sources have F814W $>18$ mag. Accordingly, for bright galaxies $(\mathrm{F} 814 \mathrm{~W} \leq 17)$, we measure stellar mass from the SDSS $g$ and $r$ AB magnitudes, while for fainter sources the stellar mass is preferentially measured from the $B$ and $V$ Vega magnitudes of Adami et al. (2006), when available; otherwise the SDSS $g r$ photometry is used.

Stellar masses are determined using the relations of Bell et al. (2003) and assuming a Kroupa et al. (1993) initial mass function (IMF). For the $g r$ photometry we use the relations

$$
\mathrm{g}_{\text {lum }}=10^{(-0.4(\mathrm{~g}-35-5.10))}
$$

and

$$
\mathrm{M}_{\star}=\mathrm{g}_{\text {lum }} \times 10^{(-0.499+1.519(\mathrm{~g}-\mathrm{r})-0.1)},
$$

where $g$ and $r$ are apparent SDSS magnitudes, 35 is the distance modulus to Coma, and 5.10 is the solar absolute magnitude in $g$-band.

Similar equations apply for the $B V$ photometry in the Vega system.

$$
\begin{gathered}
\mathrm{V}_{\text {lum }}=10^{(-0.4(\mathrm{~V}-35-4.83))} \\
\mathrm{M}_{\star}=\mathrm{V}_{\operatorname{lum}} \times 10^{(-0.628+1.305(\mathrm{~B}-\mathrm{V})-0.1)},
\end{gathered}
$$


Here $B$ and $V$ are apparent CFHT magnitudes and 4.83 (Vega mag) is the $V$-band solar magnitude. These equations were derived ${ }^{3}$ in Bell et al. (2003) using a sample of $\sim 22,700$ galaxies from the SDSS Early Data Release (Stoughton et al. 2002) distributed across 414 square degrees.

We test for consistency between the masses from the two mass calculations at bright $(\mathrm{F} 814 \mathrm{~W} \leq 17)$ magnitudes. The stellar masses from $g r$ photometry agree with masses from $B V$ photometry to within a median factor of 1.35 at intermediate and high stellar mass $\left(M_{\star} \geq 10^{9} M_{\odot}\right)$.

For the two cDs in sample S1, it is harder to derive the stellar mass for several reasons. cDs are quite different in many respects from normal galaxies. cDs have $M_{B}$ from about -22 to -25 (Vega mag) and dynamical mass-to-light ratio $M_{d y n} / L_{B}>100$ (Schneider 2006), while ellipticals have $M_{B}$ from about -15 to -23 (Vega mag) and $M_{d y n} / L_{B}>10-100$ (Schneider 2006). The stellar $M / L$ ratio is also uncertain for cDs as most of the light of a $\mathrm{cD}$ is in an outer envelope made of intracluster light and galaxy debris. Another problem is that even if one knew the correct stellar $M / L$ ratio, it is likely that SDSS photometry is missing light from extended low surface brightness envelope.

Given all these factors, it is likely that Equations 1 and 2, which are typically used to convert color to $M_{\star}$ for normal representative galaxies, are underestimating the $M / L$ ratios and stellar masses of the $\mathrm{cDs}$, so that the adopted stellar masses for the $\mathrm{cDs}$ $\left(M_{\star} \sim 3-4 \times 10^{11} M_{\odot}\right)$ are lower limits. Due to the uncertain stellar masses and nature of the $\mathrm{cDs}$, we, wherever possible, do not compare on the same footing the $\mathrm{cD}$ systems with

\footnotetext{
${ }^{3}$ The Kroupa IMF offset term reported as -0.15 in Bell et al. 2003 was calculated assuming unrealistic conditions (Bell, E., private communication). The correct value is -0.1 and is used in Borch et al. (2006).
} 
the normal massive galaxy population of $\mathrm{E}, \mathrm{S} 0$, and spirals.

\subsubsection{Selection of Final Sample of Massive Galaxies}

Figure 3.1 shows the absolute F814W magnitudes, stellar masses, and $g-r$ colors for sample S1. In this paper, we focus on massive $\left(M_{\star} \geq 10^{9} M_{\odot}\right)$ galaxies. Our rationale is that we are specifically interested in understanding the evolution of the most massive cluster galaxies through comparisons with model clusters (Section 3.5) which show mass incompleteness at galaxy stellar masses $M_{\star}<10^{9} M_{\odot}$. Figure 3.2 shows the distribution of stellar mass versus visually assigned Hubble type, which are either taken from RC3 (de Vaucouleurs et al. 1991) for the brightest galaxies or estimated from optical morphology

(Section 3.2.1). The mass cut $M_{\star} \geq 10^{9} M_{\odot}$ effectively removes most dwarf/irregular and very low surface brightness (VLSB) galaxies, and afterward we are left with 71 galaxies that consist primarily of E, S0, and spiral galaxies. Two of these galaxies are more than halfway cutoff the ACS detector, and we ignore these sources. Our working sample S2, consists of these 69 galaxies inside the central $0.6 \mathrm{Mpc}$ with stellar mass $M_{\star} \geq 10^{9} M_{\odot}$.

\subsection{Method and Analysis}

\subsubsection{Overview of Structural Decomposition Philosophy}

As outlined in Section 3.1, galaxy bulges and stellar disks hold information on galaxy assembly history. A key step in comparing the structure of galaxies across different environments is to have a consistent way to identify galaxies with an outer disk and their central bulges in both field and clusters. In this section, we discuss how our approach to structural decomposition attempts to do this.

The main galaxy structures (bulges, disks, and bars) are represented by generalized 
ellipses whose radial light distributions are described by the Sérsic profile

$$
I(r)=I_{e} \exp \left(-b_{n}\left[\left(\frac{r}{r_{e}}\right)^{1 / n}-1\right]\right),
$$

where $I_{e}$ is the surface brightness at the effective radius $r_{\mathrm{e}}$ and $b_{n}{ }^{4}$ is a constant that depends on Sérsic index $n$. Models consisting of 1-3 generalized ellipses are fit to each galaxy with GALFIT (Peng et al. 2002), which converges on the best-fit model by minimizing the $\chi^{2}$ residuals between the input image and output model image (e.g., Weinzirl et al. 2009). In a model with one or more Sérsic profiles, there is expected to be coupling between the free parameters, particularly $r_{\mathrm{e}}$ and $n$, although most previous studies have generally ignored this effect. Weinzirl et al. (2009) explores the issue of parameter coupling for barred and unbarred spiral galaxies.

The overall goal in this work is to separate galaxy components into groups of diskdominated structures versus classical bulges/ellipticals. Disk-dominated components include large-scale and small-scale inner disks (called disky pseudobulges), and such disks can contain embedded non-axisymmetric structures (spiral arms, bars, ovals/lenses, and rings). Large-scale disks form as gas with angular momentum (acquired through cold streams, halo accretion, or gas-rich mergers) loses energy and settles into a thin disk as it conserves angular momentum. Inner small-scale disks arise due to gas inflows into the inner kiloparsec triggered by internal secular processes such as bar-driven gas inflows (Kormendy 1993a; Kormendy \& Kennicutt 2004; Jogee, Scoville, \& Kenney 2005) or by external processes such as tidal interactions and minor mergers that drive gas inflow by inducing bars and exerting torques (Hernquist \& Mihos 1995).

\footnotetext{
${ }^{4}$ The precise values of $b_{n}$ are given from the roots of the equation $\Gamma(2 n)-2 \gamma\left(2 n, b_{n}\right)=0$, where $\Gamma$ is the gamma function and $\gamma$ is the incomplete gamma function.
} 
We adopt the working assumption that in intermediate and high-mass $\left(M_{\star} \geq 10^{9}\right.$ $\left.M_{\odot}\right)$ galaxies, a low Sérsic index $n$ below a threshold value $n \leq n_{\text {disk_max }}$ corresponds to a dynamically cold disk-dominated structure. Note we specify "disk-dominated" rather than "pure disk" as we refer to barred disks and thick disks. This approach is based on multiple lines of compelling evidence that are outlined below.

1. Freeman (1970) showed that many large-scale disks of S0 and spiral galaxies are characterized by an exponential light profile (Sérsic index $n \sim 1$ ) over 4-6 disk scalelengths. Since then, it has become standard practice in studies of galaxy structure to model the outer disk of S0s and spirals with an exponential (Sérsic $n=1$ ) profile (e.g., Kormendy 1977; Boroson 1981; Kent 1985; de Jong 1996; Baggett et al. 1998; Byun \& Freeman 1995; Allen et al. 2006; Laurikainen 2007; Gadotti 2009; Weinzirl et al. 2009). Not all disks are strictly exponential, however, as numerous studies have identified outer disks with down-bending truncations or up-bending anti-truncations (Freeman 1970; van der Kruit 1979; van der Kruit \& Searle 1981a, 1981b; de Grijs et al. 2001; Pohlen et al. 2002; Matthews \& Gallagher 1997; Erwin et al. 2005; Pohlen \& Trujillo 2006).

2. On smaller scales, flattened, rotationally supported inner disks with high $V / \sigma$ (i.e., disky pseudobulges) have been associated with low Sérsic index $n \lesssim 2$ (Kormendy 1993a; Kormendy \& Kennicutt 2004; Jogee, Scoville, \& Kenney 2005; Athanassoula 2005; Kormendy \& Fisher 2005; Fisher \& Drory 2008; Fabricius et al. 2012). This suggests $n_{\text {disk_max }}$ should be close to 2 .

Fisher \& Drory (2008) study all spiral galaxies in the Northern hemisphere that have imaging with $H S T$ and distance $<40 \mathrm{Mpc}$. Disky pseudobulges are identified visually 
based on the presence of disk signatures such as nuclear spirals, nuclear rings, nuclear bars, and nuclear patchiness. Figure 9 of Fisher \& Drory (2008) shows $\sim 90 \%$ of disky pseudobulges have bulge Sérsic index $n \lesssim 2$, while $\sim 90 \%$ of classical bulges have $n \gtrsim 2$.

Fabricius et al. (2012) explores the major-axis kinematics of 45 S0-Scd galaxies with high-resolution spectroscopy. They demonstrate, for the first time, a systematic agreement between the shape of the velocity dispersion profile and the bulge type as indicated by the Sérsic index. Low Sérsic index bulges have both increased rotational support (higher $<V^{2}>/<\sigma^{2}>$ values) and on average lower central velocity dispersions. Classical bulges have centrally peaked velocity dispersion profiles while disky pseudobulges in general have flat dispersion profiles and even at times show drops in the central velocity dispersion. This correlation holds if visual morphology is used for the bulge classification instead of the Sérsic index.

3. At high $(z \sim 2)$ redshift, where it is not yet possible to fully resolve galaxy substructures, it has become conventional to use the global Sérsic index $n \lesssim 2$ in massive galaxies to separate disk-dominated versus bulge-dominated galaxies (e.g., Ravindranath et al. 2004; van der Wel et al. 2011; Weinzirl et al. 2011). Weinzirl et al. (2011) further explore the distributions of ellipticities $(1-b / a)$ for the massive $z \sim 2$ galaxies with low $(n \leq 2)$ and high $(n>2)$ global Sérsic index. They find that galaxies with low global Sérsic index $n \leq 2$ have a long tail of high projected ellipticities and their distribution of projected ellipticities is much more similar to massive $z \sim 0$ spirals than to massive $z \sim 0$ ellipticals. One question that cannot be fully answered from Weinzirl et al. (2011) is whether the flattening is due to anisotropic velocity dispersions or rotation. 
However, the above suggests the anisotropy in elliptical galaxies cannot account for the degree of flattening in massive $z \sim 2$ galaxies with low Sérsic index.

4. In Section 3.3.4.2 we set $n_{\text {disk_max }}$ to 1.66 based on looking at the Sérsic $n$ of outer disks in those Coma galaxies that are barred and clearly harbor outer disk. Note that this approach generates a conservative lower limit on $n_{\text {disk_max. }}$. See Section 3.3.4.2 and Appendix A.3 for further details.

We assume that components with Sérsic $n>n_{\text {disk_max }}$ are puffy and dynamically hot classical bulge/elliptical components. Such bulges/ellipticals are formed by the redistribution of stars during galaxy collisions, particularly major mergers but also possibly minor mergers. Traditionally, classical bulges/ellipticals were considered to have a Sérsic index $n \sim 4$ and were associated with complete violent relaxation in equal mass (mass ratio 1:1), dry major mergers. However, more generally, the latest hydrodynamical simulations (e.g., Robertson et al. 2006; Naab et al. 2006; Governato et al. 2007; Hopkins et al. 2009a, b) show that in major mergers with gas fractions in the range $\sim 20-80 \%$, most stellar remnants have Sérsic $2 \lesssim n \lesssim 4$, although lower and higher values are rare but possible.

Minor mergers do have an effect on the Sérsic index of bulges. $N$-body simulations show that minor mergers consistently raise the bulge Sérsic index. Aguerri et al. (2001) show the process is very efficient for dense satellites. Starting with an exponential bulge, roughly a $25 \%$ increase in $B / D$ is associated with an increase $\Delta n=1$, whereas gentler increases result from lower-density satellites, leaving bulges with $n \lesssim 2$ (Eliche-Moral et al. 2006; Naab \& Trujillo 2006). The effect is, as expected, cumulative (Aguerri et al. 2001; Bournaud, Jog, \& Combes 2007; Naab et al 2009; Hilz et al. 2012). 
In Section 3.5, we try to connect this census of stellar mass in components with $n \leq$ $n_{\text {disk_max }}$ and $n>n_{\text {disk_max }}$ in Coma cluster galaxies with the predictions of a theoretical model for the stellar mass in dynamically cold flattened components versus in dynamically hot components built by major and mergers.

\subsubsection{Uncertainties Due to Dwarf Galaxies}

The term "dwarf elliptical" (dE) is often used to describe elliptical galaxies fainter than $M_{B}=-18$ (Sandage \& Binggeli 1984). Local Group dwarf galaxies are not particularly flattened with $b / a \sim 0.7$, yet they have Sérsic $n \sim 0.5-1$ (e.g., Caldwell 1999; Jerjen et

al. 2000). There has been much debate over the true nature of such galaxies. Early studies (Wirth \& Gallagher 1984; Sandage et al. 1985; Binggeli et al. 1988) suggested elliptical and $\mathrm{dE}$ galaxies may follow separate luminosity functions, and therefore represent different families of galaxies, that happen to overlap for $-15 \gtrsim M_{B} \gtrsim-18$. A central question is what implications do such objects have on our interpretation of galaxy structure based on Sérsic index? Here, we discuss the different interpretations of dE galaxies and quantify many such systems are in sample S2.

Several papers (e.g., Kormendy 1985; Kormendy et al. 2009; Kormendy \& Bender 2012) demonstrate that elliptical and dE galaxies separate cleanly in global galaxy parameter correlations for $r_{\mathrm{e}}$, surface brightness $\mu_{\mathrm{e}}$ at $r_{\mathrm{e}}$, and luminosity. The differences in structural parameter correlations between elliptical and $\mathrm{dE}$ galaxies are interpreted to mean separate formation mechanisms and that elliptical and $\mathrm{dE}$ galaxies are fundamentally different classes of objects. Elliptical galaxies form in major galaxy mergers while dE galaxies are late-type galaxies stripped of gas by non-merger processes. Kormendy et al. (2009) and Kormendy 
\& Bender (2012) advocate that such defunct late types should be called spheroidals (Sph) rather than dwarf ellipticals to avoid association with elliptical and "true" dwarf elliptical galaxies (i.e., galaxies that are actually miniature versions of ellipticals, like M32).

An alternate perspective is that dEs and ellipticals represent a continuous population (e.g., Sandage \& Binggeli 1984; Graham \& Guzmán 2003). The reasoning is that dE galaxies form a linear sequence with giant ellipticals for parameters including concentration (Sérsic index), central surface brightness $\left(\mu_{0}\right)$, luminosity, color, metallicity, age, and dynamics (Graham \& Guzmán 2003; Graham 2013 and references therein). Graham \& Guzmán (2003) argue that the curved or dichotomous appearance of global parameter correlations involving $r_{\mathrm{e}}$, surface surface brightness $\mu_{\mathrm{e}}$ at $r_{\mathrm{e}}$, or average surface brightness $\left\langle\mu_{\mathrm{e}}\right\rangle$ within $r_{\mathrm{e}}$ is a consequence of the well-established linear correlations between luminosity-Sérsic-index and luminosity- $\mu_{0}$ correlations that are continous for $\mathrm{dE}$ and ellipticals.

It is beyond the scope of this paper to comment further on the interpretation of $\mathrm{dE}$ /spheroidal galaxies in Coma (but see Hammer et al. 2012). Instead, we note that Figure 3.2 demonstrates the incidence of ambiguous $\mathrm{dE} /$ spheroidal galaxies in our sample is nominal and represents a small percentage $(4 / 69,6 \%)$ of the massive $\left(M_{\star} \geq 10^{9} M_{\odot}\right)$ galaxies in sample S2. Thus, the conclusions drawn in the remainder of this paper are not significantly influenced by the highly debated nature of $\mathrm{dE} /$ spheroidal galaxies.

\subsubsection{Special Steps for Structural Decomposition in Cluster Environments}

In this paper, we take several important steps to avoid potential fallacies with structural decomposition in a cluster environment. This approach allows us to identify low$n$ flattened disk-dominated structures (including both outer disks and disky pseudobulges) 
and high-n classical bulges/ellipticals. Disks represent the stellar mass associated with the dissipative settling of gas accreted through cold streams, halo accretion, and gas-rich mergers, while the high- $n$ classical bulges/ellipticals are likely built by redistribution of stars during major mergers and possibly also minor mergers (see Sections 3.1 and 3.3.1).

1. For a mass-complete sample of high-to-intermediate mass $\left(M_{\star} \geq 10^{9} M_{\odot}\right)$ galaxies, we use deep, high-resolution ( $0{ }^{\prime \prime} 1$ or $50 \mathrm{pc}$ ) images of Coma from HST ACS, which allow accurate structural decomposition.

2. We take careful steps to distinguish large-scale outer disks from non-disks (Section 3.3.1, Section 3.3.4.2, and Appendix A.3). In the field, where spirals (rather than E+S0 galaxies) dominate the bright galaxy population, it is fairly straightforward to identify the outer disk and central bulge. Since field spirals have gas and ongoing SF, the outer disk is readily identified from disk features such as spiral arms delineated by young stars, rings of SF, and distinctive dust lanes associated with stellar bars.

In clusters, however, E/S0 galaxies dominate the bright/massive galaxy population, while dwarf elliptical galaxies dominate the faint $M_{B}>-18$ (Sandage \& Binggeli 1984) and low mass galaxy population. Disk components (outer disks, inner disks, disky pseudobulges) are more difficult to identify in $\mathrm{S} 0$ and $\mathrm{dE}$ galaxies because they have little gas and ongoing SF, and are therefore devoid of the traditional tell-tale signposts of disks, such as young spiral arms, rings of SF and gas/dust lanes associated with stellar bars.

3. Once the outer disk is identified, we adopt the standard practice of considering the bulge as the central galaxy component that harbors the light in excess of the outer 
disk (e.g., Laurikainen et al. 2007, 2009, 2010; Fisher \& Drory 2008; Gadotti 2009; Weinzirl et al. 2009). Such an approach assumes that spiral galaxies contain an outer disk and a central bulge, which falls in three main categories called classical bulges, disky pseudobulges (Kormendy 1993a; Kormendy \& Kennicutt 2004; Jogee, Scoville, \& Kenney 2005; Athanassoula 2005; Kormendy \& Fisher 2005; Kormendy \& Fisher 2005; Fisher \& Drory 2008), and boxy pseudobulges (Combes \& Sanders 1981; Combes et al. 1990; Pfenniger \& Norman 1990; Bureau \& Athanassoula 2005; Athanassoula 2005; Martinez-Valpuesta et al. 2006). Some bulges are composite mixtures of the first two classes ((Kormendy \& Barentine 2010; Barentine \& Kormendy 2012).

4. During structural decomposition, we do not make a large number of a priori assumptions on the physical nature or shape of galaxy structures. This is accomplished by fitting all structures with a generalized Sérsic profile where the Sérsic index is a free parameters (Section 3.3.3). In particular, we do not require that outer disks be exponential to accommodate non-exponential disk structures (e.g., disks with down-bending truncations or up-bending anti-truncations Freeman 1970; van der Kruit 1979; van der Kruit \& Searle 1981a, 1981b; de Grijs et al. 2001; Pohlen et al. 2002; Matthews \& Gallagher 1997; Erwin et al. 2005; Pohlen \& Trujillo 2006; Maltby et al. 2012) that are rotationally supported.

5. We also model stellar bars, ovals/lenses, and nuclear point sources when needed. Accounting for such non-axisymmetric structures in $2 D$ decomposition is critical for obtaining a reliable characterization of the bulge (e.g., Laurikainen et al. 2005, 2007; Weinzirl et al. 2009). Without the bar, the bulge-to-total light ratio is often overestimated. Nuclear clusters are found in 58\% of early-type field disk galaxies (Balcells et 
al. 2007a), and failing to model such structures can severely overestimate the bulge Sérsic index (Balcells et al. 2003; Weinzirl et al. 2009).

\subsubsection{Structural Decomposition Scheme and Galaxy Classification System}

We characterize the structure of galaxies in sample S1 with structural decomposition. We fit galaxies with one, two, or three Sérsic profiles plus a nuclear point source, when needed. An overview of the decomposition scheme and decision sequence is shown in Figures 3.3 and 3.4 and is also outlined below. Additional details are given in the next subsection 3.3.4.

- Stage 1 (Single Sérsic fit with nuclear point source if needed): The single Sérsic model is adopted if either the galaxy does not show any coherent structures (e.g., inner/outer disk, bars, bulges, rings, or spiral arms) indicating the need for additional Sérsic components, or, alternatively, if the galaxy has a core - a light profile that deviates downward from the inward extrapolation of the Sérsic profile (see Appendix A.2). Such galaxies are interpreted as photometric ellipticals if the single Sérsic index is above a threshold value $n_{\text {disk_max }}$ associated with disks (Section 3.3.1, 3.3.4.2, and Appendix A.3); otherwise they are considered photometric disks. Three galaxies show convincing evidence for being cores, and these are luminous objects with high single Sérsic $n>n_{\text {disk_max }}$ (see Section 3.3.4.2, Table 3.1, Appendix A.2). See Section 3.3.4.1 for additional details on the single Sérsic fits.

- Stage 2 (Double Sérsic model with nuclear point source if needed): Galaxies showing coherent structure in the Stage 1 residuals are subjected to a two-component Sérsic + Sérsic fit, with nuclear point source if needed (see Figure 3.4). This two-component 
model is intended to model the inner $(\mathrm{C} 1)$ and outer $(\mathrm{C} 2)$ galaxy structures.

There are two possible outcomes. a) If the outer component $\mathrm{C} 2$ is an outer disk based on having Sérsic index $n \leq n_{\text {disk_max }}$, then the galaxy is considered a spiral or S0 with an outer disk having a photometric bulge and, in some cases, a large-scale bar. b) If the outer component $\mathrm{C} 2$ does not meet our definition of an outer disk, then the galaxy is considered a photometric elliptical having an outer component $\mathrm{C} 2$ with $n>n_{\text {disk_max }}$ and an inner component $\mathrm{C} 1$ of any $n$. See Section 3.3.4 for details.

- Stage 3 (Triple Sérsic model with nuclear point source if needed): Case a) in Stage 2 identifies spiral and S0 galaxies with an outer disk. These galaxies are further processed as follows: a) If there is evidence for a large-scale bar (see Section 3.3.4.2), then a triple Sérsic profile is fitted in Stage 3 for the photometric bulge, disk, and bar. The photometric bulge is defined as the excess light above the outer disk and bar. b) Otherwise, the galaxy is considered as unbarred and the double Sérsic fit for a photometric bulge and disk is adopted. In this case, the bulge is defined as the excess light above the outer disk. In both cases (a) and (b), it is important to note that the photometric bulge is allowed to have any Sérsic index $n$, thus allowing for structures with $n \leq n_{\text {disk_max }}$ (which are effectively dynamically cold central disks or so-called disky/pseudo bulges) and structures with $n>n_{\text {disk_max }}$ (which are more akin to classical bulges/ellipticals).

The decomposition scheme outlined above and in Figures 3.3 and 3.4 leads naturally to the galaxy classification system outlined in Figure 3.5, where there are five main galaxy types, G1 to G5. Systems best fitted by single Sérsic models (plus a nuclear point source if 
present) represent galaxies of type G1 and G2. Systems best fitted by two or three Sérsic profiles (plus a nuclear point source if present) represent galaxies of type G3 to G5.

1. G1: Photometric disk with $n \leq n_{\text {disk_max }}$ (plus a nuclear point source if present).

2. G2: Photometric elliptical with $n>n_{\text {disk_max }}$ (plus a nuclear point source if present).

3. G3: Unbarred S0 or spiral having an outer disk with $n \leq n_{\text {disk_max }}$ and an inner photometric bulge of any $n$ (plus a nuclear point source if present).

4. G4: Barred S0 or spiral having an outer disk with $n \leq n_{\text {disk_max }}$, a bar, and an inner photometric bulge of any $n$ (plus a nuclear point source if present).

5. G5: Photometric elliptical having an outer component with $n>n_{\text {disk_max }}$ and an inner component of any $n$.

This galaxy classification scheme has multiple advantages. Firstly, it allows us to identify low-n disk-dominated structures within galaxies, both on large scales and in the central regions, in the form of outer disks with $n \leq n_{\text {disk_max }}$ in spirals and S0s, photometric bulges with $n \leq n_{\text {disk_max }}$ in spirals and S0s (representing disky pseudobulges), and inner disks within ellipticals represented by a component C1 having $n \leq n_{\text {disk_max }}$. Furthermore, it allows a census of galaxy components with $n>n_{\text {disk_max }}$ more akin to classical bulges/ellipticals.

For readers interested in all the technical details, we provide a detailed description of the decomposition in Section 3.3.4. The more general reader may want to proceed directly to Section 3.4, where we present the outcomes from the above decomposition and associated galaxy classification schemes. 


\subsubsection{Details of Structural Decomposition}

\subsubsection{Single Sérsic Fits}

We first fit all galaxies with a single Sérsic profile before attempting the multicomponent fits. The results are listed in Table 3.2. This step is useful for measuring the total luminosity of a galaxy as well as measuring the centroid (Weinzirl et al. 2009). The Sérsic profile has seven free parameters: centroid, luminosity, half-light radius $r_{\mathrm{e}}$, Sérsic index $n$, axis ratio, position angle, and diskiness/boxiness. We fix the diskiness/boxiness so that the fitted structures are perfect ellipses. We estimate the other six parameters based on the parameters in SExtractor and allow them to optimize in the fit. The detailed image preparation and inputs for the proper operation of GALFIT are described in Appendix A.1.

Figure 3.6 compares our results for the single Sérsic fits (with no point source) with those of Hoyos et al. (2011), who also perform single Sérsic fits with GALFIT and GIM2D using Coma ACS Treasury Survey data. We compare our results with those reported by Hoyos et al. (2011) for GALFIT. Note that the galaxies in our sample requiring one Sérsic profile are distinguished in Figure 3.6. With the exception of NGC 4874 (denoted as COMAi125935.698p275733.36 in the Coma ACS Treasury Survey catalogs), our results for these sources requiring one Sérsic profile well match those derived by Hoyos et al. (2011). For NGC 4874, we measure the $r_{\mathrm{e}}$ and $n$ of NGC 4874 to be $17.3 \mathrm{kpc}$ and 3.05 , respectively, while Hoyos et al. (2011) measure $r_{\mathrm{e}}$ and $n$ to be $3.2 \mathrm{kpc}$ and 1.3 .

For sources requiring more than one Sérsic profile, our single Sérsic magnitudes agree well in general with those of Hoyos et al. (2011), except for one case (COMAi13051.149p28249.90) where Hoyos et al. (2011) underestimate the magitude by $\sim 5.5$ mag. There are also outliers in both $r_{\mathrm{e}}$ and $n$. In 9 (5) instances (including COMAi13051.149p28249.90), the difference 
in $r_{\mathrm{e}}(n)$ exceeds a factor of 1.5 .

There are two key differences in our fitting methodology (see Appendix A.1) compared with Hoyos et al. (2011). Most importantly, we entirely unmask the target galaxy and background in the segmentation-based masks so that GALFIT fits to pixels beyond what SExtractor associates with each galaxy. Hoyos et al. (2011) confine a galaxy to a customized mask generated based on the output of SExtractor. This approach misses a finite fraction of the flux in the target galaxy corresponding to a 0.1 mag offset in the worst case. This may explain why in Figure 3.6 we measure brighter magnitudes and larger $r_{\mathrm{e}}$ for

more extended galaxies, where SExtractor does not detect all of the light in the galaxy. Second, we measure and fix the background sky while Hoyos et al. (2011) keep the sky as a free parameter. Allowing the sky background to freely vary in our fits fails to account for most of the scatter between our results and those of Hoyos et al. (2011). Rather, the disagreement appears to mainly be the result of differences in image masking.

\subsubsection{Multi-Component Fits}

For the Stage 2 Sérsic + Sérsic fits, we model the 'inner' and 'outer' components (C1 and C2) with Sérsic profiles that can represent physically different components. The 'inner' component, for example, may represent a high- $n$ classical bulge built by major mergers, and perhaps by minor mergers. A second possibility is that the inner component represents a low Sérsic index structure such as a disky pseudobulge) or inner bar. Such low $n$ components are suspected to be supported by rotation (e.g. Kormendy \& Kennicutt 2004 Fig. 17; Jogee, Scoville, \& Kenney 2005; Fabricius et al. 2012).

The 'outer' component is likewise a generalized structure. By placing no restriction 
on the Sérsic index of the outer component, we allow for galaxies whose outer components are not necessarily disks. For instance, some ellipticals might have an inner accreted or extra-light (Hopkins et al. 2009a; Kormendy et al. 2009) component in the central region, and an outer component whose light profiles can be fitted by an SB profile with $n \gtrsim 2$. Second, by not fixing Sérsic index to $n=1$ for the outer component, we allow for outer disks with non-exponential surface brightness profiles such as disk truncations or disk antitruncations (see Section 3.3.2).

Sensible initial guess parameters for Stage 2 are determined from a combination of the data image, Stage 1 model, and Stage 1 residuals. Guesses for the inner Sérsic component (C1) are usually based on the Stage 1 model. The centroid of the Sérsic components (and nuclear point source if present) are fixed to the best-fit centroid from the single Sérsic model. During the fits, we allow $r_{\mathrm{e}}$ and $n$ to vary for the inner and outer components without a priori fixing the nature of these components.

With one exception, the $\chi^{2}$ in Stage 2 is always lower compared with $\chi^{2}$ in Stage 1 due to the extra Sérsic component. While the rare increase in $\chi^{2}$ from Stage 1 to Stage 2 is an indication the latter model is not reliable, the almost universal decrease in $\chi^{2}$ is not necessarily a sign that the Stage 2 fit is meaningful because, in principle, such a decrease in $\chi^{2}$ could be driven by the extra free model parameters. We consider a Stage 2 multicomponent model to be superior to the Stage 1 fit if i) $\chi^{2}$ drops, ii) the Stage 2 model parameters are well behaved (i.e., not unphysically large or small), and iii) the Stage 2 residuals are deemed by visual inspection to show a reduction in coherent structure relative to the Stage 1 residuals.

Figure 3.7 provides examples where a single Sérsic model fails to model the entire 
galaxy well and leaves behind coherent structure in the residuals. Such coherent structure is indicative of additional components such as compact central structures, rings, annuli and extended components, and bars/ovals. We illustrate in Figures 3.8 and 3.9 how some of these examples are best fitted by models with multiple Sérsic components.

If a galaxy does not require a Stage 2 model, or if the Stage 2 model fails to meet the above criteria, then the galaxy is described by a single Sérsic profile + point source, if present. Six galaxies are best represented by Stage 1. Two (COMAi13017.683p275718.93 and COMAi13018.093p275723.59) cannot be fit with multiple Sérsic models because they are interacting. In the third case, (COMAi125931.103p275718.12), the $\chi^{2}$ increases from Stage 1 to Stage 2. The final three cases (NGC 4874, NGC 4889, and COMAi125909.468p28227.35) show evidence of a core (see Appendix A.2).

Galaxies for which the Stage 2 model is deemed an improvement are interpreted as follows. Since the outer component C2 could represent a disk, we must specify criteria for identifying an outer disk. The outer component $\mathrm{C} 2$ is a disk if it satisfies at least one of the following. i) The galaxy is highly inclined such that $\mathrm{C} 2$ has a low axis ratio $b / a \leq 0.25$ that is below the axis ratios found for ellipticals. ii) The galaxy is moderately inclined and C2 shows disk signatures (e.g., bars, rings, or spiral arms) in the data images and/or Stage 2 residuals. iii) For moderately inclined galaxies without disk features that do not satisfy (i) or (ii), we require Sérsic $n$ be less than a threshold value $n_{\text {disk_max }}$.

Theoretical considerations show that pure disks have $n=1$, suggesting the threshold should be $n \sim 1$. However, real galaxy disks are not fitted perfectly by Sérsic profiles. We determine the value empirically from the maximum disk Sérsic index in galaxies satisfying (i) and (ii). Highly inclined disks show a range in Sérsic index of 0.48-0.86. Moderately 
inclined galaxies identified as having spiral arms but no bar have outer disks with Sérsic index 0.84-1.20.

In order to accurately model the outer disk of moderately inclined barred galaxies, a triple Sérsic profile (see below) is required. After taking this extra step, the outer disk Sérsic index among moderately inclined barred galaxies is $0.25-1.66$. The maximum Sérsic index among outer disks in galaxies satisfying requirement (i) and (ii) is 1.66, and we therefore set $n_{\text {disk_max }}$ to this value. Thus, outer disks span the range 0.25-1.66 in Sérsic index and have a median $n$ of 0.84 . Figure 3.10 shows the galaxy (COMAi125950.105p275529.44) on which we base our measurement of $n_{\text {disk_max }}$. Appendix A.3 discusses the uncertainties in the adopted value of $n_{\text {disk_max }}$.

Galaxies that satisfy any of requirements (i), (ii), or (iii) are deemed to have an outer disk. As discussed in Section 3.3.3 and Figure 3.4, these galaxies are morphologically either S0s or spirals. Figure 3.11 shows the distribution of $n$ for outer disks. Strikingly, the majority of outer disks have light profiles that are shallower than pure exponential disks with $n=1$; most $(67.3 \pm 7.9 \%)$ of outer disks have Sérsic index $n<1$ and many $(19.2 \pm 12.5 \%)$ have $n<0.5$. We further discuss these low- $n$ disks in Section 3.4.5. Galaxies without an outer disk are considered photometric ellipticals.

We test all galaxies having an outer disk for the presence of a large-scale bar/oval in Stage 3 by fitting a triple Sérsic profile + point source, if present. Bars/ovalsare modeled with an elongated, low Sérsic index $(n \sim 0.5)$ profile (Peng et al. 2002; Weinzirl et al. 2009). In the remainder of the text, we do not distinguish between bars and ovals, and we use "bar" to desribe both.

The initial guesses for the three-component models come from the best Stage 2 
model combined with visual inspection. The Sérsic index for the bar is initially guessed to be 0.5 , and the shape and position angle of the bar are visually estimated using the data image or the residuals of the Stage 2 fit. When selecting between the Stage 2 and Stage 3 fits, we applied the same constraints described above for the behavior of $\chi^{2}$. An additional complication is that in galaxies with unbarred outer disks, GALFIT may fit a 'bar' to any existing spiral arms, rings, or clumpy disk structure. Stage 3 fits in these cases could be discarded by noting the resulting discrepancies in appearance between the galaxy images and the Stage 3 model images. Figure 3.9 shows examples of two disk galaxies where adding the third Sérsic component removes the bar signature from the residuals.

Table 3.3 lists the results of using this procedure to choose the best-fit model. Table 3.4 lists the structural parameters from the best overall single or multi-component model. We find 6 objects are best-fit by Stage 1, 37 are best fit by Stage 2, and 26 are best fit by Stage 3. The number of Stage 3 fits implies the bar fraction among galaxies with an extended outer disk is $50.0 \pm 6.9 \%$.

\subsubsection{Nuclear Point Sources}

Nuclear point sources are found in galaxies of all Hubble types. The frequency of nuclear point sources is very sample dependent and is particularly sensitive to range of galaxy luminosity. HST studies of early-type galaxies (e.g., Ravindranath et al. 2001; Côté et al. 2006) have measured nucleation rates of $50 \%$ or more. Ravindranath et al. (2001) find about half of early-type (E, S0, S0/a) galaxies have nuclear point sources. Côté et al. (2006) show that the frequency of nucleation in ACS images of the Virgo cluster is at least $66 \%$ in galaxies with $M_{B} \leq-15$. Graham \& Guzmán (2003) discuss 13/15 examples 
of dwarf ellipticals in the Coma cluster showing evidence for nucleation. Balcells et al. (2007a) measure a frequency of 58\% for S0 to Sbc galaxies. Böker et al. (2002) measure the frequency of point sources to be $75 \%$ in spirals with Hubble types Scd to Sm.

Although nuclear point sources account for a small percentage $(<1 \%)$ of a galaxy's light, it is important to include them during multi-component structural decomposition. Neglecting nuclear point sources can have a significant effect on derived parameters of bulges (Balcells et al. 2003; Weinzirl et al. 2009). We assess the presence of nuclear point sources with visual inspection. If a compact light source is visible by eye in the residuals of the single Sérsic fit, the galaxy is flagged as having a potential point source. With this procedure, 48/69 galaxies in sample S2 have a potential nuclear point source.

Galaxies having a potential nuclear point source are fitted with an extra nuclear point source component in the best-fit single or multi-component model. GALFIT models the point source with the user-input PSF. More than half $(37 / 69,53.6 \pm 6.00 \%)$ of objects in sample S2 have a nuclear point source in the final, best-fit structural decomposition. Figure 3.12 shows examples of residual galaxy images with point sources.

Figure 3.13 shows the derived point source luminosities correlate with total galaxy magnitude such that more luminous point sources are found in brighter galaxies. Similar results been found in earlier work (e.g., Graham \& Guzmán 2003; Balcells et al. 2007a).

\subsubsection{4 cD Galaxies}

cD galaxies are defined by having extra light on cluster-sized $(\sim 1 \mathrm{Mpc})$ scales with respect to the outward extrapolation of the Sérsic profile fit to the inner $(\sim 100 \mathrm{kpc})$ portion of the galaxy. Such galaxies are luminous and are found in regions of high galaxy number 
density (Binney \& Merrifield 1998). Of the three cD galaxies in Coma, two (NGC 4874 and NGC 4889) lie in the central 0.6 Mpc and are therefore in our sample. The third cD (NGC 4839) lies is in the outer southwest region of Coma and is not part of this study. Definitive proof that NGC 4874 and NGC 4889 are cDs is the detection of intracluster light in Coma (Kormendy \& Bahcall 1974; Melnick et al. 1977; Thuan \& Kormendy 1977; Bernstein et al. 1995; Adami et al. 2005; Arnaboldi 2011).

The single Sérsic indices reported in Section 3.3.4 and Table 3.2 for the these cD galaxies are $n \sim 3-4.4$ because the decompositions also include the central core. The central core is a clear deviation from the inward extrapolation of the Sérsic profile that characterizes the outer galaxy structure. For this reason, masking the core regions (i.e., the central $\sim 2^{\prime \prime}$ ) is more physically motivated and would yield higher single Sérsic indices $n \gtrsim 8$. This is demonstrated in Appendix A.2 and Table 3.1. We note that both approaches (masking or not masking the core during the $2 D$ decomposition) lead us to the same conclusion that all of the $\mathrm{cD}$ light is associated with structures of $n \gg n_{\text {disk_max }}$ (Appendix A.2). Note in Table 3.4 we list the $\mathrm{cD}$ galaxies the structure parameters from the $2 \mathrm{D}$ decomposition where the core is masked.

The high $n \gg n_{\text {disk_max }}$ values in the $\mathrm{cD}$ galaxies are due to the extended wings in the Sérsic profile resulting from the extended low surface brightness envelope of the $\mathrm{cD}$. This extended envelope is likely made up of intra-cluster light and the cumulative debris from galaxies, consistent with the view that $\mathrm{cD}$ galaxies arise from repeated bouts of galactic cannibalism and tidal stripping of satellite galaxies in a cluster (Ostriker \& Tremaine 1975; Aragon-Salamanca et al. 1998; De Lucia \& Blaizot 2007). 


\subsection{Empirical Results on Galaxy Structure}

\subsubsection{Galaxy Types and Morphology-Density Relation in the Center of Coma}

We outlined in Section 3.3.3 and Figures 3.3-3.4 our structural decomposition scheme in which we model galaxies with up to three Sérsic profiles, plus a nuclear point source if present. We then used these decompositions to assign galaxies among types G1 to G5 as discussed in Section 3.3.3 and Figure 3.5. Table 3.3 lists the distribution of best-fit models for the sample of galaxies with stellar mass $M_{\star} \geq 10^{9} M_{\odot}$, and the breakdown of galaxies into classes G1 to G5. Table 3.4 lists the structural parameters from the best single or multi-component model. In summary, we fit 6,37 , and 26 galaxies with 1,2 , and 3 Sérsic profiles, respectively. In terms of galaxy types G1 to G5, we assign 1, 5, 25, 26, and 12 objects to classes G1, G2, G3, G4, and G5, respectively. Nuclear point sources are included when needed. We find nuclear point sources in just over half $(37 / 69,53.6 \pm 6.00 \%)$ of galaxies with stellar mass $M_{\star} \geq 10^{9} M_{\odot}$. As pointed out in Section 3.3.4.3, a similar high incidence of point sources in early type galaxies has been reported in other samples, including Coma and Virgo cluster galaxies (Ravindranath et al. 2001; Graham \& Guzmán 2003; Côté et al. 2006).

We next map classes G1 to G5 to more familiar Hubble types, namely $\mathrm{cD}$, photometric E, S0, and spiral. The results are shown in Table 3.3. The one object in class G1 (photometric disk) has a single Sérsic index $n \leq n_{\text {disk_max }}$ and a nuclear point source. We consider this object to be a pure disk. Since this object has no visible spiral arms in either the data image or the single Sérsic residual image, we consider it to be an S0 rather than a spiral. Objects assigned to class G2 (photometric ellipticals) have single Sérsic index $n>n_{\text {disk_max }}$ and include two known central cD galaxies, NGC 4874 and NGC 4889. We 
label these two sources separately as $\mathrm{cD}$ galaxies because they contain a disproportionately large fraction of the stellar mass. We consider the remaining three objects in G2 to be pure photometric ellipticals. Classes G3 (unbarred S0, spiral) and G4 (barred S0, spiral) represent S0 or spiral disk galaxies with a possible large-scale bar. We find five galaxies in either class G3 or G4 showing spiral arms in the data or residual images. Objects displaying such spiral structure are considered as spirals, while the remaining sources are labeled S0. The class G5 objects (photometric elliptical with inner and outer components) have an outer component with $n>n_{\text {disk_max }}$ and an inner component of any $n$. The subset of ellipticals with inner components of $n \leq n_{\text {disk_max }}$ areanalogous to the extra-light ellipticals discussed by Hopkins et al. (2009a) and Kormendy et al. (2009).

We find evidence of a strong morphology-density relation. In the central $0.6 \mathrm{Mpc}$ of the Coma cluster, there are $2 \mathrm{cDs}$ (NGC 4874 and NGC 4889), spirals are rare, and the morphology breakdown of $(\mathrm{E}+\mathrm{S} 0)$ :spirals is $(22.4 \%+70.2 \%): 7.4 \%$ by numbers and $(28.0 \%$ $+66.3 \%$ ):5.8\% by stellar mass. The total stellar mass cited here does not include the cDs as their stellar mass is quite uncertain (see Section 3.2).

In contrast to the central parts of Coma, lower-density environments are typically dominated by spirals. This is quantitatively illustrated by Table 3.5, which compares the results in Coma with the lower-density Virgo cluster and the field. McDonald et al. (2009) study a sample of 286 Virgo cluster member galaxies that is complete down to $M_{B}=16$ (Vega mag). At stellar mass $M_{\star} \geq 10^{9} M_{\odot}$, if M 87 is counted as a giant elliptical, the $(\mathrm{E}+\mathrm{S} 0)$ :spirals breakdown is $(34.1 \%+31.6 \%): 34.8 \%$ by numbers and $(59.2 \%+19.3 \%): 21.4 \%$ by stellar mass. There is evidence (Kormendy et al. 2009; Mihos et al. 2005, 2009) that M 87 has a cD halo, and after excluding M 87, the (E+S0):spirals breakdown changes 
slightly to $(33.5 \%+31.6 \%): 34.8 \%$ by numbers and $(57.2 \%+20.3 \%): 22.5 \%$ by stellar mass. In the field, the (E/S0):spiral morphology breakdown is $\sim 20 \%: 80 \%$ by number for bright galaxies (Dressler 1980).

\subsubsection{Census of Low- $n$ Disk-Dominated Structures Versus High- $n$ Spheroidal or Triaxial Bulges/Ellipticals}

Here and in Section 3.4.3, we discuss the stellar mass breakdown among galaxy components within each galaxy type. Our results are summarized in Tables 3.6 and 3.7. These results are calculated by applying a constant $\mathrm{F} 814 \mathrm{~W} M / L$ ratio to the fractional luminosities of the components, and we start by discussing the validity of this assumption. This approach is well-suited to the NIR where uncertainties in $M / L$ due to input stellar models, spectra, dust, and age of stellar populations is a factor of $\lesssim 3$ (see e.g., Weinzirl et al. 2009). The F814W filter is at the red end of the optical spectrum, and this approach could be more uncertain here than in the NIR.

Observational studies reveal that the stellar populations in the central regions of Coma, where our sample is located, are of intermediate or old age. Poggianti et al. (2001) measure the spread in luminosity-weighted age among Coma members to be $\sim 3-9$ Gyr at any luminosity $\left(-20.5<M_{B}<-14\right)$. Within the central Mpc, $50-60 \%$ of galaxies show no evidence of star formation in the last $\sim 10$ Gyr $(z<2)$, and only a small fraction $(5-10 \%)$ of the brightest centrally located galaxies experienced star formation in the last 3 Gyr $(z<0.35)$. Similarly, Trager et al. (2008) measure elliptical and S0 galaxies in the core of Coma to have single-stellar-population-equivalent ages of 5-8 Gyr.

Experimentation with Bruzual \& Charlot (2003) single stellar population models using a range of metallicity $\left(-0.4 \lesssim\left[Z / Z_{\odot}\right] \lesssim 0.4\right)$ and a Chabrier (2003) IMF shows that, 
given an optical depth $\tau=1$ and a stellar population $\sim 3-9$ Gyr old, the F814W $M / L$ within a galaxy may vary by up to a factor of 2.5 . Thus, assuming a constant $M / L$ for centrally located Coma galaxies is a reasonably sound practice.

As discussed in Section 3.1 and Section 3.3.1, one of our goals is to provide a census of the mass in low- $n$ disk-dominated components on different scales, ranging from outer disks of galaxies to central stellar disks, with the rationale that such a measure would probe disks built by dissipative settling of gas accreted through a variety of ways (e.g., cold streams, halo accretion, and gas-rich mergers). Similarly, a knowledge of high- $n$ classical bulges/ellipticals would tell us something about the importance of violent stellar processes such as violent relaxation in major mergers. Table 3.7 summarizes our attempt at doing this, and we discuss the results below.

1. Stellar mass in low-n flattened disk-dominated structures (44\%):

Our low $n \leq n_{\text {disk_max }}$ disk-dominated structures include outer disks, inner disks, and disky pseudobulges, and bars. The first three components consist of the largescale outer disks of S0s and spirals, the inner disks and disky pseudobulges with $n \leq n_{\text {disk_max }}$, and also the inner components of photometric ellipticals with $n \leq$ $\left.n_{\text {disk_max }}\right)$. The total stellar mass in these components is $\sim 36.5 \%$. Most $(\sim 31.2 \%)$ of this stellar mass is in the outer disks of S0s and spirals. Only $\sim 2.18 \%$ is from the disky pseudobulges of S0s and spirals. The remaining $\sim 3.11 \%$ is from the inner components of photometric ellipticals.

As pointed out in Section 3.3.4.1 and Figure 3.11 the majority of outer diskswith 
low Sérsic index; most $(67.3 \pm 7.9 \%)$ outer disks have Sérsic index $n<1$ and many $(19.2 \pm 12.5 \%)$ have $n<0.5$. We further discuss this point in Section 3.4.5.

Next we discuss the mass in stellar bars, which are disk-dominated components in the sense that they are flattened non-axisymmetric components. Bar proportions typically range from 2.5:1 to 5:1 in their equatorial plane (Binney \& Tremaine 1987). The vertical thickness of bars is harder to constrain. Some bars may be as flat as disks, though they can have thicker scale heights than their disks. In Section 3.3.4.2 we fit bars by initially guessing a low Sérsic index of 0.5 . The actual range in Sérsic index found for the 26 galaxies with bars is 0.14 to 1.05 with a median of 0.51 . This is comparable to Weinzirl et al. (2009) who found bars in field spirals have Sérsic index 0.02 to 2.19 with a median of 0.43 . The stellar mass percentage in bars is $7.46 \%$. The mass percentage among just S0s and spirals is $10.4 \%$, and this is similar to the bar mass percentage (9.58\%) found among spirals by Weinzirl et al. (2009).

2. Stellar mass in high-n classical bulges/ellipticals (56\%):

The remaining stellar mass is in components with $n>n_{\text {disk_max }}$. These components include the outer components of photometric ellipticals, the central components with $n>n_{\text {disk_max }}$ in photometric ellipticals, and the bulges of S0s and spirals with $n>$ $n_{\text {disk_max }}$. The percent stellar mass in these systems is $56 \%$. This percentage is an upper limit to the mass in classical bulges and photometric ellipticals.

Finally, we discuss how the fraction of galactic stellar mass, which we call $f_{\text {disk_dominated }}$ for convenience, varies with environment. One might naturally expect that $f_{\text {disk_dominated }}$ 
would fall from low-density to high-density environments. For the lower density field-like environments studied by Weinzirl et al. (2009), this fraction $f_{\text {disk-dominated }}$ is $\sim 89.6 \%$ for galaxies with $M_{\star} \geq 10^{10} M_{\odot}$. If the same stellar mass cut is applied in Coma, the fraction $f_{\text {disk_dominated }}$ in Coma is $\sim 42 \%$, which is lower than in the field as expected.

We also note here the results for the Virgo cluster, which has a central projected galaxy number density of $360 \mathrm{Mpc}^{-3}$, (Binggeli et al. 1987), and is a less rich cluster than Coma, where the corresponding number exceeds several $1000 \mathrm{Mpc}^{-3}$ (The \& White 1986). In Virgo Kormendy et al. (2009) find that for galaxies with stellar mass $M_{\star} \gtrsim 5 \times 10^{9} M_{\odot}$, more than $2 / 3$ of the stellar mass is in classical bulges/ellipticals, implying that $f_{\text {disk_dominated }}$ is less than $1 / 3$. At first sight, it may seem surprising that our value of $f_{\text {disk_dominated }}$ in Coma is higher than the value of $1 / 3$ for Virgo. However, we believe this apparent discrepancy is due to the fact that the Virgo study includes the giant $\mathrm{E}$ galaxy M 87, which is marginally classified as a cD (Kormendy et al. 2009), while our study excludes the $2 \mathrm{cDs}$ in the central part of Coma. If we include these $2 \mathrm{cDs}$ and adopt a conservative lower limit for their stellar mass, then the fraction $f_{\text {disk-dominated }}$ of stellar mass in the low- $n$ component would be less than $28 \%$, since the cDs add their mass to high- $n$ stellar components (see Section 3.3.4.4).

\subsubsection{Stellar Mass Distributions Within S0, E, and Spiral Galaxy Structures}

We now discuss how the stellar mass is distributed among the different Hubble types, starting with S0 and spiral galaxies. We described in Section 3.4.1 how these Hubble

types were assigned based on the presence of an outer disk with Sérsic index below the threshold $n_{\text {disk_max }}=1.66$. 
1. Mass distribution among high-n classical bulges/ellipticals versus low-n disky pseudobulges in Coma SOs and spirals:

Outer disks in spiral and S0 galaxies contribute $20.1 \%$ of the mass budget in our sample of galaxies with stellar mass $M_{\star} \geq 10^{9} M_{\odot}$. The bulges of S0 and spiral galaxies account for $21.3 \%$ of the stellar mass. Thus, bulges contain about the same overall stellar mass as outer disks.

We further note there are two kinds of bulges, namely low- $n$ disky pseudobulges and high- $n$ classical bulges (see Section 3.1 and Sections 3.3.1-3.3.3). When bulge $n \leq$ $n_{\text {disk_max }}$, the bulge is usually thought to represent a compact stellar disk also called a disky pseudobulge. Such bulges are believed to be built by dissipative settling of gas in the circumnuclear region. In comparison, when bulge $n>n_{\text {disk_max }}$, the bulge is more akin to high- $n$ classical bulges built by violent stellar processes, such as violent relaxation in major mergers and possibly also minor mergers. Tables 3.6 and 3.7 show that the latter kind of bulge with $n>n_{\text {disk_max }}$ in S0s and spirals combined contributes $\sim 19.9 \%$ of the total stellar mass. Among the mass in just S0s and spirals, high- $n$ bulges make up $\sim 44 \%$ and $\sim 32 \%$, respectively, of the stellar mass. Low- $n$ disky pseudobulges contribute $\sim 1.5 \%$ of the total stellar mass in the sample. In terms of bulge properties among SOs and spirals in our sample, the ratio $R$ of stellar mass in high-n $(n \gtrsim 1.7)$ classical bulges to low- $n(n \lesssim 1.7)$ disky pseudobulges is $\sim 13.3$.

2. Mass distributions among bulges in Coma S0s versus S0s in lower-density environments:

We next compare the bulges of Coma S0s versus S0s in lower-density environments (LDEs). The results are summarized in Table 3.8. We base this comparison on the 
results of Laurikainen et al. (2010), who derive structural parameters from $2 D$ multicomponent decompositions of 117 S0s in LDEs, that include a mix of field and Virgo environments. We note that Virgo is a significantly LDE than the center of Coma, with much lower projected galaxy number densities and halo mass (Binggeli et al. 1987). We estimate this sample of Laurikainen et al. (2010) to have stellar mass $M_{\star} \geq 7.5 \times 10^{9} M_{\odot}$. For S0s with $M_{\star} \geq 7.5 \times 10^{9} M_{\odot}$, the ratio $R$ of stellar mass in high- $n(n \gtrsim 1.7)$ classical bulges to low- $n(n \lesssim 1.7)$ disky pseudobulges is $(27.7 \% / 5.1 \%$ or 5.4) in the LDE probed by Laurikainen et al. (2010) while it is $(42.7 \% / 2.3 \%$ or 18.5) in the central 0.6 Mpc of Coma.

Note the difference in mass stored in high- $n$ and low- $n$ bulges is not due to a greater frequency of high- $n$ bulges. At this mass range $\left(M_{\star} \geq 7.5 \times 10^{9} M_{\odot}\right)$, the relative fraction of low and high- $n$ bulges is similar. In Coma, the fraction of high- $n$ bulges among these massive S0s is $78.6 \pm 7.75 \%$, and the fraction of low- $n$ bulges is $21.4 \pm$ 7.75\%. Among the S0s from Laurikainen et al. (2010), the fraction of high- $n$ and low- $n$ bulges is $77.7 \pm 3.93 \%$ and $22.3 \pm 3.93 \%$, respectively.

3. Mass distribution in outer and inner components of photometric ellipticals in Coma: By definition, photometric ellipticals have no outer disk since their outermost structures have Sérsic index $n>n_{\text {disk_max }}$ (see Figure 3.5 and Section 3.4.1). The Sérsic indices of the outer component in photometric ellipticals range from 1.83 to 6.95 , with a median value of 2.1 . The outer structures of ellipticals contain $14.4 \%$ of the total mass, which is less than the amount contained in the bulges of S0 and spiral galaxies. Photometric ellipticals may contain an inner component of any Sérsic index. These inner components span 0.31 to 2.21 in Sérsic index and they contain $3.68 \%$ of the total 
mass. Most $(8 / 12$ or $66.7 \pm 13.6 \%)$ of these inner components have $n \leq n_{\text {disk_max }}$ and represent compact inner disks analogous to the disky pseudobulges in S0s and spirals.

\subsubsection{Scaling Relations for Outer Disks and Bulges}

In this section we explore scaling relations for the bulges and outer disks of galaxies located in the central $0.6 \mathrm{Mpc}$ of the Coma cluster. We assess how these structures compare with outer disks and bulges in LDEs or low-density environments where environmental processes and merger histories are likely to be different. Here, LDEs refer to environments of much lower density than the rich Coma cluster (whose central projected galaxy number

density exceeds several 1,000 galaxies $\mathrm{Mpc}^{-3}$, The \& White 1986), and can include field, groups, and even low-density clusters similar to the Virgo cluster.

For this comparison, we use the results of Gadotti (2009), who studies face-on (b/a $\geq$ 0.9) galaxies from the SDSS Data Release 2 in a volume limited sample at $0.02 \leq z \leq 0.07$. He derives galaxy structure from 2D decompositions of multi-band gri images that account for bulge, disk, and bar components. While the sample from Gadotti (2009) includes galaxies with $M_{\star}$ as low as $\sim 5 \times 10^{9} M_{\odot}$, his sample is incomplete at $M_{\star} \lesssim 5 \times 10^{10} M_{\odot}$. The Coma sample S0s/spirals have stellar mass $10^{9} \leq M_{\star} \leq 6.2 \times 10^{10} M_{\odot}$, and for this comparison we consider only galaxies with stellar mass $5 \times 10^{9} \leq M_{\star} \leq 6.2 \times 10^{10} M_{\odot}$.

Figure 3.14 compares properties of large-scale disks (size, luminosity) with galaxy $M_{\star}$. Figure 3.14a explores the half-light radius in the $i$-band $\left(r_{\mathrm{e}}\right)$ of outer disks at a given galaxy $M_{\star}$ in Coma versus LDEs. It shows that at a given galaxy $M_{\star}$, the average disk $r_{\mathrm{e}}$ is smaller in the central 0.6 Mpc of Coma compared with LDEs by $\sim 40-80 \%$. Note, however, that the scatter in disk $r_{\mathrm{e}}$ is large, and that a $\sim 40-50 \%$ offset in average $r_{\mathrm{e}}$ is 
comparable to the $1 \sigma$ standard deviation. Nonetheless, a Kolmogorov-Smirnov test shows there is zero probability that outer disks of massive galaxies in Coma are drawn from the same distribution as outer disks in LDEs. The suggestion that outer disks in Coma are more compact is consistent with the results of previous analyses of disk structure in Coma (Gutiérrez et al. 2004; Aguerri et al. 2004).

Since the Gadotti (2009) sample is incomplete at $M_{\star} \lesssim 5 \times 10^{10} M_{\odot}$, it is important to consider whether the incompleteness in his sample is driving the perceived offset in average outer disk size. If his sample were missing compact galaxies, then this could drive the offset. This is unlikely, however, as a galaxy at a given $M_{\star}$ that is more compact will also have higher surface brightness and therefore be more detectable. Additionally, disk galaxies from Gadotti (2009) are face-on $(b / a \geq 0.9)$ while disk galaxies in the Coma sample show all inclinations. A moderate-to-highly inclined disk is more likely to have large apparent size than a face-on disk due to projection effects. If the galaxies in Gadotti (2009) were moderately to highly inclined, the offset in mean size could only be larger at a given galaxy $M_{\star}$.

Figure 3.14b makes a similar comparison for the outer disk luminosity between Coma and LDEs. We use here the ACS F814W photometry for Coma and the SDSS $i$-band photometry from Gadotti (2009). At a given stellar mass, the average outer disk luminosities are fainter by $\sim 42-70 \%$, excluding the lowest mass bin.

It is tempting to associate the difference in outer disk luminosity with a difference in outer disk mass, as one might expect that more compact disks in Coma are also less massive. This cannot be done without considering the effect of mass-to-light ratio $(M / L)$. For this comparison, it would be ideal to use the $i$-band $M / L$ ratios in the outer disks, 
$(M / L)_{d, i}$. We use the multi-band structural decompositions of Gadotti (2009) to calculate the value of $(M / L)_{d, i}$ in LDEs. For the Coma sample, we use as an upper limit of $(M / L)_{d, i}$ the galaxy-wide $(M / L)_{i}$ ratio estimated from SDSS $g r$ photometry using the prescriptions of Bell et al. (2003). The effect of the bulge is to raise the global $(M / L)_{i}$ ratio to higher values than found in the disk. As noted in Section 3.4.2, stellar populations in Coma are older, and consequently have higher mass-to-light ratios, than in LDEs. Furthermore, den Brok et al. (2011) study color gradients in bright Coma galaxies and conclude most galaxies show negative color gradients. Thus, in Coma, $(M / L)_{d, i}$ should in general be lower.

Figure $3.14 \mathrm{c}$ compares the resulting $(M / L)$ values against galaxy $M_{\star}$. In Coma, the average $(M / L)_{i}$ is larger than the average $(M / L)_{d, i}$ in LDEs by a factor of $\sim 1.5-2.1$ at a given galaxy $M_{\star}$, excluding the lowest mass bin. This difference in $(M / L)_{i}$ accounts for $\sim 52-86 \%$ of the average offset in disk luminosity. This suggests some of the difference in outer disk luminosity is driven by a real difference in outer disk mass. If it were possible to repeat this comparison using the true $(M / L)_{d, i}$ ratios in Coma, then the difference in disk luminosity would be less well accounted for by differences in $(M / L)_{d, i}$, and the suggestion of a difference in disk mass would be more robust.

Figure 3.15 examines how bulge size $\left(r_{\mathrm{e}}\right)$, bulge luminosity, bulge Sérsic index, and bulge-to-disk light ratio $(B / D)$ scale with galaxy $M_{\star}$. Figures $3.15 \mathrm{a}-\mathrm{c}$ show that bulge size, bulge luminosity, and bulge Sérsic index as a function of galaxy $M_{\star}$ are not systematically offset in Coma versus LDEs. Figure $3.15 \mathrm{~d}$ shows there is a great scatter in $B / D$ versus galaxy $M_{\star}$. Although the $B / D$ ratios at all galaxy $M_{\star}$ in Coma do not reach the low $B / D \lesssim 0.1$ found in LDEs, the mean $B / D$ values for Coma and LDEs are similar in most galaxy $M_{\star}$ bins due to bias on the mean value by larger $B / D$ values. 
Figure 3.16 further compares outer disks and their bulges. Figure 3.16a shows $B / D$ versus bulge Sérsic index. At a given bulge Sérsic index, galaxies in Coma show a systematically higher average $B / D$ ratio than galaxies in LDEs. A linear regression fit reveals similar slopes in $B / D$ versus bulge $n$ but shows a clear offset in $B / D$ for a given bulge index. A difference in average $B / D$ ratio at a given Sérsic index could be driven by differences in bulge luminosity, differences in disk luminosity, or both. Figure 3.16b indicates that at a given bulge Sérsic index the bulge luminosities in Coma and LDEs are very consistent. Figure 3.16c, on the other hand, shows a clear offset in disk luminosity ( $\sim 0.6 \mathrm{mag}$ ), indicating that differences in $B / D$ are driven by outer disk size/luminosity.

From this investigation, we have learned that at a given galaxy $M_{\star}$, the average $i$-band half-light radius of outer disks in Coma are typically smaller on the mean by $\sim$ $40-80 \%$ than outer disks in LDEs. We have also found evidence for a lower mean outer disk luminosity in Coma versus LDEs, which may translate into a lower mean outer disk stellar mass based on our inspection of the stellar mass-to-light ratios. A difference in outer disk size/luminosity in Coma may be explained in part by cluster environmental effects. We consider this point further in Section 3.4.5.

\subsubsection{Environmental Processes in Coma}

Galaxy evolution in clusters has been explained by a combination of two main principles. In the "nature" scenario, galaxies evolve rapidly and gravitational collapse proceeds faster because the dynamical timescale is shorter in higher density environments. At very early epochs, small disk galaxies formed more rapidly in the high-density central regions of proto-clusters than in the lower-density field environment. Spiral galaxies in such high- 
density proto-clusters had a "head start" to consume their gas via star formation, so that by the time the proto-cluster collapses into a present-day rich cluster of galaxies there is a relatively high abundance of S0s over spirals. Additionally, higher merger rates at early times in dense proto-clusters converted spirals into ellipticals, partly accounting for the low relative frequency of spirals.

The "nurture" scenario in contrast, dictates that cluster-specific processes (strangulation, ram-pressure stripping, and galaxy harassment) act on galaxies to transform them in a way that raises the relative frequency of early-type galaxies over spirals. Strangulation (Larson et al. 1980) is the removal a galaxy's hot gas halo, and this can suppress the further growth of large-scale disks by removing gas that would otherwise cool onto the galaxy. Ram-pressure stripping is associated with the removal cold gas, cessation of star formation, and reddening/dimming of the outer disk (Gunn \& Gott 1972; Fujita \& Nagashima 1999). Galaxy harassment (Barnes \& Hernquist 1991; Moore et al. 1996, 1998, 1999; Hashimoto et al. 1998; Gnedin 2003) is the combination of interactions with the cluster potential and high-speed, galaxy-galaxy encounters. Repeated bouts of harassment can lead to tidal stripping of mass, dynamical heating, and gas inflow with associated SF in the central regions of galaxies.

In the remainder of this section, we review the evidence for environmental processes in Coma and comment on what our results add to this picture.

\section{Existing evidence for environmental processes in Coma:}

Analysis of stellar populations and star formation history in Coma provide indirect evidence for the action of processes (starvation and ram-pressure stripping) that remove gas from from galaxies. Several studies find predominantly intermediate or old 
stellar populations in the center of the cluster (e.g., Poggianti et al. 2001; Trager et al. 2008; Edwards \& Fadda 2011).

Several examples of ram-pressure stripping have been seen in Coma (Yagi et al. 2007, 2010; Yoshida et al. 2008; Smith et al. 2010; Fossati et al. 2012). Yagi et al. (2010) image the diffuse and extended $\mathrm{H} \alpha$ cloud trails of 14 spectroscopically confirmed member galaxies that appear to be shedding gas from their outer disks while falling into the cluster potential. Some of these systems were previously reported by Yagi et al. (2007) and Yoshida et al. (2008). Smith et al. (2010) find 11 examples of Coma galaxies showing tails of UV-bright debris, pointing away from the cluster center, interpreted as stars formed in ram-pressure stripped gas. Fossati et al. (2012) further report the detection of a $65 \mathrm{kpc}$ ionized gas trail behind NGC 4848, which appears to be making its first trip through Coma.

There is also much evidence for the violent effects of tidal forces. The presence of a diffuse intra-cluster medium around Coma central galaxies NGC 4874 and NGC 4889 has long been discussed (Kormendy \& Bahcall 1974; Melnick et al. 1977; Thuan \& Kormendy 1977; Bernstein et al. 1995; Adami et al. 2005; Arnaboldi 2011). At the cluster center, the intra-cluster light represents up to $20 \%$ of the cluster galaxy luminosity (Adami et al. 2005). This central intra-cluster light is not uniform given the presence of plumes and tidal tails (Gregg \& West 1998; Adami et al. 2005), and debris fields are also found further outside the cluster center (Gregg \& West 1998; Trentham \& Mobasher 1998).

2. Reduced Growth and Truncations of Outer Disks in Coma S0s/spirals: 
In Section 3.4.4, we found that at a given galaxy stellar mass, the average half-light radius $\left(r_{\mathrm{e}}\right)$ of the outer disk in S0s/spirals is $\sim 40-80 \%$ smaller, and the average disk $i$-band luminosity is $\sim 42-70 \%$ fainter in Coma than in lower-density environments (Figure 3.14).

In Section 3.4.4, we examined the sizes $\left(r_{\mathrm{e}}\right)$ and luminosities of S0/spiral galaxy outer disks in Coma relative to the outer disks of equally massive S0s/spirals in low-density environments (LDEs). Over the stellar mass range considered here $\left(5 \times 10^{9} \leq M_{\star} \leq\right.$ $\left.6.2 \times 10^{10} M_{\odot}\right)$, the average offset at a given galaxy $M_{\star}$ in outer disk size between Coma and LDEs is $\sim 40-80 \%$. Furthermore, there is also an offset in outer disk luminosity such that in Coma outer disks are fainter by $\sim 42-70 \%$. Not all of the offset in luminosity can be attributed to differences in $M / L$, which implies outer disks in Coma are less massive.

These observations may be explained in part by cluster environmental effects (e,g., strangulation, ram-pressure stripping, tidal stripping) that suppress the growth of large-scale disks. Hot gas stripping (strangulation) can plausibly suppress disk growth by limiting the amount of gas that can cool and become part of the outer disk. Tidal stripping via galaxy harassment is predicted (e.g., Moore et al. 1999) to be particularly efficient at removing mass from extended disks. Ram-pressure stripping is most effective at removing HI gas in the outskirts of a large scale-disk. The evidence (Yagi et al. 2007, 2010; Yoshida et al. 2008; Fossati et al. 2012) suggests ram-pressure stripping happens quickly, and if so it should be effective at preventing the growth of large-scale disks after the host galaxy enters the cluster. 


\section{Low Sérsic index in S0/spiral outer disks:}

Figure 3.11 demonstrates the majority of outer disks have low Sérsic index $(67.3 \pm 7.9 \%$ with $n<1$ and $19.2 \pm 12.5 \%$ with $n<0.5)$. This effect is not artificially driven by bars because the low $n<1$ disks include barred and unbarred galaxies to similar proportions, and additionally, the disk are fitted separately from the bars in our work. Similar examples, however, have also been found in Virgo. Kormendy \& Bender (2012) find several examples of Gaussian $(n \sim 0.5)$ disks among both barred and unbarred galaxies. Such structure is characteristic of outer rings and oval disks in barred galaxies (e.g., Kormendy \& Kennicutt 2004). Gaussian-like disks among unbarred galaxies is much more surprising (Kormendy \& Bender 2012). Figure 3.11 shows that the large fraction of $n<1$ outer disks in Coma is not driven by barred galaxies alone; there are several unbarred galaxies also showing this behavior. It is not easy to compare the fraction of low $n<1$ disks in Coma versus LDEs because most work to date in LDEs (e.g., Allen et al. 2006; Laurikainen 2007, 2010; Weinzirl et al. 2009) fit the outer disk with a fixed $n=1$ exponential profile.

It is natural to suspect that environmental processes could be creating the unusual Gaussian-like disks. This was the conclusion of Kormendy \& Bender (2012). We could be seeing a stronger effect because Coma is denser than Virgo. Ram-pressure stripping and tidal stripping can plausibly reduce the Sérsic $n$ by cutting off the outskirts of the outer stellar/gaseous disk, and they are likely the mechanisms driving the Gaussian-like outer disks in Coma. Strangulation means there is less fuel to cool and grow large-scale disks. This could make the resulting outer disks smaller in scale, but it should not necessarily alter the resulting disk Sérsic index. Tidal heating of disk 
stars is expected from repeated high-speed encounters. Simulations (e.g., Bournaud, Jog, \& Combes 2007; Naab et al. 2009; Hilz et al. 2012), show that the net effect of repeated minor mergers is higher global Sérsic $n$ because they make disks thicker and more extended. Dynamical heating may, however, indirectly contribute to tidal stripping by making stars less bound and easier to strip.

\section{Bulge-to-disk ratio $(B / D)$ :}

The mean bulge Sérsic index rises with mean bulge-to-disk $(B / D)$ light ratio in both the central part of Coma and LDEs, consistent with the idea that the development of high $B / D$ ratio in galaxies is usually associated with processes, such as major mergers, which naturally results in a high $n$. Such a correlation was also found previously in field spirals (e.g., Andredakis et al. 1995; Weinzirl et al. 2009).

However, we also find that at a given bulge index, the $B / D$ light ratio is higher for Coma. This environmental effect appears to be due the fact that at a given bulge $n$ , the bulge luminosity is similar in Coma and LDEs, but the outer disks have lower luminosity by a factor of a few in Coma (Figure 3.16). As discussed in part (ii) above, this reduced disk growth is likely due to cluster environmental effects (e,g., strangulation, ram-pressure stripping, tidal stripping) that suppress the growth of large-scale outer disks. 


\subsection{Comparison of Empirical Results With Theoretical Predictions}

\subsubsection{Overview of the Models}

In this section, we compare our empirical results for Coma with simulations of

clusters. Readers interested only in the comparison between model and empirical data should skip directly to Sections 3.5.3 and 3.5.6.

The simulated clusters are derived from a semi-analytical model (SAM) based on Neistein \& Weinmann (2010). The SAM is able to produce reasonable matches (Wang, Weinmann, \& Neistein 2012) to the galaxy stellar mass function determined by (Li \& White 2009) for massive $M_{\star} \gtrsim 5 \times 10^{8} M_{\odot}$ galaxies at low redshift $(0.001<z<0.5)$ over all environments (including Virgo and Coma) probed in the northern hemisphere component of SDSS Data Release 7. A brief summary of the SAM formalism is given below.

The SAM uses merger trees extracted from the Millennium $N$-body simulation (Springel et al. 2005). The merger trees are based on subhaloes identified as bound density peaks inside friends-of-friends (FOF) groups (Davis et al. 1985). The mass of each subhalo is determined according to the number of particles it contains. The minimum halo mass is $\sim 2 \times 10^{10} h_{73}^{-1} M_{\odot}$.

Galaxies are modeled as vectors of stellar mass, cold gas, and hot gas. Baryonic physics are handled with semi-analytic prescriptions. Gas collapses $\left(f_{\text {baryon }}=0.17\right)$ with the dark matter halo and is initially shock-heated to the halo virial temperature. Cooling gas settles into a rotating disk at the center of the halo. In between merger events, the efficiencies of quiescent evolutionary processes, such as cold and hot gas accretion, gas cooling, star formation, and supernovae feedback, are modeled as functions of halo mass and redshift only. The star formation rate is proportional to the amount of cold gas, and 
the star formation efficiency is a function of halo mass and redshift. In the model, the baryonic mass (sum of stellar and cold gas mass) is used to define major and minor mergers. As we will discuss in Section 3.5.4, the results are highly sensitive to whether the stellar mass ratio or baryonic mass ratio are used.

In the merger of two DM halos, the merger timescale of galaxies is estimated from the time it would take the satellite galaxy to reach the central galaxy at the center of the halo via dynamical friction. The simulations differentiate between major and more minor mergers of galaxies. The progenitor galaxies of a major merger are required to have baryonic (stars + cold gas) mass ratios $M_{1} / M_{2} \geq 1 / 4$. Immediately after a major merger, the remnant's stellar $B / T$ ratio is always one. This is because the model assumes any existing stellar disks are destroyed, and all stars undergo violent relaxation to form a bulge/elliptical. After a major merger, an extended stellar disk is rebuilt via gas cooling, causing $B / T$ to fall. Any further major mergers will reset $B / T$ to one.

Minor mergers, occurring when progenitors have the stellar mass ratios $1 / 10<$ $M_{1} / M_{2}<1 / 4$, are another pathway for bulge formation. In the model used here, the stellar component of the satellite of baryonic mass $M_{2}$ is added to the bulge. We discuss in Section 3.5.6 what type of Sérsic $n$ it would be natural to assign to such a bulge.

During a major/minor merger, some fraction of cold gas is converted to stars in a short induced starburst $\sim 10 \mathrm{Myr}$ in duration. Stars formed in major merger-induced starbursts are considered part of the bulge (see Section 3.5.6 and Equation 3.7). This is a reasonable assumption given that a) the bursts of star formation are much shorter than the overall duration of the mergers and b) all existing stars from both progenitors are violently relaxed during final coalescence. It seems less likely the starburst stars induced in minor 
mergers should be violently relaxed since minor mergers are not very efficient at violently relaxing stars in the host galaxy. We consider this issue further in Section 3.5.6.

The efficiency of merger-induced starbursts depends on the baryonic masses of the progenitor galaxies (e.g., Mihos \& Hernquist 1994; Somerville, Primack, \& Faber 2001; Cox et al. 2008). The amount of stars produced in a merger is given by

$$
\Delta m_{\star}=\alpha_{b}\left(\frac{m_{1}}{m_{2}}\right)^{\alpha_{c}}\left(m_{1, \text { cold }}+m_{2, \text { cold }}\right),
$$

where $m_{i}$ are the sum of cold gas mass and stellar mass in the progenitors, $m_{i}$, cold are the cold gas masses, and $\alpha_{b}, \alpha_{c}$ are constants set to 0.56 and 0.7 , respectively. Note that this recipe for merger-induced star formation depends explicitly on the cold gas mass. Other approaches, in comparison, parameterize the amount of induced star formation as a function of gas fraction; in Hopkins et al. (2009c), for instance, the fraction of gas that participates in the starburst is proportional to the cold gas fraction.

Therefore, in the model used in this paper, the bulge stellar mass traces the mass assembled via major and minor mergers. Galaxies without bulges have had no resolvable merger history. Recall from Section 3.1 that classical bulges can also be assembled through the coalescence of clumps condensing in violent disk instabilities (Bournaud, Elmegreen, \& Elmegreen 2007; Elmegreen et al. 2009). The model used here does not build any bulges through this pathway.

Galaxy clusters impose additional environmental effects that complicate modeling with SAMs. The SAM used here account for stripping of hot gas (i.e., strangulation, Larson et al. 1980) by assuming hot gas is stripped exponentially with a timescale of 4 Gyr. Yet, they neglect other processes like ram-pressure, stripping/disruption of stellar mass (Moore 
et al. 1996, 1998, 1999; Gnedin 2003), dynamical friction heating by satellite (El-Zant et al. 2004), and gravitational heating by infalling substructures (Khochfar \& Ostriker 2008). Although ram-pressure can compress cold gas and can therefore spur an increase in star formation in an outer disk, as a galaxy falls further into the potential of the cluster, ram-pressure stripping removes cold gas, causing a drop in star formation that makes the disk become redder and fainter (Fujita \& Nagashima 1999). Ram-pressure can in principle impede the growth of the outer disk and increase the fractional mass/light of the bulge relative to the disk.

It is not clear how much the inclusion of ram-pressure stripping in the semianalytical models would affect our results. While hydrodynamical simulations clearly demonstrate the strong influence of ram-pressure stripping on gas mass, galaxy morphology, and star formation (e.g., Quilis et al. 2000; Tonnesen \& Bryan 2008, 2009, 2010), some semianalytic models (e.g., Okamoto \& Nagashima 2003; Lanzoni et al. 2005) suggest that accounting for ram-pressure stripping has a small affect on luminosity functions, galaxy colors, and galaxy morphology. Okamoto \& Nagashima (2003) note thatthe effect of ram-pressure stripping on star formation and galaxy color can be negated with the right star formation timescale.

Stripping/disruption of stellar material from satellites is another process that is highly relevant to clusters with deep potential wells. This process creates a population of intra-cluster stars that can contribute between $10-40 \%$ of the optical light in rich clusters (e.g.; Bernstein et al. 1995; Feldmeier et al. 2004; Zibetti et al. 2005). Low surface brightness features attributed to tidal stripping have been clearly observed in Coma (Gregg \& West 1998; Trentham \& Mobasher 1998; see also Section 3.4.5). The inclusion 
of tidal stripping in SAMs is important for addressing a wide range of systematic effects. Stellar stripping/disruption plays a role in resolving the problem of too many dwarf galaxies, which is a common issue in SAMs (e.g., Weinmann et al. 2006; Henriques et al. 2008). Stripping/disruption pulls red, low-metallicity stars, that would otherwise be deposited into the central subhaloes, from satellite galaxies and places them into the intra-cluster medium. In addition to reducing the amount of stars accreted by the central subhaloes, and therefore their stellar mass, stripping/disruption also has the effect of raising merger timescales by $10-$ $20 \%$, reducing the number of red galaxies at all mass scales, and increasing the metallicity of intermediate and high-mass galaxies (Henriques et al. 2010). Tidal stripping has been included in several SAM models (e.g., Bullock et al. 2001; Henriques et al. 2008, 2010) but is not present in our SAM.

We will come back to this issue in Section 3.5.3 where we discuss the model versus data mismatch in global galaxy stellar mass function, as well as in Section 3.5.5 where we discuss the data versus model cold gas fraction.

\subsubsection{The Mass Function and Cumulative Number Density in Coma}

In order to compare galaxies in the simulations with those in the center of Coma, we first need to identify model clusters that best represent Coma. We do this by first computing global empirical properties of the Coma cluster, such as the halo mass, galaxy stellar mass function, and radial profile of cumulative projected galaxy number density, out to at least the virial radius of the Coma cluster. We describe below how we compute these in the Coma cluster.

As the ACS coverage of Coma encompasses a fraction of the central $0.6 \mathrm{Mpc}$, we 
must use an alternative dataset to calculate the Coma mass function and cumulative number density out to larger radii. We use Data Release 7 (DR7) of the NYU Value-Added Galaxy Catalog (NYU-VAGC, Blanton et al. 2005), which provides full spatial coverage of Coma. NYU-VAGC DR7 is based on SDSS DR7 data (Abazajian et al. 2009) and provides catalogs generated from an independent, and improved, reduction of the public data (Padmanabhan et al. 2008).

We select Coma cluster member galaxies from NYU-VAGC after making key assumptions. We assume Coma cluster galaxies have radial velocity in the range $\mathrm{v}_{\min }=4620$ $\mathrm{km} / \mathrm{s}$ to $\mathrm{v}_{\max }=10,000 \mathrm{~km} / \mathrm{s}$, which is the range in radial velocity among spectroscopically confirmed members in the ACS survey. We also adopt the Coma virial radius and virial mass to be $2.9 h_{70}^{-1} \mathrm{Mpc}$ and $1.4 \times 10^{15} h_{70}^{-1} M_{\odot}$, respectively, measured by Lokas \& Mamon (2003) with a $30 \%$ accuracy, where $h_{70}^{-1}=H_{0} / 70$. For our adopted $H_{0}$ of 73 , we scale these numbers by $(73 / 70)^{-1}$, so that the virial radius and virial mass are $2.8 h_{73}^{-1} \mathrm{Mpc}$ and $1.3 \times 10^{15} h_{73}^{-1} M_{\odot}$, respectively. We select galaxies with the following criteria:

1. Radial velocity in range 4620 to $10,000 \mathrm{~km} / \mathrm{s}$.

2. Projected radius, $R_{p}$ from the cluster center (i.e., NGC 4874) less than the virial radius.

3. Brightness exceeding the SDSS spectroscopic completeness limit of $r=17.7$ mag, or $r \leq-17.3 \mathrm{mag}$ at the $100 \mathrm{Mpc}$ distance of Coma. This corresponds to a stellar mass of $1.3 \times 10^{9} M_{\odot}$ assuming a $g-r$ color of 0.67 , which is the average among Coma galaxies in the NYU-VAGC selected in this manner. 
Panel (b) of Figure 3.17 shows the resulting projected galaxy density profile for this set of Coma galaxies. The central density peaks at $\sim 3 \times 10^{4} \mathrm{Mpc}^{-3}$.

We next calculate the global galaxy stellar mass function within the virial radius. Figure $3.17 \mathrm{c}$ shows the result. This mass function includes normal massive galaxies (E, S0, spirals) as well as the two cDs (NGC 4874 and NGC 4889). As described in Section 3.2.2, for the first group of galaxies we derive the stellar mass by applying Equations 1 and 2 (or 3 and 4) to photometry from SDSS or CFHT (Adami et al. 2006) data. For the cDs, the stellar masses are more uncertain, and using the approach outlined in Section 3.2.2 we obtain a lower limit $\left(M_{\star} \sim 3-4 \times 10^{11} M_{\odot}\right)$ in stellar mass.

Using the cD galaxy stellar masses as lower limits at the high mass end of the galaxy stellar mass function in Figure 3.17c, we measure a slope $\alpha=-1.16$ and characteristic mass $M^{*}=1.25 \times 10^{11} M_{\odot}$ for the global galaxy stellar mass function of Coma inside the cluster virial radius.

\subsubsection{Global Properties of Model Clusters Versus Coma}

Next, we compare the above global properties of the Coma cluster with the simulated clusters in the theoretical model in order to identify the model clusters that best represent Coma. We consider all 160 FOF groups in the Millennium simulation having a halo mass in the range $5 \times 10^{14}-10^{16} M_{\odot}$. We refer to the most massive halo, and its gravitationally bound subhaloes, in each FOF group as a 'cluster'.

To find potential matching clusters, we identify member galaxies in each cluster in a way that is consistent with the selection of Coma member galaxies in Section 3.5.2. For each cluster, the range in radial velocity (in the $\mathrm{xy}, \mathrm{xz}, \mathrm{yz}$ projections) is measured for 
the cluster members. Then, for all massive $\left(M_{\star} \geq 10^{9} M_{\odot}\right)$ galaxies (including non-cluster members) within a projected radius $R_{\mathrm{p}}=R_{\mathrm{vir}}$ of the cluster center, the sight-line-averaged galaxy stellar mass and cumulative number density functions are calculated for each cluster using criteria analogous to the selection from NYU-VAGC data:

1. Radial velocity matching the range in line-of-sight velocities in the xy, xz, yz projections of the cluster.

2. Projected radius, $R_{p}$, from the cluster center less than the cluster virial radius.

3. Luminosity brighter than the SDSS spectroscopic completeness limit of $r \leq-17.3$ mag at the $100 \mathrm{Mpc}$ distance of Coma.

To gauge how well the simulated clusters compare with Coma in terms of global properties, we examine the match in cumulative number density, mass function, and halo parameters (virial mass and radius).

In Figure 3.17, we gauge how the global properties of Coma compare with those of all 160 cluster simulations. Figure 3.17 a shows the combinations of virial radius and halo masses of the simulated clusters. The Coma halo parameters (virial mass and radius) adopted in Section 3.5.2 are well matched to the largest and most massive model clusters.

Figure $3.17 \mathrm{~b}$ shows the radial profile of cumulative galaxy number density. The central galaxy number densities in the simulated clusters span three orders of magnitude from $\sim 10^{2}$ to $\sim 4 \times 10^{4} \mathrm{Mpc}^{-3}$, overlapping with the high central density in Coma $(\sim$ $\left.3 \times 10^{4} \mathrm{Mpc}^{-3}\right)$. The radial profile of the cluster model with the best halo parameters from Figure 3.17a is marked as a thick dotted line. This halo model does a good job at 
matching the galaxy number density profile of Coma at projected radius $R_{p}>0.7 \mathrm{Mpc}$, but not at smaller projected radii. In comparison, the model with the best matching cumulative number density profile, shown as the open circle in Figure 3.17a, is smaller by $\sim 60 \%$ in halo mass than Coma. The next nine best matches to cumulative number density also differ in halo mass by $\sim 30 \%$ or more from the halo mass in Coma, which is estimated to be accurate to within $30 \%$ (Section 3.5.2).

The bottom panel of Figure 3.17 compares the galaxy stellar mass function between Coma and the simulated model clusters. All the model clusters produce too many extremely massive $\left(M_{\star} \gtrsim 5 \times 10^{11} M_{\odot}\right)$ galaxies, which are absent in Coma. These very high-mass galaxies are not devoid of ongoing star formation like ellipticals in Coma are (Section 3.4.2). Rather, these galaxies have present day SFR of $\sim 10 M_{\odot} \mathrm{yr}^{-1}$. Furthermore, the cluster mass functions show slopes that are marginally too steep $(\alpha \sim-1.5$ versus $\alpha=-1.16)$ on the low-mass end (Section 3.5.2).

We note that when this SAM model was compared with SDSS observations of galaxies averaged over all environments at low redshift (Wang, Weinmann, \& Neistein 2012), the model galaxy stellar mass function shows a similar, but less extreme, discrepancywith the galaxy stellar mass function of Li \& White (2009) in terms of producing too many of the most masssive galaxies. Figure 3.17c includes the galaxy stellar mass function from Li \& White (2009) as a dashed line for comparison.

The large discrepancy in the galaxy stellar mass function between the model and Coma could be due to a number of factors. The model currently does not include tidal stripping/disruption of stars and ram-pressure stripping (Section 3.5.1), which would reduce the stellar mass of galaxies on all mass scales. This could potentially alleviate the overproduc- 
tion of extremely massive galaxies in the simulations and move to the model curves in Fig 18c to the left, providing a better match with the data. The importance of ram pressure stripping is also discussed in Section 3.5.5, where we find that the cold gas fraction in the model galaxies is much higher in Coma galaxies. This suggests that in the model, SF events at different epochs were fueled by a more copious supply of cold gas than is available to real Coma galaxies.

If we combine the results from all three panels of Figure 3.17, we are left with a sobering conclusion. The simulations cannot produce a model cluster that simultaneously matches multiple global properties (halo properties, galaxy number density and galaxy stellar mass function), of Coma, our local benchmark for one of the richest nearby galaxy clusters. We also realize that the approach adopted by many modelers to assume that matching theclustering (i.e., cumulative number density) and mass functions of the full galaxy population is enough to constrain the physics of galaxy formation. The discrepancy shown in Figure 3.17 suggests that this picture is rather limited because the model fails to match the mass function and halo parameters in Coma despite matching the cumulative density profile of Coma.

When comparing the data with the model, we do the comparisons with three sets of model clusters (a total of 30 model clusters) containing the 10 best matches to Coma in terms of the cumulative galaxy number density, galaxy stellar mass function, and halo parameters (halo mass and virial radius). We find that the three selection criteria lead to very similar conclusions. Figure 3.18 shows how these samples compare with Coma. As alluded to above, matching to one criterion (e.g., cumulative number density) does not ensure a good match to the other two criteria (galaxy stellar mass function and halo parameters). 


\subsubsection{Strong Dependence of Results on Mass Ratio Used to Define Mergers}

Certain galaxy properties (merger history and $B / T$ ) are highly dependent on the mass used (stellar mass, baryonic mass, halo mass) in the definition of the merger mass ratio $M_{1} / M_{2}$. For a single representative cluster model, Figure 3.19 highlights the key differences that arise when $M_{1} / M_{2}$ is defined as the ratio of stellar mass (Def 1 , left column) versus cold gas plus stars (Def 2, right column). This representative cluster was selected because it is the best matching cluster to the cumulative galaxy number density distribution in Coma (Figure 3.18).

The first row of Figure 3.19 shows the cumulative percentage of galaxies with a major merger since redshift $z$. In the left column for Def 1 , only $\sim 7 \%$ of galaxies have a major merger since $z=4$. For Def 2 , the fraction is $>35 \%$, or $\gtrsim 5$ times larger. In the second row of Figure 3.19, the histograms show the percentage of galaxies with a last major merger at redshift $z$. Most mergers happen at $z<1$. Under Def 2 (right column), the model shows a higher fraction of major mergers at $1<z<2$, compared with Def 1 . The third row shows the percentage of galaxies with a given $B / T$ value, sorted by galaxies with and without a major merger. The major merger definition has a strong influence here. In both cases, the highest $B / T$ galaxies have had major mergers. Under Def 2 (right column), more gas-rich interactions are counted as major mergers, and this leads to both a higher fraction of galaxies with high $B / T>0.6$ and a lower fraction of galaxies with intermediate $0.2<B / T<0.6$. Finally, the last row of Figure 3.19 gives the distribution of present-day $B / T$ versus redshift of the last major merger. This plot re-illustrates that under Def 2 , five times more galaxies have a major merger than for Def 1 . Correspondingly, this higher major merger rate leads to a higher fraction of galaxies with high $B / T>0.6$. 
In the following sections, we consider a model where the merger mass ratio $M_{1} / M_{2}$ depends on stellar mass plus cold gas, as this ratio is understood to be the most appropriate definition (Hopkins et al. 2009b). Traditionally, observers have tended to use stellar mass ratios in identifying mergers (e.g., Lin et al 2004; Bell et al. 2006; Jogee et al. 2009; Robaina et al. 2010) as stellar masses are readily measured for a large number of galaxies. However, with the advent of ALMA, it will be increasingly possible to incorporate the cold gas mass for a large number of galaxies.

\subsubsection{Cold Gas Mass in Coma Galaxies Versus Model Galaxies}

In the SAM used in this study, the cold gas fraction $f_{\text {gas }}$ (defined as the ratio of cold gas to the baryonic masss made of cold gas, hot gas, and stars $)$ and the ratio $\left(M_{\text {cold_gas }} / M_{\star}\right)$ of cold gas to stellar mass are both overly high, The issue of high cold gas fraction in this model was highlighted and discussed in Wang, Weinmann, \& Neistein (2012). Here, we quantify how far off the model values are compared with what is expected for a rich cluster like Coma.

For instance, $f_{\text {gas }} \sim 60-80 \%$ at $M_{\star}<2 \times 10^{10} M_{\odot}$. Figure 3.20 illustrates the degree to which the ratio $\left(M_{\text {cold_gas }} / M_{\star}\right)$ is overestimated by comparing with data from Boselli et al. (1997), who measure HI and $H_{2}$ masses for Coma cluster member galaxies and non-cluster galaxies. The top panel shows that the average ratio of cold gas to stellar mass $\left(M_{\text {cold_gas }} / M_{\star}\right)$ ranges from $\sim 1-12$ for a representative model cluster. The bottom panel shows that the ratio of $M_{H I+H_{2}} / M_{\star}$ for Coma cluster galaxies is usually $<0.1$, with a median value of 0.04. Non-cluster galaxies from Boselli et al. (1997) are more more gas rich (median $M_{H I+H_{2}} / M_{\star} \sim 0.09$ ), but the ratio of $M_{H I+H_{2}} / M_{\star}$ is still $\ll 1$. Thus, the 
amount of cold gas in the model is unrealistically high. At $10^{10} \lesssim M_{\star} \lesssim 10^{11} M_{\odot}$, the model predicts a cold gas to stellar mass ratio that is a factor $\sim 25-87$ times higher than the median in Coma cluster galaxies by Boselli et al. (1997). This issue could cause systematic effects during merger-induced starbursts, which are modeled as a function of the absolute amount of cold gas mass.

\subsubsection{Data Versus Model Predictions for Stellar Mass in Dynamically Hot and ColdComponents}

We next proceed to compare the observed versus model predictions for the relative distribution of stellar mass between high $n>n_{\text {disk_max }}$, dynamically hot classical bulges/ellipticals and low $n \leq n_{\text {disk_max }}$ disk-dominated components. The following comparisons are all made in the central 0.6 Mpc of Coma and or the model clusters.

We first start by describing how the model builds bulges and ellipticals. In the model, the total bulge stellar mass $M_{\star, \text { Bulge,model }}$ consists of stellar mass accreted in major and minor mergers, plus stellar mass from SF induced in both types of mergers. The bulge in the model is considered to be made of the following

$$
\begin{aligned}
& M_{\star, B u l g e}=\left(M_{\star_{-} B \text { Bulge_ACC }}\right)+M_{\star_{-} B \text { Bulge_SF }} \\
& =\left(M A J O R \_A C C+M I N O R_{-} A C C\right) \\
& +(M A J O R S F+M I N O R S F)
\end{aligned}
$$

where MAJOR_ACC is the total stellar mass in both progenitors in the last major merger, MINOR_ACC is the stellar mass coming in satellites from all minor mergers that occur after the last major merger, MAJORSF is the stellar mass from the SF episode in last major merger, and MINORSF is the stellar mass for bursts of SF induced in all minor mergers after the last major merger. The physics underlying each term has been discussed 
in detail in Section 3.5.1.

Next we discuss how to compare the model with the data. In the data, we have the stellar mass of Coma galaxies distributed between low $n \leq n_{\text {disk_max }}$ disk-dominated structures and high $n>n_{\text {disk_max }}$ classical bulges/ellipticals. For our sample of Coma galaxies (excluding the $2 \mathrm{cD}$ systems) with $M_{\star} \geq 10^{9} M_{\odot}$, we compute the ratio $\mathrm{R} 1_{\text {data }}$ defined below.

$$
\mathrm{R} 1_{\text {data }}=\frac{\mathrm{M}_{\star} \text { in high } \mathrm{n}>\mathrm{n}_{\text {disk } \_ \text {max }}}{\mathrm{M}_{\star} \text { of galaxies }} .
$$

The reasons for not including the cD systems were discussed in Section 3.2.2 and include the fact that $c D s$ have uncertain stellar masses and are very different from regular galaxies (E, S0s, spirals) in terms of structure, assembly, and contribution from intra-cluster light. As discussed in Section 3.4.2, $\mathrm{R} 1=56 \%$.

We next compare this ratio to the corresponding quantity in the model. The comparison is not entirely straightforward as the model does not give a Sérsic index. We therefore have to associate components in the model, given in Equation 3.7, to the corresponding high $n>n_{\text {disk_max }}$ classical bulges/ellipticals in the data. The most natural step is to assume that the stellar mass built during major mergers in Equation 3.7 is redistributed into such high- $n$ components We therefore calculate the model ratio $\mathrm{R} 22_{\text {model }}$ :

$$
\mathrm{R} 2_{\text {model }}=\frac{(\text { MAJOR_ACC }+ \text { MAJORSF })}{\mathrm{M}_{\star} \text { in galaxies }}
$$

We find that for $M_{\star} \geq 10^{9} M_{\odot}, \mathrm{R} 2_{\text {model }}$ has a wide dispersion: $\sim 23-76 \%$ for the 30 model clusters shown in Figure 3.18, with a median value of $\sim 57 \%$. The representative cluster (i.e., the best matching model to the cumulative density profile in Coma) discussed in Section 3.5.4 and Figures 3.19-3.20 has a value of $\sim 70 \%$. 
We can get some guidance on the matter of Sérsic index from Hopkins et al. (2009b), who discuss that in the general case of an unequal mass merger, the coalescence of the smaller progenitor (mass $M_{2}$ ) with the center of the primary will destroy (i.e., violently relax) the smaller galaxy and also potentially violently relax an additional mass $\leq M_{2}$ belonging to the primary. The stars that are violently relaxed in the minor merger become part of the bulge in the primary galaxy. Note, the amount of mass in the primary that is violently relaxed depends on the structure of the galaxy. In the case of a bulge-dominated primary galaxy, the stars are merely heated but not relaxed at all. For our work here, if we consider the extreme case where where all the stellar mass accreted during a minor mergers can be considered to be associated with a high- $n$ classical bulge/elliptical, we can compute and compare $\mathrm{R} 3_{\text {model }}$ to the data:

$$
\begin{aligned}
& R 3_{\text {model }}= \\
& \frac{\left(M A J O R \_A C C+M A J O R S F+M I N O R \_A C C\right)}{M_{\star} \text { in galaxies }} .
\end{aligned}
$$

We find that for $M_{\star} \geq 10^{9} M_{\odot}$, adding in the stellar mass accreted in minor mergers makes only a modest difference. $\mathrm{R} 3_{\text {model }}$ is only slightly higher than $\mathrm{R} 2_{\text {model }}$ by a few percent. $\mathrm{R} 33_{\text {model }}$ ranges from $\sim 23-82 \%$, with a median value of $\sim 61 \%$, and the representative cluster (Section 3.5.4, Figures 3.19-3.20) has a value of $\sim 71 \%$.

The comparison of $\mathrm{R} 1_{\text {data }}$ with $\mathrm{R} 2_{\text {model }}$ and $\mathrm{R} 3_{\text {model }}$ is a global comparison of the total stellar mass fraction within high-n components summed over all the galaxies with $M_{\star} \geq 10^{9} M_{\odot}$. Next, we push the data versus model comparison one step further by doing it in bins of stellar mass, as shown in Figure 3.21.

The top panel of Figure 3.21 plots the mean ratio of stellar mass fraction in dynamically hot components $\left(f_{\star, \text { hot }}\right)$ as a function of total galaxy stellar mass, for data versus 
model. For each stellar mass bin shown in Figure 3.21, the value of $f_{\star \text {,hot }}$ is calculated for each galaxy in the data and model. The mean value is taken to be

$$
\begin{aligned}
& \text { Mean fraction } f_{\star, \text { hot }} \text { in a given galaxy mass bin } \\
& f_{\star, h o t}=\sum_{i=0}^{N} \frac{M_{\star, h o t, i}}{M_{\star, i}} .
\end{aligned}
$$

In the data, $M_{\star, \text { hot }}$ is taken as the stellar mass of any high $n>n_{\text {disk_max }}$ classical bulge/elliptical component in the galaxy. The model shown here is the best cluster model matched by cumulative galaxy number density (see Figure 3.18, column 1). For this model, two lines are shown: the solid line takes $M_{\star, \text { hot }}$ as the stellar mass $\left(M A J O R \_A C C+\right.$ MAJORSF) accreted and formed during major mergers in Equation 3.7, while the dotted line also adds in the stellar mass accreted during minor mergers so that $M_{\star \text {,hot }}$ is $\left(M A J O R_{-} A C C+M A J O R S F+M I N O R \_A C C\right)$.

From the top panel of Figure 3.21, there is significant disagreement between the fractions of $f_{\star, \text { hot }}$ for the Coma data and the model. This fraction in the Coma data ranges from $\sim 30 \%$ to $54 \%$ over $10^{9} \leq M_{\star} \leq 8 \times 10^{10}$. In the cluster model, the corresponding fraction is significantly less, $\sim 7-35 \%$, over the same mass range. As shown by the second dotted model curve in Figure 3.21, adding in the stellar mass accreted in minor mergers to the model only changes the fraction by a few percent. The values of $f_{\star, \text { hot }}$ are chiefly representative of the contributions from major mergers.

The bottom panel of Figure 3.21 plots the mean ratio of stellar mass fraction $f_{\star, \text { cold }}$ in dynamically cold flattened components as a function of total galaxy stellar mass, for data versus model. We compute this mean as

Mean fraction $f_{\star, \text { cold }}$ in a given galaxy mass bin

$$
f_{\star, \text { cold }}=\sum_{i=0}^{N} \frac{M_{\star, \text { cold }, i}}{M_{\star, i}}
$$


In the data, $M_{\star, \text { cold }}$ is taken as the stellar mass of any low $n \leq_{\text {disk_max }}$ diskdominated component in the galaxy. In the model, the two lines show two different expressions for $M_{\star, \text { cold }}$. For the solid line, we take $M_{\star, \text { cold }}$ to be the mass of the outer disk $M_{\star, \text { outer_disk }}$, which is given by $\left(M_{\star}-M_{\star, \text { Bulge,model }}\right)$ and represents the difference between the bulge mass given in Equation 3.7 and the total stellar mass. One problem with this approach is that it only considers the outer disk in $M_{\star, \text { cold }}$, but it ignores small-scale nuclear disks formed in the bulge region. We tackle this problem by defining a second dotted model line, where we consider $M_{\star, \text { cold }}$ to be the mass $M_{\star, \text { all_disk }}$, of inner and outer disks. We compute the latter mass as the sum $\left(M_{\star, \text { outer_disk }}+\right.$ MINORSF $)$, where MINORSF was defined in Equation 3.7 as the mass of stars formed via induced SF during minor mergers.

From the bottom panel of Figure 3.21, it appears that the model overpredicts the mass in disks as a function of galaxy stellar mass. The fraction $f_{\star, \text { cold }}$ in Coma ranges from $\sim 45-69 \%$ over $10^{9} \leq M_{\star} \leq 8 \times 10^{10}$, after removing the $2 \mathrm{cD}$ galaxies. Over the same stellar mass range, the corresponding values in the model is $\sim 90 \%$ to $\sim 52 \%$ for just the mass in $M_{\star, \text { outer_disk }}$, and $\sim 93 \%$ to $\sim 65 \%$ for the mass in $M_{\star, \text { outer_disk }}+$ MINORSF. Note the contribution to $f_{\star, \text { cold }}$ from MINORSF $\lesssim 17 \%$ in a stellar mass given bin.

The main conclusion from Figure 3.21 is that the best-matching cluster model is underpredicting over a wide range in galaxy stellar mass $\left(10^{9} \leq M \star \lesssim 8 \times 10^{10} M_{\odot}\right)$ the mean fraction $f_{\star, \text { hot }}$ of stellar mass locked in hot components by a factor of $\gtrsim 1.5$. Similarly these model overpredicts the mean value for $f_{\star, \text { cold }}$. While the discussion in this section focused only on a single model cluster, the results and conclusions would be similar if we had analyzed alternate simulated clusters, such as those matched to cluster galaxy stellar mass function (see Figure 3.18, column 2) or halo parameters (see Figure 3.18, column 3). 
One explanation for the underprroeduction of $f_{\star, \text { hot }}$ could be that the model ignores classical bulge production via the merging of star forming clumps (Bournaud, Elmegreen, \& Elmegreen 2007; Elmegreen et al. 2009). Another possibility is that $f_{\star, \text { cold }}$ is being overproduced. The absence of cluster processes (especially ram-pressure stripping and tidal stripping) would reduce a galaxy's cold gas reservoir. This means that SF in gas that would otherwise be removed from the galaxy builds additional dynamically cold stellar mass following the last major merger. Both issues could be simultaneously important to resolving the discrepancy, and this possibility underscores the need to implement in theoretical models more comprehensive models of classical bulge assembly as well as environmental processes, such as ram-pressure stripping and tidal stripping, that affect the cold gas content of galaxies.

\subsection{Summary \& Conclusions}

We present a study of the Coma cluster in which we constrain galaxy assembly history in the central $0.6 \mathrm{Mpc}$ by performing multi-component structural decomposition on a mass-complete sample of 69 galaxies with stellar mass $M_{\star} \geq 10^{9} M_{\odot}$. Some strengths of this study include the use of superb high-resolution (0"1), F814W images from the HST ACS Treasury Survey of the Coma cluster, and the adoption of a multi-component decomposition strategy where no a priori assumptions are made about the Sérsic index of bulges, bars or disks. We use structural decomposition to identify the two fundamental kinds of galaxy structure: low- $n(n \lesssim 1.7)$ dynamically cold, disk-dominated components (large-scale disks, inner disks, disky pseudobulges, and bars) as well as high- $n(n \gtrsim 1.7)$ puffed-up, dynamically hot classical bulges/ellipticals. We explore the effect of environment by comparing the morphology density relation and properties of disks and bulges in Coma to low-density 
environments (LDEs) such as Virgo and the field. We also compare our empirical results on galaxies in the center of the Coma cluster with theoretical predictions based on combining the Millennium cosmological simulations of dark matter with baryonic physics from a semianalytical model. Our methodology and main results are summarized below.

\section{Methodology and decomposition results:}

We start with modeling galaxy structure as a single Sérsic profile, and add one or two additional Sérsic profiles only if there is evidence of of coherent structure (inner/outer disks, bulges, bars) that require extra components. In our multiple-component $2 \mathrm{D}$ structural decomposition, we define disk-dominated structures as components with a low Sérsic index $n$ below an empirically determined threshold value $n_{\text {disk_max }} \sim 1.7$ (Sections 3.3.1 and 3.3.4.2).

Galaxies with an outer disk are called spirals or S0s, and in such systems the photometric bulge is defined as the component hosting the excess light above the outer disk and bar. Outer disks have Sérsic index in the range $0.25-1.66$ with a median value of 0.84. The majority $(67.3 \pm 7.9 \%)$ of outer disks have shallow profiles with Sérsic index $n<1$, and many $(19.2 \pm 12.5 \%)$ have $n<0.5$ (Figure 3.11). We find $50.0 \pm 6.9 \%$ of outer disk galaxies are barred, and this is consistent with the bar fraction in Coma derived by Marinova et al. (2012).

Galaxies without an outer disk are considered photometric ellipticals or $\mathrm{cD}$ galaxies. Such systems in Coma have outer components whose Sérsic index ranges from 1.83 to 6.95, with a median value of 2.10. A large fraction (66.7 $\pm 13.6 \%$ ) of ellipticals contain inner disk-dominated components with Sérsic index $n \lesssim 1.7$, and these systems are 
like the extra-light ellipticals discussed by Hopkins et al. (2009a) and Kormendy et al. (2009).

Over half $(53.6 \pm 6.00 \%)$ of the galaxies in our sample require a nuclear point source in the final multi-component model. The derived point source luminosity correlates with the total galaxy luminosity (Section 3.3.4.3, Figure 3.13).

We find that the 2 cDs (NGC 4874 and NGC 4889) can be modeled with a component of high Sérsic index $n(n \gg 1.7)$. This high $n$ arises from an extended low surface brightness envelope, which is believed to result from repeated bouts of galactic cannibalism and tidal stripping of satellite galaxies in a cluster.

2. Breakdown of stellar mass in Coma between low-n disk-dominated structures and high$n$ classical bulges/ellipticals:

We make the first attempt (Section 3.4.2 and Tables 3.6-3.7) at exploring the distribution of stellar mass in Coma in terms of high- $n$, puffy and dynamically hot classical bulges/ellipticals built by processes associated with major mergers and possibly also minor mergers versus low- $n$, flattened and dynamically cold, disk-dominated structures (e.g. outer disks, inner disks, disky pseudobulges, and bars) built by by gas-rich dissipative processes. After excluding the $2 c D s$, we find that in the central $0.6 \mathrm{Mpc}$ of the Coma cluster, galaxies with stellar mass $M_{\star} \geq 10^{9} M_{\odot}$ have $56 \%$ of the cumulative stellar mass locked up in high-n $(n \gtrsim 1.7)$ classical bulges/ellipticals while the remaining $44 \%$ is in the form of low- $n(n \lesssim 1.7)$ disk-dominated structures (outer disks, inner disks, disky pseudobulges, and bars). It is particularly intriguing that there was time for secular processes to build disky pseudobulges before most spirals 
transformed into S0s.

Thus, most (at least 56\%) of the stellar mass in Coma galaxies appears to have been assembled and shaped through the redistribution of stars during violent major mergers and possibly also minor mergers. The fraction (44\%) of stellar mass in disk-dominated components is significant and underscores that even in the rich environment of the Coma cluster, an important role is played by gas-rich dissipative processes that build outer and inner disks, such as cold streams, gas accretion, settling of residual gas left over in gas-rich mergers, and gas inflows during secular processes, mergers, and tidal interactions

In citing the above ratios, we exclude the cDs because they have uncertain stellar masses and they are very different from regular galaxies in terms of structure, assembly, and contribution from intra-cluster light. If we include the cDs in our Coma study, the stellar mass in the high- $n$ components would rise from $56 \%$ to at least $72 \%$.

\section{Impact of environment: morphology-density relation:}

Using our structural decomposition to assign galaxies the Hubble types E, S0, or spiral, we find evidence of a strong morphology-density relation. In the central 0.6 Mpc of the Coma cluster, there are 2 cDs (NGC 4874 and NGC 4889), spirals are rare, and the morphology breakdown of $(\mathrm{E}+\mathrm{S} 0)$ :spirals is $(22.4 \%+70.2 \%): 7.4 \%$ by numbers and $(28.0 \%+66.3 \%): 5.8 \%$ by stellar mass (Section 3.4 .1 and Table 3.5$)$. In the center of Coma, the frequency of spiral galaxies is only $\sim 7 \%$, and they contain less than $6 \%$ of the stellar mass, while lower-density environments are typically dominated by spirals. 
4. Impact of environment: properties of outer disks of S0s/spirals:

In the central parts of Coma, a high fraction $(67.3 \pm 7.9 \%)$ of large-scale outer disks have low Sérsic index $n<1$ (Figure 3.11) compared with LDEs. This is an upper limit due to projection effects. At a given galaxy stellar mass, the average $i$-band half-light radius $\left(r_{\mathrm{e}}\right)$ of the outer disk in S0s/spirals is $\sim 40-80 \%$ smaller and the average disk $i$-band luminosity is fainter by $\sim 42-70 \%$ in Coma than in LDEs (Figure 3.14). These properties are likely indicative of environmental processes (e.g., ram-pressure stripping, tidal stripping, strangulation) that suppress disk growth or truncate disks (Section 3.4.5).

This conclusion for Coma nicely parallels studies of ram-pressure stipping (Cayette et al. 1990, 1994; Kenney et al. 2004, 2008; Chung et al. 2007, 2009) and dynamical heating (Kormendy \& Bender 2012) in the less extreme Virgo cluster.

\section{Impact of environment: properties of bulges in S0s:}

For the bulges of S0s and spirals, the ratio $R$ of stellar mass in high- $n(n \gtrsim 1.7)$ classical bulges to low- $n(n \lesssim 1.7)$ disky pseduobulges is 13.3 (Section 3.4.3, Tables 3.6-3.7). For the same galaxy stellar mass range $\left(M_{\star} \geq 7.5 \times 10^{9} M_{\odot}\right)$, the value of $R$ in S0s is 3 times higher in Coma than in lower density environments (field and Virgo S0s).

We also find that at a given bulge Sérsic index $n$, the bulge-to-total ratio $B / D$, and the $i$-band light ratio are offset to higher values in Coma compared with LDEs. This effect appears to be due to the above-mentioned lower disk luminosity in Coma. 
6. Comparison of data to theoretical predictions: We compare our empirical results on galaxies in the center of the Coma cluster with theoretical predictions based on combining the Millennium cosmological simulations of dark matter (Springel et al. 2005) with baryonic physics from a semi-analytical model (Neistein \& Weinmann 2010; Wang, Weinmann, \& Neistein 2012).

Among the 160 simulated clusters with halo mass in the range $5 \times 10^{14}-10^{16} M_{\odot}$, there is no model cluster that can simultaneously match the global properties (halo properties, cumulative galaxy number density and galaxy stellar mass function), of Coma, our local benchmark for one of the richest nearby galaxy clusters (Figures 3.17 and 3.18). However, it is striking that the model does well reproduce the spatial distributions(i.e., cumulative density profile) of Coma while failing to simultaneously match other global properties.

We confirm the suggestion of Hopkins et al. (2009b) that the galaxy merger history is highly dependent on the mass used (stellar mass, baryonic mass, halo mass) in the definition of the merger mass ratio $M_{1} / M_{2}$. In particular, we find that the cumulative percentage of galaxies with a major merger since redshift $z \sim 4$ can be a factor of $\gtrsim 5$ times larger if one uses the mass ratio of (cold gas plus stars) as opposed to the mass ratio of stars (Figure 3.19). Traditionally, observers have tended to use stellar mass ratios in identifying mergers, but with the advent of ALMA, it will be increasingly possible and important to incorporate the cold gas mass.

One limitiation of the model is that classical bulges are not produced from violent disk instabilities whereby star-forming clumps merge at the center of a galaxy (Bournaud, Elmegreen, \& Elmegreen 2007; Elmegreen et al. 2009). Another strong limitation 
of the model is that it predicts a ratio $\left(M_{\text {cold_gas }} / M_{\star}\right)$ of cold gas to stellar mass that is very high compared with what is expected for a rich cluster. For instance, at $10^{10} \lesssim M_{\star} \lesssim 10^{11} M_{\odot}$, the model predicts a $\left(M_{\text {cold_gas }} / M_{\star}\right)$ ratio of $\sim 1-12$, which is a factor $\sim 25-87$ times higher than the median in Coma cluster galaxies by Boselli et al. (1997).

For representative "best-match" simulated clusters, we compare the empirical and theoretically predicted fraction $f_{\star, \text { hot }}$ and $f_{\star, \text { cold }}$ of stellar mass locked, respectively, in high- $n$, puffy and dynamically hot, classical bulges/ellipticals versus low- $n$ dynamically cold disk-dominated components for galaxies in different stellar mass bins. We assume that hot high- $n$ components in the model stem from stellar mass accreted during major and minor mergers, and new stars formed during minor mergers. Over a wide range of galaxy stellar mass $\left(10^{9} \leq M \star \lesssim 8 \times 10^{10}\right.$, the model underpredicts the mean fraction $f_{\star, \text { hot }}$ of stellar mass locked in hot components by a factor of $\gtrsim 1.5$. Similarly the model overpredicts the mean value for $f_{\star, \text { cold }}$ (Section 3.5.6 and Figure 3.21). We speculate that the deficiency of hot stellar components in the model is due to ignoring classical bulge production via the merging of star-forming clumps. Adding this process would increase $f_{\star \text {,hot }}$ at all stellar masses. At the same time, $f_{\star \text {,cold }}$ might be being overproduced. The uncertainty here underscores the need to improve theoretical models by allowing for bulge assembly through modes other than galaxy mergers as well environmental processes, such as ram-pressure stripping and tidal stripping, which impact the cold gas content of galaxies. 
Table 3.1 Properties of Cored Ellipticals

\begin{tabular}{|c|c|c|c|}
\hline $\begin{array}{c}\text { Galaxy Name } \\
(1)\end{array}$ & $\begin{array}{c}\text { Core-Sérsic }\left(\gamma, r_{b}\right) \\
(2)\end{array}$ & $\begin{array}{l}\text { 2D Sérsic profile w/o } \\
\text { Core Masked }\left(n, r_{e}\right) \\
(3)\end{array}$ & $\begin{array}{l}\text { 2D Sérsic Profile w/ } \\
\text { w/ Core Masked }\left(n, r_{e}\right) \\
(4)\end{array}$ \\
\hline COMAi125909.468p28227.35 & $(0.16,0 . ! 13)$ & $\left(2.54,4{ }^{\prime \prime} 2\right)$ & $\left(2.54,4 .{ }^{\prime \prime} 20\right)$ \\
\hline NGC 4874 (ACS F814W) & $\left(0.15,1^{\prime \prime} 40\right)$ & $(2.89,35 . " 4)$ & $\left(11.4,875^{\prime \prime}\right)$ \\
\hline NGC 4874 (SDSS $i$-band) & $\left(0.15,2^{\prime \prime} 32\right)$ & $\left(4.30,88^{\prime \prime} .3\right)$ & $\left(4.70,107^{\prime \prime}.\right)$ \\
\hline NGC 4889 (SDSS $i$-band) & $\left(0.06,1{ }^{\prime \prime} 88\right)$ & $(3.90,42 . .9)$ & $\left(7.80,1299^{\prime \prime}\right)$ \\
\hline
\end{tabular}


Table 3.2. Single Sérsic Profile Structural Parameters

\begin{tabular}{|c|c|c|c|}
\hline $\begin{array}{c}\text { Galaxy Name } \\
\text { (1) }\end{array}$ & $\begin{array}{c}\text { F814W Magnitude } \\
\text { (2) }\end{array}$ & $\left(\begin{array}{l}r_{\mathrm{e}} \\
\mathrm{kpc}) \\
(3)\end{array}\right.$ & $(40$ \\
\hline COMAi125950.183p275445.52 & & & \\
\hline COMAi125946.943p275930.90 & 17.02 & 1.49 & 1.65 \\
\hline COMAi1 $25937.200 \mathrm{p} 275819.97$ & & 0.29 & \\
\hline COMAi125930.270p 28115.17 & 17.46 & 1.04 & 1.90 \\
\hline & & & \\
\hline COMAi13034.430p 275604.95 & 16.65 & 2.11 & \\
\hline COMAi125959.476p 275626.02 & 16.53 & & 2.30 \\
\hline COMAi $13030.954 \mathrm{p} 28630.22$ & 17.07 & 1.41 & 1.94 \\
\hline COMAi125937.010p28106.95 & 17.34 & 0.69 & 2.08 \\
\hline COMAi125845.533p 274513.75 & 16.56 & 2.14 & 2.09 \\
\hline $\begin{array}{l}\text { COMAi13035.420p275634.06 } \\
\text { (2) }\end{array}$ & 16.83 & 1.92 & 1.81 \\
\hline $\begin{array}{l}\text { COMAAi1 } 25931.103 \mathrm{p} 275718.12 \\
\text { COMAi125931.8932 } 275140.72\end{array}$ & $\begin{array}{l}16.87 \\
16.78\end{array}$ & $\begin{array}{l}2.47 \\
0.94\end{array}$ & $\begin{array}{l}1.61 \\
1.88\end{array}-1250$ \\
\hline COMAi13018.545 D28549.62 & 16.64 & 1.62 & \\
\hline COMAi13000.949p 275643.85 & 16.40 & 2.00 & 3.38 \\
\hline COMAi125844.578p 274458.31 & 17.56 & 1.65 & 5.39 \\
\hline COMAi13018.873p28033.38 & 16.58 & 0.38 & 3.29 \\
\hline COMAi13018.883p28033.55 & 16.51 & 0.45 & 3.94 \\
\hline COMAi13024.823p275535.94 & 16.41 & 2.68 & 2.15 \\
\hline $\begin{array}{l}\text { COMAi13021.673p275354.81 } \\
\text { COMAi125937.990p } 28003.52\end{array}$ & $\begin{array}{l}16.37 \\
16.53\end{array}$ & $\begin{array}{l}1.46 \\
1.16\end{array}$ & 2.57 \\
\hline $\begin{array}{l}\text { COMAi125937.990p } 28003.52 \\
\text { COMAi13011.143p28354.91 }\end{array}$ & $\begin{array}{l}16.53 \\
16.38\end{array}-120$ & $\begin{array}{l}1.16 \\
1.42\end{array}$ & $\begin{array}{l}2.00 \\
2.12\end{array}$ \\
\hline COMAi12511.543p28033.32 & 16.40 & 1.22 & 1.84 \\
\hline COMAi13051.149p28249.90 & 15.52 & 5.35 & 3.17 \\
\hline COMAi125844.318p 274501.18 & 17.54 & 1.12 & 5.05 \\
\hline COMAi125904.797p28301.16 & 16.09 & 2.16 & 2.28 \\
\hline COMAi125909.468p28227.35 & 15.94 & 1.88 & 2.54 \\
\hline COMAi125935.286p275149.13 & 15.96 & 1.06 & 1.54 \\
\hline $\begin{array}{l}\text { COMAi130411.193p } 28242.34 \\
\text { COMAi125950.105p275529.44 }\end{array}$ & $\begin{array}{l}16.83 \\
15.62\end{array}$ & $\begin{array}{l}0.86 \\
1.35\end{array}$ & $\begin{array}{l}1.66 \\
2.32\end{array}$ \\
\hline COMAi13005.405p28128.14 & $\begin{array}{l}15.02 \\
15.11\end{array}$ & $\begin{array}{l}2.35 \\
2.61\end{array}$ & 2.58 \\
\hline & 15.09 & 3.23 & 2.57 \\
\hline COMAi125940.270p 275805.71 & 15.04 & 2.52 & 5.94 \\
\hline COMAi125944.208p 275730.38 & 14.60 & 3.55 & \\
\hline COMAi125938.321p275913.89 & 14.93 & 3.67 & 3.50 \\
\hline COMAi125928.721p 28225.92 & 14.95 & 1.67 & 2.97 \\
\hline COMAi13044.632p 28602.31 & 14.86 & 1.82 & 2.84 \\
\hline $\begin{array}{l}\text { COMAi125939.659p275714.03 } \\
\text { COM } 25942.301275529 .1259\end{array}$ & $\begin{array}{l}15.05 \\
1.56\end{array}$ & 1.33 & $\begin{array}{l}3.65 \\
7.49\end{array}$ \\
\hline COMAi13017.014p28350.07 & 14.75 & 1.35 & 3.91 \\
\hline COMAi125943.721p275940.82 & 14.48 & 1.38 & 3.81 \\
\hline COMAi1 $25956.697 \mathrm{p} 275548.71$ & 14.35 & 4.71 & \\
\hline COMAi13027.966p 275721.56 & 14.51 & 1.55 & 4.68 \\
\hline COMAi13016.534p275803.15 & 14.24 & 2.98 & 4.58 \\
\hline COMAi13012.868p28431.74 & & 1.40 & \\
\hline COMAi13038.761p28052.34 & 13.87 & 5.34 & 4.31 \\
\hline $\begin{array}{l}\text { COMAi13006.395p28015.94 } \\
\text { COMAi13028.370p } 275820.64\end{array}$ & $\begin{array}{r}13.82 \\
14.24\end{array}$ & $\begin{array}{l}5.01 \\
4.29\end{array}$ & $\begin{array}{r}7.52 \\
4.04\end{array}$ \\
\hline $\begin{array}{l}\text { COMAi13028.370p275820.64 } \\
\text { COMAi13042.8320275746.95 }\end{array}$ & $\begin{array}{l}14.24 \\
14.17\end{array}$ & $\begin{array}{l}4.29 \\
2.70\end{array}$ & \\
\hline $\begin{array}{l}\text { COMAini3042.832p27575746.95 } \\
\text { COMAi13014.746p28228.69 }\end{array}$ & 14.06 & 1.58 & 4.70 \\
\hline COMAi125931.453p 28247.60 & 14.22 & 1.82 & 2.84 \\
\hline COMAi13018.093p275723.59 & 14.41 & & \\
\hline COMAi125852.097p 274706.15 & 14.02 & 1.90 & 3.29 \\
\hline
\end{tabular}


Table 3.2 (cont'd)

\begin{tabular}{|c|c|c|c|}
\hline $\begin{array}{c}\text { Galaxy Name } \\
\text { (1) }\end{array}$ & $\begin{array}{c}\text { F814W Magnitude } \\
\text { (2) }\end{array}$ & $\left(\begin{array}{l}r_{\mathrm{e}} \\
\mathrm{kpc})\end{array}\right.$ & \\
\hline 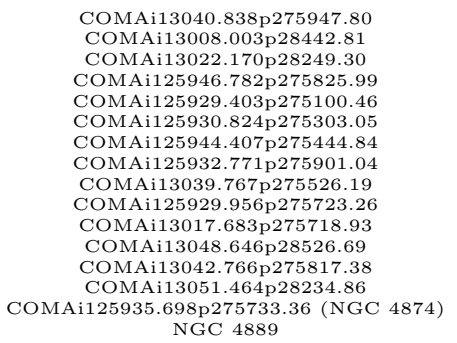 & $\begin{array}{l}14.00 \\
14.10 \\
13.84 \\
13.83 \\
13.87 \\
13.61 \\
13.67 \\
13.20 \\
13.44 \\
13.32 \\
13.28 \\
13.30 \\
13.43 \\
13.07 \\
10.96 \\
10.57\end{array}$ & $\begin{array}{l}3.15 \\
1.13 \\
2.97 \\
3.38 \\
1.68 \\
1.54 \\
2.26 \\
5.50 \\
2.93 \\
3.85 \\
2.13 \\
2.21 \\
2.37 \\
2.85 \\
17.35 \\
22.65\end{array}$ & $\begin{array}{l}3.27 \\
2.59 \\
4.08 \\
4.33 \\
4.07 \\
3.77 \\
2.96 \\
5.86 \\
3.64 \\
4.89 \\
2.85 \\
3.02 \\
4.00 \\
3.92 \\
3.05 \\
4.37\end{array}$ \\
\hline
\end{tabular}

Note. - Rows are sorted by increasing $M_{\star}$. 
Table 3.3 Distribution of Best-Fit Structural Decompositions for Stellar Mass $M_{\star} \geq 10^{9}$ $M_{\odot}$

\begin{tabular}{|c|c|c|c|c|c|c|c|}
\hline $\begin{array}{c}\text { Morphology } \\
\text { (1) }\end{array}$ & $\begin{array}{c}\text { Number Per Bin } \\
(2) \\
\end{array}$ & $\begin{array}{c}\text { Stage } 1 \\
\text { w/o } \\
\text { Point Source } \\
(3) \\
\end{array}$ & $\begin{array}{c}\text { Stage } 1 \\
\text { w/ } \\
\text { Point Source } \\
(4) \\
\end{array}$ & $\begin{array}{c}\text { Stage } 2 \\
\text { w/o } \\
\text { Point Source } \\
(5) \\
\end{array}$ & $\begin{array}{c}\text { Stage } 2 \\
\text { w/ } \\
\text { Point Source } \\
(6) \\
\end{array}$ & $\begin{array}{c}\text { Stage } 3 \\
\text { w/ } \\
\text { Point Source } \\
(7) \\
\end{array}$ & $\begin{array}{c}\text { Stage } 3 \\
\text { w/o } \\
\text { Point Source } \\
(8) \\
\end{array}$ \\
\hline All Galaxies & 69 & 3 & 3 & 14 & 23 & 15 & 11 \\
\hline \multicolumn{8}{|c|}{ In terms of galaxy types G1 to G5 } \\
\hline G1: photometric disk & 1 & 0 & 1 & 0 & 0 & 0 & 0 \\
\hline G2: photometric E & 5 & 3 & 2 & 0 & 0 & 0 & 0 \\
\hline G3: unbarred So, spiral & 25 & 0 & 0 & 8 & 17 & 0 & 0 \\
\hline G4: barred So, spiral & 26 & 0 & 0 & 0 & 0 & 15 & 11 \\
\hline $\begin{array}{l}\text { G5: photometric } \mathrm{E} \text { with } \\
\text { extra inner component }\end{array}$ & 12 & 0 & 0 & 6 & 6 & 0 & 0 \\
\hline \multicolumn{8}{|c|}{ In terms of Hubble types cD, E, So, and spiral } \\
\hline $\mathrm{cD}$ & 2 & 2 & 0 & 0 & 0 & 0 & 0 \\
\hline Photometric E & 15 & 1 & 2 & 6 & 6 & 0 & 0 \\
\hline so & 47 & 0 & 1 & 8 & 14 & 15 & 9 \\
\hline Spiral & 5 & 0 & 0 & 0 & 3 & 0 & 2 \\
\hline
\end{tabular}


Table 3.4. Structural Parameters For the Best Model

\begin{tabular}{|c|c|c|c|c|c|c|c|c|c|}
\hline $\begin{array}{l}\text { Galaxy Name } \\
\text { (1) }\end{array}$ & $\mathrm{G} n$ & $\begin{array}{l}\text { Hubble Type } \\
\text { (3) }\end{array}$ & $\begin{array}{c}\mathrm{Pts} / T, C 1 / T, C 2 / T, \operatorname{Bar} / T \\
(\%, \%, \%, \%) \\
(4)\end{array}$ & $\begin{array}{l}\text { C1 } r_{\mathrm{e}} \\
\text { (kpc) } \\
(5)\end{array}$ & $\begin{array}{c}\text { C1 } n \\
(6)\end{array}$ & $\begin{array}{l}\mathrm{C} 2 r_{\mathrm{e}} \\
(\mathrm{kpc}) \\
(7)\end{array}$ & $\begin{array}{c}\text { C2 } n \\
(8)\end{array}$ & $\begin{array}{l}\operatorname{Bar} r_{e} \\
(\mathrm{kpc}) \\
(9)\end{array}$ & $\begin{array}{c}\text { Bar } n \\
(10)\end{array}$ \\
\hline COMAi125950.183p275445.52 & G4 & So & $(0.28,1.85,86.80,11.10)$ & 0.19 & 0.76 & 1.68 & 0.91 & 0.88 & 0.42 \\
\hline COMAi1 $25946.943 \mathrm{p} 275930.90$ & G3 & so & $(0.09,7.22,92.70,0.00)$ & 0.31 & 0.98 & 1.47 & 1.01 & & \\
\hline COMAi125937.200p275819.97 & G5 & $\mathrm{E}$ & $(0.00,79.90,20.10,0.00)$ & 0.31 & 6.20 & 0.20 & 0.89 & $\ldots$ & $\ldots$ \\
\hline COMAi125930.270p 28115.17 & G5 & $\mathrm{E}$ & $(0.46,89.20,10.30,0.00)$ & 1.13 & 1.83 & 0.62 & 0.70 & & \\
\hline $\begin{array}{l}\text { COMAi13018.351p28333.32 } \\
\text { COMAi13034.430p } 275604.95\end{array}$ & $\begin{array}{l}\text { G3 } \\
\text { G3 }\end{array}$ & $\begin{array}{l}\text { So } \\
\text { so }\end{array}$ & $\begin{array}{l}(0.05,42.50,57.40,0.00) \\
(0.00,40.80,59.20,0.00)\end{array}$ & $\begin{array}{l}0.85 \\
1.15\end{array}$ & 1.24 & 1.75 & 0.52 & & \\
\hline $\begin{array}{l}\text { COMAi113334.430p273604.95 } \\
\text { COMAi125959.476p275626.02 }\end{array}$ & $\begin{array}{l}\text { G3 } \\
\text { G3 }\end{array}$ & $\begin{array}{l}\text { So } \\
\text { so }\end{array}$ & $\begin{array}{l}(0.00,40.80,59.20,0.00) \\
(0.38,42.00,57.60,0.00)\end{array}$ & $\begin{array}{l}1.15 \\
1.07\end{array}$ & $\begin{array}{l}1.80 \\
1.67\end{array}$ & $\begin{array}{l}2.83 \\
2.67\end{array}$ & $\begin{array}{l}1.00 \\
0.72\end{array}$ & $\cdots$ & \\
\hline COMAi $13030.954 \mathrm{p} 28630.22$ & G4 & so & $(0.33,2.13,75.40,22.20)$ & 0.14 & 1.11 & 1.87 & 1.20 & 0.61 & 0.85 \\
\hline COMAi125937.010p28106.95 & G5 & $\mathrm{E}$ & $(0.51,91.10,8.42,0.00)$ & 0.79 & 2.13 & 0.35 & 0.51 & $\cdots$ & \\
\hline & & & & & & & & $\ldots$ & $\ldots$ \\
\hline COMAi13035.420p275634.06 & G3 & so & $(0.06,28.10,71.80,0.00)$ & 0.73 & 1.05 & 2.51 & 0.76 & $\ldots$ & $\ldots$ \\
\hline COMAi125931.103p275718.12 & G1 & so & $(0.11,0.00,100.00,0.00)$ & & & 2.38 & 1.52 & $\hat{n}$ & $\ldots$ \\
\hline COMAi125931.893p275140.76 & G5 & $\mathrm{E}$ & $(0.19,89.40,10.40,0.00)$ & 1.04 & 2.09 & 0.62 & 0.65 & & \\
\hline $\begin{array}{l}\text { COMAi13018.545p28549.62 } \\
\text { COMAi13000.949p275643.85 }\end{array}$ & $\begin{array}{l}\text { G3 } \\
\text { G3 }\end{array}$ & $\begin{array}{l}\text { so } \\
\text { so }\end{array}$ & $\begin{array}{l}(0.14,34.90,64.90,0.00) \\
(0.35,27.70,72.00,0.00)\end{array}$ & $\begin{array}{l}0.76 \\
0.47\end{array}$ & $\begin{array}{l}0.97 \\
1.56\end{array}$ & 2.83 & 0.86 & $\cdots$ & $\cdots$ \\
\hline $\begin{array}{l}\text { COMAi1 } 3300.949 \mathrm{p} 2776433.85 \\
\text { COMAi125844.578p } 274458.31\end{array}$ & $\begin{array}{l}\text { G3 } \\
\text { G3 }\end{array}$ & $\begin{array}{l}\text { So } \\
\text { so }\end{array}$ & $\begin{array}{l}(0.35,27.70,72.00,0.00) \\
(0.60,77.30,22.10,0.00)\end{array}$ & $\begin{array}{l}0.47 \\
0.76\end{array}$ & $\begin{array}{l}1.56 \\
4.30\end{array}$ & $\begin{array}{l}2.43 \\
0.78\end{array}$ & $\begin{array}{l}1.15 \\
0.78\end{array}$ & $\cdots$ & \\
\hline COMAi13018.873p28033.38 & G4 & so & $(0.00,54.20,34.70,11.10)$ & 0.18 & 2.95 & 0.86 & 1.04 & 0.51 & \\
\hline COMAi13018.883p28033.55 & G4 & so & $(0.00,78.20,12.10,9.67)$ & 0.30 & 3.74 & 0.97 & 0.35 & 0.51 & 0.46 \\
\hline COMAi13024.823p275535.94 & G3 & spiral & $(0.23,14.20,85.50,0.00)$ & 0.58 & 1.36 & 3.06 & 1.20 & & \\
\hline COMAi13021.673p 275354.81 & G3 & so & $(0.74,30.40,68.90,0.00)$ & 0.40 & 1.14 & 1.71 & 0.56 & & \\
\hline COMAi125937.990p 28003.52 & G4 & spiral & $(0.76,22.60,67.50,9.09)$ & 0.37 & 2.38 & 1.40 & 0.50 & 0.67 & 0.33 \\
\hline COMAi13011.143p28354.91 & G3 & so & $(0.13,19.20,80.70,0.00)$ & 0.66 & 2.64 & 1.48 & 1.40 & $\ldots$ & $\ldots$ \\
\hline COMAi125911.543p28033.32 & G3 & $\begin{array}{l}\text { So } \\
\text { so }\end{array}$ & $(0.19,23.90,75.90,0.00)$ & 0.54 & 0.95 & $\begin{array}{l}1.55 \\
5.98\end{array}$ & 1.21 & $\cdots$ & $\cdots$ \\
\hline $\begin{array}{l}\text { COMAMi13051.149p28249.90 } \\
\text { COMAi12584.318p } 274501.18\end{array}$ & $\begin{array}{l}\text { G3 } \\
\text { G5 }\end{array}$ & $\underset{\mathrm{S}}{\mathrm{E}}$ & $\begin{array}{l}(0.05,16.30,83.60,0.00) \\
(0.00,100.00,0.00,0.00)\end{array}$ & $\begin{array}{l}0.96 \\
1.65\end{array}$ & $\begin{array}{l}1.57 \\
1.99\end{array}$ & $\begin{array}{l}5.98 \\
0.14\end{array}$ & $\begin{array}{l}1.35 \\
1.86\end{array}$ & & \\
\hline COMAi125904.797p28301.16 & G4 & so & $(0.09,4.23,76.80,18.90)$ & $\begin{array}{l}1.00 \\
0.21\end{array}$ & 1.22 & $\begin{array}{l}0.14 \\
2.72\end{array}$ & $\begin{array}{l}1.80 \\
1.12\end{array}$ & 0.83 & 0.85 \\
\hline & G2 & $\mathrm{E}$ & $(0.00,100.00,0.00,0.00)$ & 1.88 & 2.54 & & & & \\
\hline COMAi125935.286p 275149.13 & G5 & $\mathrm{E}$ & $(0.05,85.80,14.10,0.00)$ & 1.37 & 2.08 & 0.70 & 0.31 & $\ldots$ & $\ldots$ \\
\hline COMAi13041.193p 28242.34 & G3 & spiral & $(0.29,30.60,69.10,0.00)$ & 0.41 & 1.08 & 1.11 & 0.84 & & \\
\hline COMAi125950.105p275529.44 & G4 & so & $(0.00,26.70,66.00,7.31)$ & 0.80 & 2.37 & 1.99 & 1.66 & 0.51 & 0.28 \\
\hline COMAi13005.405p28128.14 & G4 & So & $(0.00,14.20,72.20,13.60)$ & 0.35 & 1.18 & 3.60 & 0.92 & 1.29 & 0.45 \\
\hline COMAi13018.772p275613.34 & G4 & $\begin{array}{l}\text { so } \\
\text { so }\end{array}$ & $(0.00,9.13,69.10,21.80)$ & 0.49 & 0.98 & 2.87 & 0.69 & 1.64 & 0.60 \\
\hline 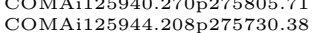 & $\begin{array}{l}\text { G4 } \\
\text { G5 }\end{array}$ & $\begin{array}{c}\text { SO } \\
\mathrm{E}\end{array}$ & $\begin{array}{l}(0.00,33.40,65.70,0.94) \\
(0.00,85.30,14.70,0.00)\end{array}$ & $\begin{array}{l}0.31 \\
5.43\end{array}$ & $\begin{array}{l}3.39 \\
5.82\end{array}$ & $\begin{array}{l}1.82 \\
1.95\end{array}$ & $\begin{array}{l}0.86 \\
0.56\end{array}$ & 0.48 & 0.14 \\
\hline COMAi125938.321p275913.89 & G3 & spiral & $(0.08,25.30,74.60,0.00)$ & $\begin{array}{l}5.43 \\
0.71\end{array}$ & $\begin{array}{l}5.82 \\
2.06\end{array}$ & 3.27 & 0.89 & & \\
\hline COMAi125928.721p28225.92 & G4 & so & $(0.00,25.30,33.70,41.00)$ & 0.40 & 1.65 & 3.28 & 0.57 & 1.54 & 1.05 \\
\hline MAi13044.632p 28602.31 & G3 & so & $(0.00,26.80,73.20,0.00)$ & 0.35 & 1.51 & 2.36 & 0.80 & & $\ldots$ \\
\hline COMAi125939.659p275714.03 & G3 & so & $(0.64,33.70,65.70,0.00)$ & 0.32 & 1.91 & 1.97 & 1.08 & $\ldots$ & $\ldots$ \\
\hline COMAi125942.301p275529.15 & G3 & so & $(0.00,25.20,74.80,0.00)$ & 0.08 & 1.53 & 0.98 & 1.48 & & \\
\hline COMAi13017.014p28350.0 & G4 & so & $(0.00,47.20,31.60,21.20)$ & 0.70 & 4.67 & & 0.58 & 0.80 & 0.64 \\
\hline COMAi125943.721p275940.82 & G3 & $\begin{array}{l}\text { So } \\
\text { So }\end{array}$ & $\begin{array}{l}(0.00,69.20,30.80,0.00) \\
(0.18,49.60,26.20 \\
(0.00)\end{array}$ & 0.76 & 3.20 & 1.83 & 0.72 & & \\
\hline $\begin{array}{l}\text { COMAAi125956.697p p275548.717 } \\
\text { COMA13027.966p } 275721.56\end{array}$ & $\begin{array}{l}\text { G4 } \\
\text { G4 }\end{array}$ & $\begin{array}{l}\text { So } \\
\text { So }\end{array}$ & $\begin{array}{l}(0.18,49.60,26.20,24.00) \\
(0.00,42.80,41.30,15.90)\end{array}$ & $\begin{array}{l}1.89 \\
0.42\end{array}$ & $\begin{array}{l}4.33 \\
2.67\end{array}$ & $\begin{array}{l}3.57 \\
3.32\end{array}$ & $\begin{array}{l}0.25 \\
0.32\end{array}$ & $\begin{array}{l}2.48 \\
1.01\end{array}$ & $\begin{array}{l}0.41 \\
0.98\end{array}$ \\
\hline COMAi13016.534p 275803.15 & G3 & so & $(0.00,84.10,15.90,0.00)$ & 3.26 & 6.16 & $\begin{array}{l}3.59 \\
3.59\end{array}$ & 0.48 & & \\
\hline $\mathrm{CC}$ & G4 & so & $(0.00,67.10,19.10,13.80)$ & 0.77 & 2.42 & 5.07 & 0.41 & 3.00 & 53 \\
\hline $\mathrm{CC}$ & G4 & so & $(0.13,16.80,71.90,11.10)$ & 0.46 & 1.6 & 3.81 & 0.85 & 2.86 & 0.61 \\
\hline COMAi13006.395p28015.94 & G3 & so & $(0.00,66.10,33.90,0.00)$ & 1.60 & 6.78 & 2.08 & 0.84 & & \\
\hline COMAi13028.370p 275820.64 & G4 & so & $(0.05,33.80,31.40,34.80)$ & 0.85 & 2.53 & 5.02 & 0.38 & 3.61 & 0.59 \\
\hline COMAi13042.832p275746.95 & G4 & so & $(0.00,43.90,46.40,9.68)$ & 0.75 & 3.01 & 3.81 & 0.47 & 1.51 & 0.39 \\
\hline $\begin{array}{l}\text { COMAi13014.746p28228.69 } \\
\text { COMAi125931.453p28247 }\end{array}$ & $\begin{array}{l}\text { G3 } \\
\mathrm{G} 3\end{array}$ & $\begin{array}{l}\text { So } \\
\text { So }\end{array}$ & $\begin{array}{l}(0.43,78.20,21.40,0.00) \\
(0.00,78,60,21.40,0.00)\end{array}$ & 0.90 & 3.68 & 2.26 & 0.47 & & $\cdots$ \\
\hline COMAi $3018.093 \mathrm{p} 275723.59$ & G2 & $\mathrm{E}$ & $(0.17,100.00,0.00,0.00)$ & $\begin{array}{l}1.01 \\
1.27\end{array}$ & $\begin{array}{l}3.51 \\
2.37\end{array}$ & & & $\ldots$ & $\cdots$ \\
\hline COMAi125852.097p274706.15 & G5 & $\mathrm{E}$ & $(0.00,50.00,50.00,0.00)$ & 2.12 & 6.95 & 1.74 & 1.41 & $\ldots$ & ... \\
\hline
\end{tabular}


Table 3.4 (cont'd)

\begin{tabular}{|c|c|c|c|c|c|c|c|c|c|}
\hline $\begin{array}{l}\text { Galaxy Name } \\
\text { (1) }\end{array}$ & $\begin{array}{l}\mathrm{G} n \\
\text { (2) }\end{array}$ & $\begin{array}{l}\text { Hubble Type } \\
\text { (3) }\end{array}$ & $\begin{array}{c}\mathrm{Pts} / T, C 1 / T, C 2 / T, \operatorname{Bar} / T \\
(\%, \%, \%, \%) \\
(4)\end{array}$ & $\begin{array}{l}\text { C1 } r_{\mathrm{e}} \\
(\mathrm{kpc}) \\
(5)\end{array}$ & $\begin{array}{l}\text { C1 } n \\
(6)\end{array}$ & $\begin{array}{l}\mathrm{C} 2 r_{\mathrm{e}} \\
(\mathrm{kpc}) \\
(7)\end{array}$ & $\begin{array}{c}\text { C2 } n \\
(8)\end{array}$ & $\begin{array}{c}\text { Bar } r_{\mathrm{e}} \\
(\mathrm{kpc}) \\
(9)\end{array}$ & $\begin{array}{l}\text { Bar } n \\
(10)\end{array}$ \\
\hline COMAi13040.838p 275947.80 & G3 & so & $(0.08,93.50,6.43,0.00)$ & 3.00 & 3.41 & 3.57 & 0.53 & $\ldots$ & $\ldots$ \\
\hline COMAi13008.003p 28442.81 & G3 & So & $(0.00,85.00,15.00,0.00)$ & 0.99 & 3.00 & 1.66 & 0.57 & & \\
\hline COMAi13022.170p 28249.30 & G4 & so & $(0.00,15.20,73.40,11.50)$ & 0.31 & 1.35 & 3.49 & 1.24 & 1.31 & 0.48 \\
\hline COMAi125946.782p 275825.99 & G4 & So & $(0.00,15.70,67.70,16.60)$ & 0.31 & 1.75 & 3.44 & 0.67 & 1.23 & 0.72 \\
\hline $\begin{array}{l}\text { COMAi125929.403p275100.46 } \\
\text { COMA125930.82427530305 }\end{array}$ & $\begin{array}{l}\text { G5 } \\
\text { G4 }\end{array}$ & $\underset{\text { spiral }}{\mathrm{E}}$ & $\begin{array}{l}(0.00,100.00,0.00,0.00) \\
(0.36,41.90,41.20 \\
16.50)\end{array}$ & $\begin{array}{l}2.37 \\
0.54\end{array}$ & $\begin{array}{l}1.86 \\
1.89\end{array}$ & 0.28 & $\begin{array}{l}2.19 \\
0.66\end{array}$ & & $\ldots$ \\
\hline $\begin{array}{l}\text { COMAii } 25930.824 \mathrm{p} 275303.05 \\
\text { COMA1 } 25944.407 \mathrm{p} 75444.84\end{array}$ & $\begin{array}{l}\text { G4 } \\
\text { G4 }\end{array}$ & $\begin{array}{l}\text { spiral } \\
\text { So }\end{array}$ & $\begin{array}{l}(0.36,41.90,41.20,16.50) \\
(0.00,34.30,62.10,3.65)\end{array}$ & $\begin{array}{l}0.54 \\
0.75\end{array}$ & $\begin{array}{l}1.89 \\
2.59\end{array}$ & $\begin{array}{l}6.41 \\
2.92\end{array}$ & 0.66 & 1.79 & 0.46 \\
\hline $\begin{array}{l}\text { COMA1 } 25944.407 \mathrm{p} 2754444.84 \\
\text { COMA } 125932.771 \mathrm{p} 275901.04\end{array}$ & $\begin{array}{l}\text { G4 } \\
\text { G4 }\end{array}$ & $\begin{array}{l}\text { So } \\
\text { So }\end{array}$ & $\begin{array}{l}(0.00,34.30,62.10,3.65) \\
(0.02,49.00,46.70,4.25)\end{array}$ & $\begin{array}{l}0.75 \\
2.21\end{array}$ & $\begin{array}{l}2.59 \\
6.05\end{array}$ & $\begin{array}{l}2.92 \\
3.01\end{array}$ & $\begin{array}{l}1.09 \\
0.83\end{array}$ & 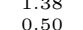 & $\begin{array}{l}0.23 \\
0.54\end{array}$ \\
\hline COMAi13039.767p275526.19 & G4 & So & $(0.01,46.30,53.40,0.34)$ & 1.28 & $\begin{array}{l}0.05 \\
3.05\end{array}$ & $\begin{array}{l}3.01 \\
3.59\end{array}$ & 1.42 & 0.89 & $\begin{array}{l}0.04 \\
0.28\end{array}$ \\
\hline COMAi1 $25929.956 \mathrm{p} 275723.26$ & G4 & so & $(0.37,24.20,25.50,49.90)$ & 0.45 & 1.75 & 5.59 & 0.33 & 3.15 & 1.02 \\
\hline COMAi13017.683p 275718.93 & G2 & $\mathrm{E}$ & $(0.21,100.00,0.00,0.00)$ & 2.13 & 2.80 & & & & $\ldots$ \\
\hline COMAi13048.646p28526.69 & G5 & $\mathrm{E}$ & $(0.11,70.20,29.70,0.00)$ & 3.12 & 5.17 & 1.87 & 1.10 & & \\
\hline COMAi13042.766p275817.38 & G4 & so & $(0.00,66.90,31.30,1.80)$ & 1.27 & 2.99 & 6.23 & 0.35 & 1.42 & 0.17 \\
\hline & G5 & $\mathrm{E}$ & $(0.00,100.00,0.00,0.00)$ & 3.90 & 2.01 & 0.61 & 2.21 & & \\
\hline COMAi125935.698p275733.36 (NGC 4874) & G2 & $\mathrm{cD}$ & $(0.00,100.00,0.00,0.00)$ & 391.3 & 11.4 & & & $\cdots$ & ... \\
\hline & & & & 57.7 & & & & & $\ldots$ \\
\hline
\end{tabular}

Note. - Rows are sorted by increasing $M_{\star}$. In Column 4 , Pts $/ T$ is the fractional light in the point source component. In Columns 4-8, the meaning of C1 and $\mathrm{C} 2$ depends on Hubble type. For $\mathrm{CD}$ and elliptical (E) galaxies, C1 is the outermost structure. For E galaxies, C2 represents the inner component of any
$n$. For So and spiral galaxies, C1 is the bulge and C2 is the outer disk. The bar component represents bars ovals in So and spiral galaxies. For cored galaxies n. For 4874 , NGC 4889, COMAi125909.468p28227.35), the reported model corresponds to the 2D fit where the cored region of the galaxy has been masked (see
(NGGle 3.1 and Appendix A.2). 
Table 3.5 Morphology-Density Relation

\begin{tabular}{cccc}
\hline $\begin{array}{c}\text { Region } \\
(1)\end{array}$ & $\begin{array}{c}\text { Mass or Mag Cut } \\
(2)\end{array}$ & $\begin{array}{c}\text { Galaxy Type } \\
(3)\end{array}$ & $\begin{array}{c}\% \text { by Numbers } \\
(4)\end{array}$ \\
\hline $\begin{array}{c}\text { Central 0.6 Mpc } \\
\text { of Coma (this work) }\end{array}$ & $M_{\star} \geq 10^{9} M_{\odot}$ & $(\mathrm{E}+\mathrm{S} 0):$ spiral & $(22.4 \%+70.2 \%): 7.4 \%$ \\
\hline
\end{tabular}

Table 3.6 Mass Breakdown Among Galaxies With $M_{\star} \geq 10^{9} M_{\odot}$ in the Central 0.6 Mpc of Coma, Excluding the Two cDs

\begin{tabular}{|c|c|c|}
\hline $\begin{array}{l}\text { Structure } \\
\text { (1) }\end{array}$ & $\begin{array}{c}\text { \% of Stellar Mass Within } \\
\text { Each Galaxy Type } \\
(2)\end{array}$ & $\begin{array}{c}\% \text { of Stellar Mass in the } \\
\text { Central 0.6 Mpc of Coma } \\
(3)\end{array}$ \\
\hline \multicolumn{3}{|c|}{ Photometric E $(\mathrm{N}=15)$} \\
\hline \multicolumn{3}{|c|}{ Spiral $(N=5)$} \\
\hline
\end{tabular}


Table 3.7 Mass Breakdown Among Galaxy Components Separated by Sérsic Index \begin{tabular}{cc}
\hline Structure & \% of Stellar Mass in the \\
Central 0.6 Mpc of Coma
\end{tabular} (1)

Disk-dominated components with $n \leq n_{\text {disk_max }}$
$(2)$

Outer disks of S0s 18.14 Outer disks of spirals 1.96

Bulges with $n \leq n_{\text {disk_max }}$ (disky/pseudo bulge) in S0s

Bulges with $n \leq n_{\text {disk_max }}$ (disky/pseudo bulge) in spirals

1.33

Inner component with $n \leq n_{\text {disk_max }}$ (inner disks) in photometric $\mathrm{E}$

Total

Bars

0.12

1.98

23.53

Bars in S0s

Bars in spirals

4.42

Total

0.40

Non-disk "hot" components with $n>n_{\text {disk_max }}$

Components with $n>n_{\text {dmax }}$ in cDs

Outer component with $n>n_{\text {disk_max }}$ in photometric $\mathrm{E}$

Inner component with $n>n_{\text {disk_max }}$ in photometric $\mathrm{E}$

Inner component with $n>n_{\text {disk_max }}$ in SOs

Inner component with $n>n_{\text {disk_max }}$ in spirals

Total

4.82

35.66

9.35

6.71

18.69

1.19

71.60

Table 3.8 Distribution of Bulge Sérsic Index in S0s

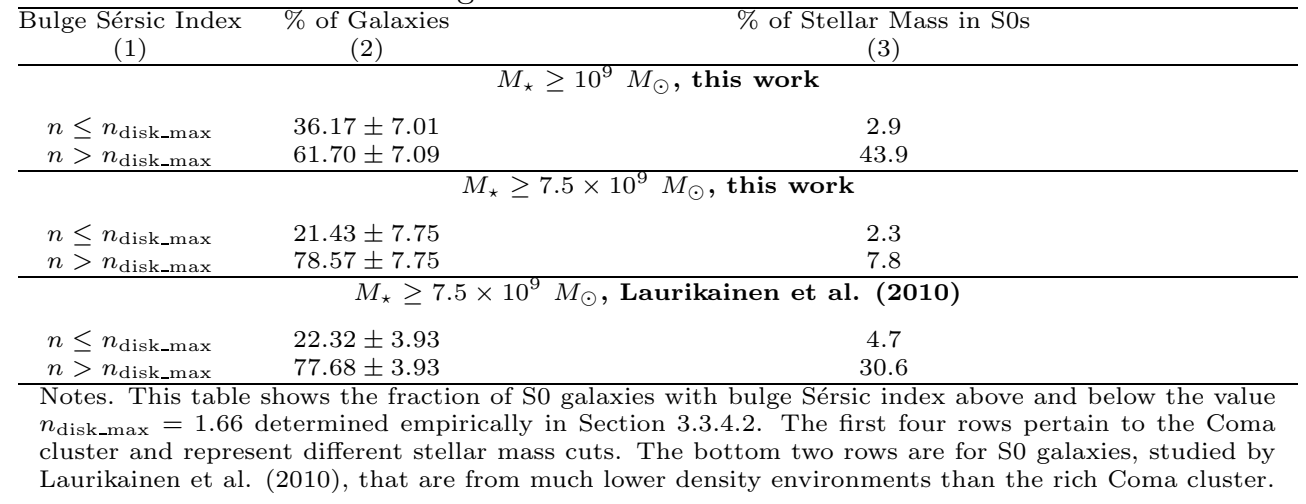



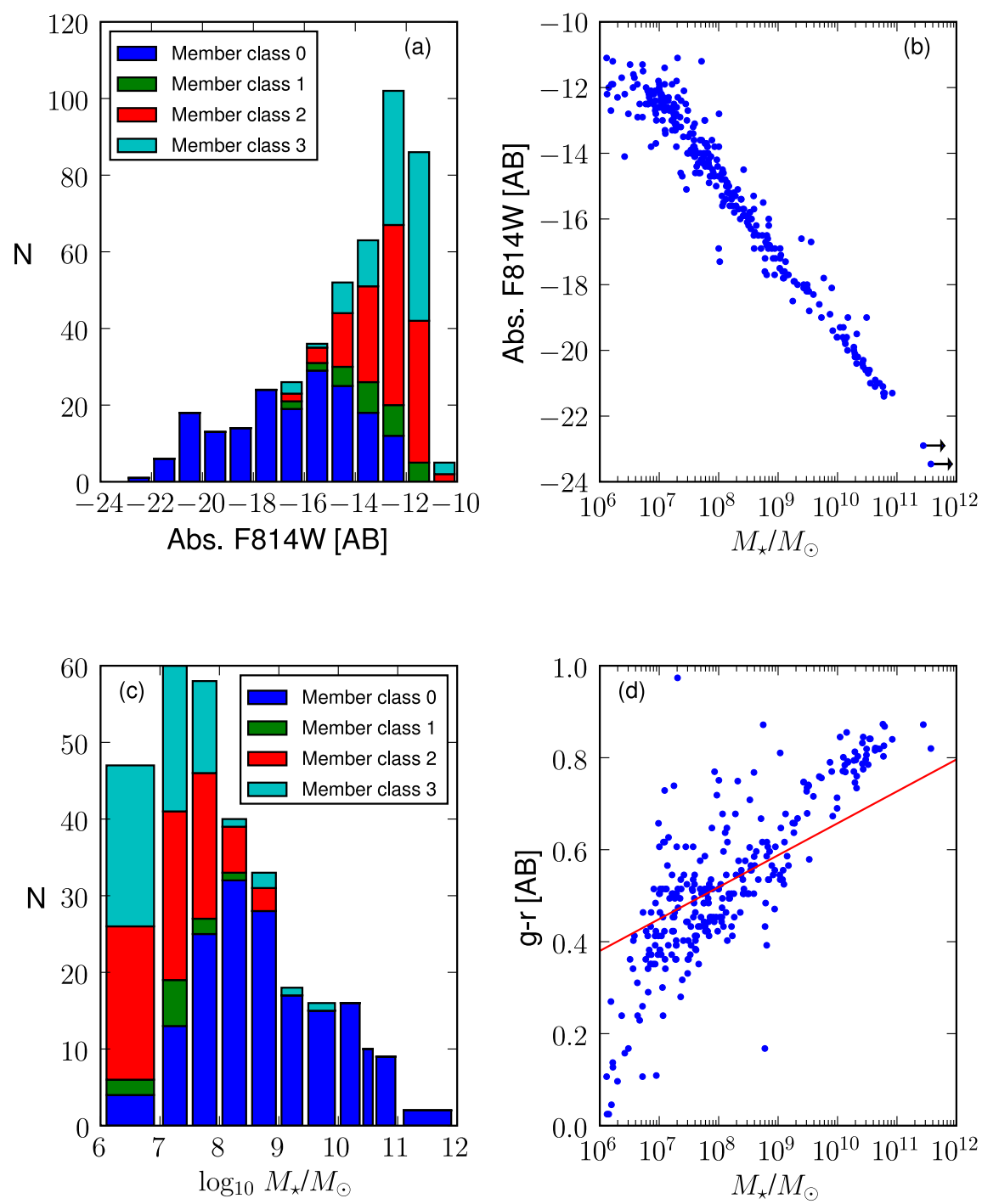

Figure 3.1 This figure shows in panels (a) to (d) the luminosity, stellar mass, and $g-r$ color, respectively, for the 447 galaxies in sample $\mathrm{S} 1$ having $\mathrm{F} 814 \mathrm{~W} \leq 24$, locations within the central 0.6 Mpc of the Coma cluster, and cluster membership rating 0-3. See Section 3.2.1 for details. In panel (b), the two most massive sources are $\mathrm{cD}$ galaxies, and the arrows indicate their adopted stellar masses are lower limits (Section 3.2.2). The solid line in panel (d) is the color-luminosity break between the red and blue sequence of galaxies from Blanton et al. (2005), which we convert from luminosity to stellar mass using the relations of Bell et al. (2003). 


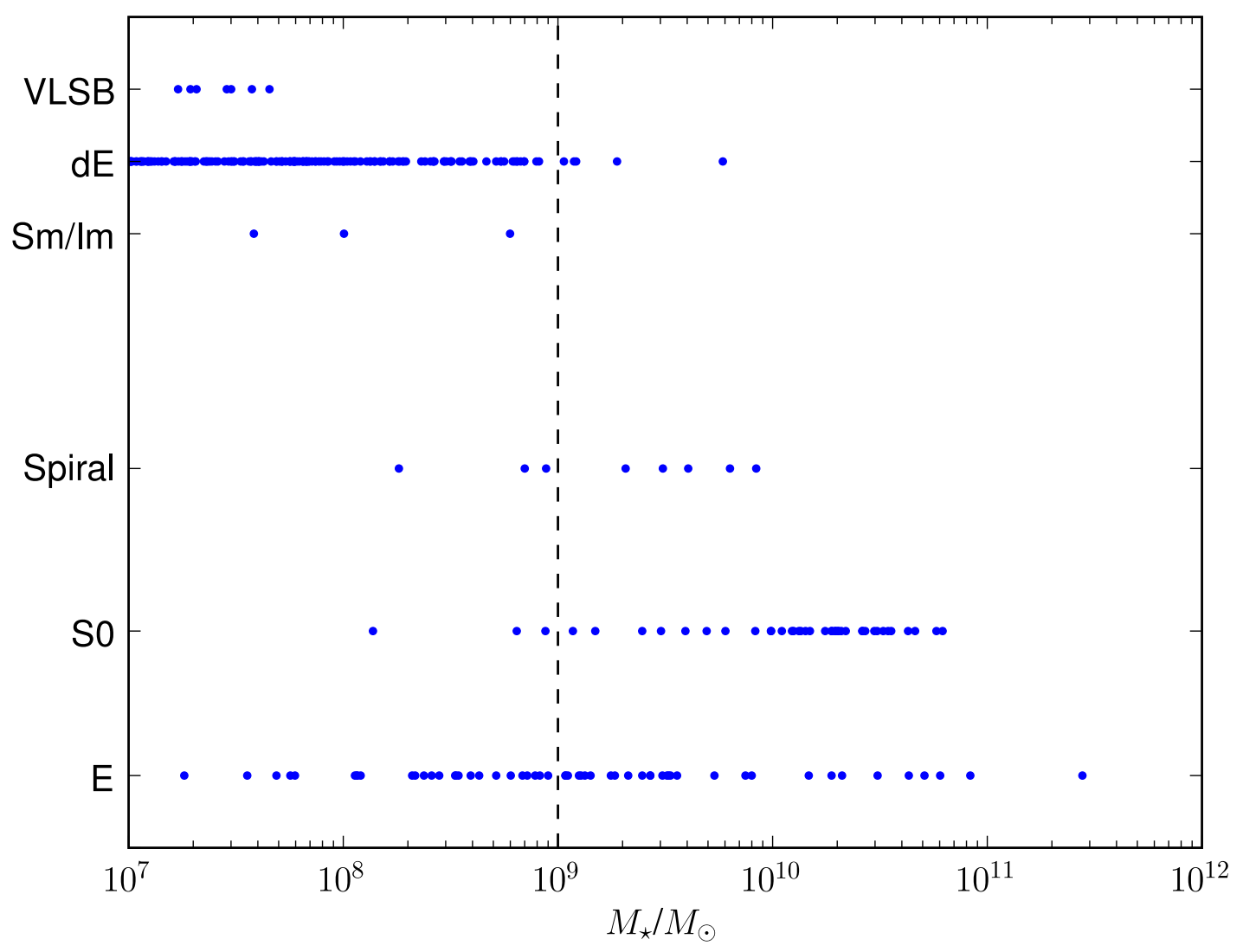

Figure 3.2 Visually determined Hubble types from the catalog of N. Trentham et al. (in preparation), with updates from Marinova et al. (2012), are plotted against stellar mass. As discussed in Section 3.2.3, we apply a mass cut (dashed line) to focus on galaxies with stellar mass $M_{\star} \geq 10^{9} M_{\odot}$. This effectively removes from the working sample most dwarf/irregular and very low surface brightness (VLSB) galaxies. 


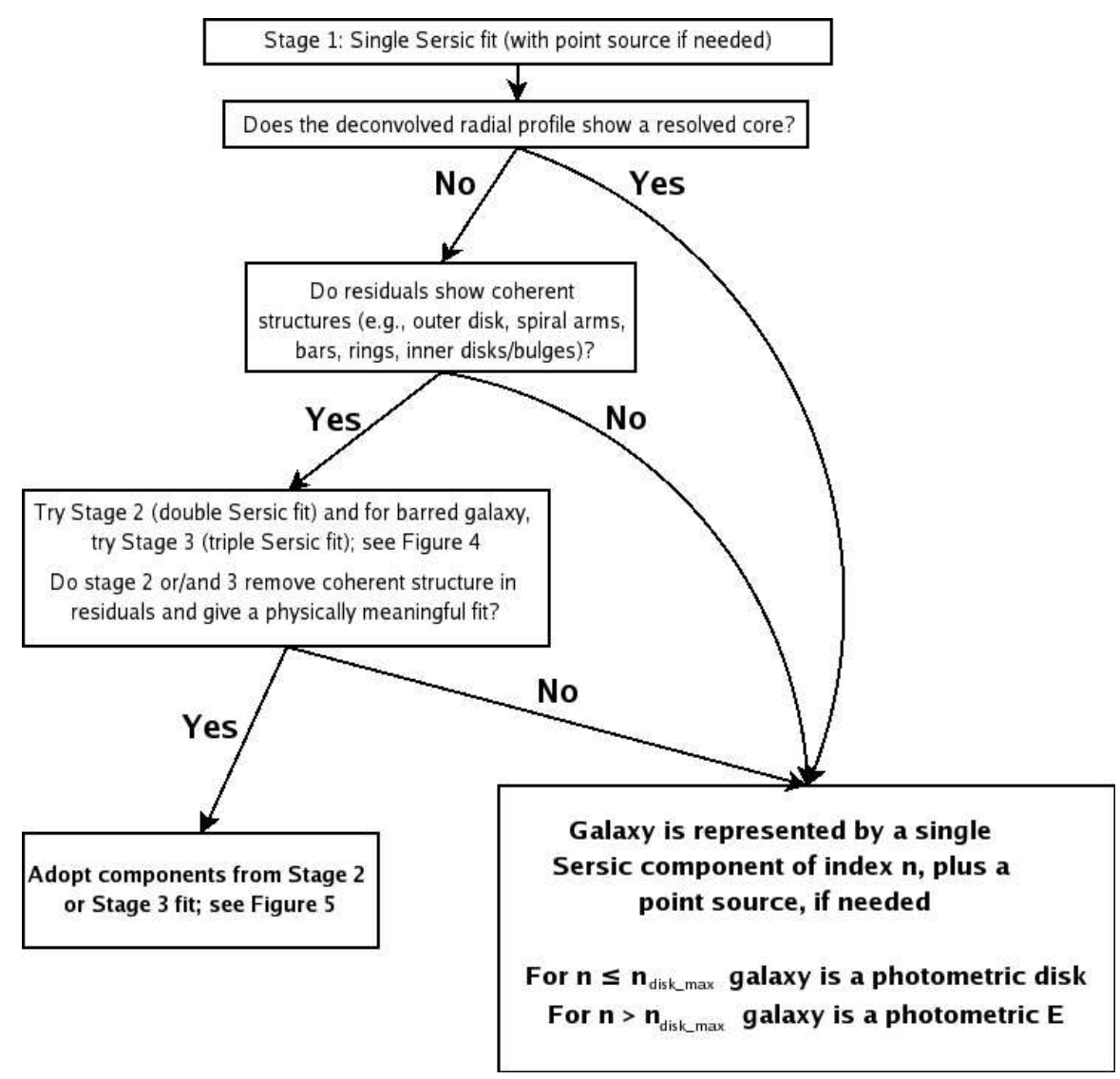

Figure 3.3 Overview of the method of decomposition. All galaxies are subjected to Stage 1 , and most are further processed in Stage 2. A galaxy best fit with a single Sérsic profile plus point source (if needed) is interpreted as a photometric elliptical or photometric disk. A galaxy with extra coherent structure that cannot be described with a single Sérsic profile is subjected to a multiple-component fit in Stage 2 and, if needed, Stage 3. Figure 3.4 describes Stage 2 and Stage 3 in more detail. 


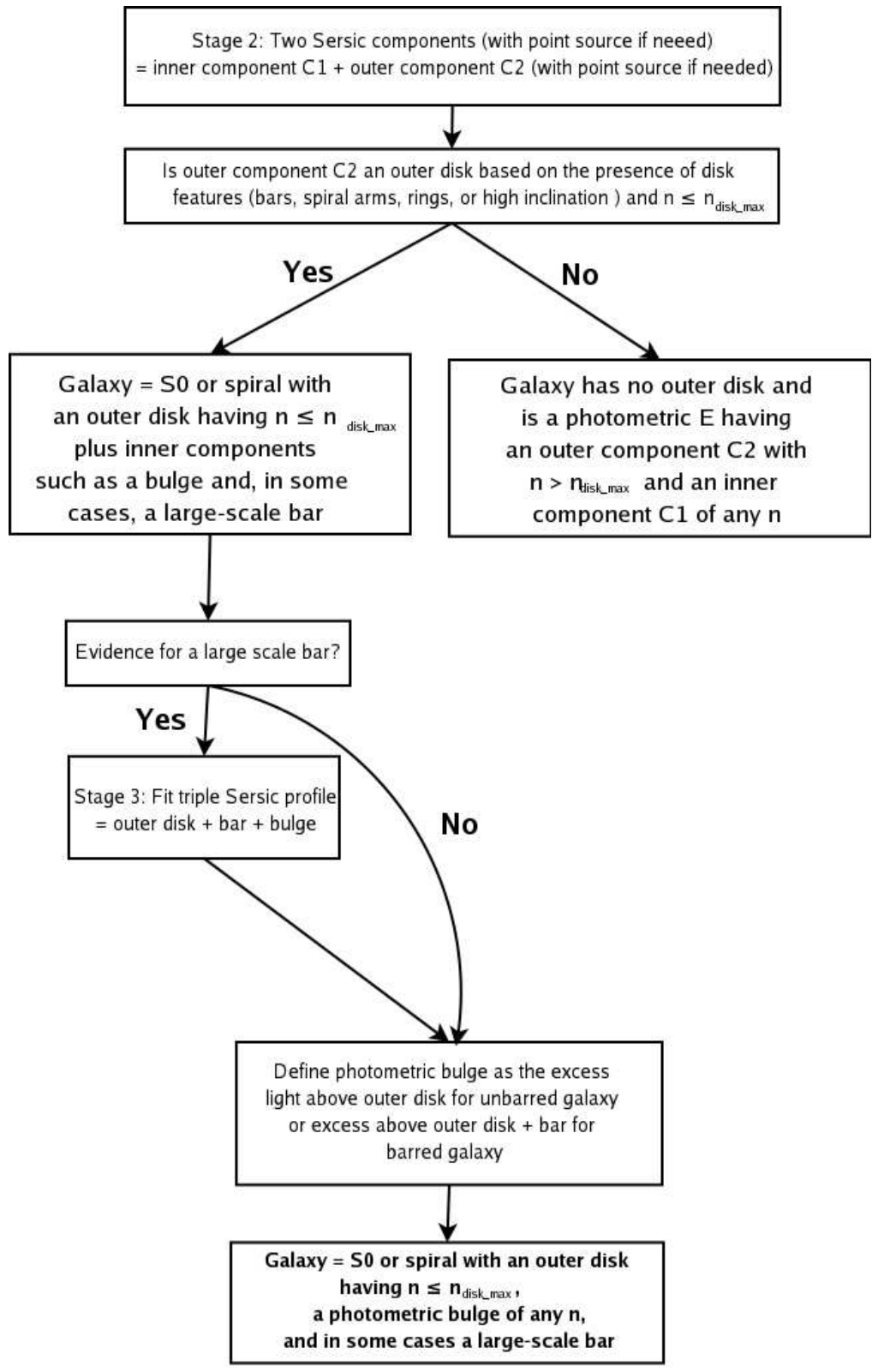

Figure 3.4 Multi-component decompositions are broken into two phases, Stage 2 and Stage 3. A galaxy without an extended outer disk is interpreted as a photometric E, while a galaxy with such a disk is labeled either an S0 or spiral. When evidence for a large scale bar is found in a galaxy with an outer disk, Stage 3 is used to model the bar component. 


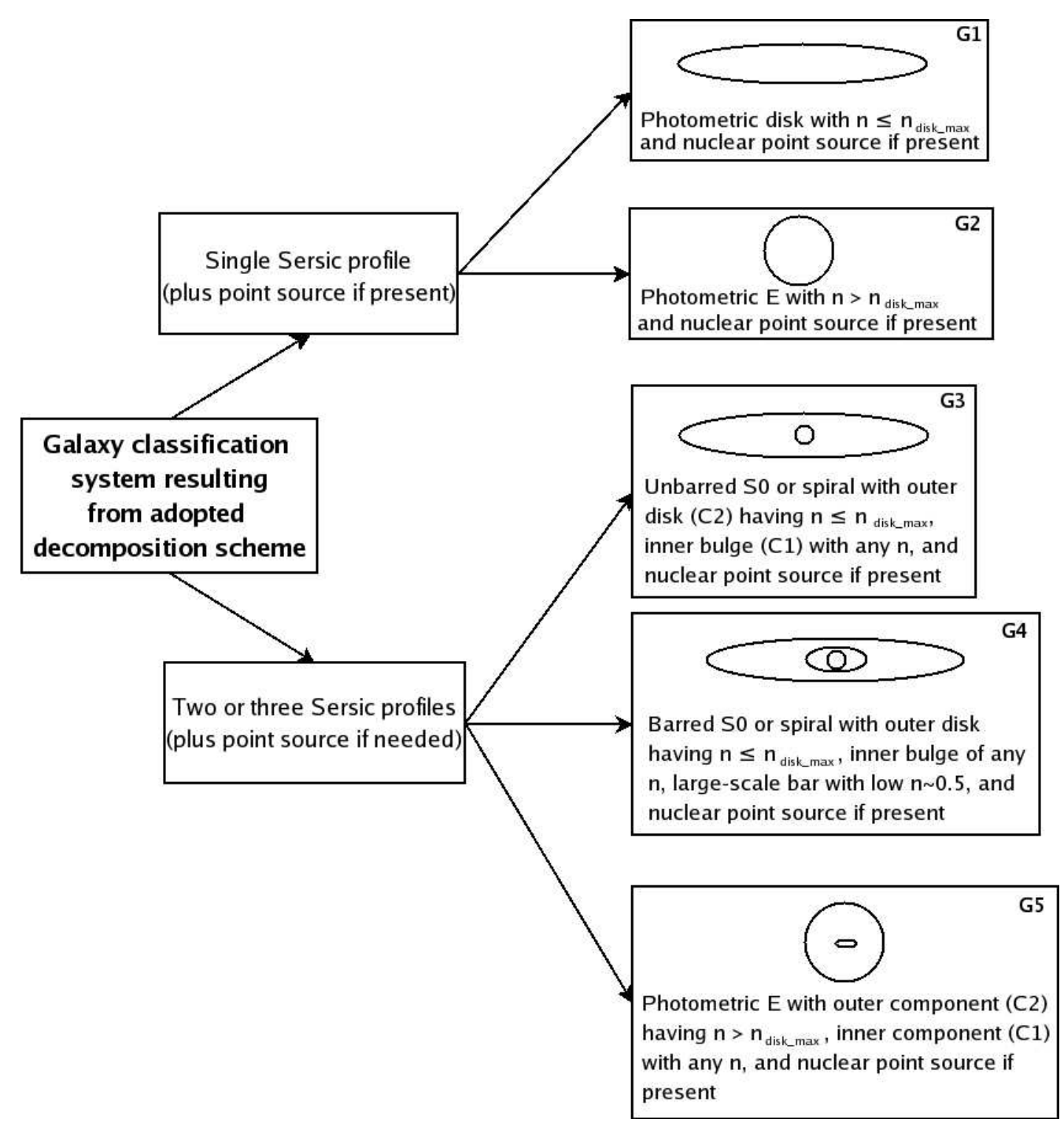

Figure 3.5 Overview of galaxy classification system. Galaxies are deemed to be best represented by a either a single or multi-component Sérsic profile (plus point source, if needed). Galaxies fitted with a single Sérsic profile are further interpreted as a pure disk (if Sérsic index $n \leq n_{\text {disk_max }}$ ) or photometric elliptical (if Sérsic index $n>n_{\text {disk_max }}$ ). When a multicomponent Sérsic profile is required, the galaxy is either an unbarred/barred S0 or spiral, or a photometric E with inner and outer components. S0s and spirals must have an outer

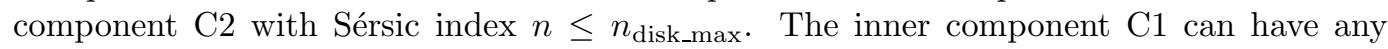
$n$. If the outer component $\mathrm{C} 2$ has Sérsic index $n>n_{\text {disk_max }}$, the galaxy is a photometric elliptical with inner component $\mathrm{C} 1$ of any $n$. The value of $n_{\text {disk_max }}$ is set to 1.66 based on several considerations. We determine $n_{\text {disk max }}$ to be the maximum Sérsic index of the outer disk in spiral and S0 galaxies showing clear signs of an outer disk, such as bars, spiral arms, rings, or high inclination. 

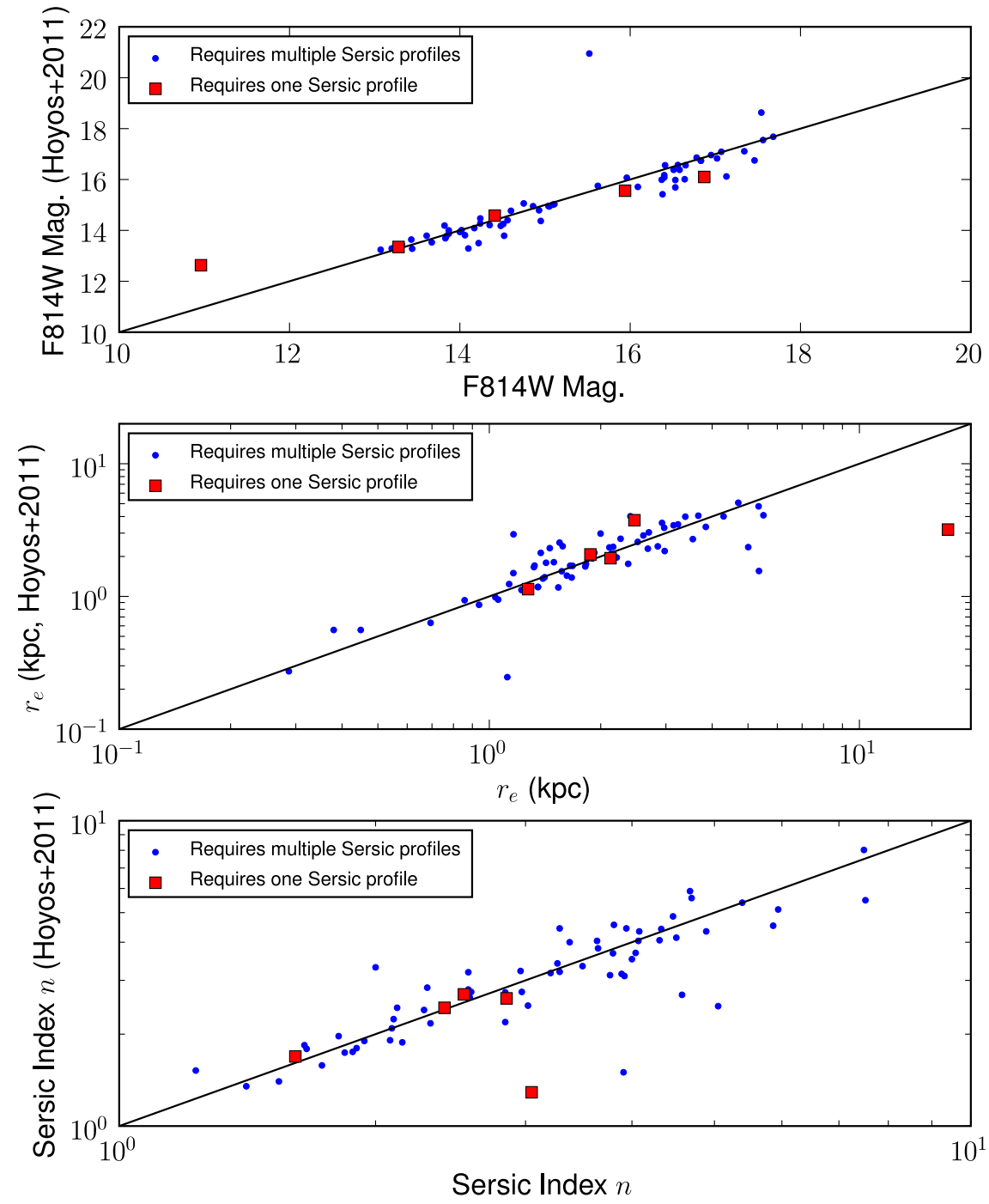

Figure 3.6 This figure compares our results for the single Sérsic fits (with no points source) with those obtained by Hoyos et al. (2011) using GALFIT on the same data. The sources in our sample requiring one Sérsic component are labeled separately from sources requiring two or three Sérsic profiles. Our derived magnitudes, $r_{\mathrm{e}}$, and $n$ for the sources requiring one Sérsic profile agree well with the parameters from Hoyos et al. (2011), with the one exception being cD galaxy NGC 4874 (COMAi125935.698p275733.36) with $n \sim 3$. Note that cD galaxy NGC 4889 requires only one Sérsic profile but it is not included here as it is not in the Hoyos et al. (2011) sample. See Section 3.3.4.1 for additional details. 

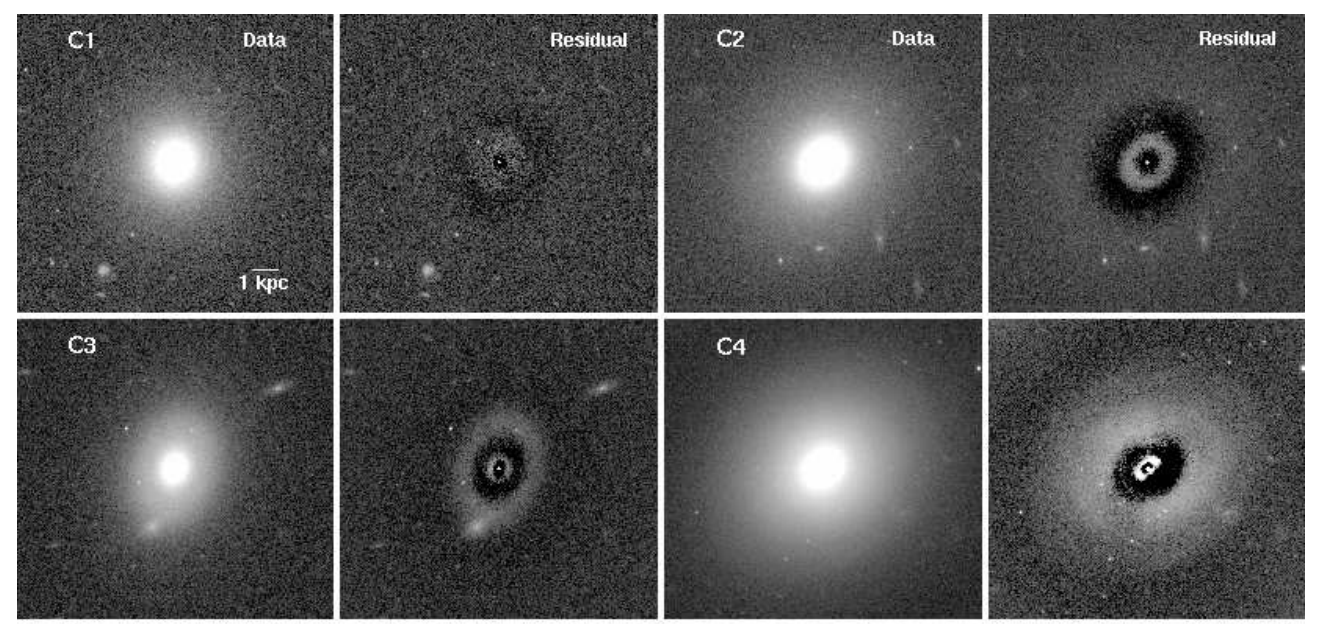

C5

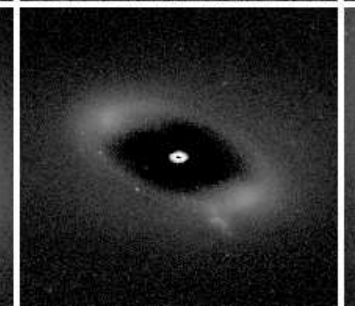

C6

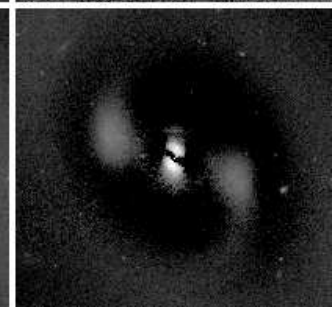

Figure 3.7 This figure shows examples $\mathrm{C} 1$ to $\mathrm{C} 6$ where a single Sérsic model (+ point source if needed) does not provide a good fit to coherent galaxy structure that is best modeled with one or more additional Sérsic profiles. Such residual structure includes central compact structures $(\mathrm{C} 2, \mathrm{C} 3, \mathrm{C} 4, \mathrm{C} 5, \mathrm{C} 6)$, rings $(\mathrm{C} 3, \mathrm{C} 4)$, annuli and extended components $(\mathrm{C} 1, \mathrm{C} 4)$, and bars/ovals $(\mathrm{C} 5, \mathrm{C} 6)$. These systems are better fitted by models with multiple Sérsic components (see Figures 3.8 and 3.9). Columns 1 and 3 show the input $I$-band images. Columns 2 and 4 show the residuals after subtracting the best single Sérsic fit. Note C1=COMAi125931.893p275140.76, C2 = COMAi125935.286p275149.13, $\mathrm{C} 3=$ COMAi13021.673p275354.81, C4 = COMAi13014.746p28228.69, C5 = COMAi125833.134p272151.73, and C6 = COMAi125930.824p275303.05. 

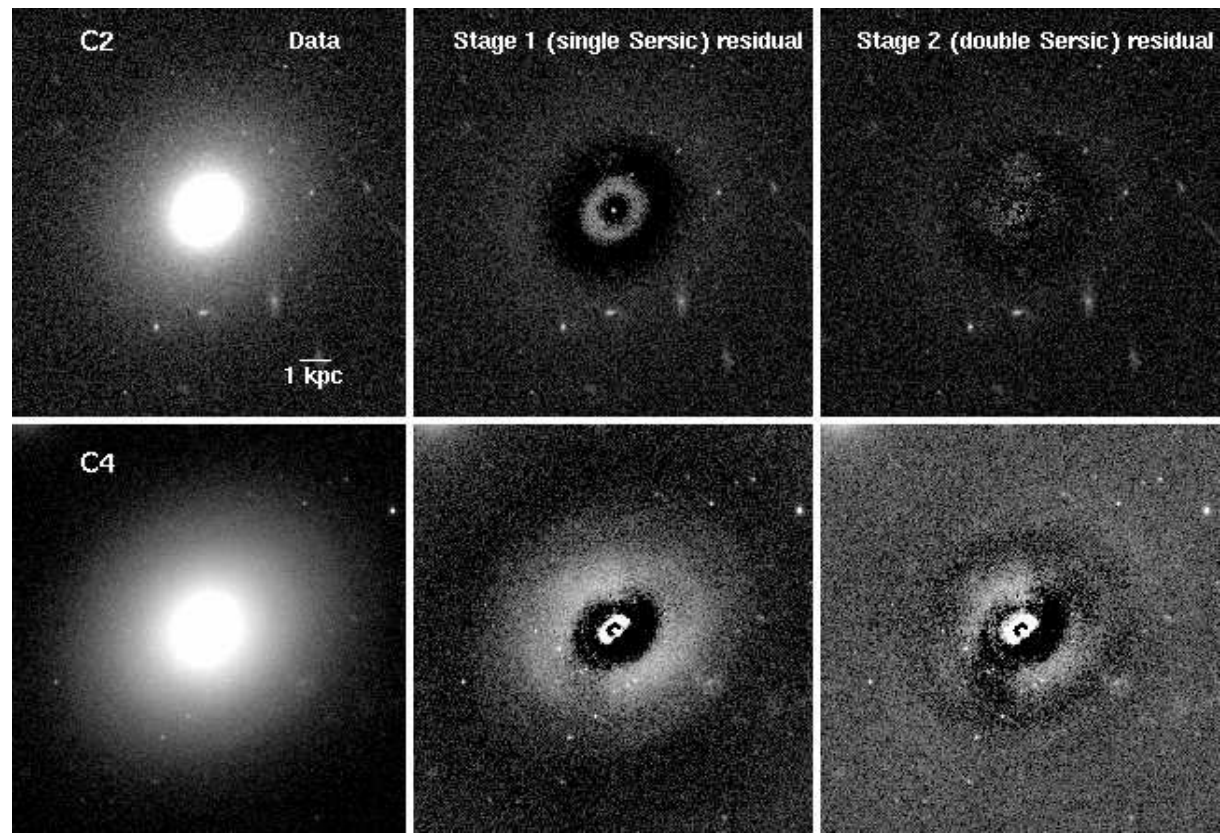

Figure 3.8 This figure shows how some of the galaxies (C2=COMAi125935.286p275149.13 and C4=COMAi13014.746p28228.69) poorly fitted by a single Sérsic model (plus point source if needed) in Stage 1 can be better fitted by two Sérsic models (plus point source if needed) in Stage 2. Each row shows the data, residual after Stage 1, and the residual after Stage 2. Galaxy $\mathrm{C} 2$ is best-fit as having an inner disk $(n=0.31)$ and an outer elliptical structure $(n=2.08)$. Galaxy C4 is best fit with an inner bulge $n=3.68$ and an outer disk $(n=0.47)$. 

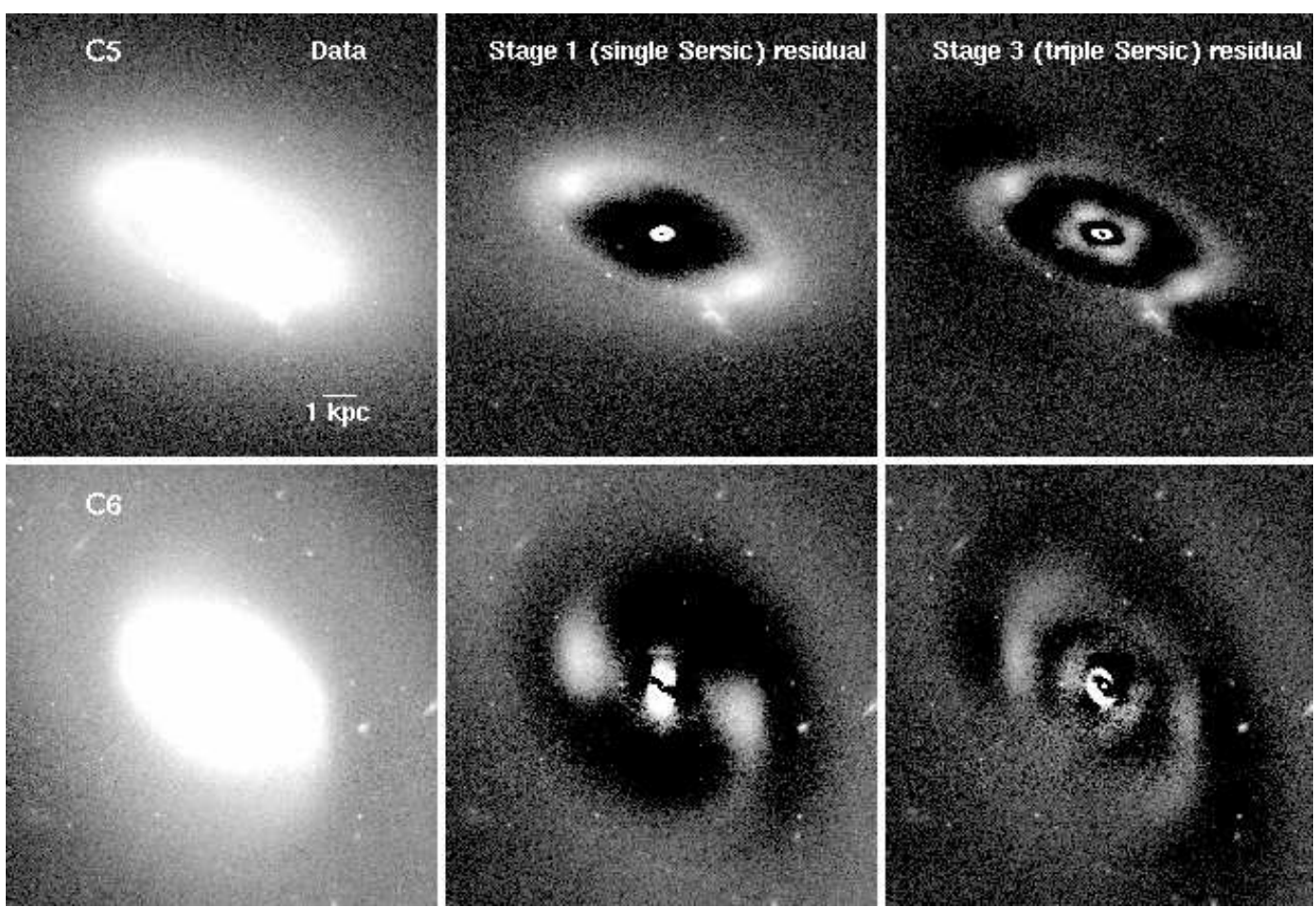

Figure 3.9 This figure shows two examples of barred galaxies (C5=COMAi125833.134p272151.73 and C6=COMAi125930.824p275303.05) poorly fitted by a Stage 1 single Sérsic model that are better fitted by a Stage 3 triple Sérsic (plus point source if needed) model. Column 1 shows the data images while columns 2 and 3 show the residuals after the Stage 1 and Stage 3 model, respectively. 


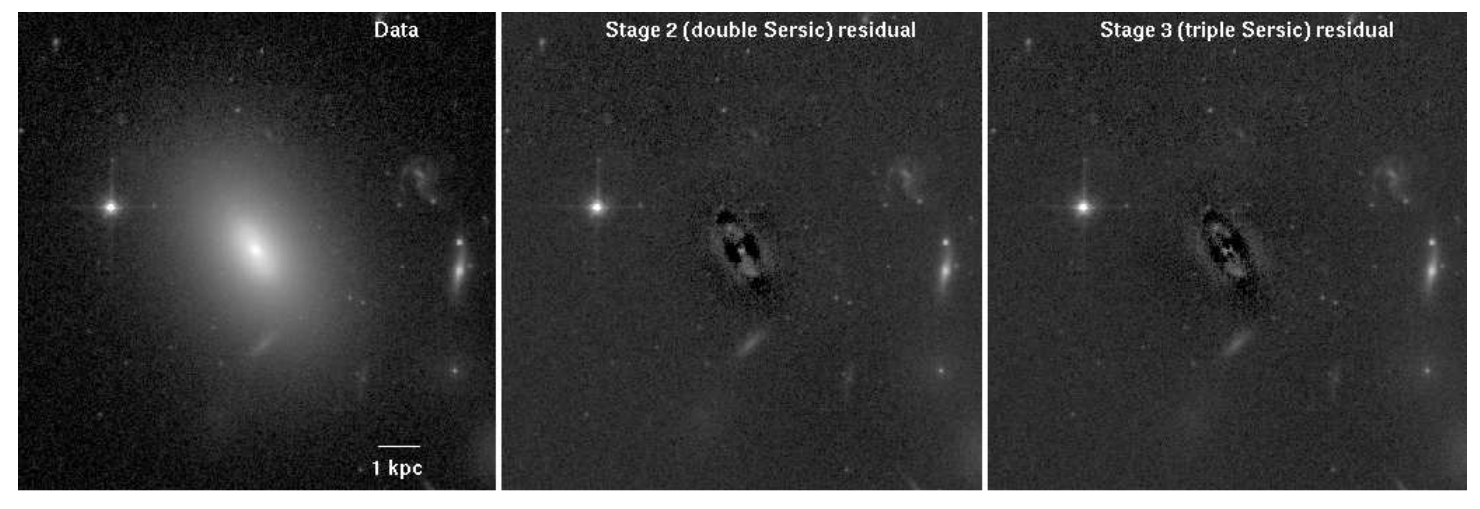

Figure 3.10 This figure shows the decomposition of moderately inclined, barred galaxy COMAi125950.105p275529.44, in which we measure the highest outer disk Sérsic index $n=1.66$. Thus, this galaxy sets the empirically determined upper limit on disk Sérsic index, $n_{\text {disk_max }}=1.66$. Column 1 shows the data images while columns 2 and 3 show the residuals for the Stage 2 and Stage 3 model, respectively. The bar signature is clearly present in the residuals. 


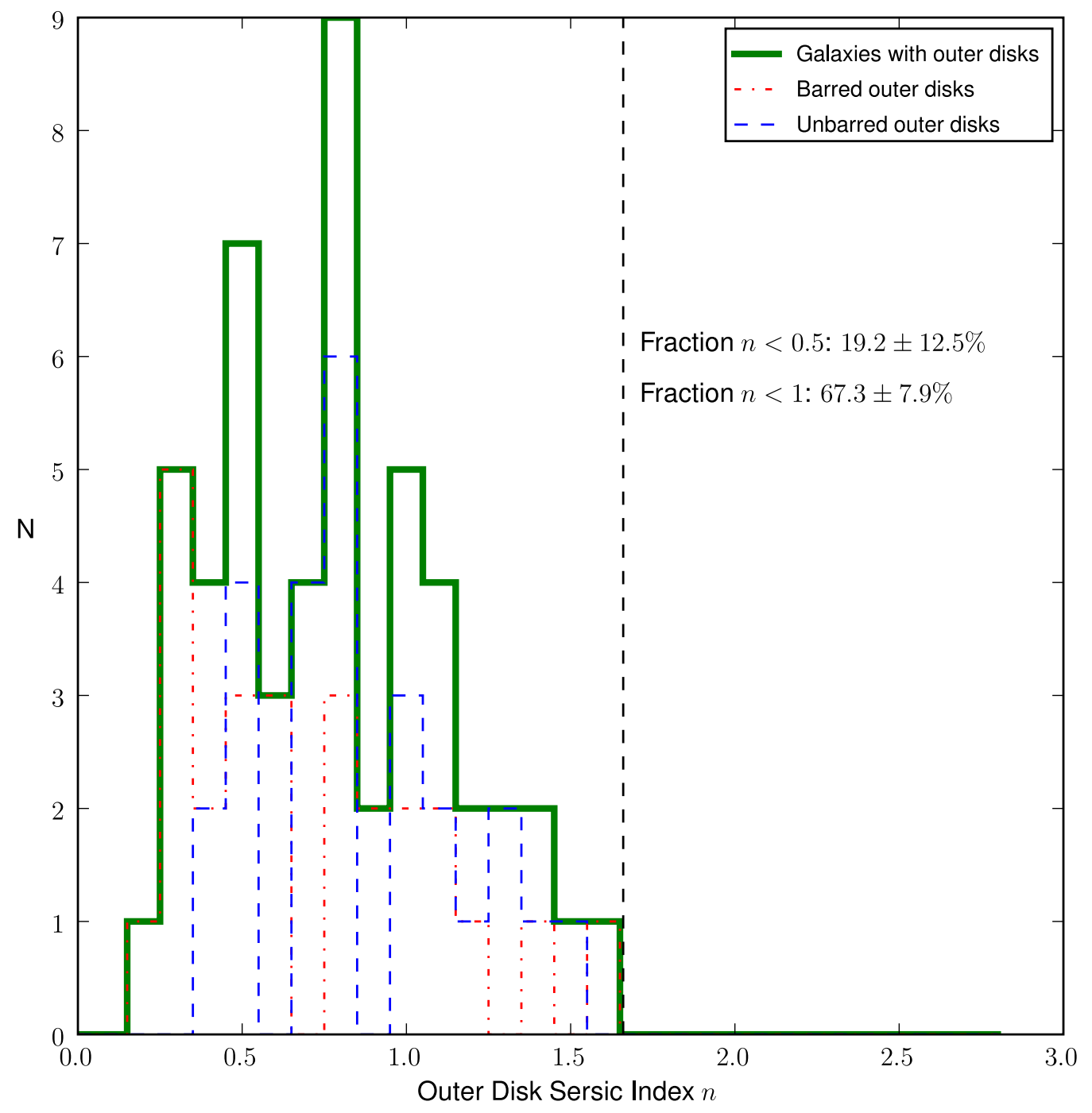

Figure 3.11 The distribution of Sérsic index for outer disks in S0/spiral galaxies. The vertical line represents the empirically determined upper limit, $n_{\text {disk_max }}$, in the Sérsic index of outer disks. The dash-dotted and dashed lines show the distributions for barred and unbarred outer disks, respectively. See Section 3.3.4.2 for details. 

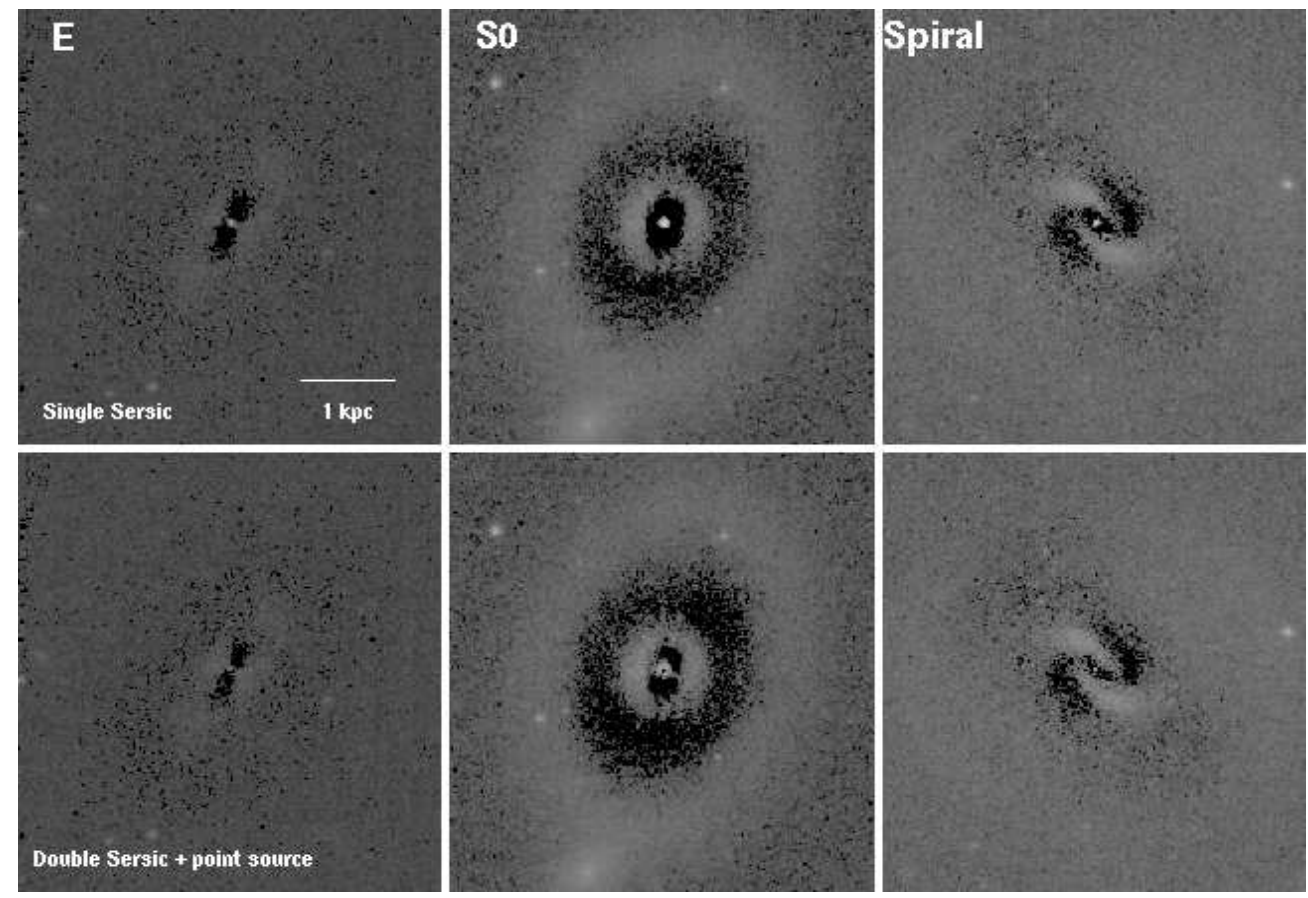

Figure 3.12 This figure compares residuals after fitting a single Sérsic model (top row) versus the best fit double Sérsic + nuclear point source model (bottom row) for an elliptical (COMAi13030.954p28630.22), S0 (COMAi13021.673p275354.81), and spiral (COMAi13041.193p28242.34). The nuclear point source is visible in the residuals in the top row. 


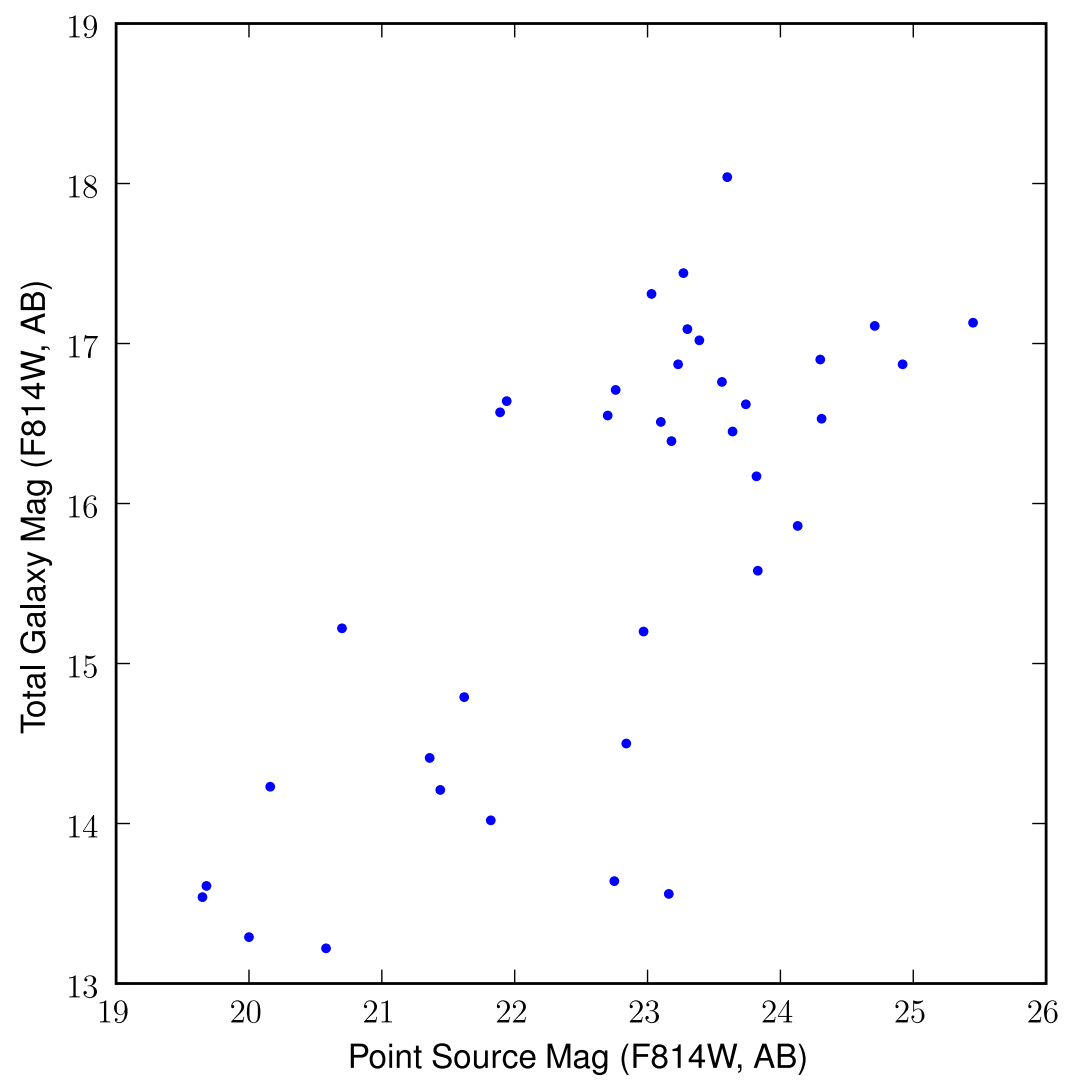

Figure 3.13 This panel shows the relation between total galaxy luminosity and point source luminosity for objects having a nuclear point source in the final, best structural decomposition. 


$$
5 \times 10^{9} \leq M_{\star} \leq 6.2 \times 10^{10} M_{\odot}
$$
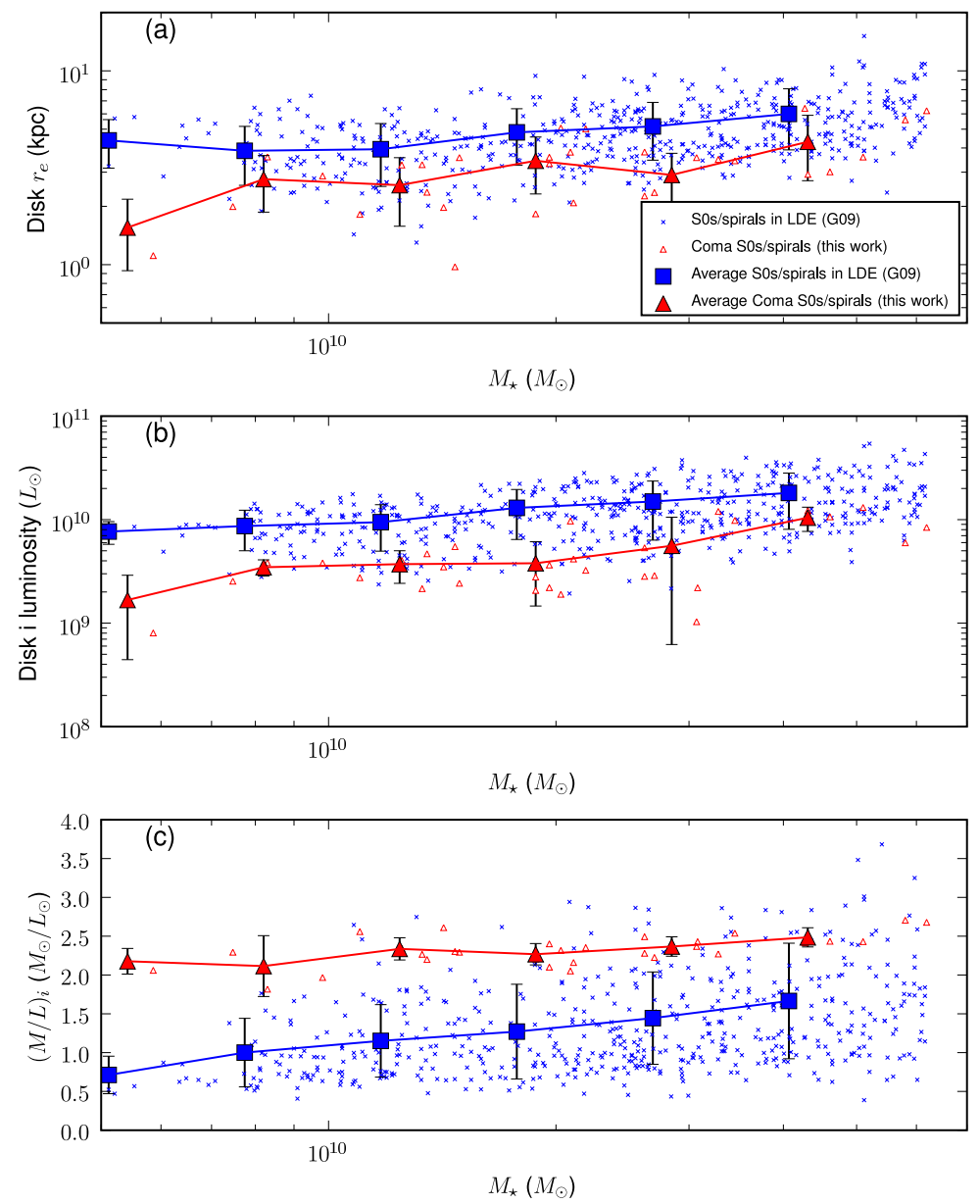

Figure 3.14 Panels (a) and (b) compare the properties of large-scale disks ( $r_{\mathrm{e}}$, luminosity) with galaxy $M_{\star}$. Massive $5 \times 10^{9} \leq M_{\star} \leq 6.2 \times 10^{10} M_{\odot}$ S0/spiral galaxies from the central 0.6 Mpc of Coma as well as low-density environments (LDEs) are considered. The LDE sample is from Gadotti (2009) and includes galaxies in SDSS Data Release 2 that are face-on $(b / a \geq 0.9)$ and have redshift $0.02 \leq z \leq 0.07$. In panel (b), the $i$-band luminosity represents the ACS F $814 \mathrm{~W}$ photometry for Coma galaxies and the SDSS $i$-band photometry for the LDE galaxies. Panel (c) compares $i$-band mass-to-light ratio $(M / L)_{i}$ with galaxy $M_{\star}$. For Coma, the galaxy wide mass-to-light ratio is plotted, while for LDEs the outer $\operatorname{disk}(M / L)_{i}$ is shown. In all panels, the mean values in galaxy $M_{\star}$ for Coma and LDEs are slightly offset for readability. The error bars on the mean values represent the $1 \sigma$ standard deviation. This figure demonstrates that at a given galaxy stellar mass, the average disk half-light radius $r_{\mathrm{e}}$ in the $i$-band is smaller in the central $0.6 \mathrm{Mpc}$ of Coma compared with LDEs. 


$$
5 \times 10^{9} \leq M_{\star} \leq 6.2 \times 10^{10} M_{\odot}
$$
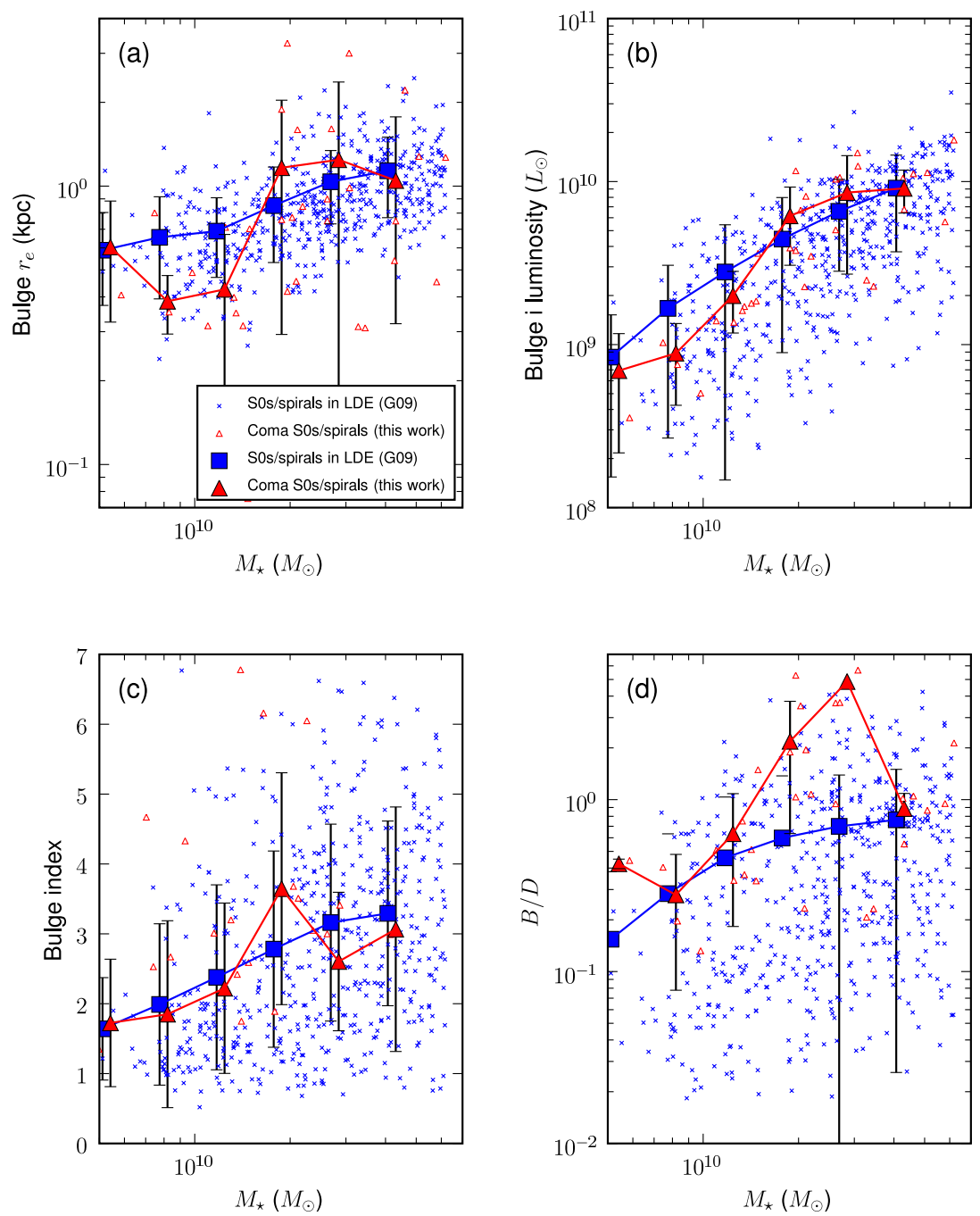

Figure 3.15 This figure is similar to Figure 3.14, except that it emphasizes S0/spiral galaxy bulges. See Section 3.4.4 and Figure 3.14 for extra details on the sample from Gadotti (2009). Panels (a), (b), (c), and (d) show bulge size $\left(r_{\mathrm{e}}\right)$, bulge $i$-band luminosity, bulge Sérsic index, and bulge-to-disk light ratio $(B / D)$, respectively, versus galaxy $M_{\star}$. In all panels, the error bars on the mean values represent the $1 \sigma$ standard deviation, and in all panels the mean values in galaxy $M_{\star}$ for Coma and LDEs are slightly offset for readability. This figure demonstrates that at a given galaxy stellar mass, there is no systematic offset between bulges in Coma and LDEs. 

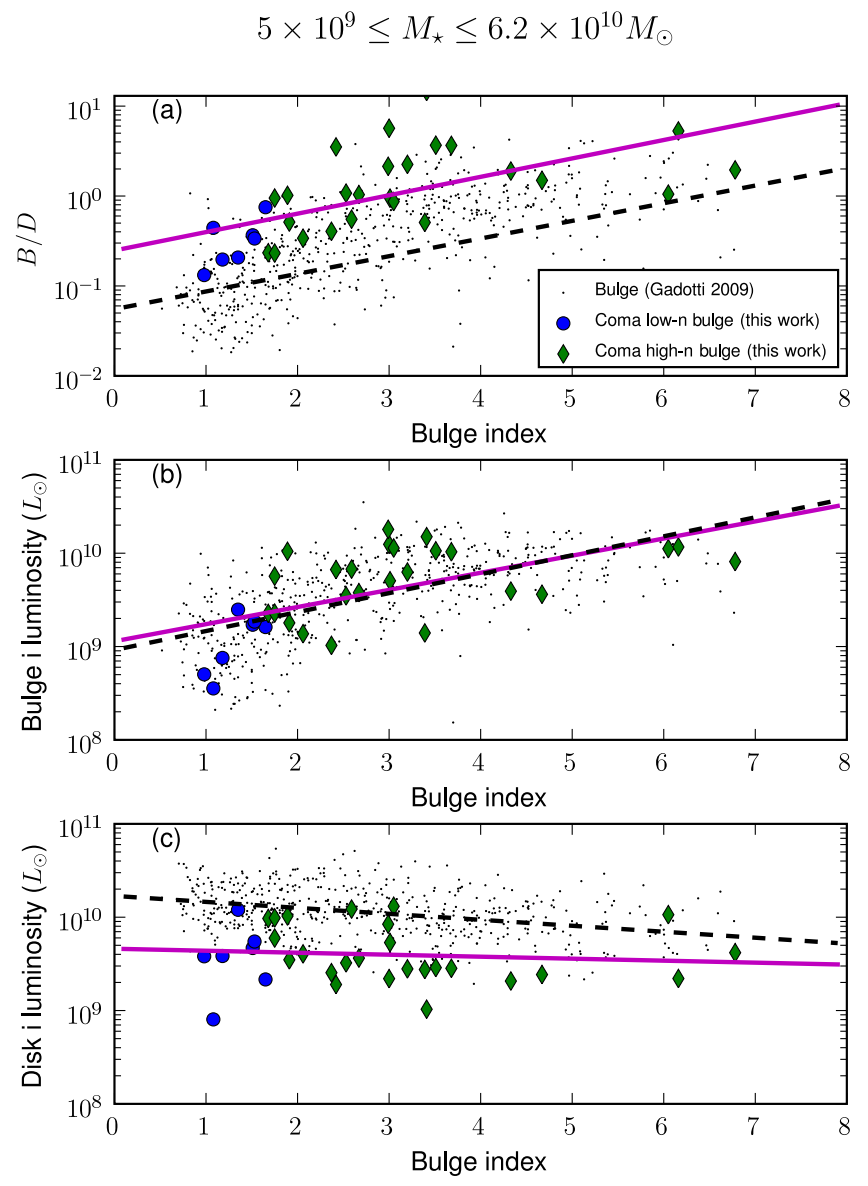

Figure 3.16 This figure shows a comparison of bulges in massive $\left(5 \times 10^{9} \leq M_{\star} \leq 6.2 \times\right.$ $10^{10} M_{\odot}$ ) S0/spiral galaxies in low-density environments (LDEs) from Gadotti (2009) versus galaxies in the central 0.6 Mpc of Coma. See Section 3.4.4 and Figure 3.14 for extra details on the sample from Gadotti (2009). Bulges in Coma are divided into groups of low Sérsic index $\left(n \leq n_{\text {disk_max }}\right)$ and high Sérsic index $\left(n>n_{\text {disk_max }}\right)$. Panel (a) shows bulge-to-disk light ratio $(B / D)$ versus bulge Sérsic index. Panels (b) and (c) show bulge and disk $i$-band luminosity, respectively, versus bulge Sérsic index. In each panel, the solid line represents the fit to Coma bulges of all Sérsic $n$, and the dashed line is the corresponding fit to bulges of all Sérsic $n$ from LDEs. The offset in $B / D$ in panel (a) appears to be driven by the offset in disk luminosity in panel (c). 

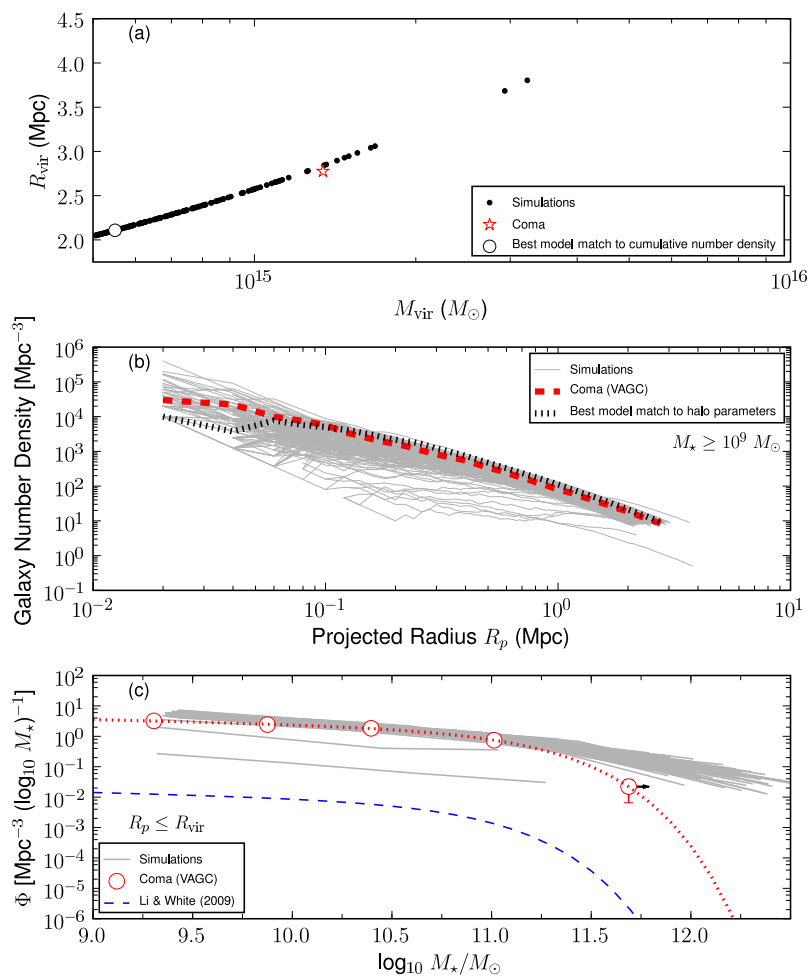

Figure 3.17 This figure shows how the global parameters of the Coma cluster compare with all 160 clusters in the Millennium simulation having a halo mass in the range $5 \times 10^{14}$ $10^{16} M_{\odot}$. The solid lines and black data points represent the simulated clusters. In panel (a), the virial mass and virial radius adopted for Coma are $2.8 h_{73}^{-1} \mathrm{Mpc}$ and $1.3 \times 10^{15} h_{73}^{-1} M_{\odot}$ (Section 3.5.2). The open circle is the model cluster having the best match to the projected galaxy number density of Coma. In panels (b) and (c), the cumulative projected galaxy number density and the galaxy mass stellar function of Coma at projected radius $R_{\mathrm{p}} \leq R_{\mathrm{vir}}$ are based on data from the NYU Value-Added Galaxy Catalog (NYU-VAGC, Blanton et al. 2005). In panel (b), the dotted line represents the cumulative galaxy number density of the model cluster best matching the Coma halo parameters. In panel (c), for the Coma galaxy stellar mass function, we measure a slope $\alpha=-1.16$ and characteristic mass $M^{*}=$ $1.17 \times 10^{11} M_{\odot}$. The last mass bin in the global mass function for Coma contains the two cD galaxies, and the arrow on this bin indicates the adopted stellar masses for the cDs are lower limits. The simulations are based on a model that produces a reasonable match to the galaxy stellar mass function of Li \& White (2009) averaged over all environments at $0.001<z<0.5$ (dashed line). However, they cannot produce a model cluster that simultaneously matches multiple global properties (halo properties, galaxy number density, and galaxy stellar mass function), of Coma, our local benchmark for one of the richest nearby galaxy clusters. 

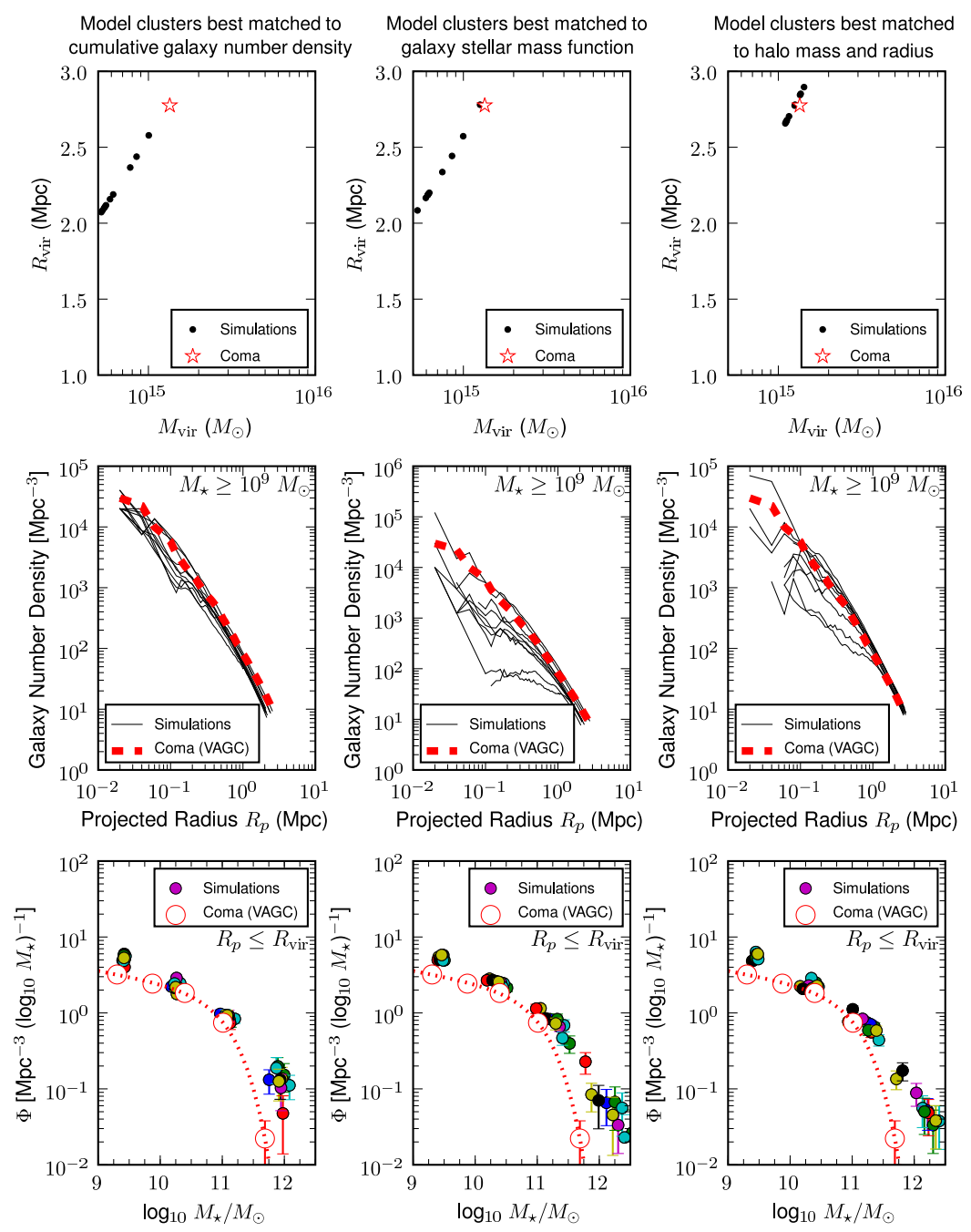

Figure 3.18 This figure shows the three sets of simulated model clusters (30 model clusters in total) chosen to best match, separately, the cumulative galaxy number density (Column 1), the galaxy stellar mass function (Column 2), and halo parameters (halo mass and virial radius, Column 3). The solid lines and solid circles in each panel represent the different simulated clusters. Rows 1, 2, and 3 show how the different simulated clusters compare with the global properties (halo mass and radius, cumulative galaxy number density, and galaxy stellar mass function) determined with data from the NYU Value-Added Galaxy Catalog (NYU-VAGC, Blanton et al. 2005) for Coma in Section 3.5.2. No model cluster simultaneously matches all three global properties. 

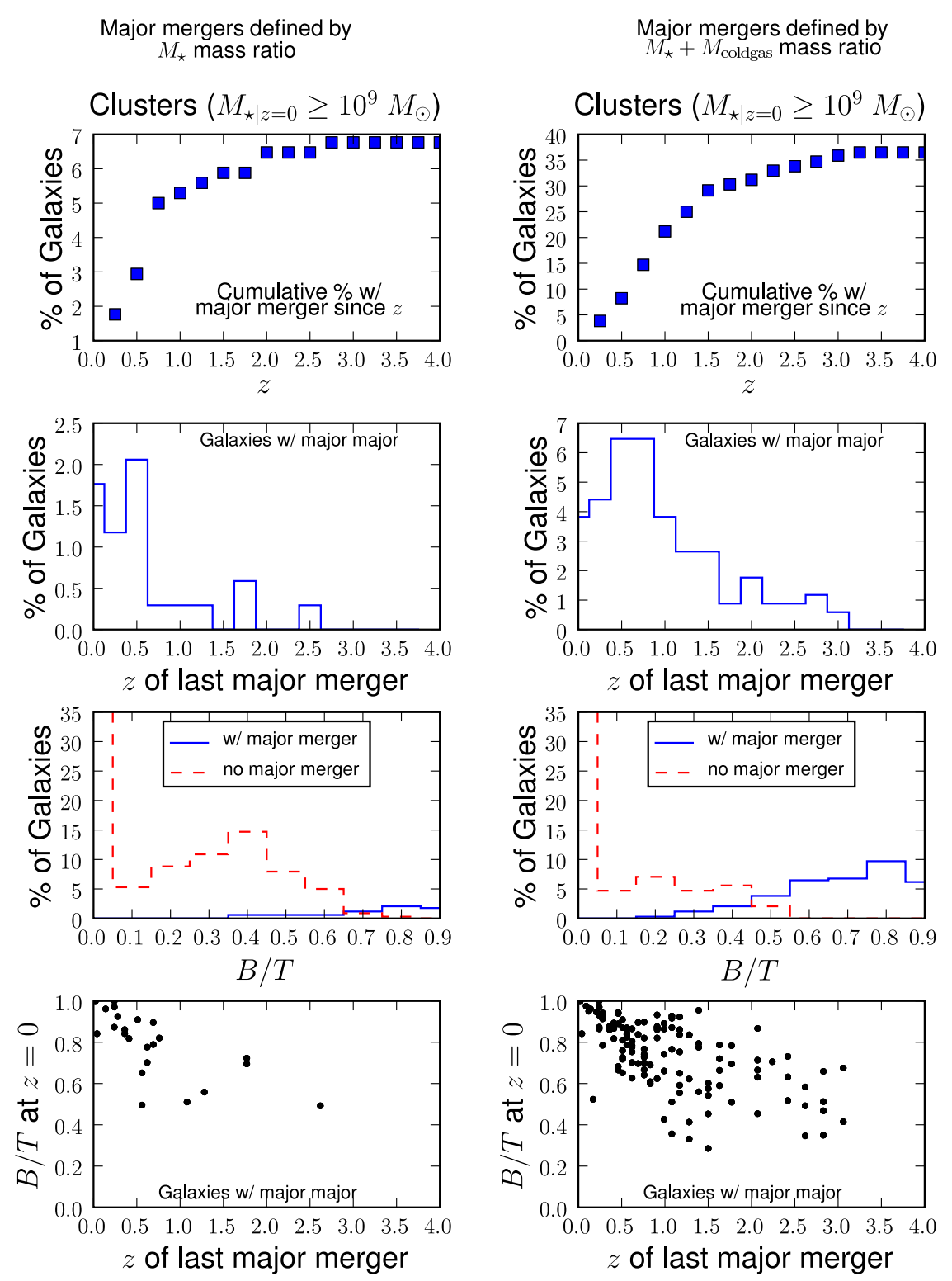

Figure 3.19 This figure highlights the effect that the definition of the merger mass ratio $M_{1} / M_{2}$ has on certain galaxy properties (merger history and $B / T$ ), for a representative model cluster (see Section 3.5.4). Note we require a major merger to have $M_{1} / M_{2} \geq 1 / 4$. The left column is the manifestation of the model cluster when $M_{1} / M_{2}$ refers to the stellar mass ratio, and in the right column the merger mass ratio represents cold gas plus stars. The two mass ratio definitions lead to vastly different merger histories and significantly affect the resulting distribution of $B / T$. 

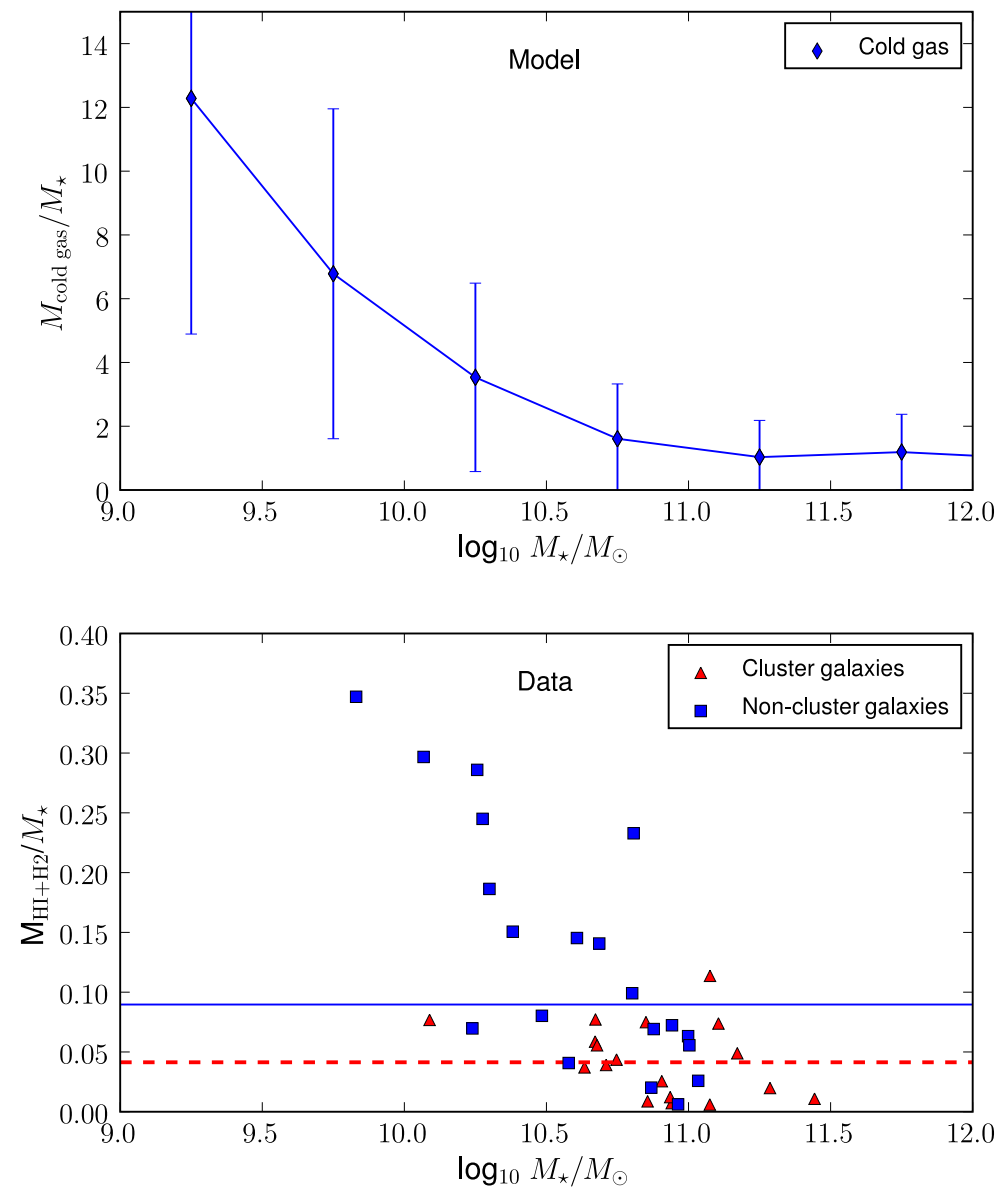

Figure 3.20 The top panel shows the ratio of cold gas to stellar mass $\left(M_{\text {cold_gas }} / M_{\star}\right)$ for the best cluster model matched by cumulative galaxy number density (see Figure 3.18, column 1). The error bars represent the $1 \sigma$ standard deviation around the mean value. The bottom panel shows the ratio of observed cold gas $\left(H I+H_{2}\right)$ to stellar mass $\left(M_{H I+H_{2}} / M_{\star}\right)$ for galaxies studied by Boselli et al. (1997) that are part of or near the Coma cluster. The dashed line is the median ratio (0.04) for Coma cluster galaxies, and the solid line is the median ratio $(0.09)$ for the non-cluster galaxies. At $10^{10} \lesssim M_{\star} \lesssim 10^{11} M_{\odot}$, the model predicts a cold gas to stellar mass ratio that is a factor $\sim 25-87$ times higher than the median value observed in Coma cluster galaxies. 

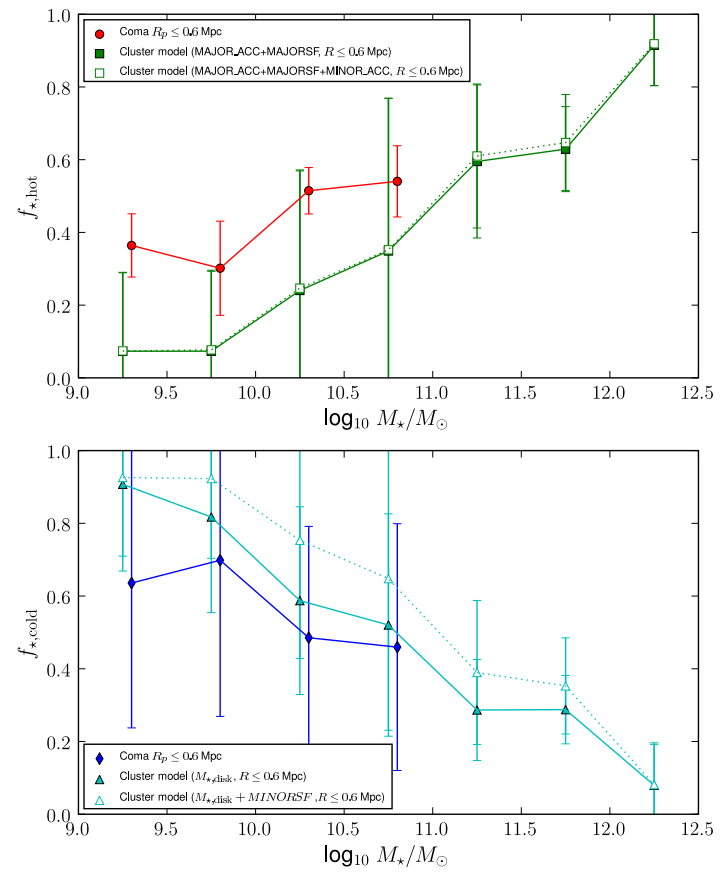

Figure 3.21 Top: The mean ratio of stellar mass fraction in dynamically hot components $\left(f_{\star, h o t}=M_{\star, h o t} / M_{\star}\right)$ is plotted as a function of total galaxy $M_{\star}$. In the data, $M_{\star, h o t}$ is taken as the stellar mass of any high $n>n_{\text {disk_max }}$ classical bulge/elliptical component in the galaxy, excluding the $\mathrm{cD}$ galaxies (see Section 3.2.2). The model shown here is the best cluster model matched by cumulative galaxy number density (see Figure 3.18, column 1). For this model, the solid line takes $M_{\star, h o t}$ as the stellar mass built during major mergers in Equation 3.7, namely (MAJOR_ACC $+M A J O R S F)$, while the dashed line considers $M_{\star, h o t}$ as (MAJOR_ACC+MAJORSF + MINOR_ACC), where the last term represents the stellar mass accreted during minor mergers. Bottom: The mean stellar mass fraction in dynamically cold flattened components $\left(f_{\star, \text { cold }}=M_{\star, \text { cold }} / M_{\star}\right)$ is plotted as a function of total galaxy stellar mass $M_{\star}$. In the data, $M_{\star, \text { cold }}$ is taken as the stellar mass of any low $n \leq n_{\text {disk_max }}$ disk-dominated component in the galaxy. The model is represented by the solid and dashed lines. With the solid line we take $M_{\star, \text { cold }}$ to be the mass of the outer disk $M_{\star, \text { outer_disk }}$, which is given by $\left(M_{\star}-M_{\star, \text { Bulge,model }}\right)$. For the dotted line, we consider $M_{\star, \text { cold }}$ to be the mass $M_{\star \text {,all_disk }}$ of inner and outer disks. We compute the latter mass as the sum $\left(M_{\star, \text { outer_disk }}+M I N O R S F\right)$, where MINORSF was defined in Equation 3.7 as the mass of stars formed via induced SF during minor mergers. In both panels, only the central $0.6 \mathrm{Mpc}$ of the clusters is considered. The error bars represent the $1 \sigma$ standard deviation on the mean. The mean values for Coma are slightly offset in $M_{\star}$ for readability. The main conclusion is that the best-matching simulated clusters are underpredicting the mean fraction of $f_{\star, \text { hot }}$ and overpredicting $f_{\star, \text { cold }}$ over a wide range in galaxy stellar mass. 


\section{Chapter 4}

\section{Structure and Assembly of the Most Massive Galaxies Present a Few Gyr $(z \sim 2-3)$ After the Big Bang}

\subsection{Introduction}

Studies of high-redshift galaxies are essential for testing and constraining models of galaxy formation. Conventional wisdom suggests galaxies are assembled and shaped by a combination of mergers, smooth accretion, and internal secular evolution. Galaxies form inside cold dark matter halos that grow hierarchically through mergers with other halos and gas accretion (Somerville \& Primack 1999; Cole et al. 2000; Steinmetz \& Navarro 2002; Birnboim \& Dekel 2003; Kereš et al. 2005; Dekel \& Birnboim 2006; Dekel et al. 2009a; Dekel et al. 2009b; Kereš et al. 2005; Kereš et al. 2009; Brooks et al. 2009; Ceverino et al. 2010), while internal secular evolution (Kormendy \& Kennicutt, 2004; Jogee et al. 2005) redistributes accreted material. Within the paradigm of hierarchical assembly, a number of issues remain. It is not known when and how the main baryonic components of modern galaxies (bulges, disks, and bars) formed, but the global stellar mass density rose

\footnotetext{
${ }^{1}$ This chapter has been previously published as Weinzirl, T., Jogee, S., Conselice, C. J., et al. 2011, ApJ, 743,87

Co-author contributions: Jogee, S. supervised the research. Bluck, A., Buitrago, F., and Conselice, C. J. provided the GOODS-NICMOS Survey data products. Papovich, C. provided estimates of infrared (8-1000 $\mu \mathrm{m}$ ) luminosity from the $24 \mu \mathrm{m}$-band flux. Papovich, C. and Ranga-Ram, C. provided advice on converting infrared luminosity to star formation rate. Bauer, A. E., Buitrago, F., Conselice, C. J., Dickinson, M., Grützbauch, R., Jogee, S., Lucas, R. A., and Mobasher, B. provided editorial comments.
} 
substantially between $z \sim 1-3$, reaching $\sim 50 \%$ of its present value by $z \sim 1$ (Dickinson et al. 2003b; Drory et al. 2005; Conselice et al. 2007; Elsner et al. 2008; Pérez-González et al. 2008).

It is also not clear how high-redshift galaxies evolve into present-day galaxies. Complex baryonic physics such as mergers, gas dissipation, and feedback are all at work to an extent. There is also mounting evidence that cold-mode accretion (Birnboim \& Dekel 2003; Kereš et al. 2005; Dekel \& Birnboim 2006; Dekel et al. 2009a; Dekel et al. 2009b; Kereš et al. 2009; Brooks et al. 2009; Ceverino et al. 2010) is important for building star-forming galaxies. This process is particularly effective in galaxies with halos of mass below $10^{12} M_{\odot}$ such that cold-mode accretion dominates the global growth of galaxies at high redshifts and the growth of lower mass objects at late times.

High-redshift galaxies are different from local galaxies. Within the framework of hierarchical assembly, early, high-redshift galaxies are expected to be smaller, at a given mass, than their present-day counterparts. The size difference is predicted to be a factor of a few at $z=2-3$ (Loeb \& Peebles 2003; Robertson et al. 2006; Khochfar \& Silk 2006a; Naab et al. 2007). Several recent studies using rest-frame optical data provide evidence for size evolution among massive galaxies (Guzman et al. 1997; Daddi et al. 2005; Trujillo et al. 2006, 2007; Zirm et al. 2007; Toft et al. 2007; Longhetti et al. 2007; Cimatti et al. 2008; Buitrago et al. 2008; van Dokkum et al. 2008, 2010; van der Wel et al. 2011). Aside from size evolution, there is some evidence that the nature of red galaxies changes at higher redshift. At $z \lesssim 1$, the red sequence primarily consists of old, passively evolving galaxies (Bell et al. 2004). Among extremely red galaxies (EROs) at $z=1-2$, less than $40 \%$ are morphologically early types (Yan \& Thompson 2003; Moustakas et al. 2004). It 
is well known that star formation rates were more intense at higher redshift (Daddi et al. 2007; Drory \& Alvarez 2008), and a link has been found between star formation, size, and morphology at $z \sim 2.5$. Toft et al. (2007) and Zirm et al. (2007) find from NICMOS rest-frame optical imaging that blue star-forming galaxies are significantly more extended than red quiescent galaxies. Additionally, examples of rapidly star-forming galaxies (SFR $\left.\sim 50-200 M_{\odot} \mathrm{yr}^{-1}\right)$ at $z \sim 2-3$, whose ionized gas kinematics are consistent with turbulent rotating disks, are found in the SINS survey (Förster Schreiber et al. 2009; Genzel et al. 2008; Shapiro et al. 2008).

Progress on understanding the evolution of massive galaxies at high redshift has been hindered by significant observational challenges. The deep optical surveys carried out by HST ACS, such as the Hubble Ultra Deep Field (HUDF, Beckwith et al. 2006) and the Great Observatories Origins Deep Survey (GOODS, Giavalisco et al. 2004), trace rest-frame optical galaxy morphology only out to $z \sim 1$. At higher $z$, bandpass shifting effects cause filters to trace progressively bluer bands, and optical filters trace rest-frame UV at $z \gtrsim 2$. UV light traces massive young stars, but manages to set few constraints about the overall mass distribution, making it difficult to probe the structure and mass of galaxy components at early epochs.

Without high-resolution, deep, rest-frame optical imaging, it is not possible to robustly compare structural parameters in galaxies across redshift. NIR imaging is required to probe the rest-frame optical at $z \sim 1-3$. Unfortunately, deep NIR imaging with $H S T$ has been completed for a limited number of galaxies over relatively small fields and small volumes at $z>1$, with most pointings being within the Hubble Deep Fields and the Hubble Ultra Deep Field due to the inefficiency of the NICMOS camera in covering large areas 
(e.g., Dickinson et al. 2004; Thompson et al. 2005; Zirm et al. 2007; van Dokkum et al. 2008). While ground-based NIR imaging surveys (e.g., Kajisawa et al. 2006; Retzlaff et al. 2010) efficiently cover wide fields at resolutions almost comparable with HST NICMOS, the depths reached are at least an order of magnitude shallower.

A large area, high-resolution, deep, space-based NIR survey would be bountiful for galaxy formation studies. The GOODS-NICMOS Survey (GNS; Conselice et al. 2011), covering $44 \operatorname{arcmin}^{2}$ of the GOODS fields with NIC3, is a strong first effort. The GOODS-North and GOODS-South are among the best-studied regions in the sky and are a natural choice for such a survey. The GOODS fields already have deep data from $H S T A C S$ (Giavalisco et al. 2004), Spitzer IRAC/MIPS (Dickinson et al. 2003a), and Chandra (Giacconi et al. 2002; Alexander et al. 2003; Lehmer et al. 2005; Luo et al. 2008), among others. GNS consists of 60 pointings centered on massive $\left(M_{\star}>10^{11} M_{\odot}\right)$ galaxies at $z>2$, observed to a depth of $H=26.8$ magnitudes. The value of GNS lies in the fact that the target fields were optimized to include massive galaxies selected by multiple methods in order to create an unbiased sample (see Conselice et al. 2011). There are additional massive galaxies in each field beyond the 60 main targets, so that there are 82 galaxies with $M_{\star} \geq 10^{11} M_{\odot}$ at $z=1-3$ across all pointings. Thus, the GNS data contain one of the largest samples of very massive galaxies at high redshift with rest-frame optical imaging, and they robustly probe massive galaxies when the Universe was less than $1 / 3$ of its current age, during the epoch of bulge and disk formation.

The goal of this work is to investigate the evolution of massive galaxies over $z=1-3$ with this unique sample. We take advantage of the existing rich ancillary data to derive star formation rates (SFR) from $24 \mu \mathrm{m}$ detections and look for AGN activity based on X-ray 
detections and mid-IR SEDs. We correlate rest-frame optical structural parameters with SFR to gain insight into how massive galaxies are expected to evolve.

The plan of this paper is as follows. We discuss the data and sample properties in $\S 4.2$. In $\S 4.3$ we describe the measurement of structural parameters, and in $\S 4.3 .2$ we make a detailed comparison with $z \sim 0$ galaxies of similar stellar mass. A detailed artificial redshifting experiment is conducted in $§ 4.3 .3 .1$ to explore the impact of instrumental and redshift-dependent effects on structural parameters. In $\S 4.4$, we measure star formation properties based on Spitzer MIPS $24 \mu \mathrm{m}$ detections and discuss how they relate to structural properties. Estimates of the mass and fraction of cold gas in massive star-forming galaxies at $z=2-3$ are presented in $\S 4.5$. In $\S 4.6$, we use a variety of techniques (X-ray properties, IR power-law, and IR-to-optical excess) to identify AGN and consider how galaxy activity relates to galaxy structure. Finally, in $\S 4.7$ and $\S 4.8$, we discuss and summarize our results. All calculations assume a flat $\Lambda$ CDM cosmology with $\Omega_{\Lambda}=0.7$ and $H_{0}=70 \mathrm{~km} \mathrm{~s}^{-1} \mathrm{Mpc}^{-1}$.

\subsection{Data and Sample}

\subsubsection{Observations and Pointing Selections for GNS}

Our data comes from the GOODS-NICMOS Survey (GNS; Conselice et al. 2011). GNS is a deep, 180-orbit survey with the HST NICMOS-3 camera in the F160W $(H)$ band that probes optical light from galaxies between $z \sim 1-3$. The coverage extends over both ACS GOODS fields and is divided between 60 pointings centered on massive $M_{\star} \geq 10^{11} M_{\odot}$

galaxies at $z>2$. Each pointing covers $51^{\prime \prime} \cdot 2 \times 51^{\prime \prime} 2$ and was observed to a depth of three orbits in nine exposures of $\sim 900$ seconds ( $\sim 135$ minutes per pointing). A total of $\sim 8300$ sources were detected across an effective area of $\sim 44 \operatorname{arcmin}^{2}$. The $5 \sigma$ limiting magnitude 
for an extended source with a $0 . ! 7$ diameter is $H=26.8(\mathrm{AB})$. The NIC-3 images were drizzled with a pixfrac of 0.7 and output platescale of 0.1 . The NIC3 camera is currently out of focus,and after detailed investigation (see Appendix B.1), we find the point spread function (PSF) spans a full width half maximum (FWHM) of $0.26-0 ! 36$ with a mean value of $0 . \prime 3$.

The 60 GNS pointings were planned by identifying massive galaxies having a photometric redshift of $1.5<z<2.9$ and stellar mass $M_{\star}>10^{11} M_{\odot}$ via three color selection criteria. The target galaxies include Distant Red Galaxies (DRGs, Papovich et al. 2006), Extremely Red Objects (EROs, Yan et al. 2004), and $B z K$-selected galaxies (Daddi et al. 2004). All of these methods are designed to select red dusty or red passively evolving galaxies. DRGs have evolved stellar populations that are identified with $J-K>2.3$ (Vega mag). EROs are selected based on Spitzer and NIR data via $f_{\nu}(3.6 \mu m) / f_{\nu}(z 850)>20$. This selection is sensitive to red populations that are either old or reddened, so EROs contain a mixture of young and old stellar populations. $B z K$ galaxies are selected based on the quantity $B z K \equiv(z-K)_{A B}-(B-z)_{A B}$. Galaxies with $B z K>-0.2$ at $z>1.4$ are identified as star-forming galaxies. Redder and possibly more evolved galaxies are identified with $B z K<-0.2$ and $(z-K)_{A B}>2.5$. The final pointings were designed to include at least one red massive galaxy and to also maximize the total number of additional galaxies (e.g., Lyman-break galaxies and sub-mm galaxies) within each pointing.

\subsubsection{Our Sample of Massive Galaxies at $\mathrm{z}=1-3$}

The sample of massive galaxies that we work with in this paper is not limited to the original color-selected massive galaxies at $z>1.5$ defining the original 60 GNS pointings. Instead, our sample of massive galaxies at $z=1-3$ is derived from the set of all galaxies 
mapped with NIC3 F160W across the 60 fields, and for which a reliable stellar mass and photometric redshift was estimated by Conselice et al. (2011), based on SED fits to the NIC3 F160W and optical imaging. A detailed description of how these quantities were estimated is in Conselice et al. (2011), and we only briefly summarize the methodology here.

The source extraction catalog for the NICMOS images across the 60 pointings of the GNS survey contains $\sim 8300$ sources with $H<28$ and $V<30$. For those galaxies detected in the $A C S B V i z$ and NICMOS $H$ bands, we use the available photometric redshifts and stellar masses from Conselice et al. (2011). Photometric redshifts were determined by fitting template spectra to the $B V i z H$ data. Stellar masses were measured by fitting the $B V i z H$ magnitudes to a grid of SEDs generated from Bruzual \& Charlot (2003) stellar population synthesis models, assuming a Salpeter $\mathrm{IMF}^{2}$. The grid includes different colors, ages of stellar populations, metallicities, dust content, and star formation histories as characterized by exponentially declining models. In general, the stellar masses derived depend on the SED used and the assumptions used in the SED modeling, such as the IMF, the metallicity, the extinction law, and star formation history (e.g., Borch et al. 2006; Marchesini et al. 2009; Conselice et al. 2011). The typical uncertainty in stellar mass across the sample is a factor of $\sim 2-3$.

In order to account for a small number (15) of additional massive $\left(M_{\star} \geq 5 \times 10^{10}\right.$ $\left.M_{\odot}\right)$ red systems, which are undetected in the GOODS ACS $B V$ and therefore do not have viable stellar masses from the above techniques, we use available masses and redshifts (Buitrago et al. 2008; Bluck et al. 2009) based on deep ground-based RIJHK data along

\footnotetext{
${ }^{2}$ In $\$ 4.4$ we use a Chabrier IMF for SFR estimates. Using a Chabrier IMF rather than a Salpeter IMF in estimating the stellar mass would lower the values by a factor of 0.25 dex or less.
} 
with ACS $i z$ data, where available. Photometric redshifts are determined with a mixture of techniques (e.g., neural networks and Bayesian techniques) described more fully in Conselice et al. (2007). Stellar masses were measured from these data with uncertainties of a factor of $\sim 2-3$ with the multi-color stellar population fitting techniques from Conselice et al. (2007, 2008). As with the larger sample described above, a stellar mass is produced by fitting model SEDs to the observed SED for each galaxy. A Salpeter IMF is assumed, and the SED grids are constructed from Bruzual \& Charlot (2003) stellar population synthesis models.

From the sample of galaxies with photometric redshifts and stellar masses determined as described above, we define the sample of massive galaxies used in this paper. We restrict our analysis to the redshift interval $z=1-3$ over which our NIC3/F160W images probe the rest-frame optical light in order to avoid bandpass shifts into the rest-frame UV. This ensures that we measure all structural parameters in the rest-frame optical across $z=1-3$, thereby reducing bandshift biases (see $\S 4.3 .1$ for a quantitative estimate). Although the mass functions calculated for GNS by Mortlock et al. (2011) show that the mass completeness limit is $\sim 3 \times 10^{9} M_{\odot}$ at $z \sim 3$, we apply a higher mass cut of $5 \times 10^{10} M_{\odot}$ as our interest is specifically with the most massive galaxies.

Our final sample consists of the $166(82)$ massive galaxies with $M_{\star} \geq 5 \times 10^{10} M_{\odot}$ $\left(M_{\star} \geq 1 \times 10^{11} M_{\odot}\right)$ and $z=1-3$. This is the largest $H S T$-based dataset with rest-frame optical imaging of massive galaxies over $z=1-3$. The galaxies with $M_{\star} \geq 10^{11} M_{\odot}$ from Buitrago et al. (2008) are part of the sample. The other previous HST NICMOS studies (e.g., Toft et al. 2007; Zirm et al. 2007; van Dokkum et al. 2008) each contain, at most, 10-20 systems with $M_{\star} \geq 10^{11} M_{\odot}$. The full distributions of apparent $H$ and $V$ magnitude, 
stellar mass, and redshift for this sample are shown in Figure 4.1.

Figure 4.2 shows a comparison of the galaxy stellar mass function (SMF) of our GNS-based sample with the published SMF of other NIR-selected samples in the literature, such as the $K$-selected samples of Fontana et al. (2006), Kajisawa et al. (2009) and Marchesini et al. (2009), as well as the IRAC-selected sample of Pérez-Gonzalez et al. (2008). This figure essentially shows that for the mass range $\left(M_{\star} \geq 5 \times 10^{10} M_{\odot}\right)$ relevant for the GNS-based sample used in our paper, there is good agreement between the SMF of our sample and those from these four studies. In particular, at $M_{\star} \geq 5 \times 10^{10} M_{\odot}$, the top panel shows that there is very good agreement with our sample, Fontana et al. (2006), and Pérez-Gonzalez et al. (2008) for three different redshift bins between $z=1.5$ and $z=3.0$. In the lower panel, at $M_{\star} \geq 5 \times 10^{10} M_{\odot}$, the average SMF from Kajisawa et al. (2009) agrees with that of our sample within a factor of $\sim 2$ over $1.5<z<2.5$. The SMF from our GNS-based sample and Marchesini et al. (2009) show good agreement at $z=2-3$, and are slightly offset at $\mathrm{z}=1.3$ to 2.0 . The small offset may not be statistically significant if one includes all the sources of error. The error bars on the GNS mass functions include Poisson errors only. Marchesini et al. (2009) show that the dominant sources of error regarding stellar mass functions are cosmic variance and systematics from the assumptions used in the SED modeling. For a discussion of the SMF for lower mass $\left(M_{\star} \leq 5 \times 10^{10} M_{\odot}\right)$ galaxies, which are not included in the sample used in this paper, we refer the reader to Mortlock et al. (2011).

In our sample of 166 massive galaxies, spectroscopic redshifts are available for 44 galaxies $(26.5 \pm 3.4 \%$ of the sample). These 44 galaxies are all bright with $V \leq 27$ and $H_{\mathrm{AB}}<23$. Among these 44 galaxies, the median photometric redshift error is $\delta z /(1+z)=$ 
0.071 (Grützbauch et al. 2011), $7 / 44(15.9 \pm 5.5 \%)$ have $\delta z /(1+z)>0.2$, and none have $\delta z /(1+z)>0.5 .^{3}$ For the remaining 122/166 $(73.5 \pm 3.4 \%)$ of our sample galaxies without spectroscopic redshifts, photometric redshifts are used. Among these 122 galaxies, $60(49.2 \pm 4.5 \%)$ are fainter than $V>27$, and the uncertainties in photometric redshifts may be larger than the median value of 0.071 cited above.

\subsubsection{Properties and Selection Biases in the Sample}

We estimate the number density of massive $\left(M_{\star} \geq 5 \times 10^{10} M_{\odot}\right)$ galaxies over $z=2-3$ to be $\sim 5 \times 10^{-4} \mathrm{Mpc}^{-3}$ (see Conselice et al. 2011 for a detailed discussion of the number density of massive galaxies in the GNS sample). The corresponding stellar mass density is $\sim 6 \times 10^{7} M_{\odot} \mathrm{Mpc}^{-3}$. The massive GNS galaxies are collectively 10-100 times more abundant than SMGs, which have space densities of $10^{-5}-10^{-6} \mathrm{Mpc}^{-3}$ at $z \sim 2-3$ (Blain et al. 2002). Rather, the number density is in agreement with published values (Daddi et al. 2005; 2007) for other passively evolving and star-forming galaxies at $z \sim 2$.

How does our sample break down in terms of the typical color-selection methods, which are usually used to identify massive high redshift galaxies? About 63\% (104/166) of this final sample is listed in existing catalogs for DRG (Papovich et al. 2006), BzK (Daddi et al. 2004), or ERO (Yan et al. 2004) galaxy populations. There are 8, 9, and 43 sources that are uniquely listed in one of the DRG, $B z k$, or ERO galaxy catalogs, respectively. An additional 44 sources are listed in two or more of these catalogs. About 37\% (62/166)

\footnotetext{
${ }^{3}$ While figure 6 of Conselice et al. (2011) shows that $\sim 15-20 \%$ of bright $\left(20<H_{\mathrm{AB}}<23\right)$ galaxies with spectroscopic redshifts are catastrophic outliers in photometric redshift with $\delta z /(1+z)>0.5$, it should be noted that there are no catastrophic outliers with such large $\delta z /(1+z)>0.5$ among the 44 galaxies with spectroscopic redshifts in our sample of massive $\left(M_{\star} \geq 5 \times 10^{10} M_{\odot}\right)$ galaxies at $z=1-3$. The outliers with $\delta z /(1+z)>0.5$ in the GNS survey have stellar masses below the cutoff value of our sample or/and lie outside its redshift range.
} 
sources were not previously identified as DRG, ERO, or $B z K$ galaxies.

What are the selection biases impacting our sample? General biases in the selection of massive galaxies in the GNS survey have been discussed in Conselice et al. (2011), and we only discuss below the points relevant for our sample.

The 60 GNS pointings were selected to include massive galaxies identified via three color methods (DRG, BzK, and IERO). Combining all three color criteria, rather than using any single one, is already a step forward compared with many earlier studies because no single criterion would isolate a complete sample of massive galaxies (e.g., van Dokkum et al. 2006; Conselice et al. 2011). These three criteria all pick massive galaxies with red observed colors, but due to the range of criteria involved, they can pick both red dusty systems and red evolved stellar populations.

Another key step that makes our study less biased towards a specific type of massive galaxy is that our working sample at $z=1-3$ is neither limited to nor defined by the original color-selected massive galaxies. Rather, it is derived from all galaxies within the survey area that are bright enough to be mapped with NIC3/F160W and for which a reliable stellar mass and photometric redshift could be determined by Conselice et al. (2011), as outlined in $§ 4.2 .2$. The first potential bias in this final sample is introduced by excluding galaxies that are undetected by NIC3/F160W. The second potential bias is introduced by excluding detected galaxies for which no reliable stellar mass and photometric redshift could be determined. For instance, ultra-dusty galaxies, may not be detected in enough of the optical bands to allow a photometric redshift to be reliably estimated.

We assess the impact of the second bias by estimating how many massive galaxies we might miss due to the lack of available photometric redshift and stellar masses. Of 
the 8300 sources detected by GNS, 1076 have no photometric redshift and stellar mass measurements. Most $(68 \%)$ of these 1076 sources are fainter $(H>25)$ than our sample of massive galaxies (Figure 4.1). Among GNS objects as bright $(H<25)$ as our sample of massive galaxies, only $8.5 \%$, or $349 / 4083$ have no redshift or stellar mass measurements. Furthermore, not all $8.5 \%$ of these bright $(H<25)$ sources will be massive, so that this fraction represents an upper limit on the sources we might not include in our sample due to the lack of a photometric redshift or stellar mass measurements.

We next discuss the impact of the first potential bias and the type of objects the GNS survey might not detect. It is relevant to ask whether we might miss galaxies with blue observed colors. We believe this is not the case for the following reasons. As discussed above, our working sample is not strongly biased against galaxies with blue observed colors because it is not limited to those massive galaxies selected by the three color methods (DRG, $B z K$, and IERO) that preferentially pick galaxies with red observed colors. Secondly, Conselice et al. (2011) explicitly show that many galaxies with blue observed $(z-H)$ colors, which would have been undetected by these color selections, do get included in this final sample of massive galaxies for the GNS survey. Nearly all known Lyman Break Galaxies or BX/BM objects (Reddy et al. 2008) at $z=2-3$ in the GNS fields are detected by the GNS NIC3/F160W imaging (Conselice et al. 2011).

In terms of rest-frame colors, rather than observed colors, it is also important to note that the galaxies detected by GNS at $z=1-2$ or $z=2-3$ include systems with both blue and red rest-frame $U-V$ colors. The rest-frame $U-V$ color ranges from about -0.4 -2.1 for galaxies in the stellar mass range $M_{\star} \sim 10^{9}-10^{12} M_{\odot}$ (Figure 4.3 ). The systems with blue rest-frame $U-V$ colors are preferentially at low masses, while GNS galaxies with 
$M_{\star} \geq 1 \times 10^{11} M_{\odot}$ at $z=2-3$ have preferentially red rest-frame $U-V$ colors, in the range of 1.0 to 1.7 . These inherently red rest-frame $U-V$ colors of the massive galaxies at $z=2-3$ could be due to a combination of old stellar populations and dusty young starforming regions. We checked that the colors are consistent with stellar population synthesis models (based on Bruzual \& Charlot (2003) and assuming a Chabrier IMF, an exponentially declining star formation history with a $100 \mathrm{Myr}$ e-folding time). We find that even without dust extinction $U-V$ color rises rapidly. Assuming solar metallicity, $U-V$ is already $\sim 1$ at an age of 0.5 Gyr and reaches $\sim 1.6$ at 2 Gyr. For the case with dust extinction and an optical depth of $1, U-V$ is $\sim 1.1$ after 0.5 Gyr and $\sim 1.8$ after 2 Gyr.

\subsection{Structural Properties of Massive Galaxies}

\subsubsection{Structural Decomposition}

We characterize the massive GNS galaxies with structural decomposition. Ideally, one would like to fit multiple components (bulge, disk, bar, nuclear point source, etc.) in the decomposition, but the $00^{\prime \prime} 3$ diameter (or full width half-maximum) of the PSF (corresponding to $\sim 2.4 \mathrm{kpc}$ at $z=1-3)$ prevents such detailed decompositions ${ }^{4}$. Instead, we choose to fit the 2D light distributions with only single Sérsic (1968) $r^{1 / n}$ profiles, which have the form

$$
I(r)=I_{e} \exp \left(-b_{n}\left[\left(\frac{r}{r_{e}}\right)^{1 / n}-1\right]\right),
$$

where $\mathrm{I}_{\mathrm{e}}$ is the surface brightness at the effective radius $r_{e}$ and $\mathrm{b}_{\mathrm{n}}$ is a constant that depends on Sérsic index $n$. Knowledge of the PSF is important for deriving structural parameters. We model the PSF (Appendix B.1) while taking into account both the variation in PSF

\footnotetext{
${ }^{4}$ For the more extended galaxies multiple components (e.g., bulge and disk) decomposition was attempted with limited success and this is discussed in $§ 4.7 .1$.
} 
with position on the NIC3 field and the dependence on the drizzle algorithm. We find a range in PSF FWHM of $\sim 00^{\prime \prime} 26-0^{\prime \prime} 36$.

It is clear that a single Sérsic profile is not a complete indicator of overall galaxy structure. For instance, in detailed images of nearby galaxies, the best-fit index $n$ for a single Sérsic profile does not always correlate with the bulge Sérsic index obtained with 2D bulge-disk or bulge-disk-bar decomposition (Weinzirl et al. 2009). However, the single Sérsic index $n$ is on average a good way to separate disk-dominated galaxies from the class of luminous spheroidal and bulged-dominated galaxies (see §4.3.3.1), and in studies of highredshift galaxies the criterion $n \lesssim 2$ is often used to separate spirals or disk galaxies from ellipticals (e.g., Ravindranath et al. 2004; Bell et al. 2004; Jogee et al. 2004; Barden et al. 2005; Trujillo et al. 2007; Buitrago et al. 2008).

The NIC3/F160W images of the 166 sample galaxies were fit with a single Sérsic component using GALFIT (Peng et al. 2002). In each image, objects that were near, but not blended with, the primary source were masked out. For the fraction $(\sim 15 \%)$ of the primary galaxies that were blended or overlapping with another galaxy identified in the source extraction catalog, the blended sources were each fitted simultaneously with a separate Sérsic profile. Some fraction of primary galaxies appeared morphologically disturbed ( $\sim 8 \%$, see Figure 4.4 and $§ 4.3 .2)$, but these were fitted with only a single Sérsic profile as they only counted as a single galaxy in the source extraction catalog.

Bandpass shifting causes the $H$-band central wavelength to move from $4000-8000$ $\AA$ over $z=1-3$. The $z=1-2$ and $z=2-3$ bins used in Figure 4.5, for example, correspond to 5333-8000 $\AA$ ( $I$-band) and 4000-5333 $\AA$ ( $B$-band), respectively. Even with the bandpass shifting, comparing the structural parameters $\left(n, r_{e}\right)$ measured in these two 
bands with each other and with parameters of $z \sim 0$ galaxies measured in rest-frame $B$ is a vast improvement over previous studies forced to compare the rest-frame UV at $z>1$ with the rest-frame optical at $z<1$. The systematic effects resulting from $H$-band changing from $B$ to $I$-band over $z=1-3$ are small, as can be inferred from studies of nearby galaxies. Graham (2001) presents bulge-disk decompositions of local $z \sim 0$ galaxies based on images in the $B$ and $I$ bands. The median ratio in $B$-band $/ I$-band disk scalelength is 1.13 , so that the disks are measured to be slightly larger in the B-band. If similar errors apply here, then the bias $r_{e}$ due to bandpass shifting is on the order of $10 \%$.

Another important consideration is the effect of potential AGN on the structural fits. When fitting high resolution images of nearby galaxies, it is well known that fitting a galaxy that hosts a point source with a single Sérsic component will lead to an artificially high Sérsic index $n$ (typically $n>4$; e.g., Weinzirl et al. 2009; Pierce et al. 2010). If a point source is added to the Sérsic model, the index $n$ of the Sérsic component falls to more reasonable values. In the case of the massive GNS galaxies at $z=1-3$, we expect that the low resolution $\left(0^{\prime \prime} 3\right.$, corresponding to $2.5 \mathrm{kpc}$ at $\left.z \sim 2\right)$ of the NIC3/F160W images will reduce the effect of potential point sources on the structural decomposition. However, for completeness, we have fitted all the galaxies at $z=1-3$ in which a potential AGN was identified via a variety of techniques (§4.6) with both a Sérsic component and a point source. The fractional luminosity of the point source components, or PSF/Total light ratio, ranges from $1-46 \%$, with a median of $10 \%$. As expected, including the point source produces generally small changes in $\left(n, r_{e}\right)$ and goes in the direction of lowering $n$ and enlarging $r_{e}$. Overall, our results are not biased by the presence of AGN. In the rest of the paper, we therefore choose to use the structural parameters for a single Sérsic component fit. 


\subsubsection{Derived Structural Properties at $z=2-3$}

The results of the structural fits to the NIC3/F160W images of the 166 sample galaxies are shown in Table 4.1, Figure 4.4, Figure 4.5, and Figure 4.6.

Figure 4.4 shows examples of massive $\left(M_{\star} \geq 5 \times 10^{10} M_{\odot}\right)$ galaxies at $z=2-3$ with different ranges of Sérsic index $n$ and effective radius $r_{e}$. The majority $(\sim 82 \%$; Table 4.1$)$ of the massive GNS galaxies at $z=2-3$ have $r_{e} \leq 4 \mathrm{kpc}$. In such systems, structural features are generally hard to discern due to resolution effects, so that systems appear fairly featureless (top 4 rows of Figure 4.4). In the small fraction of massive galaxies at $z=2-3$ with large $r_{e}>4 \mathrm{kpc}$, one can discern some structural features such as an elongated bar-like feature or a combination of a central condensation surrounded by a more extended lower surface brightness component, reminiscent of a bulge and disk (5th row). Row 6 contains morphologically disturbed systems. The fraction of such systems is small, only $\sim 8 \%$, but this is a lower limit given redshift-dependent effects such as degraded physical resolution and surface brightness dimming.

The lower two rows of Figure 4.5 shows the rest-frame optical Sérsic index $n$ and effective radius $r_{e}$ for the samples of massive galaxies at $z=1-2$ and $z=2-3$. For comparison, the top row of Figure 4.5 also shows the rest-frame optical structural parameters for $z \sim 0$ galaxies of similar stellar mass taken from Allen et al. (2006), who performed a single component Sérsic fit to $B$-band images of galaxies in the Millennium Galaxy Catalogue (MGC), a large ground-based imaging and spectroscopic survey over $37.5 \mathrm{deg}^{2}$ (Liske et al. 2003; Driver et al. 2005). It is clear from Figure 4.5, Figure 4.6, and Table 4.1 that the massive galaxies at $z=2-3$ are strikingly offset toward lower $\left(n, r_{e}\right)$ compared with the massive $\sim 0$ galaxies. 
Firstly, we find that the majority $\left(64.9 \pm 5.4 \%\right.$ for $M_{\star} \geq 5 \times 10^{10} M_{\odot}$, and $58.5 \pm 7.7 \%$ for $M_{\star} \geq 10^{11} M_{\odot}$ ) of massive galaxies at $z=2-3$ have low $n \leq 2$, while the fraction at $z \sim 0$ is five times lower. We will present evidence in $\S 4.7 .1$ that most of the massive systems with a low $n \leq 2$ harbor a massive disk component, so that our results point to the predominance of disk-dominated systems among massive galaxies at $z=2-3$.

Secondly, we also find that massive galaxies at $z=2-3$ typically have smaller $r_{e}$ than massive galaxies at $z \sim 0$. In particular, $\sim 40 \%\left(39.0 \pm 5.6 \%\right.$ for $M_{\star} \geq 5 \times 10^{10} M_{\odot}$ and $39.0 \pm 7.6 \%$ for $M_{\star} \geq 1 \times 10^{11} M_{\odot}$ ) of massive galaxies at $z=2-3$ are ultra-compact $\left(r_{e} \leq 2 \mathrm{kpc}\right)$, compared with less than one percent at $z \sim 0$. The massive ultra-compact $\left(r_{e} \leq 2 \mathrm{kpc}\right)$, galaxies at $z=2-3$ have few counterparts among $z \sim 0$ massive galaxies.

The population of galaxies with low $n \leq 2$ and the population of ultra-compact $\left(r_{e} \leq 2 \mathrm{kpc}\right)$ galaxies show limited overlap. Only $28.0 \pm 6.4 \%$ of the systems with low $n \leq 2$ are ultra-compact and the remaining majority $\left(72.0 \pm 6.3 \%\right.$ for $M_{\star} \geq 5 \times 10^{10} M_{\odot}$, and $75.0 \pm 8.8 \%$ for $\left.M_{\star} \geq 10^{11} M_{\odot}\right)$ are extended $\left(r_{e}>2 \mathrm{kpc}\right)$. Conversely, among the ultra-compact $\left(r_{e} \leq 2 \mathrm{kpc}\right)$ systems, nearly half $\left(46.7 \pm 9.1 \%\right.$ for $M_{\star} \geq 5 \times 10^{10} M_{\odot}$, and $37.5 \pm 12.1 \%$ for $\left.M_{\star} \geq 10^{11} M_{\odot}\right)$ have low $n \leq 2$.

Figure 4.7 further illustrates the striking difference between massive galaxies at $z=2-3$ and $z \sim 0$ by comparing their effective radius $r_{e}$ and their mean rest-frame optical surface brightness $<\mu_{\mathrm{e}}>$ within $r_{e}$. The value of $<\mu_{\mathrm{e}}>$ was measured from the extinction-corrected rest-frame $B$-band light within $r_{e}$ and is defined as:

$$
\mu_{\mathrm{e}}=\mathrm{B}_{\mathrm{corr}}+2.5 \log _{10}\left(2 \pi \mathrm{r}_{\mathrm{e}}^{2}\right)-10 \log _{10}(1+\mathrm{z})
$$

where $B_{\text {corr }}$ is the extinction-corrected, rest-frame apparent $B$ magnitude and $-10 \log _{10}(1+$ 
z) and is the correction for surface brightness dimming. The MGC galaxies at $z \sim 0$ are corrected only for Galactic extinction, while for the GNS galaxies the correction includes Galactic and internal extinction. The mean rest-frame optical surface brightness can be 2.0 to 6.0 magnitudes brighter for the massive galaxies at $z=2-3$ than for $z \sim 0$ massive galaxies. This is due to their smaller sizes and likely differences in the age of the stellar populations. The high mean rest-frame optical surface brightness of the massive galaxies at $z=2-3$ translates into high mean stellar mass densities, and suggests that highly dissipative events played an important role in their formation (see $\S 4.7)$.

It is worth noting that the use of deeper images for the $z \sim 0$ galaxies could make the large offset in $\left(n, r_{e}\right)$ at $z=2-3$ versus $z \sim 0$ even stronger. The MGC $B$-band images have a median sky background of $22 \mathrm{mag} / \operatorname{arcsec}^{2}$. Low surface brightness halos may be detected around some of the $z \sim 0$ galaxies in deeper exposures. This is true for some massive elliptical and $\mathrm{cD}$ galaxies, and in these cases the $\left(n, r_{e}\right)$ are significantly boosted if the halo is region is also fitted (Kormendy et al. 2009).

How do these results compare with earlier studies? While many of the earlier studies focused on small samples, this work is a step forward because of the improved number statistics that come with an unbiased and complete sample of massive galaxies. The observed apparent size evolution in our data generally agrees with results reported in other studies of massive galaxies (e.g., Daddi et al. 2005; Trujillo et al. 2007; Zirm et al. 2007; Toft et al. 2007; Buitrago et al. 2008; van Dokkum et al. 2008; 2010; Williams et al. 2010).

The ratio in $r_{e}$ of high-redshift galaxies with respect to $z \sim 0$ galaxies, or $r_{e} / r_{e, z \sim 0}$, can be modeled as a power law in redshift of the form $\alpha(1+z)^{\beta}$, where $\alpha$ and $\beta$ are constants. 
Using the $z \sim 0$ massive $\left(M_{\star} \geq 5 \times 10^{10} M_{\odot}\right)$ MGC galaxies as the normalization, we measure $\alpha$ and $\beta$ for different subsamples of the massive galaxies and summarize the results in Table 4.2. For all galaxies the slope $\beta$ is -1.30 for a fit over $z=0-3$. For disk-like $n \leq 2$ galaxies $\beta$ is also -1.30 , and for $n>2$ galaxies $\beta$ is -1.52 . For non-AGN host galaxies with $S F R_{\mathrm{IR}}$ detected above the $5 \sigma$ detection limit (see $\S 4.4$ ), $\beta$ is -1.21 , while for non-AGN host galaxies not detected by Spitzer the slope is substantially steeper (-1.67).

These results are comparable with the findings of earlier studies. Buitrago et al. (2008) show for massive $\left(M_{\star} \geq 10^{11} M_{\odot}\right)$ galaxies over $z=0-3$ that $\beta$ varies from -0.8 for $n<2$ disk-like galaxies to -1.5 for $n>2$ spheroidal galaxies. Williams et al. (2010) find $\beta$ is -0.88 for all massive $\left(M_{\star} \geq 6.3 \times 10^{10} M_{\odot}\right)$ galaxies over $z=0.5-2$. van Dokkum et al. (2010) find a slope of -1.27 for massive $\left(M_{\star} \geq 10^{11} M_{\odot}\right)$ galaxies over $\mathrm{z}=0-2$, which is a good match to our slope $(-1.30)$ for massive $\left(M_{\star} \geq 5 \times 10^{10} M_{\odot}\right)$ galaxies of all $n$ over $z=0-3$. Compared to massive $z \sim 0$ galaxies, the implied mean size evolution is a factor of $\sim 4$ from $z=2-3$ and a factor of $\sim 3$ from $z=1-2$. In order to determine whether this apparent size evolution is real, one needs to address a number of systematic effects, as outlined in the next section.

\subsubsection{Impact of Systematic Effects on Structural Properties}

In the previous section we found that the massive galaxies at $z=2-3$ are strikingly offset toward lower $\left(n, r_{e}\right)$ compared with the massive $z \sim 0$ galaxies. It is relevant to ask whether the large fraction of low $\left(n, r_{e}\right)$ systems we observe among massive galaxies at $z=2-3$, compared to massive galaxies at $z \sim 0$ is real or due to a number of systematic effects. We address the most important effects in the main text and include the others in 
Appendix 2. We consider the issues listed below:

1. Is it possible that the distribution of $\left(n, r_{e}\right)$ for massive galaxies at $z \sim 0$ and at $z=2-3$ is intrinsically similar, but that some selection effects at $z=2.5$ is making us preferentially detect the compact low $n$ systems, thereby causing an artificial excess of the latter? We argue that this is very unlikely because even if we take all the massive compact low $n$ systems at $z \sim 0$, and appropriately scale them for the difference in number density between $z \sim 0$ and $z=2.5$, we still would fall way short of reproducing the observed number densities of compact low $n$ systems. The number density of massive $\left(M_{\star} \geq 1 \times 10^{11} M_{\odot}\right)$ galaxies at $z=2.5$ is approximately $30 \%$ that at $z \sim 0$. If we take the most compact $\left(r_{e} \leq 2 \mathrm{kpc}\right)$ and low $n \leq 2$ systems at $z \sim 0$, and scale

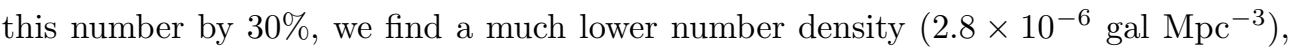
than the observed no density $\left(5.0 \times 10^{-5} \mathrm{gal} \mathrm{Mpc}^{-3}\right)$ at $z=2.5$ for such compact systems.

2. Can redshift-dependent systematic effects cause structural parameters, such as the high Sérsic index $n$ of massive galaxies at $z \sim 0$, to 'degrade' into the regime of low $n \leq 2$ values, measured in the $z=2-3$ systems. We address this issue in $\S 4.3 .3 .1$.

3. How robust are our fits to the NIC3/F610W images of the $z=2-3$ galaxies? Could some of the galaxies with a best-fit Sérsic index $n \leq 2$ have similarly good fits with much higher $n$ ? We show in Appendix B.2 and Appendix B.3 that this is unlikely. We are confident that the fraction of $n \leq 2$ systems is not being overestimated.

4. Can the offset in $\left(n, r_{e}\right)$ between the $z=2-3$ galaxies and the $z \sim 0$ galaxies be caused by systematic differences between the fitting techniques applied by us to the 
NIC3/F610W images of $z=2-3$ galaxies and the fitting techniques used by Allen et al. (2006) on the $B$-band images of the massive $z \sim 0$ galaxies in MGC? We conduct additional tests (see Appendix B.4) and conclude that this is also not the case.

\subsubsection{Artificial Redshifting}

We next investigate whether redshift-dependent systematic effects could potentially cause the offset in $\left(n, r_{e}\right)$ shown in Figure 4.5 between massive galaxies at $z \sim 0$ and $z=2-3$, by causing the $\left(n, r_{e}\right)$ of massive $z \sim 0$ galaxies to 'degrade' into the regime of low $n \leq 2$ and low $r_{e} \leq 2 \mathrm{kpc}$ exhibited by the $z=2-3$ systems.

Ideally one would investigate this question by artificially redshifting the entire MGC subsample of 385 massive $z \sim 0$ galaxies shown in Figure 4.5 out to $z \sim 2.5$, and redecomposing the redshifted galaxies. However, this is extremely time consuming, and, furthermore, many of the galaxies do not have high quality SDSS ugriz images which are needed for redshifting software (FERENGI; Barden et al. 2008) to work. We therefore decide to artificially redshift a smaller, but representative sample S1 of 255 galaxies. S1 consists of 42 massive $\left(M_{\star} \geq 5 \times 10^{10} M_{\odot}\right)$ MGC galaxies combined with 213 nearby $(z<0.05)$ massive galaxies having high quality and well-resolved SDSS imaging. We ensure the $(n$, $r_{e}$ ) of the 255 galaxies in S1 match those of the entire subsample of MGC galaxies shown in Table 4.1, Figure 4.8, and Figure 4.9. We also ensure that the distribution of Hubble types of sample S1 matches those of the MGC subsample; the MGC subsample contains $\sim 66 \% \mathrm{E} / \mathrm{S} 0$ galaxies versus $\sim 34 \%$ Spirals, while sample $\mathrm{S} 1$ is $\sim 64 \% \mathrm{E} / \mathrm{S} 0$ galaxies and $\sim 36 \%$ Spirals. Many of the galaxies in S1 are well studied and include E, S0, and Sabc galaxies from Barden et al. (2008), E galaxies in Kormendy et al. (2009), as well as S0s 
and bulge-dominated spirals from Eskridge et al. (2002).

We used FERENGI (Barden et al. 2008) to artificially redshift the SDSS ugriz images (tracing rest-frame UV-to-optical light) of $z \sim 0$ galaxies, out to $z=2.5$, and reobserve them with the NIC3 F160W filter to the same depth as the GNS survey. During this process, FERENGI mimics the effects of surface brightness dimming, instrumental resolution, transmission efficiency, and PSF effects. It also corrects for other geometrical effects of cosmological redshift by appropriately re-binning input images for the desired redshift and platescale.

Specifically, during artificial redshifting, as is standard convention, FERENGI assumes surface brightness dimming at the rate of $(1+z)^{-4}$ for the bolometric luminosity of the full redshifted rest-frame optical SED. For galaxies where only part of this redshifted rest-frame optical SED falls within the NIC3/F160W filter bandwidth, the observed flux per unit wavelength $f_{\lambda}$ relates to the rest-frame luminosity per unit wavelength at redshift $z$ via a $(1+z)^{-3}$ dependence (e.g., Weedman 1986). The exact surface brightness dimming in such a case will be set by the integral of $f_{\lambda}$ over the filter-detector response function and depends on the detailed shape of the SED (e.g., Hogg 1999; Hogg et al. 2002). In practice, when using the FERENGI software, the relevant degree of surface brightness dimming is automatically applied when FERENGI convolves the redshifted images with the NIC3 F160W PSF and then re-observes the redshifted SED with the NIC3 F160W $(H)$ filter-detector, while taking into account the filter-detector characteristics, such as bandwidth and transmission efficiency. An exposure time of three-orbits (8063 seconds) and a resolution of $0.2 /$ pixel is assumed to mimic the GNS survey. A sky background equal to the mean sky background of the GNS NIC3 images (0.1 counts/second) was added to the redshifted images. Poisson 
noise, sky noise, and read noise (29 $e^{-}$for NIC3) were then added to the redshifted images.

During artificial redshifting of local galaxies, it is standard procedure to incorporate surface brightness evolution (Barden et al. 2008) because galaxies at higher redshifts have been observed to have higher mean surface brightness after applying the standard correction for the geometrical effect of cosmological surface brightness dimming. For instance, Lilly et al. (1998) find that surface brightness for disk-dominated galaxies of similar properties increases on average by 0.8 magnitudes by $z=0.7$. Barden et al. (2005) find from the GEMS ACS survey that galaxies with $M_{V} \lesssim-20$ show a brightening of $\sim 1$ magnitude in rest-frame $V$-band by $z \sim 1$. Labbé et al. (2003) find a disk-like galaxy with spectroscopic redshift $z=2.03$ to have a rest-frame $B$-band surface brightness $\sim 2$ magnitudes brighter than nearby galaxies. Finally in our own study, the mean surface brightness within $r_{e}$ of massive galaxies at $z=2-3$ is 2 to 6 magnitudes higher than that of massive galaxies at $z \sim 0$, with a mean offset of $\sim 4.5$ magnitudes (Figure 4.7 ).

In our experiment of artificially redshifting massive galaxies from $z \sim 0$ to $z=2.5$, we applied a conservative value of 2.5 magnitudes of surface brightness evolution. This value is motivated by several considerations: a) 2.5 magnitudes of surface brightness evolution is on the conservative side as many of the massive galaxies at $z=2.5$ show even more evolution (Figure 4.7). Thus, using this value will not lead to overoptimistic recovery of faint features during the experiment; b) The adopted 2.5 magnitudes of evolution out to $z=2.5$ corresponds to one magnitude of brightening per unit redshift. This rate of brightening is comparable with those seen in studies out to $z \sim 2$ (Lilly et al. 1998; Barden et al. 2005; Labbé et al. 2003); c) Using the Bruzual \& Charlot (2003) models, one can show that the passive evolution of a single stellar population from $z=2.5$ to $z=0$, assuming an 
exponentially declining star formation history associated with an e-folding time of $100 \mathrm{Myr}$, will lead the rest-frame $B$ luminosity to decline by 2.5 to 3 magnitudes, depending on the chosen metallicity.

While we believe that 2.5 magnitudes of surface brightness evolution is a conservative and reasonable value to use during the artificial redshfiting experiment, for the sake of completeness, we have also tested the effect of applying a surface brightness evolution (brightening) of $0,1.25,2.5$, and 3.75 magnitudes between $z \sim 0$ and $z=2.5$. There is a discernible difference in the recovered morphology and structural parameters between 0 and 1.25 magnitudes of brightening, but less difference between $1.25,2.5$, or 3.75 magnitudes of brightening. More details on the use of zero surface brightness evolution are given in point 4 at the end of this section.

After artificially redshifting $\mathrm{S} 1$ from $z \sim 0$ to $z=2.5$, we fit both the original galaxy images and their redshifted counterparts with single Sérsic profiles. We compare the restframe optical structural parameters in the original and redshifted images in order to assess the influence of redshift-dependent systematic effects (e.g., surface brightness dimming, loss of spatial resolution) and see how well the structural parameters are recovered. We also compare the redshifted distribution of $\left(n, r_{e}\right)$ with the one actually observed in the GNS massive galaxies to assess whether they are similar. Note that the structural parameters are measured at $z \sim 0$ from $g$-band images, while at $z=2.5$ they are measured from the artificially redshifted images in the NIC3/F160W band so that all parameters are measured in the rest-frame blue optical light, thereby avoiding bandpass shifting problems. Our main results are outlined below.

1. Figure 4.8 shows the $\left(n, r_{e}\right)$ distribution obtained by redshifting the sample S1 (ma- 
genta points in row 1) of $255 z \sim 0$ massive galaxies to $z \sim 2.5$ (blue points in row 2). This redshifted distribution of $\left(n, r_{e}\right)$ is still significantly offset from those observed in the massive GNS galaxies at $z=2-3$ (red points in row 2).

This difference is shown more quantitatively in Figure 4.9 where results in discrete bins of $n$ and $r_{e}$ are compared. The massive galaxies at $z=2-3$ (red line) includes $64.9 \pm 5.4 \%$ of systems with low $n \leq 2$, while the corresponding fraction for the redshifted sample (blue line) is $10.6 \pm 1.9 \%$. Similarly, for the $r_{e}$ distribution of the massive galaxies at $z=2-3,39.0 \pm 5.6 \%$ have $r_{e} \leq 2 \mathrm{kpc}$, while the redshifted sample has $1.2 \pm 0.7 \%$. We therefore conclude that cosmological and instrumental effects are not able to account for the large offset shown in Figure 4.8 and Figure 4.9 between the $\left(n, r_{e}\right)$ distributions of the massive galaxies at $z=2-3$ and those at $z \sim 0$.

2. It is very interesting to look at how the structural parameters of galaxies of different morphological types change during the redshifting. Figure 4.10 compares the restframe optical structural parameters in massive E, S0, and spirals at $z \sim 0$ with the structural parameters recovered after these galaxies were artificially redshifted.

From Figure 4.10, one can see that $r_{e}$ is recovered to better than a factor of 1.5 for the vast majority of redshifted E/S0 and spirals of early-to-late Hubble types. In the case of a small fraction of $z \sim 0$ galaxies with highly extended halos or disks and associated large $r_{e}$, the recovered $r_{e}$ at $z=2.5$ can be nearly a factor of two lower than the original $r_{e}$ at $z \sim 0$. Inspection of the surface brightness profiles shows that this effect primarily happens because surface brightness dimming prevents the outer lower surface brightness components of the galaxies from being adequately recovered after redshifting. 
It is striking that even after redshifting out to $z=2.5$, practically none of the massive $z \sim 0$ galaxies fall into the regime of $r_{e} \leq 2 \mathrm{kpc}$ (shown as shaded areas) inhabited by the ultra-compact systems, which make up $40 \%\left(39.0 \pm 5.6 \%\right.$ for $M_{\star} \geq 5 \times 10^{10}$ $M_{\odot}$ and $39.0 \pm 7.6 \%$ for $M_{\star} \geq 1 \times 10^{11} M_{\odot}$ ) of the massive galaxies at $z=2-3$ (see $\S 4.3 .2)$. Thus, these massive ultra-compact $\left(r_{e} \leq 2 \mathrm{kpc}\right)$ systems at $z=2-3$ appear to truly have no analogs among $z \sim 0$ massive galaxies, in terms of their size, structure, and optical surface brightness.

The top row of Figure 4.10 shows the distribution of Sérsic index $n$ before and after redshifting out to $z=2.5$. The recovered Sérsic index $n$ can be lower or higher than the original $n$ at $z \sim 0$, but is recovered to better than a factor of two in all cases. The shaded area in the plots represents the regime of $n \leq 2$ where the majority $\left(64.9 \pm 5.4 \%\right.$ for $M_{\star} \geq 5 \times 10^{10} M_{\odot}$ and $58.5 \pm 7.7 \%$ for $\left.M_{\star} \geq 1 \times 10^{11} M_{\odot}\right)$ of massive GNS galaxies at $z=2-3$ lie (Table 4.1). It is interesting to note that massive $\mathrm{E}$ and S0s, which are spheroid-dominated and bulge-dominated systems, do not typically lie in the $n \leq 2$ regime, before or after redshifting. In contrast, a large fraction of $z \sim 0$ spirals with intermediate-to-late Hubble types ${ }^{5}$ populate the $n \leq 2$ regime, both before and after redshifting. Disk features on large and small scales (e.g., outer disk or disky pseudobulge) lead to an overall single Sérsic index $n \leq 2$ for the entire galaxy. It is possible that similar disk features are responsible at least in part, for the low $n \leq 2$ values shown by the majority $(\sim 65 \%)$ of the massive GNS galaxies at $z=2-3$. We discuss this point further in $\S 4.7$.

\footnotetext{
${ }^{5}$ The Hubble types are based on the bulge-to-total light ratio $(B / T)$, which we measured with bulge-disk and bulge-disk-bar decomposition of $z \sim 0 \mathrm{~g}$-band images.
} 
3. One important question is whether the use of deeper images of the $z \sim 0$ galaxies would change the conclusion of the redshfiting experiment. In the present experiment, we used SDSS $g$-band images, which have an exposure time of 54 seconds and a typical sky background of $22 \mathrm{mag} / \operatorname{arcsec}^{2}$. Deeper exposures of nearby galaxies may potentially detect an outer low surface brightness halo (if such a halo exists), which is missed in the SDSS images, and in that case lead us to measure larger $\left(n, r_{e}\right)$ at $z \sim 0$ with a Sérsic fit. Such halos can be found in very local massive elliptical and cD galaxies, where the measured $\left(n, r_{e}\right)$ can increase significantly if the halo is included in the fit (Kormendy et al. 2009). However, such low surface brightness halos will be dimmed out and not recovered during the artificial redshifting of these deep images, so that the $\left(n, r_{e}\right)$ parameters recovered at $z=2.5$ will be similar to those we presently obtain from the SDSS images. The net effect will be that using deeper images of local massive galaxies during the artificial redshifting will at most raise the $\left(n, r_{e}\right)$ at $z \sim 0$, but not at $z=2.5$. Thus the difference in the $\left(n, r_{e}\right)$ at $z \sim 0$ compared with $z=2.5$ will be unchanged (for systems without halos) or amplified (for systems with such halos). Our overall conclusion from the redshifting experiment regarding degradation of the profiles to $n \leq 2$ and $r_{e} \leq 2 \mathrm{kpc}$ would remain unchanged or be even stronger.

4. Finally, as one additional test, we repeated the redshifting experiment assuming zero surface brightness evolution, rather than 2.5 magnitudes of brightening, out to $z=2.5$. Even in this case there is still a large offset in the $\left(n, r_{e}\right)$ distributions of the redshifted sample S1 compared with the massive GNS galaxies. Specifically, the fraction of systems with low $n \leq 2(22.0 \pm 2.6 \%)$ is still significantly less than that for massive GNS galaxies at $z=2-3(64.9 \pm 5.4 \%)$. Likewise, there are still few systems with 
$r_{e} \leq 2 \mathrm{kpc}(1.6 \pm 0.8 \%)$ compared with the high fraction $(39.0 \pm 5.6 \%)$ found at $z=2-3$.

Thus, even without surface brightness evolution it is still true that cosmological and instrumental effects are not able to account for the large offset between massive galaxies at $z=2-3$ versus $z \sim 0$.

\subsection{Star Formation Activity}

\subsubsection{Matching GNS Galaxies to MIPS $24 \mu \mathrm{m}$ Counterparts}

The Spitzer GOODS Legacy Program (Dickinson et al. 2003a; Dickinson et al. in preparation) provides deep Spitzer MIPS $24 \mu \mathrm{m}$ observations of the GOODS fields. In the discussion below, we only consider MIPS $24 \mu \mathrm{m}$ counterparts with $f_{24 \mu m} \geq 30 \mu \mathrm{Jy}$, the $5 \sigma$ flux limit. The MIPS images have a PSF diameter of $6^{\prime \prime}(\sim 42 \mathrm{kpc}$ at $z=1-3)$, versus the NIC3/F160W PSF of 0"'3. MIPS $24 \mu \mathrm{m}$ counterparts of the massive GNS galaxies were identified by selecting the closest MIPS $24 \mu \mathrm{m}$ source within a maximum matching radius of $1 . " 5$. We initially find 84/166 massive GNS galaxies with MIPS $24 \mu \mathrm{m}$ counterparts with $f_{24 \mu m} \geq 30 \mu \mathrm{Jy}$ and further refine these matches below.

There are several potential problems with the above procedure. Firstly, it allows for the situation where a given MIPS $24 \mu \mathrm{m}$ source could be matched to several massive GNS galaxies. This would happen if some massive GNS galaxies were crowded within a radius of a few arcseconds so that the MIPS source would be within 1." 5 of all of them. This situation occurs for 2/84 $(\sim 2 \%)$ of massive galaxies with a MIPS counterpart. We reject these two cases, reducing the number of unique and secure matches from 84 to 82 .

A second possible caveat is that within the large MIPS $24 \mu \mathrm{m}$ PSF of 6 " diameter, there may be several other NIC3/F160W sources, in addition to the main massive GNS 
galaxy to which the MIPS source is matched. These extra NIC3/F160W sources may even be lower mass galaxies not in our sample of massive $\left(M_{\star} \geq 5 \times 10^{10} M_{\odot}\right)$ galaxies. In such a scenario, all the extra NIC3 sources could potentially contribute to the MIPS $24 \mu \mathrm{m}$ flux, and assigning all the $24 \mu \mathrm{m}$ flux of the MIPS counterpart to the nearest massive GNS galaxy would overestimate the $24 \mu \mathrm{m}$ flux of this galaxy. In order to assess the extent of this potential problem, we proceed as follows. For the MIPS $24 \mu \mathrm{m}$ counterpart assigned previously to each massive GNS galaxy, we determine how many extra NIC3/F160W sources with $M_{\star} \geq 10^{9} M_{\odot}$, in addition to the massive GNS galaxy, lie within a circle of diameter $6^{\prime \prime}$ (i.e., the PSF diameter) centered on the MIPS source. Of the 82 massive GNS galaxies with a secure MIPS $24 \mu \mathrm{m}$ counterpart, 30 involve cases where there are extra NIC3 sources, along with the massive GNS galaxy, inside the MIPS PSF diameter.

Next, we estimate the relative expected contributions of the massive GNS galaxy and the extra NIC3/F160W sources to the overall $24 \mu \mathrm{m}$ flux by using the stellar mass ratio of the main massive GNS galaxy (e.g., $M_{\star 1}$ ) and of the contaminating source (e.g., $M_{\star 2}$ ), scaled by a function that takes into account the different redshifts of the two sources. Specifically, for the two sources with stellar mass $M_{\star 1}$ and $M_{\star 2}$, having redshifts $z_{1}$ and $z_{2}$ and luminosity distances $D_{L 1}$ and $D_{L 2}$, the stellar mass ratio $M_{\star 1} / M_{\star 2}$ is scaled by $\left(\left(1+z_{2}\right) \times D_{L 2}^{2}\right) /\left(\left(1+z_{1}\right) \times D_{L 1}^{2}\right)$. In 8 of 30 cases, the contribution of the extra NIC3 contaminating sources to the overall $24 \mu \mathrm{m}$ flux is $>20 \%$ that of the main GNS galaxy, and spans $\sim 40 \%$ to $\sim 126 \%$. We reject these latter 8 cases rather than try to correct for the contamination, which in all cases is distributed across two or more nearby galaxies. For the remaining 22 cases, the contamination by extra NIC3/F160W sources is $<20 \%$ and we deem that our afore-described procedure of assigning all the $24 \mu \mathrm{m}$ flux of the MIPS 
counterpart to the massive GNS galaxy is reasonable.

Therefore, in summary, 74/166 $(44.6 \pm 3.9 \%)$ massive $\left(M_{\star} \geq 5 \times 10^{10} M_{\odot}\right)$ GNS galaxies have a reliable MIPS $24 \mu \mathrm{m}$ counterpart (with $f_{24 \mu m} \geq 30 \mu \mathrm{Jy}$ ) whose entire flux is assigned to the massive GNS galaxy. In contrast, 82/166 (49.4 $\pm 3.9 \%)$, massive GNS

galaxies do not have a reliable MIPS counterpart with $f_{24 \mu m} \geq 30 \mu \mathrm{Jy}$ and in these cases we can only measure upper limits on their SFR. Table 4.3 lists the fraction of massive GNS galaxies with a MIPS $24 \mu \mathrm{m}$ counterpart as a function of redshift.

\subsubsection{Star Formation Rates}

In order to estimate the SFR, the total IR luminosity $\left(L_{\mathrm{IR}}\right)$ over $8-1000 \mu \mathrm{m}$ is first estimated from the observed $24 \mu \mathrm{m}$ flux (corresponding to rest-frame wavelengths of 6-12 $\mu \mathrm{m}$ over $z=1-3$ ) by using SED templates from Chary \& Elbaz (2001). Using solely $24 \mu \mathrm{m}$ flux density to measure $L_{\mathrm{IR}}$ works well for inferred $L_{\mathrm{IR}} \lesssim 10^{12} L_{\odot}$ galaxies at $z \sim 2$, but $L_{\mathrm{IR}}$ is overestimated by a factor of $\sim 3$ in more luminous galaxies (e.g., Papovich et al. 2007). Early results from Herschel (e.g., Elbaz et al. 2010; Nordon et al. 2010; D. Lutz, private communication) suggest that at $z>1.5$, the SFRs extrapolated from $24 \mu \mathrm{m}$ fluxes may overestimate the true SFR, typically by a factor of 2 to 4 and possibly as much as a factor of 10. This overestimate could be due to a rise in the strength of $\mathrm{PAH}$ features, changes in the SEDs, or AGN contamination at $z>1.5$. Murphy et al. (2009) find that estimates of $L_{\mathrm{IR}}$ from $24 \mu \mathrm{m}$ flux density alone are incorrect because the templates used are based on local galaxies with smaller PAH equivalent widths than galaxies of similar luminosity at high-redshift. We account for this discrepancy by making a correction for 
galaxies with inferred $L_{\mathrm{IR}}>6 \times 10^{11} L_{\odot}$ using

$$
\log _{10}\left(\mathrm{~L}_{\mathrm{IR}}\right)=0.59 \times \log _{10}\left(\mathrm{~L}_{\mathrm{IR}}^{24}\right)+4.8
$$

where $L_{\mathrm{IR}}^{24}$ is the infrared luminosity inferred solely from $24 \mu \mathrm{m}$ flux density (R. Chary, private communication). The upper-left and upper-right panels of Figure 4.11 show the distribution of $24 \mu \mathrm{m}$ flux and the inferred $L_{\mathrm{IR}}$.

The obscured star formation rate can be calculated using the expression

$$
\mathrm{SFR}_{\mathrm{IR}}=9.8 \times 10^{-11} \mathrm{~L}_{\mathrm{IR}}
$$

from Bell et al. (2007). This calculation is based on a Chabrier IMF (Chabrier 2003) and assumes that the infrared emission is radiated by dust that is heated primarily by massive young stars. Uncertainties in the SFR estimates are a factor of $\sim 2$ or higher for individual galaxies.

If an AGN is present, then $\mathrm{SFR}_{\mathrm{IR}}$ only gives an upper limit to the true SFR. In $\S 4.6$, we adopt several techniques to identify AGN candidates in the sample and estimate the mean SFR for galaxies with and without a candidate AGN (see Table 4.3). The upper-right panel of Figure 4.11 shows $L_{\mathrm{IR}}$ for AGN and non-AGN, and the bottom panels show $\mathrm{SFR}_{\mathrm{IR}}$. The AGN candidates dominate the tail of highest $L_{\mathrm{IR}}$ and $\mathrm{SFR}_{\mathrm{IR}}$. Among the HyLIRGs ${ }^{6}$, 9/11 $(\sim 82 \%)$ turn out to be AGN. After excluding the AGN candidates, the mean $L_{\mathrm{IR}}$ is a factor of $\sim 8$ times lower, while the mean $\mathrm{SFR}_{\mathrm{IR}}$ is reduced a factor of $\sim 1.5$ to $\sim 2.7$, and the difference rises with redshift (Table 4.3).

How do our measurements of $\mathrm{SFR}_{\mathrm{IR}}$ compare with UV-based SFR derived in other studies of high-redshift galaxies? The left panel of Figure 4.12 plots $\mathrm{SFR}_{\mathrm{IR}}$ versus $M_{\star}$ for

\footnotetext{
${ }^{6}$ HyLIRGs are defined to have $L_{I R} \geq 10{ }^{13} L_{\odot}$
} 
the massive $\left(M_{\star} \geq 5 \times 10^{10} M_{\odot}\right)$ GNS galaxies at $z=2-3$ with $24 \mu \mathrm{m}$ flux above the $5 \sigma$ limit $(30 \mu \mathrm{Jy})$. We demonstrate that the SFR derived at $z=2-3$ for non-AGN are in approximate agreement with the UV-based SFR from Daddi et al. (2007). Drory \& Alvarez (2008) parameterize SFR as a function of mass and redshift for a wide range in stellar mass $\left(M_{\star} \sim 10^{9}-10^{12} M_{\odot}\right)$. In the right panel of Figure 4.12, the black line shows average SFR versus redshift for a $5 \times 10^{10} M_{\odot}$ galaxy as calculated by Drory \& Alvarez (2008). The mean $\mathrm{SFR}_{\mathrm{IR}}$ for massive non-AGN GNS galaxies, with $\mathrm{SFR}_{\mathrm{IR}}$ above the $5 \sigma$ limit, are higher by a factor of $\sim 1.5-4$ over $z=1-3$, with the offset worsening with redshift. This disagreement with mean $\mathrm{SFR}_{\mathrm{IR}}$ is not just a bias caused by the requirement that $\mathrm{SFR}_{\mathrm{IR}}$ exceed the $5 \sigma$ limit, which selects the most intense star-forming systems at each redshift. Even if the upper limits on $\mathrm{SFR}_{\mathrm{IR}}$ are included, our $\mathrm{SFR}_{\mathrm{IR}}$ do not show the same break and flattening seen at $z \sim 2$ by Drory \& Alvarez (2008). Finally, Bauer et al. (2011) measure dust-corrected UV-based SFR ( $\mathrm{SFR}_{\mathrm{UV} \text {,corr }}$ ) for galaxies in GNS over $1.5<z<3$. Among massive $\left(M_{\star} \geq 5 \times 10^{10} M_{\odot}\right)$ galaxies, $\mathrm{SFR}_{\mathrm{UV}}$,corr can differ by as much as a factor of 10 , but for higher $\mathrm{SFR}_{\mathrm{IR}}$ the difference is typically a factor of $\sim 2-3$.

\subsubsection{Relation Between Star Formation and Structure}

Figure 4.13 shows the distribution of $\mathrm{SFR}_{\mathrm{IR}}$ among systems of different $n$. On the LHS panel, galaxies with $\mathrm{SFR}_{\mathrm{IR}}$ below the $5 \sigma$ detection limit are shown as downward pointing arrows. The potential AGN candidates identified in $§ 4.6$ are coded separately as $\Sigma_{\mathrm{SFR}_{\mathrm{IR}}}$ is likely overestimating the true SFR in the galaxy. For the histograms on the RHS panel, the y-axis shows the fraction of massive GNS galaxies in each redshift bin, while on the $\mathrm{x}$-axis, we plot the actual value of $\mathrm{SFR}_{\mathrm{IR}}$ for systems with $\mathrm{SFR}_{\mathrm{IR}}$ above the $5 \sigma$ detection limit (indicated by the vertical line), and the upper limit for the other systems. 
The massive galaxies at $z=1-3$ display several interesting relations between their star formation activity and structure, as characterized by the Sérsic index $n$. Firstly, among the non-AGN massive $\left(M_{\star} \geq 5 \times 10^{10} M_{\odot}\right)$ galaxies at $z=2-3$, the fraction of galaxies with low $n \leq 2$ having $\mathrm{SFR}_{\mathrm{IR}}$ high enough to produce a $24 \mu \mathrm{m}$ flux above the $5 \sigma$ detection limit is $(53.4 \pm 10.9 \%)$, which is significantly higher than the corresponding fraction $(15.4 \pm 10.0 \%)$ for systems with $n>2$. Secondly, among the non-AGN massive $\left(M_{\star} \geq 5 \times 10^{10} M_{\odot}\right)$ galaxies at $z=2-3$ with $\mathrm{SFR}_{\mathrm{IR}}$ above the $5 \sigma$ detection limit, the majority $(84.6 \pm 10.0 \%$ ) have low $n \leq 2$, while none have $n>4$. The corresponding numbers for the redshift bin $z=1-2$ are $67.7 \pm 8.0 \%$ and $11.8 \pm 5.5 \%$, respectively. Thirdly, the RHS panel of Figure 4.13 shows that the high SFR tail in each redshift bin is populated primarily by $n \leq 2$ systems. While the $n \leq 2$ disky systems have a wide range of $\mathrm{SFR}_{\mathrm{IR}}\left(21\right.$ to $626 M_{\odot} \mathrm{yr}^{-1}$ at $z=1-2,53$ to $1466 M_{\odot} \mathrm{yr}^{-1}$ at $\left.z=2-3\right)$, they include the systems of the highest SFR at both $z=1-2$ and $z=2-3$. Thus, the systems with low $n \leq 2$ seem to be more actively star-forming than the systems of high $n>3$.

Most $\left(72.0 \pm 6.3 \%\right.$ of systems with low $n \leq 2$ are extended $\left(r_{e}>2 \mathrm{kpc}\right)$ so that a relation is also expected between $\mathrm{SF}$ activity and size. We thus investigate next the relationship between SFR and effective radius $r_{e}$. The distribution of $\mathrm{SFR}_{\mathrm{IR}}$ for different $r_{e}$ ranges is shown in Figure 4.14. The same convention as for Figure 4.13 is adopted, with upper limits being plotted for galaxies with $\mathrm{SFR}_{\mathrm{IR}}$ below the $5 \sigma$ detection limit, and only non-AGN systems being plotted on the RHS panel. We find that among the non-AGN massive $\left(M_{\star} \geq 5 \times 10^{10} M_{\odot}\right)$ galaxies at $z=2-3$, the fraction of ultra-compact $\left(r_{e} \leq 2\right.$ $\mathrm{kpc}$ ) objects with $\mathrm{SFR}_{\mathrm{IR}}$ above the $5 \sigma$ detection limit is only $15.0 \pm 8.0 \%$ compared with the fraction $(32.4 \pm 8.0 \%)$ for the whole sample. Thus, among non-AGN massive galaxies 
over $z=2-3$, the ultra-compact $\left(r_{e} \leq 2 \mathrm{kpc}\right)$ galaxies show a deficiency by a factor of $\sim 2.2$ of systems with $S F R_{\mathrm{IR}}$ above the detection limit, compared with the whole sample. At $z=1-2$, the deficiency is a factor of $\sim 3.5$. Furthermore, as illustrated by the RHS panel of Figure 4.14, although there are some ultra-compact $\left(r_{e} \leq 2 \mathrm{kpc}\right)$ galaxies with high $\mathrm{SFR}_{\mathrm{IR}}$, on average, the mean $\mathrm{SFR}_{\mathrm{IR}}$ of the $z=2-3$ and $z=1-2$ is significantly lower than that of more extended galaxies.

\subsection{Constraints On Cold Gas Content}

The high estimated $\mathrm{SFR}_{\mathrm{IR}}$ found in $§ 4.4$ suggest that copious cold gas reservoirs are present to fuel the star formation. For the massive GNS galaxies with $\mathrm{SFR}_{\mathrm{IR}}$ measurements above the $5 \sigma$ detection limit, we assume half of $\mathrm{SFR}_{\mathrm{IR}}$ lies within the circularized rest-frame

optical half-light radius $\left(r_{c}=r_{e} \times \sqrt{b / a}\right)$ from single component Sérsic fits, and thereby estimate that the deprojected SFR per unit area as

$$
\Sigma_{\mathrm{SFR}_{\mathrm{IR}}}=\frac{0.5 \times \mathrm{SFR}_{\mathrm{IR}}}{\pi \times r_{c}^{2}} .
$$

In galaxies that $\mathrm{AGN}$ host candidates, $\Sigma_{\mathrm{SFR}_{\mathrm{IR}}}$ is likely overestimating the true $\mathrm{SFR}$ in the galaxy (see $§ 4.4)$. If potential AGN candidates are included, $\Sigma_{\mathrm{SFR}}$ ranges from $\sim$ $0.10-360.8 M_{\odot} \mathrm{yr}^{-1} \mathrm{kpc}^{-2}$, with a mean value of $\sim 19.4 M_{\odot} \mathrm{yr}^{-1} \mathrm{kpc}^{-2}$ over $z=1-3$ After excluding the potential AGN candidates $\Sigma_{\mathrm{SFR}_{\mathrm{IR}}}$ ranges from $\sim 0.24-360.8 M_{\odot} \mathrm{yr}^{-1}$ $\mathrm{kpc}^{-2}$, with a mean value of $\sim 14.8 M_{\odot} \mathrm{yr}^{-1} \mathrm{kpc}^{-2}$. This range is comparable with that seen in BzK/normal galaxies, ULIRGS, and submillimeter galaxies (e.g., see Daddi et al. $2010 b)$.

We use a standard Schmidt-Kennicutt law (Kennicutt 1998), with a power-law index of 1.4 and a normalization factor of $2.5 \times 10^{-4}$, to estimate the cold gas surface 
density from $\Sigma_{\mathrm{SFR}}$. The results are uncertain by at least a factor of $\sim 2.5$ because different relations between molecular gas surface density and SFR surface density have been suggested for various types of star-forming systems over a broad range of redshifts (Kennicutt 2008; Gnedin \& Kravtsov 2010; Daddi et al. 2010b; Genzel et al. 2010; Tacconi et al. 2010). If potential AGN candidates are included, the resulting implied cold gas surface density

$$
\Sigma_{\text {gas }}=\left[\frac{10^{4} \times \Sigma_{\mathrm{SFR}_{\mathrm{IR}}}}{2.5}\right]^{1 / 1.4}
$$

ranges from $\sim 73-25,091 M_{\odot} \mathrm{pc}^{-2}$, with a median value of $\sim 907 M_{\odot} \mathrm{pc}^{-2}$ over $z=1-3$ (Figure 4.15). The corresponding values after excluding AGN candidates are $\sim 136-$ $25091 M_{\odot} \mathrm{pc}^{-2}$, with a median value of $\sim 607 M_{\odot} \mathrm{pc}^{-2}$ (Figure 4.15). These values are again comparable with those observed in BzK/normal galaxies, ULIRGS, and submillimeter galaxies (e.g., see Daddi et al. 2010b).

In the subsequent discussion, we only cite values obtained after excluding AGN candidates, but Figure 4.15 also shows the values for the full sample of galaxies. Next we estimate the cold gas fraction relative to the baryonic mass within $r_{c}$. For each galaxy, we use the above cold gas surface density to estimate the total cold gas mass within the circularized rest-frame optical half-light radius,

$$
M_{\text {gas }}\left(r_{c}\right)=\Sigma_{\text {gas }} \times \pi \times r_{c}^{2}
$$

$M_{\text {gas }}$ ranges from $3.4 \times 10^{9}-1.0 \times 10^{11} M_{\odot}$, with a mean value of $1.9 \times 10^{10} M_{\odot}$ (Figure 4.15) The baryonic mass $\left(M_{\text {Baryon }}\right)$ within $r_{c}$ is taken to be the sum of cold gas mass and stellar mass within $r_{c}$, and we assume that the latter term is half of the total stellar mass of the galaxy. 
The cold gas fraction $\left(f_{\text {gas }}\left(r_{c}\right)\right)$ within the circularized rest-frame optical half-light radius $r_{c}$ is defined as

$$
f_{\text {gas }}\left(r_{c}\right) \equiv M_{\text {gas }} /\left[M_{\text {gas }}+M_{\star}\right]
$$

The cold gas fraction $\left(f_{\text {gas }}\left(r_{c}\right)\right)$ ranges from $6.5-65.4 \%$, with a mean of $\sim 23 \%$ over $z=1-3$ (Figure 4.15). Figure 4.16 shows how $f_{\text {gas }}\left(r_{c}\right)$ varies as a function of stellar mass and redshift, both with and without the AGN candidates. For galaxies with $5 \times 10^{10} M_{\odot} \leq M_{\star}<10^{11}$ $M_{\odot}$ above the $5 \sigma$ detection limit, the mean $f_{\text {gas }}\left(r_{c}\right)$ (without AGN candidates) rises from $\sim 19 \%$ to $\sim 25 \%$ to $\sim 41 \%$ across the three redshift bins. In comparison, for $M_{\star} \geq 10^{11}$ $M_{\odot}$ galaxies, the mean cold gas fraction is $\sim 14 \%$ to $\sim 23 \%$. The $1 \sigma$ error bars are large and there is considerable overlap between the two mass ranges. Still, the highest cold gas fractions within the circularized rest-frame optical half-light radius at a given redshift are found among the less massive galaxies, consistent with downsizing.

Our inferred cold gas fractions $\left(f_{\text {gas }}\left(r_{c}\right)\right)$ within the circularized rest-frame optical half-light radius $r_{c}$ can be higher or lower than the total cold gas fraction of the galaxy, depending on whether the molecular gas is centrally concentrated or extended, respectively. While bearing this caveat in mind, we note that our inferred values for $f_{\text {gas }}\left(r_{c}\right)$ are consistent with previous direct measurements of the total cold gas fraction at high-redshift. Daddi et al. $\left(2008,2010\right.$ a) report gas fractions of $50-65 \%$ in massive $\left(M_{\star} \sim 4 \times 10^{10}-1 \times 10^{11} M_{\odot}\right)$ IR-selected $B z K$ galaxies at $z \sim 1.5$. Tacconi et al. (2010) also measure cold gas fraction from $\mathrm{CO}$ observations of high-redshift galaxies at $z=1.1-2.4$. For stellar masses spanning $M_{\star} \sim 3 \times 10^{10}-3.4 \times 10^{11} M_{\odot}$, they find cold gas fractions in the range of $\sim 14-78 \%$. 


\subsection{AGN in massive galaxies at $z=1-3$}

\subsubsection{Frequency of AGN}

We use a variety of techniques (X-ray properties, IR power-law, IR-to-optical excess, and mid-IR colors) to identify Active Galactic Nuclei (AGN) among the massive GNS galaxies because selection based on X-ray emission alone may fail at high redshift in the case of Compton-thick AGN where much of the soft X-ray emission is Compton scattered or absorbed by thick columns of gas $\left(N_{H} \gg 10^{24} \mathrm{~cm}^{-2}\right.$; Brandt et al. 2006). We briefly summarize here and in Table 4.4 the number of AGN identified by each of the selection methods ${ }^{7}$.

1. X-ray counterparts to the massive GNS sources were searched for in the CDF-N and CDF-S catalogs of Alexander et al. (2003) and Luo et al. (2008), as well as the ECDF-S catalogs of Lehmer et al. (2005). A total of 33/166 massive GNS galaxies had counterparts within $11^{\prime \prime} 5$ across all catalogs.

2. Following Alonso-Herrero et al. (2006) and Donley et al. (2008), we look for AGN power-law emission over $z=1-3$ using SEDs from the IRAC bands at 3.6, 4.5, 5.8, and $8.0 \mu \mathrm{m}$. The IRAC SEDs were fit with a power-law SED $\left(f_{\nu} \propto \nu^{\alpha}\right)$. There are only $3 / 166$ sources with power-law index $\alpha \leq-0.5$ that are considered power-law galaxies (PLGs) and obscured AGN candidates.

3. Heavily obscured AGN may be present in highly reddened, IR-excess galaxies. Fiore et al. (2008) identify obscured AGN candidates in IR-bright, optically faint, red galaxies

\footnotetext{
${ }^{7}$ The mid-IR selection criteria of Lacy et al. (2004) and Stern et al. (2005) were investigated but considered unreliable. Contamination from high-redshift star-forming galaxies drastically reduces their accuracy (e.g., Donley et al. 2008). Applying these methods at $z=1-3$ would add more false-positives than true AGN.
} 
over $z=1.2-2.6$ using the criteria $f_{24 \mu m} / f_{R} \geq 1000$ and $R-K>4.5$. We search for such IR-bright, optically faint systems with $f_{24 \mu m} / f_{R}>1000$ in our sample of massive galaxies. $R$-band flux is determined by linear interpolation between the ACS $V$ and $i$-band fluxes. We find 25 sources meeting this criteria, of which 16 are new AGN candidates not identified via the above two methods.

Among the 166 massive GNS galaxies at $z=1-3$, the AGN fraction is 49/166 or $29.5 \pm 3.5 \%$. When the results are broken down in terms of redshift, the AGN fraction rises with redshift, increasing from $17.9 \pm 6.1 \%$ at $z=1-1.5$ to $40.3 \pm 8.8 \%$ at $z=2-3$. The percentage of AGN among all massive GNS galaxies is higher than at $z \sim 1$, where it is reported that less than $15 \%$ of the total $24 \mu \mathrm{m}$ emission at $z<1$ is in X-ray luminous AGN (e.g., Silva et al. 2004; Bell et al. 2005; Franceschini et al. 2005; Brand et al. 2006).

\subsubsection{Relation Between AGN Activity and Structure}

We summarize the properties of the AGN host candidates and discuss their implications in terms host galaxy structure.

Figure 4.17 shows the single Sérsic $n$ versus $r_{e}$. Most $(80.6 \pm 7.9 \%)$ of the AGN hosts at $z=2-3$ have $r_{e}>2 \mathrm{kpc}$ and are not ultra-compact. AGN appear to be found preferentially in the more extended galaxies. Indeed, at $z=2-3$, the AGN fraction in ultra-compact galaxies is $\sim 2.7$ times lower than in extended galaxies $(20.0 \pm 16.3 \%$ versus $53.2 \pm 10.0 \%)$. At $z=1-2$ the deficiency is a factor of 5.6. Thus, the ultra-compact galaxies are more quiescent in terms of both AGN activity and SFR activity (see §4.4).

Furthermore, a significant fraction of these AGN $(64.6 \pm 10.7 \%)$ have disky $(n \leq 2)$ morphologies. Over half $(58.2 \pm 11.6 \%)$ of the AGN candidates are both disky and not 
ultra-compact. Similar statistics apply over $z=1-2$. The disky nature of AGN hosts at $1.5<z<3$ has been measured previously by Schawinski et al. (2011). From decomposition of the rest-frame optical light for 20 AGN imaged with HST WFC3, they measure a mean Sérsic index of 2.54 and a mean effective radius of $3.16 \mathrm{kpc}$. Their results for $\left(n, r_{e}\right)$ are consistent with our results for $z=2-3$ in Table 4.4 and Figure 4.17. Furthermore, Kocevski

et al. (2011) find from visual classification of rest-frame optical morphologies that $51.4_{-5.9}^{+5.8}$ of X-ray selected $\operatorname{AGN}\left(L_{X} \sim 10^{42-44} \mathrm{erg} \mathrm{s}^{-1}\right)$ at $1.5<z<2.5$ reside in galaxies with visible disks; only $27.4_{-4.6}^{+5.8}$ reside in pure spheroids.

If the disky AGN host candidates host massive black holes, then massive black holes are present in galaxies that are not dominated by a massive spheroid. In the local Universe, nearly all massive galaxies are believed to host a central supermassive black hole (Kormendy 1993b; Magorrian et al. 1998; Ferrarese \& Merritt 2000; Gebhardt et al. 2000; Marconi \& Hunt 2003), and the black hole mass is tightly related to the bulge stellar velocity dispersion (Ferrarese \& Merritt 2000; Gebhardt et al. 2000). This has led to the suggestion that the black hole and bulge or spheroid probably grew in tandem (e.g., Cattaneo \& Bernardi 2003; Hopkins et al. 2006a). The presence at $z=2-3$ of luminous and potentially massive black holes in high mass galaxies that do not seem to have a prominent bulge or spheroid may be at odds with this picture.

\subsection{Discussion}

\subsubsection{Do Massive Galaxies With $n \leq 2$ at $z=2-3$ Host Disks?}

We have shown in $\S 4.3 .2$ that the majority $\left(64.9 \% \pm 5.4 \%\right.$ for $M_{\star} \geq 5 \times 10^{10} M_{\odot}$, and $58.5 \% \pm 7.7 \%$ for $\left.M_{\star} \geq 10^{11} M_{\odot}\right)$ of massive galaxies at $z=2-3$ have low $n \leq 2$, while 
the fraction at $z \sim 0$ is five times lower. We also demonstrated via artificial redshifting experiments and extensive tests ( $\$ 4.3 .3$ and the Appendix) that this difference between $z=2-3$ and $z \sim 0$ is real and not driven primarily by systematic effects. Furthermore, most ( $\sim 72 \%$ ) of these with low $n \leq 2$ massive galaxies at $z=2-3$ are extended with $r_{e}>2 \mathrm{kpc}$, rather than being ultra-compact.

What is the nature of the large population of galaxies with low $n \leq 2$ at $z=2-3$ ? We present below different lines of evidence which suggest that many of these massive galaxies at $z=2-3$ with $n \leq 2$, particularly the extended $\left(r_{e}>2 \mathrm{kpc}\right)$ systems, likely host a significant disk component.

1. Some insight into the interpretation of $n \leq 2$ values can be gleaned by considering massive galaxies at $z \sim 0$. As discussed in $§ 4.3 .3 .1$ and illustrated in Figure 4.10, massive E and S0s, which are spheroid-dominated and bulge-dominated systems, are predominantly associated with $n>2$, both at $z \sim 0$ and after artificially redshifting to $z=2.5$. In contrast, spiral galaxies of intermediate to low bulge-to-total ratios, often have an overall low Sérsic index $n \leq 2$ (Figure 4.10) because they have a disk component, such as an outer disk or a central disky pseudobulge (e.g., Kormendy \& Kennicutt 2004; Jogee 1999; Jogee et al 2005), which contributes significantly to the total blue light of the galaxy. An extension of these arguments to $z=2-3$ suggests the large fraction $\sim 65 \%$ of massive galaxies at $z=2-3$ with low $n \leq 2$ is driven, at least partially, by the presence of an outer disk or central disky pseudobulge.

2. We next consider the relationship between disk structure and projected ellipticity $e$. The top panels of Figure 4.18 show the deconvolved ellipticity $e=1-b / a$ determined 
by GALFIT for the massive $\left(M_{\star} \geq 5 \times 10^{10} M_{\odot}\right)$ galaxies at $z=2-3$ with $n \leq 2$ and $n>2$. The lower left and right panels of Figure 4.18 show the distributions of deconvolved ellipticity determined by GIM2D of similarly massive spiral (Sabc and $\mathrm{Sd} / \mathrm{Irr})$ and $\mathrm{E} / \mathrm{S}^{8}$ galaxies in the MGC catalog.

The projected ellipticity distribution of massive galaxies at $z=2-3$ with $n \leq 2$ is quite different from that of $z \sim 0$ massive E/S0 galaxies. For local E/S0s, the distribution of $e$ drops sharply at $e>0.35$ and there are few systems at $e>0.5$. In contrast, for the massive galaxies at $z=2-3$ with $n \leq 2$, the $e$ distribution continues to rise out to $e \sim 0.5$. There is also a significant fraction $(\sim 58 \%)$ of systems with $n \leq 2$ having $e$ above 0.5 , specifically in the range of 0.5 to 0.75 . In effect, a Kolmogorov-Smirnov (KS) test ( Table 4.5) shows that the galaxies at $z=2-3$ with $n \leq 2$ have a $0 \%$ KS-test probability of coming from the same distribution as local massive E/S0s in MGC. These comparisons suggest that the massive galaxies at $z=2-3$ with $n \leq 2$ are very different from local bulge-dominated and spheroid-dominated E/SOs.

Among the massive systems with $n \leq 2$ at $z=2-3,28.0 \pm 6.4 \%$ are ultra-compact $\left(r_{e} \leq 2 \mathrm{kpc}\right)$. Thus, our conclusion complements the results of van der Wel et al. (2011) who analyze WFC3 images of a small sample of 14 massive $\left(M_{\star} \geq 6 \times 10^{10}\right.$ $\left.M_{\odot}\right)$, quiescent, and compact $\left(r_{e} \leq 2 \mathrm{kpc}\right)$ galaxies at $1.5<z<2.5$ and report that most $(65 \pm 15 \%)$ are disk-dominated systems. They find that 5 of 14 galaxies are flat in projection and have an ellipticity $\geq 0.45$.

What is the nature of the massive galaxies at $z=2-3$ with $n \leq 2$ ? Figure 4.18 and

\footnotetext{
${ }^{8}$ The MGC catalog assigns the 'E/S0' Hubble type and unfortunately does not allow us to identify Es separately.
} 
the KS tests in Table 4.5 show that the massive galaxies at $z=2-3$ with $n \leq 2$ are more similar to $z \sim 0$ massive Sd/Irr (KS probability of $23.5 \%$ and $D=0.317$ ) and to $z \sim 0$ massive Sabc spirals (KS probability of $4.8 \%$ and $D=0.221$ ) than to $z \sim 0$ massive $\mathrm{E} / \mathrm{S} 0$ s. However, the similarity to massive late-type spirals at $z \sim 0$ is clearly limited, since most massive galaxies at $z=2-3$ with $n \leq 2$ have smaller half-light radii ( $r_{e}$ primarily below $7 \mathrm{kpc}$; Figure 4.5 ) than any of the $z \sim 0$ massive systems. It is possible that they host less extended and thicker disks than present-day massive spirals.

Another possibility is that the massive galaxies at $z=2-3$ with $n \leq 2$ might be related to clump-cluster and chain galaxies (Cowie et al. 1995; van den Bergh et al. 1996; Elmegreen et al. 2005, 2009a, 2009b). Such galaxies very often host disk structures (Elmegreen et al. 2009a), and many of them appear to represent a population of highly clumped disk galaxies viewed at different orientations (Elmegreen et al. 2008; Elmegreen et al. 2005). While clumpy disks may be among the massive GNS galaxies with low $n \leq 2$, we cannot identify them due to resolution effects. Finally, we note that in principle a low Sérsic index could be the result of a merger that has not fully coalesced. However, as noted in $§ 4.3 .2$ most massive GNS galaxies do not visually appear to be made of multiple distorted systems in early phases of mergers. Artificial redshifting of present-day interacting systems show that our GNS images should be able to resolve systems in early phases of merging, such as NGC4568 and NGC 3396, but would be unlikely to resolve late merger phases, such as Arp 220 into two separate systems.

3. Another line of evidence for massive galaxies at $z \sim 2$ with potentially thick disks 
comes from the SINS survey (Genzel et al. 2008; Shapiro et al. 2008; Förster Schreiber et al. 2009), which provides ionized gas kinematics of $z \sim 2$ star-forming galaxies and finds examples of clumpy, turbulent, and geometrically thick systems having high velocity dispersions $(\sigma \sim 30-120 \mathrm{~km} / \mathrm{s})$. About $\sim 1 / 3$ of such systems show rotating disks kinematics. Furthermore, Förster Schreiber et al. (2011) find from HST NIC2 imaging that five star-forming galaxies with rotating disk kinematics are wellcharacterized with shallow $n \leq 1$ Sérsic profiles. Compared to these SINS galaxies, the massive GNS galaxies at $z=2-3$ are more massive on average.

4. In this work (§4.3.1), we fitted the NIC3/F160W images of the massive galaxies at $z=$ $2-3$ with single Sérsic components, rather than separate bulge and disk components because the low resolution (PSF FWHM of 0 "' 3 corresponding to $\sim 2.4$ kpc at $z=1-3$ ) of the images prevent reliable multiple component decomposition for all the galaxies, particularly the fairly compact ones. However, for the galaxies with large $r_{e} \geq 4 \mathrm{kpc}$ we attempted a bulge-plus-disk decomposition following the techniques outlined in Weinzirl et al. (2009). The decomposition was reliable only for the more extended systems within this group and yielded bulge-to-total light ratios below 0.4, indeed suggesting the presence of a significant disk component among massive galaxies at $z=2-3$ with $n \leq 2$.

5. It is also interesting to note that most $\left(\sim 72 \%\right.$ for $\left.M_{\star} \geq 5 \times 10^{10} M_{\odot}\right)$ of these massive galaxies at $z=2-3$ with low $n \leq 2$ are extended $\left(r_{e}>2 \mathrm{kpc}\right)$ rather than ultracompact systems. This is in itself does not prove that disk components exist in low $n \leq 2$ systems, but it is suggestive of such a picture. Furthermore, we found in $\S 4.4 .3$ that at $z=2-3$, the $n \leq 2$ disky systems have a wide range of $\mathrm{SFR}_{\mathrm{IR}}$ and include 
systems of the highest $\mathrm{SFR}_{\mathrm{IR}}$. This result is generally consistent with the idea that the systems with $n \leq 2$ are actively star-forming and host copious amounts of gas $(\S 4.5)$, which tends to settle in disk-like configurations.

6. For completeness, we note that in principle the presence of a massive disk component is not the only way to produce a low Sérsic index $n \leq 2$ in massive galaxies at $z=2-3$. For the ultra-compact $\left(r_{e} \leq 2 \mathrm{kpc}\right)$ massive galaxies with $n \leq 2$, it has been argued that such systems could be somewhat like a massive elliptical, which has a bright high surface brightness central component surrounded by a very extended low surface brightness envelope. If the low surface brightness envelope is somehow not detected by the NIC3/F160W images, then the latter could yield a lower $n \leq 2$, as the wings of the surface brightness profile would be effectively clipped. However, this scenario does not seem likely since our artificial redshifting experiments (§4.3.3.1) show that $z \sim 0$ massive Es are not degraded into ultra-compact systems. Furthermore, Szomoru et al. (2010) confirm the absence of a low surface brightness halo in an ultra-compact, massive galaxy at $z=1.9$ from extremely deep $\left(H \sim 28 \operatorname{mag}_{\operatorname{arcsec}}{ }^{-2}\right)$ WFC3 imaging.

In summary, based on all the above tests and arguments, we conclude that the massive galaxies at $z=2-3$ with $n \leq 2$, particularly the more extended systems with $r_{e}>2 \mathrm{kpc}$, likely host a massive disk component, which contributes significantly to the rest-frame blue light of the galaxies.

\subsubsection{Formation of Massive Galaxies By $z=2-3$}

How do the massive galaxies with ultra-compact $\left(r_{e} \leq 2 \mathrm{kpc}\right)$ and low $n \leq 2$ disky structures form by $z=2-3$ ? The surface brightness in the rest-frame $B$-band of the 
massive galaxies at $z=2-3$ is on average 4.5 magnitudes brighter than massive $z \sim 0$ galaxies (Figure 4.7). This implies a large mass surface density of young-to-intermediate-age stars had to built up in less than a few Gyr. Implied stellar mass surface densities exceed several $10^{10} M_{\odot} \mathrm{pc}^{-2}$ even for conservative mass-to-light ratios. This implies that rapid and highly dissipative gas-rich events must have led to the formation of these massive galaxies by $z=2-3$. Both gas accretion and wet major mergers at $z>2$ are likely to have played an important role because at such high redshifts, the short dynamical timescales associated with mergers, and the short cooling time associated with gas accretion imply that both mechanisms would lead to a rapid buildup of cold gas. The latter can in turn lead to rapid star formation and dense stellar remnants (e.g., Wuyts et al. 2009; Wuyts et al. 2010; Khochfar \& Silk 2011; Bournaud et al. 2011).

A further constraint on the formation pathway is provided by the structure of the massive galaxies at $z=2-3$. We have shown in $\S 4.3 .2$ that as much as $\sim 65 \%$ of the massive galaxies at $z=2-3$ have a low $n \leq 2$, and we further argued in $\$ 4.7 .1$ that most of these systems with $n \leq 2$ at $z=2-3$ likely host a massive disk component. Major mergers of low-to-moderate gas fraction (e.g., $\leq 30 \%$ ) will typically produce merger remnants with a de Vaucouleurs type profile and a Sérsic index $n>3$ (Naab, Khochfar, \& Burkert 2006; Naab \& Trujillo 2006). Mergers with moderate-to-high gas fractions are expected to produce lower Sérsic $n$ that are still in general $>2$. For instance, Figure 14 of Hopkins et al. (2009a) show the Sérsic index of major merger remnants for a range of orbits and a range of progenitors with gas fractions spanning from 10-100\%. Although some massive $\left(M_{\star} \geq 10^{11} M_{\odot}\right)$ remnants with $n \sim 1$ arise in mergers with $f_{\text {gas }} \geq 80 \%$, most remnants of gas-rich $\left(f_{\text {gas }} \geq 40 \%\right)$ mergers have a Sérsic index $n>2$. Furthermore, 
Rothberg \& Joseph (2004) find from $K$-band imaging of 52 merger remnants that $\sim 51 \%$ $(26 / 51)$ have $n>3, \sim 37 \%(19 / 51)$ have $n \sim 2-3$, and only a small fraction $(\sim 12 \%, 6 / 51)$ have $n \sim 1-2$. Thus, when considering isolated gas-rich major mergers, namely those not fed by cold streams, it is challenging to produce a population of merger remnants where $\sim 65 \%$ of the systems have $n \leq 2$

The challenge of producing a large population of disky $(n \leq 2)$ systems with high SFRs from isolated gas-rich major mergers may be an indication that the accretion of cold gas along cosmological filaments ((Birnboim \& Dekel 2003; Kereš et al. 2005; Dekel \& Birnboim 2006; Dekel et al. 2009a; Dekel et al. 2009b; Kereš et al. 2005; Kereš et al. 2009; Brooks et al. 2009; Ceverino et al. 2010) may be particularly important in the build-up of massive galaxies by $z=2-3$. As merger remnants at $z>2$ acquire gas via cold-mode accretion, a gas disk is expected to form (Khochfar \& Silk 2009a; Burkert et al. 2010). Depending on the angular momentum of the accreted gas, it can settle into a compact disk component or into an outer extended disk. Burkert et al. (2010) discuss a scenario where turbulent rotating disks can form, segregating into compact $\left(r_{e} \sim 1-3 \mathrm{kpc}\right)$ dispersiondominated $(1 \leq v / \sigma \leq 3)$ systems and more extended $\left(r_{e} \sim 4-8 \mathrm{kpc}\right)$, rotation-dominated $(v / \sigma>3)$ disks. The formation of a gas disk via cold-mode accretion and its subsequent conversion into a stellar disk, would lower the overall Sérsic index of the massive galaxies at $z=2-3$, making them more in line with the observed values.

However, many key questions remain unanswered. Can theoretical models account for the observed fractions of massive galaxies with low $n \leq 2$, as well as the fraction of galaxies with ultra-compact $\left(r_{e}>2 \mathrm{kpc}\right)$ sizes? Can the relation between structure, SFR, and AGN activity discussed in $\S 4.4 .3$ and $\S 4.6 .2$, as well as the range in SFR at a given stellar 
mass, be accounted for? We will address these questions in a future paper (Jogee et al., in preparation) where we perform detailed comparisons with different theoretical scenarios.

\subsubsection{Transformation of Massive Galaxies at $z=2-3$ Into Present-Day E and SOs}

Next we discuss the transformation of massive galaxies at $z=2-3$ into their more massive present-day descendants, which are primarily E and S0s. During this transformation, the massive galaxies will need to significantly increase $n$ since the majority $(\sim 65 \%)$ of massive galaxies at $z=2-3$ have low $n \leq 2$, while the corresponding fraction among massive systems at $z \sim 0$ is five times lower (Table 4.1 and Figure 4.5). Similarly, the galaxies will also need to significantly raise $r_{e}$, since approximately $40 \%$ of massive galaxies at $z=2-3$ are in the form of ultra-compact $\left(r_{e} \leq 2 \mathrm{kpc}\right)$ galaxies compared with less than $1 \%$ at $z \sim 0$ (Table 4.1 and Figure 4.5). In general, the massive $z=2-3$ galaxies must experience a substantial growth in $r_{e}$ by up to a factor of $\sim 6$, a dimming in rest-frame optical surface brightness within $r_{e}$ by up to 6 magnitudes (Figure 4.7), and their $n$ must increase to $n>2$. An increase in $\left(n, r_{e}\right)$ and a dimming in $\mu_{\mathrm{e}}$ can be achieved via several pathways.

A natural pathway to produce large changes in $\left(n, r_{e}, \mu_{\mathrm{e}}\right)$ is a dry major merger of two disk systems. This produces a remnant with $n \sim 4$, a higher $r_{e}$, and a lower surface brightness within $r_{e}$ than the progenitors (Naab, Khochfar, \& Burkert 2006; Naab

\& Trujillo 2006; Naab et al. 2009). In this case, the change in $n$ is produced by the transformation of galaxies with disks into systems dominated by spheroids or bulges. This type of transformation must take place from $z=2-3$ to $z \sim 0$ in many of the massive galaxies because $\sim 65 \%$ of them at $z=2-3$ have $n \leq 2$, which we argued is indicative 
of a massive disk in many cases (§4.7.1). In contrast the E/S0s at $z \sim 0$ are dominated by spheroids or bulges.

Other lines of evidence support the idea that dry major mergers play a role in making the most massive $z \sim 0$ ellipticals. The most massive local ellipticals are found to harbor cores (missing light), which are believed to be scoured by binary black holes that form in dry major mergers (Kormendy et al. 2009). From a study of the tidal features associated with bulge-dominated early-type galaxies, van Dokkum (2005) concludes that today's most luminous ellipticals form through mergers of gas-poor, bulge-dominated systems. Kriek et al. (2008) focus on massive red-sequence galaxies at $z \sim 2.3$ with little or no ongoing star formation, finding that the changes in color and number density of galaxies on the highmass end $\left(M_{\star} \geq 1 \times 10^{11} M_{\odot}\right)$ of the red sequence from $z \sim 2.3$ to the present are better explained by a combination of passive evolution and red mergers that induce little star formation, rather than by passive evolution alone.

While dry major mergers play a role in the evolution of massive galaxies, it remains debated whether they can account for the full size and mass evolution of massive galaxies. From a theoretical standpoint, the predicted dry major merger rate appears to be too low. From simulations, Khochfar \& Silk (2009b) find that only between $10 \%-20 \%$ of massive $\left(M_{\star}>6.3 \times 10^{10} M_{\odot}\right)$ galaxies have had a dry major merger in the last Gyr at any redshift $z<1$. Hopkins et al. (2010a) find from semi-empirical models that the importance of major mergers in bulge formation scales with galaxy stellar mass. Namely, an $L_{*}$ galaxy with $M_{\star} \sim 10^{11} M_{\odot}$ at $z=0$ will experience only one dry major merger at $z<2$. Shankar et al. (2010) calculate that the frequency of dry mergers increases with final stellar mass, and they find that by $z=0$ massive $\left(M_{\star}>10^{11} M_{\odot}\right)$ early-type galaxies undergo on average 
$<1$ dry major merger since their formation.

From an observational standpoint, direct measurements of the dry major merger rate at $z<1$ are highly uncertain. Bell et al. (2006) suggest that present-day spheroidal galaxies with $M_{\mathrm{V}}<-20.5$ on average have undergone anywhere between 0.5 and 2 dry major mergers since $z \sim 0.7$. The analysis carries large uncertainties as it is based on a small number $(\sim 6)$ of observed dry major mergers. Several observational studies report that between $16 \%$ to $35 \%$ of massive $\left(M_{\star}>2.5 \times 10^{10} M_{\odot}\right)$ galaxies have undergone a major merger since $z \sim 0.8$ (e.g., Jogee et al. 2009; Lotz et al. 2008; Conselice et al. 2009), but it should be noted that most of the major mergers in the above studies are star-forming systems, and there are very few dry major mergers. Robaina et al. (2010) find that galaxies with $M_{\star}>10^{11} M_{\odot}$ have undergone, on average, only 0.5 mergers since $z \sim 0.7$ involving progenitor galaxies that are both more massive than $M_{\star}>5 \times 10^{10} M_{\odot}$. Hammer et al. (2009) focus on starbursts with disturbed ionized gas morphologies and kinematics at $z \sim 0.65$, and they argue based on modeling that $\sim 6$ Gyr ago $46 \%$ of the galaxy population was involved in major mergers, most of which were gas-rich. Kaviraj et al. (2011) find that theoretically and empirically determined major merger rates at $z<1$ are too low by factors of a few to account for the fraction of disturbed systems they find among morphologically classified early-type massive $\left(M_{\star}>1 \times 10^{10} M_{\odot}\right)$ galaxies at $0.5<z<0.7$. They suggest that the overall evolution of massive early type galaxies, particularly the low-level star formation activity, may be heavily influenced by minor merging at late epochs. At higher redshifts $1<z<2$, higher major merger rates are reported than at $z<1$ (e.g., Conselice et al. 2003), but the frequency of dry major mergers is claimed to be low (Williams et al. 2011). 
An alternate pathway that could be at least as important as major mergers consists of consecutive dry minor mergers or accretion of externally formed stars such that stellar mass is cumulatively added to the outskirts of a compact galaxy (e.g., Naab et al. 2009; Feldman et al. 2010). Naab \& Trujillo (2006) show that successive minor mergers can, on average, raise the Sérsic index of the merger remnant about as effectively as major mergers. Furthermore, it is claimed from simulations and analytical arguments that dry minor mergers produce a much larger increase in size $\left(r_{e}\right)$ and a larger fall in average stellar mass densities within $r_{e}$ than do dry major mergers (Naab et al. 2009; Bezanson et al. 2009). Shankar et al. (2011) find in simulations that massive $\left(M_{\star} \geq 10^{11} M_{\odot}\right) z \sim 0$ galaxies grow primarily by dry minor mergers, especially at $z<1$. Oser et al. (2010; 2012) use cosmological simulations to study 40 individual massive galaxies with present-day stellar masses of $M_{\star}>6.3 \times 10^{10} M_{\odot}$. They find that massive galaxies at $z>2$ are dominated by "in situ" star formation fueled by in-falling cold gas within the galaxy. As cold-mode accretion becomes inefficient at $z \approx 2$, accretion of externally created stars (i.e., stellar satellites) dominates at $z<2$. For galaxies of present-day stellar mass $M_{\star}>6.3 \times 10^{10} M_{\odot}$, the average number-weighted merger mass-ratio is $\sim 1: 16$, while the average mass-weighted merger mass-ratio is $\sim 1: 5$. In other words, the mass growth since $z \sim 2$ is dominated by minor mergers with a mass ratio of 1:5. The importance of stellar accretion increases with galaxy mass and toward lower redshift, and it substantially raises the galaxy stellar mass and size. For systems with present-day stellar mass $M_{\star}>6.3 \times 10^{10} M_{\odot}$, a size evolution of up to a factor of $\sim 5-6$ occurs from $z=2$ to $z \sim 0$. However, one strong caveat of these simulations is that all their massive $\left(M_{\star}>1 \times 10^{11} M_{\odot}\right)$ galaxies at $z=2$ are ultra-compact $\left(r_{e} \leq 2 \mathrm{kpc}\right.$ ), while observations (see Fig. 4.5) show a large fraction of such massive galaxies 
at $z=2$ are extended $\left(r_{e}=3-10 \mathrm{kpc}\right)$, with a wide range in star formation rate. The increase of size and mass induced by minor mergers in these simulations is qualitatively in agreement with our results on size evolution for the ultra-compact systems and also with the inside-out growth reported by van Dokkum et al. (2010) from stacking deep rest-frame $R$-band images of massive galaxies over the redshift range of 0.6 to 2.0.

However, many questions remain unresolved. While dry minor mergers appear to be effective at inducing significant evolution in mass and size from $z \sim 2$ to $z \sim 0$ in the simulations of Oser et al. (2010; 2012), it is unclear if they can really drive the large change in Sérsic index $n$ required by the observations. Furthermore, these simulations focus only on ultra-compact $\left(r_{e} \leq 2 \mathrm{kpc}\right)$ galaxies, and are not representative of the large dominant population of more extended galaxies at $z=2-3$. Finally, it is not clear whether minor mergers can account for the changes in effective surface brightness between $z=2-3$ and $z \sim 0$

\subsection{Summary}

We present a study of the structure, activity, and evolution of massive galaxies at $z=1-3$ using deep ( $5 \sigma$ limiting magnitude of $H=26.8 \mathrm{AB}$ for an extended source of diameter $0 . .7$ ), high resolution (PSF 0.!3) NIC3/F160W images from the GOODSNICMOS Survey (GNS), along with complementary ACS, Spitzer IRAC and MIPS, and Chandra X-ray data. One of the strengths of our study is that the NIC3/F160W data provide rest-frame optical imaging over $z=1-3$ for one of the largest (166 galaxies with $M_{\star} \geq 5 \times 10^{10} M_{\odot}$ and 82 with $\left.M_{\star} \geq 10^{11} M_{\odot}\right)$, most diverse, and relatively unbiased samples of massive galaxies at $z=1-3$ studied to date. Our main results are summarized 
below.

1. Structure of massive galaxies at rest-frame optical wavelengths: We analyze the rest-frame optical structure of the massive galaxies by fitting single Sérsic profiles to the 2D light distribution in the NIC3/F160W images. We find that the rest-frame optical structures of the massive galaxies are very different at $z=2-3$ compared with $z \sim 0$, with their Sérsic index $n$ and half-light radius $r_{e}$ being strikingly offset toward lower values compared with $z \sim 0$. (Table 4.1 and Figure 4.5). Through extensive tests and artificial redshifting experiments we conclude that the offset in $\left(n, r_{e}\right)$ between massive galaxies at $z=2-3$ and $z \sim 0$ is real and not primarily driven by systematic effects related to the fitting techniques instrumental effects, or redshift-dependent effects (e.g., cosmological surface brightness dimming and the loss of spatial resolution). In effect, we find a large population of ultra-compact $\left(r_{e} \leq 2 \mathrm{kpc}\right)$ systems, as well as a dominant population of systems with low $n \leq 2$ disky morphologies at $z=2-3$. We further describe these populations below.

We find that approximately $40 \%\left(39.0 \pm 5.6 \%\right.$ for $M_{\star} \geq 5 \times 10^{10} M_{\odot}$ and $39.0 \pm 7.6 \%$ for $M_{\star} \geq 1 \times 10^{11} M_{\odot}$ ) of the massive galaxies at $z=2-3$ are in the form of ultra-compact $\left(r_{e} \leq 2 \mathrm{kpc}\right)$ galaxies compared with less than $1 \%$ at $z \sim 0$ (Table 4.1 and Figure 4.5$)$. These ultra-compact galaxies are practically unmatched among $z \sim 0$ massive galaxies, and their surface brightness in the rest-frame optical can be 4-6 magnitudes brighter (Figure 4.7).

Secondly, we find that the majority $\left(64.9 \% \pm 5.4 \%\right.$ for $M_{\star} \geq 5 \times 10^{10} M_{\odot}$, and $58.5 \% \pm 7.7 \%$ for $M_{\star} \geq 10^{11} M_{\odot}$ ) of massive galaxies at $z=2-3$ have low $n \leq 2$, while the corresponding fraction among massive systems at $z \sim 0$ is five times lower. Most $(\sim 72 \%)$ of these massive galaxies at $z=2-3$ with low $n \leq 2$ have $r_{e}>2 \mathrm{kpc}$, and therefore 
complement the ultra-compact galaxies. We further explore the meaning of a Sérsic index $n \leq 2$ at $z=2-3$, and present evidence that most of the massive galaxies with $n \leq 2$ at $z=2-3$, particularly the extended $\left(r_{e}>2 \mathrm{kpc}\right)$ ones, likely host a prominent disk, unlike the majority of massive galaxies at $z \sim 0$. Our evidence is based on rest-frame optical morphologies, ellipticities, artificial redshifting experiments, as well as bulge-to-total ratios from bulge-plus-disk decompositions of extended systems.

2. Star formation rates: We estimate star formation rates using IR luminosities (8-1000 $\mu \mathrm{m})$ derived from the Spitzer $24 \mu \mathrm{m}$ flux for massive GNS galaxies having a secure MIPS $24 \mu \mathrm{m}$ counterpart and a $24 \mu \mathrm{m}$ flux exceeding the $5 \sigma$ detection limit of $30 \mu \mathrm{Jy}$. AGN host candidates are excluded because the inferred IR luminosities overestimate the true star formation rate.

We find a strong link between galaxy structure and SFR. Among the non-AGN massive $\left(M_{\star} \geq 5 \times 10^{10} M_{\odot}\right)$ galaxies at $z=2-3$ with $\mathrm{SFR}_{\mathrm{IR}}$ high enough to yield a $5 \sigma$ (30 $\mu \mathrm{Jy})$ Spitzer $24 \mu \mathrm{m}$ detection, the majority $(84.6 \pm 10.0 \%)$ have low $n \leq 2$. While the $n \leq 2$ disky systems have a wide range of $\mathrm{SFR}_{\mathrm{IR}}\left(53\right.$ to $1466 M_{\odot} \mathrm{yr}^{-1}$ at $z=2-3$ ), they include the systems of the highest $\mathrm{SFR}_{\mathrm{IR}}$ at both $z=1-2$ and $z=2-3$. In contrast, the massive ultra-compact objects at $z=2-3$ are less likely by a factor of $\sim 2.2$ to have $\mathrm{SFR}_{\mathrm{IR}}$ above the detection limit, compared with the whole sample of non-AGN massive galaxies.

3. AGN activity: Using a variety of techniques (X-ray properties, IR power-law, and IR-to-optical excess) to identify AGN, we find that 49/166 (29.5 $\pm 3.5 \%)$ of the massive galaxies at $z=1-3$ are AGN candidates. The AGN fraction rises with redshift, increasing from $17.9 \pm 6.1 \%$ at $z=1-1.5$ to $40.3 \pm 8.8 \%$ at $z=2-3$ (Table 4.4 ). 
We find a relationship between host galaxy structure and AGN activity that complements the relationship between SFR and structure. Among massive galaxies at $z=2-3$, AGN appear to be found preferentially in galaxies that are not ultra-compact, as evidenced by the fact that most $(80.6 \pm 7.9 \%)$ AGN hosts have $r_{e}>2 \mathrm{kpc}$. In fact, at $z=2-3$, the AGN fraction in ultra-compact galaxies is $\sim 2.7$ times lower than in extended galaxies (20.0 $\pm 16.3 \%$ versus $53.2 \pm 10.0 \%)$. Thus, ultra-compact galaxies appear quiescent in terms of both SFR and AGN activity. In terms of their Sérsic index $n$, a large fraction (64.6 $\pm 10.7 \%)$ of AGN hosts at $z=2-3$ have disky $(n \leq 2)$ morphologies.

4. Cold gas content: We apply a standard Schmidt-Kennicutt law (Kennicutt 1998) to the $\mathrm{SFR}_{\mathrm{IR}}$ of the non-AGN host candidates. The high estimated $\mathrm{SFR}_{\mathrm{IR}}$ suggest that copious cold gas reservoirs are present. We estimate that the average cold gas surface density in non-AGN hosts ranges from $\sim 136$ to $\sim 25,091 M_{\odot} \mathrm{pc}^{-2}$ at $z=1-3$, with a median value of $\sim 607 M_{\odot} \mathrm{pc}^{-2}$ (Figure 4.15). The implied cold gas fraction within the rest-frame optical half-light radius ranges from $6.5 \%$ to $65.4 \%$, with a mean of $\sim 41 \%$ at $z=2-3$ (Figure 4.16). The highest gas fractions at a given redshift are found among the less massive galaxies, consistent with downsizing.

5. Formation of massive galaxies by $z=2-3$ : The massive galaxies at $z=2-3$ already have an average rest-frame optical surface brightness within $r_{e}$ that can be up to 3-6 magnitudes brighter than $z \sim 0$ massive galaxies. The associated high stellar mass densities imply that massive galaxies at $z=2-3$ must have formed via rapid, highly dissipative events at $z>2$. Both gas-rich major mergers and gas accretion at $z>2$ are viable as their associated short dynamical timescales and short gas cooling times at $z>2$ would lead to a 
rapid buildup of mass. However, the large fraction $(\sim 65 \%)$ of massive galaxies at $z=2-3$ with $n \leq 2$ and disky morphologies suggest that cold-mode accretion at $z>2$ must have played an important role in the build-up of massive galaxies by $z=2-3$, since it may be challenging to have such a large fraction of of merger remnants with low $n \leq 2$ from isolated gas-rich major mergers.

6. Transformation of massive galaxies at $z=2-3$ into present-day $E$ and S0s: In order for massive galaxies at $z=2-3$ to evolve into $z \sim 0$ massive systems (which are primarily E and S0s), they need to radically change their rest-frame optical structure and distributions of $\left(n, r_{e}\right)$. In particular they need to raise $n$ well above 2 , increase $r_{e}$ by an average factor of 3-4, and dim the average rest-frame optical surface brightness. Dry major mergers can induce changes in galaxy size, Sérsic index, and stellar surface density, but they may be too rare to account for all the needed evolution. Successive dry minor mergers have been shown to influence galaxy size, Sérsic index, and stellar surface density in a similar direction. We suggest the transformation of massive $z=2-3$ galaxies into $z \sim 0$ galaxies will occur through a combination of dry major mergers, minor mergers. We will investigate in the relative importance and efficiency of these mechanisms in a future paper. 

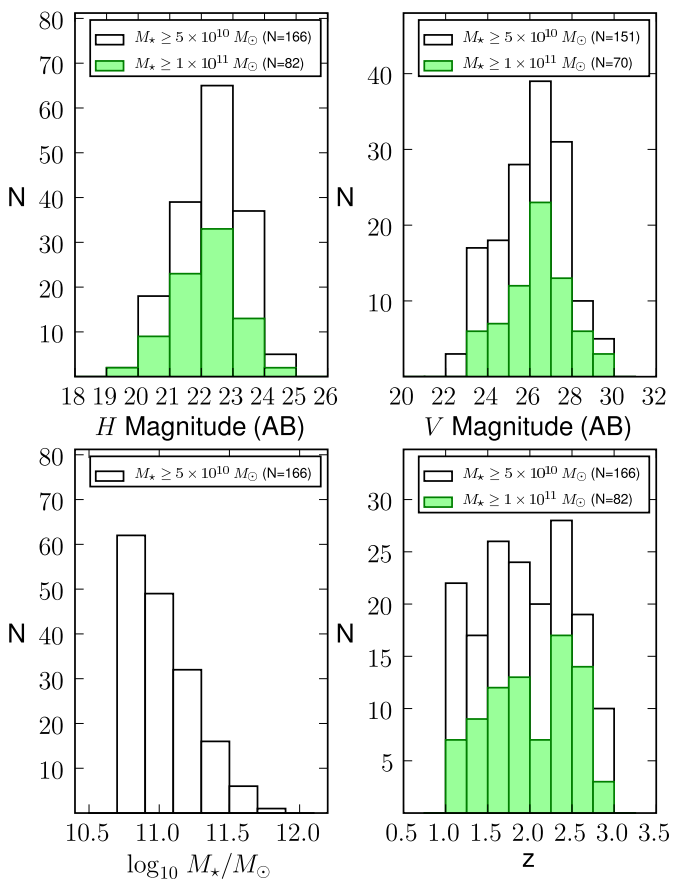

Figure 4.1 The distribution of apparent $H$ (F160W), $V$ apparent magnitude, stellar mass, and redshift for the final, complete sample of 166 galaxies with $M_{\star} \geq 5 \times 10^{10} M_{\odot}$ and redshift $z=1-3$. 

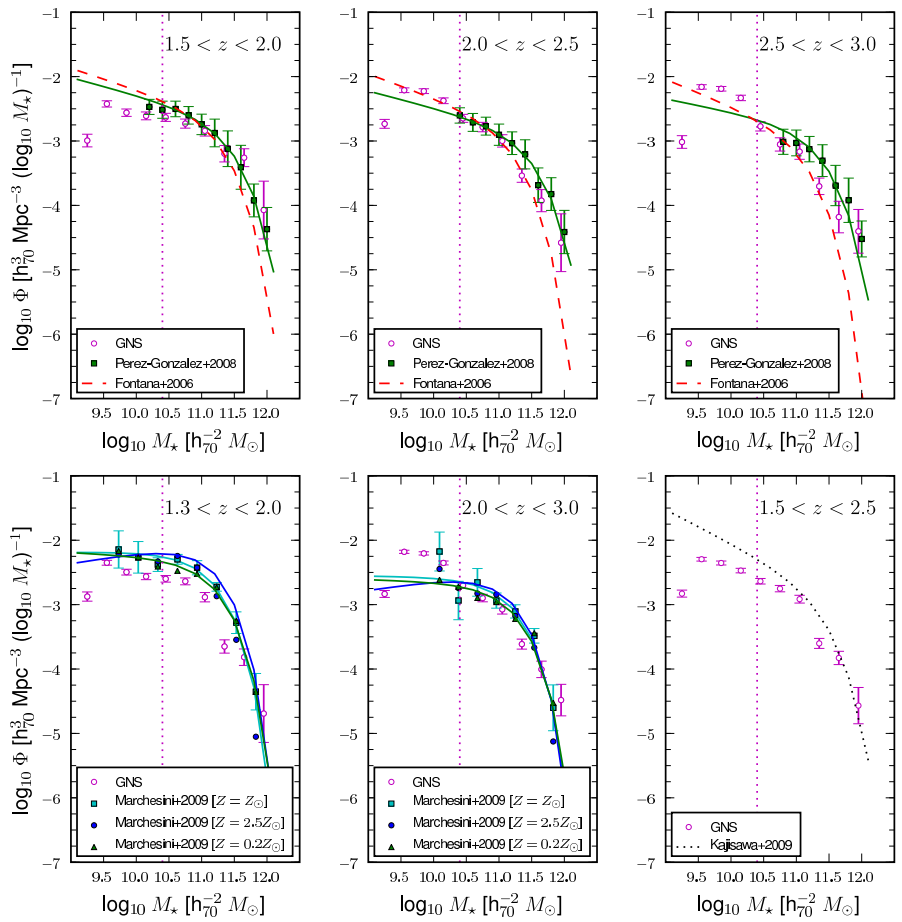

$\log _{10} M_{\star}\left[\mathrm{h}_{70}^{-2} M_{\odot}\right]$

Figure 4.2 We compare the galaxy stellar mass functions for GNS over $z=1-3$ with those from other studies that are based on $K$ or IRAC-selected samples (Kajisawa et al. 2009; Marchesini et al. 2009; Pérez-González et al. 2008; Fontana et al. 2006). The vertical line in each plot marks the mass cut $\left(M_{\star} \geq 5 \times 10^{10} M_{\odot}\right)$ for the GNS-based sample used in this paper. We include the data points with error bars from the other studies, where available, along with each Schechter function fit. Some studies (Kajisawa et al. 2009; Marchesini et al. 2009) present results for multiple sets of SED-modeling assumptions, and in these cases we show the results for the assumptions that most closely match those used for GNS by Conselice et al. (2011). For Kajisawa et al. (2009), we show the mass function calculated with Bruzual \& Charlot (2003) stellar templates. For Marchesini et al. (2009), we show the stellar mass functions calculated with Bruzual \& Charlot 2003 templates, metallicities of $0.2,1$, and $2.5 Z_{\odot}$, a Kroupa IMF, and a Calzetti extinction law, but in the above plot, we scale their mass functions by +0.2 dex along the $x$-axis to convert their Kroupa IMF to a Salpeter IMF. For the GNS mass functions, in comparison, the best metallicity is determined on a galaxy-by-galaxy basis from a set of discrete values spanning 0.005 to $2.5 Z_{\odot}$. The error bars for Marchesini et al. (2009) take into account the uncertainties due to cosmic variance, Poisson error, photometric redshifts, and stellar SED templates. The error bars from Pérez-González et al. (2008) account for Poisson error and uncertainty in photometric redshifts. In comparison, the error bars on the GNS mass functions show only Poisson error. 


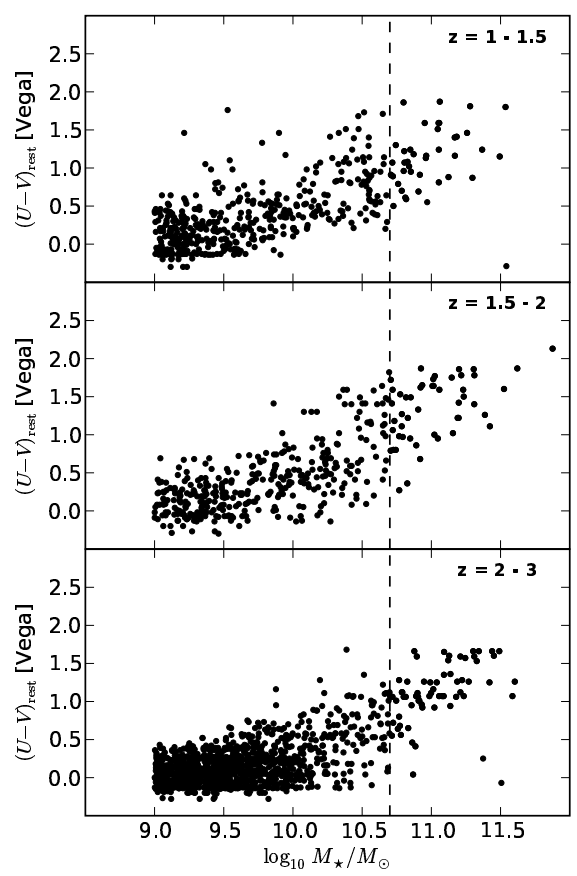

Figure 4.3 For all galaxies detected in the GOODS-NICMOS Survey (GNS) over $z=1-3$, the rest-frame $U-V$ color is plotted against $M_{\star}$ for different redshift bins. Blue systems are preferentially at low masses, while the most massive $\left(M_{\star} \geq 1 \times 10^{11} M_{\odot}\right)$ galaxies are preferentially red. The vertical line denotes $M_{\star}=5 \times 10^{10} M_{\odot}$, the mass cut we adopt for our final sample of 166 galaxies. 


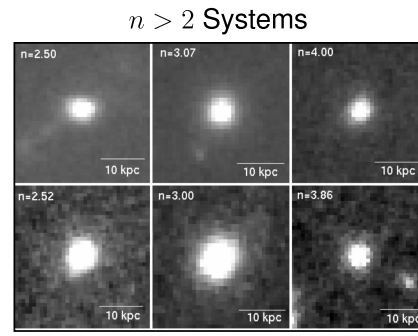

$r_{e} \leq 2 \mathrm{kpc}$
$2<r_{e} \leq 4 \mathrm{kpc}$

$n \leq 2$ Systems

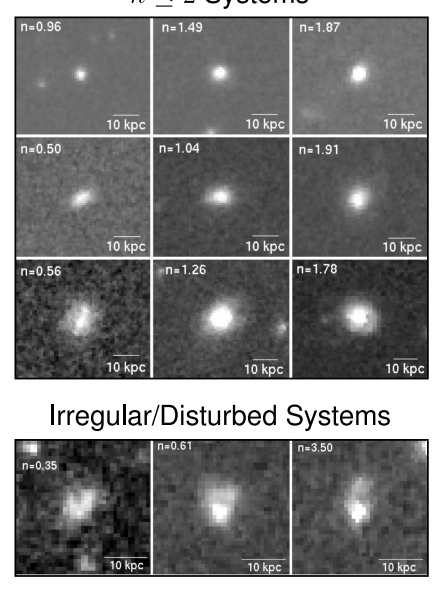

$r_{e} \leq 2 \mathrm{kpc}$

$2<r_{e} \leq 4 \mathrm{kpc}$

$4<r_{e} \leq 8 \mathrm{kpc}$

$2.7 \leq r_{e} \leq 3.6 \mathrm{kpc}$

Figure 4.4 NICMOS F160W images for representative GNS galaxies with $M_{\star} \geq 5 \times 10^{10}$ $M_{\odot}$ at $z=2-3$. The Sérsic index $n$ and effective radius $r_{e}$ referenced here are based on fitting single Sérsic components to the NICMOS images, as described in $\S 4.3$. The top panel shows example systems with Sérsic index $n>2$ and half-light radii $r_{e} \leq 4 \mathrm{kpc}$. The middle panel shows examples with $n \leq 2$ and $r_{e} \leq 8 \mathrm{kpc}$. The majority ( $\sim 2 \%$; Table 4.1$)$ of the massive GNS galaxies have $r_{e} \leq 4 \mathrm{kpc}$. In such systems, structural features are generally hard to discern due to resolution effects, so that systems appear fairly featureless (top 4 rows). In the small fraction of massive galaxies at $z=2-3$ with large $r_{e}>4 \mathrm{kpc}$, one can discern some structural features such as an elongated bar-like feature or a combination of a central condensation surrounded by a more extended lower surface brightness component, reminiscent of a bulge and disk (row 5). The bottom panel (row 6) contains systems that appear morphologically disturbed. 


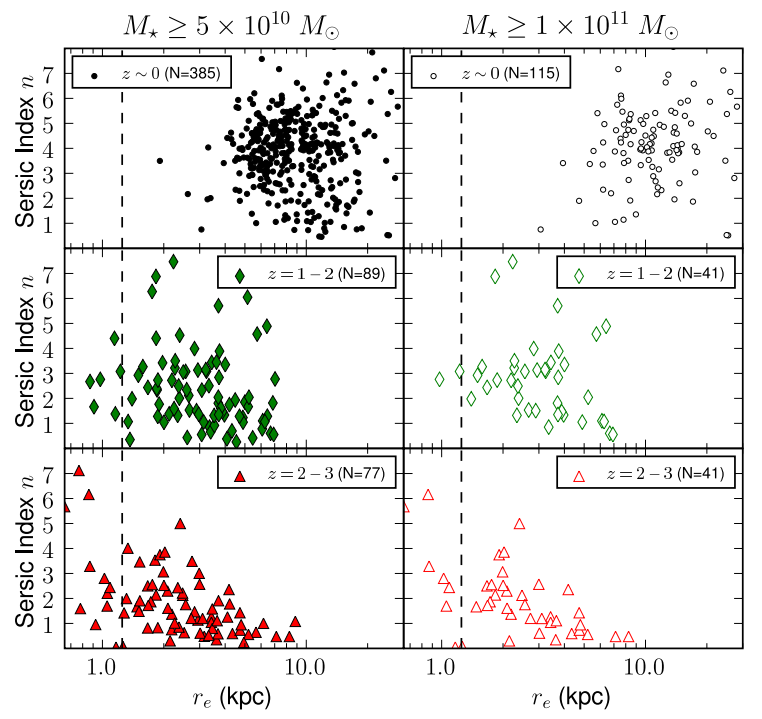

Figure 4.5 The $B$-band Sérsic index $n$ and effective radius $r_{e}$ derived from single Sérsic profile fits to massive $\left(M_{\star} \geq 5 \times 10^{10} M_{\odot}\right)$ galaxies are plotted for the three redshift bins listed in Table 4.1. In the top row, the black points represent fits to $z \sim 0$ galaxies by Allen et al. (2006) on $B$-band images of galaxies from the Millennium Galaxy Catalog (Liske et al. 2003). The lower two rows are based on our fits to the NIC3 F160W images of massive GNS galaxies at $z=1-2$ and $z=2-3$. Note that the massive galaxies at $z=2-3$ are strikingly offset toward lower $\left(n, r_{e}\right)$ compared with the massive $z \sim 0$ galaxies, and have five times more low $n \leq 2$ disky systems (see also Figure 4.6). The black dashed line represents the typical half-width half maximum of the NICMOS3 PSF at $z=1-3$ of $\sim 1.2 \mathrm{kpc}$. 

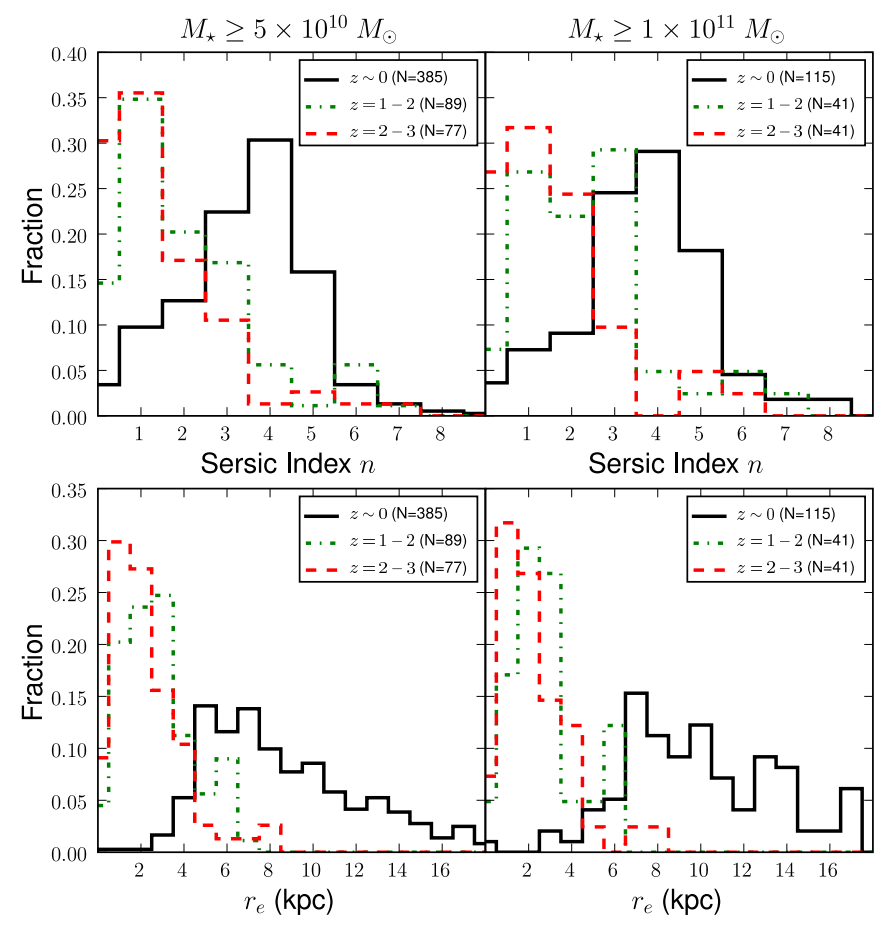

Figure 4.6 Left column: The distributions of rest-frame optical Sérsic index and effective radius $r_{e}$ based on single Sérsic profile fits to massive $\left(M_{\star} \geq 5 \times 10^{10} M_{\odot}\right)$ galaxies are plotted for the three redshift bins listed in Table 4.1: at $z \sim 0$ (solid line), based on the fits of Allen et al. (2006) on $B$-band images of galaxies from the Millennium Galaxy Catalog (Liske et al. 2003), and at $z=1-2$ (dash-dotted line) and $z=2-3$ (dashed line), based on our fits to the NIC3 F160W images of massive GNS galaxies. Note that a significant fraction $(39.0 \pm 5.56 \%)$ of massive $\left(M_{\star} \geq 5 \times 10^{10} M_{\odot}\right)$ galaxies at $z=2-3$ have $r_{e} \leq 2 \mathrm{kpc}$, compared with only $0.52 \pm 0.37 \%$ at $z \sim 0$. Note also that most $(64.9 \pm 5.4 \%)$ of massive galaxies at $z=2-3$ have low $n \leq 2$ (disky) structures compared with only $13.0 \pm 1.7 \%$ at $z \sim 0$. Right column: Same as left column but for the mass range $M_{\star} \geq 1 \times 10^{11} M_{\odot}$. 

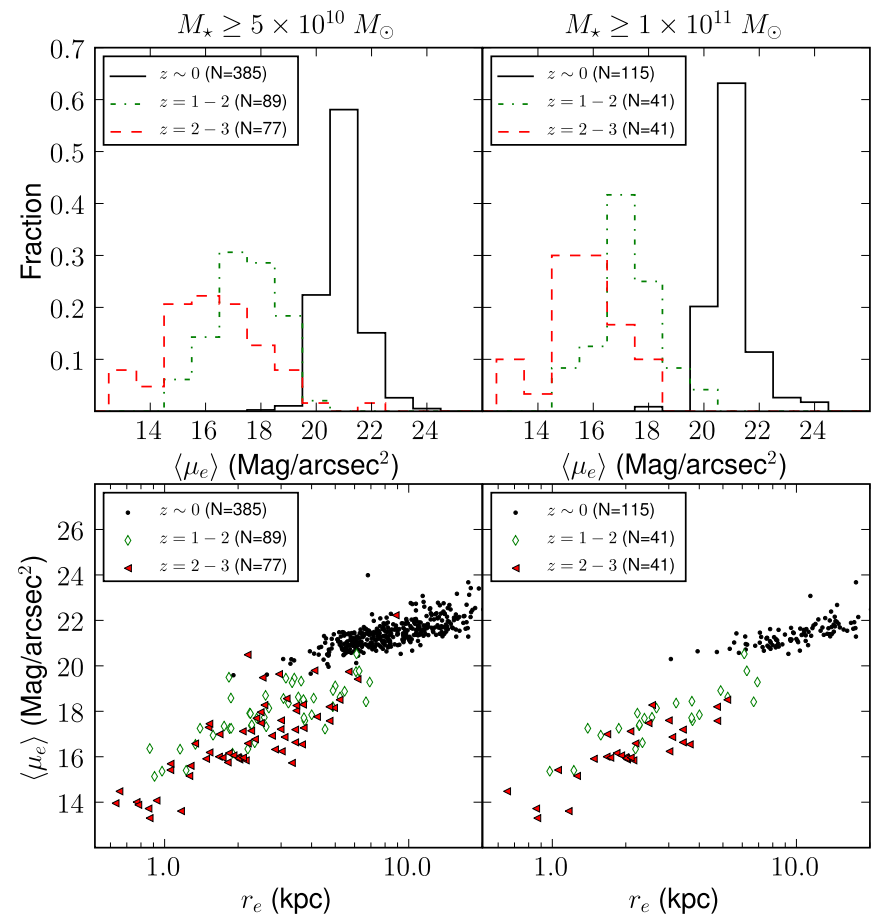

Figure 4.7 Left column: The top panel shows mean extinction-corrected rest-frame $B$-band surface brightness within the effective radius $\left(<\mu_{\mathrm{e}}>\right)$ for massive $\left(M_{\star} \geq 5 \times 10^{10} M_{\odot}\right)$ galaxies for the three redshift bins listed in Table 4.1. The solid line is for $z \sim 0$ MGC galaxies. The dash-dotted line $(z=1-2)$ and the dashed line $(z=2-3)$ are based on our fits to the NIC3 F160W images of massive GNS galaxies. The GNS galaxies at $z=2-3$ have a mean surface brightness of $16.8 \mathrm{mag} / \operatorname{arcsec}^{2}$ and are systematically brighter than the $z \sim 0$ MGC galaxies, which have a mean surface brightness of $21.3 \mathrm{mag} / \mathrm{arcsec}^{2}$. In the bottom panel, surface brightness within the effective radius is plotted against effective radius $r_{e}$ for the same redshift bins. Right column: The same plots are repeated for galaxies with $M_{\star} \geq 1 \times 10^{11} M_{\odot}$. Surface brightness is calculated with the extinction-corrected rest-frame $B$-band light and is defined as $\left\langle\mu_{\mathrm{e}}\right\rangle=B_{\text {corr }}+2.5 \log _{10}\left(2 \pi \mathrm{r}_{\mathrm{e}}^{2}\right)-10 \log _{10}(1+\mathrm{z})$, where $B_{\text {corr }}$ is the extinction-corrected, rest-frame $B$ apparent magnitude. 


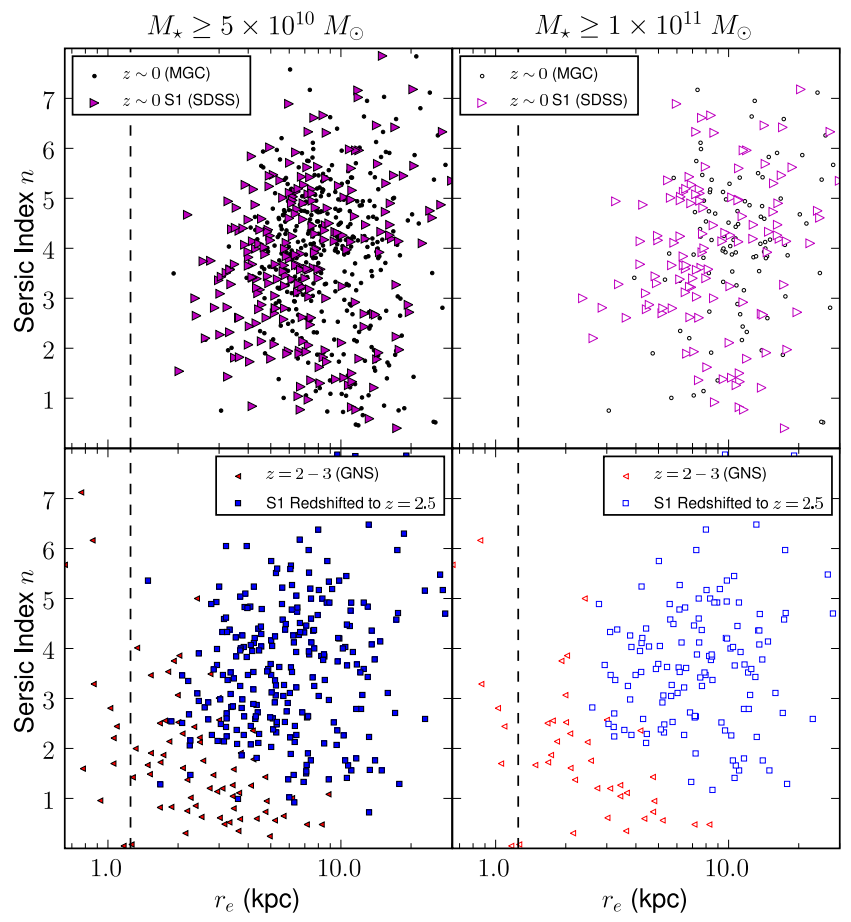

Figure 4.8 Top row: The black points show the massive $\left(M_{\star} \geq 5 \times 10^{10} M_{\odot}\right) z \sim 0$ galaxies from MGC described earlier in Figure 4.5. The magenta points denote the SDSS-based sample S1 of 255 representative massive $\left(M_{\star} \geq 5 \times 10^{10} M_{\odot}\right) z \sim 0$ galaxies used in the redshifting experiment. Note the $\left(n, r_{e}\right)$ distribution of $\mathrm{S} 1$ covers the same parameter space as that of the MGC sample. This is also shown quantitatively in Figure 4.9. Row 2: We show as blue squares the $\left(n, r_{e}\right)$ distribution obtained after redshifting S1 to $z=2.5$ and 're-observing' it with NIC3/F160W as in the GNS survey. We assume a surface brightness evolution of 2.5 magnitudes and brighten each redshifted galaxy by this amount. The actual observed $\left(n, r_{e}\right)$ distributions of the massive galaxies at $z=2-3$ in the GNS survey are significantly offset toward lower values compared with the redshifted galaxies. The black dashed line represents the typical half-width half max of the NICMOS3 PSF at $z=1-3$ of $\sim 1.2 \mathrm{kpc}$. 

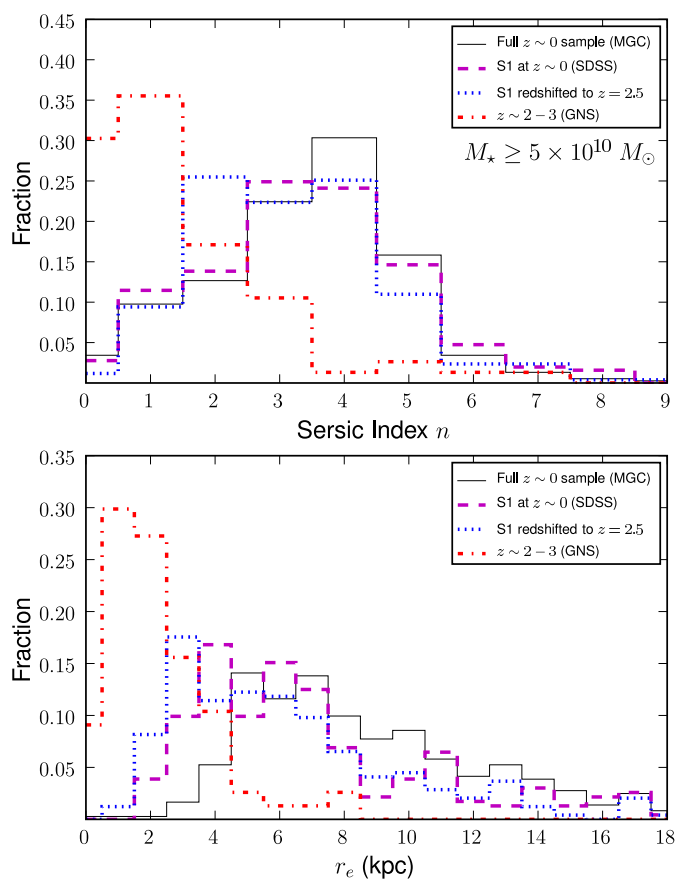

Figure 4.9 This figure illustrates the same information as in Figure 4.8 but in more quantitative terms. It shows the $n$ and $r_{e}$ distributions for the full MGC sample of massive $z \sim 0$ galaxies (black line) and the representative sample $\mathrm{S} 1$ of 255 galaxies used in the redshifting experiment (magenta line). Sample S1 does a good job of matching the full MGC sample and is typically within $\pm 10 \%$ for a given bin. We also contrast the $\left(n, r_{e}\right)$ values after redshfiting S1 to $z=2.5$ (blue line) with the actual distribution observed in the massive the GNS galaxies at $z=2-3$ (red line). While $64.9 \pm 5.4 \%$ and $39.0 \pm 5.6 \%$ of the massive $z=2-3$ galaxies have $n \leq 2$ and $r_{e} \leq 2 \mathrm{kpc}$, respectively, the corresponding fractions for the redshifted sample are $10.6 \pm 1.9 \%$ and $1.2 \pm 0.7 \%$. The results shown here are for galaxies with $M_{\star} \geq 5 \times 10^{10} M_{\odot}$, but a similar result is obtained for $M_{\star} \geq 1 \times 10^{11} M_{\odot}$. 

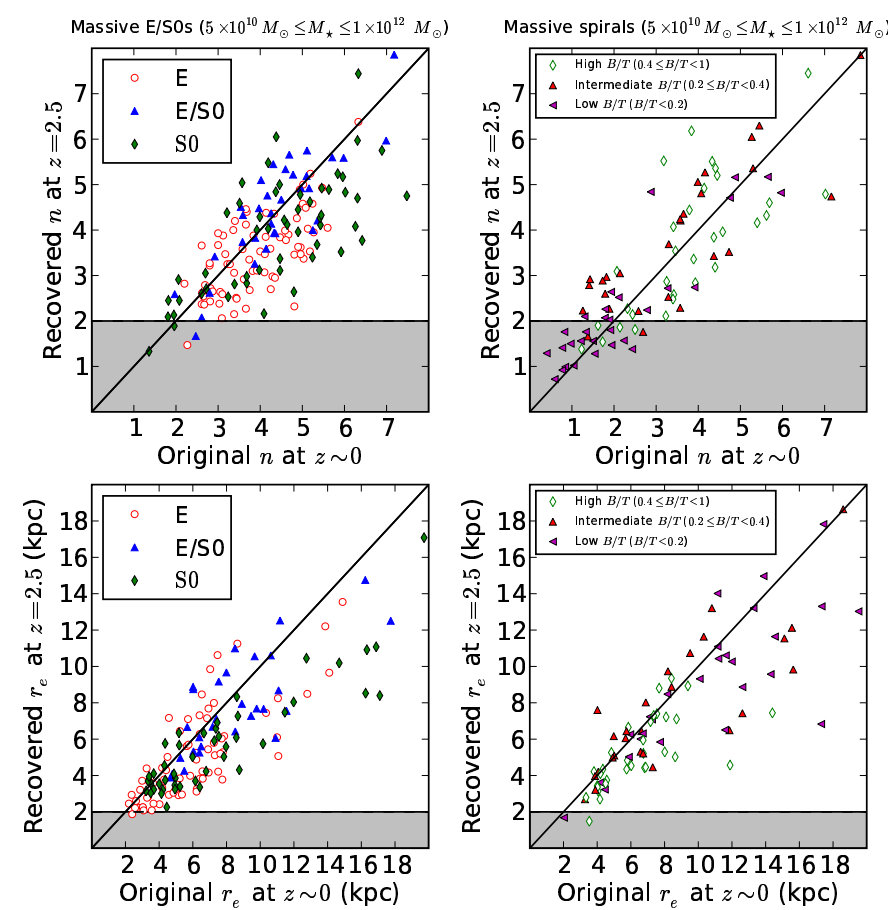

Figure 4.10 Left column: The panels compare the rest-frame optical structural parameters (Sérsic index $n$ and effective radius $\left.r_{e}\right)$ of massive $\left(M_{\star} \geq 5 \times 10^{10} M_{\odot}\right)$ elliptical and S0 galaxies at $z \sim 0$ with the structural parameters recovered after these galaxies were artificially redshifted to $z=2.5$, brightened by 2.5 magnitudes in surface brightness, and re-observed with NIC3/F160W. At $z \sim 0$, the structural parameters were measured from $g$-band images, while at $z=2.5$ they are measured from the artificially redshifted images in the NIC3/F160W band, so that all parameters are measured in the rest-frame blue optical light. The black lines represent equality, while the shaded area represents the regime of $n \leq 2$ and $r_{e} \leq 2 \mathrm{kpc}$, where $64.9 \pm 5.4 \%$ and $39.0 \pm 5.6 \%$, respectively, of massive GNS galaxies at $z=2-3$ lie (Table 4.1 and Figure 4.8). The plots show that the Sérsic index $n$ and effective radius $r_{e}$ of the massive $z \sim 0 E$ and SOs may be lower or higher after redshifting out to $z=2.5$, but they do not, in general, drop to values as low as $n \leq 2$ and $r_{e} \leq 2 \mathrm{kpc}$, and avoid the shaded area. Right column: Same as left column, but this time for massive $\left(M_{\star} \geq 5 \times 10^{10} M_{\odot}\right) z \sim 0$ spiral galaxies. The galaxies are coded by bulge-to-total light ratio $(B / T) . B / T$ was measured with bulge-disk and bulge-disk-bar decomposition of the $z \sim 0 g$-band images. The top plot shows that it is mainly massive $z \sim 0$ late-type spirals of low $B / T$ that yield Sérsic index $n$ as low as $n \leq 2$ after redshifting, and populate the shaded area where $64.9 \pm 5.4 \%$ of massive GNS galaxies at $z=2-3$ lie. However, as shown by this lower plot, the local massive spirals have much larger $r_{e}\left(r_{e} \gg 2 \mathrm{kpc}\right)$ and after artificial redshifting avoid the shaded area where $39.0 \pm 5.6 \%$ of the massive GNS galaxies at $z=2-3$ lie. 

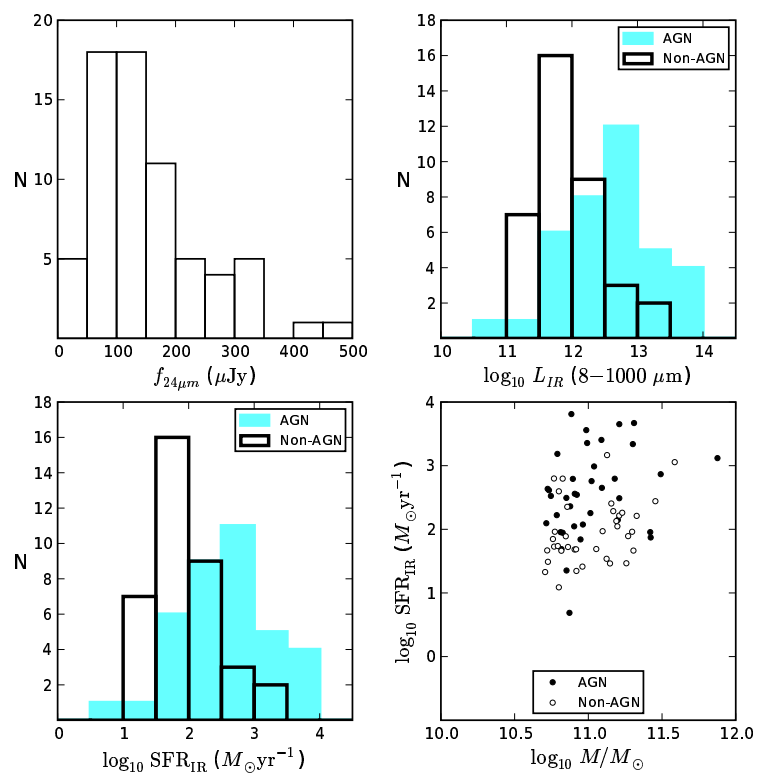

Figure 4.11 Top left: The $f_{24 \mu m}$ distribution for the massive $\left(M_{\star} \geq 5 \times 10^{10} M_{\odot}\right)$ GNS galaxies with reliable MIPS $24 \mu \mathrm{m}$ counterpart. Upper right: The inferred $L_{I R}$ distribution over $8-1000 \mu \mathrm{m}$. Lower left: The inferred $\mathrm{SFR}_{\mathrm{IR}}$ distribution based on $L_{I R}$, which is estimated using the Chary \& Elbaz (2001) templates, with a correction at $\mathrm{L}_{I R}>6 \times 10^{11}$ $L_{\odot}$. Lower right: $\mathrm{SFR}_{\mathrm{IR}}$ versus $M_{\star}$. For sources containing an AGN, the measured $L_{I R}$ and $\mathrm{SFR}_{\mathrm{IR}}$ are upper limits. The upper right and bottom panels use different coding for sources identified in $\S 4.6$ as hosting an AGN. 

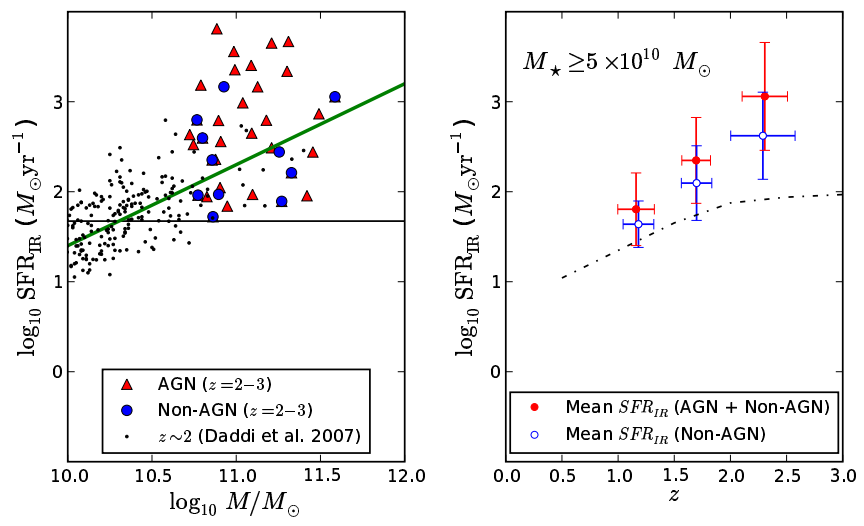

Figure 4.12 The left-hand panel shows $\mathrm{SFR}_{\mathrm{IR}}$ versus $M_{\star}$ at $z=2-3$. The AGN candidates are coded as triangle symbols, and their SFR $\mathrm{IR}$ likely overestimate their true SFR. The completeness limit in $\mathrm{SFR}_{\mathrm{IR}}$ (corresponding to the limiting $24 \mu \mathrm{m}$ flux of $\sim 30 \mu \mathrm{Jy}$ ) is shown as a black solid line. The black dots represent SFR from UV measurements by Daddi et al. (2007) for $z \sim 2$; the diagonal green line is their corresponding SFR-mass correlation at $z \sim 2$. The right-hand panel shows mean $\mathrm{SFR}_{\mathrm{IR}}$ in the different redshift bins for sources with $\mathrm{SFR}_{\mathrm{IR}}$ above the detection limit. The error bars are the $1 \sigma$ standard deviation around the mean. The black line shows average UV-based SFR versus redshift for a galaxy with $5 \times 10^{10} M_{\odot}$, as calculated by Drory \& Alvarez (2008). 

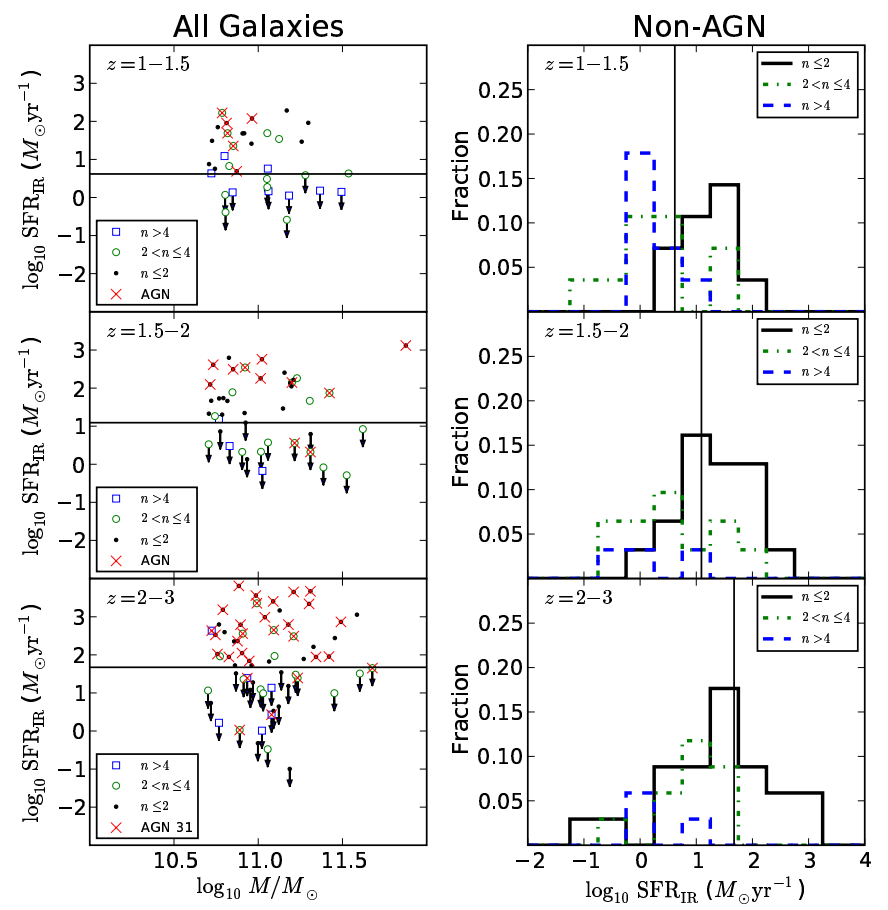

Figure 4.13 Left column: $\mathrm{SFR}_{\mathrm{IR}}$ is plotted versus $M_{\star}$, for all galaxies with $M_{\star} \geq 5 \times 10^{10}$ $M_{\odot}$, in different redshift bins spanning 1-1.4 Gyr in cosmic time. Data are sorted according to the Sérsic index $n$ calculated in $\S 4.3 .1$. AGN candidates (see $\$ 4.6)$ are labeled with red x's. Galaxies with $\mathrm{SFR}_{\mathrm{IR}}$ below the detection limit (shown as a horizontal line) are shown with downward pointing arrows because they are upper limits. At $z=2-3$ the majority $(84.6 \pm 10.0 \%)$ of massive non-AGN galaxies with $S F R_{\mathrm{IR}}$ above the detection limit have $n \leq 2$ (disky) structures. Right column: For non-AGN sources, histograms show the fraction of massive galaxies in each redshift bin with a given $\mathrm{SFR}_{\mathrm{IR}}$ for separate ranges of $n$. The vertical black lines mark the $\mathrm{SFR}_{\mathrm{IR}}$ detection limit. For sources to the left of the line, we plot upper limits for $\mathrm{SFR}_{\mathrm{IR}}$. The high SFR tail in each redshift bin is populated primarily by systems with low $n \leq 2$ (disky) structures. 

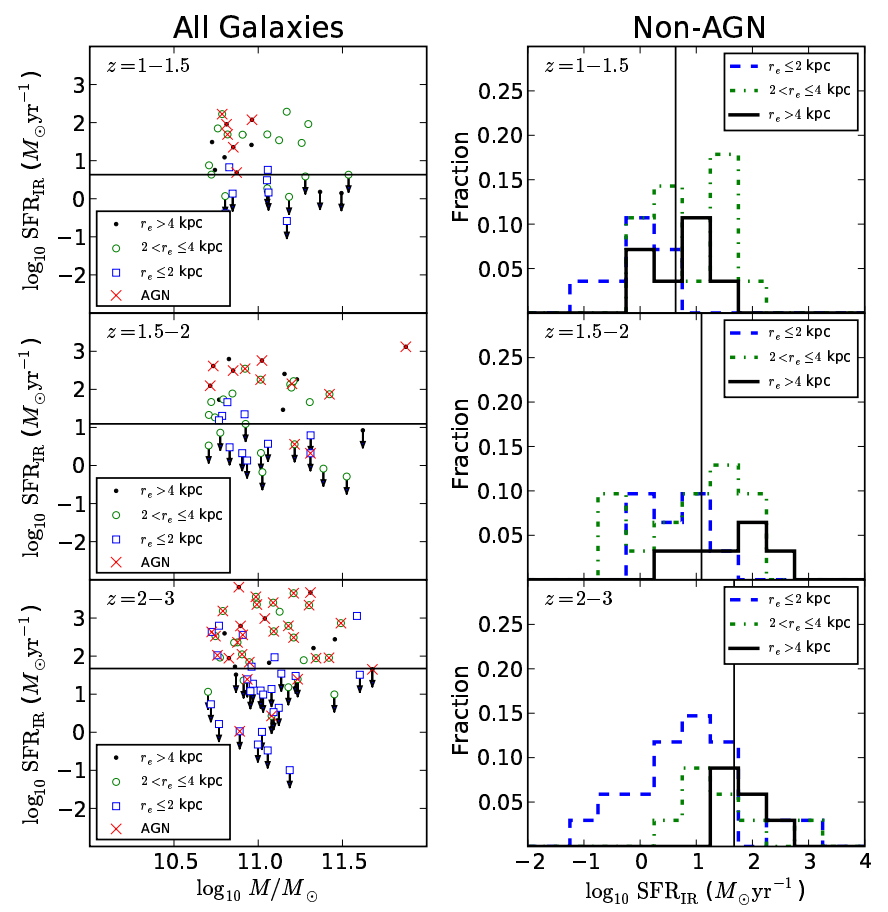

Figure 4.14 Same as Figure 4.13, but now the data are sorted by half-light radius $r_{e}$. Note that only a small fraction of the ultra-compact $\left(r_{e} \leq 2 \mathrm{kpc}\right)$ galaxies have $\mathrm{SFR}_{\mathrm{IR}}$ above the $5 \sigma$ detection limit. Some ultra-compact galaxies have high $\mathrm{SFR}_{\mathrm{IR}}$, but, on average, their mean $\mathrm{SFR}_{\mathrm{IR}}$ are lower than in more extended systems. 

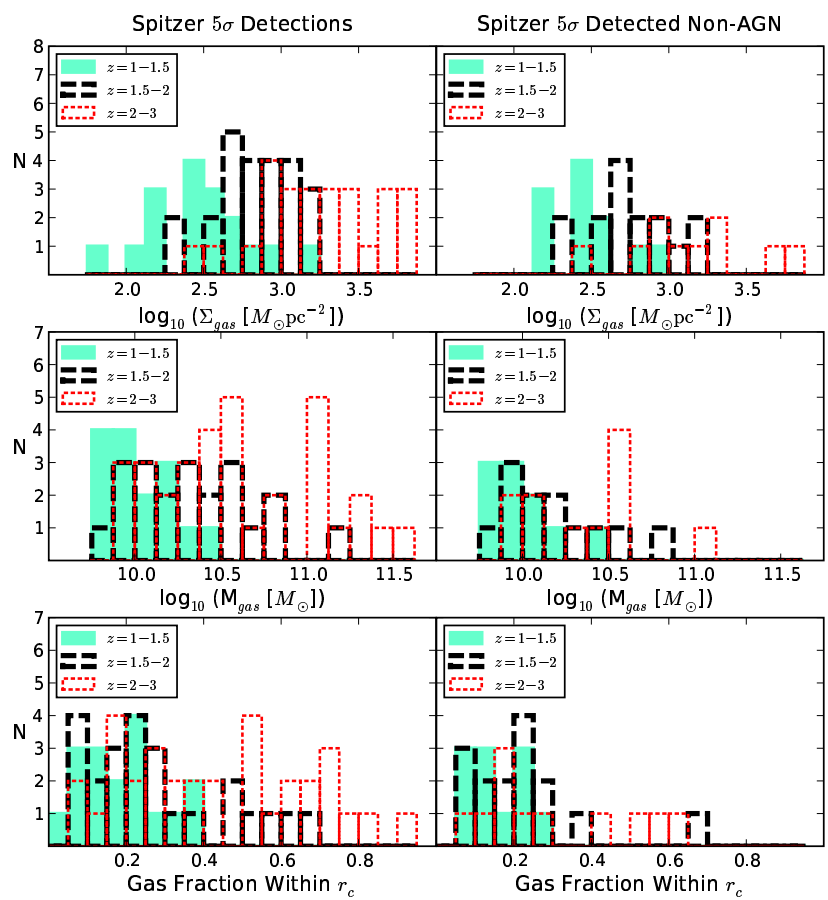

Figure 4.15 Left column: For galaxies with $\mathrm{SFR}_{\mathrm{IR}}$ above the $5 \sigma$ detection limit, the distributions of cold gas surface density $\left(\Sigma_{\text {gas }}\right)$, cold gas mass $M_{\text {gas }}$, and cold gas fraction $\left(f_{\text {gas }}\left(r_{c}\right)\right)$ within the circularized optical half-light radius $r_{c}$ are shown for different redshift ranges. $\Sigma_{\text {gas }}$ is calculated using a Schmidt-Kennicutt law (Kennicutt 1998) with power-law index 1.4 a normalization factor of $2.5 \times 10^{-4}$. The cold gas fraction $\left(f_{\mathrm{gas}}\left(r_{c}\right) \equiv M_{\mathrm{gas}} /\left(M_{\mathrm{gas}}+M_{\star}\right)\right)$ is calculated relative to the total baryonic mass within $r_{c}$. Right column: Same as left column except that only non-AGN sources are shown. 


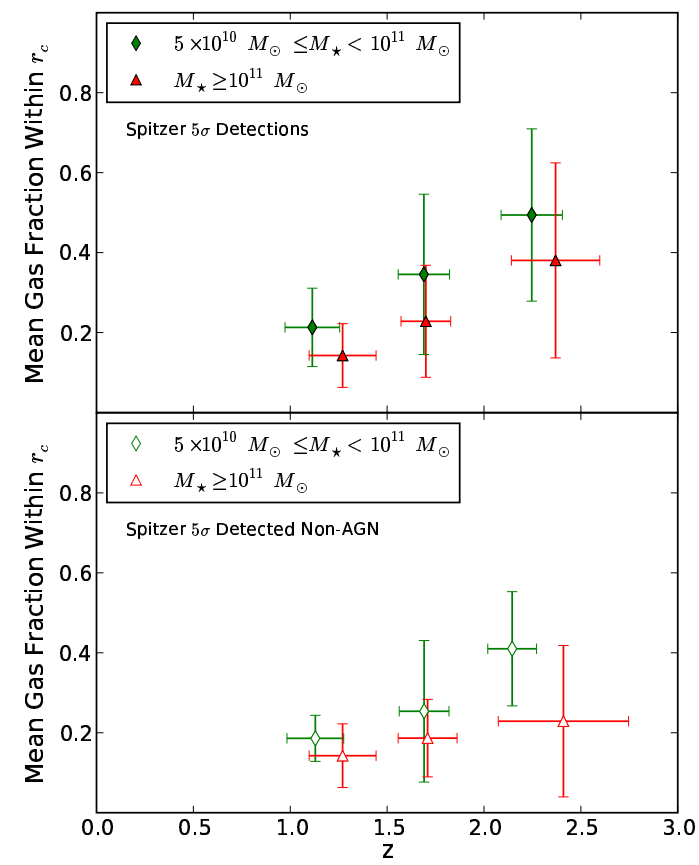

Figure 4.16 Top: For galaxies with $\mathrm{SFR}_{\mathrm{IR}}$ above the $5 \sigma$ detection limit, the mean cold gas fraction $\left(f_{\text {gas }}\left(r_{c}\right) \equiv M_{\text {gas }} /\left(M_{\text {gas }}+M_{\star}\right)\right)$ within the circularized optical half-light radius $r_{c}$ is shown in three redshift bins for all galaxies with $5 \times 10^{10} M_{\odot} \leq M_{\star}<10^{11} M_{\odot}$ and $M_{\star} \geq 10^{11} M_{\odot}$. The error bars indicate the $1 \sigma$ scatter in gas fraction and redshift. Bottom: Same as the top except that only non-AGN sources are shown. 

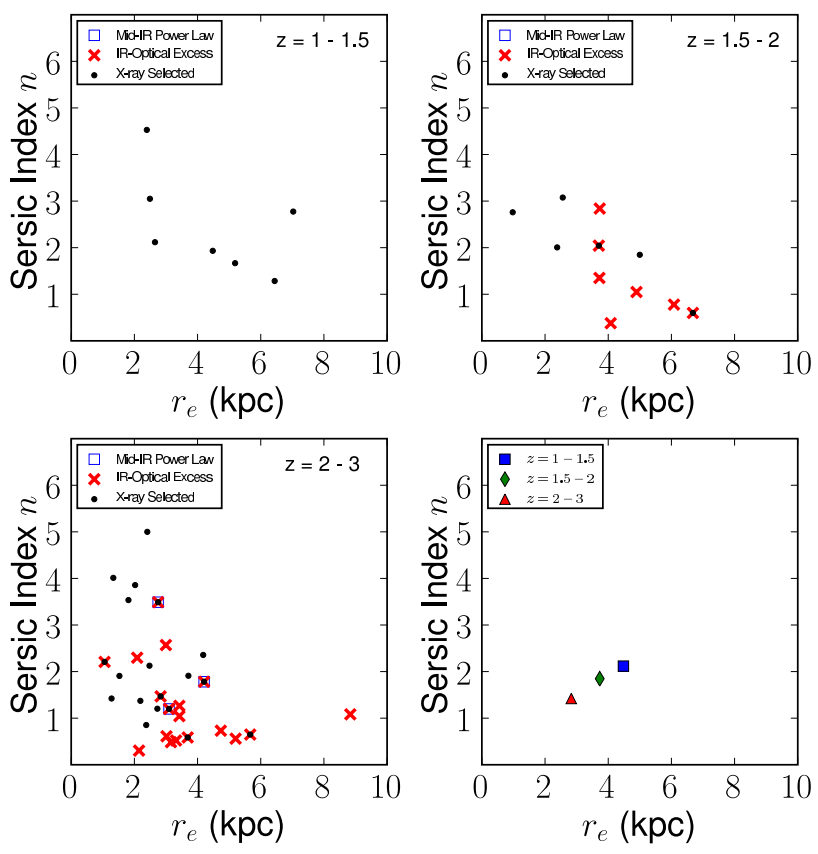

Figure 4.17 The upper and lower-left panels show single Sérsic index $n$ versus effective radius $r_{e}$ for the 49 AGN candidates selected either based on X-ray properties, mid-IR power-law, or IR-to-optical excess. The lower-right panel shows the median Sérsic index and $r_{e}$ in each redshift bin. 

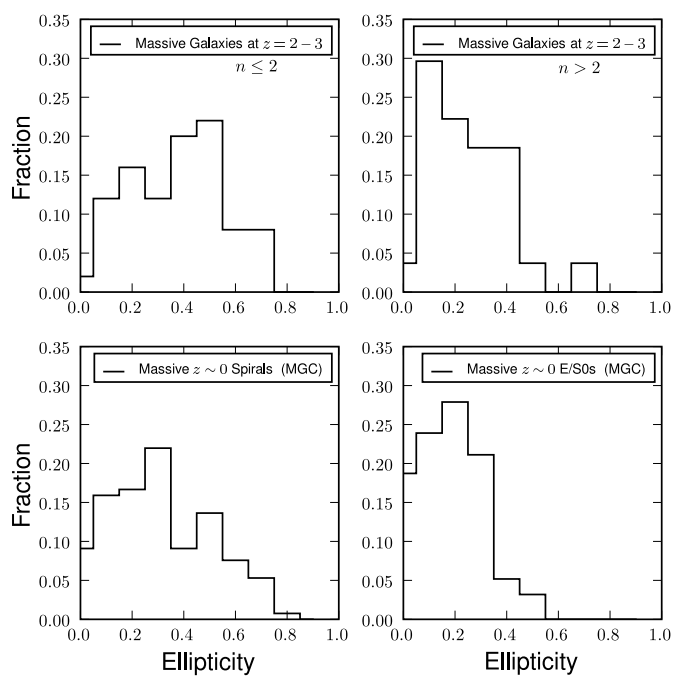

Figure 4.18 In the top panels, the deconvolved ellipticity $(1-b / a)$ measured by GALFIT is shown for massive $\left(M_{\star} \geq 5 \times 10^{10} M_{\odot}\right)$ GNS galaxies at $z=2-3$ with $n \leq 2$ and $n>2$. The bottom panels show the deconvolved ellipticity for similarly massive E/S0 and Spiral galaxies as measured with GIM2D by Allen et al. (2006). 
Table 4.1. Rest-Frame Optical Sérsic Index $n$ and $r_{e}$ in Massive $\left(M_{\star} \geq 5 \times 10^{10} M_{\odot}\right)$ Galaxies

\begin{tabular}{|c|c|c|c|c|}
\hline$z$ & Morphology & $n \leq 2$ & $n>2$ & $n>3$ \\
\hline \multicolumn{5}{|c|}{$M_{\star} \geq 5 \times 10^{10} M_{\odot}$} \\
\hline$z=2-3(N=77)$ & All & $64.9 \pm 5.44 \%$ & $35.1 \pm 5.44 \%$ & $18.2 \pm 4.40 \%$ \\
\hline$z=1-2(N=89)$ & All & $49.4 \pm 5.30 \%$ & $50.6 \pm 5.30 \%$ & $30.3 \pm 4.87 \%$ \\
\hline \multirow{4}{*}{$z \sim 0(N=385)$} & All & $\mathbf{1 3 . 0} \pm 1.71 \%$ & $87.0 \pm 1.71 \%$ & $74.3 \pm 2.23 \%$ \\
\hline & $\mathrm{E} / \mathrm{S} 0$ & $0.8 \pm 0.45 \%$ & $64.9 \pm 2.43 \%$ & $58.7 \pm 2.51 \%$ \\
\hline & Sabc & $10.4 \pm 1.56 \%$ & $20.8 \pm 2.07 \%$ & $14.8 \pm 1.81 \%$ \\
\hline & $\mathrm{Sd} / \mathrm{Irr}$ & $1.82 \pm 0.68 \%$ & $1.30 \pm 0.58 \%$ & $0.78 \pm 0.45 \%$ \\
\hline \multicolumn{5}{|c|}{$M_{\star} \geq 1 \times 10^{11} M_{\odot}$} \\
\hline$z=2-3(N=41)$ & All & $\mathbf{5 8 . 5} \pm 7.69 \%$ & $41.5 \pm 7.69 \%$ & $17.1 \pm 5.88 \%$ \\
\hline$z=1-2(N=41)$ & All & $\mathbf{3 4 . 1} \pm 7.41 \%$ & $65.9 \pm 7.41 \%$ & $43.9 \pm 7.45 \%$ \\
\hline \multirow{4}{*}{$z \sim 0(N=115)$} & All & $10.4 \pm 2.85 \%$ & $89.6 \pm 2.85 \%$ & $80.9 \pm 3.67 \%$ \\
\hline & $\mathrm{E} / \mathrm{SO}$ & $1.7 \pm 1.22 \%$ & $72.2 \pm 4.18 \%$ & $67.0 \pm 4.39 \%$ \\
\hline & Sabc & $6.09 \pm 2.23 \%$ & $13.9 \pm 3.23 \%$ & $12.2 \pm 3.05 \%$ \\
\hline & $\mathrm{Sd} / \mathrm{Irr}$ & $2.61 \pm 1.49 \%$ & $3.48 \pm 1.71 \%$ & $1.74 \pm 1.22 \%$ \\
\hline$z$ & Morphology & $r_{e} \leq 2 \mathrm{kpc}$ & $2<r_{e} \leq 4 \mathrm{kpc}$ & $r_{e}>4 \mathrm{kpc}$ \\
\hline \multicolumn{5}{|c|}{$M_{\star} \geq 5 \times 10^{10} M_{\odot}$} \\
\hline$z=2-3(N=77)$ & All & $39.0 \pm 5.56 \%$ & $42.9 \pm 5.64 \%$ & $18.2 \pm 4.40 \%$ \\
\hline$z=1-2(N=89)$ & All & $24.7 \pm 4.57 \%$ & $48.3 \pm 5.30 \%$ & $27.0 \pm 4.70 \%$ \\
\hline \multirow[t]{4}{*}{$z \sim 0(N=385)$} & All & $0.52 \pm 0.37 \%$ & $1.8 \pm 0.68 \%$ & $97.7 \pm 0.77 \%$ \\
\hline & $\mathrm{E} / \mathrm{S} 0$ & $0.26 \pm 0.26 \%$ & $1.8 \pm 0.68 \%$ & $63.6 \pm 2.45 \%$ \\
\hline & Sabc & $0.00 \pm 0.00 \%$ & $0.0 \pm 0.00 \%$ & $31.2 \pm 2.36 \%$ \\
\hline & $\mathrm{Sd} / \mathrm{Irr}$ & $0.26 \pm 0.26 \%$ & $0.00 \pm 0.00 \%$ & $2.86 \pm 0.85 \%$ \\
\hline \multicolumn{5}{|c|}{$M_{\star} \geq 1 \times 10^{11} M_{\odot}$} \\
\hline$z=2-3(N=41)$ & All & $39.0 \pm 7.62 \%$ & $41.5 \pm 7.69 \%$ & $19.5 \pm 6.19 \%$ \\
\hline$z=1-2(N=41)$ & All & $\mathbf{2 2 . 0} \pm 6.46 \%$ & $56.1 \pm 7.75 \%$ & $22.0 \pm 6.46 \%$ \\
\hline \multirow[t]{4}{*}{$z \sim 0(N=115)$} & All & $\mathbf{0 . 8 7} \pm 0.87 \%$ & $1.74 \pm 1.22 \%$ & $97.39 \pm 1.49 \%$ \\
\hline & $\mathrm{E} / \mathrm{S} 0$ & $0.00 \pm 0.00 \%$ & $1.7 \pm 1.22 \%$ & $72.2 \pm 4.18 \%$ \\
\hline & Sabc & $0.00 \pm 0.00 \%$ & $0.00 \pm 0.00 \%$ & $20.0 \pm 3.73 \%$ \\
\hline & $\mathrm{Sd} / \mathrm{Irr}$ & $0.87 \pm 3.73 \%$ & $0.00 \pm 0.00 \%$ & $5.22 \pm 2.07 \%$ \\
\hline
\end{tabular}

Note. - Rows 1-12: For a given redshift (Column 1), morphology (Column 2), and stellar mass range, Columns 3, 4, and 5 list the fraction of galaxies in three separate bins of Sérsic index $n$. Rows 13-24: Same as the above except that Columns 3, 4, and 5 reflect bins of half-light radius $r_{e}$. 
Table 4.2. Fit of Massive Galaxies to $r_{e} / r_{e, z \sim 0}=\alpha(1+z)^{\beta}$ Over $z=0-3$

\begin{tabular}{ccc}
\hline \hline $\begin{array}{c}\text { Sample } \\
(1)\end{array}$ & $\begin{array}{c}\alpha( \pm 1 \sigma) \\
(2)\end{array}$ & $\beta\left(\begin{array}{c} \pm 1 \sigma) \\
(3)\end{array}\right.$ \\
\hline All $n$ & $1.15(0.30)$ & $-1.30(0.24)$ \\
$n \leq 2$ & $1.11(0.32)$ & $-1.30(0.29)$ \\
$n>2$ & $1.20(0.31)$ & $-1.52(0.26)$ \\
Non-AGN hosts with high SFR $_{\text {IR }}{ }^{2}$ & $1.15(0.33)$ & $-1.22(0.30)$ \\
Non-AGN hosts with low SFR ${ }_{\text {IR }}{ }^{2}$ & $1.67(0.33)$ & $-1.67(0.28)$ \\
\hline
\end{tabular}

Note. - ${ }^{1}$ Non-AGN hosts with $24 \mu \mathrm{m}$ flux above the Spitzer $5 \sigma$ limit $(30 \mu \mathrm{Jy}){ }^{2}$ Non-AGN hosts with $24 \mu \mathrm{m}$ flux below the Spitzer $5 \sigma$ limit $(30 \mu \mathrm{Jy})$. 
Table 4.3. Fraction of Massive $\left(M_{\star} \geq 5 \times 10^{10} M_{\odot}\right)$ Galaxies With $24 \mu \mathrm{m}$ Detections

\begin{tabular}{ccccc}
\hline \hline$z$ & $\begin{array}{c}\mathrm{SFR}_{\min } \\
\left(M_{\odot} \mathrm{yr}^{-1}\right)\end{array}$ & $\begin{array}{c}\text { Fraction with } f_{24 \mu m} \geq 30 \mu \mathrm{Jy} \\
(\%)\end{array}$ & $\begin{array}{c}\text { Mean SFR (AGN + non-AGN) } \\
\left(M_{\odot} \mathrm{yr}^{-1}\right)\end{array}$ & $\begin{array}{c}\text { Mean SFR (Non-AGN) } \\
\left(M_{\odot} \mathrm{yr}^{-1}\right)\end{array}$ \\
$(1)$ & $(2)$ & $(3)$ & & $(4)$ \\
& & & $63.8 \pm 12.9$ & \\
\hline$z=1-1.5$ & 4.29 & $43.6 \pm 7.9 \%$ & $222.8 \pm 58.5$ & $44.0 \pm 7.3$ \\
$z=1.5-2$ & 12.4 & $48.0 \pm 7.1 \%$ & $1145.6 \pm 274.5$ & $418.8 \pm 142.9$ \\
$z=2-3$ & 47.2 & $42.9 \pm 5.6 \%$ & & \\
\hline
\end{tabular}

Note. - Column 2 estimates the detection limit on SFR given the $5 \sigma$ limit on $f_{24 \mu m}$ of $30 \mu \mathrm{Jy}$. The expected SFR $\mathrm{IR}$ at $30 \mu \mathrm{Jy}$ is determined by linear regression of the distribution of $f_{24 \mu m}$ versus $\mathrm{SFR}_{\mathrm{IR}}$ in each redshift bin. Column 3 lists the percentage of massive GNS galaxies with $f_{24 \mu m}>30 \mu \mathrm{Jy}$. Column 4 shows the mean SFR among all galaxies having $f_{24 \mu \mathrm{m}}>30 \mu \mathrm{Jy}$. Column 5 shows the mean SFR among all galaxies without any evidence for AGN activity (see $\S 4.6 .1$ ). The error bars in Column 4 and Column 5 represent the standard error on the mean. 
Table 4.4. Summary of AGN Detection and Properties

\begin{tabular}{cccccccc}
\hline \hline$z$ & Total Number & X-ray AGN & PLG & IR Excess AGN & AGN Fraction & $\begin{array}{c}\text { Median } n \\
\text { Median } r_{e} \\
(\mathrm{kpc}) \\
(8)\end{array}$ \\
& $(2)$ & $(3)$ & $(4)$ & $(5)$ & $(6)$ & $(7)$ & \\
\hline$z=1-1.5$ & 7 & 7 & 0 & 0 & $17.9 \pm 6.1 \%$ & 2.12 & 4.48 \\
$z=1.5-2$ & 11 & 6 & 0 & 5 & $22.0 \pm 5.9 \%$ & 1.85 & 3.73 \\
$z=2-3$ & 31 & 20 & 3 & 11 & $40.3 \pm 8.8 \%$ & 1.42 & 2.83 \\
\hline
\end{tabular}


Table 4.5. Summary of Kolmogorov-Smirnov Test on Ellipticity

\begin{tabular}{|c|c|c|c|}
\hline $\begin{array}{c}\text { Sample } 1 \\
(1)\end{array}$ & $\begin{array}{c}\text { Sample } 2 \\
(2)\end{array}$ & $\begin{array}{c}\text { Probability } \\
(\%) \\
(3)\end{array}$ & $\begin{array}{c}\text { KS Test D } \\
(4)\end{array}$ \\
\hline$n \leq 2 z=2-3$ & MGC E/SO & 0 & 0.489 \\
\hline$n \leq 2 z=2-3$ & MGC Spiral (Sabc + Sd/Irr) & 4.78 & 0.221 \\
\hline$n \leq 2 z=2-3$ & MGC Sd/Irr & 23.5 & 0.317 \\
\hline$n>2 z=2-3$ & MGC E/S0 & 34.3 & 0.184 \\
\hline$n>2 z=2-3$ & MGC Spiral (Sabc + Sd/Irr) & 14.0 & 0.237 \\
\hline$n>2 z=2-3$ & MGC Sd/Irr & 15.8 & 0.370 \\
\hline
\end{tabular}

Note. - Columns 1 and 2 list the two samples for which ellipticity was compared in each KS test. Column 3 lists the probability that Sample 1 and Sample 2 are drawn from the same distribution. Column 4 lists the KolmogorovSmirnov statistic specifying the maximum separation between the cumulative ellipticity distribution functions for Sample 1 and Sample 2. 


\section{Chapter 5}

\section{Summary, Implications, and Future Work}

How galaxies form and evolve is one of the classic problems in astronomy. My thesis contributes to the field of galaxy formation with a quantitative census of the relative importance of the major structural components (spheroidal or triaxial bulges/ellipticals, disks, and bars) in massive galaxies over cosmic time and across different environments in order to explore how galaxies evolve under the action of the various assembly mechanisms (major mergers, minor mergers, gas accretion, and internal secular processes) in these different regimes. This census is based on three inter-related analyses focusing on massive galaxies from $z \sim 0-3$ in both field and rich cluster environments. Important strengths of this thesis include the use of high-resolution, panchromatic imaging from some of the largest and deepest galaxy surveys with the HST, Spitzer, and Chandra space telescopes, and also the inclusion of detailed comparisons between the empirical data and hierarchical $\Lambda$ CDM-based models of galaxy evolution.

Important background information relating structural components of galaxies to physical processses is given in Chapter 1, particularly in Section 1.3. In particular, I broadly divide galaxy components into disk-dominated flattened and dynamically cold structures with Sérsic index $n \lesssim 2$ and non-disk components with Sérsic index $n \gtrsim 2$. I assume diskdominated components with $n \lesssim 2$ form via gas-rich dissipative processes. This gas can come from the galaxy halo, cosmological filaments, and gas-rich mergers. Conversely, non- 
disk components with Sérsic index $n \gtrsim 2$ are considered to include spheroidal or triaxial bulges/ellipticals with $2<n<6$ associated with stellar remnants that form in major mergers with a range of mass ratios $(1: 1$ to $1: 4)$ and gas fractions $\left(f_{\text {gas }} \sim 20-80 \%\right)$. Nondisk components may also be associated with repeated minor mergers (Section 1.3). Thus, in general, I assume that non-disk components with $n \gtrsim 2$ are built by the redistribution of stars during major mergers, and possibly, to a lesser degree, during minor mergers.

In this Chapter, I summarize the main results of this thesis (Section 5.1) as well as discuss the broader implications on galaxy assembly in (Section 5.2). In Section 5.3 I discuss some future observational and theoretical research that would advance the field.

\subsection{Key Results}

\subsubsection{Probing the Structure and Assembly of Nearby Field Spirals}

In Chapter 2, I consider the assembly history of high-mass $\left(M_{\star} \geq 10^{10} M_{\odot}\right)$ spirals in the field at $z \sim 0$. Sophisticated 2D structural decompositions, which take into-account bulges, disks, and bars, reveal that only a minority (less than 26\%) of massive field spirals host non-disk bulges. Instead, most (74\%) massive field spirals host disky/pseudo bulges with low Sérsic index $n \lesssim 2$ indicative of small-scale inner disks. Furthermore, in $66 \%$ of these spirals, the bulges have a low bulge-to-total stellar mass ratio $(B / T \leq 0.2)$. Massive field spirals overall contain $89 \%$ of their stellar mass in disk-dominated components (largescale disks, bars, and inner disky/pseudo bulges) with $n \lesssim 2$, while $11 \%$ is in the form of non-disk $n \gtrsim 2$ components, which are likely built by the redistribution of stars during major mergers and possibly to some extent in minor mergers.

In the years leading up to Weinzirl et al. (2009), on which Chapter 2 is based, 
quasi-bulgeless galaxies with low $B / T$ were regarded as a problem for $\Lambda$ CDM-based models of galaxy formation. There was mounting evidence that bulgeless and low $\mathrm{B} / \mathrm{T}$ galaxies were common locally. Böker et al. (2002) found Sd galaxies often possess no bulge. Based on analysis of several thousand late-type SDSS galaxies, Kautsch et al. (2006) and Barazza, Jogee, \& Marinova (2008) found $15-20 \%$ of edge-on and moderately inclined disks out to $z \sim 0.03$ appeared quasi-bulgeless. Of the 19 galaxies with distance $<8 \mathrm{Mpc}$ and $V c>150$ $\mathrm{km} \mathrm{s}^{-1}, 11$ have disky/pseudo bulges instead of merger-built, spheroidal or triaxial bulges (Kormendy \& Fisher 2008); even the biggest disks displayed no evidence of a merger-built bulge.

The prevalence of such quasi-bulgeless and low- $B / T$ galaxies was considered worrisome given that in the $\Lambda \mathrm{CDM}$ paradigm, galaxies having a past major merger when their mass was a large fraction of their present-day mass are expected to have a spheroidal or triaxial bulge with a significant $B / T$ and a high Sérsic index (Hopkins et al. 2009). But in reality, galaxies that experienced major mergers only in their distant past will not have a bulge with a large $\mathrm{B} / \mathrm{T}$ ratio. Depending on the typical merger history of a spiral galaxy, there could be either a large or small fraction of present-day galaxies with low $B / T$. The empirical results from Chapter 2 (Weinzirl et al. 2009) agree with the large body of earlier work (e.g., Böker et al. 2002; Kautsch et al. 2006; Barazza, Jogee \& Marinova 2007,2008; Kormendy \& Fisher 2008) regarding the abundance of quasi-bulgeless spirals. The high fraction of such proportionately low-mass bulges $(B / T \leq 0.2)$ among spirals requires that the vast majority of these spirals have not had a major merger since early times.

To test this notion, I make a detailed comparison with the $B / T$ ratios predicted by two different theoretical semi-analytic models (Khochfar \& Silk 2006b; Hopkins et al. 
2009 c) in which $\sim 87 \%$ of massive spiral galaxies have not had a major merger over the last

10 Gyr since $z=2$. In the models, a high-mass spiral can have a bulge with a present-day low $B / T \leq 0.2$ if it did not undergo a major merger since $z=2$. The observed large frequency $(\sim 66 \%)$ of high-mass $\left(M_{\star} \times 10^{10} M_{\odot}\right)$ spirals with low present-day $B / T \leq 0.2$ can be accounted for in the hierarchical models where $\sim 84-87 \%$ of high-mass spirals not having undergone a major merger since $z=2$. The good agreement in the cumulative $B / T$ distribution, especially at low $B / T$ ratios, between the observed and predicted distributions of $B / T$ leads to the conclusion that most spirals must have grown by processes other than major mergers, such as gas accretion, minor mergers, and secular processes.

\subsubsection{Exploring the Structure and Assembly of Galaxies At the Heart of the Coma Cluster}

In Chapter 3, I present a study of the structure of massive galaxies in the central $0.6 \mathrm{Mpc}$ of the Coma cluster at $z=0.02$, the richest nearby galaxy cluster, in order to constrain the assembly history of massive $\left(M_{\star} \geq 10^{9} M_{\odot}\right)$ galaxies in this rich environment. This work incorporates high-resolution (0.'1), F814W images from the HST ACS Treasury Survey of the Coma cluster (Carter al. 2008). Structural decompositions are used to assess the frequency of disk-dominated components, built by dissipative processes, versus spheroidal or triaxial bulge/elliptical components, built by violent stellar processes associated with major mergers and, to a lesser degree, minor mergers. The main empirical results are summarized below.

a) Morphology-density relation:

Using structural decompositions to assign galaxies the Hubble types E, S0, or spiral, I find strong evidence of a morphology-density relation. While low-density environments 
(LDEs) are typically dominated by spirals, the morphology breakdown in the center of Coma in terms of $(\mathrm{E}+\mathrm{S} 0)$ :spiral is $(22.4 \%+70.2 \%): 7.4 \%$ by numbers and $(28.0 \%+66.3 \%): 5.8 \%$ by stellar mass. The morphology-density relation in Coma is extreme compared with the field, where the ratio $\mathrm{E}+\mathrm{S} 0$ :spiral is about 20\%:80\% (Dressler 1980).

b) A census of disk-dominated and non-disk components:

Across massive E, S0, and spiral Coma cluster galaxies in the central $0.6 \mathrm{Mpc}$, most $(\sim 56 \%)$ of the stellar mass mass is within high-n ( $\mathrm{z} 1.7)$, puffed-up "hot" spheroidal or triaxial bulges/ellipticals, while the remaining $\sim 44 \%$ of the stellar mass forms low$n$ ( $n \lesssim 1.7)$ disk-dominated flattened "cold" components (e.g. outer disks, inner disks, disky/pseudo bulges, and bars). Thus, most of the stellar mass in Coma galaxies appears to have been assembled and shaped through the redistribution of stars during violent major mergers, and possibly, to a lesser degree, during minor mergers.

c) Impact of environmental processes on large-scale disk properties:

Compared with disk galaxies from LDEs, S0s/spirals in Coma have outer disk sizes $\left(r_{\mathrm{e}}\right) \sim 40-80 \%$ smaller at a given galaxy $M_{\star}$. The difference in size suggests there might also be a difference in outer disk $M_{\star}$ with galaxy $M_{\star}$. However, at a given galaxy $M_{\star}$, outer disk $M_{\star}$ are only $\sim 10-20 \%$ smaller on average. Across bulge $M_{\star}$, outer disks are smaller in size by $\sim 40 \%$ and less massive by $\sim 50-80 \%$.

There is also a high fraction $(67.3 \pm 7.9 \%)$ of large-scale disks with low Sérsic index $n<1$. The abundance of such $n<1$ outer disks is not driven by barred galaxies alone, and this is important because Gaussian-like outer disk structure in unbarred galaxies is unusual (Kormendy \& Bender 2012). 
These properties are likely indicative of environmental processes (e.g., ram-pressure stripping, tidal stripping, strangulation) that suppress disk growth or truncate disks, provided these processes affect outer disks more than bulges.

d) Comparison of Coma to simulated clusters:

Finally, I investigate the assembly history of Coma galaxies with simulated clusters made with dark matter merger trees from the Millennium Simulation (Springel et al. 2005) and prescriptions for baryonic physics from semi-analytic models (Neistein \& Weinmann 2010). Among the 160 clusters with halo mass in the range $5 \times 10^{14}-10^{16} M_{\odot}$, there is no single cluster that simultaneously matches the global properties (number density, mass function, and halo mass/radius) of Coma, one of the best studied local clusters.

Another important problem is that the models overpredict the abundance of cold gas at all galaxy stellar masses, but especially for stellar masses below $10^{11} M_{\odot}$. Gas fractions are as high as $\sim 60-80 \%$, and this is unrealistic for a cluster. The predicted ratios of cold gas to stellar mass exceed, by a factor of $\sim 27-85$, the median gas fraction measured in Coma cluster galaxies by Boselli et al. (1997). A perhaps related issue to the overproduction of cold gas is that the predicted model merger rates depend sensitively on whether baryonic or stellar mass is used in defining mergers.

I make a comparison with the models in terms of the global distribution of stellar mass in high- $n$, puffy and dynamically hot, spheroidal or triaxial bulges/ellipticals versus low- $n$ dynamically cold disk-dominated components. In the models, the fraction $f_{\star \text {,hot }}$ of dynamically hot stellar mass assembled by major and minor mergers ranges from $\sim 23-82 \%$, with a median value of $\sim 61 \%$. This is comparable to the measurement of $56 \%$ in Coma, and the models are qualitatively consistent with the global predominance of dynamically hot 
stellar mass. However, at a given stellar mass, the models underpredict (overpredict) the amount of stellar mass in high- $n$ hot bulge/elliptical components (low- $n$ cold disk-dominated components).

The problems the between data and models are due, at least in part, to physics missing from the models. I speculate the deficiency of hot stellar components in the models is due to not allowing for bulge assembly via the merging of clumps that form in violent disk instabilities. Additionally, the high cold gas masses may lead to too much dynamically cold stellar mass via star formation after the last major merger. Incorporating a number of environmental processes (especially ram-pressure stripping and tidal stripping) would effectively lower the cold gas masses/fractions and lead to higher fractions of dynamically hot stellar mass and improve the disagreement between data and model galaxy stellar mass functions.

\subsubsection{Structure and Assembly of the Most Massive Galaxies Present a Few Gyr $(z \sim 2-3)$ After the Big Bang}

In Chapter 4, I investigate the properties of 166 massive $\left(M_{\star} \geq 5 \times 10^{10} M_{\odot}\right)$ galaxies at $z=1-3$ with $H$-band imaging from the GOODS-NICMOS survey (Conselice et al. 2011). This is among the largest and most diverse samples of massive galaxies with HST-based rest-frame optical imaging over $z=1-3$. The main results are summarized below.

a) The radically different structure of massive galaxies at $z=2-3$ vs $z \sim 0$ :

Most massive galaxies at $z \sim 0$ are morphologically E/S0 galaxies having extended sizes and typically large $B / T$ ratios and large global Sérsic indexes. The rest-frame optical structures of the massive galaxies are strikingly offset in half-light radius and global Sérsic 
index compared with massive galaxies at $z \sim 0$. Approximately $40 \%$ of the massive galaxies at $z=2-3$ are in the form of ultra-compact $\left(r_{\mathrm{e}} \leq 2 \mathrm{kpc}\right)$ galaxies compared with less than $1 \%$ at $z \sim 0$. Also, the majority $(\sim 65 \%)$ of massive galaxies at $z=2-3$ have disky morphologies (Sérsic index $n \lesssim 2$ ), while the corresponding fraction among massive systems at $z \sim 0$ is five times lower. Detailed checks for systematic effects, including artificial redshifting tests, show that the perceived size evolution and prominent disky structure is real. Therefore, massive galaxies at $z \sim 2.5$ must grow in size (by a factor of $\sim 5$ ) and experience a change in structure (Sérsic index $n \lesssim 2$ to $n \gtrsim 2$ ) by $z \sim 0$.

b) The AGN and SFR activity of massive galaxies at $z=2-3$ :

I employ a variety of techniques (X-ray properties, IR power-law, and IR-to-optical excess) to identify AGN. There is a relationship between host galaxy structure and AGN activity suggesting AGN hosts are extended, disky structures. At $z=2-3, \sim 80 \%$ of AGN are in galaxies that are not ultra-compact $\left(r_{\mathrm{e}}>2 \mathrm{kpc}\right)$, and $\sim 65 \%$ of AGN hosts have disky $(n \lesssim 2)$ morphologies. These findings are in agreement with previous work (e.g., Schawinski et al. 2011) based on smaller samples.

Estimating the star formation rate $\left(\mathrm{SFR}_{\mathrm{IR}}\right)$, using IR luminosities $(8-1000 \mu \mathrm{m})$ derived from the Spitzer $24 \mu \mathrm{m}$ flux via SED templates, reveals that at $z=2-3$ the inferred $\mathrm{SFR}_{\mathrm{IR}}$ range from $\sim 50-1460 M_{\odot} \mathrm{yr}^{-1}$ and are extreme compared with local galaxies. There is a strong link between galaxy structure and $\mathrm{SFR}_{\mathrm{IR}}$. Among the non-AGN massive galaxies at $z=2-3$ having $\mathrm{SFR} \geq 47 M_{\odot} \mathrm{yr}^{-1}$ (corresponding to the $5 \sigma 24 \mu \mathrm{m}$ flux limit of $30 \mu \mathrm{Jy}$ ), the majority ( $\sim 85 \%$ ) have disky morphologies (Sérsic $n \lesssim 2$ ). Moreover, the high $\mathrm{SFR}_{\mathrm{IR}}$ tail in each redshift bin is populated primarily by systems with disky, $n \lesssim 2$ structures. This is consistent with such galaxies having extended outer star-forming disks. 
In contrast, the massive ultra-compact objects at $z=2-3$ are less likely by a factor of $\sim 2$ to have significant $\mathrm{SFR} \geq 47 M_{\odot} \mathrm{yr}^{-1}$.

c) Initial formation of massive galaxies at $z=2-3$ :

The rest-frame optical surface brightness and associated stellar mass densities $(\sim$

$10^{10} M_{\odot} \mathrm{pc}^{-2}$, Figure 5.1) in massive galaxies at $z=2-3$ imply they formed at $z>$ 2 in rapid and highly dissipative gas-rich events, such as gas-rich mergers or cold-mode accretion. By consulting the output of theoretical simulations, it is possible to learn the relative importance of these mechanisms. In Chapter 4, I discuss how simulations of major mergers generally produce remnants with global Sérsic index $n \gtrsim 2$, even in the case of gas-rich $\left(f_{\text {gas }}>40 \%\right)$ mergers. Thus, it is challenging for mergers to initially produce a population of massive galaxies where $65 \%$ have low Sérsic index $(n \lesssim 2)$. Cold-mode accretion at $z>2$ was likely the more important mechanism in forming these massive galaxies. Finally, it is important to note that even simulations accounting for cold-mode accretion are under-predicting the fraction of moderately extended $\left(r_{\mathrm{e}} \sim 2-10 \mathrm{kpc}\right)$, disky $(n \lesssim 2)$ galaxies at $z \sim 2$. This is discussed further in Section 5.3.

\subsection{What Has Been Learned About Galaxy Formation/Evolution?}

Many questions about how galaxies grow remain unanswered. However, this thesis contributes many insights to the problem of galaxy formation/evolution, and below I list and discuss these insights.

1. Disk-dominated structures are more common than previously thought:

The results from Chapters 2 and 4 of this thesis imply that disk-dominated structures 
are much more abundant in low-density field environments than previously thought. In Chapter 2 , it is found that nearly $3 / 4$ of bulges in $z \sim 0$ field spirals are actually disky/pseudo bulges indicative of small-scale disks. Similarly, in Chapter 4 it is determined that the majority (65\%) of massive galaxies at $z=2-3$ have globally disk-dominated structures and, most likely, extended outer disks. Such disky structures are unlikely to have formed in major mergers at $z>3$ even given the high galaxy gas fractions at early epochs.

The abundance of disk-dominated structure in field environments out to 3 Gyr after the Big Bang says something about the relative importance of galaxy assembly mechanisms in these regimes. Namely, major mergers, which build spheroidal or triaxial bulges/ellpticals via violent relaxation, appear to not be the dominant galaxy-wide mass assembly mechanism for field galaxies at $z<2$. Rather, disk-building mechanisms such as gas accretion and secular processes are implied to dominate galaxy mass assembly and evolution.

This conclusion is consistent with the emerging theme in the literature that the overall importance of major mergers has been historically overstated. For example, major mergers can induce starbursts given the presence of cold gas, thereby accounting for some of the assembled stellar mass. However, recent observational (e.g., Bell et al. 2005; Wolf et al. 2005; Jogee et al. 2009; Robaina et al. 2009; Kaviraj et al. 2013) and theoretical (e.g. Hopkins et al. 2006, 2010a) studies that add up the cumulative contribution of this mode of star formation find that major mergers have not contributed significantly to the cosmic star formation rate density over the last 8 Gyr $(z<1)$.

Additional constraints on major mergers are arrived at by direct attempts to measure 
the major merger rate across galaxy mass and redshift. High-resolution galaxy simulations (e.g., Hopkins et al. 2010a) show that while the mass assembly of spheroidal or triaxial bulges/ellipticals is dominated by major mergers (as opposed to minor mergers), the major merger rate is modest overall. The theoretical merger rates from Hopkins et al. (2010a) show that a typical galaxy with $M_{\star}=10^{10} M_{\odot}$ experiences $\sim 0.2$ major $\left(M_{1} / M_{2} \geq 0.25\right)$ mergers at $z<2$. A typical galaxy of stellar mass $M_{\star}=10^{11} M_{\odot}$ experiences $\sim 1$ major merger over the same time interval.

Observational measures of major merger fraction (e.g., Conselice et al. 2003; Bell et al. 2006; Lotz et al. 2008; Conselice 2009; Hammer et al. 2009; Jogee et al. 2009; Robaina et al. 2010; Kaviraj et al. 2011; Williams et al. 2011) yield highly uncertain major merger rates given the uncertainty in the merger timescale (e.g., 0.3-1.1 Gyr, Conselice 2009). However, it has been shown (Hopkins et al. 2010a) that theoretical and observational merger rates converge to within a factor of $\sim 2$ when the theoretical estimates are extracted by mimicking observational methods.

\section{Minor mergers are important drivers of structural evolution:}

It was concluded in Chapter 4 that most massive $\left(M_{\star} \geq 5 \times 10^{10} M_{\odot}\right)$ galaxies at $z=2-3$ are radically different in size and structure compared with similar-mass galaxies at $z \sim 0$. Whereas most massive galaxies at $z \sim 0$ are early-type E/S0 galaxies (Liske et al. 2003; Driver et al. 2005, Figure 5.1), most (65\%) of the massive galaxies at $z=2-3$ resemble more compact versions of spiral galaxies. This implies a significant structural change is necessary, namely, an increase in size by an average factor of $\sim 5$ and an increase in global Sérsic index from $n \lesssim 2$ to $n \gtrsim 2$.

It was argued in Chapter 4 that dry minor mergers are at least as important as dry 
major mergers in driving this transformation. Compared to major mergers, minor mergers have the advantages of being more frequent and also producing more efficient size growth. The first observational constraints on minor mergers at $z<1$ suggest they are at least three times more frequent than major mergers (Jogee et al. 2009; Lotz et al. 2011). Cosmological simulations by Oser et al. (2012) show that the number weighted merger ratio is 1:16 and that the average mass-weighted merger is 1:5. In terms of growth, major mergers of similarly massive progenitors will induce similar mass and size increases (i.e., a factor of $\sim 2$ ). Simple analytic considerations (Naab et al. 2009; Bezanson et al. 2009) suggest a series of minor mergers should, in comparison, produce proportionately more size growth for the amount of deposited mass (e.g., a factor of $\sim 4$ growth in size for a mass doubling).

For these reasons, I speculate in Chapter 4 that minor mergers should be at least as important as major mergers in driving the future size and structure evolution of the $z \sim 2$ massive galaxies. This scenario is compelling given that the massive galaxies at $z=2-3$ already have high central stellar mass densities comparable with present-day massive galaxies (Figure 5.2).

While minor mergers are definitely promising mechanisms for inducing size growth, several new observational and theoretical works (e.g., Hopkins et al. 2010b; Newman et al. 2012; Nipoti et al. 2012) are concluding that dry major and minor mergers alone cannot account for the required size evolution in massive galaxies from $z \sim 2$ to $z \sim 0$. Hopkins et al. (2010b) present a case study, based on high-resolution cosmological hydrodynamical simulations, that allows for size evolution via multiple channels, such as major/minor mergers, adiabatic expansion following gas loss, and evolving mass- 
to-light ratio gradients. The main result is that minor mergers dominate the size growth (accounting for a factor of $\sim 3-4$ growth) while the combination of other the mechanisms contribute at a less important but still significant level (size growth by an additional factor of $\sim 2-3$ ).

\section{Galaxy evolution in rich clusters:}

Chapter 3 examines the role of environment in the center of the Coma cluster, which is the richest nearby environment (number density $n_{g} \sim 10,000$ galaxies $\mathrm{Mpc}^{-3}$ ). Galaxy evolution is expected to proceed differently in a rich cluster due to a combination of initial conditions and cluster-specific processes (ram-pressure stripping, galaxy harassment, and strangulation). The combination of these effects must be effective in transforming late-type spiral galaxies into early-type E+S0 galaxies given that clusters at earlier epochs show proportionately more spiral galaxies (e.g., Dressler et al. 1994). Indeed, among high-mass $\left(M_{\star} \geq 10^{9} M_{\odot}\right)$ galaxies in the central 0.6 Mpc of Coma the ratio of $(\mathrm{E}+\mathrm{S} 0)$ :spiral galaxies is $(22.4 \%+70.2 \%): 7.4 \%$ by number, indicating a near-complete transformation of centrally located spirals.

Structural analysis of Coma galaxies shows that galaxy mergers were at one point much more important than they are today in the cluster, where the galaxy velocity dispersion is $\sigma_{v} \sim 1,000 \mathrm{~km} \mathrm{~s}^{-1}$. The census of galaxy structure in Coma reveals nearly $3 / 4$ of the stellar mass is stored in puffy and dynamically hot structures. This implies dynamical heating and the redistribution of stars due to major mergers and, to a lesser degree, minor mergers, were at one point very important in shaping the stellar mass distribution.

The high velocity dispersion suggests that environmental processes like ram-pressure 
stripping (which is proportional to galaxy velocity) and high-speed encounters (galaxy harassment) should have an important effect. The estimated number of interactions in Coma over a given time interval $\Delta t$ is

$$
N \approx 4\left(\frac{n_{g}}{250 M p c^{-3}}\right)\left(\frac{b}{20 k p c}\right)^{2}\left(\frac{\sigma_{v}}{1,000 k m s^{-1}}\right)\left(\frac{\Delta t}{10 G y r}\right)
$$

where $b$ is the impact parameter. Assuming $b=20 \mathrm{kpc}$ and $n_{g} \geq 200$ galaxies $\mathrm{Mpc}^{-3}$ within the central 0.6 Mpc of Coma (Chapter 3, Figure 18), then over the last $\Delta t=10$ $\operatorname{Gyr}(z<2)$ a typical galaxy in the central 0.6 Mpc of Coma should experience at least $\sim 30$ high-speed encounters. Therefore, high-speed encounters (galaxy harassment) should be important, and in Chapter 3 I speculate that tidal stripping is one of the processes possibly connected with truncated large-scale disks.

\section{Black-hole-bulge correlation:}

In Chapter 4, the relationship between AGN activity and galaxy structure is explored for massive $\left(M_{\star} \geq 5 \times 10^{10} M_{\odot}\right)$ galaxies at $z=2-3$. Most $(65 \%)$ AGN hosts are moderately extended galaxies $\left(r_{\mathrm{e}} \sim 2-10 \mathrm{kpc}\right)$ with globally disky structures. It is fascinating that most AGN host galaxies at $z=2-3$ are not spheroid-dominated systems given the well-known black-hole-bulge correlation at $z \sim 0$. In the local universe, nearly all massive galaxies are believed to host a central supermassive black hole (Kormendy 1993b; Magorrian et al. 1998; Ferrarese \& Merritt 2000; Gebhardt et al. 2000; Marconi \& Hunt 2003), and furthermore the black hole mass is tightly related to the bulge stellar velocity dispersion (Ferrarese \& Merritt 2000; Gebhardt et al. 2000). This has led to the suggestion that the black hole and bulge or spheroid probably grew in tandem (e.g., Cattaneo \& Bernardi 2003; Hopkins et al. 2006). 
Chapter 4 was written at time (years 2009-2011) at a time when the connection between black holes and disk-dominated structures (outer disks and inner disky/pseudo bulges), was being actively re-investigated. The result in Chapter 4 that a significant fraction of AGN hosts at $z=2-3$ are disk-dominated systems fits in well with the emerging conclusions based on $z \sim 0$ galaxies ( $\mathrm{Hu} \mathrm{2008;} \mathrm{Greene} \mathrm{et} \mathrm{al.} \mathrm{2008,} \mathrm{2010;}$ Nowak et al. 2010; Kormendy, Bender, \& Cornell 2011) that a) the formation of a supermassive black hole does not require a spheroidal or triaxial bulge and b) supermassive black holes in galaxies with disky/pseudo bulges are less massive than implied by the original black-hole-bulge correlation.

Kormendy, Bender, \& Cornell (2011) and Kormendy \& Ho (2013) put these observations in perspective. They emphasize there exist separate black-hole-bulge correlations, one for spheroidal or triaxial bulges/ellipticals and one for disky/pseudo bulges. These separate correlations arise through distinct black hole formation pathways. Black holes in spheroidal or triaxial bulges/ellipticals grow rapidly via global processes when mergers drive gas infall that feed quasar-like events. It is for this reason that the biggest black holes coevolve with their bulges. In contrast, black holes in bulgeless galaxies and in galaxies having a disky/pseudo bulge only become low-level Seyfert-like AGN because black hole growth is driven locally and stochastically (see Jogee 2006 for a review). Black holes in this scenario do not coevolve with large-scale disks and disky/pseudo bulges.

Thus, supermassive black holes can exist outside of merger-built bulges, and such black holes will be more Seyfert-like than quasar-like. The identification of black holes in disk-dominated galaxies at $z=2-3$ as described in Chapter 4 and also by Schawinski 
et al. (2011) is consistent with this picture; the black holes found in these galaxies have modest X-ray luminosities that match the X-ray luminosity of Seyfert AGN.

\subsection{Outlook and Future Work}

Although there is much that remains to be understood about galaxy evolution, the outlook for this field is bright. In the next decade, new facilities like the James Webb Space Telescope and the Giant Magellan Telescope will revolutionize the study of galaxy evolution. However, there is still much that can be done with the currently available technology. Below I outline some observational and theoretical work that would be valuable followup projects to this thesis.

\subsubsection{Dynamics and Gas Content of Massive Galaxies at $z=2-3$}

As discussed in Chapter 4, the large fraction (65\%) of massive galaxies at $z=$ $2-3$ with disky morphologies qualitatively supports the idea that cold gas accretion from cosmological filaments is important in building the extended disks at $z>2$. However, even cosmological simulations (e.g., Oser et al. 2012), which include a phase of rapid in-situ SF fueled by cold-mode accretion at $2<z<6$, fail dramatically to simultaneously produce the dominant observed population of extended, disky, and star forming galaxies in conjunction with the compact and quiescent galaxies.

Observations of the kinematics and dynamics of representative disky, non-disky, extended, and ultra-compact galaxies from Chapter 4 is the most direct way to assess the nature and dynamics of massive galaxies at $z=2-3$. This is doable with existing groundbased facilities such as ALMA. Mapping the distribution and kinematics of the molecular gas with high-resolution millimeter observations was not possible before the advent of ALMA 
because the available $\sim 1-2^{\prime \prime}$ resolution only permitted a global galaxy analysis. With ALMA, 0 "' 3 -resolution observations (corresponding to $\sim 2.5 \mathrm{kpc}$ at $z \sim 2$ ) of the higher order CO lines and radio continuum are possible for massive galaxies studied in Chapter 4. Such observations are sufficient to test the assumption that low $(n \lesssim 2)$ global Sérsic index at $z=2-3$ corresponds to disk-dominated structure. ALMA data would provide a measure of the resolved gas velocity field, as well the morphology of the gas, allowing a direct assessment of the dynamical state of massive galaxies at $z=2-3$. For non-interacting galaxies the gas velocity would show ordered rotation and the gas morphology would be comparatively more uniform and extended. In comparison, chaotic velocity maps coupled with centrally concentrated or chaotic distributions would serve as evidence for recent or ongoing gas-rich mergers.

\subsubsection{Chemical Evolution and Stellar Populations of Nearby Galaxies}

A complete picture of bulge and galaxy assembly requires investigation of star formation history, stellar populations, chemical abundances, and kinematics, which can provide additional ways to discriminate between galaxy assembly modes to complement analyses of structural properties (e.g., Chapters 2 and 3).

Integral field unit (IFU) data from the VIRUS-P Exploration of Nearby Galaxies (VENGA) survey (Blanc et al. 2013) of 32 nearby spirals provides some of the best data to date for constraining such parameters. VENGA is the largest and most efficient IFU survey of nearby spirals; the large $110^{\prime \prime} \times 110^{\prime \prime}$ field of view and sensitive $4^{\prime \prime}$ fibers allow extraction of $2 \mathrm{D}$ spectra from the inner bright parts to the faint outer disks of spirals. Several of the nearby spiral galaxies studied in Chapter 2 are also covered in VENGA. 
A logical extension of Chapter 2 is to derive, from the spatially resolved spectra of these galaxies, the stellar population age, metallicity, and star formation. The results will have a variety of implications, including insights on merger history and the effectiveness of secular evolution in driving gas inflow and building disky pseudobulges over several Gyr, that can be directly compared with emerging picture of galaxy assembly in this thesis.

\subsubsection{Future Progress for Hierarchical Models}

This thesis has made extensive use of hierarchical models of galaxy formation. The comparisons with theoretical models in Chapters 2 and 3 were based on theoretical measures of $B / T$ because semi-analytic models cannot make robust predictions about galaxy structure or Sérsic index. The best way to compare observational and theoretical measures of galaxy structure would be to perform structural decompositions on simulated telescope images of galaxies from cosmological simulations. It is currently possible to create such images with existing radiative transfer codes (e.g., Jonsson 2006).

Due to missing or incompletely implemented physics, theoretical models are not in general agreeing well with observations of both field galaxies at high redshift and galaxies in rich clusters at $z \sim 0$. Specifically, the simulations are failing to reproduce both the moderately extended size $\left(r_{\mathrm{e}} \sim 2-10 \mathrm{kpc}\right)$ and globally disk-dominated $(n \lesssim 2)$ structure of massive field galaxies at $z=2-3$. Oser et al. (2012) produce massive galaxies that are all too compact $\left(r_{\mathrm{e}} \leq 2 \mathrm{kpc}\right)$ when in reality only $40 \%$ of massive galaxies are like this (Chapter 4). The reason for this discrepancy may be related to the non-implementation of ejective supernovae wind feedback. Similarly, for reasons that are not clear, Ceverino \& Dekel (in prep.), using the model from Ceverino, Dekel, \& Bournaud (2010), drastically 
under produce the fraction of disk-dominated $(n \lesssim 2)$ galaxies at these redshifts. Figures 5.3 and 5.4 further illustrates the discrepancy.

As discussed in Chapter 3, models based on dark matter halo merger histories from the Millennium Simulation (Springel et al. 2005) and baryonic physics from semianalytical models (Neistein \& Weinmann 2010) are producing galaxy clusters that do no simultaneously the match global properties (cumulative number density, mass function, halo properties) of the Coma cluster. This is disheartening considering that Coma is one of best-studied galaxy clusters. A likely starting point for solving the discrepancy is a more complete accounting for of cluster-specific environmental processes (ram-pressure stripping, galaxy harassment, and strangulation) in the semi-analytical models, which currently only model galaxy strangulation. 


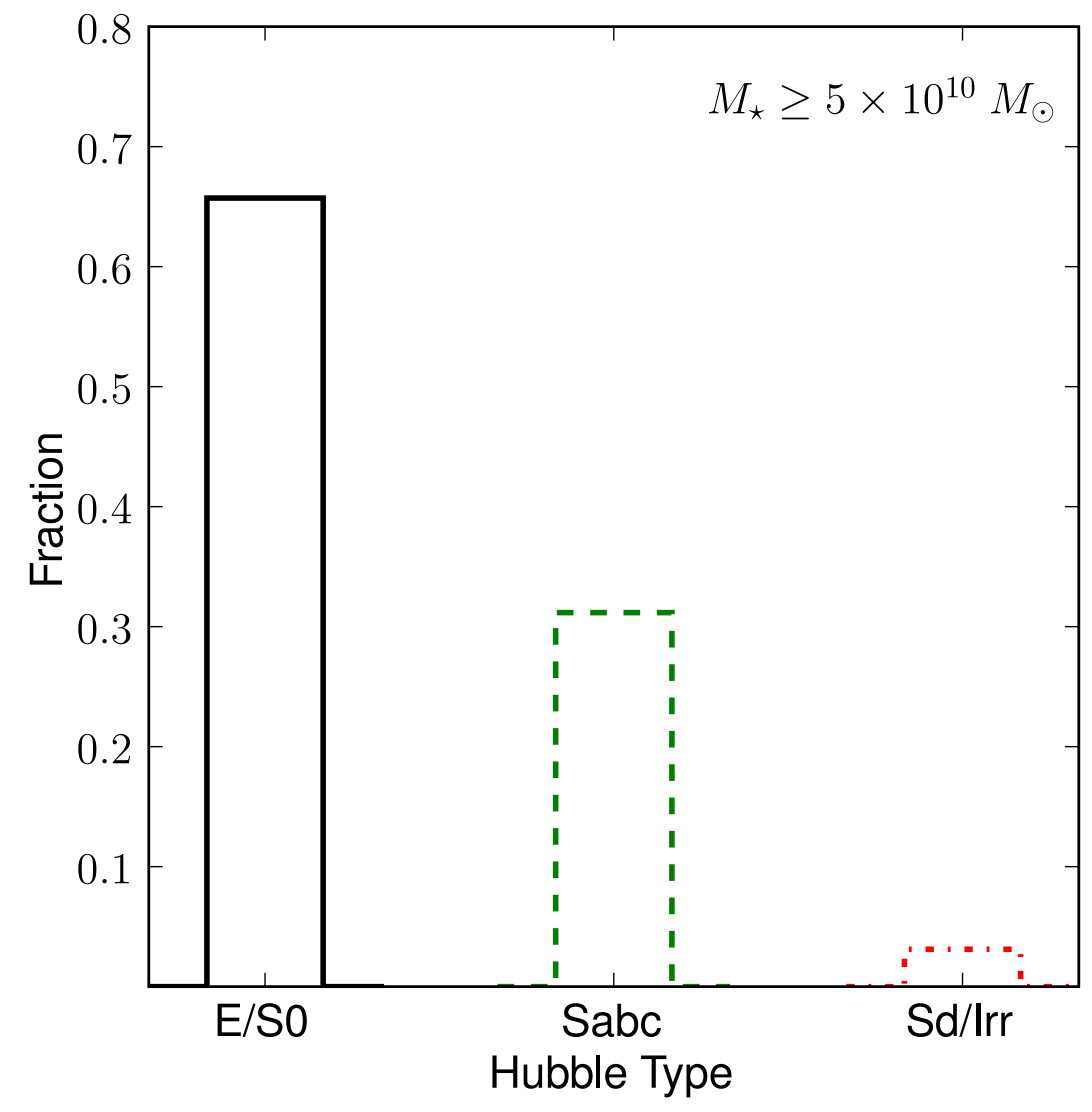

Figure 5.1 This figure shows the morphology breakdown of massive $\left(M_{\star} \geq 5 \times 10^{10} M_{\odot}\right)$ galaxies at $z \sim 0$ from the Millennium Galaxy Catalog (Liske et al. 2003; Driver et al. 2005). 


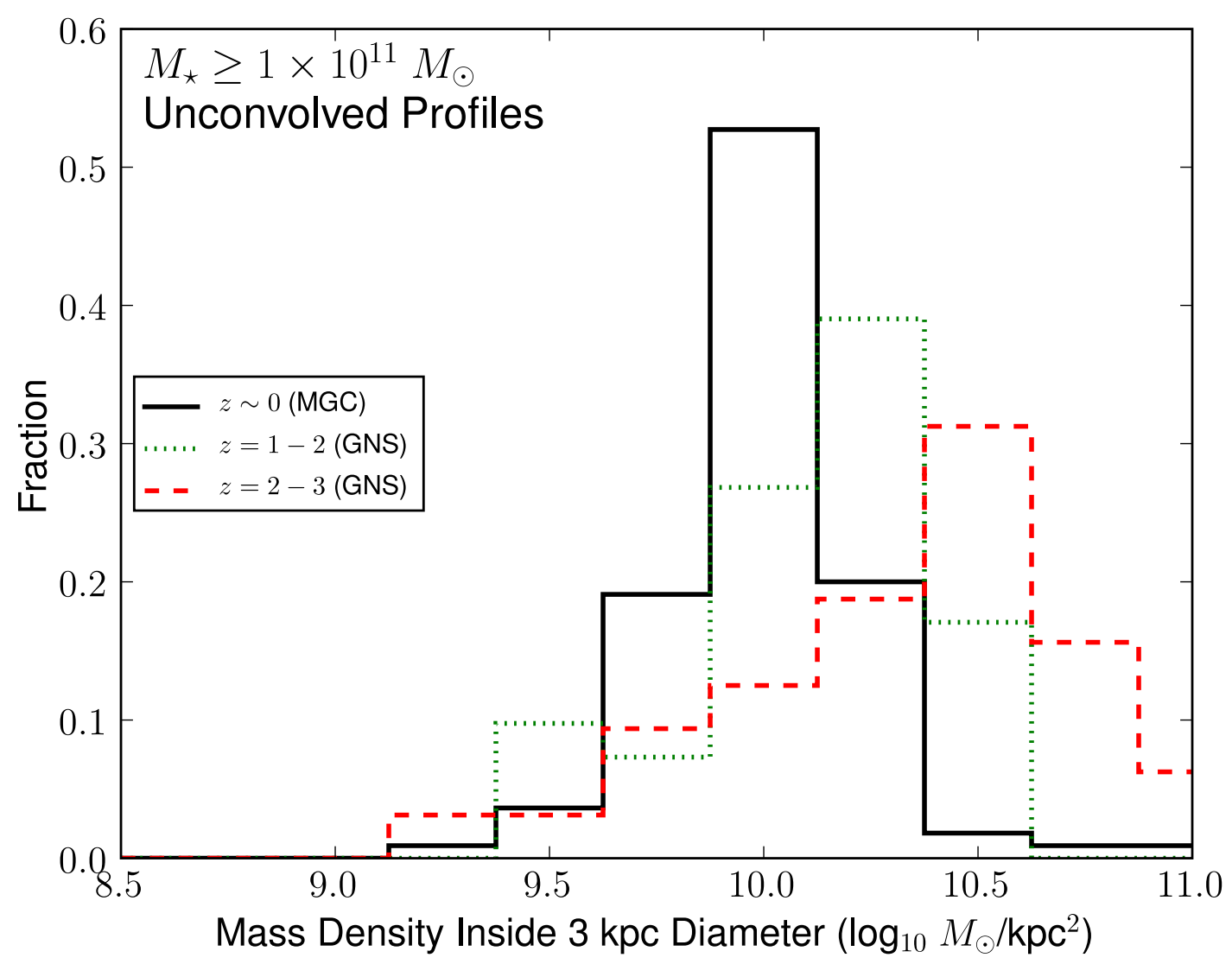

Figure 5.2 This figure shows how the surface mass densities within the central $3 \mathrm{kpc}$ of massive galaxies at $z=1-3$ (Chapter 4) compare with similarly massive galaxies at $z \sim 0$. The surface mass densities at $z=1-3$ are calculated assuming a conservative mass-to-light ratio $(M / L)$ of 1 . For the $z \sim 0$ galaxies, the $B$-band $M / L$ ratio from Driver et al. (2006) is applied. The median $B$-band $M / L$ ratio is $\sim 5$. Surface densities are determined from PSF-deconvolved model images of the galaxies. 


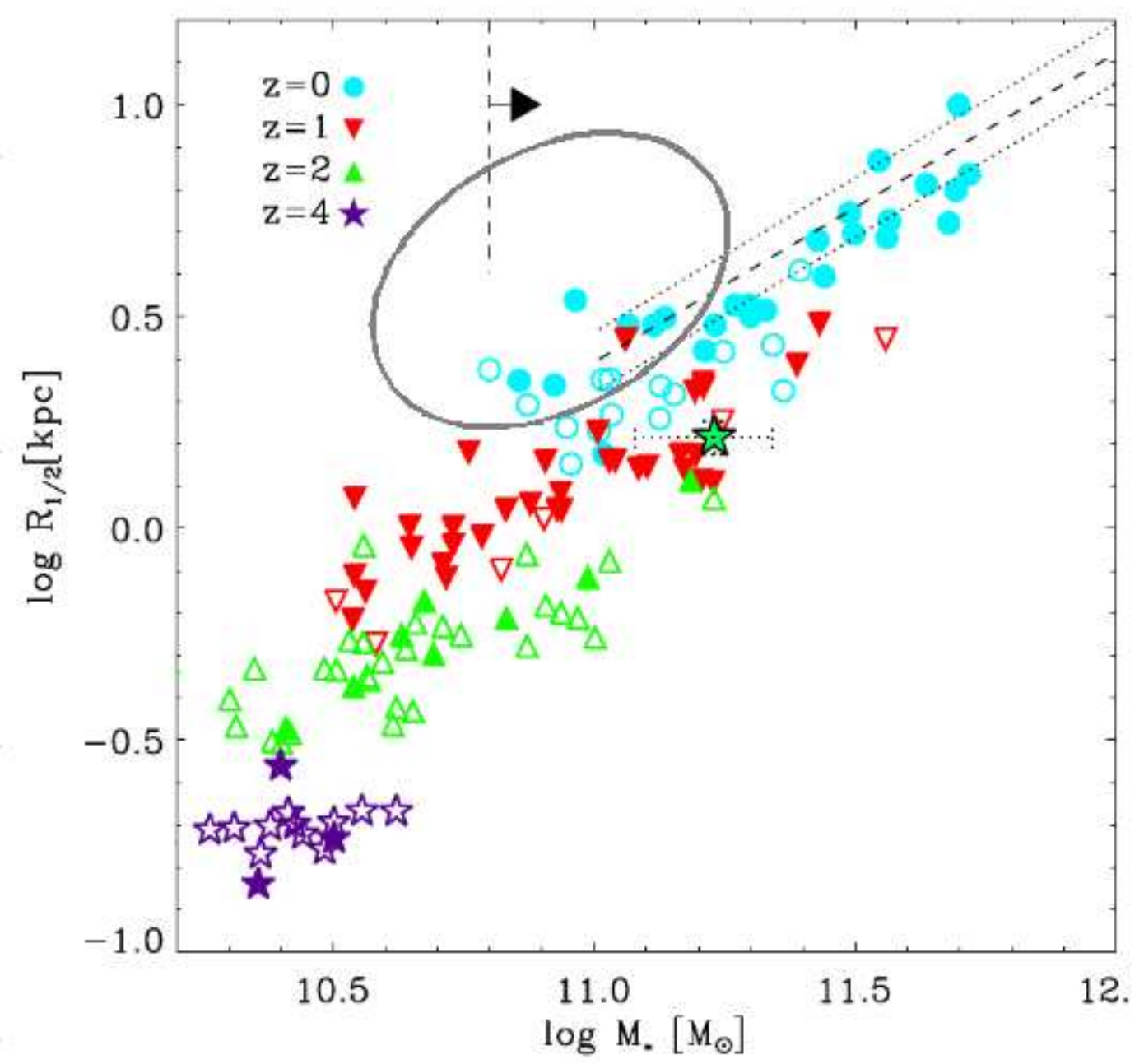

Figure 5.3 This figure, adapted from Figure 1 of Oser et al. (2012), shows the projected stellar half-mass radius versus stellar mass for galaxies at $z=1-4$ from the simulations of Oser et al. (2012). The galaxies at $z=0$ have stellar mass $M_{\star}>6.3 \times 10^{10} M_{\odot}$ (denoted by the vertical dashed line) and are taken from Nipoti et al. (2009); for these galaxies, the diagonal lines indicates the observed size-mass relation and the associated $1 \sigma$ scatter. Quiescent galaxies have solid symbols, other galaxies have open symbols. The elliptical annulus highlights the parameter space in size/stellar mass that this simulation is failing to reproduce at $z \sim 2$. 


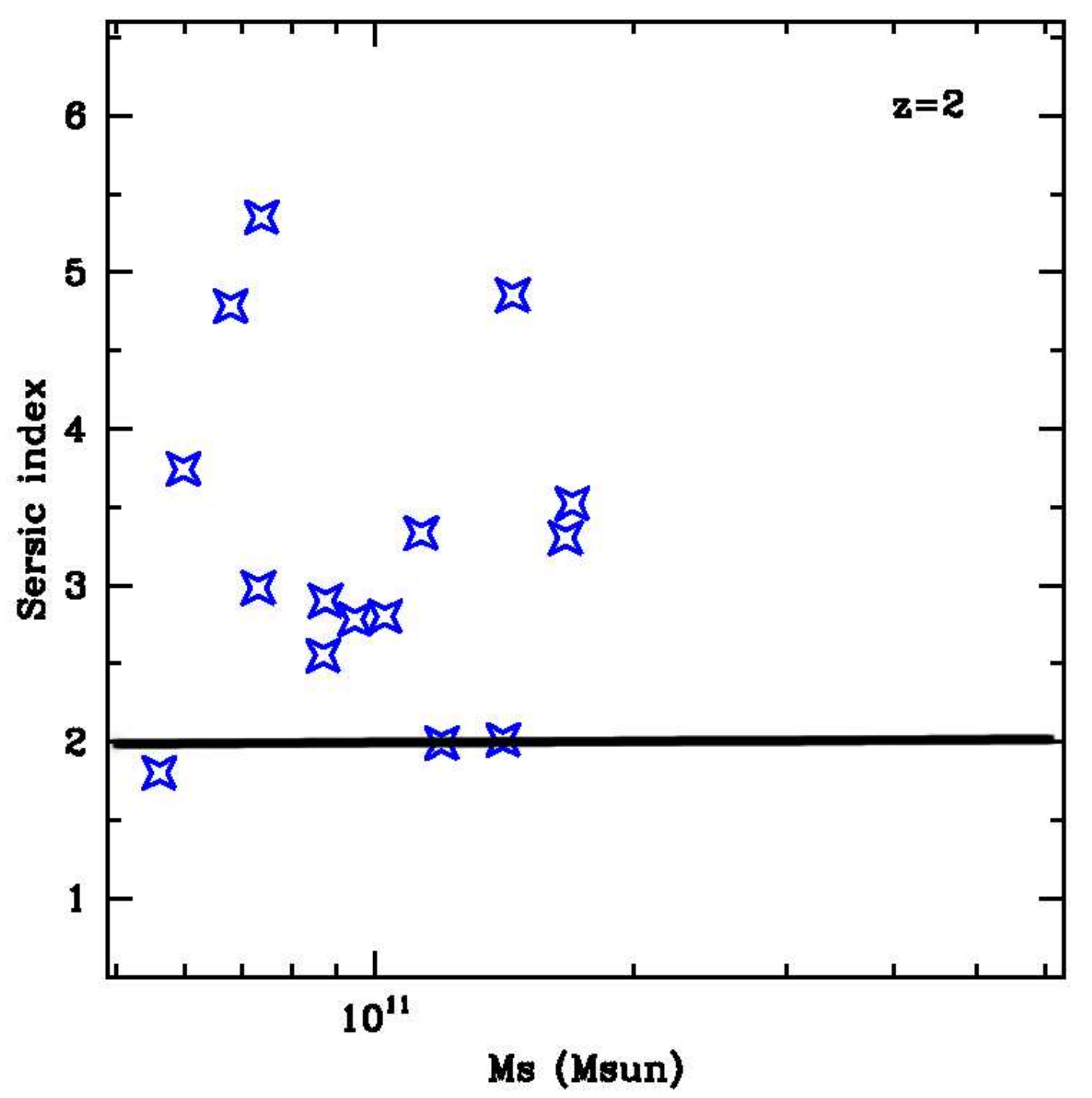

Figure 5.4 This figure demonstrates that nearly all the massive $z \sim 2$ galaxies made by Ceverino \& Dekel (in prep.), who use the model of Ceverino, Dekel, \& Bournaud (2010), have global non-disk structures with Sérsic index $n \gtrsim 2$. 
Appendices 


\section{Appendix A}

\section{Appendix to Chapter 3}

\section{A.1 Using GALFIT}

The proper operation of GALFIT depends on certain critical inputs. We briefly describe below how these important inputs are handled:

1. Point Spread Function (PSF):

Accurate modeling of the PSF is essential in deriving galaxy structural properties. GALFIT convolves the provided PSF with the galaxy model in each iteration before calculating the $\chi^{2}$. Because the PSF varies with position across the ACS/WFC chips, it is ideal to separately model the PSF for each galaxy position. We use the grid of model ACS PSFs in the F814W filter from Hoyos et al. (2011). This grid of PSFs was created with TinyTim (Krist 1993) and DrizzlyTim ${ }^{1}$.

For a given set of multidrizzle parameters, DrizzlyTim transforms $x-y$ coordinates in the final science frames back to the system of individually distorted FLT images. DrizzlyTim invokes TinyTim to create a PSF with the specified parameters (e.g., position and filter) and then places the PSF at the appropriate position in blank FLT frames. The FLT frames are passed through MULTIDRIZZLE with the same parameters as the science images. Finally, a Charge Diffusion Kernel is applied to the PSFs in

${ }^{1}$ DrizzlyTim is written by Luc Simard 
the geometrically distorted images. The grid of ACS PSFs from Hoyos et al. (2011) models a PSF for every 150 pixels in the $x$ and $y$ directions. For each galaxy in our sample we select the model PSF closest in proximity to the galaxy.

\section{Sigma Images:}

A sigma image is the $2 \mathrm{D}$ map of the $1 \sigma$ standard deviations in pixel counts of the input image. GALFIT uses the sigma image as the relative weight of pixels for calculating the goodness of fit. A sigma image can either be provided, or GALFIT can be allowed to calculate one based on the properties of the data image (image units of counts or counts/second, effective gain, read noise, number of combined exposures). We choose the latter option and allow GALFIT to calculate the sigma images.

\section{Background Subtraction:}

While it is possible for GALFIT to freely fit the background sky, this is not recommended (Peng et al. 2002). In a multiple-component fit to a galaxy with at least two components, freely fitting the sky can exaggerate or suppress the wings of the central Sérsic profile and incorrectly measure the bulge half-light radius and Sérsic index. To avoid this, for each galaxy the background sky is measured and held fixed during the fit. The sky background is based on ellipse fitting with the IRAF/ELLIPSE task. Ellipses are fit to the galaxy and the surrounding area, with the ellipses in the surrounding area being fixed to the shape and orientation of the galaxy. The gradient

along the semi-major axis is calculated, and the sky is estimated as the mean of elliptical annuli over a span in semi-major axis where the gradient reaches a prescribed small value. 


\section{Image Thumbnails and Masks:}

Thumbnail cutouts of the intermediate-mass galaxies are made to lessen the computational time for fitting. Following Hoyos et al. (2011), square image thumbnails centered on the target galaxy are made using the output from SExtractor. Image size in pixels is determined with

$$
\text { size }=4 \times \text { A_IMAGE } \times \text { KRON_RADIUS } .
$$

The image units are transformed from counts/second to counts by multiplying by the exposure time. Image masks are based on the segmentation images provided from SExtractor. The segmentation images are modified to unmask the background and target galaxy being fitted. Any bright sources that visibly overlap with the target galaxy are also unmasked so that overlapping sources can be fitted simultaneously. Masks for relatively bright sources that do not overlap with the galaxy being fitted are expanded in semi-major axis by a factor of 1.5 . We visually check by blinking the data image and modified segmentation image to verify that the unmasked region encompasses all of the target galaxy, including those with large diffuse halos that SExtractor does not capture (Hoyos et al. 2011).

\section{A.2 Identifying Core Ellipticals}

While elliptical galaxies are remarkably well-fit by Sérsic profiles over large dynamic ranges, giant elliptical galaxies contain cores, or "missing light" at small radii that constitute a downward deviation from from the inward extrapolation of the outer Sérsic profile (Graham et al. 2003; Trujillo et al. 2004; Kormendy et al. 2009). Such cores are hypothesized to form from scouring induced by binary black holes during dry, dissipationless mergers. 
Because cores, which have traditionally been identified with 1D radial light profiles, are not an obvious feature of the galaxy's 2D light distribution, global Sérsic fits will encompass any existing core. This is potentially problematic for at least two reasons. Including the core in the Sérsic fit will lower the global Sérsic index. This is of concern in this paper where the Sérsic index plays a key role in interpreting galaxy structure (Section 3.3.1). Secondly, fitting the core region may produce features in the residuals that prompt addition of extra nuclear components that have no physical justification.

We systematically search for cores in all sample galaxies. For this task, we use 1D light profiles generated from ellipse fitting of deconvolved images. The ACS images were deconvolved using a simulated PSF (Appendix A.1 for details) and 40 iterations of LucyRichardson deconvolution with the IRAF task LUCY (Lucy 1974; Richardson 1972). Our approach uses the criteria for identifying core galaxies from Trujillo et al. (2004) by fitting Sérsic and core-Sérsic profiles (Graham et al. 2003) to the 1D light profiles.

For simplicity, we use the version of the core-Sérsic profile that assumes an infinitely sharp transition between the outer Sérsic and inner power-law regions, namely

$$
\mathrm{I}(\mathrm{r})=\mathrm{I}_{\mathrm{b}}\left[\left(\mathrm{r}_{\mathrm{b}} / \mathrm{r}\right)^{\gamma} \mathrm{u}\left(\mathrm{r}_{\mathrm{b}}-\mathrm{r}\right)+\mathrm{e}^{\mathrm{b}\left(\mathrm{r}_{\mathrm{b}} / \mathrm{r}_{\mathrm{e}}\right)^{1 / \mathrm{n}}} \mathrm{e}^{-\mathrm{b}\left(\mathrm{r} / \mathrm{r}_{\mathrm{e}}\right)^{1 / \mathrm{n}}} \mathrm{u}\left(\mathrm{r}-\mathrm{r}_{\mathrm{b}}\right)\right]
$$

Here, $r_{b}$ denotes the division between the outer Sérsic and inner power-law profiles, $I_{b}$ is the intensity at this radius, $\gamma$ is the inner power-law slope, and $u(x-a)$ is the Heaviside step function. Parameters $n$ and $r_{\mathrm{e}}$ refer to the shape and half-light radius of the outer Sérsic profile. Additionally, $b$ is a constant that depends on several free parameters $\left(r_{b}, \gamma, r_{\mathrm{e}}\right.$, and $n)$.

We require a core galaxy to meet the following criteria: 1) the core-Sérsic model 
provide a better fit than the Sérsic profile; 2) the cores are well-resolved so that the break radius $r_{b}$ is greater than the second innermost data point in the profile; 3) the inner powerlaw slope $\gamma$ is less than the logarithmic slope of the Sérsic profile $(1 / n)$ in the core region.

Three sample galaxies meet the above criteria for having a core. Two of these are the central cD galaxies NGC 4874 and NGC 4889. Table 3.1 summarizes the $r_{b}$ and $\gamma$ measured from the core-Sérsic fit.

We further explore the best way to handle these cored galaxies in the 2D luminosity decompositions. Two natural approaches are to fit the whole galaxy, including the core, or to mask the galaxy over $r \leq r_{b}$. Masking is more physically motivated because the central core is a clear deviation from the inward extrapolation of the Sérsic profile that characterizes the outer galaxy structure. We try both approaches and summarize the results in Table 3.1. Applying a mask versus no mask has a nominal effect on COMAi125909.468p28227.35, but there is a significant increase in the $r_{\mathrm{e}}$ and $n$ of the $\mathrm{cD}$ galaxies when their larger core regions are masked.

Performing the 2D fit with the core masked is more physically motivated, and we consider these models to represent the best fits for the $\mathrm{cD}$ galaxies. It is worth noting, however, that our result from Section 3.3.4.4 that $100 \%$ of the mass in the cDs is associated with structures of $n \gg n_{\text {disk_max }}$. remains unchanged irrespective of which approach (mask or no mask) we take.

\section{A.3 Systematic Effects on $n_{\text {disk_max }}$}

Our effort in this paper to make a census (Section 3.4.2) of low- $n$, dynamically cold disk-dominated structures versus high- $n$, dynamically hot classical bulge/elliptical compo- 
nents depends fundamentally on the upper limit, $n_{\text {disk_max }}$ (Section 3.3.1), measured for the Sérsic index of a disk in Section 3.3.4.2. In our approach, all structures with Sérsic index $n \leq n_{\text {disk_max }}$ are considered disk dominated, while all other structures with higher Sérsic index are considered classical components built in major mergers, and possibly also minor mergers.

The value of $n \leq n_{\text {disk_max }}$ is set by the moderately inclined barred galaxy (COMAi125950.105p275529.44) having the highest outer disk Sérsic index. The accuracy of $n_{\text {disk_max }}$ depends on how representative the sample is as well as the robustness of the multi-component structural decompositions. Figure 3.10 shows for this galaxy the data image and residuals of the multi-component decompositions. While this galaxy was identified as an ambiguous E/S0 galaxy in Figure 2 of Marinova et al. (2012), the barred nature of this galaxy seems clear based on the image residuals produced by our improved method (Sections 3.3.2 and 3.3.3) of structural decomposition.

The value of $n_{\text {disk_max }}$ is subject to sky subtraction errors because it is measured from the outermost Sérsic profile of disk galaxies, and this is likely the dominant systematic effect on $n_{\text {disk_max }}$. As described in Appendix A.1, we measure the background sky value with a robust method and hold the sky fixed at this value during the fit. To test the importance of the sky subtraction, we refitted COMAi125950.105p275529.44 while adjusting the mean sky background by $\pm 1 \sigma$. This produces a range in outer disk $n$ of $n \sim 1.57-1.77$, which spans $\sim 0.1$ indices above and below the adopted $n_{\text {disk_max }}$ value of 1.66 . Based on the narrow error bars for $n_{\text {disk_max }}$, we do not expect the uncertainty to have a significant impact on our conclusions.

For completeness, we explore for an alternate value of $n_{\text {disk_max }}$ the relative stellar 
mass fractions that would be interpreted as belonging to disk-dominated structures versus classical bulges/ellipticals. The value $n_{\text {disk_max }}=2$ is in line with estimates of the Sérsic index of small-scale disks (e.g., Fisher \& Drory 2008; Weinzirl et al. 2009) yet is still above the anticipated range in $n_{\text {disk_max }}$ due to sky subtraction errors in this study. With this higher $n_{\text {disk_max }}$, we would find that $\sim 51 \%$ stellar mass is in disk-dominated components while $\sim 49 \%$ is still in classical bulges/ellipticals assembled in major and minor mergers. These values are somewhat different from the corresponding values (44\% in disk-dominated structures versus $56 \%$ in non-disks) derived in Section 3.4 .2 excluding the $2 \mathrm{cD}$ galaxies. Choosing a higher $n_{\text {disk_max }}$ would increase the importance of disk-building processes relative to processes that build classical bulges/ellipticals. For $n_{\text {disk_max }}=2$, these processes would be interpreted to be about equally important. 


\section{Appendix B}

\section{Appendix to Chapter 4}

\section{B.1 PSF Modeling}

Knowledge of the PSF is important to assess data quality and for deriving structural parameters. NIC3 is out of focus, so the PSF can deviate from the theoretically expected one. PSF convolution with GALFIT is commonly performed with a user-provided bright, unsaturated star. Not all of the GNS tiles contain suitably bright, unsaturated stars. It is not advisable to adopt a set of PSF stars from a subset of pointings because the NIC3 PSF depends on position within the NIC3 field and is also subject to interpolation artifacts introduced by drizzle that are dependent on the adopted dither pattern (J. Krist, private communication).

As a result, the best available option for handling PSF convolution is to make synthetic NIC3 PSFs with Tiny Tim (Krist 1995). For each galaxy, Tiny Tim PSFs were generated for all the galaxy's positions in the individual, undrizzled exposures. Telescope breathing was accounted for with each PSF by refining the Tiny Tim parameters to match the Pupil Alignment Mechanism (PAM) value recorded in the headers of the undrizzled frames. Blank, zero-valued frames retaining the WCS information of the undrizzled frames were made. The synthetic PSFs were inserted into the blank frames precisely where each galaxy would be in the individual frames. The blank frames were drizzled together in the same way as the data with a pixfrac of 0.7 and a final output platescale of $0.1 /$ pixel. This 
process was repeated for all 166 massive $\left(M_{\star} \geq 5 \times 10^{10}\right)$ galaxies in our sample.

This approach accounts both for variation in PSF with position on the NIC3 field and for the dependence on the drizzle algorithm. The range of FWHM in the final drizzled synthetic PSFs is $\sim 0{ }^{\prime \prime} 26-00^{\prime \prime} 36,{ }^{1}$ with a mean value of $0{ }^{\prime \prime} 3$. The mean PSF diameter of the science images $\left(0^{\prime \prime} 3\right)$ is $2.5 \mathrm{kpc}$ at $z=2$, under the adopted cosmology.

\section{B.2 Tests on Robustness of Fits and Parameter Coupling}

How robust are the results that a dominant fraction of the massive galaxies at $z=2-3$ have a low $n \leq 2$ and that a large fraction are ultra-compact? In particular, how non-degenerate are the fits? Could some of the galaxies with an $n \leq 2$ have similarly good fits with higher $n$ ?

First, one should note that the errors quoted by GALFIT on the structural parameters cannot be used to assess the robustness of the fits because the errors quoted by GALFIT underestimate the true parameter errors (Häussler et al. 2007), which are dominated by the systematics of galaxy structure, and in particular, by parameter coupling and degeneracy.

The task of assessing the coupling between model parameters is complicated when models have a large number of free parameters. The single Sérsic profiles fit to the NICMOS galaxy images have 7 free parameters (centroid, luminosity, $r_{e}, n$, axis ratio, and position angle). While GALFIT selects a best-fit by minimizing $\chi^{2}$ for a given set of input guesses, it is not clear whether the minimized $\chi^{2}$ is an absolute minimum or local minimum. Investigating the $\chi^{2}$ values for all combinations of fit parameters over the full multi-dimensional

\footnotetext{
${ }^{1}$ The range in PSF FWHM comes from differing positions in the NIC3 field and the PAM values used to create the synthetic PSFs.
} 
parameter space is prohibitively time consuming and computationally expensive. Instead, we will adopt a simpler approach of focusing on strong coupling between $r_{e}$ and $n$, and exploring how $\chi^{2}$ varies as these parameters are moved away from the initial solution picked by GALFIT.

One important point should be noted when using changes in $\chi^{2}$, or $\Delta \chi^{2}$, for fits to different models. When errors are normally distributed, the multi-dimensional ellipsoids for a given $\Delta \chi^{2}$ contour can be associated with a statistical confidence level (e.g., $\Delta \chi^{2} \sim 1$ corresponds to a $68 \%$ confidence level). However, since the errors in the GALFIT models are not normally distributed, but are instead dominated by the systematics of galaxy structure, this means that we cannot a priori assign a confidence level to a given $\Delta \chi^{2}$. As outlined in the test below, we can still use the shape of $\Delta \chi^{2}$ as a function of $n$ or $r_{e}$ as a guide to the quality of fit in the sense that sharp rises in $\chi^{2}$ as $n$ is varied away from the best-fit value are taken as indicative of poorer fits. But, we cannot a priori say how much poorer the fits are in a statistical sense. This is a well-known and hard problem in structural fitting. We will return to this point in Section 4.B.3.

We carry out the test below for all galaxies in our sample We denote as $\chi_{\min , 0}^{2}$, the value of $\chi^{2}$ obtained when GALFIT fits the galaxy with $n$ and $r_{e}$ as free parameters. The associated best-fit parameters are $n_{\min , 0}$ and $r_{e, \min , 0}$. We then fit single Sérsic profiles with $n$ fixed at discrete values (0.5-10), while allowing all other parameters to freely vary. The initial inputs to these fits were the same as those used to generate the model in which $n$ is a free parameter. We let GALFIT find the best-fit for each of these fixed $n$ models by minimizing $\chi^{2}$, and we record for each such best-fit the following quantities: the fixed value of $n$, the best-fit value of $r_{e}$, and the associated minimum in $\chi^{2}$ called $\chi_{\min }^{2}$. We then 
evaluate how the difference $\chi_{\min }^{2}-\chi_{\min , 0}^{2}$ varies as a function of $r_{e}$ and $n$, as we move to values away from $n_{\min , 0}$ and $r_{e, \min , 0}$.

The test was carried out for all galaxies. Figure B.1 shows the results of the test for four representative galaxies with $n \sim 1-4$. The first column of Figure B.1 shows how $\left(\chi_{\min }^{2}-\chi_{\min , 0}^{2}\right)$ changes when $n$ is varied away from $n_{\min , 0}$ at discrete values $(0.5-10)$ and GALFIT is allowed to vary all other parameters to get a best-fit that yields $\chi_{\min }^{2}$. The second column shows the corresponding best-fit $r_{e}$ for that $\chi_{\min }^{2}$. Red stars in the plots denote $n_{\min , 0}$ and $r_{e, \min , 0}$, which are associated with $\chi_{\min , 0}^{2}$. The shape of $\chi_{\min }^{2}-\chi_{\min , 0}^{2}$ is asymmetric for $n$ and $r_{e}$. The coupling between $n$ and $r_{e}$ means $\left(\chi_{\min }^{2}-\chi_{\min , 0}^{2}\right)$ varies in a similar way with both $n$ and $r_{e}$.

We can see that in Figure B.1, the absolute minimum $\chi^{2}$ values occur at the $n_{\min , 0}$ and $r_{e, \min , 0}$ values, which GALFIT picked when it was allowed to freely fit the galaxies without fixing $n$. Shifting $n$ away from $n_{\min , 0}$ (denoted by the red stars) by \pm 1 can increase $\chi_{\min }^{2}$ by several $10 \mathrm{~s}$ or $100 \mathrm{~s}$ of $\chi^{2}$ units. While only 4 representative galaxies are shown in Figure B.1, we show results for the whole sample in Figure B.2. This figure illustrates that the distribution of $\left(\chi_{\min }^{2}-\chi_{\min , 0}^{2}\right)$ for $n_{\min , 0}-1$ (top panel) and $n_{\min , 0}+1$ (bottom panel), and demonstrates for the whole sample, $\chi_{\min }^{2}$ generally changes substantially when $n$ is shifted away from $n_{\min , 0}$. We draw two primary conclusions from Figure B.1:

1. For galaxies with $n_{\min , 0}>2$ (rows 3 and 4 ), $\chi_{\min }^{2}-\chi_{\min , 0}^{2}$ rises sharply at lower $n<n_{\min , 0}$, suggesting that lower $n$ values are unlikely to yield a good fit for such systems. At $n>n_{\min , 0}, \chi_{\min }^{2}-\chi_{\min , 0}^{2}$ rises less sharply, but the rise is still substantial as demonstrated in by the high-magnification inset plots in rows 3 and 4 of column 1 . 
2. The most important point to take from Figure B.1 is that for galaxies with $n_{\min , 0}<2$ (as in rows 1 and 2 ), $\chi_{\min }^{2}-\chi_{\min , 0}^{2}$ rises rapidly at higher $n>n_{\min , 0}$, thereby making it unlikely that a higher $n>2$ would provide a similarly good fit. Thus, we have a great degree of confidence that we are not highly overestimating the number of $n \leq 2$ galaxies in the sample.

\section{B.3 Recovery of Parameters From Simulated Images}

Section 4.B.2 tests how well parameters are recovered in real galaxies, but we cannot a priori assign a confidence level to a given $\Delta \chi^{2}$ because the errors in the GALFIT models are not normally distributed. However, we can run an extra complementary test where we use simulated idealized galaxies whose $\left(n, r_{e}\right)$ are a priori known. The drawback of using idealized galaxies as opposed to the real galaxies fitted in $\S 4.3 .1$ is that the former lack the complexity of real galaxies, since they are simply generated from GALFIT models and exactly described by a functional form, such as a Sersic model with a specified $\left(n, r_{e}\right)$. However, the advantage is that we do know the $\left(n, r_{e}\right)$ values a priori and can therefore compare these values with those obtained once these idealized galaxies are inserted into frames with noise properties corresponding to the NIC3 GNS images of our sample galaxies at $z=1-3$.

This test is performed by simulating 1000 galaxy images, each with a unique set of Sérsic parameters: surface brightness at the effective radius $\mu_{e}$, effective radius $r_{e}$, Sérsic index $n$, axis ratio $\mathrm{b} / \mathrm{a}$, and position angle $\mathrm{PA}$. The parameters are chosen randomly from uniform distributions spanning the parameter space of the observed galaxies. The ranges in $\mu_{e}, r_{e}, n, \mathrm{~b} / \mathrm{a}$, and PA are 16 to $32 \mathrm{mag} / \operatorname{arcsec}^{2}, 0^{\prime \prime} 05$ to $1^{\prime \prime} 0,0.5$ to $10,0.3$ to 1.0 , and 
-90 to 90 degrees, respectively. The chosen range in input $\mu_{e}$ mimics the effect of surface brightness dimming, and the range in $r_{e}$ ensures the simulated objects span the angular size of the real GNS galaxies. The simulated galaxies were created with GALFIT and convolved with a drizzled PSF image. They were set within a sky background equivalent to the mean NIC3 sky background within GNS (0.1 counts/sec). Source noise, sky noise, and read noise $\left(29 e^{-}\right)$were added to the frames.

The simulated images were then re-fit with GALFIT to derive $\left(n, r_{e}\right)$. Initial guess parameters for $\left(\mu_{e}, r_{e}, n, \mathrm{~b} / \mathrm{a}, \mathrm{PA}\right)$ were generated randomly from uniform distributions

spanning $\pm 1.5 \mathrm{mag} / \operatorname{arcsec}^{2}$ in $\mu_{e}, \pm 00^{\prime \prime} 3$ in $r_{e}, \pm 2$ indices in $n, 0.3$ to $1 \mathrm{in} \mathrm{b/a,} \mathrm{and}-90$ to 90 degrees in PA. Figure B.3 shows the recovery in $\left(n, r_{e}\right)$ plotted against surface brightness. The dashed vertical lines represent the minimum, median, and maximum $\mu_{e}$ for the observed massive galaxies. Figure B.3 shows $\left(n, r_{e}\right)$ are well recovered across the full range in observed $\mu_{e}$. The recovery as a function of $\mu_{e}$ severely degrades only at several mag/ $\operatorname{arcsec}^{2}$ fainter than observed $\mu_{e}$. In $\sim 95 \%$ of cases, $n$ and $r_{e}$ are recovered to within $10 \%$ of their input values for the range of observed $\mu_{e}$ among the massive galaxies in our sample.

\section{B.4 Tests on MGC Fits}

The structural parameters for the massive galaxies at $z \sim 0$, are derived by Allen et al. (2006) by using the GIM2D code (Simard et al. 2002) to fit single Sérsic component to the MGC $B$-band images. We derived the structural parameters for the massive galaxies at $z=1-3$, by using the GALFIT code (Peng et al. 2002) on the NIC3/F610W images (§4.3.1). One might wonder whether the dramatic shift in Figure 4.5 of the $z=2-3$ galaxies toward lower $\left(n, r_{e}\right)$ compared with the $z \sim 0$ MGC galaxies may be caused by systematic 
differences between the fitting techniques used by us versus those by Allen et al. (2006). This would be the case only if the fits by Allen et al. (2006) give systematically higher ( $n$, $\left.r_{e}\right)$ than ours for the same galaxies. As we show below this is not the case.

In order to address this issue, we have applied GALFIT to a subset of $B$-band MGC images and compared our resulting structural parameters with the GIM2D-based results given in the MGC catalogue. The comparison (top row of Figure B.4) shows that the GIM2D-based fits of Allen et al. (2006) are not biased to higher $\left(n, r_{e}\right)$ compared with our GALFIT-based fits for the $z \sim 0$ MGC galaxies. In fact, for large $r_{e}$, the GIM2D-based values may even be lower. in many cases.

These results are consistent with extensive comparisons of single component Sérsic fits from GALFIT and GIM2D conducted by Häussler et al.(2007) on both simulated and real galaxy data. They concluded that both codes provide reliable fits with little systematic error for galaxies with effective surface brightnesses brighter than that of the sky, as long as one is not dealing with highly crowded fields.

Another possible source of difference between the structural parameters of the $z \sim 0$ and $z=2-3$ massive galaxies might be the fact that Allen et al. (2006) fitted the $z \sim 0$ massive galaxies with only a single Sérsic component, and did not include an extra point source component in galaxies with evident nuclear sources. It seems unlikely that the much larger fraction of higher $\left(n, r_{e}\right)$ systems at $z \sim 0$ in Figure 4.5 is mainly driven by this effect. To illustrate this, we have fitted the $z \sim 0$ MGC galaxies in the top row of Figure B.4 with a combination of a single Sérsic component and a point source model using GALFIT. The bottom row of Figure B.4 shows the results. The values of $r_{e}$ are not changed systematically. The Sérsic index is lowered by the addition of the point source, but only $20 \%$ of the sources 
with $n>2$ in the single Sérsic fit have $n \leq 2$ after including the point source. Since not all $z \sim 0$ MGC galaxies in Figure 4.5 will have nuclear sources, the fraction of sources impacted will be even less. We thus conclude that the presence of a point source in some of the $z \sim 0$ MGC galaxies and the inclusion of such a point source in the model fits, are not sufficient to shift the $z \sim 0$ MGC galaxies into the parameter space occupied by the $z=2-3$ massive galaxies in Figure 4.5. 


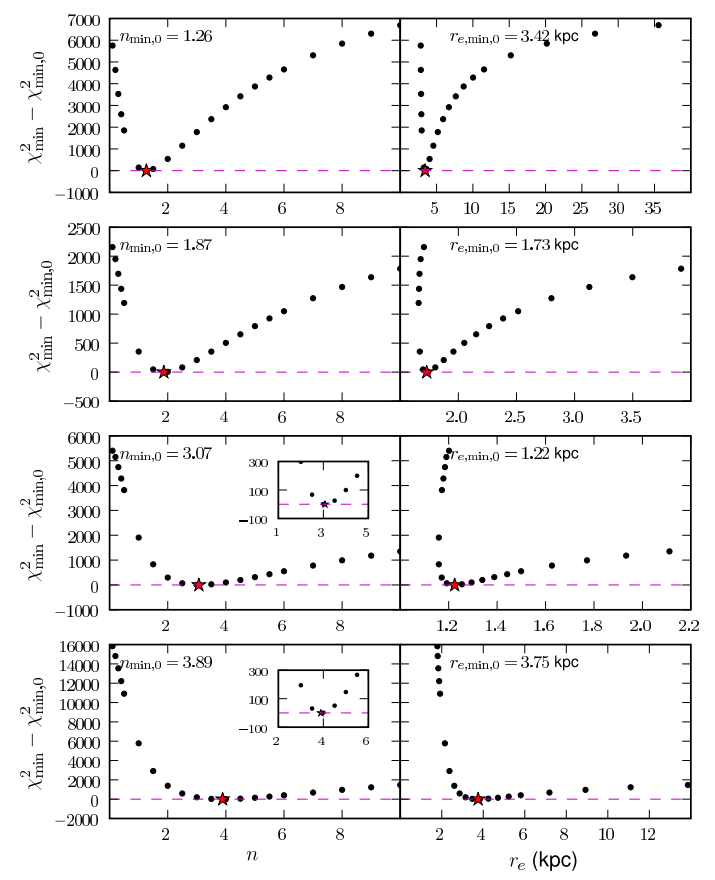

Figure B.1 For four representative galaxies with $n \sim 1-4$, the first and second columns show the difference $\chi_{\min }^{2}-\chi_{\min , 0}^{2}$ versus $n$ and $r_{e}$, respectively. $\chi_{\min , 0}^{2}$ is the minimum $\chi^{2}$ obtained when all parameters are freely fit, and $\chi_{\min }^{2}$ is the minimum $\chi^{2}$ when $n$ is held at discrete values $(0.5-10)$. The $r_{e}$ in the second column are the best-fit results for a given $n$ and $\chi_{\min }^{2}$. The red stars mark the best-fit $n_{\min , 0}$ and $r_{e, \min , 0}$ corresponding to $\chi_{\min , 0}^{2}$. The insets in rows 3 and 4 of column 1 show a magnified view around the minimum in $\chi_{\min }^{2}-\chi_{\min , 0}^{2}$. Note that for galaxies with $n_{\min , 0}<2$ (rows 1 and 2 ), $\chi_{\min }^{2}-\chi_{\min , 0}^{2}$ rises sharply at higher $n>n_{\min , 0}$, thereby making it unlikely that a higher $n>2$ would provide a similarly good fit. 


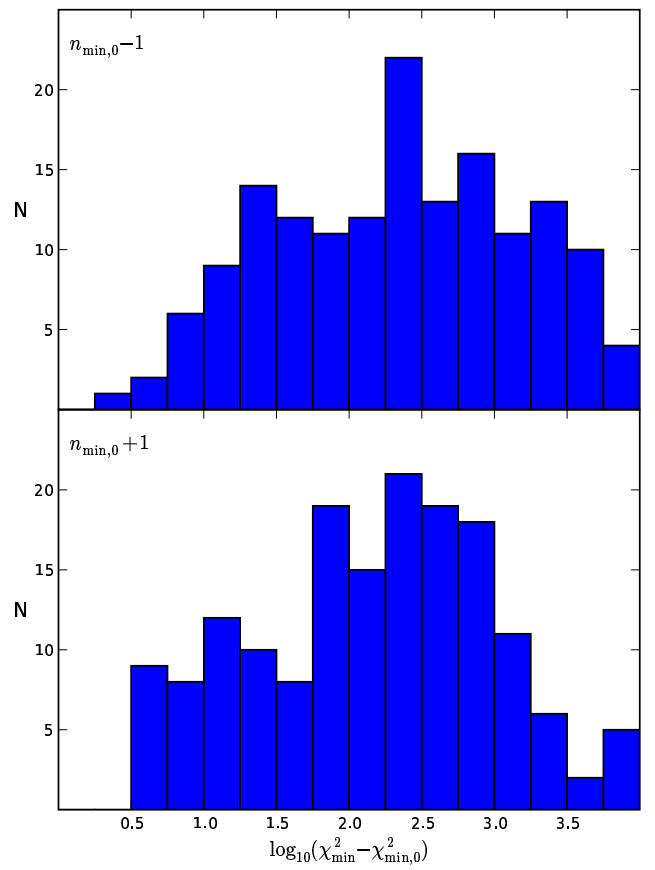

Figure B.2 The quantity $\chi_{\min }^{2}-\chi_{\min , 0}^{2}$ from Figure B.1 is shown for all massive GNS galaxies well fitted with a single Sérsic profile. The top panel evaluates $\chi_{\min }^{2}-\chi_{\min , 0}^{2}$ at $n_{\min , 0}-1$, and the bottom panel evaluates $\chi_{\min }^{2}-\chi_{\min , 0}^{2}$ at $n_{\min , 0}+1$, where $n_{\min , 0}$ is the best-fit Sérsic index corresponding to $\chi_{\min , 0}^{2}$. 


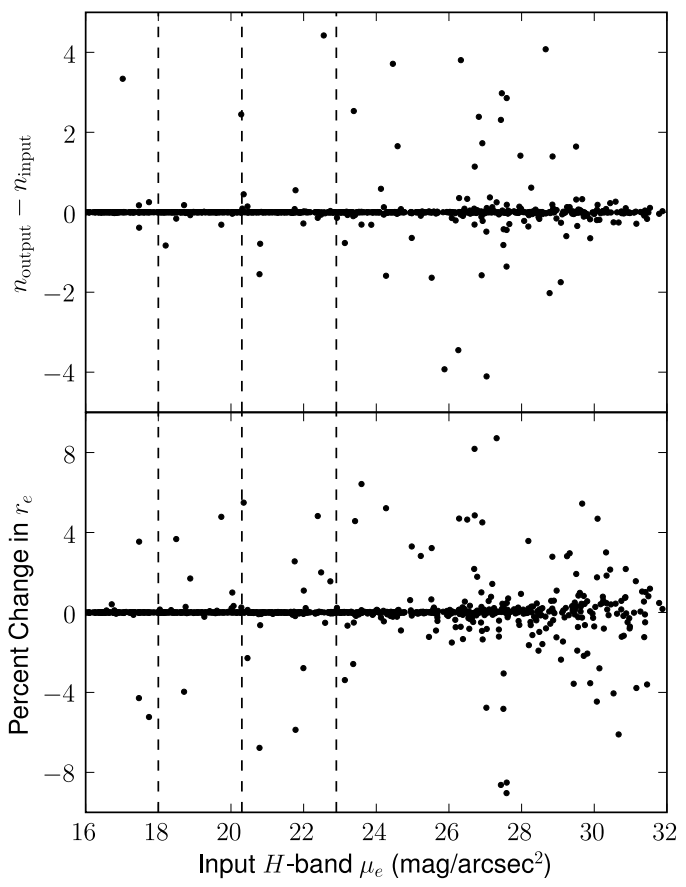

Figure B.3 For the simulations described in $§$ B.3, the difference between input and output Sérsic index $n$ and effective radius $r_{e}$ are plotted against effective surface brightness $\mu_{e}$, the surface brightness at $r_{e}$. The vertical lines correspond to the range in $\mu_{e}$ in the NIC3/F160W band for the massive galaxies at $z=1-3$ in our sample. 

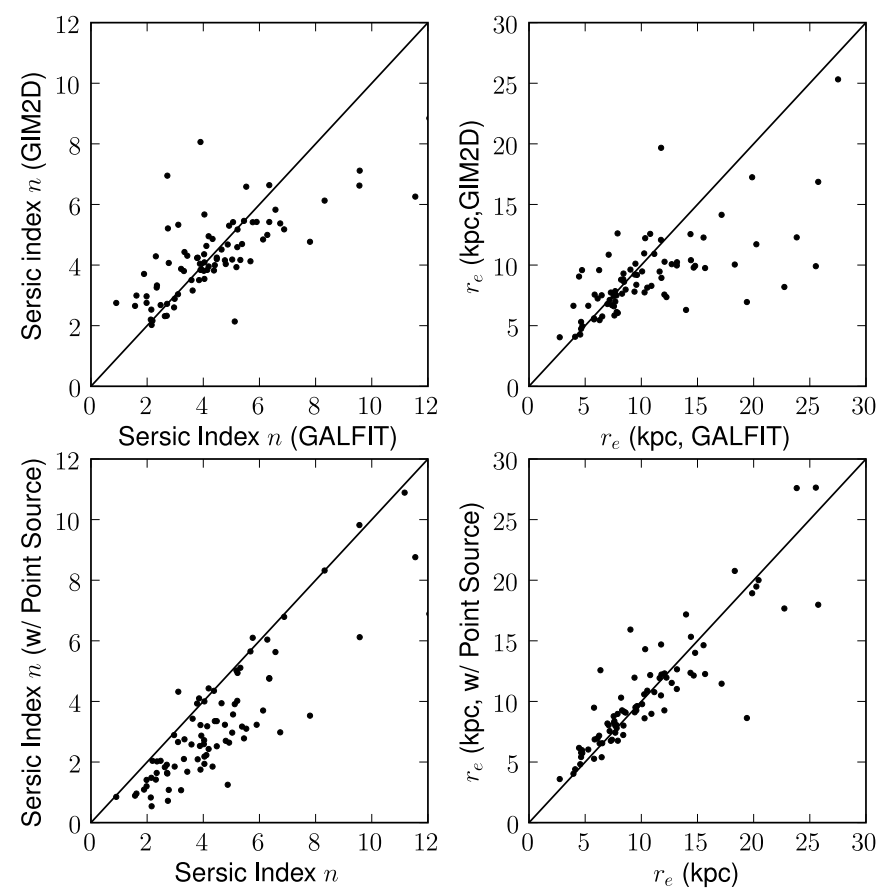

Figure B.4 Top row: We demonstrate for a subset of $z \sim 0$ galaxies in the MGC catalog that the GIM2D-based $\left(n, r_{e}\right)$ values from Allen et al. (2006) are not biased to higher values compared with our GALFIT-based fits for the same galaxies. All fits are performed on the $B$-band images from MGC. Bottom row: We show the effects of adding a point source in the GALFIT models fitted to the $z \sim 0$ MGC galaxies. The values obtained using a model made of a Sérsic component plus a point source are plotted along the $\mathrm{y}$-axis, while the $\mathrm{x}$-axis shows the values obtained with a single Sérsic component. The values of $r_{e}$ are not changed systematically. The Sérsic index is lowered by the addition of the point source, but only $20 \%$ of sources with $n>2$ in the single Sérsic fit have $n \leq 2$ after including the point source. 


\section{Bibliography}

[1] Abazajian, K. N., Adelman-McCarthy, J. K., Agüeros, M. A., et al. 2009, ApJS, 182, 543

[2] Adami, C., Scheidegger, R., Ulmer, M., et al. 2006, A\&A, 459, 679

[3] Adami, C., Slezak, E., Durret, F., et al. 2005, A\&A, 429, 39

[4] Adelman-McCarthy, J. K., Agüeros, M. A., Allam, S. S., et al. 2008, ApJS, 175, 297

[5] Aguerri, J. A. L., Balcells, M., \& Peletier, R. F. 2001, A\&A, 367, 428

[6] Aguerri, J. A. L., Méndez-Abreu, J., \& Corsini, E. M. 2009, A\&A, 495, 491

[7] Alexander, D. M., Bauer, F. E., Brandt, W. N., et al. 2003, AJ, 126, 539

[8] Allen, P. D., Driver, S. P., Graham, A. W., et al. 2006, MNRAS, 371, 2

[9] Alonso-Herrero, A., Pérez-González, P. G., Alexander, D. M., et al. 2006, ApJ, 640, 167

[10] Andredakis, Y. C., Peletier, R. F., \& Balcells, M. 1995, MNRAS, 275, 874

[11] Andredakis, Y. C., \& Sanders, R. H. 1994, MNRAS, 267, 283

[12] Aragon-Salamanca, A., Baugh, C. M., \& Kauffmann, G. 1998, MNRAS, 297, 427

[13] Arnaboldi, M. 2011, paper presented at the ESO Workshop on Fornax, Virgo, Coma et al.: Stellar Systems in High Density Environments, /sci/meetings/2011/fornax_virgo2011/talks_pdf/Arnaboldi_Magda.pdf 
[14] Athanassoula, E. 2002, ApJL, 569, L83

[15] Athanassoula, E. 2005, MNRAS, 358, 1477

[16] Baggett, W. E., Baggett, S. M., \& Anderson, K. S. J. 1998, AJ, 116, 1626

[17] Balcells, M., Graham, A. W., Domínguez-Palmero, L., \& Peletier, R. F. 2003, ApJL, 582, L79

[18] Balcells, M., Graham, A. W., \& Peletier, R. F. 2007a, ApJ, 665, 1084

[19] Balcells, M., Graham, A. W., \& Peletier, R. F. 2007b, ApJ, 665, 1104

[20] Barazza, F. D., Jogee, S., \& Marinova, I. 2007, IAU Symposium, 235, 76

[21] Barazza, F. D., Jogee, S., \& Marinova, I. 2008, ApJ, 675, 1194

[22] Barden, M., Jahnke, K., Haüßler, B. 2008, ApJS, 175, 105

[23] Barden, M., Rix, H.-W., Somerville, R. S., et al. 2005, ApJ, 635, 959

[24] Barentine, J. C., \& Kormendy, J. 2012, ApJ, 754, 140

[25] Barnes, J. E. 1988, ApJ, 331, 699

[26] Barnes, J. E., \& Hernquist, L. E. 1991, ApJL, 370, L65

[27] Barrett, P. E., \& Bridgman, W. T. 1999, Astronomical Data Analysis Software and Systems VIII, 172, 483

[28] Bauer, A. E., Conselice, C. J., Pérez-González, P. G., et al. 2011, MNRAS, 417, 289

[29] Beckwith, S. V. W., Stiavelli, M., Koekemoer, A. M., et al. 2006, AJ, 132, 1729 
[30] Bell, E. F., McIntosh, D. H., Barden, M., et al. 2004, ApJL, 600, L11

[31] Bell, E. F., McIntosh, D. H., Katz, N., \& Weinberg, M. D. 2003, ApJS, 149, 289

[32] Bell, E. F., Naab, T., McIntosh, D. H., et al. 2006, ApJ, 640, 241

[33] Bell, E. F., Papovich, C., Wolf, C., et al. 2005, ApJ, 625, 23

[34] Bell, E. F., Phleps, S., Somerville, R. S., et al. 2006, ApJ, 652, 270

[35] Bell, E. F., Zheng, X. Z., Papovich, C., et al. 2007, ApJ, 663, 834

[36] Benedict, F. G., Smith, B. J., \& Kenney, J. D. P. 1996, AJ, 111, 1861

[37] Berentzen, I., Shlosman, I., \& Jogee, S. 2006, ApJ, 637, 582

[38] Bernstein, G. M., Nichol, R. C., Tyson, J. A., Ulmer, M. P., \& Wittman, D. 1995, AJ, 110,1507

[39] Bezanson, R., van Dokkum, P. G., Tal, T., et al. 2009, ApJ, 697, 1290

[40] Binggeli, B., Sandage, A., \& Tammann, G. A. 1988, ARAA, 26, 509

[41] Binggeli, B., Tammann, G. A., \& Sandage, A. 1987, AJ, 94, 251

[42] Binney, J., \& Merrifield, M. 1998, Galactic Astronomy (Princeton: Princeton Univ. Press)

[43] Binney, J., \& Tremaine, S. 1987, Princeton, NJ, Princeton University Press, 1987, 747 p.,

[44] Birnboim, Y., \& Dekel, A. 2003, MNRAS, 345, 349 
[45] Blain, A. W., Smail, I., Ivison, R. J., Kneib, J.-P., \& Frayer, D. T. 2002, Physics Reports, 369, 111

[46] Blanc, G. A., Weinzirl, T., Song, M., et al. 2013, AJ, 145, 138

[47] Blanton, M. R., Schlegel, D. J., Strauss, M. A., et al. 2005, AJ, 129, 2562

[48] Block, D. L., Bournaud, F., Combes, F., Puerari, I., \& Buta, R. 2002, A\&A, 394, L35

[49] Bluck, A. F. L., Conselice, C. J., Bouwens, R. J., et al. 2009, MNRAS, 394, L51

[50] Blumenthal, G. R., Faber, S. M., Primack, J. R., \& Rees, M. J. 1984, Nature, 311, 517

[51] Böker, T., Laine, S., van der Marel, R. P., et al. 2002, AJ, 123, 1389

[52] Boroson, T. 1981, ApJS, 46, 177

[53] Borch, A., Meisenheimer, K., Bell, E. F., et al. 2006, A\&A, 453, 869

[54] Boselli, A., Gavazzi, G., Lequeux, J., et al. 1997, A\&A, 327, 522

[55] Bottinelli, L., Gouguenheim, L., Paturel, G., \& Teerikorpi, P. 1995, A\&A, 296, 64

[56] Bournaud, F., Elmegreen, B. G., \& Elmegreen, D. M. 2007, ApJ, 670, 237

[57] Bournaud, F., Jog, C. J., \& Combes, F. 2007, A\&A, 476, 1179

[58] Bournaud, F., Dekel, A., Teyssier, R., et al. 2011, ApJL, 741, L33

[59] Brand, K., Dey, A., Weedman, D., et al. 2006, ApJ, 644, 143

[60] Brandt, W. N., Alexander, D. M., Bauer, F. E., \& Vignali, C. 2006, Physics of Active Galactic Nuclei at all Scales, 693, 185 
[61] Brooks, A. M., Governato, F., Quinn, T., Brook, C. B., \& Wadsley, J. 2009, ApJ, 694, 396

[62] Bruzual, G., \& Charlot, S. 2003, MNRAS, 344, 1000

[63] Buitrago, F., Trujillo, I., Conselice, C. J., et al. 2008, ApJL, 687, L61

[64] Bullock, J. S., Kravtsov, A. V., \& Weinberg, D. H. 2001, ApJ, 548, 33

[65] Bureau, M., \& Athanassoula, E. 2005, ApJ, 626, 159

[66] Burkert, A., Genzel, R., Bouché, N., et al. 2010, ApJ, 725, 2324

[67] Burkert, A. M., \& D'Onghia, E. 2004, Penetrating Bars Through Masks of Cosmic Dust, 319, 341

[68] Burkert, A., \& Naab, T. 2004, Coevolution of Black Holes and Galaxies, 421

[69] Burstein, D. 1979a, ApJS, 41, 435

[70] Burstein, D. 1979b, ApJ, 232, 74

[71] Buta, R., Block, D. L., \& Knapen, J. H. 2003, AJ, 126, 1148

[72] Buta, R., Vasylyev, S., Salo, H., \& Laurikainen, E. 2005, AJ, 130, 506

[73] Byun, Y. I., \& Freeman, K. C. 1995, ApJ, 448, 563

[74] Caldwell, N. 1999, AJ, 118, 1230

[75] Caon, N., Capaccioli, M., \& D’Onofrio, M. 1993, MNRAS, 265, 1013

[76] Cappellari, M., Emsellem, E., Bacon, R., et al. 2007, MNRAS, 379, 418 
[77] Cappellari, M., Emsellem, E., Krajnović, D., et al. 2011a, MNRAS, 413, 813

[78] Cappellari, M., Emsellem, E., Krajnović, D., et al. 2011b, MNRAS, 416, 1680

[79] Carter, D., Goudfrooij, P., Mobasher, B., et al. 2008, ApJS, 176, 424

[80] Cattaneo, A., \& Bernardi, M. 2003, MNRAS, 344, 45

[81] Cayatte, V., Kotanyi, C., Balkowski, C., \& van Gorkom, J. H. 1994, AJ, 107, 1003

[82] Cayatte, V., van Gorkom, J. H., Balkowski, C., \& Kotanyi, C. 1990, AJ, 100, 604

[83] Ceverino, D., Dekel, A., \& Bournaud, F. 2010, MNRAS, 404, 2151

[84] Chabrier, G. 2003, ApJL, 586, L133

[85] Charlot, S. 1996, The Universe at High-z, Large-Scale Structure and the Cosmic Microwave Background, 470, 53

[86] Charlot, S., Worthey, G., \& Bressan, A. 1996, ApJ, 457, 625

[87] Chary, R., \& Elbaz, D. 2001, ApJ, 556, 562

[88] Chiboucas, K., Tully, R. B., Marzke, R. O., et al. 2010, ApJ, 723, 251

[89] Chung, A., van Gorkom, J. H., Kenney, J. D. P., Crowl, H., \& Vollmer, B. 2009, AJ, 138,1741

[90] Chung, A., van Gorkom, J. H., Kenney, J. D. P., \& Vollmer, B. 2007, ApJL, 659, L115

[91] Cimatti, A., Cassata, P., Pozzetti, L., et al. 2008, A\&A, 482, 21

[92] Cole, S., Lacey, C. G., Baugh, C. M., \& Frenk, C. S. 2000, MNRAS, 319, 168 
[93] Colless, M., \& Dunn, A. M. 1996, ApJ, 458, 435

[94] Combes, F., Debbasch, F., Friedli, D., \& Pfenniger, D. 1990, A\&A, 233, 82

[95] Combes, F., \& Sanders, R. H. 1981, A\&A, 96, 164

[96] Conselice, C. J. 2009, MNRAS, 399, L16

[97] Conselice, C. J., Bershady, M. A., Dickinson, M., \& Papovich, C. 2003, AJ, 126, 1183

[98] Conselice, C. J., Bluck, A. F. L., Buitrago, F., et al. 2011, MNRAS, 413, 80

[99] Conselice, C. J., Bundy, K., Trujillo, I., et al. 2007, MNRAS, 381, 962

[100] Conselice, C. J., Mortlock, A., Bluck, A. F. L., Grützbauch, R., \& Duncan, K. 2013, MNRAS, 430, 1051

[101] Conselice, C. J., Rajgor, S., \& Myers, R. 2008, MNRAS, 386, 909

[102] Côté, P., Piatek, S., Ferrarese, L., et al. 2006, ApJS, 165, 57

[103] Cowie, L. L., Hu, E. M., \& Songaila, A. 1995, AJ, 110, 1576

[104] Cox, T. J., Jonsson, P., Somerville, R. S., Primack, J. R., \& Dekel, A. 2008, MNRAS, 384,386

[105] Daddi, E., Bournaud, F., Walter, F., et al. 2010, ApJ, 713, 686

[106] Daddi, E., Cimatti, A., Renzini, A., et al. 2004, ApJ, 617, 746

[107] Daddi, E., Dannerbauer, H., Elbaz, D., et al. 2008, ApJL, 673, L21

[108] Daddi, E., Dickinson, M., Morrison, G., et al. 2007, ApJ, 670, 156 
[109] Daddi, E., Elbaz, D., Walter, F., et al. 2010, ApJL, 714, L118

[110] Daddi, E., Renzini, A., Pirzkal, N., et al. 2005, ApJ, 626, 680

[111] Davis, M., Efstathiou, G., Frenk, C. S., \& White, S. D. M. 1985, ApJ, 292, 371

[112] Debattista, V. P., \& Sellwood, J. A. 1998, ApJL, 493, L5

[113] Debattista, V. P., \& Sellwood, J. A. 2000, ApJ, 543, 704

[114] de Grijs, R., Kregel, M., \& Wesson, K. H. 2001, MNRAS, 324, 1074

[115] de Jong, R. S. 1996, A\&A Suppl., 118, 557

[116] Dekel, A., \& Birnboim, Y. 2006, MNRAS, 368, 2

[117] Dekel, A., Birnboim, Y., Engel, G., et al. 2009a, Nature, 457, 451

[118] Dekel, A., Sari, R., \& Ceverino, D. 2009b, ApJ, 703, 785

[119] De Lucia, G., \& Blaizot, J. 2007, MNRAS, 375, 2

[120] den Brok, M., Peletier, R. F., Valentijn, E. A., et al. 2011, MNRAS, 414, 3052

[121] Depoy, D. L., Atwood, B., Byard, P. L., Frogel, J., \& O’Brien, T. P. 1993, Proc. SPIE, 1946,667

[122] D’Onghia, E., Burkert, A., Murante, G., \& Khochfar, S. 2006, MNRAS, 372, 1525

[123] de Souza, R. E., Gadotti, D. A., \& dos Anjos, S. 2004, ApJS, 153, 411

[124] de Vaucouleurs, G. 1948, Annales d'Astrophysique, 11, 247

[125] de Vaucouleurs, G. 1953, MNRAS, 113, 134 
[126] de Vaucouleurs, G., de Vaucouleurs, A., \& Corwin, H. G., Jr. 1976, Second reference catalogue of bright galaxies. Containing information on 4,364 galaxies with references to papers published between 1964 and 1975., by de Vaucouleurs, G.; de Vaucouleurs, A.; Corwin, H. G., Jr.. Austin, TX (USA): University of Texas Press, $6+396$ p.,

[127] de Vaucouleurs, G., de Vaucouleurs, A., Corwin, H. G., Jr., Buta, R. J., Paturel, G., \& Fouque, P. 1991, Third Reference Catalogue of Bright Galaxies, Volume 1-3, (New York: Springer-Verlag)

[128] de Zeeuw, P. T., Bureau, M., Emsellem, E., et al. 2002, MNRAS, 329, 513

[129] Dickinson, M., Giavalisco, M., \& GOODS Team 2003, The Mass of Galaxies at Low and High Redshift, 324

[130] Dickinson, M., Papovich, C., Ferguson, H. C., \& Budavári, T. 2003, ApJ, 587, 25

[131] Dickinson, M., Stern, D., Giavalisco, M., et al. 2004, ApJL, 600, L99

[132] Donley, J. L., Rieke, G. H., Pérez-González, P. G., \& Barro, G. 2008, ApJ, 687, 111

[133] Dressler, A. 1980, ApJS, 42, 565

[134] Dressler, A., Oemler, A., Jr., Butcher, H. R., \& Gunn, J. E. 1994, ApJ, 430, 107

[135] Driver, S. P., Allen, P. D., Graham, A. W., et al. 2006, MNRAS, 368, 414

[136] Driver, S. P., Liske, J., Cross, N. J. G., De Propris, R., \& Allen, P. D. 2005, MNRAS, 360,81

[137] Drory, N., \& Alvarez, M. 2008, ApJ, 680, 41

[138] Drory, N., Bender, R., \& Hopp, U. 2004, ApJL, 616, L103 
[139] Drory, N., Salvato, M., Gabasch, A., et al. 2005, ApJL, 619, L131

[140] Edwards, L. O. V., \& Fadda, D. 2011, AJ, 142, 148

[141] Efstathiou, G., Ellis, R. S., \& Peterson, B. A. 1988, MNRAS, 232, 431

[142] Elbaz, D., Hwang, H. S., Magnelli, B., et al. 2010, A\&A, 518, L29

[143] Eliche-Moral, M. C., Balcells, M., Aguerri, J. A. L., \& González-García, A. C. 2006, A\&A, 457, 91

[144] Eliche-Moral, M. C., Prieto, M., Gallego, J., et al. 2010, A\&A, 519, A55 1 G

[145] Elmegreen, B. G. 1994, ApJL, 425, L73

[146] Elmegreen, B. G., Bournaud, F., \& Elmegreen, D. M. 2008, ApJ, 688, 67

[147] Elmegreen, B. G., \& Elmegreen, D. M. 1985, ApJ, 288, 438

[148] Elmegreen, B. G., Elmegreen, D. M., Chromey, F. R., Hasselbacher, D. A., \& Bissell, B. A. 1996 , AJ, 111, 2233

[149] Elmegreen, B. G., Elmegreen, D. M., Fernandez, M. X., \& Lemonias, J. J. 2009, ApJ, 692, 12

[150] Elmegreen, D. M., Elmegreen, B. G., Marcus, M. T., et al. 2009, ApJ, 701, 306

[151] Elmegreen, D. M., Elmegreen, B. G., Rubin, D. S., \& Schaffer, M. A. 2005, ApJ, 631, 85

[152] Elsner, F., Feulner, G., \& Hopp, U. 2008, A\&A, 477, 503

[153] El-Zant, A. A., Kim, W.-T., \& Kamionkowski, M. 2004, MNRAS, 354, 169 
[154] Emsellem, E., Cappellari, M., Krajnović, D., et al. 2007, MNRAS, 379, 401

[155] Emsellem, E., Cappellari, M., Krajnović, D., et al. 2011, MNRAS, 414, 888

[156] Emsellem, E., Cappellari, M., Peletier, R. F., et al. 2004, MNRAS, 352, 721

[157] Erwin, P., Beckman, J. E., \& Pohlen, M. 2005, ApJL, 626, L81

[158] Erwin, P., Gutiérrez, L., \& Beckman, J. E. 2012, ApJL, 744, L11

[159] Eskridge, P. B., Frogel, J. A., Pogge, R. W., et al. 2000, AJ, 119, 536

[160] Eskridge, P. B., Frogel, J. A., Pogge, R. W., et al. 2002, ApJS, 143, 73

[161] Fabricius, M. H., Saglia, R. P., Fisher, D. B., et al. 2012, ApJ, 754, 67

[162] Feldmann, R., Carollo, C. M., Mayer, L., et al. 2010, ApJ, 709, 218

[163] Feldmeier, J. J., Ciardullo, R., Jacoby, G. H., \& Durrell, P. R. 2004, ApJ, 615, 196

[164] Ferrarese, L., \& Merritt, D. 2000, ApJL, 539, L9

[165] Fiore, F., Grazian, A., Santini, P., et al. 2008, ApJ, 672, 94

[166] Fisher, D. B., \& Drory, N. 2008, AJ, 136, 773

[167] Fisher, D. B., \& Drory, N. 2010, ApJ, 716, 942

[168] Fisher, D. B., \& Drory, N. 2011, ApJL, 733, L47

[169] Fontana, A., Salimbeni, S., Grazian, A., et al. 2006, A\&A, 459, 745

[170] Förster Schreiber, N. M., Genzel, R., Bouché, N., et al. 2009, ApJ, 706, 1364

[171] Förster Schreiber, N. M., Shapley, A. E., Erb, D. K., et al. 2011, ApJ, 731, 65 
[172] Fossati, M., Gavazzi, G., Boselli, A., \& Fumagalli, M. 2012, A\&A, 544, A128

[173] Franceschini, A., Manners, J., Polletta, M. d. C., et al. 2005, AJ, 129, 2074

[174] Freeman, K. C. 1970, ApJ, 160, 811

[175] Fujita, Y., \& Nagashima, M. 1999, ApJ, 516, 619

[176] Gadotti, D. A. 2009, MNRAS, 393, 1531

[177] Gadotti, D., \& Kauffmann, G. 2007, IAU Symposium, 241, 507

[178] Gebhardt, K., Bender, R., Bower, G., et al. 2000, ApJL, 539, L13

[179] Genzel, R., Burkert, A., Bouché, N., et al. 2008, ApJ, 687, 59

[180] Genzel, R., Tacconi, L. J., Gracia-Carpio, J., et al. 2010, MNRAS, 407, 2091

[181] Gnedin, O. Y. 2003, ApJ, 589, 752

[182] Giacconi, R., Zirm, A., Wang, J., et al. 2002, ApJS, 139, 369

[183] Giavalisco, M., Ferguson, H. C., Koekemoer, A. M., et al. 2004, ApJL, 600, L93

[184] Gnedin, N. Y., \& Kravtsov, A. V. 2010, ApJ, 714, 287

[185] Governato, F., Willman, B., Mayer, L., et al. 2007, MNRAS, 374, 1479

[186] Grützbauch, R., Chuter, R. W., Conselice, C. J., et al. 2011, MNRAS, 412, 2361

[187] Graham, A. W. 2001, AJ, 121, 820

[188] Graham, A. W. 2013, Planets, Stars and Stellar Systems. Volume 6: Extragalactic Astronomy and Cosmology, 91 (arXiv:1108.0997) 
[189] Graham, A. W., Erwin, P., Trujillo, I., \& Asensio Ramos, A. 2003, AJ, 125, 2951

[190] Graham, A. W., \& Guzmán, R. 2003, AJ, 125, 2936

[191] Graham, A. W., \& Worley, C. C. 2008, MNRAS, 388, 1708

[192] Greene, J. E., Ho, L. C., \& Barth, A. J. 2008, ApJ, 688, 159

[193] Greene, J. E., Peng, C. Y., Kim, M., et al. 2010, ApJ, 721, 26

[194] Gregg, M. D., \& West, M. J. 1998, Nature, 396, 549

[195] Gunn, J. E., \& Gott, J. R., III 1972, ApJ, 176, 1

[196] Gutiérrez, C. M., Trujillo, I., Aguerri, J. A. L., Graham, A. W., \& Caon, N. 2004, ApJ, 602, 664

[197] Guzmán,, R., Gallego, J., Koo, D. C., et al. 1997, ApJ, 489, 559

[198] Hammer, D. M., Hornschemeier, A. E., Salim, S., et al. 2012, ApJ, 745, 177

[199] Hammer, D., Verdoes Kleijn, G., Hoyos, C., et al. 2010, ApJS, 191, 143

[200] Hammer, F., Flores, H., Puech, M., et al. 2009, A\&A, 507, 1313

[201] Hashimoto, Y., Oemler, A., Jr., Lin, H., \& Tucker, D. L. 1998, ApJ, 499, 589

[202] Häussler, B., McIntosh, D. H., Barden, M., et al. 2007, ApJS, 172, 615

[203] Heiderman, A., Jogee, S., Marinova, I., et al. 2009, ApJ, 705, 1433

[204] Henriques, B. M., Bertone, S., \& Thomas, P. A. 2008, MNRAS, 383, 1649

[205] Henriques, B. M. B., \& Thomas, P. A. 2010, MNRAS, 403, 768 
[206] Hernquist, L., \& Mihos, J. C. 1995, ApJ, 448, 41

[207] Hilz, M., Naab, T., \& Ostriker, J. P. 2013, MNRAS, 429, 2924

[208] Ho, L. C., Filippenko, A. V., \& Sargent, W. L. W. 1997, ApJS, 112, 315

[209] Hogg, D. W. 1999, arXiv:astro-ph/9905116

[210] Hogg, D. W., Baldry, I. K., Blanton, M. R., \& Eisenstein, D. J. 2002, arXiv:astro$\mathrm{ph} / 0210394$

[211] Hopkins, P. F., Bundy, K., Croton, D., et al. 2010a, ApJ, 715, 202

[212] Hopkins, P. F., Bundy, K., Hernquist, L., Wuyts, S., \& Cox, T. J. 2010b, MNRAS, 401, 1099

[213] Hopkins, P. F., Cox, T. J., Dutta, S. N., et al. 2009a, ApJS, 181, 135

[214] Hopkins, P. F., Cox, T. J., Younger, J. D., \& Hernquist, L. 2009b, ApJ, 691, 1168

[215] Hopkins, P. F., Hernquist, L., Cox, T. J., Robertson, B., \& Springel, V. 2006a, ApJS, 163,50

[216] Hopkins, P. F., Somerville, R. S., Hernquist, L., et al. 2006b, ApJ, 652, 864

[217] Hopkins, P. F., Somerville, R. S., Cox, T. J., et al. 2009c, MNRAS, 397, 802

[218] Hopkins, P. F., Younger, J. D., Hayward, C. C., Narayanan, D., \& Hernquist, L. 2010c, MNRAS, 402, 1693

[219] Hoyos, C., den Brok, M., Verdoes Kleijn, G., et al. 2011, MNRAS, 411, 2439

[220] Hu, J. 2008, MNRAS, 386, 2242 
[221] Hubble, E. 1929, Proceedings of the National Academy of Science, 15, 168

[222] Hubble, E. P. 1926, ApJ, 64, 321

[223] Hunt, L. K., \& Malkan, M. A. 1999, ApJ, 516, 660

[224] Jerjen, H., Binggeli, B., \& Freeman, K. C. 2000, AJ, 119, 593

[225] Jogee, S. 1999, Ph.D. Thesis,

[226] Jogee, S. 2006, Physics of Active Galactic Nuclei at all Scales, 693, 143

[227] Jogee, S. 2009, IAU Symposium, 254, 67

[228] Jogee, S., Barazza, F. D., Rix, H.-W., et al. 2004, ApJL, 615, L105

[229] Jogee, S., Kenney, J. D. P., \& Smith, B. J. 1999, ApJ, 526, 665

[230] Jogee, S., Miller, S. H., Penner, K., et al. 2009, ApJ, 697, 1971

[231] Jogee, S., Scoville, N., \& Kenney, J. D. P. 2005, ApJ, 630, 837

[232] Jonsson, P. 2006, MNRAS, 372, 2

[233] Julian, W. H., \& Toomre, A. 1966, ApJ, 146, 810

[234] Kajisawa, M., Ichikawa, T., Tanaka, I., et al. 2009, ApJ, 702, 1393

[235] Kajisawa, M., Konishi, M., Suzuki, R., et al. 2006, PASJ, 58, 951

[236] Kauffmann, G., Colberg, J. M., Diaferio, A., \& White, S. D. M. 1999, MNRAS, 303, 188

[237] Kauffmann, G., Heckman, T. M., White, S. D. M., et al. 2003, MNRAS, 341, 33 
[238] Kautsch, S. J., Grebel, E. K., Barazza, F. D., \& Gallagher, J. S., III 2006, A\&A, 445, 765

[239] Kaviraj, S., Tan, K.-M., Ellis, R. S., \& Silk, J. 2011, MNRAS, 411, 2148

[240] Kenney, J. D. P., Carlstrom, J. E., \& Young, J. S. 1993, ApJ, 418, 687

[241] Kenney, J. D. P., Tal, T., Crowl, H. H., Feldmeier, J., \& Jacoby, G. H. 2008, ApJL, 687, L69

[242] Kenney, J. D. P., van Gorkom, J. H., \& Vollmer, B. 2004, AJ, 127, 3361

[243] Kennicutt, R. C., Jr. 1998, ApJ, 498, 541

[244] Kennicutt, R. C., Jr. 2008, Pathways Through an Eclectic Universe, 390, 149

[245] Kent, S. M. 1985, ApJS, 59, 115

[246] Kereš, D., Katz, N., Fardal, M., Davé, R., \& Weinberg, D. H. 2009, MNRAS, 395, 160

[247] Kereš, D., Katz, N., Weinberg, D. H., \& Davé, R. 2005, MNRAS, 363, 2

[248] Khochfar, S., \& Burkert, A. 2001, ApJ, 561, 517

[249] Khochfar, S., \& Burkert, A. 2005, MNRAS, 359, 1379

[250] Khochfar, S., \& Silk, J. 2006a, ApJL, 648, L21

[251] Khochfar, S., \& Silk, J. 2006b, MNRAS, 370, 902

[252] Khochfar, S., \& Silk, J. 2009a, MNRAS, 397, 506

[253] Khochfar, S., \& Silk, J. 2009b, ApJL, 700, L21 
[254] Khochfar, S., \& Silk, J. 2011, MNRAS, 410, L42

[255] Knapen, J. H., Beckman, J. E., Heller, C. H., Shlosman, I., \& de Jong, R. S. 1995, ApJ, 454, 623

[256] Knapen, J. H., Shlosman, I., \& Peletier, R. F. 2000, ApJ, 529, 93

[257] Kocevski, D. D., Faber, S. M., Mozena, M., et al. 2012, ApJ, 744, 148

[258] Koda, J., Milosavljević, M., \& Shapiro, P. R. 2009, ApJ, 696, 254

[259] Kormendy, J. 1977, ApJ, 217, 406

[260] Kormendy, J. 1985, ApJ, 295, 73

[261] Kormendy, J. 1993a, Galactic Bulges, 153, 209

[262] Kormendy, J. 1993b, The Nearest Active Galaxies, 197

[263] Kormendy, J. 2008, IAU Symposium, 245, 107

[264] Kormendy, J., \& Bahcall, J. N. 1974, AJ, 79, 671

[265] Kormendy, J., \& Barentine, J. C. 2010, ApJL, 715, L176

[266] Kormendy, J., Bender, R., \& Cornell, M. E. 2011, Nature, 469, 374

[267] Kormendy, J., \& Cornell, M. E. 2004, Penetrating Bars Through Masks of Cosmic Dust, 319, 261

[268] Kormendy, J., Drory, N., Bender, R., \& Cornell, M. E. 2010, ApJ, 723, 54

[269] Kormendy, J., \& Fisher, D. B. 2005, Rev. Mex. AA Ser. Conf., 23, 101 (arXiv:0507525) 
[270] Kormendy, J., \& Fisher, D. B. 2008, Formation and Evolution of Galaxy Disks, 396, 297

[271] Kormendy, J., Fisher, D. B., Cornell, M. E., \& Bender, R. 2009, ApJS, 182, 216

[272] Kormendy, J., \& Ho, L. C. 2013, arXiv:1304.7762

[273] Kormendy, J., \& Kennicutt, R. C., Jr. 2004, ARAA, 42, 603

[274] Krajnović, D., Bacon, R., Cappellari, M., et al. 2008, MNRAS, 390, 93

[275] Krajnović, D., Emsellem, E., Cappellari, M., et al. 2011, MNRAS, 414, 2923

[276] Kriek, M., van der Wel, A., van Dokkum, P. G., Franx, M., \& Illingworth, G. D. 2008, ApJ, 682, 896

[277] Krist, J. 1995, Astronomical Data Analysis Software and Systems IV, 77, 349

[278] Kroupa, P., Tout, C. A., \& Gilmore, G. 1993, MNRAS, 262, 545

[279] Labbé, I., Rudnick, G., Franx, M., et al. 2003, ApJL, 591, L95

[280] Lacy, M., Storrie-Lombardi, L. J., Sajina, A., et al. 2004, ApJS, 154, 166

[281] Lanzoni, B., Guiderdoni, B., Mamon, G. A., Devriendt, J., \& Hatton, S. 2005, MNRAS, 361, 369

[282] Larson, R. B., Tinsley, B. M., \& Caldwell, C. N. 1980, ApJ, 237, 692

[283] Laurikainen, E., Salo, H., \& Buta, R. 2004, ApJ, 607, 103

[284] Laurikainen, E., Salo, H., \& Buta, R. 2005, MNRAS, 362, 1319

[285] Laurikainen, E., Salo, H., Buta, R., \& Knapen, J. H. 2007, MNRAS, 381, 401 
[286] Laurikainen, E., Salo, H., Buta, R., \& Knapen, J. H. 2009, ApJL, 692, L34

[287] Laurikainen, E., Salo, H., Buta, R., Knapen, J. H., \& Comerón, S. 2010, MNRAS, 405,1089

[288] Lehmer, B. D., Brandt, W. N., Alexander, D. M., et al. 2005, ApJS, 161, 21

[289] Li, C., \& White, S. D. M. 2009, MNRAS, 398, 2177

[290] Lilly, S., Schade, D., Ellis, R., et al. 1998, ApJ, 500, 75

[291] Lin, L., Koo, D. C., Willmer, C. N. A., et al. 2004, ApJL, 617, L9

[292] Liske, J., Lemon, D. J., Driver, S. P., Cross, N. J. G., \& Couch, W. J. 2003, MNRAS, 344,307

[293] Loeb, A., \& Peebles, P. J. E. 2003, ApJ, 589, 29

[294] Łokas, E. L., \& Mamon, G. A. 2003, MNRAS, 343, 401

[295] Longhetti, M., Saracco, P., Severgnini, P., et al. 2007, MNRAS, 374, 614

[296] López-Sanjuan, C., Balcells, M., Pérez-González, P. G., et al. 2010, ApJ, 710, 1170

[297] Lotz, J. M., Davis, M., Faber, S. M., et al. 2008, ApJ, 672, 177

[298] Lotz, J. M., Jonsson, P., Cox, T. J., et al. 2011, ApJ, 742, 103

[299] Lucy, L. B. 1974, AJ, 79, 745

[300] Luo, B., Bauer, F. E., Brandt, W. N., et al. 2008, ApJS, 179, 19

[301] Magorrian, J., Tremaine, S., Richstone, D., et al. 1998, AJ, 115, 2285 
[302] Maltby, D. T., Gray, M. E., Aragón-Salamanca, A., et al. 2012, MNRAS, 419, 669

[303] Marchesini, D., van Dokkum, P. G., Förster Schreiber, N. M., et al. 2009, ApJ, 701, 1765

[304] Marconi, A., \& Hunt, L. K. 2003, ApJL, 589, L21

[305] Marinova, I., \& Jogee, S. 2007, ApJ, 659, 1176

[306] Marinova, I., Jogee, S., Weinzirl, T., et al. 2012, ApJ, 746, 136

[307] Martinez-Valpuesta, I., Shlosman, I., \& Heller, C. 2006, ApJ, 637, 214

[308] Matthews, L. D., \& Gallagher, J. S., III 1997, AJ, 114, 1899

[309] McDermid, R. M., Emsellem, E., Shapiro, K. L., et al. 2006, MNRAS, 373, 906

[310] McDonald, M., Courteau, S., \& Tully, R. B. 2009, MNRAS, 394, 2022

[311] Melnick, J., Hoessel, J., \& White, S. D. M. 1977, MNRAS, 180, 207

[312] Menéndez-Delmestre, K., Sheth, K., Schinnerer, E., Jarrett, T. H., \& Scoville, N. Z. 2007, ApJ, 657, 790

[313] Mihos, J. C., Harding, P., Feldmeier, J., \& Morrison, H. 2005, ApJL, 631, L41

[314] Mihos, J. C., \& Hernquist, L. 1994, ApJL, 425, L13

[315] Mihos, J. C., \& Hernquist, L. 1996, ApJ, 464, 641

[316] Mihos, J. C., Janowiecki, S., Feldmeier, J. J., Harding, P., \& Morrison, H. 2009, ApJ, 698,1879 
[317] Mihos, J. C., Walker, I. R., Hernquist, L., Mendes de Oliveira, C., \& Bolte, M. 1995, ApJL, 447, L87

[318] Mobasher, B., Bridges, T. J., Carter, D., et al. 2001, ApJS, 137, 279

[319] Moore, B., Katz, N., Lake, G., Dressler, A., \& Oemler, A. 1996, Nature, 379, 613

[320] Moore, B., Lake, G., \& Katz, N. 1998, ApJ, 495, 139

[321] Moore, B., Lake, G., Quinn, T., \& Stadel, J. 1999, MNRAS, 304, 465

[322] Moorthy, B. K., \& Holtzman, J. A. 2006, MNRAS, 371, 583

[323] Mortlock, A., Conselice, C. J., Bluck, A. F. L., et al. 2011, MNRAS, 413, 2845

[324] Moustakas, L. A., Casertano, S., Conselice, C. J., et al. 2004, ApJL, 600, L131

[325] Murphy, E. J., Chary, R.-R., Alexander, D. M., et al. 2009, ApJ, 698, 1380

[326] Naab, T., \& Burkert, A. 2003, ApJ, 597, 893

[327] Naab, T., Johansson, P. H., \& Ostriker, J. P. 2009, ApJL, 699, L178

[328] Naab, T., Johansson, P. H., Ostriker, J. P., \& Efstathiou, G. 2007, ApJ, 658, 710

[329] Naab, T., Khochfar, S., \& Burkert, A. 2006, ApJL, 636, L81

[330] Naab, T., \& Trujillo, I. 2006, MNRAS, 369, 625

[331] Navarro, J. F., \& Steinmetz, M. 2000, ApJ, 538, 477

[332] Neistein, E., \& Weinmann, S. M. 2010, MNRAS, 405, 2717

[333] Newman, A. B., Ellis, R. S., Bundy, K., \& Treu, T. 2012, ApJ, 746, 162 
[334] Nipoti, C., Treu, T., Leauthaud, A., et al. 2012, MNRAS, 422, 1714

[335] Nordon, R., Lutz, D., Shao, L., et al. 2010, A\&A, 518, L24

[336] Nowak, N., Thomas, J., Erwin, P., et al. 2010, MNRAS, 403, 646

[337] Odewahn, S. C. 1996, IAU Colloq. 157: Barred Galaxies, 91, 30

[338] Ocvirk, P., Pichon, C., \& Teyssier, R. 2008, MNRAS, 390, 1326

[339] Okamoto, T., \& Nagashima, M. 2003, ApJ, 587, 500

[340] Oser, L., Naab, T., Ostriker, J. P., \& Johansson, P. H. 2012, ApJ, 744, 63

[341] Oser, L., Ostriker, J. P., Naab, T., Johansson, P. H., \& Burkert, A. 2010, ApJ, 725, 2312

[342] Ostriker, J. P., \& Tremaine, S. D. 1975, ApJL, 202, L113

[343] Padmanabhan, N., Schlegel, D. J., Finkbeiner, D. P., et al. 2008, ApJ, 674, 1217

[344] Papovich, C., Moustakas, L. A., Dickinson, M., et al. 2006, ApJ, 640, 92

[345] Papovich, C., Rudnick, G., Le Floc'h, E., et al. 2007, ApJ, 668, 45

[346] Peebles, P. J. E., \& Nusser, A. 2010, Nature, 465, 565

[347] Peng, C. Y., Ho, L. C., Impey, C. D., \& Rix, H.-W. 2002, AJ, 124, 266

[348] Pérez-González, P. G., Rieke, G. H., Villar, V., et al. 2008, ApJ, 675, 234

[349] Pfenniger, D., \& Norman, C. 1990, ApJ, 363, 391

[350] Pierce, C. M., Lotz, J. M., Primack, J. R., et al. 2010, MNRAS, 405, 718 
[351] Pohlen, M., Dettmar, R.-J., Lütticke, R., \& Aronica, G. 2002, A\&A, 392, 807

[352] Pohlen, M., \& Trujillo, I. 2006, A\&A, 454, 759

[353] Press, W. H., \& Schechter, P. 1974, ApJ, 187, 425

[354] Quilis, V., Moore, B., \& Bower, R. 2000, Science, 288, 1617

[355] Quinn, P. J., Hernquist, L., \& Fullagar, D. P. 1993, ApJ, 403, 74

[356] Rauch, M., Becker, G. D., Haehnelt, M. G., et al. 2011, MNRAS, 418, 1115

[357] Ravindranath, S., Ferguson, H. C., Conselice, C., et al. 2004, ApJL, 604, L9

[358] Ravindranath, S., Ho, L. C., Peng, C. Y., Filippenko, A. V., \& Sargent, W. L. W. 2001, AJ, 122, 653

[359] Reddy, N. A., Steidel, C. C., Pettini, M., et al. 2008, ApJS, 175, 48

[360] Rees, M. J., \& Ostriker, J. P. 1977, MNRAS, 179, 541

[361] Reese, A. S., Williams, T. B., Sellwood, J. A., Barnes, E. I., \& Powell, B. A. 2007, AJ, 133, 2846

[362] Retzlaff, J., Rosati, P., Dickinson, M., et al. 2010, A\&A, 511, A50

[363] Richardson, W. H. 1972, Journal of the Optical Society of America (1917-1983), 62, 55

[364] Robaina, A. R., Bell, E. F., Skelton, R. E., et al. 2009, ApJ, 704, 324

[365] Robaina, A. R., Bell, E. F., van der Wel, A., et al. 2010, ApJ, 719, 844

[366] Robertson, B., Bullock, J. S., Cox, T. J., et al. 2006, ApJ, 645, 986 
[367] Robertson, B., Cox, T. J., Hernquist, L., et al. 2006, ApJ, 641, 21

[368] Rothberg, B., \& Joseph, R. D. 2004, AJ, 128, 2098

[369] Salucci, P., Yegorova, I. A., \& Drory, N. 2008, MNRAS, 388, 159

[370] Sandage, A., \& Binggeli, B. 1984, AJ, 89, 919

[371] Sandage, A., Binggeli, B., \& Tammann, G. A. 1985, AJ, 90, 1759

[372] Sérsic, J. L. 1968, Cordoba, Argentina: Observatorio Astronomico, 1968,

[373] Schawinski, K., Treister, E., Urry, C. M., et al. 2011, ApJL, 727, L31

[374] Schechter, P. 1976, ApJ, 203, 297

[375] Schlegel, D. J., Finkbeiner, D. P., \& Davis, M. 1998, ApJ, 500, 525

[376] Schneider, P. 2006, Extragalactic Astronomy and Cosmology, by Peter Schneider. Berlin: Springer, 2006.

[377] Shankar, F., Marulli, F., Bernardi, M., et al. 2010, MNRAS, 405, 948

[378] Shankar, F., Marulli, F., Bernardi, M., et al. 2013, MNRAS, 428, 109

[379] Shapiro, K. L., Genzel, R., Förster Schreiber, N. M., et al. 2008, ApJ, 682, 231

[Sersic(1968)] Sérsic, J. L. 1968, Cordoba, Argentina: Observatorio Astronomico, 1968

[380] Sheth, K., Elmegreen, D. M., Elmegreen, B. G., et al. 2008, ApJ, 675, 1141

[381] Silva, L., Maiolino, R., \& Granato, G. L. 2004, MNRAS, 355, 973

[382] Simard, L. 1998, Astronomical Data Analysis Software and Systems VII, 145, 108 
[383] Simard, L., Willmer, C. N. A., Vogt, N. P., et al. 2002, ApJS, 142, 1

[384] Simien, F., \& de Vaucouleurs, G. 1986, ApJ, 302, 564

[385] Simmons, B. D., \& Urry, C. M. 2008, ApJ, 683, 644

[386] Smith, R. J., Lucey, J. R., Hammer, D., et al. 2010, MNRAS, 408, 1417

[387] Somerville, R. S., \& Kolatt, T. S. 1999, MNRAS, 305, 1

[388] Somerville, R. S., \& Primack, J. R. 1999, MNRAS, 310, 1087

[389] Somerville, R. S., Primack, J. R., \& Faber, S. M. 2001, MNRAS, 320, 504

[390] Springel, V., Di Matteo, T., \& Hernquist, L. 2005, MNRAS, 361, 776

[391] Springel, V., \& Hernquist, L. 2005, ApJL, 622, L9

[392] Springel, V., White, S. D. M., Tormen, G., \& Kauffmann, G. 2001, MNRAS, 328, 726

[393] Steinmetz, M., \& Navarro, J. F. 2002, NewA, 7, 155

[394] Stern, D., Eisenhardt, P., Gorjian, V., et al. 2005, ApJ, 631, 163

[395] Szomoru, D., Franx, M., van Dokkum, P. G., et al. 2010, ApJL, 714, L244

[396] Tacconi, L. J., Genzel, R., Neri, R., et al. 2010, Nature, 463, 781

[397] The, L. S., \& White, S. D. M. 1986, AJ, 92, 1248

[398] Thompson, R. I., Illingworth, G., Bouwens, R., et al. 2005, AJ, 130, 1

[399] Thuan, T. X., \& Kormendy, J. 1977, PASP, 89, 466

[400] Toft, S., van Dokkum, P., Franx, M., et al. 2007, ApJ, 671, 285 
[401] Toomre, A. 1977, in Evolution of Galaxies and Stellar Populations, ed. B. M. Tinsley \& R. B. G. Larson, D. Campbell, 401

[402] Toomre, A. 1981, Structure and Evolution of Normal Galaxies, 111

[403] Tonnesen, S., \& Bryan, G. L. 2008, ApJL, 684, L9

[404] Tonnesen, S., \& Bryan, G. L. 2009, ApJ, 694, 789

[405] Tonnesen, S., \& Bryan, G. L. 2010, ApJ, 709, 1203

[406] Trager, S. C., Faber, S. M., \& Dressler, A. 2008, MNRAS, 386, 715

[407] Trentham, N., \& Mobasher, B. 1998, MNRAS, 293, 53

[408] Trentham, N., \& Tully, R. B. 2002, MNRAS, 335, 712

[409] Trujillo, I., Asensio Ramos, A., Rubiño-Martín, J. A., et al. 2002, MNRAS, 333, 510

[410] Trujillo, I., Conselice, C. J., Bundy, K., et al. 2007, MNRAS, 382, 109

[411] Trujillo, I., Erwin, P., Asensio Ramos, A., \& Graham, A. W. 2004, AJ, 127, 1917

[412] Trujillo, I., Förster Schreiber, N. M., Rudnick, G., et al. 2006, ApJ, 650, 18

[413] Tully, R. B. 1988, Cambridge and New York, Cambridge University Press, 1988, 221 p.,

[414] Véron-Cetty, M.-P., \& Véron, P. 2006, A\&A, 455, 773

[415] van den Bergh, S., Abraham, R. G., Ellis, R. S., et al. 1996, AJ, 112, 359

[416] van der Kruit, P. C. 1979, A\&A Suppl., 38, 15 
[417] van der Kruit, P. C., \& Searle, L. 1981a, A\&A, 95, 105

[418] van der Kruit, P. C., \& Searle, L. 1981b, A\&A, 95, 116

[419] van der Wel, A., Rix, H.-W., Wuyts, S., et al. 2011, ApJ, 730, 38

[420] van Dokkum, P. G. 2005, AJ, 130, 2647

[421] van Dokkum, P. G., Franx, M., Kriek, M., et al. 2008, ApJL, 677, L5

[422] van Dokkum, P. G., Quadri, R., Marchesini, D., et al. 2006, ApJL, 638, L59

[423] van Dokkum, P. G., Whitaker, K. E., Brammer, G., et al. 2010, ApJ, 709, 1018

[424] Wadadekar, Y., Robbason, B., \& Kembhavi, A. 1999, AJ, 117, 1219

[425] Walker, I. R., Mihos, J. C., \& Hernquist, L. 1996, ApJ, 460, 121

[426] Wang, L., Weinmann, S. M., \& Neistein, E. 2012, MNRAS, 421, 3450

[427] Weedman, D. W. 1986, Cambridge and New York, Cambridge University Press, 1986, 226 p.,

[428] Weinberg, M. D. 1985, MNRAS, 213, 451

[429] Weinmann, S. M., van den Bosch, F. C., Yang, X., et al. 2006, MNRAS, 372, 1161

[430] Weinzirl, T., Jogee, S., Conselice, C. J., et al. 2011, ApJ, 743, 87

[431] Weinzirl, T. et al. 2013, submitted

[432] Weinzirl, T., Jogee, S., Khochfar, S., Burkert, A., \& Kormendy, J. 2009, ApJ, 696, 411 
[433] White, S. D. M., \& Rees, M. J. 1978, MNRAS, 183, 341

[434] Williams, R. J., Quadri, R. F., \& Franx, M. 2011, ApJL, 738, L25

[435] Williams, R. J., Quadri, R. F., Franx, M., et al. 2010, ApJ, 713, 738

[436] Wirth, A., \& Gallagher, J. S., III 1984, ApJ, 282, 85

[437] Wolf, C., Bell, E. F., McIntosh, D. H., et al. 2005, ApJ, 630, 771

[438] Wuyts, S., Cox, T. J., Hayward, C. C., et al. 2010, ApJ, 722, 1666

[439] Wuyts, S., Franx, M., Cox, T. J., et al. 2009, ApJ, 700, 799

[440] Yagi, M., Komiyama, Y., Yoshida, M., et al. 2007, ApJ, 660, 1209

[441] Yagi, M., Yoshida, M., Komiyama, Y., et al. 2010, AJ, 140, 1814

[442] Yan, H., Dickinson, M., Eisenhardt, P. R. M., et al. 2004, ApJ, 616, 63

[443] Yan, L., \& Thompson, D. 2003, ApJ, 586, 765

[444] Yoshida, M., Yagi, M., Komiyama, Y., et al. 2008, ApJ, 688, 918

[445] Zibetti, S., White, S. D. M., Schneider, D. P., \& Brinkmann, J. 2005, MNRAS, 358, 949

[446] Zirm, A. W., van der Wel, A., Franx, M., et al. 2007, ApJ, 656, 66 


\section{Vita}

Timothy Michael Weinzirl graduated from Shakopee Senior High School in 2002. At

Drake University in Des Moines, IA received a B.S. in Physics and Astronomy along with a minor in Mathematics, graduating summa cum laude in 2006. After college, he came to the University of Texas at Austin to pursue graduate studies in Astronomy, starting Fall 2006. While in graduate school, his research earned awards including the Outstanding Master's Thesis award from the College of Natural Sciences in 2009, as well as the Rodger Doxsey Travel prize from the American Astronomical Society in 2012. Timothy is a lifer in the Iron Game.

Permanent address: 2515 Speedway, Stop C1400

Austin, Texas 78712-1205

This dissertation was typeset with $\mathrm{AT}_{\mathrm{E}} \mathrm{X}^{\dagger}$ by the author.

\footnotetext{
${ }^{\dagger} \mathrm{LAT}_{\mathrm{E}} \mathrm{X}$ is a document preparation system developed by Leslie Lamport as a special version of Donald Knuth's TEX Program.
} 